Dörner, Olaf [Hrsg.]; Iller, Carola [Hrsg.]; Schüßler, Ingeborg [Hrsg.]; Felden, Heide von [Hrsg.]; Lerch, Sebastian [Hrsg.]

\title{
Erwachsenenbildung und Lernen in Zeiten von Globalisierung, Transformation und Entgrenzung
}

\author{
Opladen; Berlin; Toronto : Verlag Barbara Budrich 2020, 370 S. - (Schriftenreihe der Sektion \\ Erwachsenenbildung in der Deutschen Gesellschaft für Erziehungswissenschaft (DGfE))
}

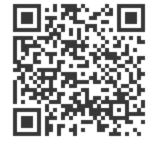

Quellenangabe/ Reference:

Dörner, Olaf [Hrsg.]; Iller, Carola [Hrsg.]; Schüßler, Ingeborg [Hrsg.]; Felden, Heide von [Hrsg.];

Lerch, Sebastian [Hrsg.]: Erwachsenenbildung und Lernen in Zeiten von Globalisierung, Transformation und Entgrenzung. Opladen; Berlin; Toronto : Verlag Barbara Budrich 2020, 370 S. - (Schriftenreihe der Sektion Erwachsenenbildung in der Deutschen Gesellschaft für Erziehungswissenschaft (DGfE)) URN: urn:nbn:de:0111-pedocs-205899 - DOI: 10.25656/01:20589

https://nbn-resolving.org/urn:nbn:de:0111-pedocs-205899

https://doi.org/10.25656/01:20589

in Kooperation mit / in cooperation with:

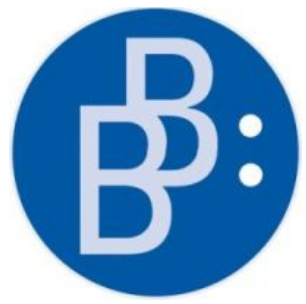

https://www.budrich.de

\section{Nutzungsbedingungen}

Dieses Dokument steht unter folgender Creative Commons-Lizenz: http://creativecommons.org/licenses/by-sa/4.0/deed.de - Sie dürfen das Werk bzw. den Inhalt vervielfältigen, verbreiten und öffentlich zugänglich machen sowie Abwandlungen und Bearbeitungen des Werkes bzw. Inhaltes anfertigen, solange sie den Namen des Autors/Rechteinhabers in der von inm festgelegten Weise nennen und die daraufhin neu entstandenen Werke bzw. Inhalte nur unter Verwendung von Lizenzbedingungen weitergeben, die mit denen dieses Lizenzvertrags identisch, vergleichbar oder kompatibel sind.

Mit der Verwendung dieses Dokuments erkennen Sie die Nutzungsbedingungen an.

\section{Terms of use}

This document is published under following Creative Commons-License: http://creativecommons.org/licenses/by-sa/4.0/deed.en - You may copy distribute and transmit, adapt or exhibit the work or its contents in public and alter, transform, or change this work as long as you attribute the work in the manner specified by the author or licensor. New resulting works or contents must be distributed pursuant to this license or an identical or comparable license.

By using this particular document, you accept the above-stated conditions of use.

\section{Kontakt / Contact:}

\section{peDOCS}

DIPF | Leibniz-Institut für Bildungsforschung und Bildungsinformation Informationszentrum (IZ) Bildung

E-Mail: pedocs@dipf.de

Internet: www.pedocs.de

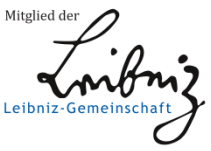



Erwachsenenbildung und Lernen in Zeiten von Globalisierung, Transformation und Entgrenzung 
Schriftenreihe der Sektion Erwachsenenbildung in der Deutschen Gesellschaft für

Erziehungswissenschaft (DGfE) 


\section{Olaf Dörner \\ Carola Iller}

Ingeborg Schüßler

Heide von Felden

Sebastian Lerch (Hrsg.)

Erwachsenenbildung und

Lernen in Zeiten von

Globalisierung, Transformation

und Entgrenzung

Verlag Barbara Budrich

Opladen • Berlin • Toronto 2020 
Bibliografische Information der Deutschen Nationalbibliothek

Die Deutsche Nationalbibliothek verzeichnet diese Publikation in der Deutschen

Nationalbibliografie; detaillierte bibliografische Daten sind im Internet über

https://portal.dnb.de abrufbar.

(C) 2020 Dieses Werk ist bei der Verlag Barbara Budrich GmbH erschienen und steht unter der Creative Commons Lizenz Attribution-ShareAlike 4.0 International (CC BY-SA 4.0): https://creativecommons.org/licenses/by-sa/4.0/.

Diese Lizenz erlaubt die Verbreitung, Speicherung, Vervielfältigung und Bearbeitung bei Verwendung der gleichen CC-BY-SA 4.0-Lizenz und unter Angabe der UrheberInnen, Rechte, Änderungen und verwendeten Lizenz. www.budrich.de

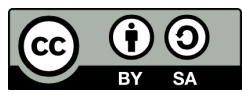

Dieses Buch steht im Open-Access-Bereich der Verlagsseite zum kostenlosen Download bereit (https://doi.org/10.3224/84742345).

Eine kostenpflichtige Druckversion kann über den Verlag bezogen werden. Die Seitenzahlen in der Druck- und Onlineversion sind identisch.

$\begin{array}{ll}\text { ISBN } & 978-3-8474-2345-4 \text { (Paperback) } \\ \text { eISBN } & 978-3-8474-1518-3 \text { (PDF) } \\ \text { DOI } & 10.3224 / 84742345\end{array}$

Lektorat: Dr. Andrea Lassalle, Berlin

Satz: Anja Borkam, Jena

Umschlaggestaltung: Bettina Lehfeldt, Kleinmachnow - www.lehfeldtgraphic.de

Druck: Paper \& Tinta, Warschau

Printed in Europe 


\section{Inhalt}

Einleitung

Jürgen Wittpoth

Gesellschaftlicher Wandel als „Herausforderung“"? Über einige

Tücken zeitdiagnostischer Orientierung von Erwachsenenbildung

Heide von Felden

Zur Rezeption von Appellen des Lebenslangen Lernens

Jörg Dinkelaker

Unterschiede im Entgrenzten. Zur Frage nach den Konturen von

Erwachsenenbildung/Weiterbildung

Jörg Schwarz, Sabine Schmidt-Lauff

(Spät-)Moderne Zeitregime, kollektive Zeitpraktiken und subjektives

Lernzeiterleben

Anita Pachner, Christina Baust

Transformation ermöglichen: Potentiale von Reflexion und

Perspektivverschränkung für die Professionalisierung von Lehrenden.

Evaluationsstudie zu einem reflexionszentrierten Fortbildungsangebot der Erwachsenenbildung für Lehrkräfte

Matthias Rohs, Ricarda Bolten

Einstellungen von Erwachsenenbildner*innen zur digitalen

Transformation der Weiterbildung

Denise Klinge

Selbstexpertisierung mittels Software? - Zum Umgang mit Sonderwissen in der Entwicklungs- und Repräsentationspraxis von ,Lernund Bildungsapps“

Jana Wienberg

Erlebte Resonanz im Weiterbildungsgeschehen - eine empirische

Annäherung 
Beatrix Niemeyer

Weggehen, um dazuzugehören? Kritische Überlegungen zum Konzept von Lernmobilität

Sebastian Zick

\#FreeInterrail, Mobilität und Bildung - Narrativanalytische

Perspektiven auf EUropäische Mobilitätsförderung

Eva Heinrich

Veränderung von Praktiken beim Wiedereinstieg in eine entgrenzte

Arbeitswelt

\section{Christian Müller}

Die Rekonstruktion betrieblicher Weiterbildungsentscheidungen in

Klein- und Kleinstunternehmen

Martin Reuter, Stefan Koscheck, Andreas Martin

Qualitätsmanagement und organisationale Felder in der

Weiterbildung

Lisa Lorenz, Eike Asche

Zwischen Fluidität und Kontinuität - Planung von Bildungsangeboten für die Digitalisierung

Gregor Dutz, Lisanne Heilmann

Lesekompetenz und politische Partizipation: Empirische Befunde aus PIAAC-L

\section{Alexandra Maßmann}

Weiterbildungsteilnahme und subjektives Alter - Kann das subjektive Alter helfen, die Funktion und Motivation einer Teilnahme zu erklären?

Frank Behr, Christiane Hof

Habituelle Grundlagen des Studierverhaltens. Annäherungen an die Bedingungen universitären Lernens 
Franziska Schulze-Stocker, Christian Schäfer-Hock

Transformation von Hochschulen - Transformation von

Bildungswegen junger Erwachsener: Frühwarnsysteme an

Hochschulen in Deutschland

Susanne Maria Weber, Marc-André Heidelmann

Lernziele bestimmen? Kompetenzen erfassen? Prozesse imaginieren?

Zur „Messung“ individueller und kollektiver Transformation im

Kontext Organisationspädagogischer Professionalisierung

Nikolaus Meyer, Dieter Nittel

Transformation von Erziehung und Bildung: Begleitung als Merkmal des pädagogisch-organisierten Systems des lebenslangen Lernens ......

Steffi Robak, Christian Kühn, Jessica Preuß, Marion Fleige,

Stephanie Freide

Kulturelle Erwachsenenbildung im Zeichen digitaler Transformation:

Theoretische Perspektiven und forschungsleitende Zugänge

Bernd Käpplinger, Anika Denninger, Torsten Denker

Heimat, Globalisierung und Erwachsenenbildung: Befunde einer

Regionalstudie

Stefanie Bauer

Transformations- und Lernprozesse alleinerziehender Mütter.

Eine qualitative Studie

Johannes Wahl

Transformation des Subjekts im Kontext des Übergangs in die

Hochschule

Joachim Ludwig

Geht das unter die Haut? Zur Rolle der Gefühle in der

Erwachsenenbildung

Tim Stanik, Lisa Marie Fritsch

Theoretische Begründungen und empirische Erfassbarkeit von

Teilnehmerorientierung in der institutionalisierten

Erwachsenenbildung 
Klaus Buddeberg, Christopher Stammer

Schließt der digitale Wandel ältere Erwachsene und gering

literalisierte Erwachsene aus?

Angaben zu den AutorInnen 
Olaf Dörner, Heide von Felden, Carola Iller, Sebastian Lerch, Ingeborg Schüßler

\section{Einleitung}

Die Jahrestagung der Sektion Erwachsenenbildung der Deutschen Gesellschaft für Erziehungswissenschaft (DGfE) fand 2018 an der Johannes GutenbergUniversität Mainz zum Thema ,Erwachsenenbildung und Lernen in Zeiten von Globalisierung, Transformation und Entgrenzung" statt. Damit wurde die Perspektive der Erwachsenenbildung auf gesellschaftliche Veränderungen gerichtet, wie sie in Debatten über Globalisierung, Transformation und Entgrenzung thematisiert werden. Im Zuge der Globalisierung und Transnationalisierung werden auf gesellschaftlicher Ebene einerseits deren Phänomene direkter auf die Nationalstaaten bezogen, andererseits verlieren, verbunden mit Entgrenzungen der Arbeits- und Wissensgesellschaft, nationalstaatliche Grenzen an Bedeutung. Dies wirkt sich auch auf die Bildung und das Lernen von Individuen sowie auf das Handeln in Organisationen aus, und zwar in Bezug auf Leben und Arbeit. Es entstehen insbesondere durch die gestiegene zeitlichen und örtlichen Mobilität in Zeiten von Digitalisierung und Globalisierung neue Arbeitsmarktstrukturen sowie neue Möglichkeiten und Notwendigkeiten einer Umgestaltung der Lebensführung.

Auch die erziehungswissenschaftlichen und soziologischen Diskurse verändern sich. Für die Erwachsenen- und Weiterbildung ist zu beobachten, dass einerseits gesellschaftliche Transformationen positiv aufgenommen werden. Andererseits werden neoliberale und ökonomische Tendenzen und Diskurse, z. B. zum Lebenslangen Lernen, die Steuerungsprozesse in kulturellen und sozialen Bereichen beeinflussen oder gar dominieren, kritisch betrachtet. Gleichzeitig gilt der Ressource, Wissen “ eine besondere Aufmerksamkeit angesichts gesellschaftlicher Veränderungs- und Wandlungsprozesse, insbesondere des Wandels von der Produktions- zur Dienstleistungs- und schließlich zur Wissensgesellschaft. Nicht selten werden in diesem Zusammenhang zusätzliche Belastungen thematisiert, die dadurch entstünden, dass persönliche Lernanstrengungen bei gleichzeitig kürzerer Halbwertzeit des Wissens einen zusätzlichen Druck auf die Individuen ausüben (können). Dabei wirken vor allem auch institutionalisierte temporale Ordnungen und kollektive Zeitpraktiken auf das subjektive Lernzeiterleben, wie empirische Untersuchungen zeigen.

Auf institutioneller Ebene sind also Phänomene der Transformation im Sinne einer weitgehenden Neuordnung des gesamten Bildungs- und Beschäftigungssystems festzustellen. Flexibilisierungsprozesse in der Erwerbsarbeit, die Diffusion von Arbeit und Freizeit sowie die zunehmende Notwendigkeit lebenslangen Lernens führen zu neuen Zeitregimen, zur Restrukturierung von 
Öffentlichem und Privatem oder zur Destandardisierung von (Bildungs-)Biografien. Übergänge und Transitionen zwischen Lebensphasen sowie zwischen Bildungsabschnitten im Lebenslauf der Individuen unterliegen Wandlungsprozessen, die sich auch in organisationaler und institutioneller Hinsicht niederschlagen. Normalitätsannahmen bezüglich des Lebenslaufs mit altersphasenspezifischen Anforderungen werden durch flexible Lebensentwürfe in multilokalen und multikulturellen Familien-, Beziehungs-, Ausbildungs- und Berufsstrukturen in Frage gestellt.

Auf individueller Ebene geht dieser Wandel einher mit Verharrungen, Verweigerungen, aber auch mit Neuverortungen und Reflexionen, die je persönlich als Chance oder Zumutung erlebt werden. Gesellschaftlicher Wandel ist also mit Unbestimmtheiten und Kontingenzen verbunden, die sowohl Unsicherheit und neue Anforderungen an Orientierungsleistungen mit sich bringen können als auch Widersprüche, Ambivalenzen und nicht kalkulierbare Folgen von Entscheidungen. Auf individueller Ebene gilt es, diese Unwägbarkeiten zu bewältigen bzw. auch subjektive und kollektive Lern- und Lebensbedingungen (mit) zu gestalten.

Die Erwachsenenbildung ist gefordert, sich diesen vielfältigen Herausforderungen mit ihren Auswirkungen auf Lehren und Lernen, Handlungsfelder, Disziplin und System zu stellen. Mit der Etablierung des bildungspolitischen Konzeptes des Lebenslangen Lernens sind ein weiter Lernbegriff und eine erste Antwort auf diese Umwälzungen gegeben, die Lernen nicht nur im institutionellen Rahmen platzieren (formales Lernen), sondern Lernen auch in der Lebenswelt und an allen gesellschaftlichen Orten sowie über die gesamte Lebenszeit als beiläufiges und inzidentelles Lernen stattfinden lassen (informelles Lernen). Damit werden auch neue Formate für Erwachsenenbildung geschaffen. Die Diskussionen der Tagung legen zudem nahe, die Typisierung von formalem, non-formalem und informellem Lernen dahingehend zu befragen, ob sie die Vielfalt und Breite des Lernens Erwachsener erfasst oder alternative Differenzierungen z. B. im Hinblick auf Vermittlungs- und Aneignungsaktivitäten und -kontexte erforderlich sind.

Sowohl in institutionellen Lernarrangements als auch beim selbstgesteuerten Lernen kommt dem Umgang mit neuen Medien eine bedeutende Rolle zu. Neuere Lerntheorien im Sinne von konstruktivistischen oder phänomenologischen Ansätzen betonen darüber hinaus die subjektive Aneignungsleistung durch die Lernenden und stellen die Steuerung der Lernprozesse durch Lehrende in den Hintergrund. Angesichts der gegenwärtigen gesellschaftlichen Herausforderungen von Komplexität und Ambivalenz, von Individualisierung und Globalisierung, von Lebensbewältigung und Lebenskunst geht es beim Lernen nicht nur um die Aneignung neuen Wissens, sondern auch darum, sich die eigene Perspektive im Vergleich zu anderen zu vergegenwärtigen, sich vermehrt in fremde Positionen hineinzuversetzen und damit mehrere Perspektiven 
gleichzeitig zu denken. Diese Offenheit erfordert von Seiten der Erwachsenenbildung auch die Beratung und Begleitung der Individuen. Um Reflexions- und Transformationsprozesse zu ermöglichen sowie angemessen zu fördern und zu begleiten, ist daher zugleich eine entsprechende Professionalisierung des Weiterbildungspersonals erforderlich, wozu auf der Tagung konkrete Vorschläge vorgestellt und diskutiert wurden.

Während die Orientierung am Kompetenzbegriff bei Formulierungen wie Ambiguitätstoleranz, Perspektivwechsel oder Kommunikationsfähigkeit stehen bleibt, werden etwa mit dem transformativen Lernen bzw. dem Bildungsbegriff grundlegende Veränderungen des Selbst- und Weltverhältnisses fokussiert: Bildung beinhaltet die Idee, dass Menschen durch die Aneignung von Wissen und Kulturleistungen und in Auseinandersetzung mit anderen ihre Persönlichkeit und Weltsicht (weiter-)entwickeln und somit zur Selbstbestimmung und Selbstverantwortung sowie Mitbestimmung und Solidarität befähigt werden.

Dies ist ohne die kritische Reflexion normativer Werte und die Ermutigung sowie Befähigung zum sozial verantwortlichen Gestaltungshandeln nicht denkbar. In diesem Sinn überschreitet Bildung jeweils gesellschaftliche Funktionalisierung und hat einen genuin gesellschaftskritischen Impetus gegenüber einer Vereinnahmung durch Funktionalität und ökonomische Verwertbarkeit. Dies ist unter den oben beschriebenen gesellschaftlichen Voraussetzungen von stetigem Wandel und Kontingenz eine besondere Herausforderung.

Auch die verschiedenen Übergänge im Lebensverlauf zwischen unterschiedlichen Institutionen (u. a. Schule, Ausbildung, Studium, Beruf, nachberufliche Phase) oder Lebenszusammenhängen (u. a. Familiengründung, Krankheit/Gesundheit, Verlust von nahestehenden Menschen) bringen eine Reihe von Lernanforderungen mit sich. Allgemein gesprochen muss man mit Neuem umgehen, es in bestehende Deutungs-, Emotions- und Handlungsmuster integrieren bzw. diese entsprechend transformieren, um mit den nacheinander oder nebeneinander auftretenden Herausforderungen adäquat umgehen zu können. Gerade Übergänge zu bewältigen bedeutet, dass der Zusammenhang zwischen formalen und informellen Lernerfahrungen prägend ist, da in der eigenen Lebenswelt Veränderungen vorgenommen werden. Dafür ist ein ,Denken in Übergängen' zugrunde zu legen, welches in der Forschung und der professionellen Begleitung sowohl gesellschaftliche Transformationen und institutionelle Strukturen als auch subjektive Konstruktionen miteinander verbindet.

Die Jahrestagung der Sektion Erwachsenenbildung 2018 in Mainz stellte einen Raum für Analysen und Untersuchungen zu Phänomenen von Globalisierung, Transformation und Entgrenzung und deren Auswirkungen auf die Erwachsenenbildung und insbesondere auf das Lernen Erwachsener bereit. Dabei zeigte sich, dass das Thema von der Erwachsenenbildung in unterschiedlichen Perspektiven und Dimensionen bearbeitet wird. Auf der Tagung wurden 
Beiträge zu den übergeordneten Themen ,Transformation', ,Professionalisierung', ,Arbeit und Betrieb', ,digitale Medien', ,politische Bildung', ,Orte', „Hochschule‘, ,Teilnahme‘ und ,Übergänge‘ vorgestellt. Nicht alle Beiträge wurden für die vorliegende Buchveröffentlichung eingereicht. Dennoch zeigt diese Veröffentlichung die Bandbreite und Multiperspektivität von theoretischen und empirischen Arbeiten, die sich mit den aktuellen gesellschaftlichen Einflüssen auf die Erwachsenenbildung beschäftigen.

Zur Gewährleistung der wissenschaftlichen Qualität der Beiträge wurde ein Review-Verfahren eingesetzt. Wir möchten an dieser Stelle allen Gutachterinnen und Gutachtern herzlich für ihre Arbeit danken.

Wir wünschen allen Leserinnen und Lesern interessante neue Anregungen und viel Freude bei der Lektüre. 
Jürgen Wittpoth

\section{Gesellschaftlicher Wandel als „Herausforderung“? Über einige Tücken zeitdiagnostischer Orientierung von Erwachsenenbildung}

Selbstverständlich steht es der Wissenschaft von der Erwachsenenbildung ebenso gut an wie der Erwachsenenbildung selbst, sich immer wieder der Umstände, unter denen sie stattfinden und auf die sie Einfluss nehmen wollen, zu vergewissern, um ihre Positionen und Praktiken gegebenenfalls neu justieren zu können. Die Frage ist, wie diese Vergewisserung sinnvollerweise vonstatten geht.

Zeitdiagnostisch orientiert ist sie immer dann, wenn sie sich auf Darstellungen, manchmal auch bloß Topoi bezieht,

- die einen Epochenbruch unterstellen, also mit einer Einst-und-jetzt-Figur arbeiten (explizit kommt das im Präfix ,post` zum Ausdruck: etwa postindustriell, postkapitalistisch, postmodern) und

- die je gegenwärtige Gesellschaften oder Zustände vor allem durch ein Merkmal kennzeichnen (also Arbeits-, Risiko-, Erlebnis-, Wissensgesellschaft, aber auch Globalisierung oder ganz aktuell Digitalisierung).

Der erste wesentliche Effekt dieser Art, sich auf Gesellschaft zu beziehen, ist, dass das Bisherige als Veraltetes, Überwundenes ,abgehakt" wird, obwohl es doch in der Regel allenfalls einem partiellen Gestaltwandel unterliegt, also in relevanten Hinsichten gegenwärtig bleibt. Der zweite Effekt ist, dass Aufnahmen in der Totale, die alles über einen Leisten schlagen, kaum Differenzierungen zulassen und für Ambivalenzen oder Gegenläufigkeiten unempfänglich sind. So bleibt für die meines Erachtens entscheidende Frage, für wen mit welchen Folgen das je unterstellte ,Neue' relevant wird und für wen nicht, gar kein Raum.

Wichtig für uns und andere gesellschaftspolitisch sensible Diskurse ist dabei, dass Betrachtungen, die an Diagnosen oder Topoi solcher Art umstandslos anschließen, in aller Regel von deren Mängeln infiziert sind. Insbesondere befreit der Anschluss sie von den Mühen, ihr eigenes Tun im Detail auf Schwächen (und Stärken), auf Veränderungsbedarf (und Bewahrenswertes) hin zu untersuchen und erst dann den Befunden gemäß Änderungen vorzunehmen. Wenn die Welt, in der wir leben, plötzlich ,ganz anders' ist, dann muss - so die überstürzte, der Nachfrage kaum standhaltende Annahme - die Art, in der wir uns auf sie beziehen, das selbstverständlich auch sein. 
Bevor Sie nun aber meinen, wir selbst täten so etwas nicht, sei darauf hingewiesen, dass der skizzierte Reflexionstypus in der Erwachsenenbildung eine anhaltend große Rolle spielt, da er ihr in die Wiege gelegt ist. Bereits der Aufruf zur Gründung der Gesellschaft für Verbreitung von Volksbildung 1871 beginnt mit: „Ein neues Zeitalter hat [...] begonnen“ (Aufforderung 1871: 1), um dann auszuführen, dass aus diesem ohne Volksbildung nichts Gutes werden könne. Und in den etwa vierzig Jahren, die ich in den Erwachsenenbildungsdiskurs involviert bin, habe ich viele Diagnosen dieser Art kommen und gehen sehen: Ich bin gerade noch ein Kind der Industriegesellschaft, die alsbald von der Dienstleistungsgesellschaft, zum Teil gepaart mit der Informationsgesellschaft abgelöst wurde; seit Anfang der 1970er Jahre gab es dann eine Fülle ,postindustrieller' Gesellschaften, in denen das ,Post' immer wieder anders akzentuiert wird, und viele andere mehr. An der jüngsten, Reckwitz' Gesellschaft der Singularitäten, in der er versucht, das Lebensgefühl im Prenzlauer Berg auf die gesamte Gesellschaft hochzurechnen (vgl. Reckwitz 2017), ließen sich vorzüglich die angesprochenen Schwächen zeigen (gleichwohl darf angenommen werden, dass Anschlüsse im erwachsenenpädagogischen Diskurs bald auftauchen werden). ${ }^{1}$

Allein darüber (in vergleichender Perspektive) nachzudenken, lohnte sich, ist hier aber nicht möglich. Ein Hinweis nur: Haben Sie schon mal etwas von „Zahlheilkunde in der Erlebnis-“ oder „Steuerrecht in der Wissensgesellschaft“ gehört? Die Neigung, an Zeitdiagnosen anzuschließen, versteht sich nicht von selbst. Meist erfolgt der Anschluss in legitimierender, begründender Manier, und je dramatischer der angenommene Wandel, desto unverzichtbarer die Weiterbildung. Wahrscheinlich sind wir noch nicht so weit, dass sich das, was wir tun, ,von selbst versteht".

Gegen diese verbreitete Praxis möchte ich in zwei Weisen Einwände formulieren:

1. in einer systematischen, die ich hier nur kurz ansprechen kann, und

2. in einer kaleidoskopartigen, der ich etwas mehr Raum geben werde - zumal es vom Charakter her wohl auch besser zu dieser Veranstaltung passt.

Dabei muss alles viel schneller gehen, als es mir lieb ist.

1 Zur Diskussion einiger in der Erwachsenenpädagogik und -bildung stark rezipierter Beispiele vgl. Wittpoth 2001. 
Mit Blick auf systematische Zugänge ist für mich Adornos Eröffnungsvortrag zum 16. Soziologentag 1968 (vgl. Adorno 1969) immer noch unübertroffen (zudem leicht verfügbar und nur 10 Seiten lang). Er diskutiert die Frage: ,Spätkapitalismus oder Industriegesellschaft?` (die seinerzeit gängige Zeitdiagnose) und löst sie nicht einfach zu einer Seite hin auf, sondern beantwortet sie im Sinne des, sowohl als auch'. Demnach lässt sich von einer Industriegesellschaft sprechen, wenn man die Produktivkraftentwicklung vor Augen hat. Schaut man auf die Produktionsverhältnisse (für die, die sich nicht mehr erinnern: Es geht darum, wer über die Produktionsmittel verfügt und wer zur Sicherung seines Lebensunterhalts auf den Verkauf der Ware Arbeitskraft angewiesen ist), haben wir es mit einem lupenreinen Kapitalismus zu tun: „Produziert wird heute wie ehedem um des Profits willen. Über alles zur Zeit von Marx Absehbare hinaus sind die Bedürfnisse [...] vollends zur Funktion des Produktionsapparates geworden“ (ebd.: 18). Dabei sind „die Produktivkräfte durch die Produktionsverhältnisse vermittelt; so vollständig vielleicht, dass diese darum als das Wesen [der Gesellschaft im zeitdiagnostischen Blick; JW] erscheinen. [...] Sie sind dafür verantwortlich, dass in irrem Widerspruch zum Möglichen die Menschen in großen Teilen der Erde darben müssen." (ebd.)

Mit Hilfe dieser Unterscheidung - Produktivkräfte/Produktionsverhältnisse - kann man nun das ganze Arsenal an Zeitdiagnosen durchkämmen, die seitdem vorgelegt worden sind (etwa die verschiedenen Varianten der Wissenschafts- und der Wissensgesellschaft, die für die Erwachsenenbildungsdiskussion ab Mitte der 1990er Jahre eine entscheidende Rolle spielen). Zwischenzeitlich tritt in einzelnen Fällen an die Stelle der Produktivkräfte Kultur (Gesellschaft ist dann wesentlich durch Veränderungen und Kämpfe im Bereich der Kultur gekennzeichnet); das gilt z.B. für die eine Hälfte der Risikogesellschaft (das Individualisierungstheorem) (vgl. Beck 1986), die Erlebnisgesellschaft (vgl. Schulze 1992) und schließlich für die bereits erwähnte Gesellschaft der Singularitäten.

Was im Kern, substantiell - es lohnt sich, an der Unterscheidung von Wesen und Erscheinung festzuhalten - überdauert, ist die kapitalistische Produktionsweise mit ihren besonderen Folgen für die Entwicklung der Gesellschaft und das Leben der Menschen. Das gilt uneingeschränkt - durch die Entgrenzung und das erweiterte Casino noch zugespitzt - bis heute; wer Adorno nicht mag oder Empiriker ist, möge sich bei Piketty (2014) in Das Kapital im 21. Jahrhundert vergewissern (ein Buch, um das es nach kurzem weltweitem Hype eigentümlich still geworden ist; über die obszöne Verteilung des Reichtums spricht man wohl nicht so gern).

Daraus ergeben sich auch für die Erwachsenenbildung einige sehr grundlegende Konsequenzen (d.h. es ist kein bloßer Streit um Worte): 
1. In einem profit- (heute würde man wohl von Rendite sprechen) und tauschwertorientierten Konkurrenzkapitalismus kann man zunächst davon ausgehen, dass bestimmte Aktivitäten der Weiterbildung - nämlich alles, was individuell oder institutionell als ,verwertbar' erscheint - , laufen' (ob wir das wollen, für kurios halten oder was auch immer: es geschieht, und da geschieht ja mittlerweile eine ganze Menge; aktuell unter den Schlagworten Digitalisierung, Industrie bzw. Arbeit 4.0 - wenn Sie meinen, sagen zu können, was damit konkret gemeint ist: in Japan hat man schon einen draufgesetzt, dort denkt man über die ,Society 5.0“ nach). Man weiß nicht genau, was sein wird, aber dramatisch wird es sicher, also kümmert man sich vorsichtshalber um Weiterbildung - darüber werde ich nicht sprechen.

2. Man kann - in Klassengesellschaften - weiterhin davon ausgehen (und wir wissen es, seitdem gezählt und gemessen wird), dass nicht alle (genauer: stets nur bestimmte Gruppen) von dem, was im genannten Sinne ,läuft', profitieren.

Schließlich gibt es etwas, das nicht, von selbst' läuft, und zwar all das, was sich der dominanten Verwertungslogik - zumindest auf den ersten, kurzfristig ausgerichteten Blick - entzieht: das allen Gemeine, dessen Wahrung der öffentlichen Verantwortung übertragen wird. Um dieses Allgemeine ist es in vielerlei Hinsicht nicht gut bestellt, und die Probleme, die sich hier - auch für die Erwachsenenbildung - auftun, sind nicht unabhängig von der besonderen Verfasstheit der Gesellschaft zu verstehen (incl. der fortwährend prekären Beschäftigung vieler Akteure in diesem Bereich) und - darum geht es im Folgenden - diese Probleme sind keineswegs neu.

\section{2}

Hier setzt mein - notwendig selektives - Kaleidoskop ein. Es geht um einige Stationen, um Beispiele für anhaltend Wesentliches, Überdauerndes, das in wirksamen Texten zum Ausdruck kommt, zu dem Manches getan wurde, aber zweifellos noch viel zu tun ist, und es geht um die Bedeutung der öffentlichen Sphäre.

1960, also vor beinahe sechzig Jahren, legt der Deutsche Ausschuss für das Erziehungs- und Bildungswesen sein wegweisendes Gutachten Zur Situation und Aufgabe der deutschen Erwachsenenbildung vor (vgl. Deutscher Ausschuss 1966). Dieser Text ist in allen seinen Bestandteilen von der Erfahrung des Nationalsozialismus durchdrungen und von - mehr als nur - dem Wunsch beseelt, es möge ,nie wieder Faschismus und nie wieder Krieg' geben. Das 
bedeutet auch: nie wieder Rassismus, Nationalismus, Chauvinismus und Populismus. Aufklärung im klassischen, an Kant angelehnten Sinne wird als wirksames Mittel verstanden, dies zu gewährleisten. Ich erinnere immer wieder gern an das klassisch moderne Verständnis: „Gebildet im Sinne der Erwachsenenbildung wird jeder, der in der ständigen Bemühung lebt, sich selbst, die Gesellschaft und die Welt zu verstehen und diesem Verständnis gemäß zu handeln" (ebd.: 870). Dafür günstige Voraussetzungen zu schaffen, ist für mich eine zeitlose, weithin konsensuelle Aufgabe, in deren Sinne seitdem einiges geschehen, aber auch noch reichlich ,Luft nach oben' ist.

1972, vor gut fünfundvierzig Jahren, erregt ein Bericht weltweite Aufmerksamkeit, in dem die Grenzen des Wachstums mit Blick auf Industrialisierung, Bevölkerungsentwicklung und Umweltverschmutzung eindrücklich dargestellt werden (vgl. Meadows u.a. 1973). Die Autoren erklären die ,Absicht, zur Reflexion über die globale Problematik der Menschheit anzuregen' (vgl. ebd.: 10). Sie nehmen an, viel zu wenige Menschen seien ,aktiv darum bemüht, Probleme überhaupt erst zu verstehen und nach Lösungsmöglichkeiten zu suchen“ (ebd.: 11), und plädieren für neue Denkgewohnheiten und eine Änderung des Verhaltens (vgl. ebd.: 170). Das lässt sich als ein klassischer Anwendungsfall für das verstehen, was dem Deutschen Ausschuss am Herzen lag.

1987, vor gut dreißig Jahren, wurde der Bericht der Brundtland-Kommission Unsere gemeinsame Zukunft vorgelegt, der den Begriff Sustainability bzw. nachhaltige Entwicklung populär gemacht hat (vgl. Weltkommission 1987). Es geht darum, ökonomische, ökologische, demographische und Entwicklungsprobleme als global interdependent und die Suche nach Problemlösungen als gemeinsame Aufgabe zu verstehen. Naheliegenderweise ist dabei viel von Umverteilung und Mäßigung die Rede, und es wird die Perspektive der Generationengerechtigkeit stark gemacht. Die Kommission hat seinerzeit die nächsten Jahrzehnte als entscheidend angesehen; drei davon sind schon herum, ohne dass im Sinne des Berichtes viel geschehen wäre.

Seit 1995, also beinahe fünfundzwanzig Jahren, müssen wir annehmen (und beachten es bis etwa 2015 gar nicht), dass es um die Literalität der Erwachsenen in Deutschland nicht gut bestellt ist. Dem International Adult Literacy Survey der OECD zufolge scheitern mehr als 50\% daran, „Texte auf zusammengehörende Elemente zu untersuchen, die einfache Schlussfolgerungen verlangen oder spezifizierte Bedingungen erfüllen müssen. [...] verschiedene Informationen, die in einem oder mehreren unterschiedlichen Sätzen oder Absätzen zu finden sind, [zu] identifizieren. [...] Informationen über Absätze oder Textteile hinweg zu integrieren, zu vergleichen oder gegenüber zu stellen“ (OECD 1995: 40). Ein konkretes Bespiel ist eine Fahrradmontageanleitung, mit deren Hilfe überprüft werden kann, ob der Sattel in der richtigen Position angebracht ist, also kein ,Beipackzettel' oder so was.

Etwa zeitgleich (!) mit der IALS-Studie wird weltweit ein letztlich zynisches Programm des Lifelong Learning for all auf den Weg gebracht, das quasi 
paradigmatisch über eine - nicht weiter reflektierte - Zeitdiagnose begründet wird: In Wissensgesellschaften müssten nun endlich alle lebenslang lernen (nicht nur die, die bereits inkludiert sind), und dazu seien das informelle bzw. selbstgesteuerte Lernen besonders geeignete Formen (gern in Verbindung mit Informations- und Kommunikationstechnologien - heute nennt man das ELearning). Dabei richtet sich die Botschaft insbesondere an diejenigen, die am allerwenigsten über die für diese Lernform notwendigen Voraussetzungen verfügen (vgl. Wittpoth 2009, 2014, 2016).

Schaut man nun von heute aus zurück, dann denke ich mit Blick auf die Hoffnungen des Deutschen Ausschusses an

- „Vogelschiss“ (Gauland zur Bedeutung des Nationalsozialismus in der 1000-jährigen deutschen Geschichte Anfang Juni 2018),

- „Antiabschiebeindustrie und Asyltourismus“ (Dobrindt, Soeder) und schließlich an eine der widerlichsten Entgleisungen:

- „Absaufen, absaufen, absaufen ...“ (Pegida Ende Juni 2018 bei einer Hetzrede gegen die Dresdner Hilfsorganisation Mission Lifeline, deren Rettungsschiff sich zu jenem Zeitpunkt mit Flüchtlingen auf einer Irrfahrt durch das abgeschottete Mittelmeer befand).

Darin kommt zunächst zweifelsfrei Politikversagen zum Ausdruck. Aber das ist es nicht allein. Nicht die Tatsache, dass es törichte Menschen gibt, die sich in ihrer Ignoranz sonnen und schreckliche Positionen vertreten, ist relevant, sondern der Umstand, dass sie dies in der Annahme tun (können), damit bis in die Mitte der Gesellschaft hinein Gefolgsleute zu finden. Insofern geht es auch um das Versagen einer kritischen - mit zunehmendem Alter traue ich mich immer mehr zu sagen: bürgerlichen - Öffentlichkeit, die wesentlichen Anteil an der Bestimmung des Sagbaren hat. Was die Verfasser*innen des 1960er Gutachtens dazu sagen würden, möchte ich mir gar nicht vorstellen.

Der Club of Rome hat 2017 einen neuen Bericht vorgelegt: Wir sind dran Was wir ändern müssen, wenn wir bleiben wollen (v. Weizsäcker/Wijkmann 2017). Darin werden zwar einige Annahmen der Grenzen des Wachstums von 1972 korrigiert, die Grundaussagen aber als nach wie vor angemessen bestätigt. Gefordert wird nun - wenn man auf Personen schaut wie hier Ernst Ulrich von Weizsäcker: eindrucksvoll unverdrossen - eine „,neue Aufklärung“ (vgl. ebd.: 124ff.). Dabei greifen die Autoren zum einen implizit Aspekte der (alten) Debatte über die Dialektik der Aufklärung auf und plädieren dafür, Rationalität und Spontaneität in ein neues produktives Verhältnis zu bringen. Zum anderen verweisen sie auf veränderte, zugespitzte Rahmenbedingungen: Wir haben es nicht länger mit einer „leeren“, sondern mit einer „vollen Welt“ (vgl. ebd.: 110ff.) und mit einem ,arrogant gewordenen Kapitalismus“ (vgl ebd.: 135ff.) - im Sinne der globalen Durchsetzung einer radikalen Marktphilosophie (anders: eines besinnungslosen Neoliberalismus) - zu tun. 
Davon weiterhin unbeeindruckt werden öffentlich - in Zustimmung erheischender Absicht - die Grenzen des Wachstums schlicht bestritten.

$\mathrm{Zu}$ Sustainability: Vor einigen Wochen habe ich einen (leider nicht zurückgelegten) Zeitungsartikel gelesen, der mich ziemlich getroffen hat. Der Tenor war - mit Blick auf die völlige Hilflosigkeit angesichts der Fluchtbewegungen - ,25 Jahre Lebenslüge der Republik‘. Ich hatte Anfang der 1990er Jahre mit explizitem Bezug auf die Nachhaltigkeitsdebatte - noch im Weiterbildungszentrum der Ruhr-Universität Bochum Veranstaltungen mit entwicklungspolitisch sensiblen Rundfunk- und Fernsehredakteur*innen sowie Wissenschaftler*innen unterschiedlicher Couleur durchgeführt. Es ging uns darum, Möglichkeiten zu eruieren, der Perspektive nachhaltiger Entwicklung im öffentlichen Bewusstsein den Stellenwert zu geben, den sie u.E. dringend bekommen musste. In dem Zusammenhang war auch von der ,Festung Europa die Rede - allerdings im Sinne eines Schreckensbildes. Durch berufliche Veränderungen und anderes bedingt, habe ich mich mit dem Thema nicht mehr vordringlich beschäftigt. Ob ich etwas erreicht hätte, weiß ich nicht, aber ich hätte es versuchen sollen. Denn wenn heute von der ,Bekämpfung der Fluchtursachen" - nicht nur bei Populisten - die Rede ist, dann bleibt diese Rede bei gleichzeitig ungehemmtem EU-Export z.B. von tiefgefrorenem Geflügelabfall, der den Aufbau kleinräumiger Produktionsstrukturen in Afrika verhindert - auf beschämende Weise hinter dem zurück, was uns seinerzeit gegenwärtig war (und bis heute ist).

$\mathrm{Zu}$ Literacy: Nach annähernd zwanzig Jahren Ignoranz replizieren PIAAC (vgl. Rammstedt 2013) und leo (vgl. Grotlüschen/Riekmann 2012) den 1995er Literacy-Survey und kommen im Wesentlichen zu denselben Ergebnissen wie damals. Das hat in Deutschland immerhin dazu geführt, dass man sich nun stärker für Alphabetisierung (also für die Menschen unter und auf Kompetenzstufe 1) interessiert, und - damit Sie mich nicht missverstehen - das ist gut so! Kaum Aufmerksamkeit erfahren allerdings die 34\% auf Stufe 2, deren Lesekompetenz kaum ausreichen dürfte, sich in komplexen Umwelten zu orientieren (oder auch nur die WAZ oder den Trierischen Volksfreund reflektiert zu lesen). Das wäre - demokratietheoretisch - schon unter den bislang geltenden Umständen kritisch. In einer Zeit, in der bestreitbares Wissen immer mehr durch ad hoc organisiertes Halbwissen verdrängt wird, das zudem in geschlossenen Räumen (sogenannten sozialen Netzwerken) zirkuliert, empfinde ich das als zutiefst beunruhigend (zumal auch die Kompetenzen im Bereich des Problemlösens mit Hilfe digitaler Technologien bei $45 \%$ der Menschen eher gering ausgeprägt sind; vgl. Rammstedt 2013). ${ }^{2}$

2 Spätestens im Lichte der CiLL-Untersuchung, die in ihrem Ansatz komplett an PIAAC anschließt, scheint allerdings eine kritische Prüfung dringend geboten. Denn ihr zufolge bleibt für die 66- bis 80-Jährigen die höchste Stufe der Lesefähigkeit plötzlich verschlossen, und nur noch etwa $17 \%$ erreichen ein Niveau, das gesellschaftliche Teilhabe 
Hinzugenommen werden muss ein Befund, für den sich ebenfalls kaum jemand interessiert:

Wir wissen (vielleicht sollte ich sagen: können wissen) seit mindestens fünfzehn Jahren (durch die PISA-Messungen), dass das Schulsystem je nach Betrachtung Jahr für Jahr bis zu 25\% ,Bildungsarme“ (das sind Zertifikatsarme plus Kompetenzarme, deren Zertifikat etwas verbürgt, was es nicht gibt) entlässt, die wegen mangelnder literaler Praxis im Laufe ihres Lebens eher noch ,ärmer' (und zahlreicher) werden dürften (vgl. exempl. Allmendinger/Liebfried 2003; Tenorth 2008). Offensichtlich kann das Schulsystem das mit ihm institutionalisierte Leistungsversprechen in einem kaum nachvollziehbaren Ausmaß nicht einlösen. Damit ist das Programm lebenslangen Lernens in erheblichen Teilen fehladressiert, zu einer , heiligen Ordnung' geworden, die das alltägliche Leben weiter Kreise der Bevölkerung gar nicht erreicht (allenfalls als Drohgebärde). Statt Weiter-Bildung - im Sinne des Anschlusses an einen soliden Sockel - wäre für nennenswerte Teile (deren Umfang zu entschlüsseln sich lohnte) der Bevölkerung repetitive, nachholende Bildung geboten, von deren Realisierungsmöglichkeiten wir noch keinerlei Vorstellung haben.

Für all das - und Vieles, was ich in diesem Sinne noch sagen könnte - ist Erwachsenenbildung nicht verantwortlich. Ihre Möglichkeiten sind und bleiben begrenzt. Das bedeutet auch, dass sie sich bisweilen öffentlich und lautstark gegen unredliche Inanspruchnahmen zur Wehr setzen muss (dazu gehört für mich, um eines der Tagungsstichworte aufzunehmen, auch die pauschale Behauptung: „Wir brauchen digitale Volkhochschulen“ Staatsministerien für Digitalisierung Dorothee Bär in der Frankfurter Rundschau vom 27.8.18: 4). Sich mit Problemen der genannten Art in der permanenten Rück-Versicherung auseinanderzusetzen, ist aber geboten; zu fragen: Was haben wir (aus guten Gründen) als Aufgabe angenommen, was ist gelungen, was nicht, wo liegen Möglichkeiten der Verbesserung und wo müssen wir nachdrücklich einen Offenbarungseid leisten? ${ }^{3}$ Das gilt zumindest, solange man noch in Anspruch nimmt, einen Beitrag zur Entwicklung und Pflege von Citizenship zu leisten, und solange man - in zeitdiagnostischer Manier - so großkalibrige Themen wie Globalisierung auflegt. Zudem hat Erwachsenenbildung sich zu all den genannten Problembereichen immer wieder in gestaltender Absicht geäußert und auch manches geleistet (z.B. mit Blick auf die Art, in der in der Republik

ermöglicht (nur sie haben ,Textverständnis ‘ und können ,rhetorische Strukturen erfassen', vgl. Friebe u.a. 2014: 62). Dass ich dies - als dieser Gruppe Zugehöriger - für sehr unwahrscheinlich halte, erscheint hoffentlich nicht (nur) als Rede ,pro domo'.

3 Das betrifft insbesondere das anhaltende Problem der Beteiligung. Seit 150 Jahren gilt systematisch immer wieder schlüssig begründet - Erwachsenenbildung ,selbst für den geringsten Mann aus dem Volke“ als unverzichtbar (vgl. etwa Der Bildungs-Verein 1871: 1). Erreicht haben wir bis heute maximal 50\%, woran auch die semantischen Kunststückchen der ,Lifelong Learning for all'-Bewegung nichts ändern. 
etwa mit Umweltfragen umgegangen wird). Schließlich ist die demokratietheoretische Begründung von Erwachsenenbildung meines Erachtens nach wie vor eine der stärksten. Ein bisschen was muss schon durch den Kopf gehen. Und: Wenn man sich für all das für nicht zuständig erklärt, wozu soll öffentliche Erwachsenenbildung dann noch nützlich sein?

Schließen möchte ich zunächst mit wenigen knappen Hinweisen auf sehr aktuelle - mit den bisher angesprochenen in vielerlei Hinsichten zusammenhängende - Probleme, die zugleich weit in die Geschichte zurückweisen:

Menschen, die für rechtspopulistisches Gedankengut empfänglich sind, werden uns, die wir für Aufklärung eintreten, auf die Seite ,derer da oben', der kulturellen (!) Eliten schlagen, die eh nur Unwahrheiten verbreiten und - vor allem - nur an ihrem eigenen Vorteil interessiert sind (dabei wissen wir nicht, wie weite Kreise diese Sicht zieht; ich befürchte mittlerweile: sehr weite). Das ist substantiell durchaus verschieden von der Kontroverse um das Verhältnis von Arbeiterbildung und Arbeiterpolitik um 1870 herum; strukturell erscheinen wir aber - wie Liebknecht das aus der Perspektive der Arbeiterbewegung seinerzeit formuliert hat - auch heute wieder als die falschen Freunde (vgl. Liebknecht 1872: 13).

In dieser Linie bezeichnen einige Allgemeine Pädagog*innen und Bildungssoziolog*innen in für meinen Geschmack deutlich zu schlanker Manier das lebenslange Lernen als ,bloße Ideologie‘. Anspruchsvoller wird das zugrundeliegende Problem in der Soziologie etwa unter dem Begriff des scholastischen Irrtums (vgl. Bourdieu 2001: 64ff.) diskutiert, sehr differenziert und engagiert unter dem Begriff des Paternalismus in der Ethik (vgl. exempl. Otto/Ziegler 2010). Es ist an der Zeit, dass wir uns mit der Selbst- und Fremdwahrnehmung unserer Rolle auseinandersetzen, danach fragen, in welchem Verhältnis das, was wir anzubieten haben, zu dem steht, was als (gesellschaftliches) Problem zur Lösung ansteht (dabei sind Rückblicke bei Weitem hilfreicher als kühne Zukunftsentwürfe).

In der Politik lässt sich hierzu ein eigentümlicher Perspektivwechsel beobachten. Konnte man anfangs noch in der Bezugnahme auf rechtspopulistische Umtriebe über das ganze parteipolitische Spektrum hinweg vor allem massive Kritik an geistiger und moralischer Verblendung oder Verwahrlosung beobachten (woran dann ,klassisch ' die Forderung nach mehr Aufklärung in der Tradition des Gutachtens anschließt), wird mittlerweile übereinstimmend angemahnt, die ,Probleme der Menschen' ernst zu nehmen und aufzugreifen. An die Stelle der einen Verkürzung (Geisteshaltung) ist eine andere (Lebenslage) getreten. Aber: Wo liegen gegenwärtige, das Leben jedes Einzelnen wie die Gesellschaft nachhaltig berührende Probleme? Empirisch gut belegen lassen sich die Bereiche Klimawandel, soziale Spaltung, Infrastrukturprobleme im Blick auf Wohnen, Verkehr, Bildung, Betreuung, europäische Integration, Gestaltung der Außenwirtschaftsbeziehungen (und tatsächlich auch: geregelte 
Einwanderung). Seitens der Politik wird im Wesentlichen angeboten: Grenzkontrollen, ,Ankerzentren', ,Rückführungen', Heimat, Leitkultur. Auch das ist fraglos Politikversagen, aber wiederum nicht nur. Denn Angebote der genannten Art werden (in vielen Fällen wider besseres Wissen) denen gemacht, die sie - man darf hier wohl mit Fug und Recht sagen: irrtümlich - für angemessen halten.

Damit beugt Politik sich der Problemsicht von Menschen, die definitiv nicht ,in dem ständigen Bemühen leben, sich selbst, die Gesellschaft und die Welt zu verstehen und diesem Verständnis gemäß zu handeln“ (s.o.; Deutscher Ausschuss 1966: 870). Und die gängige Problemverschiebung kommt einer Suspendierung von Reflexivität und Humanität gleich, die - nicht nur - 1960 an prominenter Stelle standen.

Mein allerletzter Hinweis bezieht sich auf die No-Billag-Initiative, über die im März dieses Jahres eine Volksabstimmung in der Schweiz stattgefunden hat. Es ging um die Abschaffung der Rundfunk-,Zwangsgebühren'. Ich habe in ausführlichen Fernsehdokumentationen gesehen, wie deren Protagonisten sich über öffentliche Aufgaben und deren Finanzierung geäußert haben, und war angesichts der Grobheit und Schamlosigkeit erschüttert. Zugleich habe ich Schweizer Freunde tatsächlich in Angst erlebt, weil sie das Gefühl hatten, das da etwas fürchterlich aus dem Ruder läuft. Die Initiative ist schließlich (unerwartet deutlich) mit 71\% abgewehrt worden, aber der Schreck steckt den Menschen noch in den Gliedern (zumal es in einer möglichen zweiten Runde um die Halbierung der Beiträge gehen könnte und dies große Erfolgsaussichten hat). Diese Initiative ist für mich paradigmatisch für das Verständnis öffentlicher Aufgaben (zu denen die Erwachsenenbildung in Teilen gehört) in populistischen, rechten Kreisen, die sich mindestens in dieser Hinsicht mit Neoliberalen gut verstehen (übrigens hat die rechtspopulistische Schweizerische Volkspartei, in der die Initiative starke Unterstützung erfahren hat, seit einigen Jahre mit etwa 30\% den größten Stimmenanteil aller Parteien in der Schweiz).

In Deutschland hat das Bundesverfassungsgericht jüngst eine Klage gegen die Rundfunkgebühren abgewiesen. In seiner Begründung hebt es auf ein sehr klares, ambitioniertes Verständnis des öffentlichen Auftrages ab. Dem dauerhaft gerecht zu werden, wird ohne Anstrengungen in den Rundfunk- und Fernsehanstalten kaum möglich sein. Das gilt entsprechend für die öffentliche Erwachsenenbildung. Ich möchte Attacken wie die des Spiegel-der titelte „Volksverdummung auf Steuerzahlerkosten“ (vgl. Der Spiegel Nr. 34, 17.8. 2018: 98ff.) - nicht überbewerten, aber es wäre gut, so etwas überzeugend parieren zu können. Dabei wird man sich zunächst auf Gewachsenes und Gegenwärtiges beziehen müssen und dann auch auf sorgfältig kleingearbeitete Folgen gesellschaftlichen Wandels.

Erwachsenenbildung wird in dem Maße gedeihen, in dem eine aufgeklärte Öffentlichkeit ihrem Bemühen mit expliziter Wertschätzung begegnet. Diese 
zu pflegen und wach zu halten ist eine ehrwürdige, alte und fortwährende Aufgabe, in ,welcher Gesellschaft auch immer' wir leben mögen.

\section{Literatur}

Adorno, Theodor W. (1969): Einleitungsvortrag zum 16. Deutschen Soziologentag. In: Adorno, Theodor W. / Deutsche Gesellschaft für Soziologie (Hrsg.): Spätkapitalismus oder Industriegesellschaft?: Verhandlungen des 16. Deutschen Soziologentages in Frankfurt am Main 1968. Stuttgart: Ferdinand Enke, S. 12-26. Online verfügbar unter: https://www.ssoar.info/ssoar/handle/documnet/18714 [12.7.2019]

Allmendinger, Jutta / Leibfried, Stephan (2003): Bildungsarmut. In: Aus Politik und Zeitgeschichte, H. 22-23, 2003, S. 12-18. Online verfügbar unter: http://www.bpb.de/apuz/27610/bildungsmisere [12.01.2016]

Aufforderung zur Gründung einer, Gesellschaft für Verbreitung von Volksbildung، (1871). In: Dräger, Horst: Volksbildung in Deutschland im 19. Jahrhundert, Bd. 2, Bad Heilbrunn: Klinkhardt 1984, S. 50-55.

Beck, Ulrich (1986): Risikogesellschaft. Frankfurt am Main: Suhrkamp.

Bourdieu, Pierre (2001): Meditationen. Frankfurt am Main: Suhrkamp.

Der Bildungs-Verein, Zentralblatt für das freie Fortbildungswesen in Deutschland, Nr. 1, September 1871.

Deutscher Ausschuss für das Erziehungs- und Bildungswesen (1966): Zur Situation und Aufgabe der deutschen Erwachsenenbildung. In: Empfehlungen und Gutachten des Deutschen Ausschusses für das Erziehungs- und Bildungswesen 1953-1965. Stuttgart: Klett, S. 875-928.

Friebe, Jens / Schmidt-Hertha, Bernhard / Tippelt, Rudolf (Hrsg.) (2014): Kompetenzen im höheren Erwachsenenalter. Bielefeld: W. Bertelsmann.

Grotlüschen, Anke / Riekmann, Wibke (Hrsg.) (2012): Funktionaler Analphabetismus in Deutschland. Münster u.a.: Waxmann.

Liebknecht, Wilhelm (1872): Wissen ist Macht - Macht ist Wissen. Online verfügbar unter: https://gutenberg.spiegel.de/buch/kleine-politische-schriften-1354/3 [12.7.2019]

Meadows, Dennis / Meadows, Donella / Zahn, Erich / Milling, Peter (1973): Die Grenzen des Wachstums. Reinbek: Rowohlt.

OECD (1995): Grundqualifikationen, Wirtschaft und Gesellschaft. Paris.

Otto, Hans-Uwe / Ziegler, Holger (Hrsg.) (2010): Capabilities - Handlungsbefähigung und Verwirklichungschancen in der Erziehungswissenschaft (2. Aufl.). Wiesbaden: VS Verlag.

Piketty, Thomas (2014): Das Kapital im 21. Jahrhundert. München: Beck.

Rammstedt, Beatrice (2013) (Hrsg.): Grundlegende Kompetenzen Erwachsener im internationalen Vergleich. Ergebnisse von PIAAC 2012. Münster: Waxmann. 
Reckwitz, Andreas (2017): Die Gesellschaft der Singularitäten. Berlin: Suhrkamp. Schulze, Gerhard (1992): Erlebnisgesellschaft. Frankfurt am Main / New York: Campus.

Tenorth, Heinz-Elmar (2008): Der Skandal, der nicht publiziert wurde. In: Frankfurter Allgemeine Zeitung. 15. Dezember 2008, S. 37. Online verfügbar unter: http:/www.faz.net/frankfurter-allgemeine-zeitung/feuilleton/der-skandal-dernicht-publiziert-wurde-1744866.html [12.01.2016]

Weizsäcker, Ernst Ulrich v. / Wijkmann, Anders u.a. (2017): Wir sind dran. Gütersloh: Gütersloher Verlagshaus.

Weltkommission für Umwelt und Entwicklung (1987): Unsere gemeinsame Zukunft. Greven: Eggenkamp.

Wittpoth, Jürgen (Hrsg.) (2001): Erwachsenenbildung und Zeitdiagnose. Bielefeld: W. Bertelsmann.

Wittpoth, Jürgen (2009): Leben Lernen lebenslang. Wenn das Unvermeidbare zum Programm wird. In: Ricken, Norbert / Röhr, Henning / Ruhloff, Jörg / Schaller, Klaus (Hrsg.): Umlernen. München: Fink, S. 291-301.

Wittpoth, Jürgen (2014): Bedingtheiten, Formen und Reichweiten des Lernens. In: Faulstich, Peter (Hrsg.): Lerndebatten. Bielefeld: Transcript, S. 259-282.

Wittpoth, Jürgen (2016): Selbstgesteuertes Lernen, Grundbildung und Reflexivität. In: Zimmermann, Therese / Jütte, Wolfgang / Horváth, Franz (Hrsg.): Arenen der Weiterbildung. Bern: HEP-Verlag, S. 80-92 


\section{Zur Rezeption von Appellen des Lebenslangen Lernens}

\section{Einleitung}

Spätestens seit den 1990er Jahren hat sich auch in der Bundesrepublik Deutschland auf bildungspolitischer Ebene das Konzept des Lebenslangen Lernens durchgesetzt, nachdem vorher dem Konzept gegenüber hierzulande eine gewisse Rezeptionssperre zu beobachten war (vgl. Kraus 2001). Grundsätzlich gab es bereits seit den 1970er Jahren unter dem Begriff Lebenslanges Lernen Diskussionen über Bildungsziele und Bildungssysteme in der europäischen Gemeinschaft, die sehr unterschiedliche Interessen offenbarten. Für die Zeit seit den 1970er Jahren beschreibt Hans G. Schütze vier Modelle Lebenslangen Lernens, das er „Chamäleon“ nennt:

- „,- ein sozialpolitisch-emanzipatorisches Modell - mit dem Schwerpunkt auf egalitären Bildungs- und Lebens-Chancen (,Lernen für alle');

- - ein non-utilitaristisches, kulturelles Bildungsmodell, das Selbstverwirklichung, Erhöhung der eigenen Urteils- und Kritikfähigkeit und Teilhabe am kulturellen Leben der Gemeinschaft in den Mittelpunkt stellt (,Lernen, um sich zu bilden');

- - ein liberales oder post-modernes Modell, das lebenslanges Lernen als ein adäquates Lernsystem für eine demokratische, egalitäre und multi-kulturelle Gesellschaft sieht, das im Prinzip allen Bürgern offen steht (,Lernmöglichkeit für alle, die lernen wollen und können');

- - ein Humankapitalmodell, in dem Lebenslanges Lernen vor allem oder ausschließlich Weiterbildung und Entwicklung von beruflichen Qualifikationen bedeutet (,Lernen für einen sich wandelnden Arbeitsmarkt ${ }^{\circ}$ )“ (Schütze 2005: 321f.).

Seit den ausgehenden 1990er Jahren haben die Globalisierung und die Durchsetzung des Neoliberalismus tiefgehende Veränderungen der gesellschaftlichen Situation bewirkt. Gesellschaftliche Transformationsprozesse und eingreifende Veränderungen auf dem Arbeitsmarkt führen zu höheren Flexibilisierungsanforderungen an die Menschen. Das ökonomische Denken greift auch auf kulturelle und soziale Bereiche wie Bildung, Kultur und Gesundheit über, die seitdem vermehrt durch Kosten-Nutzen-Berechnungen und unter Effizienzgesichtspunkten gesteuert werden. In diesem Zusammenhang hat sich 
das Konzept des Lebenslangen Lernens bildungspolitisch auch in Deutschland als geeignetes Steuerungsinstrument angeboten, weil es einerseits die angesprochene Bedeutungsvielfalt transportiert und andererseits das Humankapitalmodell bereits in seiner Bestimmung beinhaltet. Das Konzept wird seitdem als Teil einer europäischen Strategie staatlicher Stellen und eines öffentlichen Diskurses, in dem auch Gutachten, Stellungnahmen und Studien von gesellschaftlichen Gruppen eine bedeutende Stimme haben, in einer Top-down-Strategie verbreitet (vgl. u.a. Dohmen 1996, OECD 1996, UNESCO 1997, BMBF 2001, Kommission der Europäischen Gemeinschaften 2001, Vereinigung der bayerischen Wirtschaft 2003).

Fraglich ist aber, wie Menschen in Deutschland Lebenslanges Lernen verstehen und wie sie darauf reagieren. Grundsätzlich ist durchaus nicht geklärt, ob alle Menschen ein Leben lang lernen wollen. Die Phänomene des Weiterbildungswiderstands sind hinreichend belegt (vgl. Holzer 2017). Zudem haben Studien aufgezeigt, dass auf neue Herausforderungen häufig mit vorhandenen Aneignungsstrukturen reagiert wird, d.h. dass in der Aneignung eine Herausforderung negiert und nivelliert, somit Umlernen oder Dazulernen verweigert wird (vgl. Alheit u.a. 2003).

Die Wirkungen der bildungspolitischen Offensive des Lebenslangen Lernens in Deutschland auf die Menschen in ihren eigenen Deutungen und Entscheidungen für ihre Lern- und Bildungsprozesse sind bislang kaum erforscht. Diese Forschungslücke sollte unser Projekt füllen und untersuchen, ob und wie Personen sich die Appelle des Lebenslangen Lernens aneignen, entsprechende Orientierungen aufbauen und Entscheidungen treffen. So lautet die Forschungsfrage: „Wie rezipieren Menschen in Deutschland Appelle des Lebenslangen Lernens?" Im Folgenden stelle ich die theoretische und methodische Herangehensweise und einige Ergebnisse des von der Deutschen Forschungsgemeinschaft (DFG) finanziell geförderten Projektes vor (vgl. Felden, von 2019).

\section{Theorie}

Das Konzept des Lebenslangen Lernens wird in der wissenschaftlichen Diskussion sehr unterschiedlich eingeschätzt. Neben einer grundsätzlichen $\mathrm{Zu}-$ stimmung zur Notwendigkeit Lebenslangen Lernens gibt es auch eine Reihe von Kritikpunkten an diesem Konzept. So werden der lebenslange Zwang zum Lernen und die Sicht auf die Menschen unter wirtschaftlichen Kosten-Nutzen Erwägungen verurteilt. Einige Autorinnen und Autoren verbinden ihre Kritik mit Überlegungen zur Gouvernementalität nach Foucault (vgl. Pongratz 2009, Klingovsky 2009, Rothe 2011). Diese kritische Perspektive haben auch wir 
angelegt, um den Appellcharakter des Lebenslangen Lernens in seinen spezifischen Auswirkungen auf die Menschen zu erfassen. Im Folgenden seien deshalb wesentliche Bestimmungen Foucaults (vgl. Foucault 2004, Bröckling u.a. 2000) kurz wiedergegeben:

Foucault prägte den Begriff „Gouvernementalität“, um den Zusammenhang von Regieren und Einflussnahme, der Produktion von Wissen und der Konstitution von Subjektivität zu verdeutlichen. Er unterscheidet Herrschaftsbzw. Regierungstechniken von Selbsttechniken und untersucht deren Wechselwirkung. Dabei geht er davon aus, dass Regierungstechniken sich der Prozesse bedienen, in denen das Individuum auf sich selbst einwirkt, so dass der gesellschaftliche Einfluss darin besteht, die Individuen zu einem Handeln zu veranlassen, das sie als ihr eigenes Interesse ansehen. In diesem Sinn bedeutet Subjektivierung Unterwerfung und Freisetzung zugleich. Die Gesamtheit dieser Techniken lässt sich nach Foucault als Dispositiv beschreiben. Damit sind Regelungen, Bedingungen und Strukturen gemeint, unter denen das Handeln der einzelnen erfolgt. Als Dispositiv der Regierungsform des Neoliberalismus sieht Foucault die Organisation von Freiheit und ihrer Gefährdung für das Allgemeinwohl. Weil liberale Freiheiten ständig gefährdet sind, geht mit dem Neoliberalismus eine Kultur der Gefahr einher, die Unsicherheiten und Ängste vor sozialem Abstieg oder Erwerbslosigkeit bewirkt (vgl. Lemke 2004). Merkmal des Neoliberalismus ist die Übertragung ökonomischer Prinzipien auch auf soziale Bereiche wie Bildung, Gesundheit oder Kultur. Die Menschen werden als Unternehmer ihrer selbst angesehen und einerseits durch den prinzipiellen Mangel an Möglichkeiten der Existenzsicherung für alle zum Streben und Übervorteilen der anderen aktiviert und zum anderen angehalten, dafür die Verantwortung selbst zu übernehmen - Responsibilisierung genannt (vgl. ebd.).

Das Konzept des Lebenslangen Lernens lässt sich in dieses Dispositiv einordnen, da die Appelle Lebenslangen Lernens darauf zielen, die Einzelnen in einen Wettbewerb miteinander um begrenzte Chancen zu bringen. Indem u.a. darauf hingewirkt wird, möglichst schnell die Ausbildungen zu durchlaufen, dabei Auslandsaufenthalte und Fremdsprachenkenntnisse, sehr gute Lernergebnisse und Netzwerkverbindungen vorzuweisen, wird suggeriert, dass Lebenslanges Lernen eine Sicherheit für eine erfolgreiche Karriere oder gegen drohende Arbeitslosigkeit oder den sozialen Abstieg bedeute. Trifft das allerdings nicht zu, dann wird dem Einzelnen attestiert, nicht erfolgreich genug zu sein, so dass er für die Folgen selbst verantwortlich gemacht wird. 


\section{Methode}

Sich theoretisch auf Foucault und seinen Ansatz der Gouvernementalität zu stützen, beinhaltet für die empirische Umsetzung die Schwierigkeit, Subjektivierung empirisch zu fassen. Ulrich Bröckling benennt neben dem Ziel auch die Grenzen seiner Untersuchung Das unternehmerische Selbst:

„Selbstverständlich erlaubt die Vermessung des unternehmerischen Kraftfelds keine Aussagen darüber, wie die Menschen sich tatsächlich in ihm bewegen. [...] Untersucht wird also ein Regime der Subjektivierung, nicht was die diesem Regime unterworfenen und in dieser Unterwerfung sich selbst als Subjekte konstituierenden Menschen tatsächlich sagen oder tun. [...] Bildlich ausgedrückt: Untersucht wird die Strömung, welche die Menschen in eine Richtung zieht, und nicht, wieweit sie sich davon treiben lassen, sie nutzen, um schneller voran zu kommen, oder aber versuchen, ihr auszuweichen oder gegen sie anzuschwimmen“" (Bröckling 2013, S. 10f.).

Unser Forschungsprojekt will nun gerade auch einen Blick darauf werfen, wie sich die Menschen in dieser Strömung bewegen, also wie sie auf das Regime der Subjektivierung reagieren. Unter Zuhilfenahme des Begriffs „Anrufung“ nach Althusser (vgl. Althusser 1977) wird deutlich, dass Subjekte aufgrund ihrer Angewiesenheit auf Sozialität und des Bedürfnises nach Anerkennung auf Anrufungen eingehen, also prinzipiell offen dafür sind, auf Wirkungen des gesellschaftlichen Kraftfeldes zu reagieren (vgl. Butler 2001).

Weil aber nach unserer Auffassung für die empirische Ebene die Begriffe Dispositiv und Subjektivierung zu komplex und abstrakt sind, sprechen wir stattdessen von ,Appellen des Lebenslangen Lernens' und ,Rezeptionen durch die Individuen'. Damit soll einerseits der Aufforderungscharakter von Lebenslangem Lernen als Dispositiv im Begriff Appell zugespitzt und andererseits die Aneignung von gesellschaftlichen Phänomenen durch die Individuen als Teil von Subjektivierung im Begriff Rezeption ausgedrückt werden.

Andrea Bührmann und Werner Schneider haben 2007 einen Vorschlag zur Erforschung von Dispositiven vorgelegt (vgl. Bührmann/Schneider 2007) und unterscheiden dabei einerseits ,Subjektformierungen und Subjektpositionierungen“, die diskursiv vermittelt werden und andererseits „Subjektivierungsweisen", die als Selbstdeutung und Selbstverständnis der Individuen zu erfassen sind (vgl. ebd., S. 10). Diesen Vorschlag haben wir aufgegriffen und uns auf der empirischen Ebene dafür entschieden, die Rezeption der Appelle des Lebenslangen Lernens als Subjektivierungsweisen zu betrachten und mithilfe der Durchführung narrativer Interviews, die im exmanenten Nachfrageteil durch einen Leitfaden ergänzt wurden, zu untersuchen. Damit wollten wir Er- 
zählungen generieren und den Menschen weitgehend eigene Relevanzsetzungen für ihre Schilderungen ermöglichen, sie aber auch zur Antwort auf konkrete Nachfragen animieren.

In den Jahren 2014 und 2015 haben wir insgesamt 15 Interviews mit Menschen aus drei unterschiedlichen Alterskohorten (20-34 Jahren, 35-49 Jahren, 50-64 Jahren) und zwei unterschiedlichen Bildungsabschlüssen (mittlerer Bildungsabschluss und hoher Bildungsabschluss) geführt, davon neun Interviews aufgrund ihrer maximalen Differenz innerhalb der Alterskohorte ausgewählt und einer ausführlichen Auswertung unterzogen. Es ging uns dabei darum, in der zur Verfügung stehenden Zeit eine weitgehende Vielfalt an Rezeptionen aufzuzeigen. Die Interviews haben wir grundsätzlich in Modifikation des narrationsstrukturellen Verfahrens nach Fritz Schütze ausgewertet (vgl. Schütze 1983, 1984, Felden, von 2017). Unsere Modifikation besteht darin, als Perspektiven auf die Interviewtexte nicht nur die von Schütze genannten kognitiven Figuren Biographieträger und ihre Beziehungen, Erfahrungsverkettung, soziale Welten und die Gesamtgestalt der Lebensgeschichte (vgl. Schütze 1984, S. 81) anzuwenden, sondern darüber hinaus die Interaktionssituation, die Zeitgestaltung und die Wege im Raum zu betrachten und das Interview in dieser Perspektivenvielfalt auszuwerten (vgl. auch Lucius-Hoene/Deppermann 2004).

\section{Ergebnisse}

Im Folgenden wird aus Platzgründen lediglich ein Einblick in unsere Ergebnisse gegeben; die vollständigen Ergebnisse sind in der Monographie nachzulesen (vgl. Felden, von 2019).

\section{Inhalte der Rezeption}

Die Interviewpersonen verstehen unter Lebenslangem Lernen teilweise ein bildungspolitisches Konzept, auch in seiner neoliberalen Ausrichtung, teilweise ein Bildungskonzept, das ihnen durch Reflexionen über das eigene Leben Erkenntnisse über sich selbst vermittelt. Sie schließen damit eventuell an zwei der Modelle an, die Hans G. Schütze 2005 formuliert hat, nämlich an das Humankapitalmodell und an das kulturelle Bildungsmodell (vgl. Schütze 2005: 321f.). Nahezu alle Interviewpersonen legen einen weiten Lernbegriff an, der formales, non-formales und informelles Lernen umfasst. 


\section{Rezeptionshaltungen und Rezeptionen}

Die interviewten Personen zeigen zustimmende und ablehnende Haltungen gegenüber den bildungspolitischen Appellen des Lebenslangen Lernens, die wir auf analytischer Ebene zu folgenden Rezeptionshaltungen verdichtet haben:

Zustimmende Haltungen:

a) Identifikation mit den Appellen: Die Appelle des Lebenslangen Lernens werden vorbehaltlos angenommen, weil man sich mit den Normen der aktuellen Gesellschaft identifiziert und davon überzeugt ist, damit ein erfolgreiches und anerkanntes berufliches und privates Leben zu führen.

b) Anpassung an die Appelle: Unter Anpassung ist eine nur bedingte Zustimmung zu verstehen. Man unterwirft sich den gesellschaftlichen Erwartungen und Anforderungen und erfüllt sie mehr oder weniger bewusst. Damit ist teilweise ein Sich-Arrangieren mit den Bedingungen, teilweise ein Ringen mit der Situation, teilweise der Versuch gesellschaftlicher Teilhabe verbunden.

Ablehnende Haltungen:

c) Abwehr gegen die Appelle: Die Appelle des Lebenslangen Lernens werden mehr oder weniger bewusst abgewehrt, teils durch expliziten Widerstand, teils durch Ignorieren, Sich-Entziehen oder Einrichten in Nischen. Abwehr bezieht sich dabei eher auf eine Handlungsebene, die sich den Interviews entnehmen lässt.

d) Kritik an den Appellen: Die Appelle des Lebenslangen Lernens werden bewusst abgelehnt. Teilweise geht damit eine allgemeinere Gesellschaftskritik oder eine Ablehnung bestimmter gesellschaftlicher Normen einher. Diese Haltung drückt sich eher in einer kognitiven Stellungnahme auf einer Wissens-Ebene aus.

e) Eigen-Deutung: Der Begriff Eigen-Deutung soll ausdrücken, dass Lebenslanges Lernen vorrangig als Lernen aus eigenen Lebenserfahrungen verstanden und damit mit Reflexion und teilweise selbstbestimmter Bildung verbunden wird.

Diese Rezeptionshaltungen sind analytische Verdichtungen, so dass den meisten Personen mehrere Rezeptionshaltungen zugeschrieben werden können. Dabei sind zueinander passende Haltungen festzustellen, wie beispielsweise die Kritik an den Appellen und die Eigen-Deutung Lebenslangen Lernens, die wir als gleichförmige Rezeptionen bezeichnet haben. Daneben aber lassen sich auch ambivalente Rezeptionen entdecken, etwa die gleichzeitige Anpassung an die Appelle und die Kritik der Appelle des Lebenslangen Lernens. Diese Ambivalenzen weisen auf ein Ringen zwischen individuellen Interessen und 
gesellschaftlichen Anforderungen hin. Um einen konkreteren Einblick in unsere Ergebnisse zu geben, seien drei Beispiele exemplarisch ausgewählt, die unterschiedliche Rezeptionen zeigen.

\section{Beispiel 1: Sven Leisner ${ }^{1}$}

Sven Leisner ist zur Zeit des Interviews 28 Jahre alt und beruflich in einer Organisation auf mittlerer Managementebene tätig. Während seines Studiums eines sozialwissenschaftlichen Faches im Rahmen eines BA-Studiums in Deutschland, eines MA-Studiums im europäischen Ausland und eines Auslandssemesters in einem weiteren europäischen Ausland hält er sich an kurze Studienzeiten und handelt überwiegend im Sinne der bildungspolitischen Appelle des Lebenslangen Lernens, die ihm explizit in seiner Schul- und Studienzeit vermittelt wurden, um keine Karrieremöglichkeit zu versäumen. In seiner Formulierung hat er auf den Druck, während des Studiums möglichst im Ausland gewesen zu sein, so reagiert: „Mir war schon klar, dass ich das gerne machen möchte, und da hab' ich so das, ne? was eh erwartet wurde, mit dem Angenehmen dann verknüpfen können“ (Zeile 1289-1290). Interessanterweise formuliert er hier die Übereinstimmung von gesellschaftlichen Erwartungen und eigenen Interessen explizit und gibt damit unbewusst ein Beispiel dafür, wie - mit Foucault zu sprechen - ,die Herrschaftstechniken über Individuen sich der Prozesse bedienen, in denen das Individuum auf sich selbst einwirkt“" (Foucault 1993, S. 203f., zit. nach Lemke 2001, S. 11).

Auch hinsichtlich der Inhalte und Prüfungsmodalitäten im Studium berücksichtigt Sven Leisner seine eigenen Interessen und kommentiert: „Man muss es so'n bisschen aus Eigennutz tun, weil ansonsten ähm das System auch mal schnell zuschlagen kann, und dann is' man abgehängt" (Zeile 1152-1170). Die Verbindung von Eigeninteressen und dem Erfüllen von bildungspolitischen Appellen ist das Konzept, mit dem er sich vor bösen Überraschungen in der Zukunft schützt. So kann er sich den Eindruck eigener Handlungsfähigkeit bewahren. Auf die direkten Fragen zum Thema Lebenslanges Lernen reagiert Sven Leisner mit Kritik am bildungspolitischen Diskurs des Lebenslangen Lernens. Er vermisst die Verantwortung des Staates für eine wirkliche Chancengleichheit, moniert fehlende Ressourcen wie Zeit und Mittel zum Lernen für die Menschen und mahnt, Bildung nicht weiterhin unter einem Verwertungs- und Nutzenkalkül zu betrachten. Bei Sven Leisner lassen sich insofern die Rezeptionshaltungen Anpassung, Kritik und zum Teil Eigen-Deutung feststellen. Insgesamt zeigt er eine ambivalente Rezeption und Subjektivierungsweise.

1 Alle Namen sind pseudonymisiert. 


\section{Beispiel 2: Steffen Kaiser}

Steffen Kaiser ist zur Zeit des Interviews 34 Jahre alt. Nach seinem Abitur und einem Studium der Diplom-Wirtschaftswissenschaft ist er seit ca. sieben Jahren als Teamleiter in einem Unternehmen tätig. Das Interview mit ihm beinhaltet nur eine kurze Haupterzählung und auch in den Antworten auf die Nachfragen wenig narrative Anteile, so dass es eher ein Leitfadeninterview ist. Er achtet darauf, das Bild eines erfolgreichen, zufriedenen sowie pragmatisch und lösungsorientiert handelnden Managers zu verkörpern und lässt sich nicht auf eine Darstellungsebene des Erzählens und der Prozessorientierung ein. In der Beschreibung seiner Tätigkeit fällt auf, dass er nur positive Aspekte erwähnt:

„Ja, also ich bin jetzt hier äh in dem äh Unternehmen, wo ich bin mehr als zufrieden. Das-, äh stetig, wie gesagt, wachsen dort auch die Aufgaben und die Verantwortung. Aber nich' so in dem Sinne, dass man sich da irgendwie überlastet fühlen würde, sondern äh *positiver* [betont] Stress würd' ich's mal nennen. [...] Also ich lieb' es eher, dass-, dass man sagt: ,Ich hab' den Schreibtisch voll, und ich muss mir jetzt äh selbst überlegen, von der Priorität her, welche Aufgaben muss ich abarbeiten und welche müssen gegebenenfalls liegenbleiben oder muss man halt vielleicht delegieren. [...] Und wie gesagt, wir ham eigentlich dort in dem Bereich ham wir viel-, viel, viele Aufgaben, auch vielfältige Aufgaben. [...] Nein, bei uns ist wirklich, jeden Tag ist alles eigentlich 'n bisschen anders" (Zeile 548-593).

Diese Überbetonungen von Vielfältigkeit und ständigen Veränderungen lassen darauf schließen, dass er damit etwas Spezifisches darstellen möchte. Eventuell ist es ihm wichtig, sich als belastbar und allen Herausforderungen und Anforderungen gegenüber uneingeschränkt gewachsen darzustellen. Zudem erinnern die Vokabeln „Priorität" und ,delegieren“ im Zusammenhang mit der Bewältigung von Aufgaben an Seminare zum Zeit- oder Selbstmanagement. Das gesamte Interview zeigt, dass es ihm wichtig ist, schnell und pragmatisch zu handeln und Probleme mithilfe von Managementstrategien zu lösen. Die gesellschaftlichen Appelle des Lebenslangen Lernens, jederzeit flexibel und lernbereit zu sein, ständige Weiterbildungen zu absolvieren, Mehrarbeit durch intrinsische Motivation zu akzeptieren und selbstgesteuert auch auf informeller Ebene zu lernen, sieht er als sportliche Herausforderungen, die er gern akzeptiert. Insgesamt zeigt sich Steffen Kaiser als Mensch, der sich mit den Diskursen des Lebenslangen Lernens vollkommen identifiziert und absolut die Botschaften der Appelle erfüllt. Seine Subjektivierungsweise ist die der Identifikation, so dass man nach Foucault von einer Entsprechung von Selbst- und Regierungstechnologien sprechen kann. 


\section{Beispiel 3: Janine Quandt}

Janine Quandt ist zur Zeit des Interviews 40 Jahre alt. Nach ihrem Abitur studierte sie zunächst ein geisteswissenschaftliches, dann ein sozialwissenschaftliches Fach, das sie mit dem Diplom abschloss. Nach ihrem Einstieg in den Beruf und einigen unkomplizierten Berufswechseln ist sie derzeit seit sieben Jahren in ihrer Arbeitsstelle tätig. Sie hat aus ihrem Elternhaus eine hohe Leistungsorientierung und gleichzeitig die Botschaft, für andere auf Kosten des eigenen Selbst sorgen zu sollen, mitbekommen. Für sie bedeutet Lebenslanges Lernen ein Lernen aus den Erfahrungen des eigenen Lebens und ein Bewältigen der Lernaufgaben ihres Lebens. Aus den Lern- und Bildungsprozessen, die aus dem narrativen Interview mit ihr herauszuarbeiten sind, wird anhand der Verarbeitung einer unglücklichen Beziehung, der Abgrenzung gegenüber den Anforderungen $\mathrm{zu}$ großer Arbeitsbelastung und dem Kauf einer Immobilie deutlich, dass sie die Appelle des überhöhten Leistungsstrebens und der Vernachlässigung eigener Bedürfnisse zum großen Teil überwunden hat. Sie selbst leitet davon ab, dass sie gesellschaftlichen Anforderungen gegenüber resistent sei und sie abwehren könne. Von neoliberalen Appellen des Lebenslangen Lernens fühlt sie sich nicht betroffen, kritisiert sie aber im Hinblick auf die Einflüsse auf die nächste Generation. Sie lässt sich vorwiegend den Rezeptionshaltungen Eigen-Deutung und teilweise denen der Kritik und Abwehr zuordnen, somit der Subjektivierungsweise einer gleichförmigen Rezeption.

\section{Alterskohorten}

Die Auswertungen der Interviews haben ergeben, dass die Rezeptionen Lebenslangen Lernens zwischen der jüngeren Alterskohorte einerseits und der mittleren und älteren Alterskohorte signifikant unterschiedlich sind. Während sich die jüngere Kohorte mit den gesellschaftlichen Appellen zu kurzen Studienzeiten, häufigen Auslandsaufenthalten, ständiger Lernbereitschaft, grundlegender Konkurrenz, erfolgreicher beruflicher Karriere, Leistungsbereitschaft und Selbstverantwortung direkt und explizit auseinandersetzen muss, ist bei den meisten Personen der mittleren und der älteren Kohorte der subjektive Eindruck verbreitet, das eigene Leben selbst gestalten zu können und gesellschaftliche Vorgaben eher abzuwehren. Bei den Personen der jüngeren Kohorte zeigen sich neben der Rezeptionshaltung der Identifikation auch Haltungen der Anpassung, des vorauseilenden Gehorsams gegenüber Anforderungen der Gesellschaft sowie Phänomene der Kritik und des Leidens, so dass deutlich wird, dass ein Teil der Interviewpersonen sich durch ihre ambivalenten Rezeptionen einem besonderen Druck ausgesetzt fühlt. 
Die meisten Personen der mittleren und der älteren Kohorten verstehen Lebenslanges Lernen vor allem als Lernen aus dem eigenen Leben. Teilweise sind Personen vertreten, die eine aufklärerische Politisierung während der Studienzeiten, Emanzipationsprozesse und eine Bildungsaufbruch-Stimmung erlebt haben. Hier zeigen sich sowohl Haltungen der Eigen-Deutung als auch der Kritik und der Abwehr, also eher gleichförmige Rezeptionen. Daneben existiert die Haltung der Anpassung an gesellschaftliche Appelle des Lebenslangen Lernens dann, wenn Befürchtungen einer gesellschaftlichen Exklusion abgewehrt werden sollen.

\section{Schluss}

Ausgehend von der theoretischen Grundlegung des Ineinandergreifens von Regierungs- und Selbsttechniken im Sinne der Gouvernementalität nach Foucault haben wir in unserer empirischen Erhebung Interviewtexte generiert, die sowohl erzählerischen Charakter haben als auch manifeste Antworten auf Nachfragen beinhalten. So konnten wir durch den Vergleich von latenten Sinnstrukturen und manifesten Aussagen in den Interviewtexten auch vorbewusste Erfahrungsebenen herausarbeiten. Dass bildungspolitische Appelle des Lebenslangen Lernens auch implizit wirken, wird insbesondere unter bestimmten gesellschaftlichen Bedingungen deutlich, etwa, wenn eine Karriere angestrebt oder ein gesellschaftlicher Abstieg befürchtet wird. Zudem können Leidensprozesse, Widersprüche in der Darstellung oder intendierte Selbstdarstellungen als Hinweise auf nicht bewusste Einflüsse gelten und entsprechend aus den Interviews herausgearbeitet werden.

Nach Foucault stellt das Dispositiv der jeweiligen gesellschaftlichen Situation ein Kraftfeld dar, das Individuen zu Subjekten macht, indem sie auf bestimmte Zusammenhänge reagieren. Das tun sie u.a. durch Subjektivierungsweisen, unterschiedliche Rezeptionshaltungen und Rezeptionen. Unsere Untersuchungen haben insgesamt gezeigt, dass die Interviewpersonen Lebenslanges Lernen sehr unterschiedlich verstehen und nur zu einem geringen Teil die Appelle des Lebenslangen Lernens ohne Einschränkungen gutheißen. Für die Erwachsenenbildung bedeutet das, dass die widerständigen Rezeptionshaltungen der Interviewpersonen aber nicht etwa Weiterbildungs-Widerstand zum Ausdruck bringen (vgl. Holzer 2017). Vielmehr drücken sie aufgrund der Koppelung mit Eigen-Deutungen eine Kritik an neoliberalen Formen des Lebenslangen Lernens aus, nicht aber am Lernen aus den Erfahrungen des eigenen Lebens. Darüber hinaus ist ein wichtiger Befund für die Erwachsenenbildung, dass die Appelle des Lebenslangen Lernens gerade auf die jüngere Kohorte einen enormen Druck ausüben. Wie damit umzugehen ist, kann allerdings 
nicht mit der Forschungsperspektive der Gouvernementalität beantwortet werden, sondern ist weiteren Untersuchungen vorbehalten.

\section{Literatur}

Alheit, Peter/ Dausien, Bettina/ Kaiser, Manuela/Truschkat, Inga (2003): Neue Formen (selbst)organisierten Lernens im sozialen Umfeld. Qualitative Analyse biographischer Lernprozesse in innovativen Lernmilieus. QUEM-Materialien 43. Berlin: Arbeitsgemeinschaft Betriebliche Weiterbildungsforschung e.V.

Althusser, Louis (1977): Ideologie und ideologische Staatsapparate. In: Ders.: Ideologie und ideologische Staatsapparate. Aufsätze zur marxistischen Theorie. Hamburg, Berlin: VSA-Verlag, S. 108-153.

BMBF Bundesministerium für Bildung und Forschung (2001): Aktionsprogramm ,Lebensbegleitendes Lernen für Alle'. Bonn: BMBF.

Bröckling, Ulrich (2013): Das unternehmerische Selbst. Soziologie einer Subjektivierungsform. 5. Auflage. Frankfurt/Main: Suhrkamp.

Bröckling, Ulrich/ Krasmann, Susanne/ Lemke, Thomas (Hrsg.) (2000): Gouvernementalität der Gegenwart. Frankfurt/Main: Suhrkamp.

Bührmann, A. D./ Schneider, W. (2007): Mehr als nur diskursive Praxis? - Konzeptionelle Grundlagen und methodische Aspekte der Dispositivanalyse. In: Forum Qualitative Sozialforschung, Volume 8, Nr. 2, Art. 28, S. 1-20. http://nbnresolving.de/um:nbn:de:0114-fqs0702281 zugegriffen: 3. März 2019.

Butler, Judith (2001): Psyche der Macht. Das Subjekt der Unterwerfung. Frankfurt/Main: Suhrkamp.

Dohmen, G. (1996): Das lebenslange Lernen. Leitlinien einer modernen Bildungspolitik. Bonn: BMBWFT.

Felden, Heide von (2017): Zur Erforschung von Lern- und Bildungsprozessen über die Lebenszeit aus biographieanalytischer Perspektive. In: Hof, Christiane, Rosenberg, Hannah (Hrsg.): Lernen im Lebenslauf. Theoretische Perspektiven und empirische Zugänge. Wiesbaden: VS-Verlag, S. 45-61.

Felden, Heide von (2019): Identifikation, Anpassung, Widerstand. Rezeptionen von Appellen des Lebenslangen Lernens. Wiesbaden: VS-Verlag.

Foucault, Michel (2004): Geschichte der Gouvernementalität I: Sicherheit, Territorium, Bevölkerung. Geschichte der Gouvernementalität. In: Sennelart, Michel (Hrsg.): Die Geburt der Biopolitik. 2 Bände. Frankfurt/Main: Suhrkamp.

Holzer, Daniela (2017): Weiterbildungs-Widerstand. Eine kritische Theorie der Verweigerung. Bielefeld: Transcript.

Klingovsky, Ulla (2009): Schöne neue Lernkultur. Transformationen der Macht in der Weiterbildung. Eine gouvernementalitätstheoretische Analyse. Bielefeld: Transcript. 
Kommission der Europäischen Gemeinschaften (2001): Einen europäischen Raum des lebenslangen Lernens schaffen. Brüssel: Europäische Union.

Kraus, Karin (2001): Lebenslanges Lernen - Karriere einer Leitidee. Bielefeld: Bertelsmann.

Lemke, Thomas (2001). Gouvernementalität. In: Kleiner, M. S. (Hrsg.): Michel Foucault. Eine Einführung in sein Denken. (S. 108-122). Frankfurt/Main, New York: Campus.

Lemke, Thomas (2004): „Eine Kultur der Gefahr“ - Dispositive der Unsicherheit im Neoliberalismus. In: Widerspruch, 24. Jg., Nr. 46, S. 89-98.

Lucius-Hoene, Gabriele / Deppermann, Arnulf (2004). Rekonstruktion narrativer Identität. Ein Arbeitsbuch zur Analyse narrativer Interviews, 2. Auflage. Wiesbaden: VS-Verlag.

OECD (1996): Lifelong learning for all - Meeting of the Education Committee at ministerial level. Paris: OECD.

Pongratz, Ludwig A. (2009): Bildung im Bermuda-Dreieck: Bologna - Lissabon Berlin. Eine Kritik der Bildungsreform. Paderborn u.a.: Schöningh.

Rothe, Daniela (2011): Lebenslanges Lernen als Programm. Eine diskursive Formation in der Erwachsenenbildung. Frankfurt/Main, New York: Campus.

Schütze, Fritz (1983): Biographieforschung und narratives Interview. In: Neue Praxis 3. Neuwied, S. 283-293.

Schütze, Fritz (1984): Kognitive Figuren des autobiographischen Stehgreiferzählens. In: Kohli, Martin/Robert, Günther (Hrsg.): Biographie und soziale Wirklichkeit. Stuttgart: Metzlersche Verlagsbuchhandlung, S. 78-117.

Schütze, Hans G. (2005): Bedeutungen und Begründungen von „Lebenslangem Lernen“. Revolutionäres Bildungskonzept oder Modebegriff? In: Jütter, Wolfgang/Negt, Oskar/Thörmer, Heinz (Hrsg.): Leitlinien politischen Handelns. Hannover: Offizin, S. 313-330.

UNESCO Commission on Education for the Twenty-first Century (1996): Learning - the treasure within. Paris. (Deutsche Ausgabe: Delors, J. et. al. (1997). Lernfähigkeit: unser verborgener Reichtum. In: Deutsche UNESCO-Kommission (Hrsg.). UNESCO-Bericht zur Bildung für das 21. Jahrhundert. Neuwied, Kriftel, Berlin: Luchterhand.

Vereinigung der Bayerischen Wirtschaft (2003). Bildung neu denken! Das Zukunftsprojekt. Opladen: Leske und Budrich. 
Jörg Dinkelaker

\section{Unterschiede im Entgrenzten. Zur Frage nach den Konturen von Erwachsenenbildung/Weiterbildung}

Angesichts der Entgrenzung von Lernerwartungen und bildungspolitischen Gestaltungsansprüchen im Feld des institutionalisierten Lernens Erwachsener werden die Konturen dessen, was als Erwachsenenbildung bezeichnet wird, unscharf. Es kann nicht länger als selbstverständlich geklärt gelten, welche Lernsituationen der Erwachsenenbildung/Weiterbildung zugerechnet werden (sollen) und welche nicht. Unklar wird auch, in welchem Verhältnis der mit dieser Zurechnung konturierte Ausschnitt aus dem Gesamtzusammenhang des Lernens Erwachsener zu anderen Formen der Institutionalisierung des Lernens im Erwachsenenalter steht.

Eine Klärung dieser Frage setzt voraus, dass verschiedene Formen des Lernens Erwachsener theoretisch fundiert unterschieden und systematisch aufeinander bezogen werden können, so dass dann diskutierbar wird, welche dieser Formen zur Erwachsenenbildung gezählt werden sollen und welche nicht.

Im vorliegenden Beitrag vertrete ich die These, dass die gegenwärtig gängige Gliederung des Feldes in mehr oder weniger formalisierte Lernkontexte dezidiert nicht dazu geeignet ist, eine befriedigende Klärung dieser Frage herbeizuführen, dass vielmehr ihre Verwendung diese Klärung sogar systematisch verunmöglicht. Zunächst führe ich Gründe dafür an, warum diese Orientierung an Graden der Formalität bzw. Informalität von Lernkontexten keine geeignete Typisierung zur Erschließung der Vielfalt und Breite des Lernens Erwachsener darstellt. (1)

In einem zweiten Schritt plädiere ich dafür, neue Möglichkeiten der kategorialen Sortierung des Feldes zu erproben, und stelle selbst einen solchen Differenzierungsversuch vor, der sich an Unterschieden in der Relationierung von Vermittlungs- und Aneignungsaktivitäten orientiert. (2)

Schließlich wende ich mich vor diesem Hintergrund der Frage nach der Bestimmung der Konturen von Erwachsenenbildung zu. Hierfür prüfe ich drei gängige Definitionsweisen daraufhin, welche Ausschnitte aus dem zuvor so sortierten Feld mit ihnen jeweils eingegrenzt werden. Dabei zeichnen sich je nach Definitionsweise sehr unterschiedliche Umrisse ab. (3) 


\section{Warum die Unterscheidung zwischen mehr oder weniger formalisierten Kontexten eine differenzierte Betrachtung des Lernens Erwachsener in seiner Breite verunmöglicht}

Mit der Durchsetzung des Lebenslangen Lernens als Paradigma des Lernens Erwachsener hat es sich mittlerweile weitestgehend durchgesetzt, das interessierende Feld im Hinblick auf Grade der Formalisierung zu betrachten (Hof 2009). In der durch politische Verständigungs- und Aushandlungsprozesse dominierten Diskussion der Europäischen Union wurde hierfür die ternäre Unterscheidung von formalen, non-formalen und informellen Lernkontexten eingeführt (Kommission der europäischen Gemeinschaften 2000). Im Folgenden möchte ich zeigen, warum es die Orientierung an dieser Unterscheidung systematisch verunmöglicht, die Vielfalt der Kontexte des Lernens Erwachsener in einer den empirischen Phänomenen angemessenen Weise und theoretisch fundiert aufzuschlüsseln. ${ }^{1}$

\section{Formen des Lernens Erwachsener}

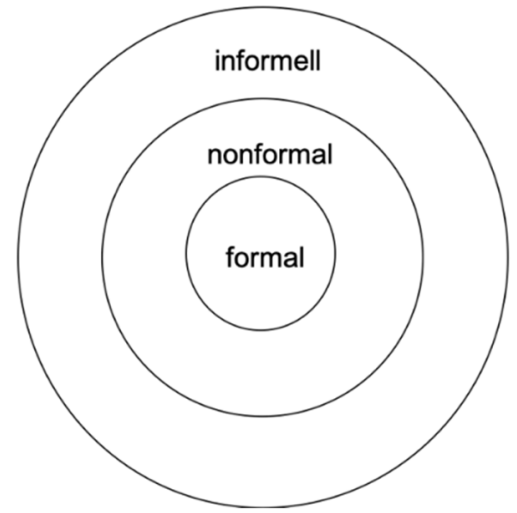

Abb. 1: Definition von Lernformen ex negativo (eigene Darstellung)

In Abbildung 1 sind die anhand von Formalitätsgraden differenzierten drei Formen des Lernens Erwachsener in konzentrischen Kreisen angeordnet. Dies soll das Zentrums-Peripherie-Verhältnis veranschaulichen, das mit der Verwendung dieser Kategorisierung etabliert wird.

1 Vgl. auch die von der hier vorgestellten Argumentation abweichenden Überlegungen bei Overwien 2005, Egetenmeyer 2008, Kaufmann 2012. 
Die Hierarchie in dieser Ordnung ergibt sich daraus, dass nur eine der drei Kategorien, nämlich die innerste, eine dezidierte Bestimmung erfährt: das formale Lernen. Die beiden anderen Kategorien werden dagegen dadurch gebildet, dass benannt wird, welche Merkmale, die der als zentral definierten Lernform zukommen, bei ihnen nicht vorhanden sind.

Das spezifische Format der Institutionalisierung von Lernen, das das „,formale" Lernen kennzeichnet, wird so zur Norm, aus der die Definitionen aller anderen Formate durch Abziehen bestimmter Merkmale abgeleitet werden. Im EU-Memorandum für Lebenslanges Lernen wird das formale Lernen entsprechend als das „Hauptsystem der allgemeinen und beruflichen Bildung“ (Kommission 2000, 9) bezeichnet. Das nonformale Lernen sei dagegen dadurch gekennzeichnet, dass es nicht diesem Hauptsystem zugeordnet werden kann, was sich u.a. darin zeige, dass ,,in der Regel“ keine Zertifikate vergeben würden. Das informelle Lernen wird vom formalen und non-formalen Lernen wiederum dadurch abgegrenzt, dass es nicht in Bildungsveranstaltungen stattfindet. Durch welche Merkmale das non-formale und informelle Lernen aber darüber hinaus positiv bestimmt sind, darüber finden sich, wenn überhaupt, nur widersprüchliche definitorische Aussagen. Eine solche Strategie des Negativ-Definierens ist insofern elegant, als sie es erlaubt, tatsächlich alle Phänomene im betrachteten Feld einer der drei Kategorien zuzuordnen. Scheinbar ist es mit dieser Begriffsstruktur möglich, das gesamte Feld systematisch zu erfassen. Dieser Anschein ist allerdings damit erkauft, dass ein Großteil des so definierten Feldes einer Kategorie zugeordnet wird, die man mit gleichem Recht auch mit „Sonstiges“ hätte bezeichnen können. Unter informellem Lernen kann schließlich alles subsumiert werden, was nicht entweder dem formalen oder dem non-formalen Lernen zugeschlagen werden kann.

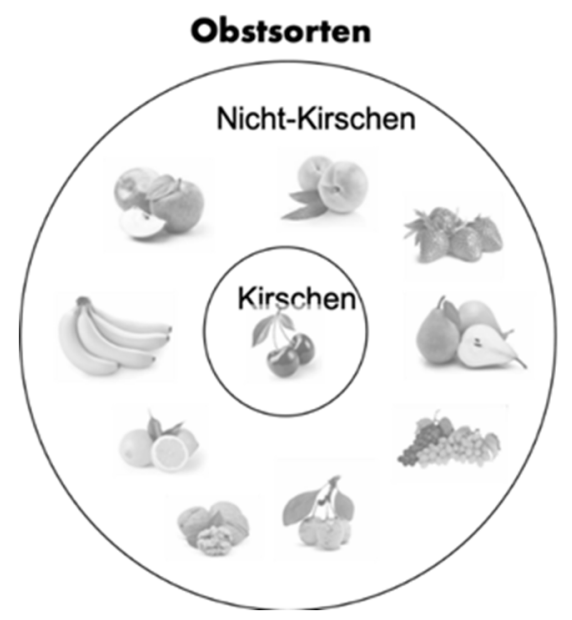

Abb. 2: Definition von Obstsorten ex negativo (eigene Darstellung) 
Ein grundsätzliches Problem solcher definitiones ex negativo besteht darin, dass durch sie unter Umständen sehr unterschiedliche Phänomene unter eine Kategorie gefasst werden, die möglicherweise wenig oder auch nichts miteinander verbindet, außer gerade dieses, dass ihnen ein bestimmtes Bündel von Merkmalen nicht zukommt. Würde man beispielsweise alle Obstsorten entlang der Unterscheidung von Kirschen und Nicht-Kirschen kategorisieren (vgl. Abb. 2), würden wir nicht nur Äpfel mit Birnen vergleichen, was entgegen der Unterstellung des Volksmunds sogar noch einigermaßen Sinn ergäbe. Wir würden zudem auch alle Varianten des Kernobsts mit Beerenobst, Schalenobst und Südfrüchten in einen Topf werfen. Zudem hätten wir die Möglichkeit verloren, überhaupt die Kategorie des Steinobstes zu bilden, weil ja per definitionem vorab bereits ausgeschlossen wurde, dass Kirschen, Pfirsiche und Mirabellen möglicherweise mehr miteinander gemein haben als andere Obstsorten. Kirschenliebhaber mögen diese Einwände wenig interessieren, weil für sie letztlich eben nur von Bedeutung ist, ob es sich bei der vorliegenden Frucht um das von ihnen präferierte Obst handelt oder nicht. Auch diejenigen, die Kirschen nicht vertragen, können sicherlich gerade mit dieser Unterscheidung etwas anfangen, weil sie so zielsicher die Früchte identifizieren können, die sie meiden müssen. In gleicher Weise überzeugt auch die Unterscheidung von formalen und non-fomalen bzw. von formalen und informellen Lernkontexten nur dort, wo Fragen der Zertifizierung und der Veranstaltungsförmigkeit des Lernens in den Mittelpunkt gestellt und vorab definitorisch als die entscheidenden Fragen bestimmt werden. Sowohl diejenigen, die sich dezidiert mit Zertifikaten und Bildungsveranstaltungen beschäftigen, können hieran anschließen als auch diejenigen, die sich eine Abkehr von der Fixierung auf diese Formen wünschen.

Solange die Gegenbegriffe zum formalen Lernen aber lediglich über die Abwesenheit von Merkmalen definiert werden, versäumen wir es, Kategorisierungen zu entwickeln, die sich aus einem systematischen Vergleich der positiv bestimmbaren Merkmale von Formaten des Lernens ableiten. Vor diesem Hintergrund erstaunt es nicht, dass diejenigen, die sich der Restkategorie des informellen Lernens vertieft zuwenden, um zu klären, worin die Spezifik dieser vermeintlich distinkten Form des Lernens besteht, zu uneinheitlichen und widersprüchlichen Ergebnissen gelangen. So lautet beispielsweise das Fazit eines Überblicksaufsatzes zum informellen Lernen: „Insgesamt wird vielfach nur scheinbar über denselben Gegenstand debattiert und Forschungsergebnisse lassen sich nicht ohne Weiteres vergleichen" (Overwien 2005, S. 242). Es ist des Weiteren auch nicht überraschend, wenn sich im empirischen Vergleich von Kontexten des formalen, des non-formalen und des informellen Lernens nur schwer systematische Unterschiede aufzeigen lassen. So halten Helen Colley, Phil Hogdkinson und Janice Malcom als Ergebnis ihrer vergleichenden Untersuchung fest: „When we examined a range of different contexts in which learning took place against the issues that supposedly distinguished informal 
(respectively) non-formal from formal learning, we discovered that what we termed attributes of informality and formality were present in all of them." (Colley/Hogdkinson/Malcom 2003, S. 64)

\section{Ein Differenzierungsvorschlag basierend auf Varianten der Relationierung von Vermittlung und Aneignung}

Wie könnten Kategorien zur Differenzierung des über Bildungsveranstaltungen hinausreichenden entgrenzten Lernens Erwachsener gebildet werden, ohne dass bereits vorab ein bestimmtes Format des Lernens als Normalform gesetzt und alle anderen Formate lediglich hinsichtlich ihrer Entsprechung zu ihr bzw. der Abweichung von ihr gedeutet werden? Voraussetzung hierfür wäre eine Vergleichsgröße, die selbst nicht bereits eine der verglichenen Formen ist. Benötigt würde ein tertium comparationis. Vorstellbar wären eine ganze Reihe von Vergleichsgrößen, anhand derer sich die Breite des Lernens Erwachsener differenzierend aufschließen ließe. ${ }^{2}$ Ich will im Folgenden lediglich einen, aus der Entgrenzungsdebatte der 1990er Jahre hervorgegangenen, Vorschlag aufgreifen und einige seiner Konsequenzen ausloten. Es handelt sich um das im Projekt „Umgang mit Wissen“ (Kade/Seitter 2007) entwickelte theoretische Konzept der pädagogischen Kommunikation (Kade 2004).

Ausgangspunkt der im Horizont dieser Theorie vorgenommenen Typisierung von Formaten der Institutionalisierung von Lernen ist die Annahme, dass sich soziale Situationen des Lernens Erwachsener dadurch konstituieren, dass Vermittlung und Aneignung als zwei Operationsweisen voneinander unterschieden und aufeinander bezogen werden (vgl. Abb. 3). Immer dann, wenn bzw. überall dort, wo der Unterschied zwischen Vermittlung und Aneignung und das Verhältnis zwischen Vermittlung und Aneignung als bedeutsam berücksichtigt werden, wird Lernen zu einer relevanten Größe.

Vgl. etwa Reischmann 2009. 


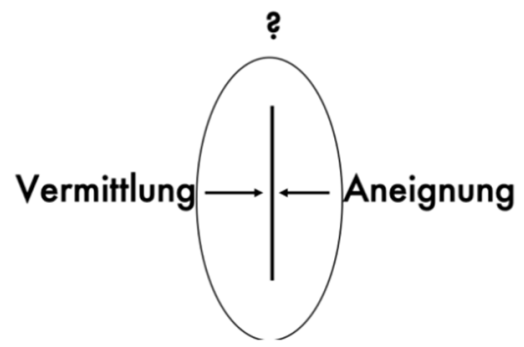

Abb. 3: Pädagogische Kommunikation als Relationierung von Vermittlung und Aneignung (eigene Darstellung)

Varianten der Institutionalisierung des Lernens im Erwachsenenalter lassen sich im Horizont dieser Grundbestimmung dort identifizieren, wo die Unterscheidung und Relationierung von Vermittlungs- und Aneignungsoperationen in unterschiedlicher Art und Weise erwartbar (gemacht) werden. Auf dieser Grundlage lassen sich unterschiedliche soziale Settings des Umgangs mit Lernen ausmachen (vgl. Tabelle 1). Settings der Interaktion unter Anwesenden sind dadurch gekennzeichnet, dass die vermittelnden und die aneignenden Personen zur selben Zeit am selben Ort körperlich anwesend sind, so dass sie sich unmittelbar wechselseitig wahrnehmen und sich in Folge dessen auch wechselseitig aufeinander beziehen können. Für Settings im Format der medialen Kommunikation ist dagegen die räumliche Distanz zwischen Vermittelnden und Aneignenden konstitutiv. Verbunden ist damit auch die Möglichkeit einer zeitlichen Entkopplung der Vermittlungs- und Aneignungsaktivitäten. Dennoch ist wie bei der Interaktion unter Anwesenden auch für die mediale Kommunikation konstitutiv, dass ein gemeinsamer Bezug auf dieselben Mitteilungen stattfindet. Settings im Format der indirekten Relationierung sind dagegen durch die Abwesenheit sowohl von (medialer) Kommunikation als auch von unmittelbarer wechselseitiger Wahrnehmung gekennzeichnet. Vermittlung und Aneignung werden zwar auch in diesem Format noch aufeinander bezogen, aber nur indirekt, vermittelt über das Arrangieren und Ausgestalten von Lernverhältnissen. Das Wesen der Relationierung zwischen Vermittlung und Aneignung besteht hier gerade darin, dass kein kommunikativer Austausch stattfindet. $^{3}$

3 Die hier verwendete Unterscheidung sozialer Settings der Relationierung von Vermittlung und Aneignung ergibt sich aus der Verknüpfung eines Vorschlags zur Differenzierung von Vermittlungsformen von Christiane Hof und eines Vorschlags zur Differenzierung von Settings der Wissensvermittlung von Jochen Kade und Wolfgang Seitter. Zur Diskussion dieser Vorschläge vgl. Dinkelaker 2018, S. $92 \mathrm{ff}$. 
Zur Frage nach den Konturen von Erwachsenenbildung/Weiterbildung 43

\begin{tabular}{|l|c|c|c|}
\hline & $\begin{array}{c}\text { ohne direkte } \\
\text { Kommunikation }\end{array}$ & $\begin{array}{c}\text { Interaktion unter } \\
\text { Anwesenden }\end{array}$ & $\begin{array}{c}\text { mediale } \\
\text { Kommunikation }\end{array}$ \\
\hline vermittlungsfokussiert & zB Lernstätten & $\begin{array}{c}\text { zB Bildungs- } \\
\text { veranstaltungen }\end{array}$ & $\begin{array}{c}\text { zB Mediale } \\
\text { Wissensvermittlung }\end{array}$ \\
\hline aneignungsfokussiert & zB Krisen & zB Beratung & zB Zertifikate \\
\hline
\end{tabular}

Tab. 1: Verwendete Kategorisierung im Grundriss "Lernen Erwachsener“

Mein Interesse an der vergleichenden Betrachtung möglicher Sortierungen von Formen des Lernens Erwachsener entstand im Zuge der Arbeit an einem einführenden Grundriss zum Lernen Erwachsener (Dinkelaker 2018). Der Rückgriff auf diese Differenzierung von Settings der Relationierung von Vermittlung und Aneignung diente dort dazu, verschiedene Formate der Institutionalisierung des Lernens Erwachsener einander vergleichend gegenüberzustellen (vgl. Tabelle 1).

Neben den bereits seit dem 19. Jahrhundert etablierten, vermittlungsdominierten Formaten der Lernstätte, der Bildungsveranstaltung und der medialen Wissensvermittlung lassen sich mit dieser Kategorisierung auch die erst seit der zweiten Hälfte des 20. Jahrhunderts pädagogisierten, stärker aneignungsfokussierten Formate wie die biographische Krise, die Beratung oder das Zertifikat in ihrer jeweiligen Spezifik und in ihrer Relation zum Gesamtzusammenhang des Felds einordnen.

Über die in diesem einführenden Band exemplarisch dargestellten eindeutig lernbezogenen Settings hinaus wären allerdings bei der Diskussion von Formaten des Lernens Erwachsener auch noch solche hybriden Settings zu berücksichtigen, in denen sich das Geschehen der Vermittlung und Aneignung von Wissen mit anderen Formen des Umgangs mit Wissen so vermischt, dass gar nicht mehr eindeutig entschieden werden kann, ob es im gegebenen Moment eigentlich noch um die Strukturierung von Lernen geht oder doch schon um etwas anderes (vgl. Dinkelaker 2008). Der Umgang mit Lernen wird in solchen Situationen flexibel in Vollzüge des sozialen Lebens eingebunden, was nicht nur eine engere Verschränkung von Lernen und Alltag ermöglicht, sondern zugleich eine reflexive Verständigung über Ansprüche und Grenzen des Lernens erschwert. Solchen hybriden Settings kommt im Kontext des sogenannten informellen Lernens eine wesentliche Bedeutung zu. Wird auch diese Unterscheidung von explizit-intensiven und hybrid-uneindeutigen Settings (Kade/Seitter 2007) mit berücksichtigt, so ergibt sich ein Tableau von 12 je unterschiedlich strukturierten Settings der Institutionalisierung des Lernens im Erwachsenenalter (vgl. Tabelle 2). 


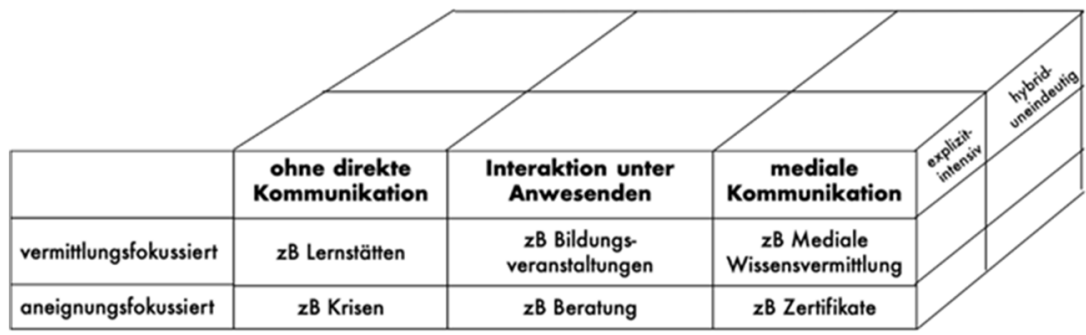

Tab. 2: Dimensionen der Differenzierung von Formen des Lernens Erwachsener

Betrachtet man die mit einer solchen Sortierung erschlossene Vielfalt von Institutionalisierungsvarianten, so dürften die Einschränkungen anschaulich werden, die sich ergeben, wenn allein das aus dem Angelsächsischen importierte Schema des formal learning als Schablone verwendet wird, um Lernkontexte miteinander zu vergleichen. Ein in sehr spezifischer Weise organisiertes Format der Verknüpfung von Bildungsveranstaltung und Zertifikat wird hier als Normalform definiert. Davon abweichend wird mit dem non-formal learning die eigentlich in der deutschen Erwachsenenbildung prominentere Variante von Bildungsveranstaltungen ohne Verknüpfung mit Zertifikaten als die weniger zentrale, aber noch zentrumsnahe Form des Lernens definiert. Alle anderen Formate (rechnerisch sind das 10 weitere Varianten) fallen dagegen unterschiedslos unter die undifferenzierte Sammelkategorie des informellen Lernens, obwohl sie sich, was die Vermittlungs- und Aneignungsverhältnisse angeht, systematisch voneinander unterscheiden.

\section{Varianten der Bestimmung der Konturen von Erwachsenenbildung}

Im Horizont der vorgestellten Kategorisierungsmatrix (Tabelle 2) lässt sich die Frage nach den Konturen von Erwachsenenbildung in veränderter Weise diskutieren. Um dies zu veranschaulichen, prüfe ich exemplarisch drei gängige Bestimmungen von Erwachsenenbildung daraufhin, welche Ausschnitte aus dem so differenzierten Feld des Lernens Erwachsener durch sie umrissen werden:

Das die Disziplin m.E. immer noch dominierende, aus den 1970er Jahren stammende Common-Sense-Verständnis von Erwachsenenbildung basiert auf der Fokussierung auf ein spezifisches Format von Lernsituationen. Erwachsenenbildung/Weiterbildung ist nach diesem Verständnis dasjenige Geschehen, 
in dem das organisierte Lernen in Bildungsveranstaltung vorbereitet und ausgestaltet wird. Dieses häufig unausgesprochene Grundverständnis prägt bis heute weite Teile der erziehungswissenschaftlichen Analyse: Texte zur Makrodidaktik befassen sich mit der Planung, Texte zur Mikrodidaktik mit der Durchführung von Bildungsveranstaltungen. Texte zur Professionalisierung der Erwachsenenbildung beziehen sich, sofern nicht dezidiert auf Abweichungen hingewiesen wird, auf eine dieser beiden Ebenen erwachsenenpädagogischen Handelns. Weiterbildungsorganisationen werden als Anbieter von Bildungsveranstaltungen verstanden. Auch Teilnahmestatistiken und Weiterbildungsgesetze orientieren sich am Modell des veranstaltungsförmigen Lernens. Es sprechen m.E. durchaus gute Gründe dafür, den Begriff der Erwachsenenbildung in dieser Weise auf das Lernen in Bildungsveranstaltungen zu begrenzen. Die Rolle einer in dieser Weise verstandenen Erwachsenenbildung ließe sich darin bestimmen, mit Hilfe von Veranstaltungsangeboten in das vielfältig strukturierte Feld des Lernens Erwachsener so zu intervenieren, dass Lernen qualifiziert, gesteuert und reflektiert stattfinden kann. Mit einer solchen Eingrenzung auf die Etablierung und Ausgestaltung von Bildungsveranstaltungen bekäme die Erwachsenenbildung eine geklärte Aufgabe und damit auch eine geklärte Wissensbasis. Man müsste aber zugleich anerkennen, dass in ihr lediglich ein Format des organisierten Lernens Erwachsener neben mehreren anderen verantwortet wird und dass sie damit auch nicht den Anspruch erheben kann, die einzige Form professionell begleiteten Lernens Erwachsener darzustellen. Die Erwachsenenbildung müsste dementsprechend ihre Außenverhältnisse neu konzipieren und in gezielten Austausch mit anderen, ebenfalls das Feld des Lernens Erwachsener prägenden Akteuren treten, etwa mit den Gestalter_innen von Lernstätten (z.B. Mandel 2016), von medialer Wissensvermittlung (z.B. Wichter/Stenschke 2004) oder von Beratungsangeboten (Gieseke/Nittel 2016).

Will man dagegen die Grenzen der Erwachsenenbildung weiter stecken und prinzipiell alle Settings des Erwachsenenlernens auch als potentielle Formate der Erwachsenenbildung in Betracht ziehen - was mittlerweile immer häufiger geschieht -, bedarf es eines anderen, nicht länger settingbezogenen Kriteriums, um Erwachsenenbildung vom Rest des Lernens Erwachsener abzugrenzen. Häufig wird an dieser Stelle Organisation als entscheidendes Merkmal herangezogen. So könnte man hinsichtlich jedes Settings gezielt organisierte und beiläufig sich ergebende Lernsituationen voneinander unterscheiden. Lehr-Lern-Situationen können von Bildungsanbietern organisiert werden oder sich im Kontext anderer sozialer Zusammenhänge ergeben. Beratung kann von einer Einrichtung angeboten werden, sie kann aber auch beiläufig aus dem beruflichen oder privaten Alltag heraus entstehen. Lernstätten können eigens als solche arrangiert werden oder es können sich spezifische Orte quasi naturwüchsig mit spezifischen Lernbedeutsamkeiten aufladen. Bildungsbe- 
deutsame biographische Übergänge können organisatorisch strukturiert werden oder sich krisenhaft unvorhergesehen ereignen. Würden wir Erwachsenenbildung nach diesem Verständnis tatsächlich als das ,organisierte Lernen Erwachsener" in seiner ganzen Breite begreifen, so würden auch zahlreiche Organisationen als Anbieter der Erwachsenenbildung in den Blick kommen, die bislang üblicherweise nicht hinzugezählt werden: Nicht nur Museen, Betriebe, Vereine und Beratungsstellen, auch kommerzielle Vermarkter von Vermittlungsangeboten wie zum Beispiel Plattformen, die selbst produzierte (Lehr-)Videos der Öffentlichkeit zugänglich machen (z.B. YouTube), müssten zu den Anbietern von Erwachsenenbildung gezählt werden. Die Aufgabe der Erwachsenenbildung würde in einem solchen Verständnis darin bestehen, das Auftreten von Lernsituationen jeder Art in aller Vielfalt möglichst überall erwartbar zu machen. Ein spezifischer normativer Anspruch des Eingreifens in das Feld würde sich mit einem solchen Verständnis allerdings erübrigen.

Wo die Breite von Formaten des Lernens berücksichtigt, zugleich aber eine pädagogisch begründete Strukturierung des Lernens beansprucht wird, würde dagegen eine Grenze zwischen Erwachsenenbildung und dem alltäglichen Lernen Erwachsener entlang der Reichweite einer wertgebundenen erwachsenenpädagogischen Profession gezogen. Unter Erwachsenenbildung würden all diejenigen Ausprägungen des Lernens Erwachsener verstanden, die professionell pädagogisch begleitet werden. Diese Spezifikation ist letztlich enger als eine organisationsbezogene, da zwar professionelles pädagogisches Handeln notwendig Organisation voraussetzt, aber die Organisation von Lernsituationen durchaus ohne Anspruch auf pädagogische Professionalität möglich ist. Entscheidend wäre in diesem professionsbezogenen Verständnis nicht, welches der unterschiedlichen Formate des Lernens etabliert würde. Entscheidend wäre vielmehr, inwiefern die etablierten und begleiteten Settings im Sinne eines normativen Anspruchs der Bildung Erwachsener strukturiert würden. Die Rolle der Erwachsenenbildung im Feld des Lernens Erwachsener bestünde in der gezielten Intervention und in der Reflexion auf Situationen des Lernens Erwachsener im Horizont erwachsenenpädagogischer Ansprüche. Eine solche Spezifikation von Erwachsenenbildung wäre mit der Anrufung einer erwachsenenpädagogischen Profession verbunden, was angesichts der gegenwärtigen Situation der Berufsgruppe der Erwachsenenbilder_innen (vgl. Nittel/Schütz/ Tippelt 2014) nur als kontrafaktischer Entwurf zu denken ist.

Vergleicht man die drei hier exemplarisch diskutierten Bestimmungen von Erwachsenenbildung, so wird deutlich, dass sich die mit ihnen eingegrenzten Ausschnitte aus dem Feld des Lernens Erwachsener erheblich voneinander unterscheiden. Offensichtlich kann sehr Unterschiedliches gemeint sein, wenn von „Erwachsenenbildung“ die Rede ist. Darüber hinaus erweisen sich alle drei Verständnisse von Erwachsenenbildung hinsichtlich der vorgestellten Matrix des Lernens Erwachsener als problematisch. Das herkömmliche veranstaltungszentrierte Verständnis ermöglicht zwar eine eindeutige Abgrenzung der 
Erwachsenenbildung vom übrigen Feld, zieht damit aber die Grenzen so eng, dass es viele der Settings nicht mit einschließt, denen in der Erwachsenenbildung eine wachsende Bedeutung zukommt. Das erweiterte, organisationszentrierte Verständnis ermöglicht zwar eine Erschließung der Vielfalt organisierter Lernsituationen, erweitert damit aber die Grenzen weit über den Bereich des pädagogisch verantworteten Lernens hinaus. Ein professionszentriertes Verständnis würde es dagegen ermöglichen, den Anspruch pädagogischer Interventionen in das Feld flexibel in unterschiedlichsten Formaten zu verfolgen, hätte aber zur Voraussetzung, kontrafaktisch das Vorhandensein einer bedeutenden erwachsenenpädagogischen Profession zu unterstellen.

Die hier dargestellte Problematik der Konturierung von Erwachsenenbildung wird überhaupt erst klar beschreibbar, wo das Feld des Lernens Erwachsener nicht bereits tautologisierend in formale und informelle Kontexte, Erwachsenenbildung und Nicht-Erwachsenenbildung gegliedert, sondern zunächst differenzierend in den es strukturierenden Dimensionen erschlossen wird. Erst im Horizont einer solchen begrifflichen Differenzierung wird es möglich, die Spannungsverhältnisse zu beschreiben und damit auch diskutierbar zu machen, in denen sich eine Modellierung der Bedeutung der Erwachsenenbildung für das Lernen Erwachsener bewegt. Angesichts der gegenwärtigen, weitreichenden Entgrenzungs- und Grenzverschiebungsdynamiken scheint mir daher eine kritische Diskussion der Dimensionen dringend geboten, in denen wir das Lernen Erwachsener und die Erwachsenenbildung/Weiterbildung ausleuchten, um ihre theoretischen Implikationen und praktischen Konsequenzen in den Blick nehmen zu können.

\section{Literatur}

Colley, H. / Hogdkinson, P. / Malcom, J. (2003): Informality and formality in learning: A report for the Learning and Skills Research Centre. London: Learning and Skills Research Centre.

Dinkelaker, J. (2008): Kommunikation von (Nicht-)Wissen. Eine Fallstudie zum Lernen Erwachsener in hybriden Settings. Wiesbaden: VS-Verlag.

Dinkelaker, J. (2018): Lernen Erwachsener. Stuttgart: Kohlhammer.

Dohmen, G. (2001): Das informelle Lernen. Die internationale Erschließung einer bisher vernachlässigten Grundform menschlichen Lernens für das Lebenslange Lernen aller. Bonn: BMBF.

Egetenmeyer, R. (2008): Informal Learning in betrieblichen Lernkulturen. Eine interkulturelle Vergleichsstudie. Baltmannsweiler: Schneider Verlag Hohengehren. 
Gieseke, W./Nittel, D. (Hrsg.): Handbuch Pädagogische Beratung über die Lebensspanne. Weinheim und Basel: Beltz Juventa.

Hof, C. (2003): Wissensvermittlung. Zur Differenz von personalen, medialen und strukturalen Formen der Wissensvermittlung. In: Nittel, D. / Seitter, W. (Hrsg.): Die Bildung des Erwachsenen. Erziehungs- und sozialwissenschaftliche Zugänge. Festschrift für Jochen Kade. Bielefeld: W. Bertelsmann, S. 25-34.

Hof, C. (2009): Lebenslanges Lernen. Eine Einführung. Stuttgart: Kohlhammer.

Kade, J. (2004): Erziehung als pädagogische Kommunikation. In: Lenzen, D. (Hrsg.): Irritationen des Erziehungssystems. Frankfurt/M.: Suhrkamp, S. 199-232.

Kade, J. / Seitter, W. (2007): Umgang mit Wissen. Recherchen zur Empirie des Pädagogischen. 2 Bände. Opladen: Barbara Budrich.

Kaufmann, K. (2012): Informelles Lernen im Spiegel des Weiterbildungsmonitorings. Wiesbaden: Springer VS.

Kommission der Europäischen Gemeinschaften (2000): Memorandum über lebenslanges Lernen. Brüssel: Kommission der Europäischen Gemeinschaften.

Mandel, B. (2016): Teilhabeorientierte Kulturvermittlung: Diskurse und Konzepte für eine Neuausrichtung des öffentlich geförderten Kulturlebens. Bielefeld: Transcript.

Nittel, D. / Schütz, J. / Tippelt, R. (2014): Pädagogische Arbeit im System des lebenslangen Lernens. Ergebnisse komparativer Berufsgruppenforschung. Weinheim: Beltz

Overwien, B. (2005): Stichwort: Informelles Lernen. In: Zeitschrift für Erziehungswissenschaft 8, H. 3, S. 339-355.

Reischmann, J. (2009): Formen des Lernens Erwachsener. In: Fuhr, T. / Gonon, P. / Hof, C. (Hrsg.): Handbuch der Erziehungswissenschaft. Band II/2: Erwachsenenbildung/Weiterbildung. Schöningh: Paderborn, S. 851-862.

Schäffter, O. (2003): Selbstorganisiertes Lernen. Eine Herausforderung für die institutionalisierte Erwachsenenbildung. In: Witthaus, U. / Wittwer, W. (Hrsg.): Selbst gesteuertes Lernen. Theoretische und praktische Zugänge. Bielefeld: W. Bertelsmann Verlag.

Tietgens, H. (1986): Erwachsenenbildung als Suchbewegung. Annäherungen an eine Wissenschaft von der Erwachsenenbildung. Bad Heilbrunn/Obb.: Klinkhardt.

Werder, L. v. (1980): Alltägliche Erwachsenenbildung. Weinheim: Beltz.

Wichter, S. / Stenschke O. (2004): Theorie, Steuerung und Medien des Wissenstransfers. Frankfurt/M.: Peter Lang. 
Jörg Schwarz, Sabine Schmidt-Lauff

\section{(Spät-)Moderne Zeitregime, kollektive Zeitpraktiken und subjektives Lernzeiterleben}

Für die Erwachsenenbildung sind Zeitverhältnisse konstitutiv: Alles Lernen verbraucht Zeit, Lehr-Lerngeschehen werden zeitlich i.d.R. chronometrisch organisiert, Bildung unterliegt der jeweiligen Historizität usw. (vgl. ausführlich Schmidt-Lauff 2008; 2012). Vor allem in Bezug auf den zuletzt genannten Aspekt steht die Erwachsenenbildung in der Verantwortung, auf sogenannte ,Transformationen und Entgrenzungen' nicht nur zu reagieren, sondern sich zu diesen möglichst reflexiv (gestaltend) zu verhalten. Unter der spezifischen Perspektive einer ,strukturell und kulturell höchst bedeutsame[n] Transformation der Temporalstrukturen" (Rosa 2005: 24) wollen wir zeigen, wie das komplexe Verhältnis zwischen gesellschaftlichen Transformations- und Entgrenzungsprozessen, Bildungsfreistellungsregelungen (als moderne Lernzeitinstitutionen für ein Anspruchsrecht auf Weiterbildung) und entsprechenden Angeboten $\mathrm{zu}$ untersuchen ist. Ziel ist zunächst die Extraktion zeitlicher Kontextualisierungen von Lernen im Erwachsenenalter mit dem besonderen Blick auf Bildungsfreistellungen. Über eine Programmanalyse des Angebotsfelds werden zugrundeliegende kollektive Zeitpraktiken von Transformationen sichtbar, indem scheinbar simple Zeitordnungen wie Dauer, Umfang, zeitliche Lage und Intensität bei genauerer Betrachtung sich zeitlich entgrenzende Prozesse freilegen.

Dazu werden wir zunächst skizzieren, welche zeitlichen Transformationen spätmoderne Zeitregime für das Lernen Erwachsener in der organisierten Erwachsenenbildung generell bewirken. Im zweiten Teil entwickeln wir eine relationstheoretische Perspektive auf Zeitlichkeiten und insbesondere auf deren Einbettung in Entgrenzungstendenzen. Hieraus lassen sich Foci für die empirische Untersuchung von Zeit in der Erwachsenenbildung ableiten, die wir anhand des Forschungsdesigns eines aktuellen Projektes diskutieren. Anhand von Ergebnissen aus dessen erstem, kontextualisierendem Schritt einer Angebotsanalyse präsentiert sich das Untersuchungsfeld zunächst als zeitlich klar strukturiert und begrenzt. Diesem Eindruck sollen in einem abschließenden Ausblick jedoch erste Hinweise auf gleichzeitige temporale Entgrenzungen in der Alltagspraxis des Bildungsurlaubs gegenübergestellt werden. 


\section{Transformation und ihre Folgen für Zeitregime in der Erwachsenenbildung}

Die Legitimation der Erwachsenenbildung ist eng mit einem auf Dauer gestellten und akzelerierten sozialen Wandel (Schäffter 2001), der Deinstitutionalisierung übergreifender Temporalstrukturen (Faulstich 2003) sowie der zunehmenden subjektiven Erfahrung von Kontingenz (Pfeiffer 2007) verknüpft, indem ihr eine zentrale Rolle für die (individuelle) Bewältigung dieser Herausforderungen zugewiesen wird. Die Karriere des Konzepts ,lebenslanges Lernen' kann so als Resultat, Ausdruck und Reaktion auf die Transformation oder gar Erosion temporaler Strukturen in modernen Gesellschaften verstanden werden (Schmidt-Lauff 2008). Die darin angelegte Entgrenzung von Lern- und Bildungszeit ermöglicht eine zeitliche Entzerrung von Lernprozessen über den Lebenslauf und eine andere Vernetzung formaler Lernformate und informellen Lernens, in der nicht selten auf ein Kurzzeit-Lernen (Bilger et al. 2017) und ein Lernen just-in-time und on-demand abgehoben wird (z.B. als arbeitsprozessbegleitendes Lernen). Erwachsenenbildung wird dann aber ,zu einem Faktor neben anderen, die zur Beschleunigung beitragen" (Käpplinger 2018: 9). Zugleich werden Bildungskarrieren offener und flexibler und Lernwünsche lassen sich in Lebensphasen mit (vermeintlich) ,mehr Zeit ${ }^{\circ}$ aufschieben (Schmidt-Lauff 2008). Die Bereitschaft für eine solche zeitliche Entgrenzung wird den Akteuren aber auch explizit abverlangt. So fordert der Rat der EU (2011), ,Erwachsene stärker dafür zu sensibilisieren, dass Lernen eine lebenslange Aufgabe ist, die sie in ihrem Leben in regelmäßigen Abständen und insbesondere bei Arbeitslosigkeit oder beruflichen Übergängen zu bewältigen haben".

Hierin zeigt sich der Zumutungscharakter der Programmatik lebenslangen Lernens, das als wichtigste Chance, den biographischen Risiken kontingenter gesellschaftlicher Entwicklungen zu begegnen, dargestellt wird (kritisch dazu: Dörpinghaus/Uphoff 2012; Schmidt-Lauff 2017). Im Zusammenhang mit der Beschleunigungsthese Hartmut Rosas (2005) stellt sich verstärkt die Frage, wie nicht nur gesamtgesellschaftlich ,Lernzeiten im Verhältnis zu anderen Tätigkeitsbereichen in die gesellschaftlichen Formen der Zeitverwendung eingefügt werden“ können (Pruschansky 2001: VII). Darüber hinaus wird für die Individuen die Integration und Ausgestaltung von Lernzeit zu einer allgegenwärtigen Herausforderung. Dieser Herausforderungscharakter schlägt sich in empirischen Untersuchungen zur Weiterbildungsbeteiligung als zentrale Barriere nieder: Als Hauptursache für eine Nicht-Teilnahme an Weiterbildungsaktivitäten werden am häufigsten (mit insgesamt $73 \%$ der Nennungen) explizit zeitbezogene Begründungen (z.B. fehlende Zeit wegen beruflicher bzw. familiärer Verpflichtungen) gegeben (Bilger et al. 2017: 269). 
Empirische Daten zur zeitlichen Strukturierung von Bildungsangeboten zeigen, dass $76 \%$ aller Weiterbildungsaktivitäten bis zu einer Woche dauern, nur knapp ein Viertel erstreckt sich über mehrere Wochen (7\%) bzw. Monate (13\%) (Bilger et al. 2017: 35). Pro Weiterbildungsaktivität lässt sich ein durchschnittlicher Umfang von 42 Stunden feststellen, was einem durchschnittlichen jährlichen Umfang der Weiterbildungsteilnahme von 72 Stunden pro Kopf entspricht (Bilger et al. 2017: 36). Diese Zeitangaben beziehen sich jedoch auf die reine Unterrichtszeit, die tatsächlich zu tätigenden Zeitinvestitionen liegen deutlich höher: Für die berufliche Weiterbildung zeigen Müller und Wenzelmann (2018: 9), dass die bloße Unterrichtszeit lediglich knapp zwei Drittel des Zeitaufwands ausmacht. Hinzu kommen Vor- und Nachbereitung (18\%), Fahrten (9\%), Prüfungsvorbereitung (5\%) sowie Information und Organisation (4\%). Für jede Zeitstunde im Unterricht müssen also durchschnittlich etwa 34 Minuten zusätzlich aufgewendet werden. Für $68 \%$ aller Weiterbildungsaktivitäten nutzen TeilnehmerInnen ,Freizeit' (im Sinne von NichtArbeitszeit), was in 16\% der Fälle als stark belastend erlebt wird und in 22\% der Fälle zu (empfundenem) Zeitdruck bei anderen Aktivitäten führt. Im Gegensatz hierzu wird eine finanzielle Eigenbeteiligung (die in 32\% der Aktivitäten vorlag) nur in 2\% der Fälle als einschränkend und in 1\% der Fälle als belastend empfunden (Müller/Wenzelmann 2018: 10).

Zeit stellt also erstens eine knappe Ressource in Hinblick auf die Weiterbildungsbeteiligung dar. Trotz der in ökonomisierten Diskursen um Flexibilisierung z.B. durch Digitalisierung von Bildung anzutreffenden Verheißungen eines Lernens ,jederzeit \& überall` bleibt Zeit letztlich eine zentrale Hürde für die Verwirklichung lebenslangen Lernens. Dies verweist zweitens auf die Institutionalisierung von Zeitfenstern für Weiterbildung: Im Gegensatz zum Lernen im Kindesalter hat sich das Lernen im Erwachsenenalter historisch vergleichsweise spät und v.a. in Abhängigkeit von der Ausweitung von Freizeit spezifische Zeitfenster erobern können (Seitter 2010). Strukturell steht das Lernen Erwachsener noch immer in ständiger Konkurrenz nicht nur zur Erwerbsarbeit, sondern auch zur Reproduktionsarbeit und zur Freizeit. Dies zeigt sich besonders deutlich auch in den seit mehr als fünfzig Jahren andauernden kontroversen Debatten um Bildungsurlaubsregelungen in Deutschland. Schließlich resultieren drittens spezifische Zeitqualitäten für das Lernen im Erwachsenenalter aus formalen Rahmungen: Wie kann sich ein Lernen ,,aus Passion“ und nicht lediglich „en Passant“, wie Käthe Meyer-Drawe (2012: 9) kritisch anmerkt, unter solchen transformativen Beschleunigungs- und Entgrenzungstendenzen überhaupt noch entwickeln? 


\section{Zeit und Bildung aus relationaler Perspektive}

Da quantitativ messbare Zeit in Bezug auf das Lernen Erwachsener lediglich ein Oberflächenphänomen darstellt, bedürfen die zugrundeliegenden Vorstellungen von Zeit und Zeitlichkeit einer theoretischen Reflexion. Obwohl innerhalb der Soziologie schon früh das Konzept der sozialen Zeit in Abgrenzung zur physischen Zeit eingeführt und seine Bedeutung für die (empirische) Forschung betont wurde (Sorokin/Merton 1937), lässt sich in sozialwissenschaftlichen Untersuchungen nach wie vor häufig eine Reproduktion objektiver Zeitkonzepte feststellen. „Time is often considered as neutral (e.g., the clock), however, the meanings [!] it takes (e.g., being fast/slow; early/late; retarded/advanced; or mature/immature) remains socially constructed" (Alhadeff-Jones 2017: 2).

Zeit als soziale Konstruktionsleistung zu würdigen, erfordert ein Abstandnehmen von Vorstellungen, die Zeit als substanzielles Phänomen, als naturgesetzlich gegebene und objektiv bestimmbare Größe konzipieren, die häufig selbstverständlich und unhinterfragt und zumeist als unabhängige Variable in sozialwissenschaftliche Untersuchungen eingeht. Dafür ist eine relationale erkenntnistheoretische Position hilfreich, die in der Frage ,[...] whether to conceive of the social world as consisting primarily in substances or processes, in static ,things' or in dynamic, unfolding relations" (Emirbayer 1997: 281) für letztere Option votiert: Weder einzelne Entitäten noch übergreifende Strukturen bilden den Ausgangspunkt der Herstellung der sozialen Welt, vielmehr werden die sich in dynamischen Prozessen entfaltenden Relationen zwischen ihnen zur primären Analyseeinheit. Als Wegbereiter einer solchen Soziologie hat sich Norbert Elias mit Zeit aus einer wissenssoziologischen Perspektive auseinandergesetzt, die Wissen (also auch: das Wissen von Zeit) als „Ergebnis des langen, anfangslosen Lernprozesses der Menschheit" (Elias 1988: XII) begreift und damit Anschlussstellen für relationale erziehungs- und bildungswissenschaftliche Fragestellungen eröffnet.

„Der Ausdruck ,Zeit‘ verweist also auf dieses ,In-Beziehung-Setzen“ von Positionen oder Abschnitten zweier oder mehrerer kontinuierlich bewegter Geschehensabläufe. Die Geschehensabläufe selbst sind wahrnehmbar. Die Beziehung stellt eine Verarbeitung von Wahrnehmungen durch wissende Menschen dar. Sie findet ihren Ausdruck in einem kommunizierbaren sozialen Symbol, dem Begriff ,Zeit` [...]“(Elias 1988: XVIIf.)

Mit Blick auf die Erwachsenenbildung ermöglicht eine relationale Konzeption von Zeit, das „Spiel zwischen Zeitrealitäten und Zeitbewusstsein“ (Wendorff 1980: 456), die Beziehungen zwischen Zeitstrukturen und Zeiterfahrung, zwischen kollektiven Zeitpraktiken und subjektivem Zeiterleben in den Mittelpunkt zu stellen. Dadurch kommt das wechselseitige Konstitutionsverhältnis 
von Zeit und Bildung in den Blick (Schmidt-Lauff 2018). Die Vielschichtigkeit der resultierenden Bezüge werden im Modell ,zeittheoretischer Implikationen für die (Erwachsenen-)Bildung“" (Schmidt-Lauff 2008, 2012, 2014, 2018) differenziert und diskutiert.

Praxistheoretische Zugänge sind geeignet, diese Perspektive auf Zeit weiter zu fundieren, da sie nicht nur der Überwindung des Gegensatzes subjektivistischer und objektivistischer Positionen und der Etablierung einer relationalen statt substanzialistischen Perspektive auf die soziale Welt verpflichtet sind. Sie weisen zudem der Zeit eine zentrale Bedeutung für die spezifische Logik sozialer Praxis zu: Die prinzipielle Irreversibilität, die flüchtige Gegenwärtigkeit und der mit dieser verbundene Dringlichkeitscharakter sind konstitutiv für die Vorstellung einer prozessualen Hervorbringung, welche die Vergangenheit als restriktive, praktische Möglichkeitsräume sinnhaft begrenzende Grundlage dieser Praxis erkennt und zugleich ihre prinzipielle Kontingenz und $\mathrm{Zu}-$ kunftsoffenheit betont (Reckwitz 2003). Aus praxistheoretischer Perspektive stellt Zeit (übrigens ebenso wie Raum) keine bloße Randbedingung des Sozialen dar, sie wird sozial hergestellt und wirkt ihrerseits an diesen Herstellungsprozessen mit. Dadurch erlangt sie auch eine strategische Dimension: „Soziale Praktiken entfalten sich nicht nur in der Zeit, sie spielen zugleich mit der Zeit“" (Schmidt 2012: 53).

Um zu untersuchen, wie nun in konkreten Bildungsräumen Herstellung und Aneignung von Zeit ineinandergreifen, kann der Feldbegriff Pierre Bourdieus (Bourdieu 2004) fruchtbar gemacht werden. Eine relational-praxistheoretische Perspektive geht davon aus, dass sich eine spezifische soziale Praxis in der Relationierung inkorporierter Denk-, Wahrnehmungs- und Handlungsstrukturen der Akteure mit institutionalisierten Strukturen des jeweiligen Feldes konstituiert und sie ihrerseits diese Strukturen (re-)produziert. So kommt das Zusammenspiel von praktischer Herstellung, subjektiver Aneignung und Institutionalisierung spezifischer Zeitstrukturen in konkreten Praxisvollzügen in den Blick (Schwarz 2016). Das Feldkonzept kann auf unterschiedlichste Formen sozialer Strukturbildung angewendet werden, von gesellschaftlichen, Teilsystemen' wie dem Bildungssystem bis zu Praxiszusammenhängen auf ,Mikroebene" wie einer Weiterbildungsveranstaltung. Als seinerseits relationales Analyseinstrument (Schwarz 2018) stellt es dabei stets die (gerade in Bezug auf eine so grundlegende Kategorie wie Zeit) vielfältigen Relationen zwischen differenten Feldern systematisch in Rechnung. 


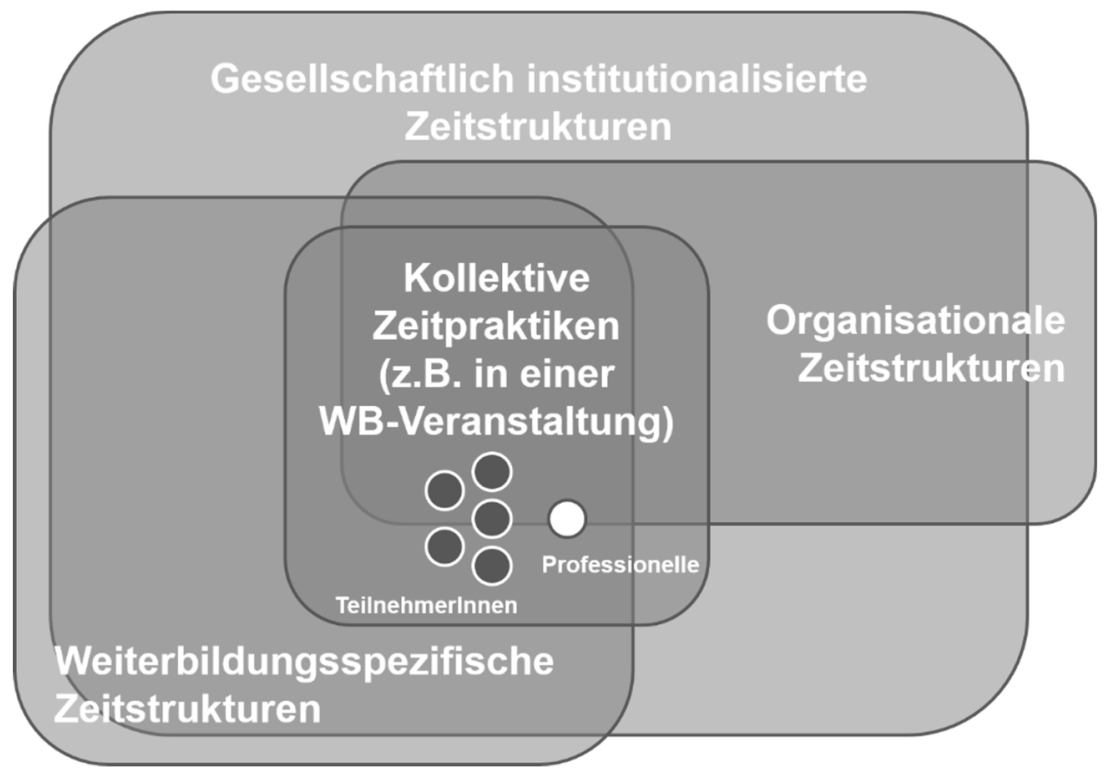

Abbildung 1: Interferierende temporale Vollzugsordnungen(Quelle: Eigene Darstellung)

Im Sinne einer relationalen Temporaltheorie für die Erwachsenenbildung gilt es also, von der „Mehrschichtigkeit“ (Schmidt-Lauff et al. 2018) des zu betrachtenden Phänomens Zeit auszugehen und die differenten, aber zugleich interferierenden temporalen Vollzugsordnungen in den Blick zu nehmen. Die Zeitpraktiken in konkreten Kursen der Erwachsenenbildung etablieren je spezifische Temporalitäten, die aber rekonstruktiv erschlossen und interpretativ auf institutionalisierte Zeitstrukturen und übergreifende Muster gesellschaftlichen Wandels bezogen werden können. Erst auf dieser Grundlage lässt sich schließlich untersuchen, in welchen Beziehungen die kollektiv etablierten Zeitpraktiken zum Lernzeiterleben der TeilnehmerInnen stehen und wie sich diese Relationen wiederum auf Lern- und Bildungsprozesse auswirken. 


\section{Hintergrund: Subjektives Lernzeiterleben und kollektive Zeitpraktiken}

Ziel unseres aktuellen explorativen Forschungsprojekts „Subjektives Lernzeiterleben und kollektive Zeitpraktiken "1 ist es, diese relationale theoretische Perspektive auf Zeit in ein multiperspektivisch-multimethodisches Forschungsdesign zu überführen und damit einerseits zur Theoriebildung, andererseits zur Methodenentwicklung im Bereich erwachsenenpädagogischer Zeitforschung beizutragen. Im Kern steht der Versuch, das Zusammenspiel kollektiver Zeitpraktiken und subjektiven Lernzeiterlebens $\mathrm{zu}$ fokussieren und schließlich Hinweise auf die Lernförderlichkeit spezifischer Zeitqualitäten zu erhalten.

Vor dem Hintergrund des Interesses an institutionalisierten Zeitfenstern für das Lernen Erwachsener fokussieren wir empirisch Bildungsurlaubsveranstaltungen und damit eine spezifische, (vermeintlich) stark strukturierte Zeitform, denn die landesgesetzlichen Bildungsfreistellungsregelungen legen zentrale temporale Gestaltungsaspekte fest. ${ }^{2}$ Die Erhebung verbindet (teilnehmende) Beobachtungen in den Kursen, die darauf zielen, die kollektive, praktische Herstellung spezifischer Zeitlichkeiten zu rekonstruieren, mit qualitativen Einzelinterviews mit den Teilnehmenden, die auf das subjektive Zeiterleben und die Rekonstruktion zugrunde liegender zeitbezogener Denk-, Wahrnehmungsund Handlungsschemata gerichtet sind. Die Auswertung orientiert sich an der dokumentarischen Methode (Bohnsack 2014) und daran anschließender Überlegungen zur Analyse von Einzelinterviews (Nohl 2012) und Beobachtungsprotokollen (Vogd 2004).

Um aber dem oben formulierten Anspruch einer relationalen Einbettung dieser Daten gerecht werden zu können, gilt es zunächst, die institutionalisierten Temporalstrukturen des Feldes näher zu untersuchen. Über die Auseinandersetzung mit aktuellen Diskursen um und die konkrete gesetzliche Regelungsvielfalt von Bildungsfreistellungen hinaus wurde deshalb in einem ersten, kontextualisierenden Untersuchungsschritt eine Programmanalyse (Nolda 2018; Käpplinger 2008) durchgeführt, die darauf zielt, die regionale Angebotslandschaft in ihren temporaltheoretisch relevanten Dimensionen abzubilden und daraus Kategorien für die weitere Untersuchung abzuleiten. Einbezogen

1 Gefördert im Rahmen der internen Forschungsförderung der Helmut-Schmidt-Universität / Universität der Bundeswehr Hamburg von 7/2018-6/2019.

2 Deshalb findet die Untersuchung auch innerhalb eines Bundeslandes statt. Die Wahl Hamburgs ermöglicht einerseits den Vergleich mit für andere Bundesländer bereits vorliegenden Ergebnissen und folgt andererseits forschungspragmatischen Erwägungen. 
wurden alle im Kursportal WISY ${ }^{3}$ registrierten Veranstaltungen des Untersuchungszeitraums (09/18-12/18) mit Förderung nach dem Hamburgischen Bildungsurlaubsgesetz. Dies waren insgesamt 275 Angebote, wovon 215 dem Förderbereich berufliche Bildung und 60 dem Förderbereich politische Bildung zugeordnet werden konnten.

\section{Zeitordnungen im ,Bildungsurlaub‘}

Die sich in den Programmen objektivierende temporale Ordnung des Feldes lässt sich zunächst entlang einiger Kennzahlen darstellen: Die durchschnittliche Dauer der Kurse betrug 5 Tage (Median; AM: 5,66 Std.; SD: 3,5), ihr durchschnittlicher Zeitumfang 40 Std. (Median; AM: 56,88 Std; SD: 92,46). Die Dauer markiert zugleich unterschiedliche Formate: Die Hälfte aller Bildungsurlaubsveranstaltungen findet im klassischen fünftägigen Kursformat statt, zweiwöchige Formate (à 10 Tage) machen weitere 14\% der Kurse aus. Jene 12 Veranstaltungen, die exakt 7 bzw. 14 Tage dauern, sind sämtlich Bildungsreisen der politischen Bildung: Hier wird offenbar die innerhalb der Freistellung nutzbare Zeit maximiert. Dies zeigt sich auch an ihrer zeitlichen Lage: Während fünftägige Kurse überwiegend montags beginnen, dominiert bei den 7- bzw. 14-tägigen Veranstaltungen der Sonntag. Insgesamt ein Viertel der Angebote dauert weniger als fünf Tage, was ein Hinweis auf die Attraktivität kürzerer Veranstaltungen in Reaktion auf soziale Beschleunigung sein kann (Nahrstedt/Brinkmann/Kadel 1997; Käpplinger 2018). Diese These wird gestützt durch den Befund, dass immerhin 15\% der Veranstaltungen bereits im Titel mit Bezeichnungen wie „Intensiv-“ bzw. ,Kompaktkurs“ auf eine besonders zeiteffiziente Gestaltung hinweisen. Hierbei ist in Rechnung zu stellen, dass viele der Bildungsurlaubsangebote zugleich für TeilnehmerInnen ohne Bildungsfreistellung, geöffnet ${ }^{\star}$ sind.

Die zeitliche Intensität (als Verhältnis der Dauer zum Stundenumfang) unterscheidet sich zwischen den beiden Förderbereichen: Während in der politischen Bildung die Dauer durchschnittlich länger ist als in der beruflichen Bildung, liegt der Stundenumfang etwas niedriger. Im Mittel umfasst ein Unterrichtstag damit in der politischen Bildung 6,44 Stunden, in der beruflichen Bildung hingegen 7,83 Stunden. Tendenziell nutzt die politische Bildung also mehr Tage mit weniger Tagesstunden; zugespitzt ließe sich sagen: Sie lässt

3 Das Weiterbildungsinformationssystem ist eine von der Behörde für Schule und Berufsbildung der Hansestadt Hamburg geförderte, unabhängige Datenbank aller beruflichen, sprachlichen oder politischen Weiterbildungsangebote, die in Hamburg stattfinden: https://hamburg.kursportal.info/ 
sich mehr Zeit. Die berufliche Bildung weist dagegen eine äußerst konsequente Orientierung am 8-Stunden-Tag und der 5-Tage-Woche als generalisierten Zeitinstitutionen der Arbeitswelt auf, die auch die Ausgestaltung der gesetzlichen Regulation des Bildungsurlaubs beeinflusst hat. Dies stellt somit eine wichtige Legitimationsbasis für Lernzeiten im Bildungs-, Urlaub', ganz konkret in Aushandlungen mit Arbeitgebern, aber auch generell innerhalb der einflussreichen Zeitkulturen der Arbeitswelt, dar.

Diese Ergebnisse der Programmanalyse kontextualisieren nun in mehrfacher Hinsicht die weitere Untersuchung: Erstens treten dabei Kategorien und kategoriale Differenzen innerhalb des Feldes zutage, die das weitere methodische Vorgehen, insbesondere das Sampling und die Fallauswahl informieren. So bestärken etwa die zeitstrukturellen Unterschiede zwischen politischer und beruflicher Bildung die Entscheidung, beide Bereiche vergleichend zu untersuchen. Zweitens dienen die Ergebnisse einer analytischen Kontextualisierung, indem sie im Rahmen der rekonstruktiven Analyse von Beobachtungen und Interviews die Einordnung in feldbezogene Logiken zeitlicher Strukturierung ermöglichen. Drittens schließlich lässt sich die Relation der in Programmen objektivierten und in den in Kursen praktizierten temporalen Strukturen selbst zum Gegenstand der Analyse zu machen.

\section{Ausblick}

Den letztgenannten Punkt möchten wir abschließend anhand eines knappen Beispiels aus der Beobachtung eines Angebots der beruflichen Bildung verdeutlichen: Dort bestätigt sich im Kursgeschehen zunächst der Eindruck einer Orientierung an etablierten Zeitstrukturen. Auch der Tagesablauf folgt typischen temporalen Mustern und die Kursleiterin versichert, die TeilnehmerInnen über die kommenden zwei Wochen hinweg Schritt für Schritt auf die anstehende Prüfung vorzubereiten. Doch bereits im Verlauf der ersten Unterrichtseinheit wird klar, dass neben die wohlstrukturierte Unterrichtszeit auch eine selbstorganisierte Lernzeit treten muss, deren Notwendigkeit sich im Umfang der auf den Tischen ausgebreiteten Lernmaterialien widerspiegelt. Dies konkretisiert sich in der Empfehlung der Kursleiterin, jeden Tag eine Stunde für die Vor- und Nachbereitung einzuplanen. Die praktische Entgrenzung der zunächst scheinbar klar umgrenzten Zeitbedarfe stimmt die TeilnehmerInnen zunehmend besorgt: In der anschließenden Vorstellungsrunde äußern sie daher zur Frage nach Erwartungen und Wünschen, dass der Unterricht selbst möglichst hinreichend auf die Prüfung vorbereiten möge und man „hoffentlich nicht jeden Abend noch drei Stunden lernen“ müsse. 
Wie dieses kurze Beispiel verdeutlicht, darf sich die Untersuchung von Zeit in der Erwachsenenbildung nicht auf ihre quantitative Messbarkeit und ihre Bedeutung als (knappe) Ressource beschränken. Fraglos ist diese Dimension aber wesentlich, um zu verstehen, wie sich Zeitstrukturen von Bildungsurlaubsveranstaltungen an übergeordneten temporalen Ordnungen orientieren und wie sich erst auf dieser Grundlage die (immer auch Konfliktpotenzial bergende) Dynamik praktischer Aushandlung der Be- und Entgrenzung von Lernzeiten entfaltet. Der hier entwickelte theoretische und methodische Zugang kann somit einen Beitrag dazu leisten, dem komplexen Zusammenwirken institutionalisierter temporaler Ordnungen, kollektiver Zeitpraktiken und subjektiven Lernzeiterlebens in der Erwachsenenbildung auf die Spur zu kommen.

\section{Literatur}

Alhadeff-Jones, Michel (2017): Time and the rhythms of emancipatory education: rethinking the temporal complexity of self and society. London: Routledge.

Bilger, Frauke / Behringer, Friederike / Kuper, Harm / Schrader, Josef / DIE (Hrsg.) (2017): Weiterbildungsverhalten in Deutschland 2016: Ergebnisse des Adult Education Survey. Bielefeld: wbv.

Bohnsack, Ralf (2014): Rekonstruktive Sozialforschung: Einführung in qualitative Methoden. 9. Auflage. Opladen: Barbara Budrich.

Bourdieu, Pierre (2004): Über einige Eigenschaften von Feldern. In: Soziologische Fragen. Frankfurt a.M.: Suhrkamp, S. 107-114.

Dörpinghaus, Andreas / Uphoff, Ina Katharina (2012): Zeit und Bildung. Über die Selbstaffektion in der Erfahrung. In: Schmidt-Lauff, Sabine (Hrsg.): Zeit und Bildung. Annäherungen an eine zeittheoretische Grundlegung. Münster: Waxmann, S. 61-70.

Elias, Norbert (1988): Über die Zeit. Frankfurt a.M.: Suhrkamp.

Emirbayer, Mustafa (1997): Manifesto for a Relational Sociology. In: American Journal of Sociology 103, 2, S. 281-317.

Faulstich, Peter (2003): Weiterbildung: Begründungen lebensentfaltender Bildung. Hand- und Lehrbücher der Pädagogik. München: De Gruyter Oldenbourg.

Käpplinger, Bernd (2008): Programmanalysen und ihre Bedeutung für pädagogische Forschung. In: Forum Qualitative Sozialforschung 9, 1.

Käpplinger, Bernd (2018): Zeit für Weiterbildung und zeitgemäße Weiterbildung. In: BWP 1, S. 6-10.

Meyer-Drawe, Käte (2012): Lernen aus Passion. In: Felden, Heide von / Hof, Christiane / Schmidt-Lauff, Sabine (Hrsg.): Erwachsenenbildung und Lernen. Baltmannsweiler: Schneider Verlag Hohengehren, S. 9-20.

Müller, Norman / Wenzelmann, Felix (2018): Berufliche Weiterbildung: Aufwand und Nutzen für Individuen. Bonn: BIBB. 
Nahrstedt, Wolfgang / Brinkmann, Dieter / Kadel, Vera (1997): Neue Zeitfenster für Weiterbildung? Arbeitszeitflexibilisierung und temporale Muster der Angebotsgestaltung. Bielefeld: Institut f. Freizeitwissenschaft u. Kulturarbeit.

Nohl, Arnd-Michael (2012): Interview und dokumentarische Methode: Anleitungen für die Forschungspraxis. 4. Auflage. Wiesbaden: Springer VS.

Nolda, Sigrid (2018): Programmanalyse in der Erwachsenenbildung/Weiterbildung - Methoden und Forschungen. In: Tippelt, Rudolf / Hippel, Aiga von (Hrsg.): Handbuch Erwachsenenbildung/Weiterbildung. 6. Auflage. Wiesbaden: Springer VS, S. 433-449.

Pfeiffer, Ursula (2007): Kontinuität und Kontingenz: Zeitlichkeit als Horizont systematischer Überlegungen in der Erziehungswissenschaft. Bad Heilbrunn: Klinkhardt.

Pruschansky, Stephanie (2001): LebensLangesLernen: Expertisen zu Lebenslangem Lernen, Lebensarbeitszeiten, Lebensweiterbildungskonten. Berlin: BBJ Verlag.

Rat der Europäischen Union (2011): Entschließung des Rates über eine erneuerte europäische Agenda für die Erwachsenenbildung. https://eur-lex.europa.eu/legal-content/DE/TXT/?uri=CELEX:32011G1220(01)

Reckwitz, Andreas (2003): Grundelemente einer Theorie sozialer Praktiken: Eine sozialtheoretische Perspektive. In: Zeitschrift für Soziologie 32, 4, S. 282-301.

Rosa, Hartmut (2005): Beschleunigung. Die Veränderung der Zeitstrukturen in der Moderne. Frankfurt a. M.: Suhrkamp.

Schäffter, Ortfried (2001): Weiterbildung in der Transformationsgesellschaft: zur Grundlegung einer Theorie der Institutionalisierung. Baltmannsweiler: Schneider-Verlag Hohengehren.

Schmidt, Robert (2012): Soziologie der Praktiken: Konzeptionelle Studien und empirische Analysen. Berlin: Suhrkamp.

Schmidt-Lauff, Sabine (2008): Zeit für Bildung im Erwachsenenalter: Interdisziplinäre und empirische Zugänge. Münster: Waxmann.

Schmidt-Lauff, Sabine (2012): Zeit und Bildung. Annäherungen an eine zeittheoretische Grundlegung. Münster: Waxmann.

Schmidt-Lauff, Sabine (2014): Zeit und Bildung. Eine temporaltheoretische Sicht auf Übergänge. In: von Felden, Heide / Schäffter, Ortfried / Schicke, Hildegard (Hrsg.): Denken in Übergängen. Wiesbaden: Springer VS, S. 19-36.

Schmidt-Lauff, Sabine (2017): Vergesellschaftete Zeit - Überlegungen zu Bildung heute. In: Journal für politische Bildung 2, S. 10-16.

Schmidt-Lauff, Sabine (2018): Zeittheoretische Implikationen in der Erwachsenenbildung. In: Tippelt, Rudolf / Hippel, Aiga von (Hrsg.): Handbuch Erwachsenenbildung/Weiterbildung. Wiesbaden: Springer, S. 319-338.

Schmidt-Lauff, Sabine / Hocke, Simone / Hufer, Klaus-Peter / Schäffter, Ortfried (2018): Die Bedeutung temporaler Phänomene - ein kritischer Aufriss politischen Zeit-Bewusstseins. In: Grotlüschen, Anke/Schmidt-Lauff, Sabine / Schreiber-Barsch, Silke / Zeuner, Christine (Hrsg.): Das Politische in der Erwachsenenbildung. Frankfurt a.M.: Wochenschau Verlag. 
Schwarz, Jörg (2016): Umkämpfte Professionalität: Eine praxistheoretische Analyse zur Institutionalisierung pädagogischer Praxisformen im Feld der Gründungsberatung. Marburg: Philipps-Universität. http://dx.doi.org/10.17192/ z2016.0940

Schwarz, Jörg (2018): Netzwerke und Felder. In: Weber, Susanne Maria / Schröder, Christian / Truschkat, Inga / Herz, Andreas (Hrsg.): Organisation und Netzwerke. Wiesbaden: Springer VS.

Seitter, Wolfgang (2010): Zeitformen (in) der Erwachsenenbildung. Eine historische Skizze. In: Zeitschrift für Pädagogik 56, 3, S. 305-316.

Sorokin, Pitirim A. / Merton, Robert K. (1937): Social Time: A Methodological and Functional Analysis. In: American Journal of Sociology 42, 5, S. 615-629.

Vogd, Werner (2004): Ärztliche Entscheidungsprozesse des Krankenhauses im Spannungsfeld von System- und Zweckrationalität. Berlin: VWF.

Wendorff, Rudolf (1980): Zeit und Kultur: Geschichte des Zeitbewusstseins in Europa. Opladen: Westdeutscher Verlag. 


\section{Transformation ermöglichen: Potentiale von Reflexion und Perspektivverschränkung für die Professionalisierung von Lehrenden. Evaluationsstudie zu einem reflexionszentrierten Fortbildungsangebot der Erwachsenenbildung für Lehrkräfte}

\section{Förderung des professionellen Umgangs mit heterogenen Lerngruppen ${ }^{1}$}

Gesellschaftsanalysen wie die der Reflexiven Modernisierung konstatieren, dass (berufliches) Handeln zunehmend unter Bedingungen von Unsicherheit, Kontingenz und immer neuen Herausforderungen erfolgt (vgl. Beck et al. 1996, Schön 1983). So erleben viele Lehrkräfte den Umgang mit zunehmender Heterogenität in ihren Schulklassen als Verunsicherung und Herausforderung (vgl. Biederbeck/Rothland 2017). Um mit dieser Herausforderung professionell umgehen zu können, ist eine reflexive Auseinandersetzung mit den eigenen Deutungs- und Handlungsmustern, die im Umgang mit heterogenen Lerngruppen zum Tragen kommen, und ggf. auch eine Anpassung und Veränderung dieser im Sinne eines transformativen Lernens notwendig (vgl. Biederbeck/Rothland 2017, Koller 2017). Die Erwachsenenbildung ist hier gefordert, berufsbegleitende, transformative Lernprozesse mithilfe innovativer Lernarchitekturen und Lernbegleitungen anzuregen und zu unterstützen (vgl. Mezirow 1997, Terhart 2015).

Die Transformation bestehender Deutungs- und Handlungsmuster setzt eine intensive Auseinandersetzung mit der eigenen Praxis voraus und bedarf der Fähigkeit zur theoriegeleiteten (Selbst-)Reflexion sowie des Austauschs mit Kolleg*innen (vgl. Arnold 1985, Siebert 2012). Dazu eigenen sich in besonderem Maße reflexionszentrierte Lernumgebungen, in denen die Teilnehmenden ihre Handlungspraxis theoriegeleitet und im Austausch mit anderen Professionellen analysieren und weiterentwickeln können (vgl. Hof 2007). Für

1 Das diesem Bericht zugrunde liegende Vorhaben wurde mit Mitteln des Bundesministeriums für Bildung und Forschung unter dem Förderkennzeichen 01JA1611 gefördert. Die Verantwortung für den Inhalt dieser Veröffentlichung liegt bei den Autorinnen. 
die empirisch fundierte Gestaltung solcher Fortbildungsangebote fehlen jedoch noch Erkenntnisse darüber, inwiefern im Rahmen reflexionszentrierter Lernumgebungen transformatives Lernen angeregt werden kann (vgl. Koller 2017). Um dieser Frage nachzugehen, wurde in einem Projekt der BMBF-geförderten „Qualitätsoffensive Lehrerbildung“ ein reflexionszentriertes Fortbildungskonzept zum „Professionellen Umgang mit heterogenen Lerngruppen“ konzipiert und realisiert. Im Beitrag sollen die Lernprozesse der Teilnehmenden nachgezeichnet werden, um herauszufinden, inwiefern Veränderungsprozesse der Deutungs- und Handlungsmuster der Teilnehmenden angeregt werden konnten und stattgefunden haben.

Der Artikel ist wie folgt aufgebaut: Im zweiten Teil wird das methodischdidaktische Konzept der reflexionszentrierten Fortbildung theoretisch fundiert und es wird erläutert, wie mithilfe der theoriegeleiteten Reflexion und des Erfahrungsaustauschs transformative Lernprozesse angeregt werden sollen. Der dritte Teil widmet sich dem methodischen Design der triangulativen Studie bestehend aus der inhaltsanalytischen Auswertung der Beobachtungsprotokolle einerseits und der Reflexionsblogs andererseits. Im vierten Teil werden die Ergebnisse der Studie ausgeführt, die dann im fünften Teil interpretiert werden. Der Artikel schließt mit einer Diskussion der Ergebnisse sowie einem Ausblick auf sich anschließende, offene Fragestellungen in Teil sechs.

\section{Veränderung ${ }^{2}$ von Deutungs- und Handlungsmustern}

\section{Der Deutungsmusteransatz und der Conceptual Change Ansatz}

Der Deutungsmusteransatz ist ein erwachsenenpädagogisches Prinzip (vgl. Arnold 1985), das auf er ,wissenssoziologische[n] und kognitionstheoretische[n] Erkenntnis" (Siebert 2012: 130) basiert, dass die Welt, so wie wir sie wahrnehmen, ,eine interpretierte Wirklichkeit ist“" (Siebert 2012: 130), die von unseren individuellen Deutungen geprägt wird (vgl. Siebert 2012). Deutungsmuster ${ }^{3}$ beeinflussen die Wahrnehmung und Bewertung von Situationen und

2 Veränderung wird hier auch im Sinne einer Erweiterung oder Anpassung der Deutungsund Handlungsmuster verstanden.

3 Es wurden neben der Forschungsliteratur zum Deutungslernen zudem interdisziplinäre und internationale Befunde zu den eng verwandten Konstrukten „Überzeugungen“ und „Konzeptionen“ berücksichtigt. 
dementsprechend auch das Handeln von Menschen. Oftmals sind sie unbewusst wirksam. Durch ein Bewusstwerden dieser Deutungsmuster kann das Individuum ihre Brauchbarkeit für das professionelle Handeln kritisch prüfen und sie ggf. anpassen oder verändern (vgl. Siebert 2012, Schüßler 2000). Dabei wird professionelles Lehrhandeln als die Fähigkeit verstanden, „breit gelagerte, wissenschaftlich vertiefte und damit vielfältig abstrahierte Kenntnisse in konkreten Situationen angemessen anwenden zu können oder umgekehrt betrachtet: in eben diesen Situationen zu erkennen, welche Bestandteile aus dem Wissensfundus relevant sein können“ (Tietgens 1988: 37ff.). Heterogenitätssensibles Lehrhandeln zeichnet sich weiter dadurch aus, dass Lehrkräfte sich mit der Konstruktion von Unterschieden, d.h. ihrer Differenzierungspraxis auseinandersetzen (vgl. Emmerich/Goldmann 2018).

Für die Auseinandersetzung mit und Veränderung von Deutungsmustern sind sowohl die ergebnisorientierte (Selbst-)Reflexion als auch ein auf Perspektivverschränkung angelegter Austausch mit anderen professionellen Akteuren essentiell (vgl. Greif 2008, Pachner 2013, 2014, Siebert 2012). Gemäß dem theoretisch fundierten und im Rahmen einer hochschuldidaktischen Weiterbildung empirisch überprüften Conceptual Change Ansatz von Ho und Kolleg*innen $(2001)^{4}$ setzen Veränderungsprozesse auf der Ebene von Deutungsund Handlungsmustern folgende Prozesse voraus:

1. einen (selbst-)reflexiven Prozess des Bewusstmachens der eigenen Deutungs- und Handlungsmuster,

2. die Konfrontation mit Unstimmigkeiten der eigenen Deutungs- und Handlungsmuster, aus denen heraus ggf. ein Wunsch nach Veränderung resultiert,

3. das Kennenlernen von alternativen Deutungs- und Handlungsmustern sowie

4. die Bereitschaft das neu entwickelte Deuten und/oder Handeln auch in die Praxis zu transferieren (vgl. Ho 2000)

\section{Das Fortbildungskonzept}

Die Fortbildungsreihe „Professioneller Umgang mit heterogenen Lerngruppen - das eigene Lehrhandeln analysieren, fördern und weiterentwickeln" richtet sich an Lehrkräfte der Sekundarstufen und umfasst drei Präsenztermine und

4 Inhaltlich ähnliche Modelle bzw. Ansätze zur Deutungsmustertransformation sind das Fallarbeitskonzept von Müller (1998) sowie die Phasen des Transformationsprozesses nach Mezirow (1997). Der Ansatz von Ho und Kolleg*innen wurde ausgewählt, weil er für die Gestaltung des Fortbildungskonzeptes prägnanter und anschlussfähiger erscheint und zudem bereits empirisch überprüft ist. 
zwei Anwendungsphasen. Die Ziele der Fortbildung sind es, zum einen die Teilnehmenden dabei zu unterstützen, sich ihrer eigenen (intuitiven) Deutungs- und Handlungsmuster im Umgang mit Heterogenität und ihrer damit einhergehenden Differenzierungspraxis (vgl. Emmerich/Hormel 2013) bewusst zu werden. Zum anderen sollen sie dazu angeregt werden, diese kritisch zu hinterfragen und auszudifferenzieren (vgl. Schüßler 2000).

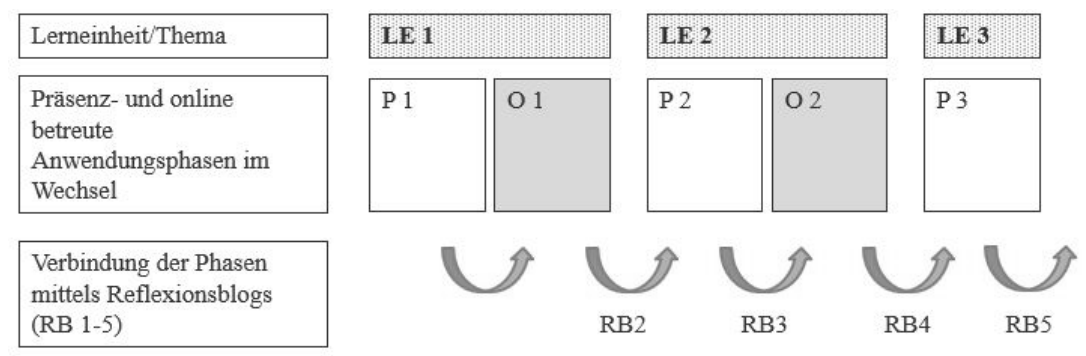

Entwicklungsphasen (vgl. Ho et al., 2001):

RB 2: Selbstreflexion

P 2: Konfrontation und Kennenlernen von Alternativen (Diskussion der Teilnehmendenfälle)

RB 3: Transfer anbahnen

RB 4: Selbstreflexion

P 3: Konfrontation und Kennenlernen von Alternativen (Diskussion der Teilnehmendenfälle)

RB 5: Transfer anbahnen

Abb. 1: Ablauf der Fortbildung inklusive zweimaligem Durchlauf durch die Entwicklungsphasen nach Ho et al. (2001) (vgl. Pachner 2018).

Um ein nachhaltiges transformatives Lernen zu ermöglichen, werden die Entwicklungsphasen nach Ho und Kolleg*innen (2001) im Konzept zweimal durchlaufen (s. Abb. 1). (Selbst-)Reflexion, der Austausch mit Kolleg*innen und der Praxistransfer im Sinne einer Erprobung neuen Handelns sind dabei die zentralen methodisch-didaktischen Elemente. Die (Selbst-)Reflexion der eigenen Deutungs- und Handlungspraxis soll mithilfe der sogenannten Reflexionsblogs (RB) angeregt werden. Darin werden die Teilnehmenden dazu aufgefordert, Fälle ${ }^{5}$ aus ihrer Unterrichtspraxis zu beschreiben und Reflexionsfragen dazu zu beantworten. In den Präsenzsitzungen werden diese Fälle mit den anderen Teilnehmenden analysiert und diskutiert, sodass neue Perspektiven auf die eigene Unterrichtspraxis eingenommen und alternative Deutungs- und Handlungsmuster kennengelernt werden können. In den Anwendungsphasen haben die Teilnehmenden die Möglichkeit, die erarbeiteten alternativen Deutungs- und Handlungsmuster in ihren Unterricht zu transferieren und zu erproben.

5 Siehe auch die Ausführungen zur Fallarbeit bei Müller (1998). 


\section{Die Evaluationsstudie}

\section{Das triangulative Erhebungsdesign}

Im Rahmen der Studie wurde das Ziel verfolgt, die Lernprozesse der Teilnehmenden nachzuzeichnen und zwar entlang der Forschungsfrage: Inwiefern können Deutungs- und Handlungsmuster im Rahmen einer reflexionszentrierten Fortbildung gezielt weiterentwickelt werden? Um die Forschungsfrage aus verschiedenen Perspektiven zu beleuchten, wird unterschiedliches qualitatives Datenmaterial trianguliert (vgl. Flick 2009). Dies sind zum einen die schriftlich fixierten Protokolle der teilnehmenden Beobachtung in den Präsenzsitzungen und zum anderen die Reflexionsblogs der Lernenden. Während die Beobachtungsprotokolle direkten Einblick in die Fortbildungspraxis und den dort stattfindenden Austausch (Konfrontation und Kennenlernen von Alternativen) geben sollen, wird mithilfe der Reflexionsblogs die Selbstreflexion der Teilnehmenden (auch im Hinblick auf das Anbahnen des Transfers) erfasst (vgl. Ho 2000).

\section{Stichprobe und Materialauswahl}

Es haben 15 Lehrkräfte an der Fortbildung teilgenommen. Davon wurden sieben Teilnehmende in die Stichprobe einbezogen, da diese besonders aktiv an allen Präsenzterminen und Anwendungsphasen beteiligt waren, was Voraussetzung für die Auseinandersetzung mit den eigenen Deutungs- und Handlungsmustern ist (vgl. Ho et al. 2001). Für diese sieben Lehrkräfte liegen sowohl Daten in den Beobachtungsprotokollen als auch in den individuellen Reflexionsblogs vor.

\section{Deduktive und induktive Kategorienbildung}

Um die Entwicklungsprozesse der Lehrkräfte im Fortbildungsverlauf sichtbar zu machen, wurden die Beobachtungsprotokolle und die Reflexionsblogs mittels qualitativer Inhaltsanalyse (Mayring 2015) ausgewertet. Auf Basis des Ansatzes von Ho und Kolleg*innen (2001) wurde in einem ersten, deduktiven Zugriff das Material daraufhin untersucht, ob die einzelnen Entwicklungspha- 
sen von den Teilnehmenden durchlaufen wurden. Anschließend wurde mithilfe induktiver Kategorienbildung eruiert, welche Art von Veränderung bei den einzelnen Teilnehmenden im Rahmen der jeweiligen Entwicklungsphasen stattgefunden hat.

\section{Die Lernprozesse der Teilnehmenden im Fortbildungsverlauf}

\section{Die Entwicklungsphasen der Teilnehmenden}

Die Ergebnisse der deduktiven Inhaltsanalyse (siehe Abb. 2, nummerierte Pfeile) zeigen, dass alle sieben Lehrkräfte, die aktiv an der Fortbildungsreihe beteiligt waren, die vier Entwicklungsphasen des Conceptual Change Ansatzes von Ho und Kolleg*innen (2001) zweimal durchlaufen haben (die Abbildung 2 zeigt nur die Phasen 1 bis 4 und nicht die Wiederholung dieser Phasen in den Schritten 5 bis 8). Das heißt, sie haben sich mit ihren Deutungs- und Handlungsmustern reflexiv auseinandergesetzt und diese dem Ansatz entsprechend auch verändert. Allerdings lassen die Ergebnisse keine Rückschlüsse darüber $\mathrm{zu}$, welcher Art die Veränderungen bei den einzelnen Teilnehmenden sind. In Zusammenhang mit den Ergebnissen der induktiven Inhaltsanalyse (Subkategorien der deduktiven Analyse) können hingegen differenzierte Einblicke in die Lernprozesse der einzelnen Teilnehmenden gewonnen werden. 


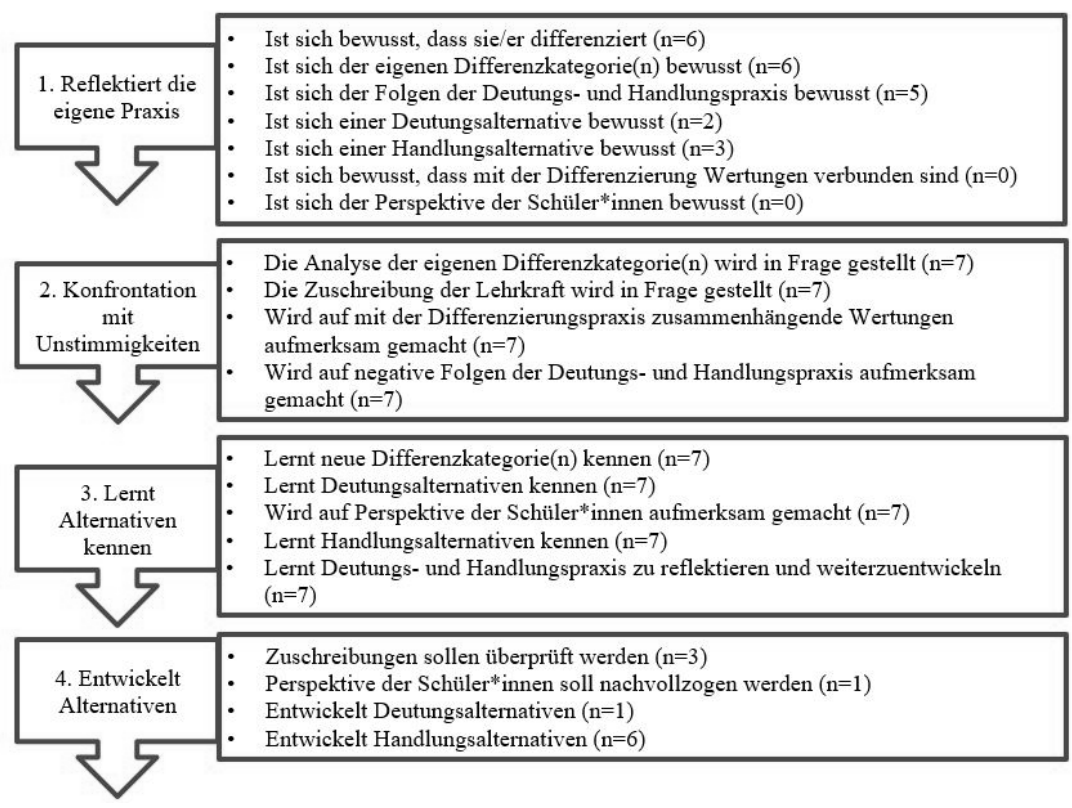

Abb. 2: Ergebnisse der deduktiven und induktiven Inhaltsanalyse $(n=7)$ (eigene Darstellung).

Anmerkung: Die in der 1. Entwicklungsphase mit $n=0$ bezeichneten Entwicklungsschritte sind erst im zweiten Durchlauf (also Phasen 5 bis 8) zu beobachten.

\section{Die Ausprägungen der Veränderungen innerhalb der Entwicklungsphasen}

Im Folgenden werden die Ergebnisse der induktiven Kategorienbildung dargestellt. Sie beschreiben, welcher Art und Ausprägung die Veränderungen waren, die innerhalb der einzelnen Entwicklungsphasen nach Ho und Kolleg*innen (2001) bei den Teilnehmenden zu beobachten waren. ${ }^{6}$

6 Vereinzelt werden die induktiven Kategorien auch in anderen Fortbildungs- bzw. Entwicklungsphasen des Ansatzes sichtbar: So konnten bei einer Teilnehmerin bereits im Austausch Reflexion und ein erweitertes Bewusstsein ihrer Deutungs- und Handlungspraxis festgestellt werden. Diese Ausprägungen der Bewusstheit/Bereitschaft wurden dann in der folgenden Phase kodiert. 


\section{Entwicklungsphase 1: Reflexion der eigenen Deutungs- und Handlungspraxis}

In der Reflexion der eigenen Deutungs- und Handlungspraxis (1. Anwendungsphase) zeigt sich, dass sich nach der ersten Präsenzsitzung fast alle Teilnehmende (sechs von sieben) darüber bewusst sind, dass sie differenzieren, d.h. Unterschiede zwischen den Schüler*innen machen:

Ich merke, wie ich sie kategorisiere, wie ich ihnen Eigenschaften zuschreibe aufgrund von Vorerfahrungen, die ich mit ihnen gemacht habe, und dann ihre Handlungen im Unterricht unterschiedlich bewerte (,Hurra, sie meldet sich', ,Mist, sie ruft schon wieder rein' etc.) (TN3, RB2, Z.16).

Sechs Teilnehmende können außerdem Differenzkategorien benennen, die ihrem Beobachten und Handeln zugrunde liegen:

Ich habe einen Schüler als „sehr langsam“ abgespeichert. Ich habe mich verstärkt um diesen Schüler gekümmert (TN7, RB2, Z.12).

Hingegen lässt sich das Bewusstsein, dass die eigene Differenzierungspraxis mit wertenden Zuschreibungen einhergeht, wie z.B. eine Differenzierung nach „guten“ und „schlechten“ Schüler*innen, erst im zweiten Durchlauf durch die vier Entwicklungsphasen ( 5 bis 8 ) bei einigen Teilnehmenden erkennen. Auch fällt es den Teilnehmenden schwer, sich in die Schüler*innen hineinzuversetzen und deren Handlungslogik nachzuvollziehen; diese Ausprägung ist ebenfalls erst im zweiten Durchlauf zu beobachten. So sind zu Beginn die Vermutungen, warum sich einzelne Schüler*innen verhalten, wie sie sich verhalten, oftmals wenig rational begründet und mit Vorurteilen behaftet:

[...] zudem habe ich ihren Bruder, der dieselben Probleme im Deutschen hat, etwas vernachlässigt, wahrscheinlich, weil er selbstbewusster ist und sich nicht total demoralisieren lässt, auch wenn er wahrscheinlich auf die gleichen Probleme stößt (TN6, RB2, Z.24f.).

Über die Folgen, die ihre eigene Deutungs- und Handlungspraxis hat bzw. haben kann, stellen fünf der Teilnehmenden begründete Vermutungen an. So ist sich eine Teilnehmerin durchaus bewusst darüber, dass sie einem Flüchtlingskind besonders viel Aufmerksamkeit schenkt und die anderen dadurch hintenanstellt, was ihr selbst nicht gefällt. Dennoch fällt ihr zu Beginn die Analyse des eigenen Handelns und die Ableitung von Deutungs- und Handlungsalternativen schwer (vgl. TN 6, RB2, 9ff.). Anfangs sind nur zwei Teilnehmende in der Lage, alternative Situationsdeutungen anzustellen:

Ich hätte nicht nach langsam und schnell unterscheiden müssen. Es hätte auch die Möglichkeit gegeben, den Schüler*innen zwei unterschiedlich schwierige Aufgabentypen zu geben (TN7, RB2, Z.20). 
Alternative Vorgehensweisen oder Handlungen für die jeweiligen Situationen können drei Teilnehmende benennen:

Es wäre möglich gewesen, die beiden Schüler*innen aktiv mit einzubeziehen auf Kosten von denen, die begeistert, aktiv und sehr raumgreifend dabei waren und viel gesagt haben (TN3, RB2, Z.24).

\section{Entwicklungsphasen 2 und 3: Konfrontation mit Unstimmigkeiten der eigenen Deutungs- und Handlungspraxis und Kennenlernen von Deutungs- und Handlungsalternativen}

In der zweiten Präsenzsitzung werden im Rahmen der gemeinsamen Fallarbeit Unstimmigkeiten der Deutungs- und/oder Handlungspraxis einzelner Teilnehmender diskutiert. Dabei werden die Analyse der Differenzkategorie sowie ihre Zuschreibungen durch die Lehrkraft in Frage gestellt. Darüber hinaus werden die mit der Differenzierungspraxis verbundenen Wertungen und negative Folgen der Deutungs- und Handlungspraxis für die Schüler*innen diskutiert. Das folgende Beispiel zeigt die Diskussion der Teilnehmenden darüber, ob die Bevorzugung eines Flüchtlingskindes gerechtfertigt ist:

TN1: Für mich klingt das wie eine Art Mitleidsbonus, das kann ich gut nachvollziehen, mache ich auch manchmal.

TN2: Würde nicht sagen Mitleidsbonus, das ist eher eine Ermunterung.

TN3: Ich finde, das ist Bevorzugung gegenüber anderen Schülern. Der Fokus liegt auf einem bestimmten Manko des Mädchens, das die anderen nicht haben. Das ist die Brille, mit der die Situation betrachtet wird.

Fallstellerin: Stimmt, das war doof. (TN6, TB2, Z.184ff.)

Anschließend werden anhand der Fallbeispiele alternative Deutungen und Handlungen sowie alternative Differenzkategorien besprochen, die Perspektive der Schüler*innen rekonstruiert und es wird von den Dozierenden aufgezeigt, wie die eigene Deutungs- und Handlungspraxis kontinuierlich reflektiert und weiterentwickelt werden kann.

\section{Entwicklungsphase 4: Entwickeln und Erproben von Deutungs- bzw. Handlungsalternativen}

Drei der Teilnehmenden nehmen sich anschließend für die zweite Anwendungsphase vor, ihre Zuschreibungen zu den Schüler*innen zu überprüfen: 
Ich möchte auf meine, Schubladen` im Kopf achten. Kenne ich ,meine Pappenheimer" wirklich so gut? Oder verhalten sich meine Schüler unter Umständen anders, wenn ich sie anderes behandle, anders herausfordere? (TN2, RB3, Z.29)

Einer der Teilnehmenden möchte versuchen, die Perspektive der Schüler*innen einzunehmen, um ihr Handeln nachvollziehen und besser verstehen zu können. Ein weiterer Teilnehmender möchte eine Deutungsalternative testen und sechs Teilnehmende möchten alternative Handlungsweisen ausprobieren:

Ich möchte mich verstärkt um die schwachen Schüler kümmern und ihnen einen größtmöglichen Lernfortschritt ermöglichen. Dabei möchte ich stärkere Schüler gezielt mit geeigneten Aufgabenstellungen versorgen (TN7, RB3, Z.27).

Die nun folgenden Phasen stellen den zweiten Durchlauf der vier von Ho und Kolleg*innen (2001) beschriebenen Entwicklungsphasen dar.

\section{Entwicklungsphase 5: Reflexion der alternativen Deutungs- bzw. Handlungspraxis}

In der darauffolgenden Reflexion der alternativen Deutungs- und Handlungsweisen zeigen sich erste Veränderungen in der Deutungs- und Handlungspraxis der Teilnehmenden: Vier der Teilnehmenden ist nun klar, dass mit ihrer Differenzierungspraxis Wertungen verbunden sind. Eine Lehrkraft erkennt etwa ihre Defizitorientierung im Umgang mit einem Mädchen mit Fluchthintergrund im Fremdsprachenunterricht. Die eigentlich sehr gute Schülerin wird aufgrund mangelnder Deutschkenntnisse als besonders hilfebedürftig eingestuft (vgl. TN6, RB4, Z.42). Der Lehrkraft gelingt es nach der Analyse besser, d.h. differenzierter, die Perspektive der Schülerin einzunehmen, und sie versteht, warum diese immer wieder ihre Aufmerksamkeit einfordert:

Es ist der einzige Unterricht, in dem sie zeigen kann, was sie kann [gemeint ist die unterrichtete Fremdsprache, Anm. d. A.], ein geschützter Raum, der auf einmal wegfällt (TN6, TB2, Z.85).

Sieben Teilnehmende leiten aus der Anwendungsphase Handlungsalternativen ab und sechs sind sich der Folgen ihrer Deutungs- und Handlungspraxis nun bewusster. 


\section{Entwicklungsphase 6 und 7: Konfrontation mit Unstimmigkeiten der eigenen Deutungs- und Handlungspraxis und Kennenlernen von Deutungs- und Handlungsalternativen}

Im Rahmen der kollegialen Beratung in der letzten Präsenzphase erhalten die Teilnehmenden Feedback von Kolleg*innen und Dozierenden zu ihrer in der zweiten Anwendungsphase erprobten Deutungs- und Handlungspraxis. Dabei werden sowohl die Deutungen als auch die Intentionen der Lehrkräfte in Frage gestellt sowie negative Folgen der Deutungs- und Handlungspraxis diskutiert. Die Teilnehmenden lernen alternative Deutungen und Handlungen kennen und werden auf die Perspektive der Schüler*innen aufmerksam gemacht. Auch wird die Möglichkeit, die eigene Deutungs- und Handlungspraxis kontinuierlich zu reflektieren und weiterzuentwickeln erläutert:

Dozierende: Ich finde spannend, dass hier die Selbstbeobachtung hilft, bestehende Konstrukte zu hinterfragen und dann neu auszutesten. Durch Reflexion wird sichtbar, dass ein anderes Vorgehen, als es theoretisch richtig wäre, funktioniert. Das Konstrukt ,der Schüler ist langsam` wird überprüft (TB2, Z.222).

\section{Entwicklungsphase 8: Weiterentwickeln von Deutungs- bzw. Handlungsalternativen und Anbahnung von Transfer}

Nach der letzten Präsenzsitzung reflektieren die Teilnehmenden ihren Lernprozess. Im Rahmen dieser Reflexion lassen sich - über die bereits stattgefundenen Veränderungen hinaus - folgende Veränderungen der Deutungsmuster feststellen. Fünf der Teilnehmenden sind sich nun Deutungsalternativen bewusst und zwei, dass mit ihrer Differenzierung Wertungen verknüpft sind. Die Perspektive ihrer Schüler*innen können zwei Lehrkräfte rekonstruieren und dadurch besser nachvollziehen. Diese Veränderungen im Bewusstsein über die eigenen Deutungsmuster zeigen sich auch in den Handlungsabsichten der Teilnehmenden: Fünf der Teilnehmenden resümieren, dass sie ihre Zuschreibungen dauerhaft aufgeben möchten:

Ich versuche, sie immer mal wieder ,neutral' anzuschauen, mit ,mal sehen, wie ist es heute?'. Das geht nicht durchgehend, aber immer mal wieder, situationsweise. Dabei kam mein Blick auch auf Entwicklungen, die die Schüler*innen gemacht haben. Ich denke nicht immer, jemand ist so', sondern: ,interessant, heute ist das so' (TN3, RB5, Z.49).

Sechs Teilnehmende möchten ihre Deutungs- und Handlungspraxis kontinuierlich reflektieren und weiterentwickeln: 
Man muss sich mal hinsetzen und das tun und bewusst machen. Es braucht Zeit. Gelegenheiten, wenn man sie bekommt, nutzen. Mehr außerunterrichtliches Engagement reinstecken, mehr einsammeln und Zuhause hinsetzen und über die Klasse zu reflektieren. Nicht nur Unterricht methodisch vorzubereiten, sondern auch über Schüler reflektieren, wie geht es denen in meinem Unterricht (TN4, RB5, Z.155).

Alle sieben Teilnehmenden möchten die erprobten Handlungsalternativen, die sich für sie als sinnvoll erwiesen haben, auch weiterhin beibehalten:

Ich habe gelernt, dass sogar manche Störungen daher rühren, dass ich zu sehr manche Schüler*innen unterstützen wollte und dabei andere vergessen habe. Dies versuche ich zu berücksichtigen und habe daher auch weniger Störungen im Unterricht (TN6, RB5, Z.56).

\section{Veränderung von Deutungs- und Handlungsmustern zum Umgang mit Heterogenität im Rahmen der Fortbildung}

Die Frage, inwiefern im Rahmen der Fortbildung Deutungs- und Handlungsmuster weiterentwickelt werden können, lässt sich auf Basis der Ergebnisse der qualitativen Inhaltsanalyse prozessbezogen nachvollziehen. Mithilfe der deduktiven Kategorienbildung konnte festgestellt werden, dass sieben der 15 Teilnehmenden die Entwicklungsphasen Reflexion, Konfrontation, Kennenlernen von Alternativen und Transfer in die Praxis (vgl. Ho et al. 2001) zweimal durchlaufen und dementsprechend auch ihre Deutungs- und/oder Handlungsmuster verändert haben. Die aus dem Material heraus gebildeten induktiven Kategorien zeigen darüber hinaus, was im Rahmen dieser Prozesse bei den einzelnen Teilnehmenden passiert. So lassen sich unterschiedliche Ausprägungen der Bewusstheit der eigenen Deutungs- und Handlungsmuster im Umgang mit Heterogenität finden. Erfreulicherweise kann bei allen aktiven Teilnehmenden im Laufe der Fortbildung diesbezüglich ein Zuwachs beobachtet werden, d.h. es wurden neue Ausprägungen der Bewusstheit dazugewonnen, wenn auch in unterschiedlichem Maße. Das Bewusstsein über die eigene Deutungs- und Handlungspraxis wurde weiter ausdifferenziert.

Gleichzeitig lassen sich unterschiedliche Absichten, die eigenen Deutungsund Handlungsmuster weiterzuentwickeln und Alternativen in der eigenen Unterrichtspraxis umzusetzen, finden. Während manche Teilnehmenden sich auf die Entwicklung von Handlungsalternativen fokussieren, stehen bei anderen die Überprüfung und Veränderung von Zuschreibungen und die Einnahme der 
Perspektive der Schüler*innen im Vordergrund. Wieder anderen streben einen „reflexiven Habitus“ an und wollen ihre Deutungs- und Handlungspraxis kontinuierlich reflektieren und weiterentwickeln.

Die Ergebnisse der qualitativen Inhaltsanalyse zeigen, dass das reflexionszentrierte Fortbildungskonzept eine Auseinandersetzung mit und auch Veränderung von individuellen Deutungs- und Handlungsmustern ermöglichen kann (vgl. Arnold 1985, Ho et al. 2001). Die ergebnisorientierte (Selbst-)Reflexion (Greif 2008, Pachner 2013, 2014), der auf Perspektivverschränkungen angelegte Austausch (vgl. Siebert 2012) und die integrierten Erprobungsphasen wurden von den Teilnehmenden als Anlässe dafür genutzt, sich neuer Aspekte ihrer Deutungs- und Handlungsmuster bewusst zu werden und diese entsprechend weiterzuentwickeln (vgl. Ho et al. 2001). Diese Prozesse deuten auf eine Veränderung impliziter Deutungs- und Handlungsmuster hin, einer wichtigen Voraussetzung für transformatives Lernen (vgl. Arnold 1985).

\section{Diskussion und Ausblick}

Die Ergebnisse dieser Studie bieten einen ersten Einblick in Prozesse der Auseinandersetzung mit und die Veränderung von Deutungs- und Handlungsmustern zum Umgang mit Heterogenität im Rahmen einer erwachsenenpädagogisch fundierten Fortbildung. ${ }^{7}$ In weiterführenden fallbasierten und qualitativrekonstruktiven Analysen wäre es nun vielversprechend, mögliche Verbindungen zwischen den unterschiedlichen Ausprägungen und Aspekten der Bewusstheit über die eigenen Deutungs- und Handlungsmuster einerseits und der Bereitschaft und Absicht der Teilnehmenden, das eigene Beobachten und Handeln weiterzuentwickeln und Alternativen in die eigene Unterrichtspraxis umzusetzen andererseits zu untersuchen und ggf. auch Entwicklungsstufen dabei $\mathrm{zu}$ rekonstruieren.

Um herauszufinden, warum manche Teilnehmende nicht alle Entwicklungsphasen durchlaufen haben, sind zudem quantitative Analysen mit einer größeren Fallzahl notwendig. Im Rahmen einer größer angelegten Studie könnten so systematisch Gelingensbedingungen erarbeitet werden, die Aus-

Auch wenn das heterogenitätssensible Lehrhandeln in der Erwachsenenbildungspraxis und -forschung unter anderen Begriffen verhandelt wird, ist ein kritisch-reflexiver Umgang mit der eigenen Differenzierungspraxis ein bedeutendes Kriterium professioneller erwachsenenpädagogischer Praxis (vgl. Siebert 2012). Dementsprechend sind die Ergebnisse auch übertragbar auf Professionalisierungsangebote für (andere) Zielgruppen der Erwachsenenbildung. 
kunft über die Bedingungskonstellationen und Einflussfaktoren auf das erfolgreiche Lernen in reflexionszentrierten Lernumgebungen geben. Beispielsweise könnten neben dem methodisch-didaktischen Setting auch Merkmale der Teilnehmenden wie die motivationale Orientierung ausschlaggebend für erfolgreiche Veränderungsprozesse sein.

Es wäre dabei außerdem ein wichtiges Anliegen herauszufinden, in welcher Form möglichst viele Lehrkräfte bezüglich eines reflektierten Umgangs mit Heterogenität bzw. Differenz fortgebildet werden können etwa im Rahmen von Schulentwicklungsmaßnahmen. Mit der hier vorgestellten Studie konnte gezeigt werden, dass reflexionszentrierte Fortbildungsangebote, die auf eine Sensibilisierung bzgl. der eigenen Deutungs- und Handlungsmuster abzielen, eine wertvolle Unterstützung für die Lehrkräfte bieten können, ihr Lehrhandeln immer wieder neu situationsangemessen zu gestalten.

\section{Literatur}

Arnold, Rolf (1985): Deutungsmuster und pädagogisches Handeln in der Erwachsenenbildung. Bad Heilbrunn: Klinkhardt.

Beck, Ulrich / Giddens, Anthony / Lash, Scott (1996): Reflexive Modernisierung: Eine Kontroverse. Frankfurt am Main: Suhrkamp.

Biederbeck, Ina / Rothland, Martin (2017): Professionalisierung des Umgangs mit Heterogenität. In: Bohl, T. / Budde, J. / Rieger-Ladich, M. (Hrsg.): Umgang mit Heterogenität in Schule und Unterricht. Grundlagentheoretische Beiträge, empirische Befunde und didaktische Reflexionen. Bad Heilbrunn: Klinkhardt, S. 223-235.

Emmerich, Marcus / Goldmann, Daniel (2018): Tübinger Fortbildung zu Heterogenität in Unterricht und Schule (TüFHUS). Verfügbar unter: https://uni-tuebingen.de/de/126119, [Stand 25.01.2019].

Emmerich, Marcus / Hormel, Ulrike (2013): Heterogenität - Diversity - Intersektionalität. Zur Logik sozialer Unterscheidungen in pädagogischen Semantiken der Differenz. Wiesbaden: Springer.

Flick, Uwe (2009): Triangulation in der qualitativen Forschung. In: Flick, U./von Kardorff, E./Steinke, I. (Hrsg.): Qualitative Forschung. Ein Handbuch. Hamburg: Rowohlt, S. 309-318.

Greif, Siegfried (2008): Coaching und ergebnisorientierte Selbstreflexion. Göttingen [u.a.]: Hogrefe.

Ho, Angela (2000): A conceptual change approach to staff development: a model for programme design. In: International Journal of Academic Development 5, 1, S. 30-41. 
Ho, Angela / Watkins, David / Kelly, Mavis (2001): The conceptual change approach to improving teaching and learning: An evaluation of a Hong Kong staff development program. In: Higher Education 42, S. 143-169.

Hof, Christiane (2007): Ein empirisch fundierter Vorschlag zur Typisierung von Lernumgebungen. In: Kaiser, A. / Kaiser, R. / Hohmann, R. (Hrsg.): Lernertypen - Lernumgebung - Lernerfolg. Bielefeld: W. Bertelsmann, S. 35-59.

Koller, Hans-Christoph (2017): Bildung as a Transformative Process. In: Laros, A. / Fuhr, T. / Taylor, E. W. (Hrsg.): Transformative Learning meets Bildung. An International Exchange. Rotterdam: Sense, S. 33-42.

Mayring, Philipp A. E. (2015): Qualitative Inhaltsanalyse. Grundlagen und Techniken (12. Aufl.). Weinheim und Basel: Beltz.

Mezirow, Jack (1997): Transformative Erwachsenenbildung. Baltmannsweiler: Schneider-Verlag Hohengehren.

Müller, Kurt R. (1998): Erfahrung und Reflexion: „Fallarbeit“ als Erwachsenenbildungskonzept. In: Grundlagen der Weiterbildung, 6, S. 273-277.

Pachner, Anita (2013): Selbstreflexionskompetenz. Voraussetzung für Lernen und Veränderung in der Erwachsenenbildung? In: Magazin erwachsenenbildung.at. Das Fachmedium für Forschung, Praxis und Diskurs, 20, S. 1-9. Verfügbar unter: http://www.erwachsenenbildung.at/magazin/13-20/meb13-20.pdf, [Stand 25.01.2019].

Pachner, Anita (2014): Die Metakompetenz „Selbstreflexion“ und ihre Bedeutung für pädagogisch Tätige und deren Professionalitätsentwicklung. In: Heyse, V. (Hrsg.): Aufbruch in die Zukunft. Erfolgreiche Entwicklungen von Schlüsselkompetenzen in Schulen und Hochschulen. Münster: Waxmann, S. 434-447.

Pachner, Anita (2018): Digital unterstütze Lernprozesse - Chancen und Herausforderungen für die Rolle der Lehrenden. Forum Erwachsenenbildung, 51(3), S. 19-23.

Schön, Donald A. (1983): The reflective practitioner. How professionals think in action. New York: Basic Books.

Schüßler, Ingeborg (2000): Deutungslernen. Erwachsenenbildung im Modus der Deutung - Eine explorative Studie zum Deutungslernen in der Erwachsenenbildung. Baltmannsweiler: Schneider-Verlag Hohengehren.

Siebert, Horst (2012): Didaktisches Handeln in der Erwachsenenbildung. Didaktik aus konstruktivistischer Sicht (7. überarb. Aufl.). Augsburg: ZIEL.

Terhart, Ewald (2015): Umgang mit Heterogenität: Anforderungen an Professionalisierungsprozesse. In: Fischer, Ch. (Hrsg.): (Keine) Angst vor Inklusion. Herausforderungen und Chancen gemeinsamen Lernens in der Schule (Münstersche Gespräche zur Pädagogik). Münster: Waxmann, S. 69-85.

Tietgens, Hans (1988): Professionalität für die Erwachsenenbildung. In: Gieseke, W. (Hrsg.): Professionalität und Professionalisierung. Bad Heilbrunn: Klinkhardt, S. 28-75. 

Matthias Rohs, Ricarda Bolten

\section{Einstellungen von Erwachsenenbildner*innen zur digitalen Transformation der Weiterbildung}

\section{Digitale Transformation in der Erwachsenenbildung}

Die Erwachsenenbildungsforschung hat sich früh mit den Herausforderungen der „neuen“ bzw. digitalen Medien auseinandergesetzt (z.B. Faulstich, 1985; Hüther, 1987; Terlinden, 1988) ${ }^{1}$; zum einen auf Angebotsebene und zum anderen mit den Möglichkeiten des Einsatzes digitaler Medien zur Unterstützung von Lehr-/Lernprozessen und den damit verbundenen Auswirkungen auf die Qualifikationsanforderungen der Beschäftigten. Die Integration „neuer“ bzw. digitaler Medien in die erwachsenenpädagogische Praxis war aber - bis auf Ausnahmen - vergleichsweise zurückhaltend (vgl. Troesser, 1985). Neben den fehlenden Ressourcen für die technische Ausstattung könnte dabei eine Skepsis gegenüber der technologischen Entwicklung eine Rolle gespielt haben. So warnte der Deutsche Volkshochschulverband davor ,alte und gescheiterte unterrichtstechnologische Modelle ohne ausreichende soziale Phase des Lernens wieder aufleben zu lassen“ (zitiert nach Troesser 1985: S. 111).

Insgesamt waren die Diskurse der Erwachsenenbildung zur Medien- und Technikentwicklung in den 1980er und 1990er Jahren von gegenläufigen Positionen geprägt: zwischen „Kulturpessimismus und Technikeuphorie“ (Schmid, 1986, S. 95) oder zwischen „Technologiefetischismus oder Maschinenstürmerei“" (Terlinden, 1988, S. 36).

Diese Muster sind bis heute erkennbar. Trotz einer starken Bedeutungszunahmen des Themas zeigt sich bisher eine vergleichsweise zögerliche Auseinandersetzung (vgl. Happel, 2018; Jörissen, 2013). Dabei sind auch deutliche Unterschiede zwischen den verschiedenen Weiterbildungssegmenten auszumachen (Schmid, Goertz, \& Behrens, 2018).

Der folgende Beitrag widmet sich der Frage nach den Gründen für die unterschiedlichen, in der Breite zögerlichen Adaptionsgeschwindigkeiten bei der Nutzung digitaler Medien in der Erwachsenenbildungspraxis. Dabei stehen die Lehrenden als zentrale Akteure sowie deren Einstellungen zur digitalen Trans-

1 Zunächst noch unter dem Stichwort „Neue Medien“, welche neben der Nutzung von Personalcomputern z.B. auch BTX oder Satellitenfernsehen umfassten. 
formation allgemein und insbesondere in Bezug auf die Lehrpraxis im Vordergrund, da diese Perspektive im Bereich der Erwachsenenbildung/Weiterbildung bisher noch nicht untersucht wurde, ihr jedoch grundsätzlich eine große Bedeutung zugesprochen werden kann.

Unter digitaler Transformation werden dabei die Auswirkungen einer Digitalisierung (als technischer Prozess) auf die Strukturen, Prozesse, Angebote, Geschäftsmodelle, Kompetenzanforderungen u.a. in der Erwachsenenbildung/ Weiterbildung verstanden. Dabei wird davon ausgegangen, dass mit der Digitalisierung - zumindest in Teilbereichen - grundsätzliche Veränderungen der Weiterbildungspraxis und des Selbstverständnisses der Professionellen in diesem Feld einhergehen können (Transformationen) wie z.B. Veränderungen des Aufgabenspektrums der Erwachsenenbildner*innen, der angebotenen Formate, der Kommunikation mit den Teilnehmenden u.a. (vgl. Rohs, 2019a).

\section{Einstellungen und Überzeugungen von Erwachsenenbildner*innen zur digitalen Transformation}

Den Einstellungen von Lehrenden wird neben den medienpädagogischen Kompetenzen und den Ressourcen eine zentrale Rolle für die Integration digitaler Medien in die unterrichtliche Praxis zugesprochen (z.B. Hew \& Brush, 2006; Knezek \& Christensen, 2016). Bei Einstellungen handelt es sich um ein psychologisches Konstrukt, welches durch eine große Heterogenität der definitorischen Zugänge charakterisiert ist (vgl. Banaji \& Heiphetz, 2010). Eine verbreitete Definition von Eagly and Chaiken (1993) beschreibt Einstellungen (attitudes) als ,,a psychological tendency that is expressed by evaluating a particular entity with some degree of favor or disfavor" (ebd., S. 1). In dem hier behandelten Kontext bedeutet dies eine Befürwortung oder Ablehnung digitaler Medien zur Unterstützung von Lehr-/Lernprozessen.

Einstellungen unterscheiden sich von Überzeugungen (beliefs), d.h. ,,individual mental constructs, which are subjectively true for the person" (Skott, 2015, S. 18). Überzeugungen von Lehrenden ,include their educational beliefs about teaching and learning (i.e., pedagogical beliefs), and their beliefs about technology“ (Hew \& Brush, 2006, S. 229). Diese Überzeugungen prägen entsprechende Einstellungen zur Nutzung digitaler Medien.

Für den Bereich der Weiterbildung liegen (soweit bekannt) keine expliziten Untersuchungen $\mathrm{zu}$ den technologiebezogenen Einstellungen und Überzeugungen der Professionellen in diesem Feld vor, jedoch wurden in verschiedenen Untersuchungen einzelne diesbezügliche Aspekte erfragt. So waren sich 
fast 60 Prozent der befragten Lehrenden der Erwachsenenbildung $(n=260)$ in einer Bertelsmann-Studie (U. Schmid et al., 2018) einig, dass digitale Medien die Attraktivität von Erwachsenenbildungseinrichtungen erhöhen, und etwas mehr als die Hälfte der Erwachsenenbildner*innen waren der Meinung, dass digitale Medien den Zugang zur Bildung in ländlichen Gebieten motivieren und fördern können. Im Gegensatz dazu erwarteten nur etwas mehr als ein Drittel der Befragten, dass die Nutzung digitaler Medien zur Verbesserung der Unterrichtsqualität (37 Prozent) und der Lernergebnisse (36 Prozent) beiträgt. Besonders skeptisch zeigten sich die Lehrenden in Bezug auf positive Auswirkungen digitaler Medien für sozial benachteiligte Lernende. Außerdem wird nur von etwa einem Drittel (31 Prozent) der Lehrenden erwartet, dass digitale Medien ihre Arbeitsbelastung reduzieren (ebd.). Letzteres erscheint von besonderer Bedeutung, da ein Großteil der Erwachsenenbildner*innen in Deutschland unter prekären Bedingungen und in ständigem Zeitmangel arbeitet (Martin \& Langemeyer, 2014).

Eine Studie aus der Schweiz, bei der 338 Weiterbildungseinrichtungen befragt wurden, kommt zu ähnlichen Ergebnissen (Sgier, Haberzeth, Schüepp, 2018). So hat sich gezeigt, dass einem großen Teil der digitalen Lehranwendungen nur ein geringer didaktischer Nutzen zugesprochen wird. Zwar gaben 60 Prozent der Befragten an, dass die Nutzung digitaler Medien die individuelle Unterstützung der Lernenden verbessere, aber nur 39 Prozent erwarteten eine Verbesserung der Lernergebnisse. Analog zur Bertelsmann-Studie zeigt sich auch, dass die Auswirkungen digitaler Lehr-/Lernprozesse für „Lernungewohnte" als schwierig eingeschätzt werden. Außerdem werden die Qualifikationsanforderungen an die Lehrkräfte bei der Nutzung digitaler Medien als hoch eingeschätzt (71 Prozent) und nur wenige Befragte nehmen die Nutzung digitaler Medien als „Entlastung“ wahr (27 Prozent) (ebd.).

Insgesamt zeigt sich zwar eine Offenheit gegenüber digitalen Medien, jedoch wird deren Nutzen sehr differenziert eingeschätzt. Vor allem für Personen mit Benachteiligungen wird er als eher gering bewertet. Außerdem stellt technologieunterstütztes Lernen nach Meinung der Befragten hohe Anforderungen an Kompetenzen und Zeit der Lehrenden. In diesem Konflikt - sehr spezifischer Nutzen und hoher Aufwand - kann eine mögliche Ursache für einen teilweise noch geringen Digitalisierungsgrad der Weiterbildung liegen, insbesondere dort, wo der ökonomische Druck hoch und die Nachfrage der Lernenden gering ist. Einschränkend ist dabei zu erwähnen, dass der Kompetenzstand der befragten Lehrenden dabei unberücksichtigt bleibt, d.h. auf welchem Kompetenzniveau die Befragten zu den gemachten Einschätzungen gekommen sind, ist nicht bekannt. 


\section{Erhebung zur Einstellung von Erwachsenenbildner*innen zur digitalen Transformation}

Aus einer 2017 durchgeführten Umfrage zur medienpädagogischen Kompetenz von Lehrenden in der Erwachsenenbildung wurden die Items, die sich auf den Bereich der medienbezogenen Einstellungen beziehen, gesondert in Bezug zu medienbezogenen Einstellungstypen und sozio-ökonomischen Faktoren betrachtet, um mögliche Einflussfaktoren auf die Einstellungen der Lehrenden zu identifizieren. Hintergrund dieser gesonderten Betrachtung der Items war die Vermutung, dass die medienbezogenen Einstellungen einen Einfluss auf die Nutzung digitaler Medien in der beruflichen Praxis von Lehrenden in der Erwachsenenbildung haben (Bolten, 2018; Rohs \& Bolten, 2017).

\section{MEKWEP-Kompetenzmodell}

Medienbezogene Einstellungen können als Teil der medienpädagogischen Kompetenz von Erwachsenenbildner*innen angesehen werden. Grundlage für die hier vorgestellte Studie ist ein medienpädagogisches Kompetenzmodell, das im Rahmen des Projektes MEKWEP ${ }^{2}$ entwickelt wurde. Das Modell wurde durch die Synthese einer Literaturrecherche von medienpädagogischen Kompetenzmodellen (vor allem im Bereich der Lehrerbildung, z.B. Blömeke, 2001; Blömeke, 2005; Herzig, 2007; Mayrberger, 2012), der Analyse allgemeiner Kompetenzmodelle aus der Erwachsenenbildung sowie auf Grundlage von Interviews und Expertenfokusgruppen entwickelt. Das Kompetenzverständnis orientiert sich an einem Zusammenwirken von Wissenskomponenten mit motivationalen und volitionalen Faktoren (vgl. Weinert, 2001). Das medienpädagogische Kompetenzmodell (vgl. Abb. 1) besteht aus vier Kompetenzfacetten: medienbezogene Feldkompetenz, medienbezogene Fachkompetenz, mediendidaktische Kompetenz sowie medienbezogene Einstellungen und Selbststeuerung (Schmidt-Hertha, Rohs, Rott, Bolten, 2017).

2 MEKWEP: Medienpädagogische Kompetenz des beruflichen Weiterbildungspersonals zur Unterstützung des Einsatzes digitaler Medien in formalen, non-formalen und informellen Lernsettings - weitere Informationen unter: www.die-bonn.de/mekwep (25.06. 2019) 


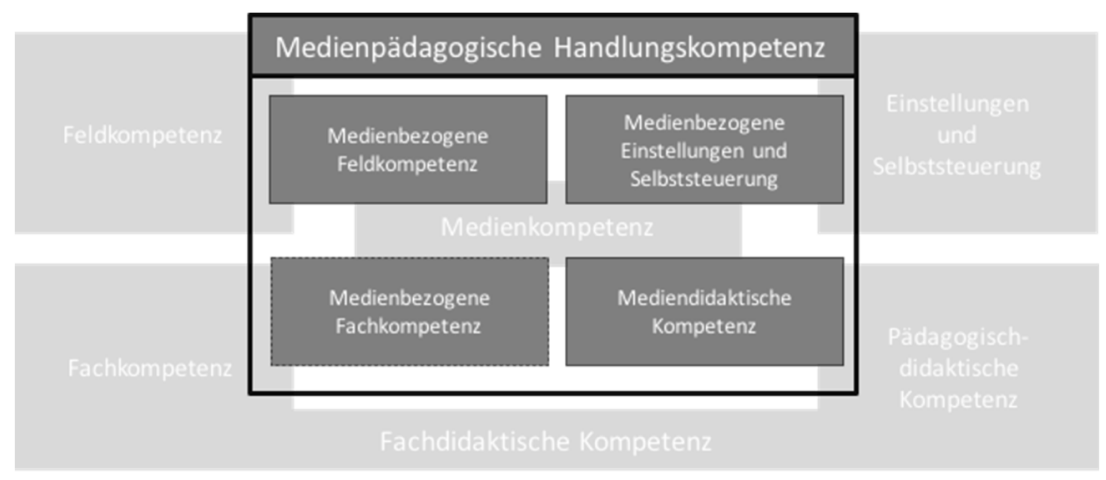

Abbildung 1: Medienpädagogische Handlungskompetenz von Lehrenden in der Erwachsenenbildung (Schmidt-Hertha et al., 2017, S. 36).

Die vorliegende Studie konzentriert sich auf die Kompetenzfacette der medienbezogenen Einstellungen und Selbststeuerung. Diese Facette umfasst die persönliche Einstellung der Lehrenden zu Medien und medienbezogenen Entwicklungen in der Gesellschaft. Darüber hinaus werden hier auch selbstregulatorische Aspekte zugeordnet, die Lehrende benötigen, um beispielsweise ihren Alltag unter den Bedingungen der Digitalisierung in einer Balance zwischen Arbeit und Privatleben zu gestalten.

Für die Bildung der Einstellungstypen wurden Selbsteinschätzungsskalen zu medienbezogenen Einstellungen (31 Items) herangezogen. Die Items wurden aufgrund ihrer inhaltlichen Aussage in fünf Gruppen zu Einstellungstendenzen unterteilt:

Positiv chancenorientiert (11 Items): Lehrende mit einer positiven und chancenorientierten Einstellung beschreiben den Einsatz von Medien als einen Mehrwert für den Lehr-/Lernprozess. Medien werden in der Lehre zur Unterstützung von Lernprozessen eingesetzt und die Wege zu Lerninhalten werden vereinfacht. Lehrende nutzen gerne Medien in ihrem Unterricht und sehen z.B. das Internet als Möglichkeit, auf Lernmaterialien zuzugreifen. Sie sind jedoch nicht sehr kritisch und verwenden Medien, wenn dies erwartet wird, ohne diesen Einsatz konsequent zu reflektieren.

Kritisch reflektiert (6 Items): Diese Einstellung zeichnet sich durch den kritisch-reflektierten Einsatz digitaler Medien aus. Quellen werden vor ihrer Verwendung geprüft und nur dann verwendet, wenn sie einen Mehrwert für die Lernenden bringen. Digitale Medien werden für eine vielfältige Gestaltung des Unterrichts eingesetzt und auch, um den Lernenden neue Wege des Lernens zu zeigen.

Vorsichtig und ablehnend (5 Items): Die vorsichtige und ablehnende Einstellung ist durch eine vermeidende Haltung gegenüber digitalen Medien gekennzeichnet. Die Lehrenden haben Angst vor der Nutzung digitaler Medien 
und befürchten, dass der Einsatz digitaler Medien ihre Rolle als Lehrperson überflüssig mache. Digitale Medien gelten als unwichtig für die Lehre und technologische Entwicklungen sollten eher zurückgedrängt werden.

Pauschal ablehnend (5 Items): Die pauschal ablehnenden Lehrenden zeigen eine positive Einstellung gegenüber analogen und eine negative Haltung gegenüber digitalen Medien. Analoge Medien gelten als vertrauenswürdig und für die Nutzung digitaler Medien wird keine Notwendigkeit gesehen. Diese Einstellung ist auch von der Angst geprägt, dass digitale Medien die Rolle des Lehrers/der Lehrerin überflüssig machen könnten.

Begründet ablehnend (4 Items): Die Einstellung der begründet Ablehnenden ist dadurch gekennzeichnet, dass die Nutzung digitaler Medien abgelehnt wird, aber nicht aus Angst, sondern weil diese als für den Unterricht der Lehrenden überflüssig angesehen werden und das breite Spektrum an Lernmöglichkeiten zudem für die Lernenden überfordernd sein kann. Darüber hinaus halten diese Lehrenden digitale Kommunikationskanäle mit den Lernenden für zu unpersönlich.

\section{Erhebung und Ergebnisse}

Basierend auf dem beschriebenen medienpädagogischen Modell (siehe Abb. 1) wurde 2017 eine Online-Umfrage unter Erwachsenenbildner*innen zur Untersuchung ihrer medienpädagogischen Kompetenzen und medienbezogenen Einstellungen durchgeführt. Neben den medienpädagogischen Items wurden sozio-ökonomische Daten, Informationen über die Beschäftigungsgeschichte sowie die alltägliche und berufliche Nutzung digitaler Medien erhoben. Zur Betrachtung der Einstellungstypen wurden in der Analyse die Ergebnisse von 626 Befragten ausgewertet.

Zum vorliegenden Datensatz ist anzumerken, dass es eine Verzerrung hinsichtlich der Verteilung der Weiterbildungseinrichtungen gibt. Diese begründet sich durch einen besonders starken Rücklauf von Lehrenden aus Volkshochschulen, an denen durchschnittlich mehr Frauen lehrend tätig sind, wodurch prozentual mehr Frauen an der Umfrage teilnahmen, als es die allgemeine Verteilungsstruktur erwarten lassen würde. Darüber hinaus zeigten zwei Bildungsuntergruppen (Hauptschule: $n=5$; Polytechnische Schule: $n=11$ ) eine geringe Anzahl von Fällen, weswegen diese von der statistischen Analyse ausgeschlossen wurden. Die meisten der Befragten sind weiblich $(64 \%)$ und an Volkshochschulen tätig (69\%). 76\% der Befragten haben ein Abitur als höchsten Schulabschluss. Aufgrund der Vorannahme, dass sozio-ökonomische Faktoren einen Einfluss auf die medienbezogenen Einstellungen der Lehrenden haben, wurden auf Grundlage von Hypothesen Bereiche identifiziert, die in der Analyse genauer betrachtet wurden (Alter, Bildung, Geschlecht, Haupt- und 
Nebenbeschäftigung sowie die Teilnahme an einer medienpädagogischen Weiterbildung). So wurde z.B. angenommen, dass ein höherer Bildungsabschluss einen positiven Einfluss auf eine kritisch reflektierte Auseinandersetzung mit digitalen Medien hat. Auch wurde vermutet, dass die Teilnahme an einer medienpädagogischen Weiterbildung positiven Einfluss auf einen kritisch reflektierten Medienumgang hat.

Zur Analyse der medienbezogenen Einstellungen der Lehrenden wurden die gebildeten Einstellungstypen auf interne Konsistenz getestet. Die interne Konsistenz der additiven gemittelten Indizes wurde mit Cronbachs Alpha gemessen. Tabelle 1 gibt einen Überblick über alle abhängigen berechneten Indizes:

Tabelle 1: Überblick über abhängige Variablen

\section{Einstellungen von Erwachsenenbildner*innen gegenüber digitalen Medien}

\begin{tabular}{ll}
\hline Positive chancenorientiert & Skalen von 11 Items, Cronbach's- $\alpha=0.807$ \\
\hline Kritisch reflektiert & Skalen von 6 Items, Cronbach's- $\alpha=0.770$ \\
\hline Vorsichtig ablehnend & Skalen von 5 Items, Cronbach's- $\alpha=0.685$ \\
\hline Pauschal ablehnend & Skalen von 5 Items, Cronbach's- $\alpha=0.615$ \\
\hline Begründet ablehnend & Skalen von 4 Items, Cronbach's- $\alpha=0.575$ \\
\hline
\end{tabular}

Die Einstellungstypen wurden durch die Durchführung von mittleren T-Vergleichen und multivariaten Regressionsanalysen auf Unterschiede zwischen verschiedenen Untergruppen hin analysiert (vgl. Tabelle 2). Die Ergebnisse der mittleren Vergleichstests zeigten teilweise Zusammenhänge zwischen Einstellungstypen und sozio-ökonomischen Faktoren. Grau schattierte Bereiche zeigen mit $\mathrm{P}<0,05$ hochsignifikante Mittelwertunterschiede.

Demnach gibt es keine signifikanten mittleren Unterschiede für das Geschlecht. Die negativen dimensionalen Skalen zeigen nur für die „Hauptbeschäftigung“ und ",medienpädagogische Weiterbildung“ signifikante mittlere Unterschiede. Außerdem sind Lehrerende mit Abitur und diejenigen, die an einer Weiterbildung zu digitalen Medien teilgenommen haben, digitalen Medien gegenüber deutlich positiver und chancenorientierter eingestellt. Hier bestätigt sich möglicherweise ein Zusammenhang zwischen medienbezogenem bzw. medienpädagogischem Wissen und einer positiveren Einstellung gegenüber digitalen Medien. Darüber hinaus waren jüngere Menschen signifikant kritischer und reflektierter als die ältere Kohorte (Ø: 1.8007 / 2.1889). Dieses Ergebnis entspricht insofern den Erwartungen, als die Gruppe der Jüngeren (Jüngere: unter 30 Jahren; Ältere: über 61 Jahre) tendenziell über detaillierteres Medienwissen verfügt und damit auch die mit der Mediennutzung verbundenen Gefahren besser einschätzen kann (vgl. Initiative D21, 2018). In gleicher 
Weise ist es auch wenig überraschend, dass Menschen mit höherem Schulabschluss positiver und chancenorientierter (Ø: 2.1541 / 2.4490) sowie kritisch reflektierender (Ø: 2.0737 / 2.1450) sind. Auch diese Ergebnisse bestätigen die Befunde anderer Studien, nach denen Personen mit hohem Bildungsniveau eher zur Gruppe der „Digitalen Vorreiter*innen“" gehören (ebd.).

Darüber hinaus waren Menschen, die nicht an einer Volkshochschule arbeiten (Ø: 1.9759 / 2.1923) und nicht hauptbeschäftigt in der Erwachsenenbildung arbeiten (Ø: 1.9701 / 2.2462), deutlich kritisch reflektierter. Gleichzeitig waren Lehrende, die hauptberuflich tätig waren, weniger pauschal ablehnend (Ø: 5.0537 / 4.8814) sondern begründeten ihre Ablehnung (Ø: 4.3685 / 4.1949). Ein ähnliches Muster zeigte sich bei Lehrenden, die an einer medienpädagogischen Weiterbildung teilgenommen hatten. Diese Untergruppe war positiver und chancenorientierter (Ø: 2,0995 / 2,2987) sowie kritisch reflektierter (Ø: 1,9233 / 2,3716), gleichzeitig weniger vorsichtig (Ø: 5,0855 / 4,8353) und pauschal ablehnend (Ø: 5,0720 / 4,8163) sowie begründet ablehnend (Ø: 4,3916 / 4,1237) gegenüber digitalen Medien eingestellt.

Insgesamt lässt sich schlussfolgern, dass es Hinweise auf systematische und strukturelle Unterschiede zwischen den Untergruppen gibt. Schulabschluss, Beschäftigungskontext und Kompetenzentwicklung korrelieren und beeinflussen die Einstellung zu digitalen Medien. Ein hohes Bildungsniveau ist dabei nicht gleichzusetzen mit blinder Euphorie, sondern mit einer kritischen-reflexiven Offenheit, bei der die Chancen digitaler Medien wahrgenommen werden, mögliche Risiken aber bekannt sind und sich im Nutzungsverhalten niederschlagen. Da sich dieser Zusammenhang auch nach dem Besuch einer medienpädagogischen Weiterbildung zeigte, kann dies auch als Indiz dafür genommen werden, dass die medienpädagogische Professionalisierung einen Effekt darauf hat, dass Lehrende dem Einsatz digitaler Medien offener gegenüberstehen und Chancen der Digitalisierung eher wahrnehmen bzw. den Medieneinsatz auch begründeter ablehnen. 


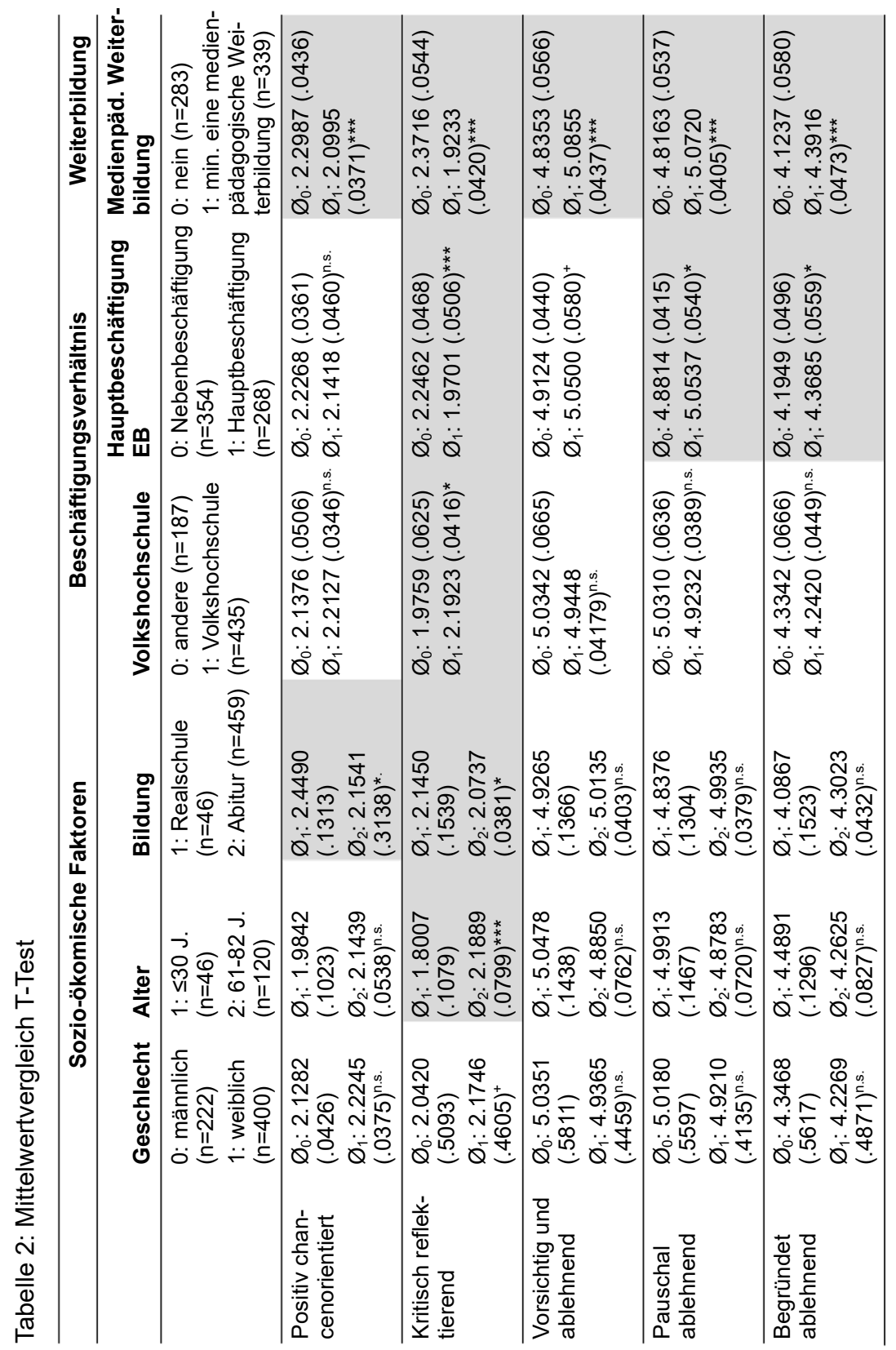




\section{Ausblick}

Aus den Ergebnissen der Studie lässt sich der Schluss ziehen, dass eine medienpädagogische Professionalisierung der Lehrenden eine wichtige Voraussetzung für eine kritisch-reflektierte Nutzung digitaler Medien ist. Das Wissen über die Potenziale digitaler Medien, aber auch die damit verbundenen Risiken stehen dabei im Zusammenhang mit der Einstellung und haben somit auch Einfluss auf die Nutzung digitaler Medien in der Lehrpraxis. Hieraus ergibt sich die Forderung nach einer stärkeren Berücksichtigung medienpädagogischer Inhalte in der Aus- und Weiterbildung von Lehrenden in der Erwachsenenbildung sowie insgesamt nach einer Ausweitung des Angebots (vgl. Rohs, 2019b). Dabei spielen auch kostenlose und qualitätsgesicherte Online-Angebote wie Informationsseiten ${ }^{3}$ oder MOOCs ${ }^{4}$ eine wichtige Rolle, um informelle Lernprozesse zu unterstützen und aktuelle Entwicklungen aufzugreifen. Bisher gibt es jedoch kaum Orientierungsmöglichkeiten bezüglich der relevanten medienpädagogischen Kompetenzen, welche in der Erwachsenenbildung notwendig sind (Rohs, Bolten, \& Kohl, 2017). Die (breitere) Verankerung entsprechender Kompetenzbeschreibungen in den Kerncurricula der Erwachsenenbildung sowie sektoralen Kompetenzmodellen wäre daher von großer Bedeutung.

\section{Literatur}

Banaji, M. R. \& Heiphetz, L. (2010). Attitudes. In S. T. Fiske, D. T. Gilbert \& G. Lindzey (Hrsg.), Handbook of Social Psychology (5. Aufl., S. 348-388). New York: Wiley.

Blömeke, S. (2001). Analyse von Konzepten zum Erwerb medienpädagogischer Kompetenz. Folgerungen aus den Ansätzen von Dieter Baacke und Gerhard Tulodziecki. In B. Bachmair, D. Spanhel, C. de Witt (Hrsg.), Jahrbuch Medienpädagogik 2 (S. 27-47). Opladen: Leske + Budrich.

Blömeke, S. (2005). Medienpädagogische Kompetenz. Theretische Grundlagen und erste empirische Befunde. In A. Frey, R. S. Jäger, U. Renold (Hrsg.), Kompetenzdiagnostik - Theorien und Methoden zur Erfassung und Bewertung von beruflichen Kompetenzen (S. 76-97). Landau: Empirische Pädagogik.

Bolten, R. (2018). Der mediale Habitus als System von Grenzen medienpädagogischen Handelns von Lehrenden in der Erwachsenenbildung. Medienpädagogik, 32. doi: $10.21240 / \mathrm{mpaed} / 32 / 2018.10 .26 . \mathrm{X}$

3 Z.B. https://wb-web.de (25.06.2019)

4 Z.B. https://erwachsenenbildung.at/ebmooc/module.php (25.06.2019) 
Eagly, A. H. \& Chaiken, S. (1993). The psychology of attitudes. Orlando, Florida: Harcourt Brace Jovanovich College Publishers.

Faulstich, P. (1985). Roboterutopie und Computerkultur. Perspektiven der Erwachsenenbildung. Hessische Blätter für Volksbildung, 35(4), S. 301-306.

Happel, J. (2018). Vertrauensbildung im Netzgestrüpp. Forum Erwachsenenbildung, 51(3), S. 42.

Herzig, B. (2007). Medienpädagogik als Element professioneller Lehrerausbildung. In W. Sesink, M. Kerres, H. Moser (Hrsg.), Jahrbuch Medienpädagogik 6 (S. 283-297). Wiesbaden: VS Verlag.

Hew, K. F. \& Brush, T. (2006). Integrating technology into K-12 teaching and learning: current knowledge gaps and recommendations for future research. $E d$ ucational Technology Research and Development, 55(3), S. 223-252. doi: 10.1007/s11423-006-9022-5

Hüther, J. (1987). Neue Medien und medienpädagogische Praxis in der Erwachsenenbildung. In L. J. Issing (Hrsg.), Medienpädagogik im Informationszeitalter (S. 193-206). Weinheim: Deutscher Studien Verlag.

Initiative D21. (2018). D21 Digital Index 2017/2018. Berlin.

Jörissen, B. (2013). Unbestellte Bildungsfelder - Wo bleiben die neuen Formate der Erwachsenen- und Weiterbildung? Forum Erwachsenenbildung, 46(2), S, $16-21$.

Knezek, G. \& Christensen, R. (2016). Extending the will, skill, tool model of technology integration: adding pedagogy as a new model construct. Journal of Computing in Higher Education, 28(3), S. 307-325. doi: 10.1007/s12528-016-91202

Martin, A. \& Langemeyer, I. (2014). Demografie, sozioökonomischer Status und Stand der Professionalisierung - das Personal in der Weiterbildung im Vergleich. In Deutsches Institut für Erwachsenenbildung (Hrsg.), Trends der Weiterbildung. DIE-Trendanalyse 2014 (S. 43-67). Bielefeld: W. Bertelsmann.

Mayrberger, K. (2012). Medienpädagogische Kompetenz im Wandel - Vorschlag zur Gestaltung des Übergangs in der Lehrerbildung am Beispiel mediendidaktischer Kompetenz. In R. Schulz-Zander, B. Eickelmann, H. Moser, H. Niesyto \& P. Grell (Hrsg.), Jahrbuch Medienpädagogik 9 (S. 389-412). Wiesbaden: Springer VS.

Rohs, M. (2019a). Erwachsenenbildung und Digitale Transformation. In M. Rohs, I. Schüßler, H.-J. Müller \& M. Schiefner-Rohs (Hrsg.), Pädagogische Perspektiven auf Transformationsprozesse. Reflexionen auf Rolf Arnolds Forschen und Wirken (S. 175-190). Bielefeld: wbv.

Rohs, M. (2019b). Medienpädagogische Professionalisierung von Weiterbildungspersonal. In E. Haberzeth \& I. Sgier (Hrsg.), Digitalisierung und Lernen. Gestaltungsperspektiven für das professionelle Handeln in der Erwachsenenbildung und Weiterbildung (S. 119-136). Bern: HEP.

Rohs, M. \& Bolten, R. (2017). Medienpädagogische Professionalisierung von Erwachsenenbildner*innen. Eine biographische Annäherung. Online unter https://kluedo.ub.uni-kl.de/frontdoor/deliver/index/docId/4547/file/_Beitraege_zur_EB_4_Medienkompetenz.pdf 
Rohs, M., Bolten, R., Kohl, J. (2017). Medienpädagogische Kompetenzen in Kompetenzbeschreibungen für Erwachsenenbildner*innen. Online unter http://nbnresolving.de/urn/resolver.pl?urn:nbn:de:hbz:386-kluedo-46905 (25.06.2019)

Schmid, U., Goertz, L., Behrens, J. (2018). Monitor Digitale Bildung. Die Weiterbildung im digitalen Zeitalter. Gütersloh: Bertelsmann Stiftung.

Schmid, W. (1986). Weiterbildung und neue Medientechniken. In J. Hüther \& R. Terlinden (Hrsg.), Neue Medien in der Erwachsenenbildung. Handbuch für Praktiker (S. 95-107). München: Max Hübner.

Schmidt-Hertha, B., Rohs, M., Rott, K., Bolten, R. (2017). Fit für die digitale (Lern-)Welt?: Medienpädagogische Kompetenzanforderungen an Erwachsenenbildner/innen. DIE-Zeitschrift, 24(3), S. 35-37.

Sgier, I., Haberzeth, E., Schüepp, P. (2018). Digitalisierung in der Weiterbildung. Ergebnisse der jährlichen Umfrage bei Weiterbildungsanbietern (Weiterbildungsstudie 2017/2018). Zürich: SVEAB \& PHZH.

Skott, J. (2015). The promises, problems, and prospects of research on teachers' beliefs. In H. Fives \& M. G. Gill (Hrsg.), International Handbook of Research on Teachers' Beliefs (S. 13-30). New York/London: Routledge.

Terlinden, R. (1988). Aufgaben der Erwachsenenbildung in Zusammenhang mit den „Neuen Medien“. Arbeit mit Erwachsenen (1), S. 35-38.

Troesser, M. (1985). Neue Medien und Weiterbildung. In H.-G. Rolff \& P. Zimmermann (Hrsg.), Neue Medien und Lernen: Herausforderungen, Chancen und Gefahren (S. 108-117). Weinheim/Basel: Beltz.

Weinert, F. E. (2001). Concept of competence: A conceptual clarification. In D. S. Rychen \& L. H. Salganik (Hrsg.), Defining and selecting key competencies (S. 45-65). Seattle: Hogrefe \& Huber Publishers. 


\section{Selbstexpertisierung mittels Software? - Zum Umgang mit Sonderwissen in der Entwicklungs- und Repräsentationspraxis von ,Lern- und Bildungsapps‘}

\section{Einleitung}

Kommerzielle Apps, d.h. Programme für mobile Geräte wie Smartphones, bieten beispielsweise Fremdsprachenerwerb oder die Verbesserung der Gedächtnisleistung und sind im Google Play Store unter dem Schlagwort „Lernen“ und im Apple Store unter dem Schlagwort „Bildung“ zu finden. Meist sammeln sie Daten bzw. die Eingaben der Nutzer*innen, bereiten diese in Bezug auf einen (Lern-)Gegenstand auf und stellen entsprechende Handlungsaufforderungen an den*die Nutzer*in.

Dieser Umgang mit Apps kann im Rahmen der Erwachsenenbildung dem informellen, bzw. selbstgesteuerten Lernen zugeordnet werden. Rohs (2013) attestiert entsprechend dem ,informellen mobilen Lernen“ ein „,situatives, intrinsisch motiviertes, selbst bestimmtes [sic!] und ortsunabhängiges Lernen mit digitalen Medien“ (ebd.: 82). Gründe für die zunehmende Relevanz des informellen Lernens in der deutschen Erwachsenbildung seit den 1990ern werden neben Individualisierungstrends und der Entwicklung von Computertechnologie vor allem in der zunehmenden Rezeption konstruktivistischer Lerntheorien und bildungspolitischen Bestrebungen hinsichtlich des lebenslangen Lernens gesehen (Gnahs 2016, S. 108-109).

Von Seiten der Anbieter*innen solcher mobilen Programme werden zumeist umfassende selbstbestimmte Lernmöglichkeiten und im Rahmen von Self-Tracking-Apps sogar Selbsterkenntnis versprochen. Ein Konzept, das ich zur Beschreibung dieser Programmatik schon an anderer Stelle eingebracht habe, ist das der „Selbstexpertisierung“ (Klinge 2018), welches ich hier nicht nur als „selbstbezogenes Wissen“ (Heyen 2019) verstehe, sondern als eine Form des Umgangs mit wissenschaftlichem bzw. spezialisiertem Wissen jenseits der Vermittlung durch Expert*innen. Wissen und Lernprozesse sollen über Rückkopplungsschleifen mit dem Programm generiert und initiiert werden. Die Betrachtung dieser Programmatik spielt für die Disziplin der Erwachsenbildung insbesondere eine Rolle, als dass sie den Rahmen für informelle Lernprozesse bietet und reflexives Lernen im Sinne einer Ermöglichungsdidaktik zumindest suggeriert (zum Konzept dieser Didaktik u.a. Arnold 2016). 
Die Frage, die sich vor diesem Hintergrund jedoch stellt, ist, wie das Wissen über einen Lern- und Erkenntnisgegenstand und seine Repräsentation in die Apps gelangen und in Algorithmen bzw. Programm umgesetzt werden, um Datenerhebung und Rückkopplungsschritte zu steuern. Denn Wissen und Vermittlung werden in der Regel federführend von Designer*innen, Programmierer*innen und Unternehmer*innen und höchstens in Kooperation mit Fachexpert*innen auf dem Gebiet des Entwicklungsgegenstandes modelliert und konstruiert.

Im Folgenden werden zuerst Arbeiten zu Expertenwissen und Technologie vorgestellt (2) und im Anschluss wissenssoziologische und systemische Überlegungen zum Expertenwissen diskutiert (3). Es folgt eine Analyse von Webpräsentationen einiger Apps und narrativer Interviews mit Entwickler*innen zu der Frage, wie das Sonderwissen in Apps einfließt und repräsentiert wird (4). Im Anschluss werden die Ergebnisse diskutiert (5).

\section{Expertenwissen und ,neue‘ Technologie}

Im Rahmen neuerer technologischer Möglichkeiten wird oftmals die Veränderung von Expertenwissen diagnostiziert. Es verschiebe sich immer stärker hin zum digital vernetzen und geteilten Wissen (Witt 2013, S. 21) und oszilliere dabei weniger in Personen als in anderen medialen Formen, wie der ,virtuellen Schwarmintelligenz" sozialer Netzwerke (Müller 2012, S. 884). Auch das Expert*innen-Lai*innen-Verhältnis habe sich verändert: Lai*innen hätten sich „,von reinen Wissenskonsumenten zu selbstbewußten [sic!] Nutzern der (widersprüchlichen) Expertisen gewandelt" (Hitzler 1998, S. 43). Aus machtheoretischer Perspektive merken Maasen und Duttweiler (2012) jedoch an, dass sich zwar die „Führung in Richtung Lenkung der [technologieunterstützten] Selbstführung verschoben" habe, jedoch nicht die Abhängigkeit von Expertenwissen, welches Praktiken bestimmt und Bedeutungen etabliert (ebd., S. 422, Einfügung DK). Dabei wirkt Macht auf zwei Ebenen: Technologien können genutzt werden, um Netzwerke aufzubauen (wie es in umfassendem Maße Facebook demonstriert) und daraus resultierend zeigt sich auf der Ebene der Nutzbarkeit der Technologien, dass Grenzen und Möglichkeiten des Handelns im Sinne einer „Take-it-or-leave-it-Wahl“ aufgezeigt werden (Bijker 1996, S. 142-143).

Auch wenn allen Expert*innen gemein ist, dass sie Wissen manipulieren, organisieren und auch mit der Vermittlung verändern (Stehr 1998, S. 24-26), so treffen wir bei der Technik- und Softwareentwicklung zum einen auf die Besonderheit, dass Akteur*innen verschiedener Bereiche, wie Manager*in- 
nen, Informatiker*innen, Forscher*innen, Designer*innen etc. mit entsprechend unterschiedlichen Wissensbeständen in Bezug auf den Entwicklungsgegenstand aufeinandertreffen (Furger und Heintz 1997, S. 536). Zum anderen verfügen die primären Entwickler*innen zumeist in Bezug auf den Anwendungskontext ihrer Programme über keinen professionellen Hintergrund (bspw. als Gesundheitsexpert*in, Sprachtrainer*in usw.) und etablieren dennoch Bedeutungen, Wissensbestände und Praktiken. „Die technischen Dinge haben einen ,Aufforderungscharakter"“ (Hörning 2001, S. 14), welcher in Design und Programm der Technologien sedimentiert ist.

Die Entwicklungsbranchen arbeiten dabei in der Logik der ,flüssigen Technokratie“: Die als starr und verhärtet beurteilten Formen des (Expert*innen-)Wissens sollen durch scheinbar transparente ,informationstechnisch optimierte Wissensvermittlungs-, Beteiligungs- und Mediationsverfahren“" ersetzt werden (Schrage 2012, S. 824). So lassen sich vermehrt organisationale Praktiken finden, die Medientechnologien in den Mittelpunkt stellen, wie dies Schäffer beispielweise anhand der Software „Liquid Feedback“ der Piratenpartei als Medium der Willensbildung herausarbeitet (vgl. Schäffer 2014, S. 205). Vertrauen wird nicht mehr in opake Formen der Expertise gesetzt, sondern in auch für Lai*innen zugängliche Informationstechnologie, welche netzwerkförmigen Austausch ermöglicht (vgl. Schrage 2014: 824). Laien sind dabei durch nutzer*innenfreundliches Design immer mehr in der Lage, komplizierte Technologien reflexionsfrei im Alltag zu benutzen, verlieren jedoch durch jene „kulturellen Banalisierungsprozesse“ das Bewusstsein für die Anwesenheit und das Wirken der Technologien in der Lebenswelt (Schmidt-Tiedemann 1996, S. 35-36). Dadurch schreibt sich das in die Technologien und Programme implementierte Wissen in der Praxis immer weiter fort.

\section{Sonderwissen und "Expertensysteme“}

Im Folgenden werden zwei Konzepte betrachtet, die m.E. für die Untersuchung von Wissensproduktion in der Technologieentwicklung relevant sind: aus wissenssoziologischer Perspektive das „Sonderwissen“ und aus systemischer das der „Expertensysteme“.

Wie bereits erwähnt, wird in der Software- bzw. App-Entwicklung Wissen überwiegend jenseits der Professionen der Anwendungsgebiete erschlossen. Sprondel (1979) bezeichnet Wissen in Bezug auf einen Gegenstand, das Expert*innen als sozial institutionalisierte Expertisenträger*innen verwalten, als „Sonderwissen“. Jenes Wissen ist für die „öffentliche[ ] Meinung“ relevant, insofern die Expert*innen sozial anerkannt sind (Schütz 1972, S. 100-101). Das Deutungsmonopol von Professionen als institutionalisiertes Expertentum 
wird jedoch ins Wanken gebracht, wenn es den Expert*innenengruppen nicht gelingt, Innovationen und Wissenserzeugung zu vereinnahmen (Hitzler 1998, S. 37). Dabei könnte man in Konstellationen der Technik- und Softwareentwicklung fragen, ob nicht genau solche Irritationsmomente entstehen, wenn Entwickler*innen Wissenstechnologien bezogen auf einen für sie professionsfremden Gegenstand produzieren. Für die Betrachtung komplexerer Entwicklungsumgebungen und Akteursgefügen stoßen allerdings Professionalisierungs- und Expertentheorien, die sich mit dem Wissen von Personen oder Institutionen beschäftigen, an ihre Grenzen. Im Folgenden wird daher für die Betrachtung von Sonderwissen in der Technik- und Softwareentwicklung die systemische Theorie der Expertensysteme von Giddens (1996) herangezogen.

Giddens (1996) sieht die „Konsequenzen der Moderne“ u.a. in der „Entbettung“ (,disembedding“ im englischen Originaltext) sozialer Systeme und Beziehungen aus ihren Abhängigkeiten von Ort und Zeit und stattdessen ihre Ordnung durch „abstrakte Systeme“ (Giddens 1996, S. 33). Einen Teil dieser Ordnung bildet das (den modernen Umgang mit Wissen kennzeichnende) Vertrauen in „Expertensysteme“ als „Systeme technischer Leistungsfähigkeit oder professioneller Sachkenntnis“ (ebd.: 40), die kontinuierlich (im Gegensatz zu einzelnen Personen) Umfelder prägen und auf das Handeln wirken. Sie garantieren gewissermaßen, dass die Erwartungen an sie auch über Raum- und Zeitgrenzen hinweg erfüllt werden, auch wenn man die Systeme nicht vollständig versteht (Giddens 1996, S. 33-42). Vertrauen in ihr Funktionieren existiert dabei ,,in der allgemeinen Einsicht, daß [sic!] menschliches Tun - wozu auch die Auswirkungen der Technik auf die materielle Welt gerechnet werden sollen nicht durch das Wesen der Dinge oder durch göttlichen Einfluß [sic!] vorgegeben, sondern eine gesellschaftliche Leistung ist" (Giddens 1996, S. 49). Zugangspunkte, um dieses Vertrauen aufrechtzuerhalten, bilden dabei Repräsentanten der abstrakten Systeme als ,gesichtsabhängige Bindungen“" (ebd.: 103) und ermöglichen „Rückbettung sozialer Beziehungen“(Giddens 1996, S. 112). Giddens nennt hier u.a. die Flugbegleiter*innen, die stellvertretend für Flugverkehr und -technologie auftreten und vermitteln, dass alles normal verläuft (vgl. ebd.: 110). Bei den Expert*innensystemen erfolgt dabei eine strenge Trennung zwischen „Darbietungen , auf der Bühne“ und der Arbeit, hinter den Kulissen “", weil die Ambiguitäten des Expert*innenhandelns und der Umgang und die Produktion von Wissen schwer vermittelbar ist (Giddens 1996, S. 110).

Dieser Beitrag geht in Anlehnung an die Funktionsweise dieser Expert*innensysteme und die Herstellung von Vertrauen in diese abstrakten Systeme zum einen der Frage nach, wie das Sonderwissen auf der „Bühne“ kommerzielle erfolgreicher Apps verhandelt und - um die Metapher der gesichtsabhängigen Bindungen zu strapazieren - welche Maske dem Wissen aufgesetzt wird. Zum anderen soll die Rekonstruktion der Entwicklungspraxis der Apps ,hinter den Kulissen“ Aufschluss darüber geben, wie das „Sonderwissen“" in Bezug auf den Lerngegenstand generiert und verhandelt wird. 


\section{Diskursives Wissen auf den Webseiten und implizites Konstruktionswissen der Entwickler*innen}

Um die Repräsentation von Sonderwissen genauer zu untersuchen, wurde die Art, wie Inhalte auf den Webseiten der Firmen kuratiert werden, hinsichtlich der Frage analysiert, welcher modus operandi sich im diskursiven, kommunikativen Wissen finden lässt (Nohl 2016). Im Sampling enthalten waren Websites von kommerziellen Apps mit einer hohen Nutzer*innenquote, die den Fremdsprachenerwerb, Gedächtnistraining und themenspezifischen Kenntnisgewinn versprechen, aber auch Meditations- und Self-Tracking-Apps. Für die Analyse des Umgangs mit Wissen ,hinter den Kulissen“ wurden narrative Interviews (Nohl 2012) mit Entwickler*innen (hauptsächlich von Self-TrackingApps) geführt und analysiert, um das implizite Konstruktionswissen zu rekonstruieren.

\section{Diskursiv-kommunikatives Wissen auf den Websites: Werbung mit wissenschaftlicher Praxis, Hauswissenschaftler*nnen und der Wissenschaftspraxis mittels der App}

Zunächst einmal lässt sich festhalten, dass auf den Websites aller untersuchten Apps auf wissenschaftliches bzw. Expertenwissen verwiesen wird. So beansprucht die Sprachlern-App „Memrise“, dass ihre Inhalte von „Linguistik Experten“ (Memrise Startseite) geschaffen wurden, und auch „Babbel“ wirbt mit „Sprachlernexperten“, die an der Erstellung der Lerninhalte beteiligt waren (Babbel Startseite). Aber auch andere Genres, beispielsweise Gehirn-JoggingApps, werben mit ihrer „Wissenschaftliche[n] Basis“ (Memorando Startseite). Die Firma „Peak“ bezeichnet sich sogar als leidenschaftlich in Bezug auf Forschung bzw. Wissenschaft und kann mit namenhaften Universitäten aufwarten (Abb. 1). Und auch Fitness- und Gesundheits-Apps wie „7 Minutes Workout“ 
(Abb. 2) oder eine andere App für die Meditationspraxis „7Mind“ (7Mind Startseite) werben mit einer wissenschaftlichen Fundierung.

Abbildung 1: Gehirnjogging-App „Peak“: www.peak.net (abgerufen am 21.01.

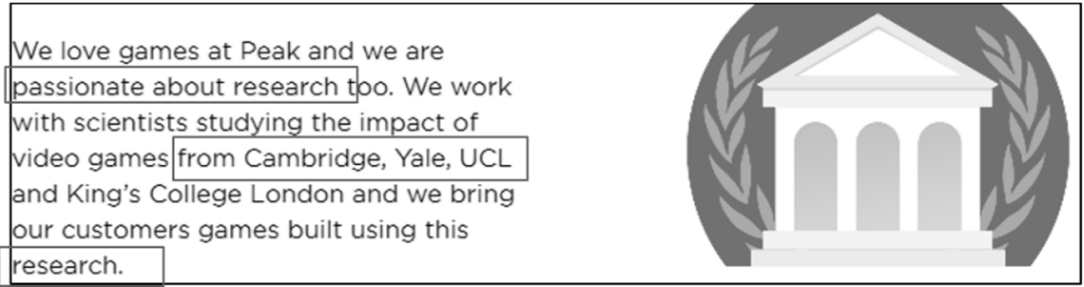

2019, Hervorhebungen durch die Autorin).

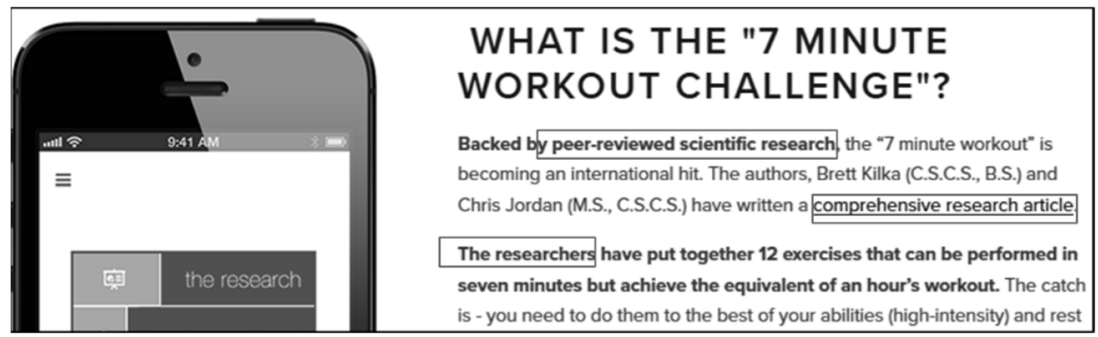

Abbildung 2: Fitness-App „7 Minute Workout“: www.7minworkoutapp.net/ \# about (abgerufen am 21.01.2019 Hervorhebungen durch die Autorin)

Auf den meisten Websites gibt es jedoch noch eine Sonderseite, auf welcher mit Informationen zur ,Wissenschaft hinter“ der jeweiligen App geworben und auf diese umfassend Bezug genommen wird. Die dezidierten Aufbereitungen des Sonderwissens enden zumeist darin, zu erklären, wie das Wissen konkret in Funktionen des Programms umgesetzt wurde (bspw. bei Memrise: www.memrise.com/de/science/). Es lassen sich in vielen Darstellungen auch Anleihen an den Fitnessdiskurs finden, welche die Effektivität des Programms erklären, wobei Effektivität generell eine große Rolle spielt. So zeigt „Peak“ (Gehirnjogging-App) auf der Internetseite ein Gehirn - vermeintlich schlau, da es eine Brille trägt -, welches Hanteltraining absolviert (Abb. 3), um den Trainingseffekt zu symbolisieren. 


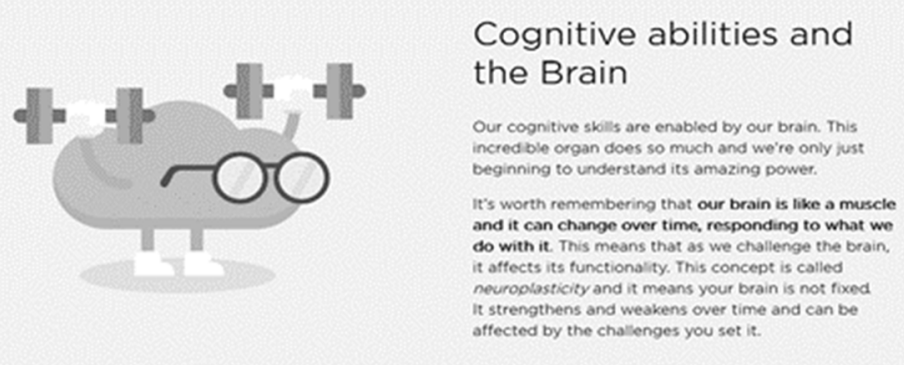

Abbildung 3: Gehirnjogging-App Peak http://www.peak.net/science (abgerufen am 21.01.2019)

Auch das Sprachprogramm „Babbel““,trainiert dein Gehirn“: Unter der Überschrift „Lernen, wie es zu dir passt“", wird erläutert, dass mittels ,,wissenschaftlicher Methoden“ individuelle Lernvoraussetzungen (hier Lerntypen) eingefriedet werden, so dass es für das Programm keine Rolle spielt, wer es benutzt. ${ }^{1}$ Zusammenfassen lässt sich, dass auf allen analysierten Websites der Anbieter*innen die Bewerbung wissenschaftlicher Praxis und der Nutzbarmachung des Sonderwissens für das Produkt zu finden ist.

Bei genauerer Analyse der Websites hinsichtlich der „Wissenschaft hinter“" den Apps findet man insbesondere bei den Sprachlern- und den GehirnjoggingApps Verweise auf eigene Veröffentlichungen, Studien und Wissenschaftler*innen - also auf Hauswissenschaftler*innen und Hausstudien - welche sich als ein Rekurrieren auf Wissenschaft und Sonderwissen als soziales und kulturelles Kapital der Firmen lesen lässt. So verweist die Sprachlern-App "Memrise“ auf ihre namentlich aufgeführten wissenschaftlichen Berater*innen, welche als „,weltweit führende[...] Forscher“" „helfen schneller zu lernen“ (www.memrise.com/de/science). Die Symbolhaftigkeit des Kapitals zeigt sich auf vielen Websites auch in der Bildsprache: Auf der Webseite „Veröffentlichungen unserer Mitarbeiter und über unsere Produkte" der Sprachlern-App „Duolingo“" wird bspw. mit bunten Mikroskopen und Reagenzgläsern die Wissenschaftlichkeit illustriert ${ }^{2}$ (Abb. 4) und Wissenschaftler*innen werden in weißen Kitteln präsentiert (Abb. 5, aber auch http://memorado.de/gci).

1 https://about.babbel.com/de/about-us/: „Babbel trainiert dein Gehirn so, dass du kontinuierlich und effizient lernst, und spricht alle verschiedenen Lerntypen an. Egal [sic!] ob du lieber schreibst, liest, sprichst oder hörst - wissenschaftliche Methoden ermöglichen es dir, neu Gelerntes effektiv aufzunehmen und im Langzeitgedächtnis zu speichern."

2 Die am 24.09.2018 abgerufene Seite existiert nicht mehr. Stattdessen wird unter dem Reiter ,Forschung“ über die „,duolingo ai“, also über das System der künstlichen Intelligenz berichtet; inklusive „Research“, „Publications“ und „Data Sets“, welche man sich herunterladen kann. 


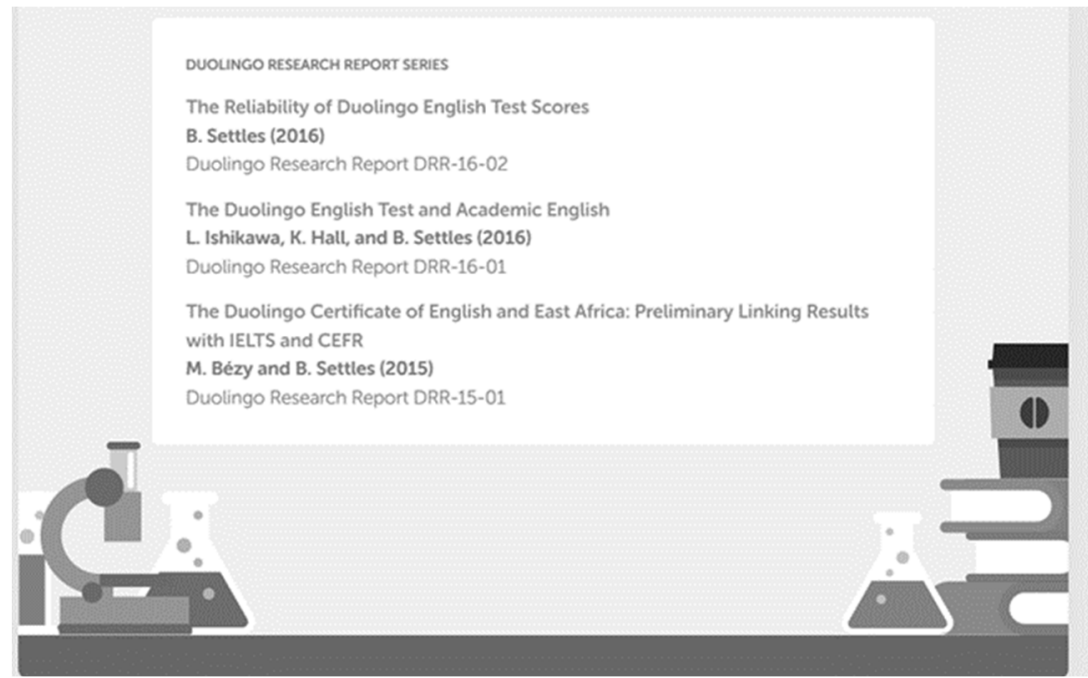

Abbildung 4: Sprachlern-App „Duolingo“: „Veröffentlichungen unserer Mitarbeiter und über unsere Produkte" (abgerufen am 24.09.2018)

Interessant ist auch, dass auf einigen Seiten der Gehirnjogging-Apps Wissenschaftler*innen eingeladen werden, mit der Firma zu kooperieren (Abb. 5), wofür sie im Gegenzug u.a. auf die Nutzer*innendaten des Programms zugreifen können (,Memorando“ verweist auf der oben genannten ,gci“-Seite explizit auf die „Möglichkeit des Zugangs zu limitierten und anonymisierten Statistiken").

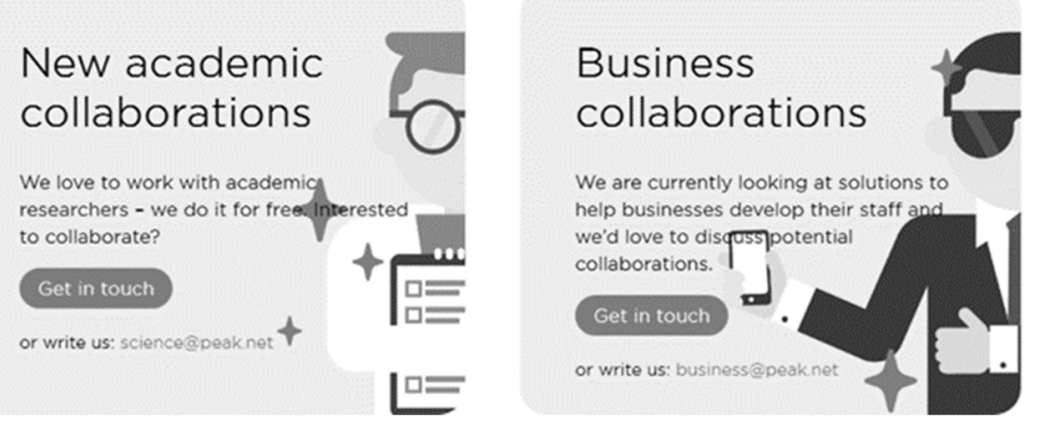

Abbildung 5: Anwerben von Partner*innen auf der Seite von Peak http://www. peak.net/science (abgerufen am 21.01.2019)

Daten, welche mithilfe der Apps erhoben wurden, werden u.a. kooperierenden Wissenschaftler*innen zur Nutzung angeboten, es werden aber auch eigene 
Studien durchgeführt. So präsentiert die Gehirnjogging-App „Memorando“ eine Studie, welche mit Nutzer*innendaten arbeitet, wobei allerdings nicht ganz klar ist, wer der*die Autor*in ist und wo die Ergebnisse veröffentlicht wurden. Die Analyse soll jedoch ,wertvolle Informationen für Arbeitgeber“ (www.memorado.de/gci\#tour-studies) über die Produktivität ihrer Mitarbeiter bieten und somit unmittelbar ökonomisch verwertbar sein. Auch in der vorinstallierten iPhone-App „Health“ steht die Erzeugung von Wissen mithilfe der (Datensammlung) der App in Bezug zu Sonderwissen an erster Stelle. Mit den Tools „ResearchKit“ und „CareKit“ können die eigenen Daten u.a. in Studien einfließen oder Symptome und Medikation selbst überwacht werden (Apple Health Webseite). Wichtig ist hier also nicht das Wissen, welches für die Entwicklung der App verwendet wird, sondern hauptsächlich, welches Datenwissen mithilfe dieser App gewonnen werden kann. Eine Gegenüberstellung von ,traditionellem ‘ wissenschaftlichem Wissen und der Möglichkeit umfassenderer Wissenserzeugung mithilfe der App setzt sich in der detaillierten Darstellung fort.

\section{Analyse der Interviews: Quellen des Sonderwissens, Einfluss multipler Sonderwissensbestände aus anderen Branchen und Schaffung eigenen Sonderwissens über die App}

In den Interviews mit Entwickler*innen bezüglich der Konstruktion von Apps berichten jene über verschiedene Quellen des Sonderwissens wie Gespräche mit Praktiker*innen oder veröffentlichte Studien zu den jeweiligen Themen. Im Interview mit einem Fitness-App-Entwickler ließ sich beispielsweise rekonstruieren, dass Ergebnisse einer Studie der World Health Organisation genutzt wurden, um Zielvorgaben für das Programm zu formulieren (vgl. Transkript Sperling ${ }^{3}$ ]. Dabei sollen genaue Standards aus dem Sonderwissen abgeleitet bzw. quantifizierte Standards übertragen werden. So auch ein anderer interviewter Entwickler:

„Die Standards sind eigentlich aus der aus der Medizin. [...] also die Erkenntnis einfach aus diesem mitgewachsenen Jahrhunderte im Hintergrund daran bedienen wir uns. und das kannst du auch in jeder Fachliteratur irgendwo nachlesen von Medizinern ähm und da gibt's unglaublich viel Inhalte 4 “" (Transkript Fink)

3 Die Entwickler*innen wurden für diesen Artikel mit Vogelnamen maskiert.

4 Die Transkriptausschnitte wurden nach den Regeln von TIQ (Talk in Qualitative Social Research) erstellt. Punkte und Kommata sind daher Marker für Intonation und keine 
Die Güte der Quellen scheint dabei an einer Art Common Sense über die Standardisierung des Praxisbereiches und der historisch gewachsenen Inhalte gemessen zu werden. Entwicklungspraktisch werden die Inhalte für die App teilweise selbstreferenziell kuratiert, indem vorhandene Inhalte nach Beliebtheit bei den Nutzer*innen gefiltert und für die App komprimiert werden.

Im Konstruktionsprozess der Apps werden aber auch Akteur*innen aus der Praxis einbezogen und ihr Sonderwissen abgefragt oder es wird mit ihnen kooperiert. So musste beispielsweise in der Entwicklung einer anderen Gesundheits-App, die nicht wie die obere im Freizeit-, sondern Medizinbereich eingesetzt werden sollte, zum einen eine „behutsame“ Kommunikation mit Therapeut*innen und Ärzt*innen gefunden werden, ,dass die einerseits uns ihr Wissen zur Verfügung stellen. also und sie auch davon überzeugt sind dass das System das kann" (Transkript Drossel). Zum anderen musste das Praxiswissen in die Machbarkeit der Technologie übersetzt werden, was dieses Wissen automatisch verändert: „Häufig kommt dann so ne etwas abgewandelte Version raus, weil wir natürlich dann sagen, dass man die ein oder andere Übung nicht unbedingt optimal abbilden kann“( (ebd.).

Ein weiteres Ergebnis der Analyse der narrativen Interviews ist, dass das Sonderwissen der Entwicklungsbranche - der Designer*innen, Informatiker*innen, Unternehmer*innen - den Prozess und damit den Gegenstand wesentlich bestimmt. In fast allen Prozessen sind insbesondere Wissensbestände des Designs eingeflossen, wie beispielsweise das anfängliche Ersinnen von Nutzerdummys, ,Personas“ oder „user stories“. Dabei werden mögliche Wissensbedürfnisse und Probleme konstruiert, die die zu entwickelnde App adressieren sollte. Es wird also eine Handlungspraxis imaginiert und diese erdachten Anwendungen auf technische Umsetzbarkeit und Produktionskosten geprüft. Der Einfluss der Sonderwissensbestände der jeweils beteiligten Branchen geht so weit, dass ein modularisiertes Arbeiten ähnlich der Fließbandarbeit stattfindet. So wird beispielsweise von Entwicklungsprozessen einer App berichtet, in welchen zuerst die Form der App mit Blindtext gestaltet wurde, bevor die Redaktion Inhalte für diesen Rahmen erstellte. Die Inhalte wurden dann wieder an die Entwickler*innen gegeben, die diese implementierten (vgl. Transkript Fink).

In den Interviews ließ sich ähnlich wie auf den Internetseiten ein großes Vertrauen in die Erzeugung von Wissen mithilfe der Apps finden: Für eine Fitness-App werden beispielsweise Nutzer*innendaten gesammelt, um die Algorithmen zu unterfüttern und robuster zu gestalten, welche dann individuellere Rückmeldungen durch das System ermöglichen sollen und so eine Art eigenes Sonderwissen erzeugen (vgl. Transkript Sperling). Teilweise geht das Vertrauen in die Wissenserzeugung mittels der Apps weiter als das Vertrauen in das Wissen der Praxisexpert*innen. In der Entwicklung einer Stress-App

Satzzeichen. Die hier genutzten Ausschnitte wurden tlw. ,bereinigt“ (,ähm“, ,äh“ und Wortwiederholungen wurden gelöscht). 
wird beispielsweise die Verfügung und Sichtbarkeit der eigenen (Gesundheits)Daten mit emanzipativer Wissenserzeugung in Verbindung gebracht, während Ärzt*innen eher nach Gewohnheit als nach wissenschaftlichen Erkenntnissen arbeiten würden (vgl. Transkript Amsel). Dem datengetriebenen Expertensystem wird also mehr vertraut als dem Expertensystem menschlicher Praktiken der Wissenserzeugung.

\section{Informelles Lernen mithilfe der Wissenskonstruktionen kommerzieller Programme}

In der Zusammenschau der Ergebnisse lässt sich - in Anlehnung an den Begriff Data-Mining - ein ,Mining von Sonderwissen“ verschiedener Bereiche rekonstruieren, um es für die Apps nutzbar zu machen. Das Sonderwissen sollte hinter den Kulissen, also im Rahmen der App-Entwicklung, gut in Technologie, übersetzbar' sein und vor den Kulissen, also im Kontakt mit den Nutzer*innen und Kund*innen, als soziales und kulturelles Kapital verwertbar sein. Weiterhin ließ sich sowohl auf der Ebene der Konstruktion als auch auf der der Repräsentation eine Orientierung an der Erzeugung von Sonderwissen mittels der Apps rekonstruieren. Zusammenfassend scheinen sich hier eigene Expertensysteme zu etablieren, die nicht nur eigenes Sonderwissen (mittels der Nutzer*innendaten und -statistiken), sondern auch eigene Standards bezüglich des Wissens generieren, welche sich teilweise explizit von bisherigen wissenschaftlichen Standards bzw. von der bisherigen Praxis der Handlungsbereiche abgrenzen.

Es stellt sich vor diesem Hintergrund die Frage nach den Irritationsmomenten für die jeweiligen Professionen und deren institutionalisiertes Wissen (Hitzler, 1998), wenn Innovation und Wissenserzeugung von Firmen vereinnahmt werden, die das Wissen als ,Baustoff' benutzen und weniger reflexive, komplexe und uneindeutige Wissensbestände aktivieren und anerkennen.

Natürlich verfolgen die Firmen, die ,Lern- und Bildungsapps ' anbieten, wirtschaftliche Interessen. Dieses Konzept des selbstgesteuerten informellen Lernens passt, so kann man mit Gnahs (2016) argumentieren, sehr gut zur „,neoliberalen Logik“, in welcher ,möglichst viel in kürzester Zeit zu erledigen und möglichst keine Zeit zu verschwenden" sei, indem man überall selbstständig und zu jeder Zeit lernen kann (ebd.: S. 111). Es stellt sich die Frage, wie sich die Praxis des informellen Lernens Erwachsener im Umgang mit Apps entwickelt, die eine solche Art der Kuratierung von Wissensbeständen vornehmen. Gerade wenn das informelle Lernen in der Erwachsenbildung mit konstruktivistischen Lerntheorien in Zusammenhang gebracht wird (u.a. Rohs 
2013, S. 83-84), ist zu fragen, ob in diesen Lernsettings nicht das abgelöst geglaubte behavioristische Lernen wieder Einzug hält (siehe zur Konstruktion des behavioristischen Lernens in der Technologie auch Klinge 2018).

Was Esposito (2013) bei der Wissenserzeugung durch Maschinen und der Konstruktion von entsprechenden Nutzungsmodellen problematisiert, ist das Fehlen der doppelten Kontingenz von Kommunikation (aber auch der Wissenserzeugung). Problematisch für Lern- und Bildungsprozesse wird es, wenn Dinge dazu konstruiert sind, Reize hervorzurufen: „Der Mensch antwortet nicht mehr auf die Herausforderungen durch die Dinge. Er reagiert vielmehr auf eine bereits zugerichtete Welt" (Dörpinghaus und Uphoff 2012, S. 165).

\section{Literatur}

7Mind Startseite: Willkommen bei dir. http://www.7mind.de/ [Zugriff: 21.01. 2019].

Apple Health Webseite: ResearchKit und CareKit. Mehr Möglichkeiten für Forschung, Ärzte und dich. https://www.apple.com/de/researchkit/ [Zugriff: 21.01.2019].

Arnold, Rolf (2016): „Didaktik“ informellen Lernens. In: Rohs, M. (Hrsg.): Handbuch Informelles Lernen. Wiesbaden: Springer Fachmedien Wiesbaden, S. 483-493.

Babbel Startseite: Sprich Sprachen, wie du es schon immer wolltest. https://de.babbel.com/ [Zugriff: 21.01.2019].

Bijker, Wiebe E. (1996): Demokratisierung der Technik - Wer sind die Experten? In: Kerner, M. (Hrsg.): Aufstand der Laien. Expertentum und Demokratie in der technisierten Welt. Aachen: Thouet Verlag, S. 133-155.

Dörpinghaus, Andreas / Uphoff, Ina Katharina (2012): Die andere Zeit der Dinge. Ein metaphysischer Grenzübertritt. In: Dörpinghaus, A. / Nießeler, A. (Hrsg.): Dinge in der Welt der Bildung. Bildung in der Welt der Dinge. Würzburg: Königshausen \& Neumann, S. 153-166.

Esposito, Elena (2013): Digital prophecies and web intelligence. In: Hildebrandt, M./Vries, K. de (Hrsg.): Privacy, Due Process and the Computational Turn. The Philosophy of Law Meets the Philosophy of Technology. Hoboken: Taylor and Francis, S. 121-142.

Furger, Franco / Heintz, Bettina (1997): Technologische Paradigmen und lokaler Kontext. Das Beispiel der EREMETH. In: Schweizerische Zeitschrift für Soziologie 23, 3, S. 533-566.

Giddens, Anthony (1996): Konsequenzen der Moderne. Frankfurt am Main: Suhrkamp. 
Gnahs, Dieter (2016): Informelles Lernen in der Erwachsenenbildung/Weiterbildung. In: Rohs, M. (Hrsg.): Handbuch Informelles Lernen. Wiesbaden: Springer Fachmedien, S. 107-122.

Heyen, Nils (2019): Von der Selbstvermessung zur Selbstexpertisierung. Zur Produktion von selbstbezogenem Wissen durch Personal Science. In: Heyen, N. / Dickel, S. / Brüninghaus, A. (Hrsg.): Personal Health Science. Persönliches Gesundheitswissen zwischen Selbstsorge und Bürgerforschung. Wiesbaden: Springer VS, S. 23-42.

Hitzler, Ronald (1998): Reflexive Kompetenz - Zur Genese und Bedeutung von Expertenwissen jenseits des Professionalismus. In: Schulz, W. K. (Hrsg.): Expertenwissen. Soziologische, psychologische und pädagogische Perspektiven. Opladen: Leske + Budrich, S. 33-47.

Hörning, Karl. H. (2001): Experten des Alltags. Die Wiederentdeckung des praktischen Wissens. Weilerswist: Velbrück Wissenschaft.

Klinge, Denise (2018): Die (implizite) Pädagogik von Self-Tracking. Handlungspraxis und Vermittlungsweisen der EntwicklerInnen im Spannungsfeld von Entrepreneurship, Technik und Design. In: Houben, D. / Prietl, B. (Hrsg.): Datengesellschaft. Einsichten in die Datafizierung des Sozialen. 1. Auflage. Digitale Gesellschaft, Band 17. Bielefeld: Transcript, S. 133-153.

Maasen, Sabine / Duttweiler, Stefanie (2012): Neue Subjekte, neue Sozialitäten, neue Gesellschaften. In: Maasen, S. et al. (Hrsg.): Handbuch Wissenschaftssoziologie. Wiesbaden: Springer VS, S. 417-428.

Memorando Startseite: Fit im Kopf. Fit durchs Leben. http://memorado.de/ [Zugriff: 21.01.2019].

Memrise Startseite: Entfessle deine sprachlichen Superkräfte mit Memrise. https://www.memrise.com/de/ [Zugriff: 21.01.2019].

Müller, Hans Peter (2012): Wozu (noch) Intellektuelle? In: Macht und Ohnmacht der Experten. Sonderheft Merkur Nr. 760/761. Stuttgart: Klett-Cotta, S. $878-885$.

Nohl, Arnd-Michael (2012): Interview und dokumentarische Methode - Anleitungen für die Forschungspraxis. 4., überarbeitete Auflage. Wiesbaden: VS Verlag für Sozialwissenschaften.

Nohl, Arnd-Michael (2016): Dokumentarische Methode und die Interpretation öffentlicher Diskurse. In: Zeitschrift für Diskursforschung, 2, S. 115-136.

Rohs, Matthias (2013): Informelles Mobiles Lernen. In: Witt, C. de / Sieber, A. (Hrsg.): Mobile Learning. Potenziale, Einsatzszenarien und Perspektiven des Lernens mit mobilen Endgeräten. Wiesbaden: Springer VS, S. 75-97.

Schäffer, Burkhard (2014): Die Piratenpartei. Ein organisationskultureller Blick auf eine auf neue Medien fokussierte Bildungsorganisation. In: Weber, S. M. et al. (Hrsg.): Organsiation und das Neue. Beiträge der Kommission Organisationspädagogik, Band 15. Wiesbaden: VS Verlag für Sozialwissenschaften, S. 203-214.

Schmidt-Tiedemann, Joachim K. (1996): Experten und Bürger - Über die Teilung der Verantwortung für Technikfolgen. In: Kerner, M. (Hrsg.): Aufstand der 
Laien. Expertentum und Demokratie in der technisierten Welt. Aachen: Thouet Verlag, S. 27-43.

Schrage, Dominik (2012): Flüssige Technokratie. In: Macht und Ohnmacht der Experten. Sonderheft Merkur Nr. 760/761. Stuttgart: Klett-Cotta, S. 817-825.

Schütz, Alfred (1972): Der gut informierte Bürger. Ein Versuch über die soziale Verteilung des Wissens. In: Schütz, A. (Hrsg.): Gesammelte Aufsätze 2. Studien zur soziologischen Theorie. Den Haag: Martinus Nijhoff, S. 85-101.

Sprondel, Walter M. (1979): „Experte“ und „Laie“. Zur Entwicklung von Typenbegriffen in der Wissenssoziologie. In: Alfred Schütz und die Idee des Alltags in den Sozialwissenschaften. Stuttgart: Enke, S. 140-154.

Stehr, Nico (1998): Wissensberufe. In: Schulz, W. K. (Hrsg.): Expertenwissen. Soziologische, psychologische und pädagogische Perspektiven. Opladen: Leske + Budrich, S. 17-31.

Witt, Claudia de (2013): Vom E-Learning zum Mobile Learning - wie Smartphones und Tablet PCs Lernen und Arbeit verbinden. In: Witt, C. de / Sieber, A. (Hrsg.): Mobile Learning. Potenziale, Einsatzszenarien und Perspektiven des Lernens mit mobilen Endgeräten. Wiesbaden: Springer VS, S. 13-26. 


\section{Erlebte Resonanz im Weiterbildungsgeschehen - eine empirische Annäherung}

\section{Einleitung}

Der Beitrag befasst sich mit dem zunehmenden Zeitdruck und der steigenden Aufgabenmenge in modernen Arbeits- und Lebenswelten. Die heutige Gesellschaft unterliegt einem allumfassenden Beschleunigungsphänomen, das unweigerlich dazu führt, dass alles immer schneller und immer mehr wird, wodurch sich die Individuen zunehmend unter Zeitdruck gesetzt fühlen. Zahlreiche Beschleunigungstendenzen eröffnen Beschäftigten in der Arbeitswelt neue Handlungsmöglichkeiten und stellen sie gleichzeitig vor neue, beschleunigungsbedingte Anforderungen (gekennzeichnet durch eine hohe Flexibilität und Mobilität, Selbststeuerung, hohes Aufgabenpensum, starke zeitliche Beanspruchungen).

Die heutige institutionalisierte Erwachsenenbildung ist von der Beschleunigung in doppelter Weise betroffen: „Einerseits reagiert sie auf den sozialen Wandel, andererseits ist sie Teil dieser Prozesse“ (Zeuner 2008: 541). Ständiges Thema bzw. häufiger Inhalt in Literatur, Kursen und Seminaren ist das Zeit-Lernen (in Form von Zeit- und Selbstmanagementseminaren). Auf pragmatischer Ebene dient die Aneignung technokratischer Methoden zur Priorisierung, zum Zeitsparen, zur Effizienzsteigerung und Optimierung von Tätigkeiten und Handlungsverläufen in der Zeit (Schmidt-Lauff 2012: 52f.).

Hartmut Rosas Aussage ,[...] wenn Beschleunigung das Problem ist, dann ist Resonanz vielleicht die Lösung" (Rosa 2016b: 13) folgend, wird jenes Beschleunigungsphänomen vor dem Hintergrund erlebter Resonanz und Entfremdung im Weiterbildungsgeschehen untersucht.

Im Rahmen des Beitrags wird unter resonanztheoretischer Perspektive die Frage aufgeworfen, welche Resonanz- und Entfremdungspotenziale sich im Weiterbildungsgeschehen aus Teilnehmendensicht (empirisch) identifizieren lassen. Einführend werden die Grundzüge der Resonanztheorie nach Hartmut Rosa erläutert. Hierzu wurden leitfadengestützte Interviews mit Teilnehmenden von Weiterbildungsveranstaltungen durchgeführt. Anschließend an die Darstellung der Erhebung, die Auswertung des empirischen Datenmaterials und die Darstellung von Relationalen Resonanzstrategien (RRS) werden exemplarisch zwei ausgewählte Relationalen Resonanzstrategien vorgestellt. 
Der Beitrag schließt mit einer kritischen Betrachtung der Zwischenergebnisse und einem Ausblick auf weiterführende Forschung.

\section{Grundzüge der Resonanztheorie nach Hartmut Rosa}

Im Folgenden wird die Grundidee der Resonanztheorie nach Hartmut Rosa skizziert. Aufbauend auf den theoretischen Überlegungen wird anschließend das methodische Vorgehen kurz erörtert. Weiterführend sollen die Zwischenergebnisse dargestellt und kritisch betrachtet werden.

Der Soziologe Hartmut Rosa entfaltet in Orientierung an philosophischen und psychologischen Theorien die „Grundlagen der Weltbeziehung“, welche er aus der Perspektive der Anthropologie sowie der Sozialtheorie ausbreitet. Hierauf basierend formuliert er eine Gesellschaftskritik. Rosa leitet Resonanz sozialtheoretisch aus dem gleichnamigen akustisch-physikalischen Vorgang $\mathrm{ab}$, wobei er unter Berücksichtigung der Beschleunigungstendenzen auch metaphorische Elemente mit einbezieht. In einem heuristischen Konzept der Resonanz fasst er die Zeitdimension als ein essentielles soziales und historisches Konstrukt. Vor diesem Hintergrund begreift Rosa die Moderne als eine durch exponentielles Wachstum (siehe zu ,Postwachstumsökonomie‘ Paech 2012) und Beschleunigungstendenzen gekennzeichnete Zeit, die sich durch einen spezifischen Modus der Reproduktion gesellschaftlicher Struktur und Identität auszeichnet und nicht primär - wie in der Steigerungslogik des Kapitalismus und den Theoriediskursen von Max Weber und Niklas Luhmann - durch Rationalisierung und funktionale Differenzierung bestimmt wird (Rosa 2016a: 44ff; vgl. Döbler 2016). Der Kritik des Erziehungswissenschaftlers Micha Brumlik und auch des Jenaer Soziologen Peter Schulz folgend, rekonstruiert Rosa eine Kritik der Resonanzverhältnisse einer (Welt-)Gesellschaft, die sich aufgrund , ,[...] zunehmender, effektiverer und beschleunigter Kontrolle sowie durch die Unterordnung aller Lebensvollzüge unter die Abstraktionen des Kapitalverhältnisses auszeichnen“" (Brumlik 2016: 122; Schulz 2015: 103). ${ }^{1}$

Folgt man Rosa, bildet der Mensch über die Lebensspanne das Bewusstsein aus, ein Subjekt zu sein, welches mit der Umwelt in eine wechselseitige Beziehung tritt. Der Autor spricht dem Menschen nicht nur eine generelle (in ihm

1 Brumliks Kritik an Rosas Resonanztheorie bezieht sich zudem darauf, dass die Resonanztheorie nicht das (in der Kritischen Theorie verankerte) immanente Interesse an der Aufhebung des gesellschaftlichen Unrechts in einer ,unversöhnlichen“ Art, impliziert (wie es u.a. bei Horkheimer 1937 und Adorno 2003 vorzufinden ist) (Brumlik 2016: 122). 
veranlagte) Sehnsucht zu, sondern umschreibt dieses Phänomen als einen Grundmodus des ,In-der-Welt-Seins`. Dieses impliziert eine grundsätzliche Plastizitäts- und Flexibilitätsthese, Lernfähigkeit im Sinne eines erfahrungsbasierten Lernens in (sozialen) Beziehungsgefügen. ${ }^{2}$

Rosas Begriffsverständnis von Resonanz umfasst eine ,[...] durch Affizierung und Emotion, intrinsisches Selbstinteresse, [Selbstkonzept] und Selbstwirksamkeitserwartung gebildete Form der Weltbeziehung, in der sich Subjekt und Welt gegenseitig berühren und zugleich transformieren" - das heißt, die Welt erscheint ,als ein antwortendes, atmendes, tragendes, in manchen Momenten sogar wohlwollendes, entgegenkommendes oder, gütiges', Resonanzsystem" (Rosa 2012: 9). Hierbei wird explizit die Antwortbeziehung der Subjekte und der Welt ins Zentrum der Betrachtung gerückt und in einem Beziehungsmodus im ,Dreiklang von Leib, Geist und erfahrbarer Welt' analysiert. Folgt man Rosas Verständnis von Resonanz, dann kann dieses als ein Moment der ,Verflüssigung' - im Sinne einer nicht starren oder indifferenten Lage der beiden Pole ,Selbst' und ,Welt ${ }^{\star}$ gedeutet werden, die die ,Anverwandlung von Weltausschnitten' (erst) eröffnet. Jene Resonanzmomente können in gesellschaftlichen Sphären erlebt werden - beispielweise im Weiterbildungsgeschehen. Der Einklang von Leib und Seele oder Subjekt und Welt ermöglicht demzufolge einen Resonanzraum.

Hierbei wird Resonanz nicht mit einem hohen Ausmaß an Harmonie oder gar der Widerspruchslosigkeit der Antworten gleichgesetzt, sondern ist vielmehr als dynamisch-dialektisches Verhältnis von Subjekt und Welt zu verstehen. So lassen sich Entfremdung und Resonanz nicht als Gegensätze deuten, sondern vielmehr als komplementäre Beziehungsmodi, welche in Bildungsund Anwandlungsprozessen in ein dialektisches Verhältnis treten (Beljan 2017: 397). Vor diesem Hintergrund kann Bildung als ein dynamischer Prozess begriffen werden, welcher sowohl resonante als auch entfremdende Momente implizieren muss (Rosa 2016a: 316):

Resonanz entspricht somit keiner bloßen Echobeziehung, sondern kann als ein antwortender und (gegebenenfalls) transformierender Prozess begriffen werden, wobei er zwischen ,stummen' (das heißt gleichgültigen oder latent feindlichen) und ,resonanten "Weltverhältnissen unterscheidet.

Das Phänomen der ,Entfremdung ' tritt nach Rosa für das Subjekt dann ein, wenn es keine Umweltbeziehungen (zu Subjekten und/oder Objekten) herstellen und ausschließlich auf äußere Bedingtheiten und Anforderungen reagieren kann. Rosa spricht in diesem Zusammenhang von einer „De-Kontextualisie-

2 Dies spricht meines Erachtens für die auf der Verhaltensebene verortete Zuschreibung der Erlernbarkeit und der Kontextabhängigkeit (Kultur, Werte, Normen, Beziehungsfelder); d.h., dass Resonanz nicht ausschließlich als eine gegebene Grundsehnsucht verstanden werden kann, sondern vielmehr auch als ein wechselseitig prägender und sich ausformender Prozess im sozialen Gefüge. 
rung von Erlebnissen“ (Rosa 2014: 341), welche die Integration narrativer Erlebnisse und das Zueinander-in-Beziehung-setzen sowie einen Selbstbezug verhindert. Er sieht in der Entfremdung die Folge eines Verlusts der „Transformation von Erlebnissen in genuine Erfahrung im Sinne Benjamins" (Rosa 2014: 470). ${ }^{3}$

Rosas Entfremdungsbegriff lehnt sich dabei dem Begriffsverständnis von Rahel Jaeggi an. Rosa umreißt ,Entfremdung“ als einen ,Zustand [...], in welchem Subjekte Ziele verfolgen oder Praktiken ausüben, die ihnen einerseits nicht von anderen Akteuren oder äußeren Faktoren aufgezwungen wurden, [...] welche sie aber andererseits nicht ,wirklich“ wollen oder unterstützen“" (Rosa 2011: 234; Jaeggi 2005: 63ff.).

Auf dieser Annahme aufbauend deutet Rosa den Zustand der Entfremdung als eine Form oder Beziehung der Beziehungslosigkeit, eine Entkoppelung des Subjekts von seiner Umwelt. Entfremdung kann demzufolge als ein Verstummen der Resonanz zwischen Mensch und Welt verstanden werden. Momente der Entfremdung stellen jedoch ,,[...] nur dann [eine] kritikwürdige Störung in den Weltbeziehungen dar, wo jene als Resonanzsphären konzipierten Weltbereiche Anwandlungsprozesse systematisch und dauerhaft verhindern, abwehren oder sich ihnen widersetzen" (Beljan 2017: 157). Vor dem Hintergrund von Bildungsprozessen bilden Phasen der angenommenen sowie bejahten Entfremdung ein konstitutives Übergangsstadium: Durch das Heraustreten des Subjekts aus seinen gewohnten Weltverhältnissen, durch Entfremdungserfahrungen können sich Selbst und Welt einander anverwandelnd und durch Ausbildung neuer Resonanzverhältnisse in eine neue Beziehung eintreten (Beljan 2017: 157). Nach Rosas Auffassung bildet Entfremdung die Voraussetzung für eine transformierende Anverwandlung von Selbst und Welt sowie für die Etablierung stabiler Resonanzachsen (Rosa 2016a: 323).

Die Dialektik von zuvor geschilderter ,Resonanz' und ,Entfremdung ‘ wird in der Entfaltung von Rosas Resonanzverständnis auf mehrdimensionale Weise ersichtlich: Der Resonanztheoretiker setzt die unterschiedlichen Dimensionen, welche den Beschleunigungstendenzen ausgesetzt sind, in Verbindung und skizziert die Formen der Entfremdung. Hierbei ersetzt Entfremdung das eigene Handeln; an die Stelle der Produkte tritt die ,Dingwelt ' und anstelle der Entfremdung von der Natur tritt die Entfremdung von den zwei Dimensionen Raum und Zeit. Die Dimension der sozialen Beziehungen und der Selbstbezug bilden in Rosas Konzept eine geschlossene Dimension.

3 Die zunehmende Fragmentierung von Zeitstrukturen verursacht durch technische Beschleunigung, Beschleunigung des sozialen Wandels sowie des Lebenstempos können in der Konsequenz dazu führen, dass die Subjektivität als Ganze zunehmend krisenhaft wird. Folglich kann die Stabilität der Identität unter Umständen nicht (ausreichend) hergestellt werden, so dass sich eine „transitorische“, „situativ[e]“ (Rosa 2014: 362ff.) Identität entwickelt, die als reine Wandlungsfähigkeit und nicht als die kohärente Verknüpfung „narrativer Muster“ (Rosa 2014: 35) angesehen wird. 
Aus Sicht des Autors stellt eine auf sozio-historische Kontingenz und kulturelle Prägung gerichtete, gelungene Lebensform ein ,dialektisches Wechselverhältnis zwischen Entfremdungs- und Resonanzmomenten der Weltbeziehung [dar]“ (Rosa 2016a: 298), wobei Rosa bezüglich des Spannungsfelds bzw. der ,Balance‘ beider Momente nur vage Argumentationen anführt.

\section{Erlebte Resonanz im Weiterbildungsgeschehen - eine empirische Annäherung}

Das zuvor erläuterte Beschleunigungsphänomen wurde vor dem Hintergrund erlebter Resonanz und Entfremdung im Weiterbildungsgeschehen ${ }^{4}$ aus Teilnehmendensicht untersucht.

Die zentrale Fragestellung der Untersuchung lautet: Welche Resonanz- und Entfremdungspotenziale lassen sich im Weiterbildungsgeschehen identifizieren?

Ziel der Untersuchung ist die Identifikation von Resonanz- und Entfremdungspotenzialen im Weiterbildungsgeschehen in einer Kontrastierung von zwei Weiterbildungsanbietern aus dem Bereich der betrieblichen Weiterbildung und der konfessionellen Erwachsenenbildung. Die Analyse für die Erwachsenenbildungspraxis zielt u.a. auf folgende Aspekte:

- Reflexion bzw. Bewusstwerdung von Resonanzerfahrungen im Weiterbildungsgeschehen,

- Reflexion bzw. Bewusstwerdung von Widersprüchen und Entfremdung, Identifikation von kollektiven Erfahrungsräumen/-momenten,

- Identifikation und Bildung von „Relationalen Resonanzstrategien“ (RRS) zur Gestaltung von resonanzfördernden Erwachsenenbildungsangeboten.

Hierzu wurden leitfadengestützten Interviews $(\mathrm{N}=20)$ mit Teilnehmenden eines konfessionellen Erwachsenenbildungsanbieters und mit Teilnehmenden eines betrieblichen Weiterbildungsanbieters (Global Player) im Anschluss an die jeweiligen Bildungsveranstaltungen durchgeführt.

In einer konstatierenden Analyse wurden die Untersuchungsergebnisse gegenübergestellt. Es wurden strukturierte, leitfadengestützte Interviews mit Teilnehmenden aus verschiedenen Veranstaltungen der beiden Anbieter durchgeführt.

4 Es wurden das Erleben, die Wahrnehmung und Performanz von resonanten und entfremdeten Momenten im Weiterbildungs- bzw. Kursgeschehen aus Teilnehmendensicht unter Berücksichtigung unterschiedlicher resonanztheoretischer Dimensionen untersucht. 
Der Leitfaden ist, neben einem einleitenden und einem abschließenden offenen Frageteil, in drei übergeordnete Themenblöcke unterteilt: 1. Soziale Beschleunigung, 2. Resonanz und 3. Entfremdung. Die Kategorien Resonanz und Entfremdung implizieren jeweils die Aspekte einer a) ,stofflichen Dimension “, einer b) „performanten, interaktionalen und didaktischen Dimension “ und einer c) ,,leiblichen/körperlichen Dimension “ im Weiterbildungsgeschehen.

Die Auswahl von zwei sehr unterschiedlichen Bildungsanbietern und eine damit vermutlich verbundene bzw. unterstellte konträre Intention der Anbieter (Ökonomisierungsgedanken und Instrumentalisierung von Bildung vs. altruistische Motive, Bildung vornehmlich zur Persönlichkeitsentwicklung) sowie deren Sicht auf Erwachsenenbildung/Weiterbildung sollen der Kontrastierung der Ergebnisse dienen. Hierzu wurden überwiegend persönlichkeitsfördernde Bildungsangebote in die Untersuchung einbezogen.

Die Auswertung der qualitativen Interviewbefragung erfolgte nach der Grounded Theory (Strauss/Corbin 1996: 148). ${ }^{5}$ Die methodologischen Analyseschritte orientierten sich an den Vorgaben des offenen und axialen Kodiervorgangs und dienten in einem zweiten Schritt der Bildung von Kodier-Familien bzw. sogenannten „Strategien-Familien“" nach Glaser (Glaser in Mey/ Mruck 2007) und somit der Herausbildung der Relationalen Resonanzstrategien. ${ }^{6}$

Exemplarisch werden im Folgenden erste Zwischenergebnisse der qualitativen Analyse vorgestellt am Beispiel der Herausbildung von Relationalen Resonanzstrategien im Umgang mit Beschleunigung und Digitalisierung sowie den Relationalen Resonanzstrategien im Umgang mit Leiblichkeit.

\section{Relationale Resonanzstrategien}

Das bereits eigens entwickelte heuristische Modell der Relationalen Resonanzstrategien (RRS), welches aus den Erhebungsdaten generiert wurde, dient der

5 Alle Interviews wurden digital aufgezeichnet und mit Hilfe der Transkriptionssoftware f4 abgebildet sowie in MAXqda ausgewertet. Es wurden anonymisierte Volltranskriptionen der Interviews durchgeführt.

6 Die bislang ausgewerteten Interviews wurden einer Doppelkodierung und zudem in Interpretations- und Diskussionsgruppen einer kommunikativen Validierung unterzogen. 
1) Relationale Resonanzstrategien im Umgang mit Beschleunigung und Digitalisierung

$\rightarrow$ Temporale \& technoide Strategien

- VORLAUFEN

- MITLAUFEN

- INNEHALTEN (ANHALTEN)

- GEGENLAUFEN

2) Relationale Resonanzstrategien im Umgang mit Stofflichkeit

$\rightarrow$ Stoffliche Strategien

- DAS GROSSE GANZE WAHRNEHMEN

- FRAGMENTE WAHRNEHMEN

- DAS VERMITTELNDE WAHRNEHMEN

- DAS ÄUSSERE WAHRNEHMEN

3) Relationale Resonanzstrategien in der Performanz und Interaktion $\rightarrow$ Soziale, performante Strategien

- INTERAKTIONALE VERSCHMELZUNG \& STABILISIERENDES LERNEN

- SOZIALE ZWECKGEBUNDENHEIT \& ERPROBENDES LERNEN

- AUTOPOEITISCHE DISTANZ ZU ANDEREN \& SELBSTREFLEXIVES LERNEN

- VERDINGLICHUNG DES KOLLEKTIVS \& ZIELGERICHTETES LERNEN

4) Relationale Resonanzstrategien im Umgang mit Körper und Geist $\rightarrow$ Leibliche Strategien

- SUCHE NACH KONGRUENZ

- ANERKENNUNG VON VULNERABILITÄT

- AMBIVALENTE SELBSTOPTIMIERUNG

- ÄQUIVOKE INKONGRUENZ

Abb.1: Übersicht der Relationalen Resonanzstrategien (RRS) (Wienberg im Erscheinen)

Beschreibung von erlebter Resonanz und Entfremdung im Lehr-Lern-Geschehen. Hierbei geht es um die Herausbildung und dem (bewussten) Einsatz von 
förderlichen Strategien zur Erhöhung der Ermöglichung von Resonanz. Der resonanztheoretische Ansatz soll auf der theoretischen Ebene für die Erwachsenenbildung/Weiterbildung fruchtbar und anschlussfähig an aktuelle Diskurse gemacht werden (Wienberg 2018; 2019).

Bei der Auswertung des Datenmaterials kristallisierten sich vier übergeordnete RRS heraus: (Abb. 1).

Exemplarisch werden im Folgenden zwei Resonanzstrategien vorgestellt: Zum einen die ,temporalen Resonanzstrategien im Umgang mit Beschleunigung" und zum anderen die ,leiblichen Resonanzstrategien im Umgang mit Körper und Geist".

\section{Relationale Resonanzstrategien im Umgang mit Beschleunigung und Digitalisierung}

Der Beschleunigungstheorie folgend unterliegen moderne Gesellschaften einem allumfassenden Beschleunigungsphänomen - insbesondere vor dem Hintergrund einer wachsenden Technologisierung und Digitalisierung durch u.a. die rasante Steigerung von Mobilität und Kommunikationsmöglichkeiten und eine damit einhergehende große zeitliche und räumliche Flexibilisierung. Hierbei spielen mögliche Interdependenzen im Spannungsfeld zwischen Kultur und Technik u.a. im Hinblick auf das Wissensmanagement eine zentrale Rolle. Mit dieser lebensbereichsübergreifenden Beschleunigung und einer zunehmenden Technologisierung kann auch auf individueller Ebene ein umfassender sozialer, kultureller und ökonomischer Wandel einhergehen (Blossfeld et al. 2017). Beispielsweise können sich soziale Praktiken, Kommunikationsstrukturen und Lebensformen verändern (Rosa 2016b: 42f.). Zudem können sich durch eine vermeintliche Beschleunigung des Lebenstempos eine Steigerungsund Wettbewerbslogik verschärfen und zugleich die Optionenvielfalt erhöhen. 


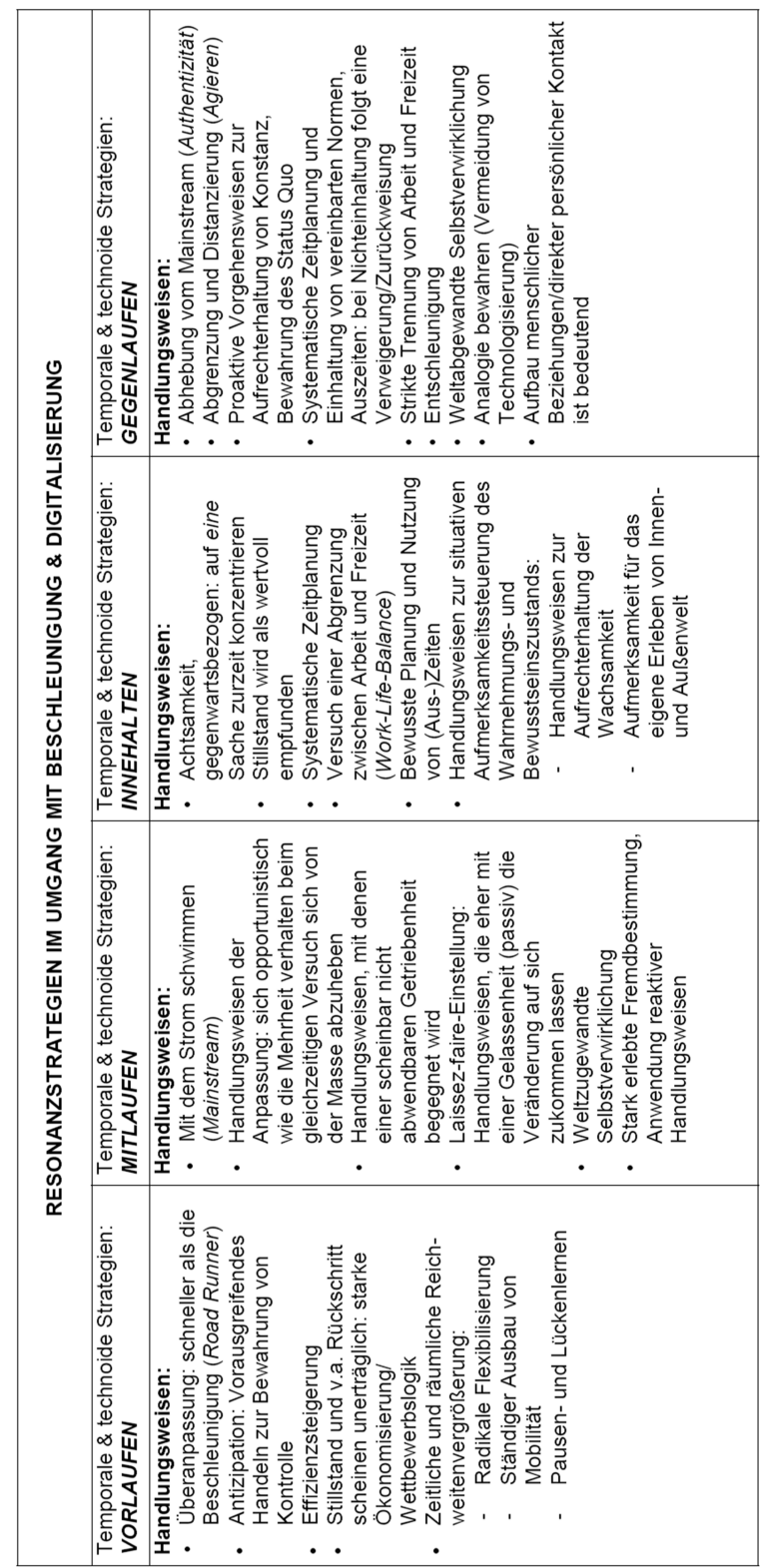

Abb. 2: Relationale Resonanzstrategien im Umgang mit Beschleunigung und Digitalisierung (Wienberg im Erscheinen) 
Folgende zentrale Aspekte wurden im Spannungsfeld der Beschleunigung und Digitalisierung von den Befragten genannt. Die Verortung, die im Material identifizierte Wahrnehmung, das Erleben und der Umgang mit Beschleunigungs- und Digitalisierungsphänomenen erstrecken sich kontextbezogen auf einem Kontinuum:

- Reichweite: Zum einen wurde eine wahrgenommene Reichweitenvergrößerung, lebensbereichsübergreifende Entgrenzung und Flexibilisierung benannt. Zum anderen wurden unterschiedliche Formen, Ausprägungen und Ausmaße räumlicher und zeitlicher Begrenzungen, Stagnationen bzw. vorherrschende Standardisierungen sowie Ein- und Begrenzungen erfahren.

- Einfluss: Die wahrgenommene Einflussnahme auf das Geschehen sowie auf Verhaltens- und Handlungsmöglichkeiten erstrecken sich in sehr differenzierten Ausprägungen von einem hohen Maß an Selbstwirksamkeit(serwartung) bis hin zu geäußerten Befürchtungen des Kontrollverlusts bezüglich des eigenen Verhaltens, Agierens und Reagierens.

- Sinn: Die im Material auffindbaren Äußerungen hinsichtlich des Empfindens und des subjektiven Erlebens bewegen sich auf der Verhältnis- und Verhaltensebene in der Ausdeutung des jeweiligen Sinnbezugs äußerst divergent: Diese Wahrnehmungen reichen von einer starken, sinnstiftenden Verinnerlichung von Normen, Werten und Zielen etc. bis hin zu einer (fremdbestimmten) Auferlegung, häufig verbunden mit dem Empfinden von Inkongruenz.

- (Selbst-)Zweck: In der Befragung zeigten sich unterschiedlich starke Ausprägungen der Wahrnehmung einer (Selbst-)Zweckgebundenheit: Deutungen und Handlungsformen richten sich hierbei sowohl auf die Aufrechterhaltung bzw. Erlangung von Individualität und (individuelle und kollektive) Aushandlungs- und Abgrenzungsprozesse als auch auf die Ausrichtung auf den sogenannten (,ökonomisierten“) Mainstream.

In der Analyse konnten Momente der Reflexion bzw. Bewusstwerdung von Resonanzerfahrungen sowie von Widersprüchen und Entfremdung im Weiterbildungsgeschehen sichtbar gemacht werden. Zudem wurden kollektive und individuelle RRS im Lehr-Lern-Geschehen aus Teilnehmendensicht identifiziert. Die Handlungsweisen, die aus der Befragung der Weiterbildungsteilnehmenden generiert wurden, werden im Folgenden exemplarisch anhand von Interviewausschnitten (Zitaten) entfaltet: 


\section{Relationale Resonanzstrategien im Umgang mit Leiblichkeit}

Die Phänomenologie versucht mit ihrem Konzept der Leiblichkeit, eine Bedingung der Möglichkeit und Wirklichkeit sowie Sozialität als eine dem Menschen inhärente Fähigkeit darzulegen. Diese Fähigkeit ist ambiguitär - d.h. weder über Identität noch Alterität ist vollständig zu verfügen (Meyer-Drawe 1987). Die chiastische Verschränkung von Welt und Subjekt ist im Modus der Leibgebundenheit begründet. Sie ermöglicht, den Dualismus der getrennten Sphären des Körpers einerseits und des Geistes andererseits zu überwinden.

Aus resonanztheoretischer Sicht bzw. in den Ausdeutungen von Hartmut Rosa stellt die menschliche Weltbeziehung eine leibliche, körperliche und verkörperte Weise dar, sich auf die Welt zu beziehen, die Welt zu erfahren und sich zur Welt „zu verhalten“ (Rosa 2016a: 71). Der Mensch ist eingebettet in Gesellschaften und Kulturen, weshalb man nicht verkennen darf, dass die soziale und kulturelle Existenz auch immer eine leibliche Erfahrung ist und dass Körperlichkeit und Leiblichkeit u.a. die Folge sozialer Prozesse sind (Butler 2005: 33f.; Vlieghe 2014: 1030; Burghard 2017: 560). Während Menschen miteinander kommunizieren, interagieren unbewusst auch ihre Körper, wodurch komplexe Wechselbeziehungen entstehen. Als ein Wesen, dessen Leib in Abhängigkeit von der Gesellschaft existiert, werden Menschen somit auch durch Menschen leiblich beeinflusst (Merleau-Ponty 1994). Im Lehr-Lern-Geschehen nimmt demzufolge Leiblichkeit eine „Scharnierfunktion“ ein. ${ }^{7}$

Folgende zentralen Aspekte wurden im Spannungsfeld von Leiblichkeit von den Befragten geäußert. Die Verortung, die im Material identifizierte Wahrnehmung, das Erleben und der Umgang mit Leiblichkeit erstrecken sich kontextbezogen auf einem Kontinuum:

- Anerkennung: Hierbei handelt es sich um eine erfahrene Leiblichkeit und Körperlichkeit bzw.einer Wahrnehmung eines Dualismus von Körper und Geist. Zum einen wurde über eine wahrgenommene Ganzheitlichkeit bzw. Unauflösbarkeit von Körper und Geist berichtet. Zum anderen wurde eine deutliche Fragmentierung des leiblichen Erlebens und der Wahrnehmung beschrieben. Ebenso spielen Aspekte der Entität oder auch der Vulnerabilität in diesen Erlebens- und Wahrnehmungsbereich hinein.

7 Anzumerken ist, dass der Leiblichkeitsbegriff vielfältige Aspekte anerkennt und impliziert, beispielsweise nehmen Emotionen, Sexualität und Geschlechtlichkeit eine entscheidende Rolle ein (jene Facetten der Leiblichkeit, welche nicht augenscheinlich aus dem Datenmaterial aufgrund von Fragestellung und Interviewsetting hervorgehen). 


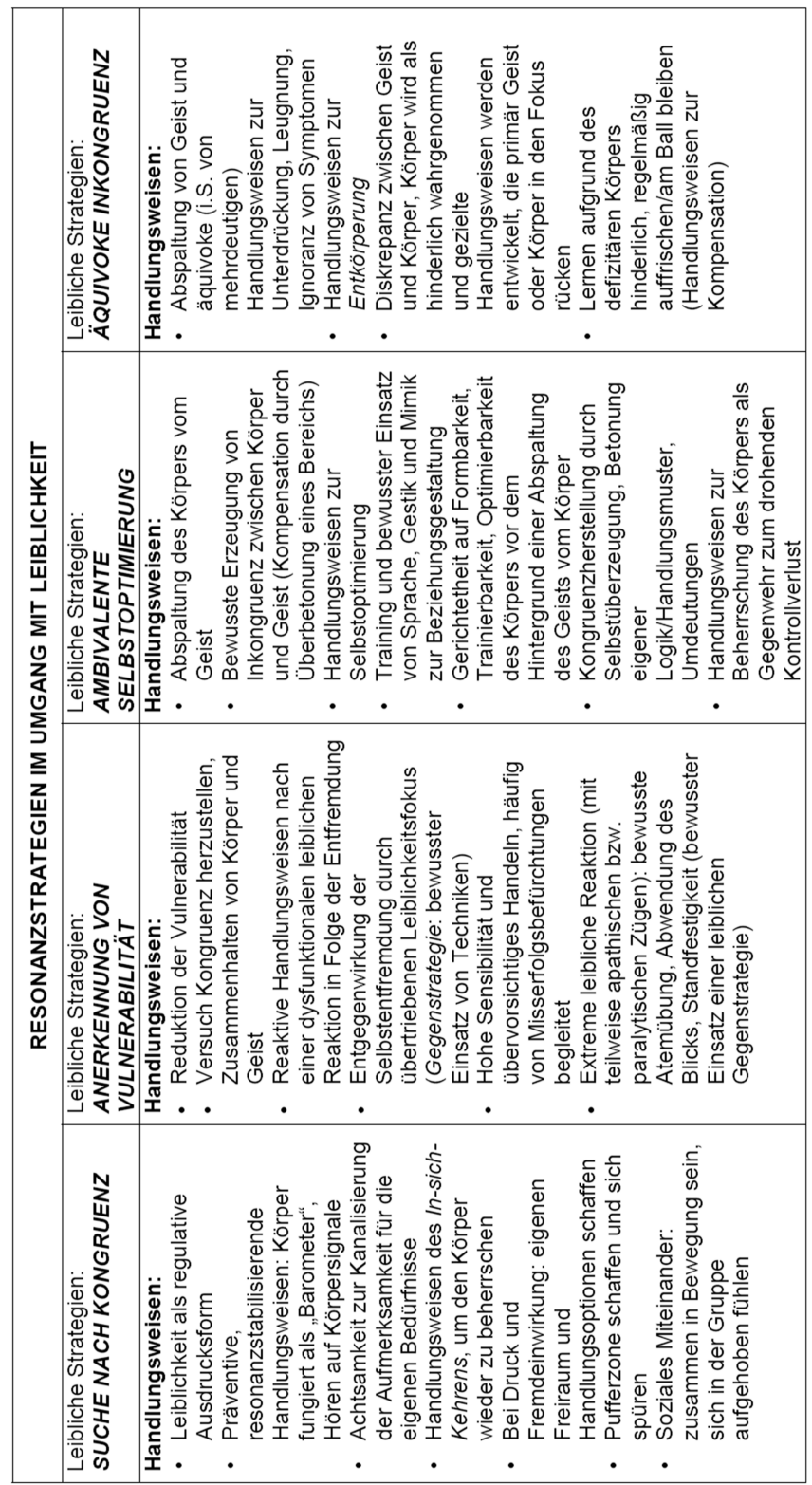

Abb. 3: Relationale Resonanzstrategien im Umgang mit Körper und Geist (Wienberg im Erscheinen) 
- Wahrnehmung \& Erleben: Die Befragten berichteten, inwiefern Kongruenz als auch Inkongruenz hinsichtlich der eigenen Wahrnehmung und des Erlebens jeweils deutlich wurde: Deutungen und Handlungsformen richten sich hierbei sowohl auf die Aufrechterhaltung von bisher "Geglaubtem“ und auf den Schutz vor „Neuem“ als auch auf die Erlangung von Individualität und persönliche Entwicklung. Kongruenz wurde hierbei durch Selbstüberzeugung bzw. eine Betonung eigener Logik/Handlungsmuster oder Umdeutungen hergestellt. Zudem wurde als Kompensation versucht, bewusst Inkongruenz zu erzeugen, z.B. durch Überbetonung eines Bereichs.

- Einfluss: Die wahrgenommene Einflussnahme auf das Geschehen sowie auf Verhaltens- und Handlungsmöglichkeiten erstreckt sich in sehr differenzierten Ausprägungen von einem hohen $\mathrm{Ma}$ an Selbstwirksamkeit(serwartung) bis hin zu geäußerten Befürchtungen bezüglich des eigenen Verhaltens, Agierens und Reagierens die Kontrolle zu verlieren.

- Gerichtetheit: Die aus dem Material gesichteten Äußerungen weisen hinsichtlich des Verhaltens, der Performanz und der Interaktion sehr unterschiedliche Grade der Intentionalität als auch der Ungerichtetheit bzw. Intuition auf: Die Handlungsformen reichen hierbei von einer starken Gerichtetheit und Bewusstwerdung bis hin zu ungerichteten und/oder intuitiven Handlungen. In diesem Zusammenhang zeigen die Befragten eine intentionale Welt-Bezogenheit, welche nicht nur eine kognitive, sondern auch evaluative und existenzielle Beziehung zur Welt beinhaltet.

Im Folgenden werden die Handlungsweisen, die durch die Reflexion bzw. Bewusstwerdung von Resonanzerfahrungen sowie von Widersprüchen und Entfremdung im Weiterbildungsgeschehen identifiziert wurden, dargestellt und mit Interviewpassagen (Zitaten) veranschaulicht:

\section{Resümee und Ausblick}

Meines Erachtens fungiert die Erwachsenenbildung und Weiterbildung als eine Art „Scharnier“ zwischen den Bildungsbereichen und den Transitionen innerhalb einer Bildungsbiografie: zwischen der Schule und dem Beruf, der Erwerbstätigkeit und Phasen der Nicht-Erwerbstätigkeit oder auch des Wiedereinstiegs oder der Neuorientierung sowohl im Rahmen von beruflicher als auch allgemeiner Erwachsenenbildung/Weiterbildung. Erlebte Momente von Resonanz oder auch Entfremdung nehmen in diesem Zusammenhang eine weichenstellende Rolle für das Lernen über den Lebenslauf - und somit für die Erwachsenenbildung/Weiterbildung - ein. 
Veränderte Anforderungen an das Subjekt ergeben sich darüber hinaus u.a. aus den Auswirkungen des digitalen Wandels, die eine flexiblere Orts- und Zeitnutzung von Bildungsangeboten oder auch veränderte Settings zur Ausübung von Bildungsaktivitäten mit sich bringen. Auch eine zunehmende Steigerungslogik könnte der Anverwandlung von (subjektivem) Resonanzerleben entgegenstehen, da theoriekonform grundsätzlich von einem Moment des Unverfügbaren und deshalb Unbezahlbaren bzw. Unverdinglichten ausgegangen wird. Es verlangt vielmehr ein ,Sich-einlassen auf die Dinge sowie die Bereitschaft, sich selbst zu verändern, sich gleichsam aufs Spiel zu setzen, zugleich ist sie nur dort möglich, wo Selbstwirksamkeit erfahrbar wird" (Rosa 2016a: 433).

Die Relationalen Resonanzstrategien als ein heuristisches Modell scheinen zur Beschreibung von Momenten erlebter Resonanz und Entfremdung sowie resonanzermöglichenden Strategien beizutragen. Hierbei geht es um die Herausbildung und den (bewussten) Einsatz von förderlichen Strategien, um Möglichkeiten von Resonanz zu vergrößern. Lebensnah lässt es sich meines Erachtens wie folgt ausdeuten: Wer beim Anblick von Nordseewellen bereits Resonanz verspürt hat, kann dieses erneute Erleben zwar nicht sicherstellen, jedoch kann die Möglichkeit an der Nordsee zu sein und Wellen zu sehen die Wahrscheinlicht des individuellen Resonanzerlebens durchaus erhöhen. (meines Erachtens)

Abschließend lässt sich konstatieren, dass die zuvor beschriebenen zentralen Aspekte der Erwachsenenbildung, welche sich mitunter vom Lernen im schulischen Kontext unterscheiden, in der resonanztheoretischen Diskussion in der Resonanzachse ,lebenslanges Lernen“ Berücksichtigung finden. Dieses vollzieht sich zum einen in der Ausgestaltung und Ausdeutung des Bildungsbegriffs. Jenem Bildungsverständnis liegt die Idee zu Grunde, dass Bildung ein lebensgeschichtlicher Vorgang sei, in dessen Verlauf die Individuen versuchen, Identität für sich immer wieder neu herzustellen und erweiterte Handlungsfähigkeit zu entwickeln (Holzkamp 1993). Zudem sollte in der Betrachtung von Lehr-Lern-Geschehen stärker der Aspekt der Leiblichkeit berücksichtigt werden.

Die bisherige Forschung lässt eine starke Ausrichtung der Analysen auf individuelle Prozesse erkennen. Aus erwachsenenpädagogischer Sicht erscheint jedoch weiterführend eine Übertragung auf institutionelle und kollektive Prozesse sinnvoll, insbesondere im Hinblick auf die (berufliche bzw. betriebliche) Erwachsenenbildung/Weiterbildung. 


\section{Literatur}

Adorno, Theodor W. (2003): Gesammelte Schriften: Herausgegeben von Rolf Tiedemann. Frankfurt am Main: Suhrkamp.

Beljan, Jens (2017): Schule als Resonanzraum und Entfremdungszone. Eine neue Perspektive auf Bildung. Weinheim: Beltz.

Blossfeld, Hans-Peter et al. (2017): Bildung 2030 - veränderte Welt. Fragen an die Bildungspolitik. Gutachten. Vereinigung der Bayerischen Wirtschaft e.V. (Hrsg.). Münster: Waxmann.

Brumlik, Micha (2016): Resonanz oder: Das Ende der kritischen Theorie. Blätter für deutsche und internationale Politik, 5/2016, S. 120-123.

Burghard, Anna Bea (2017): Körperlichkeit und Leiblichkeit. In: Böller, Karin (Hrsg.) (2017): Kompendium Kinder- und Jugendhilfe, Wiesbaden: Springer, S. 553-557.

Butler, Judith (2005): Giving an Account of Oneself. New York: Fordham University Press.

Döbler, Marie-Kristin (2016): Rezension vom 10.08.2016 zu: Hartmut Rosa: Resonanz. Eine Soziologie der Weltbeziehung. Suhrkamp Verlag (Berlin) 2016. H-Soz-Kult, 10.08.2016. URL: http://www.hsozkult.de/publicationreview/id/r ezbuecher-26084. Abruf: 21.03.2019.

Holzkamp, Klaus (1993): Lernen. Subjektwissenschaftliche Grundlegung. Frankfurt am Main, New York: Campus Verlag.

Horkheimer, Max (1937): Traditionelle und kritische Theorie. URL: http://lesekreis.blogsport.de/images/MaxHorkheimerTraditionelleundkritischeTheorie.pdf. Abruf: 21.03.2019.

Jaeggi, Rahel (2005): Entfremdung. Zur Aktualität eines sozialphilosophischen Problems. Frankfurt am Main: Campus.

Merleau-Ponty, Maurice (1994): Keime der Vernunft. Vorlesungen an der Sorbonne. Herausgegeben von Bernhard Waldenfels. München: Fink.

Mey, Günter/Mruck, Katja (2007): Grounded Theory Methologie. Bemerkungen zu einem prominenten Forschungsstil. In: Historical Social Research. 19/2007, S. 11-39.

Meyer-Drawe, Käte (1987): Leiblichkeit und Sozialität. Phänomenologische Beiträge zu einer pädagogischen Theorie der Inter-Subjektivität. 2. Auflage. München: Fink.

Oevermann, Ulrich (2002): Klinische Soziologie auf der Basis der Methodologie der objektiven Hermeneutik. URL: https://www.ihsk.de/publikationen/Ulrich Oevermann-Manifest_der_objektiv_hermeneutischen_Sozialforschung.pdf. Abruf: 21.03.2019.

Paech, Niko (2012): Befreiung vom Überfluss. Auf dem Weg in die Postwachstumsökonomie. München: Oekom Verlag.

Rosa, Hartmut (2009): Kritik der Zeitverhältnisse. Beschleunigung und Entfremdung als Schlüsselbegriffe der Sozialkritik. In: Jaeggi, Rahel / Wesche, Tilo (Hrsg.): Was ist Kritik? Frankfurt am Main: Suhrkamp, S. 23-54. 
Rosa, Hartmut (2011): Entfremdung in der Spätmoderne. Umrisse einer Kritischen Theorie der sozialen Beschleunigung. In: Koppetsch, Cornelia (Hrsg.): Nachrichten aus den Innenwelten des Kapitalismus. Wiesbaden: Springer, S. 221-252.

Rosa, Hartmut (2012): Weltbeziehungen im Zeitalter der Beschleunigung. Frankfurt am Main: Suhrkamp.

Rosa, Hartmut (2014): Beschleunigung. Die Veränderung der Zeitstrukturen in der Moderne. 10. Auflage. Frankfurt am Main: Suhrkamp.

Rosa, Hartmut (2016a): Resonanz. Eine Soziologie der Weltbeziehung. 3. Auflage. Berlin: Suhrkamp.

Rosa, Hartmut (2016b): Beschleunigung und Entfremdung. Entwurf einer Kritischen Theorie spätmoderner Zeitlichkeit. 5. Auflage. Berlin: Suhrkamp.

Schmidt-Lauff, Sabine (2012): Grundüberlegungen zu Zeit und Bildung. In: Schmidt-Lauff, Sabine (Hrsg.): Zeit und Bildung. Annäherungen an eine zeittheoretische Grundlegung. Münster: Waxmann, S. 11-60.

Strauss, Anselm / Corbin, Juliet (1996): Grundlagen qualitativer Sozialforschung. Weinheim: Beltz.

Schulz, Peter (2015): Zur Ambivalenz der kritischen Soziologie Hartmut Rosas. De Gruyter-Zeitschrift für kritische Sozialtheorie und Philosophie 2015; 2(1), S. 101-117.

Vlieghe, Joris (2014): Foucault, Butler and corporeal experience: Taking social critique beyond phenomenology and judgement. In: Philosophy and Social Criticism, Vol.14, Issue 10, S. 1019-1035.

Wienberg, Jana (2018): Eine resonanztheoretische Betrachtung von Beratung. In: Dörner, Olaf / Iller, Carola / Schüßler, Ingeborg / Maier-Gutheil, Cornelia / Schiersmann, Christiane (Hrsg.): Beratung im Kontext des Lebenslangen Lernens. Konzepte, Organisation, Politik, Spannungsfelder. Opladen, Berlin, Toronto: Barbara Budrich Verlag, S. 35-46.

Wienberg, Jana (2019): Resonanz in der Arbeitswelt - keine Frage des Alters. In: Grotlüschen, Anke/Schmidt-Lauff, Sabine/Schreiber-Barsch, Silke/Zeuner, Christine (Hrsg.): Das Politische in der Erwachsenenbildung. Frankfurt am Main: Wochenschau Verlag, S. 190-194.

Zeuner, Christine (2008): Erwachsenenbildung. In: Faulstich-Wieland, Hannelore/Faulstich, Peter (Hrsg.): Erziehungswissenschaft. Ein Grundkurs. Hamburg: Rowohlt Verlag, S. 532-555. 


\section{Weggehen, um dazuzugehören? Kritische Überlegungen zum Konzept von Lernmobilität ${ }^{1}$}

\section{Vorbemerkung}

Internationale Begegnungen, Auslandsjahre, Erasmus-Semester, Fachkräfteaustausch - es ist kaum möglich, den vielfältigen Mobilitätsaufforderungen $\mathrm{zu}$ entgehen, ohne als träge und desinteressiert oder gar als fremdenfeindlich und chauvinistisch zu gelten. Auslandserfahrung scheint regelmäßiger Bestandteil von Lebensläufen geworden zu sein. Allerdings, was als allgemeiner Anspruch transportiert wird, spricht keineswegs alle auf gleiche Weise an. Der Diskurs um Bildungs- bzw. Lernmobilität zeigt feine Risse, die im Folgenden in den Blick genommen werden sollen. Dabei ist die Fragestellung darauf gerichtet, welche Forschungs- (und Handlungs-)Desiderate sich daraus für das Feld der Erwachsenenbildung ableiten lassen. Nach einem einleitenden Blick auf die Bedeutung von Mobilität im Kontext aktuellen gesellschaftlichen Wandels wird der EUropäische Mobilitätsimperativ einer kritischen Revision unterzogen und die Argumente vorgestellt, mit denen Auslandsaufenthalte zu Lernzwecken - so die offizielle Diktion - beworben werden. Abschließend illustrieren empirische Erträge aus der begleitenden Feldforschung eines Mobilitätsförderungsprojekts exemplarisch das Potential einer subjektorientierten Mobilitätsforschung, um die inhärenten Paradoxien und Ambivalenzen des Konzepts von Lernmobilität freizulegen.

\section{Mobilität als Gesellschaftsdiagnose}

Konzepte von Mobilität sind offen für vielfältige Deutungen. So kann der Begriff Mobilität die individuelle Bereitschaft zum Ortswechsel ebenso bezeichnen wie die Entwicklung einer korrespondierenden Infrastruktur und deren soziale Implikationen oder die vielfältigen Varianten von Bildungserfahrungen.

1 Es handelt sich um die überarbeitete Fassung meines Vortrags ,Learning Mobility. Mobilität als Programm - programmatische Entgrenzung?", gehalten auf der Jahrestagung der Sektion Erwachsenenbildung am 27.9.2019 in Mainz. 
Vorstellungen von Mobilität beziehen sich auf die Bewegung im Raum als Ortswechsel ebenso wie auf die Disposition zur Beweglichkeit oder auf die physischen Bedingungen und die Fähigkeit, sich (vorwärts) bewegen zu können.

Bewegungsmetaphern prägen aktuelle Gesellschaftsdiagnosen und Theorien, sie beschreiben einen Modus der Lebensführung moderner Gesellschaften. Bildungsprozesse werden als Horizonterweiterung oder Grenzüberwindung konzipiert. Bewegungsmetaphern unterliegen dabei ihrerseits einem Bedeutungswandel; Bilder und Geschichten, mit denen auf soziale oder individuelle Bewegung und Beweglichkeit Bezug genommen wird, reflektieren in ihren Konnotationsverschiebungen wandelnde gesellschaftliche Kontexte (Baumann 1996).

,$[\ldots]$ in the same way as the pilgrim was the most fitting metaphor for the modern life strategy preoccupied with the daunting task of identity-building, the stroller, the vagabond, the tourist and the player offer jointly the metaphor for the postmodern strategy moved by the horror of being bound and fixed" (Baumann 1996: 25f.).

Baumann differenziert zwischen Spaziergängern, Vagabunden, Touristen und Spielern als postmodernen Bewegungstypen, die auf je unterschiedliche Weise allesamt Variationen von Unverbindlichkeit repräsentieren. Sie bewegten sich durch die Welt, ohne irgendwo dazuzugehören, ohne sich einzubringen oder zu engagieren. Ihr Weltbezug bestünde in der Betrachtung, nicht in der Anteilnahme oder dem Engagement. Verbindlichkeit würde vermieden, soziale Beziehungen erschienen als vorübergehend, folgen- und voraussetzungslos, wodurch dem Verständnis gesellschaftlicher Zusammenhänge die politische Dimension entzogen würde (Baumann 1996: 24). Mobilität als UnterwegsSein, als demonstrierte Beweglichkeit oder zur Schau gestellte Haltung ist ihm Anforderung und Diagnose, Programm und Analyse postmoderner Gesellschaftsverhältnisse. Diese Überlegungen sind in mehrfacher Hinsicht herausfordernd. Mittels Bewegungsmetaphern lassen sich festgelegte, definierte Grenzen als prozesshaft begreifen. Raum-Zeit-Verhältnisse und soziale Zusammenhänge lassen sich im Hinblick auf eine mobile Gesellschaft weiterdenken, deren soziale Konstruktion wiederum zum Forschungsgegenstand wird. So skizziert Cresswell (2006: 219ff.) beispielsweise neue Orte, die in einer mobilen Welt entstünden als Transitbereiche, z.B. auf internationalen Flughäfen, wo sich alle nur vorübergehend aufhalten und nur in der Gemeinsamkeit des Unterwegsseins miteinander verbunden sind.

Der von Baumann wiederholt thematisierte Zusammenhang zwischen Mobilität und Identitätsbildung (Baumann 1996; 2014; o. J.) öffnet die Perspektive für die weitergehende Überlegung zu den sozialen und individuellen Folgen veränderter Mobilitätsmuster. Die Relativierung sozialer und materieller Bindungen geht für ihn mit der Sorge um sozialen Zusammenhalt und der 
Frage nach den veränderten Bedingungen für politisches Handeln einher. Aus dieser Diagnose einer wachsenden Unverbindlichkeit, dem ,horror of being bound and fixed" (Baumann 1996: 26), begründet sich m.E. die Relevanz einer kritischen Mobilitätsforschung, denn selbstverständlich bleiben die Institutionen und Angebote der Erwachsenen- und Weiterbildung von den Anrufungen des Mobilitätsimperativs nicht unberührt. Die Vermeidung von Verbindlichkeit und die Auflösung sozialer Zusammenhänge, die Baumanns Perspektive auf Mobilität kennzeichnen, stehen auch einem Bildungsprogramm, das auf Emanzipation, politische Teilhabe und gesellschaftliches Engagement gerichtet ist, entgegen. Diese Sichtweise steht freilich in direktem Gegensatz zu den Begründungen und Zielrichtungen, mit denen auf bildungspolitischer Ebene Mobilitätsförderungsprogramme argumentiert werden, wie im nächsten $\mathrm{Ab}$ schnitt zu sehen ist.

\section{Mobilität zu Lernzwecken}

Der im EU-Diskurs zumeist verwendete englische Begriff der ,learning mobility“ wird in offiziellen Dokumenten mit „Mobilität zu Lernzwecken“ übersetzt. Vereinfachend wird im Folgenden „Lernmobilität“ synonym verwendet. Damit werden in der offiziellen Diktion Auslandsaufenthalte bezeichnet, die explizit zum Kompetenzerwerb oder zu Bildungszwecken unternommen werden, und zwar zunächst unabhängig davon, ob dies in formalen oder non-formalen Zusammenhängen geschieht.

„Die Mobilität zu Lernzwecken - also ein Auslandsaufenthalt mit dem Ziel, neues Wissen sowie neue Fähigkeiten und Kompetenzen zu erwerben - ist eine der grundlegenden Möglichkeiten, mit denen junge Menschen ihre künftige Beschäftigungsfähigkeit erhöhen, ihr interkulturelles Bewusstsein und ihre persönliche Entwicklung voranbringen sowie ihre Kreativität und ihren Bürgersinn stärken können. Europäer, die als junge Lernende mobil sind, sind zumeist auch später im Arbeitsleben mobil.“ (Empfehlungen des Rates vom 28.6.2011, Jugend in Bewegung - die Mobilität junger Menschen zu Lernzwecken fördern. Amtsblatt der Europäischen Union 2011/C 199/01: 1)

Lernmobilität gilt demzufolge als unverzichtbarer Baustein der Europäischen Wissensgesellschaft (EU-2020 strategy: http://ec.europa.eu/europe2020/index_en.html) und jeder junge Mensch sollte daran teilhaben können:

„Every young person should have the opportunity to take part in some form of mobility, whether this be during their studies or training, in the form of 
a work placement, or in the context of voluntary activities“ (2008: European Platform of Learning Mobility: 2).

Dieser programmatische Mobilitätsdiskurs, der ausschließlich positive Effekte postuliert, ist mit konkreten Förderinstrumenten hinterlegt, von denen Erasmus + sicher das bekannteste ist. Die europäischen Fördermittel für Mobilität zu Lernzwecken sollen in der laufenden Förderperiode (2014 bis 2020) von 14,7 Mrd. auf ca. 30 Mrd. verdoppelt werden. Nationale Agenturen in den Mitgliedsländern unterstützen, beraten und organisieren umfangreiche Begleitmaßnahmen.

Folgt man der Argumentation in den entsprechenden Programmdokumenten, so ist Lernmobilität politisches Programm und individuelle Chance zugleich, Anforderung und Versprechen in einem. Lernmobilität erscheint als eine der vier europäischen Freiheiten, skizziert wird eine dreifache Gewinnsituation, in der die Europäische Gemeinschaft, Wirtschaft und Arbeitgeber und Individuen gleichermaßen vom Lernen im Ausland profitieren. Auslandserfahrungen brächten einen individuellen Nutzen, der sich vor allem in einer verbesserten Beschäftigungsfähigkeit ausdrückt. In der EUropäischen Mobilitätsförderung lediglich ein weiteres Instrument der Zurichtung flexibler Arbeitskräfte zu sehen, greift dennoch zu kurz, denn auch eine wirtschaftspolitisch motivierte Mobilitätsfördermaßnahme kann subjektiv bereichernde Bildungserfahrungen ermöglichen. Bildungseffekte durch Befremdungen und die Überwindung von Orientierungskrisen, wie sie während eines längeren Auslandsaufenthalts zwar nicht notwendig, jedoch sehr wahrscheinlich vorkommen, können subjektiv gewinnbringend, Horizont erweiternd sein. Jugend für Europa, die vom Bundesfamilienministerium (BMFSFJ) beauftragte Nationale Agentur für die Umsetzung der EU-Programme unter Erasmus+, betont diese individuelle Dimension der Lernerfahrungen durch grenzüberschreitende Mobilität:

„Gesellschaftliche Teilhabe bedeutet heute auch, über Grenzen hinweg leben zu können. Erfahrungen in verschiedenen Kontexten stärken die Persönlichkeit des Einzelnen in der globalisierten Welt. Grenzüberschreitende Mobilität eröffnet jungen Menschen neue Perspektiven. Studien zeigen: Solche Erfahrungen motivieren nicht nur zu lebenslangem Lernen. Sie steigern auch das europäische Bewusstsein und führen dazu, dass Jugendliche mehr gesellschaftliche Verantwortung übernehmen." (https://www.jugendfuereuropa.de/themen/lern erfahrungen-durch-grenzueberschreitende-mobilitaet/)

Obwohl Mobilitätsförderprogramme wie Erasmus+ sich vom Anspruch her explizit an die Allgemeinheit aller jungen Europäer*innen richten und ihre Möglichkeiten allen jungen Menschen in der Europäischen Union offenstehen sollen, setzt sich diese Form von Lernmobilität nur langsam durch. Zudem sind die Teilnahmequoten weder sozial noch geografisch gleich verteilt. Sie reichen 
von 3\% in der Türkei und $10 \%$ in Bulgarien, Großbritannien und Rumänien bis zu fast $40 \%$ in Zypern und Luxemburg. In den meisten anderen Ländern lag die Beteiligung an Erasmus-Aktivitäten bis vor kurzem noch unter 20\% (European Commission, 2011: 24). Studierende an Universitäten beteiligen sich deutlich stärker als Auszubildende in der beruflichen Bildung. Trotz aller unterstützenden Fördermaßnahmen bestehen die unsichtbaren Barrieren offensichtlich fort, mit denen sozial benachteiligte Jugendliche in Europa davon abgehalten werden, lernmobil zu werden. Dafür werden vor allem finanzielle Gründe geltend gemacht: lediglich 15\% der Auslandsstudierenden bzw. -lernenden hat diesen Aufenthalt über ein Förderprogramm wie Erasmus finanziert, die große Mehrheit reist und bildet sich auf eigene Kosten (European Commission, 2011: 6). Als weiterer wichtiger Hinderungsgrund werden familiäre Verpflichtungen genannt (ibid.: 25).

Der als allgemein postulierte Mobilitätsanspruch adressiert also keineswegs alle Jugendlichen in Europa in gleicher Weise. Das dreifache Gewinnversprechen gilt nicht für alle Beteiligten; der Zugang zu den Förderprogrammen ist nicht für alle gleich weit geöffnet und die Verwertungschancen sind ebenfalls unterschiedlich hoch. Zu den Paradoxien der Mobilitätsförderung gehört die exklusive Wirkung, die das Konzept der Lernmobilität entfaltet. Auch wenn formale ebenso wie non-formale Kontexte mit einbezogen werden, ist die Anerkennung einer Maßnahme doch darauf angewiesen, dass ein - wie auch immer gearteter - Lernprozess konstatierbar ist. Als Lernmobilität gilt somit vor allem, was im Rahmen eines Förderprogramms geordnet und strukturell gerahmt ist, weitgehend auf formale Bildungsstrukturen rückbezogen und direkt oder indirekt mit Credit Points bemessen werden kann. Deutlich wird dies im Vergleich mit anderen Formaten des Unterwegsseins - touristisches Reisen, Auslandsjahre, Ferienlager oder auch Flucht und Migration mögen ebenfalls Bildungserfahrungen ermöglichen, interkulturelle Kompetenzen vermitteln, Fremdsprachenkenntnisse ausbilden und Persönlichkeitsentwicklung fördern, ohne dass dafür entsprechende Anerkennungsstrukturen bereitgehalten sind und sich dadurch Chancen auf Teilhabe an Gesellschaft und Erwerbsleben erhöhen.

\section{Auf die Spur kommen ... Feldzugänge und methodologische Reflexionen}

Erwachsenen- und Weiterbildung ist nun von den beschriebenen Entwicklungen in mehrfacher Hinsicht betroffen. In der Praxis werden nicht nur Reiseangebote und die Organisation von Lernmobilität zu pädagogischen Aufgaben, 
sondern auch die Bewältigung ihrer Folgen und die Herstellung und Verwaltung, das Management entsprechender Passungen, Anerkennungsverfahren und Bewertungsstrukturen, mit denen der Ordnungsrahmen hergestellt und aufrechterhalten wird. Wie in anderen Gesellschaftsbereichen auch, sind die sozialen Folgen von Mobilität als allseitige Aufforderung zur permanenten Beweglichkeit und fortgesetzten Beschleunigung, die von Baumann befürchtete Bedrohung fragmentierter sozialer Verhältnisse, individuell zu bewältigen. So ist Pädagogik im Allgemeinen und Erwachsenen- und Weiterbildung auf je spezifische Weise angefragt, die Widersprüche und Verwerfungen, auf die Individuen und Gesellschaft in mobilen Verhältnissen treffen, pädagogisch zu bearbeiten. Ratgeberliteratur und Thematisierungen von Zeit(-mangel) verweisen darauf; die zunehmenden Angebote fernöstlicher Meditations- und Achtsamkeitsübungen, die in einer scheinbar immer hektischeren Welt Anleitung zum Innehalten geben, lassen sich möglicherweise ebenfalls als Reflex auf die Mobilitätsgesellschaft interpretieren.

Forschungsarbeiten zum Zusammenhang von Bildung und Reisen, insbesondere Untersuchungen zu Lernmobilität sind bislang oft durch einen affirmativen Bezug zum Gegenstand geprägt. Fragestellung und Erkenntnisinteresse sind zumeist darauf gerichtet, die Teilnahme an Austauschprogrammen zu erhöhen und die Reichweite der Maßnahmen zu erweitern; Reisen und Bildung, Auslandsaufenthalt und Persönlichkeitsentwicklung werden unhinterfragt in eins gesetzt (vgl. u.a. Becker/Goldkamp/Kroos 2012; Brandtner/Wisser 2016). Dabei zeichnen sich vor dem Hintergrund der bisherigen Ausführungen komplexe Forschungsdesiderate ab: So wäre u.a. zu fragen, wie und warum Bildung durch Reisen zustande kommt und wie sich mobile Menschen in einer mobilen Welt aufeinander beziehen, wie also gesellschaftlicher $\mathrm{Zu}-$ sammenhalt zwischen allseits mobilen Subjekten entsteht und erhalten wird. Untersuchungen darüber, wie soziale Ungleichheiten beim Zugang zu Reise-, Lernmobilitäts- oder ganz allgemein Auslandserfahrungen re-/produziert werden, sind ebenfalls noch weitgehend Desiderat. Offen bleiben bislang auch Fragen danach, wie sich Raum-Zeit-Verhältnisse durch vermehrtes Reisen bzw. wachsende Mobilität verändern und welche Wechselwirkungen sich beispielsweise im Verhältnis von Gast und Gastgebern - mit dem sozialen Raum ergeben, in dem Reisende immer, wenngleich nur auf Zeit, ankommen. Im Rekurs auf Baumann stellen sich in einer bewegten Gesellschaft, deren Mitglieder unterwegs sind, Fragen nach Zugehörigkeit und Beteiligung. Wenn Bewegung zum allumfassenden Paradigma wird und Räumlichkeit nicht mehr als geschlossener Ort vorstellbar bleibt, wie werden dann Zugehörigkeiten konstruiert? Und bleibt die implizite Setzung, dass die spezifische Kombination von Distanzierung, Erfahrung, Entfremdung und Befreiung unweigerlich persönliche Entwicklung oder Re-Konstruktionen des Subjekt-Welt-Verhältnisses mit sich bringt, weiterhin gültig? 


\section{Erste Einsichten}

Auch die Frage nach dem empirischen Zugang einer kritischen Mobilitätsforschung, die das Wechselverhältnis von gesellschaftlicher Transformation und subjektiver Bewältigung für den Bereich der Lernmobilität rekonstruiert, ist von Bedeutung. Eine solche Perspektive auf die soziale Konstruktion von Mobilität (Cresswell 2006) thematisiert Fragen nach dem Zugang zu grenzüberschreitenden, interkulturellen Bildungsmöglichkeiten ebenso wie Formen ihrer Anerkennung in formalen, institutionellen und in sozialen, lebensweltlichen Kontexten. Sie verweist darüber hinaus auf die soziale Konstruktion differenter Formen von Mobilität, die in Institutionalisierungs- und Anerkennungspraxen zum Ausdruck kommt. So mögen beispielsweise Teilnehmende am Weltwärts-Programm und spanische Ausbildungsmigrant*innen gleichermaßen vor persönliche Herausforderungen gestellt sein - sind aber bei der subjektiven Aneignung ihrer jeweiligen Bildungserfahrungen auf unterschiedliche Anerkennungskontexte verwiesen. Wo aber findet sich ein empirischer Zugang zu den skizzierten Fragestellungen? Welche Forschungszugänge sind fruchtbar zu machen, um sich auf das bewegte Feld einer kritischen Mobilitätsforschung $\mathrm{zu}$ begeben?

Einen exemplarischen Einblick ermöglichen die Interviews, die im Rahmen eines deutsch-dänischen INTERREG-Projekts mit Jugendlichen gesammelt wurden (JUMP - Jobs durch Ausbildung, Mobilität und Praxis), die in den vergangenen zwei Jahren an niedrigschwelligen, kurzen, pädagogisch begleiteten Austauschmaßnahmen zwischen deutschen Bildungsträgern und dänischen Produktionsschulen teilgenommen haben. Die Jugendlichen wurden zu ihren Mobilitätserfahrungen befragt, ihre Erzählungen eröffnen subjektive Perspektiven auf die Zumutungen, die der Mobilitätsimperativ transportiert und die Verunsicherungen, die mit der Teilnahme an Auslandsaktivitäten ausgelöst werden. Sie ermöglichen Einsichten in Biografien von jungen Erwachsenen, für die Zugehörigkeit immer eine fragile Kategorie und Partizipation selten eine Option war (Niemeyer, Revsbech-Jensen, Sommer, Zick, in Vorbereitung). Wenn die Lebensbedingungen zu Hause prekär sind, sei es durch Gewalterfahrungen, Krankheit, instabile Herkunftsfamilien o.ä., Sicherheit im Alltag und Stabilität in sozialen Beziehungen nur mit Mühe aufrechtzuerhalten sind, stellt bereits die Reise ins Nachbarland ein Risiko dar, dass ein - aus der Teilnehmerperspektive - nur diffus zu erkennenden Kompetenzgewinn kaum aufwiegt. Wenn die bisherige Erfahrungswelt in einem Umkreis von wenigen Kilometern konzentriert war, erscheinen weitere Entfernungen bedrohlich. Für die Teilnehmer*innen an diesem Projekt stellt die Aufforderung zur Teilnahme an einer Lernmobilitätsmaßnahme eine große Herausforderung dar; vor dem Hintergrund ihrer Lebensgeschichten erscheint die Bedeutung von Auslandserfahrungen stark relativiert. 
Anke $^{2}$ beispielsweise ist nach der frühen Trennung ihrer Eltern mit ihrer Mutter ,umgezogen, umgezogen, umgezogen“. Jeder Umzug ging mit einem Schulwechsel einher, jedes Mal mussten neue Anforderungen bewältigt werden. Wenn sie spricht, wird deutlich, dass Mobilitätserfahrungen nicht notwendig mit Horizonterweiterungen gleichzusetzen sind. Vielmehr erfordern Ortswechsel stets auch eine individuelle Anpassungsleistung. Wenn Anlass und Ziel nicht selbst bestimmt, sondern von außen aufgegeben sind, schränkt Mobilität die Möglichkeiten des eigenen Handelns ein, anstatt sie zu erweitern. Lernmobilität wird vor diesem biografischen Hintergrund eher nicht als Chance wahrgenommen.

Die Zumutung eines Austauschs oder Praktikums im Nachbarland wird nicht notwendig zum biografischen Risiko, vielmehr werden durch eine solche Herausforderung durchaus auch Bewältigungsstrategien aktualisiert, wie etwa von Claus aus Dänemark, dem ein Praktikum in Deutschland angeboten wird. Claus reizt die Chance und er ist an einer Arbeit im Nachbarland interessiert, aber er ist sehr vorsichtig und traut sich das Praktikum selbst zunächst nicht zu. In dieser Phase entwickelt er, mit pädagogischer Begleitung, eine gezielte Vorbereitungsstrategie. „Dann habe ich meinen Hausarzt angerufen und gefragt, welche Impfungen man für Deutschland braucht." Er macht eine Vorbereitungsfahrt zu seinem zukünftigen Arbeitsort, prägt sich den Weg ein, stellt eine Liste der notwendigen Dinge zusammen, die er für ein Leben in Deutschland notwendig findet. Während des Praktikums selbst, das in einer Fahrradwerkstatt stattfindet, erleichtern ihm seine Fachkenntnisse die Eingewöhnung im Arbeitsprozess, auch wenn ihn zunächst Sprachschwierigkeiten irritieren.

Martina muss mit gesundheitlichen Einschränkungen leben und sagt: „Wenn ich zu lange von meiner Familie entfernt bin, dann muss ich zurück, dann schaff ich's nicht.“ Für sie ist ein Praktikum in Dänemark undenkbar: „Ich könnte nie in einer anderen Stadt sein.“ Dennoch nimmt sie an kurzfristigen Austauschaktivitäten teil. Dabei macht sie die positive Erfahrung, auch außerhalb ihrer Familie Anerkennung und Unterstützung zu finden. „Was Neues sehen, andere Sprache, neue Menschen kennenlernen, ... kommt man auch mal raus. Das tut gut", lautet ihre Bilanz.

\section{Fazit}

Eine weitergehende Analyse der Mobilitätsgeschichten, die hier nur angerissen werden konnten, rückt einige der gängigen Annahmen zur biografischen und sozialen Bedeutung von Lernmobilität in ein anderes Licht. Es wird deutlich,

2 Alle Namen geändert. 
wie voraussetzungsvoll das Konzept von Lernmobilität ist. Die Disposition zur permanenten Horizonterweiterung und Grenzüberwindung lässt die Dimensionen der Grenzen, denen sich die Teilnehmer*innen im Hinblick auf ihre mentale, physische, psychische, geografische, motorische, soziale Beweglichkeit gegenübersehen, unberücksichtigt. Die Konzeption von Lernmobilitätsangeboten für besondere Zielgruppen erfordert folglich besondere Ermöglichungsstrukturen, die, so lässt sich zunächst in praktischer Hinsicht folgern, insbesondere emotionale Unterstützung und Begleitung mit beinhalten sollten.

Eine solcherart subjektorientierte Mobilitätsforschung lässt u.a. die emotionalen Kosten von Lernmobilität sichtbar werden. Aus dieser Perspektive werden einige der Selbstverständlichkeiten, die der EU-Diskurs um Lernmobilität transportiert, in Frage gestellt. Die persönlichkeitsstärkende Wirkung von Auslandserfahrungen kann nicht in jeder Lebenssituation und unter allen Umständen aktualisiert werden, ist aber ebenso wenig ausschließlich beängstigend und entmutigend. Vielmehr geht aus den meisten Erzählungen hervor, dass die Reise und selbst ein kurzer Aufenthalt im Nachbarland einen Möglichkeitshorizont eröffnen kann. Gleichzeitig wird deutlich, dass die individuelle Mobilitätsdisposition andere soziale Zugehörigkeitskategorien unter Umständen in Bewegung bringen, nicht aber notwendig überwinden kann. Weggehen ist keine Voraussetzung für Zugehörigkeit, Dabeisein ist nicht dasselbe wie dazugehören.

In theoretischer Hinsicht treten so einige der Paradoxien hervor, die sich um dem Mobilitätsimperativ entfalten und damit Desiderata für die weitere Forschung markieren: Auslandsaufenthalte im Kontext von Lernmobilität können befreiend und einschränkend zugleich sein, sie erfordern notwendig Anpassungsleistungen, können als Verunsicherung und Bestärkung, als Horizonterweiterung und als Entwurzelung erlebt werden. Situations- und zielgruppenangemessene Anerkennungspraktiken und Unterstützungsstrukturen sind dabei von wesentlicher Bedeutung.

\section{Literatur}

Baumann, Zygmunt (1996): From Pilgrim to Tourist - or a Short History of Identity. In: Hall, Stuart; du Gay, Paul (Hrsg.): Questions of Cultural Identity. London: Sage Publications, S. 18-36.

Baumann, Zygmunt (2014): Glocalization and Hybridity. In: Glocalism. Journal of culture, politics and innovation. Issue 2014, 1; http://www.glocalismjournal.net/ Issues/HYBRIDITY/Articles/Glocalization_And_Hybridity.kl [Zugriff: 20.4. 2017]. 
Baumann, Zygmunt (o.J.): Europe of strangers. http://www.transcomm.ox.ac.uk/ working\%20papers/bauman.pdf [Zugriff: 6.2.2019].

Becker, Carsten; Goldkamp, Sina; Kroos, Daniela (2012): Mobilität - Kompetenzzuwachs für Benachteiligte, Nationale Agentur Bildung für Europa (Hrsg.), Bonn.

Bergh, Charles; Milmeister, Marianne; Weis, Christiane (2013): Learning mobility in the youth field: starting to set a framework. In: Friesenhahn, Günter J.; Schild, Hanjo; Wicke, Hans-Georg; Balogh, Judit (Hrsg.): Learning mobility and nonformal learning in European contexts. Policies, approaches and examples. Strasbourg: Council of Europe Publishing. S. 12-24.

Brandtner, Hans; Wisser, Ulrike (2016): Grenzüberschreitungen. Europäische Mobilitätsangebote für sozial benachteiligte junge Menschen. JUGEND fur Europa. Nationale Agentur Erasmus+ JUGEND IN AKTION (Hrsg.), Bonn.

Cairns, David (2015): Analytical Paper: Learning Mobility and Social Inclusion. Youth Partnership. University Institute of Lisbon.

Cresswell, Tim (2006): On the Move: Mobility in the Modern Western World. London: Routledge.

European Commission, Flash Barometer, Youth on the move. Analytical report, Field-work: January 2011. Publication: May 2011. Flash EB Series \#319b.

Europäische Union (2011), Empfehlungen des Rates vom 28.6.2011, Jugend in Bewegung - die Mobilität junger Menschen zu Lernzwecken fördern. Amtsblatt der Europäischen Union 2011/C 199/01.

EPLM - European Platform on Learning Mobility in the Youth Field: Position Paper. https://pjp-eu.coe.int/documents/1017981/8321545/EPLM+Position+Paper.pdf/83bad147-8688-4357-ba1c-b55e165810e0 [Zugriff: 23.3.2016].

EU 2020-strategy. http://ec.europa.eu/europe2020/index en.html [Zugriff 30.1. 2019].

European Union 2014. Erasmus+. A guide to European Union opportunities in education, training, youth and sport. Luxembourg: Publications Office of the European Union.

Friesenhahn, Günter J.; Schild, Hanjo; Wicke, Hans-Georg; Balogh, Judit (Hrsg.) (2013): Learning mobility and non-formal learning in European contexts. Policies, approaches and examples. Strasbourg: Council of Europe Publishing.

Hlavin-Schulze, Karin (1998): Man reist ja nicht, um anzukommen. Reisen als kulturelle Praxis. Frankfurt am Main, New York: Campus.

Honneth, Axel (2013): Strukturwandel der Anerkennung. Paradoxien sozialer Integration in der Gegenwart. Frankfurt am Main, New York: Campus.

Jugend für Europa: Lernerfahrungen durch grenzüberschreitende Mobilität. https://www.jugendfuereuropa.de/themen/lernerfahrungen-durch-grenzueberschreitende-mobilitaet/ [Zugriff: 30.1.2019].

Kristensen, Soren (2013): Mobility as a pedagogical tool for young people with fewer opportunities. In: Friesenhahn, Günter J.; Schild, Hanjo; Wicke, HansGeorg; Balogh, Judit (Hrsg.): Learning mobility and non-formal learning in European contexts. Policies, approaches and examples. Strasbourg: Council of Europe Publishing. S. 97-102. 
Kronauer, Martin (2002): Exklusion. Die Gefährdung des Sozialen im hoch entwickelten Kapitalismus. Frankfurt am Main, New York: Campus.

Lifelong Learning Platform: Learning mobility for all. http://lllplatform.eu/policyareas/skills-and-qualifications/learning-mobility-for-all [Zugriff: 30.1.2019].

Marotzki, Winfried (1991): Bildung, Identität und Individualität. In: Benner, Dietrich; Lenzen, Dieter (Hrsg.): Erziehung, Bildung, Normativität. Weinheim: Juventa. S. 79-94.

Niemeyer, Beatrix; Revsbech-Jensen, Christine; Sommer, Finn; Zick, Sebastian (in Vorbereitung), Mobility Stories. Lerngeschichten von Mobilitätserfahrungen benachteiligter Jugendlicher in Deutschland und Dänemark. 

Sebastian Zick

\section{\#Freelnterrail, Mobilität und Bildung - Narrativanalytische Perspektiven auf EUropäische Mobilitätsförderung}

\section{Von \#Freelnterrail, Bildungsreisen und Mobilität zu Lernzwecken}

„Imagine it's your 18th birthday and you find a personal letter from the EU in your mailbox. In it: a voucher to travel Europe. Your life will change! " (FreeInterrail 2015). Mit dieser pathetischen Formel wirbt seit 2015 die zivilgesellschaftliche Initiative \#FreeInterrail für die Umsetzung ihres Ziels: Alle 18-jährigen Bürger*innen eines EU-Mitgliedsstaates sollen ein von der EU-Kommission finanziertes Interrailticket erhalten, um damit eine Reise durch Europa antreten zu können.

Der transformative Charakter (your life will change!), welcher der potentiellen Europareise unterstellt wird, ist dabei anschlussfähig an verbreitete und wirkmächtige Imaginationen des Zusammenhangs von Bildung und Reisen. Reisehistorisch zeigen sich seit der Renaissance unterschiedliche Reiseformen, innerhalb derer räumliche Bewegung in je unterschiedlicher Form Anlass und Möglichkeit für ,geistige“ Bewegung zu versprechen scheint. Ob bei Pilgerreisen, Gesellenwanderungen oder bei der Grand Tour - stets gehen mit den jeweiligen Reisetypen für je differente soziale Gruppen Versprechungen individueller Entwicklung einher, welche als (je unterschiedliche) Bildungsprozesse übersetzbar sind (vgl. Hlavin-Schulze 1998; Zick 2018). Die implizite und bildungsprozesstheoretisch anschlussfähige Grundannahme, welche eine Verbindung von räumlicher und geistiger Bewegung nahelegt, fußt dabei darauf, dass das Medium der Reise die Erfahrung ,irritierender Fremdheit“ (Schäfer 2011) ermöglicht, was Subjekte von ihren alltäglichen Selbst- und Weltverhältnissen distanziert und ihnen dadurch transformative Bildungserfahrungen ermöglicht (vgl. Koller 2012). Reisen lassen sich von dieser Perspektive ausgehend als Bildungschancen par excellence betrachten.

Strukturierte Mobilitätsangebote finden sich sowohl im Rahmen formaler (Klassenfahrten, Auslandssemester etc.) als auch im Kontext informeller Bildung (Studienreisen, Bildungsurlaub, Gruppenreisen etc.). Gerade im Bereich des formalen Bildungs- und Ausbildungswesens tritt die Europäische Union durch ihr breites Spektrum an möglichen Auslandsaufenthalten als wirkmächtige Förderin und Forderin (vgl. Liesner 2006) von Mobilität in Erscheinung. 
Unter dem Dach von Erasmus + organisieren nationale Agenturen in spezifischen Bildungsbereichen die Durchsetzung des politischen Ziels der EU, „die Mobilität junger Menschen zu Lernzwecken [zu] fördern“ (Kommission 2009). Mobilität zu Lernzwecken wird dabei in Deutschland auf vier unterschiedliche Mobilitätsgruppen (Hochschulmobilität, Schulmobilität, Berufsbildung/Erwachsenenbildung, Jugendarbeit) aufgeteilt und entsprechend in je unterschiedlichen Agenturen (DAAD, PAD, BIBB, Jugend für Europa) bearbeitet.

Entgegen der für EUropäische Förderstrukturen üblichen Einordnung von Mobilitätsangeboten in formale Bildungsbereiche fokussiert die Initiative \#FreeInterrail den Sektor informeller Bildung und orientiert sich dabei in ihrem Gestus eher an klassischen Idealen des Bildungsreisens. Auf welche Form des bildenden Reisens \#FreeInterrail abzielt, in welcher Weise das politische EUropa auf diese Adressierung reagiert und welche Erzählungen über den $\mathrm{Zu}-$ sammenhang von Bildung und Mobilität dafür eingesetzt werden, ist Gegenstand des folgenden Beitrags.

Der Beitrag verfolgt dabei ein inhaltliches sowie ein methodisches Anliegen:

Inhaltlich stehen die Konstruktionen eines spezifischen Bildungswertes des Reisens und dessen politische Förderung und Forderung am Beispiel von \#FreeInterrail im Vordergrund.

Methodisch plädiere ich im Rahmen des Beitrags dafür, das Repertoire der Beforschung von Diskursen um Bildung und Mobilität und die damit verbundenen politischen Setzungen um eine politikwissenschaftlich inspirierte narrativanalytische Perspektive zu erweitern.

Hierzu erfolgt zunächst ein deskriptiver Überblick über die Entwicklungsgeschichte der Initiative \#FreeInterrail und ihrer EUropäischen Rezeption (2.). Darauf folgen methodologische Überlegungen zur Implementierung eines der Politikwissenschaft entlehnten narrativanalytischen Verfahrens zur Untersuchung politischer Diskurse hinsichtlich ihrer pädagogisch relevanten Implikationen (3.). Potentiale von Narrativanalysen für Erziehungswissenschaft und Erwachsenenbildung werden im Folgenden anhand der Untersuchung einer Debatte des Europäischen Parlaments zum Thema \#FreeInterrail erprobt (4.). Der Beitrag endet mit einer bilanzierenden Perspektive auf die Verbindungslinien von Europa, Mobilität und Bildung, welche in der politischen Rezeption von \#FreeInterrail sichtbar wurden, und skizziert, welche Konsequenzen der Beforschung politischer Diskurse um Bildung und Mobilität sich für Erwachsenenbildung daraus ergeben (5.). 


\section{2 \#Freelnterrail}

Der Darstellung der Initiatoren folgend ${ }^{1}$, entwickelten die deutschen Aktivisten Vincent-Immanuel Herr und Martin Speer die Idee zu \#FreeInterrail während eines Interrailtrips im Jahr 2014. In der Folgezeit publizierten Herr\&Speer eine Vielzahl an Artikeln, in welchen sie ihr Konzept eines kostenfreien Interrailtickets für alle 18-jährigen Europäer*innen vorstellten. Im September 2015 erschien in der ZEIT ein Essay mit dem Titel „Lasst uns reisen. Kostenloses Interrail für Junge als EU-Projekt“", in dem sie eine von Abschottung, aufkommendem Nationalismus und Vertrauensverlust gekennzeichnete Identifikationskrise Europas konstatieren. Als Gegenmittel schlagen sie ihr \#FreeInterrail-Konzept vor. „Was also tun, um diese Ängste und Vorurteile abzubauen? Reisen!“ (ZEIT 34/2015). Sie entwerfen dabei die Idee einer Parallelstruktur zum bestehenden Erasmus-Programm, das sich vor allem durch seine Inklusivität von Erasmus abheben sollte. „Europa sollte für alle Jungen erfahrbar werden - unabhängig von ihrem Bildungsgrad und ihren finanziellen Möglichkeiten“ (ebd.). Darüber hinaus initiieren die Aktivisten eine an die Europäische Kommission gerichtete Petition mit dem Titel „Europa retten? Schickt die junge Generation auf Reisen!“. In der Petition wird beschrieben, wie ,\#FreeInterrail [...] Europa retten und ein festes Fundament für Frieden und Solidarität schaffen" (FreeInterrail 2015) könne. Den starken Fokus auf die Weiterentwicklung Europas demonstriert der Untertitel der Initiative: „Moving Europe forward! “

Im September 2016 erfährt die Idee gesteigerte mediale Aufmerksamkeit als Manfred Weber, Präsident der EVP-Fraktion im Europäischen Parlament (EP), in seiner Reaktion auf die State-of-the-Union-Rede von Kommissionspräsident Jean-Claude Juncker die Idee von \#FreeInterrail aufgreift. Weber setzt diesen Vorschlag ans Ende einer Thematisierung der Krise Europas, welche er nach dem gerade zurückliegenden Brexit-Votum durch eine Stärkung der Sichtbarkeit des „European way of life“ zu überwinden gedenkt. Als mögliches Exempel hierfür nennt er die Idee von \#FreeInterrail, mit der die junge Generation Europas adressiert werden soll. Diese Fürsprache für \#FreeInterrail führte zu einer Parlamentsdebatte im Oktober 2016, welche unten (4.) narrativanalytisch untersucht wird. Als Ergebnis dieser Debatte wurde im November 2016 von der Kommission die Absicht erklärt, ein den Ideen von \#FreeInterrail entsprechendes Pilotprojekt zu initiieren. Nach der Einführung des ersten Pilotprojekts Move2Learn, Learn2Move, welches aufgrund des geringen

1 Soweit mir bekannt ist, gibt es bislang keine systematischen Studien zum Phänomen \#FreeInterrail, so dass eine Beschreibung der Entwicklung nur anhand der Selbstbeschreibung der Initiative möglich ist. 
Budgets und der komplizierten Bewerbungsprozesse auf Ablehnung der Initiatoren stieß, wurde im Jahr 2018 unter dem Titel \#DiscoverEU ein zweites Pilotprojekt gestartet. In den beiden ersten Bewerbungsrunden wurden ca. 27.000 Tickets auf Grundlage der Teilnahme an einem Wettbewerb verteilt. ${ }^{2}$ Die Initiative soll in den folgenden Jahren fortgeführt und ausgeweitet werden.

\section{Narrativanalytik in der Erziehungswissenschaft - Potential und Verfahren}

Der politische Diskurs um \#FreeInterrail soll im Folgenden hinsichtlich seiner Implikationen von Europa, Mobilität und Bildung analysiert werden. Dabei plädiere ich für einen Zugang, der Aussagen in der Sphäre des Politischen als ,Erzählungen' betrachtet und dabei den analytischen Fokus auf die Untersuchung politischer Narrative legt, welche für erziehungswissenschaftliche Fragestellungen relevant sind. Für die behandelte Thematik fokussiert das Vorgehen entsprechend die Frage, welche Erzählweisen über den Zusammenhang von Bildung und Mobilität sich in der Debatte um \#FreeInterrail nachvollziehen lassen.

Eine Perspektive auf das „Erzählen als politische Praktik“ (Gadinger et al. 2014: 9) versteht Erzählungen als Akte der Produktion von Sinn und Legitimität im politischen Diskurs. Erzählungen werden nicht auf ihre reproduzierende und repräsentative Wirkung von Welt reduziert, sondern über ihre Praxis des „Fürwahrhaltens“ des Erzählten (Münch 2016: 9) als kreative Akte der Modellierung dieser betrachtet (vgl. Viehöver 2014: 69).

Hierzu haben in der deutschsprachigen Politikwissenschaft Gadinger et al. (2014) einen postfundamentalistisch verorteten Ansatz entwickelt, welcher als Variante (und nicht als Abgrenzung von) diskursanalytischer Forschung betrachtet werden kann. Ihr Fokus liegt auf der Analyse von „Erzählungen als zentrales diskursstrukturierendes Regelsystem“ (Münch 2016: 84) des Politischen. Empirischer Gegenstand der Analyse können politische Reden, Programmatiken, Strategiepapiere o. Ä. sein. Wesentlich ist für narrativanalytische Verfahren die Frage nach der Plausibilisierungskraft politischer Erzählungen. Hierfür relevant ist u.a. die innerhalb von Erzählungen hergestellte ,kulturelle Modellierung von Zeit“ (Yildiz et al. 2015: 425) mit ihren Konstruktionen, Diagnosen und Entwürfen von Vergangenheiten, Gegenwarten und Zukünften und deren jeweiligen Verbindungen. Politische Zukunftsentwürfen benötigen

2 Von der Idee einer Partizipation aller Jugendlichen ist das Projekt noch weit entfernt. Für Deutschland sind derzeit 1.944 Tickets vorgesehen, was bei derzeit ca. 746.00018 Jährigen einer Quote von 0,26\% entspricht. 
aus narrativanalytischer Perspektive ,[e]in Wissen von der Zukunft [...] ebenso wie eine Verständigung über sie" (Horn 2014: 22f), wobei dieses Wissen nicht ,ohne Rückgriff auf Erzählungen [auskommt], die von der Zukunft aus auf die Gegenwart ,zurückblicken' oder [...] aus der Kenntnis bisheriger Verläufe eine Voraussage über das Kommende extrapolieren“ (ebd.). Im Zentrum der narrativanalytischen Verfahrenstechnik stehen zudem Metaphern, Rollen und Plots (vgl. Yildiz et al. 2015: 426), mit welchen nachvollzogen werden soll, in welcher Weise und auf der Konstruktion welcher Wissensbestände Akteure ihren Erzählungen narrativ Plausibilität verleihen und dabei politische Möglichkeitsräume strukturieren.

Während narrativanalytische Ansätze politikwissenschaftlich inzwischen etabliert sind (vgl. Gadinger et al. 2014), sind sie in erziehungswissenschaftlicher und erwachsenenbildungsbezogener Forschung bislang kaum in Erscheinung getreten. Erzählungen werden bisher primär in biografietheoretischen Ansätzen im Kontext biographisch-narrativer Interviews als Möglichkeit subjektive Deutungsmuster bzw. biografische Konstruktionen der Wirklichkeit (Alheit/Dausien 2000) zu rekonstruieren Gegenstand qualitativ-empirischer Analysen. Wenn es hingegen um die empirische Beforschung (bilungs-)politisch relevanter Programmatiken, Zielsetzungen und Ordnungsvorstellungen geht, spielt der Narrativbegriff bislang kaum eine Rolle. Im Rahmen erziehungswissenschaftlicher Diskursforschung orientieren sich Analysen des Politischen häufig an Foucaults Konzept der Gouvernementalität (vgl. Fegter et al. 2015: 9; Rothe 2015), um ,,pädagogische Machtverhältnisse nicht für sich, sondern im Rahmen gesamtgesellschaftlicher ,Programme des Regierens' zu betrachten" (Fegter et al. 2015: 9). Die Etablierung eines narrativanalytischen Ansatzes zur Analyse pädagogisch relevanter Politiken stünde diesen diskursanalytischen Felderkundungen nicht entgegen. Vielmehr könnte darin eine Ergänzung diskursanalytischen Instrumentariums in Erziehungswissenschaft und Erwachsenenbildung bestehen, mit welcher politische Diskurse mittels der Fokussierung auf Erzählungen methodisch auf ihre innernarrativen Konstruktionen von Wissen über pädagogisch relevante Gegenstandsfelder hin befragt werden. So ließe sich befragen, wie welchen Begriffen von Bildung im Kontext von Mobilitätspolitik mittels Erzählungen politisch Geltung verschafft wird.

Die Potentiale einer solchen Erweiterung diskursanalytischer Verfahren sollen im Folgenden anhand der exemplarischen Analyse einer parlamentarischen Debatte erprobt und vorgestellt werden. 


\section{Narrative von Europa, Mobilität und Bildung}

Anhand der Debatte des Europäischen Parlaments vom 4.10.2016 „Zum 18. Geburtstag ein Interrail-Pass für Europa“ sollen im Folgenden leitende Narrative in der Verbindung von Europa, Mobilität und Bildung nachgezeichnet werden. Analysiert wurden hierzu anhand des offiziellen Parlamentsprotokolls die Redebeiträge der jeweils ersten Sprecher*innen der einzelnen Fraktionen. Bereits das Zustandekommen der Debatte zeugt indes davon, dass die von \#FreeInterrail ausgegange Adressierung der EU als jene Organisation, von welcher die postulierte Krise europäischer Identifikation bearbeitet werden sollte, vom EU-Parlament angenommen wird. Dies ist zumindest insofern erstaunlich, als dass es in der zu implementierenden Initiative um ein Anliegen geht, welches strukturell über die üblichen Beschäftigungsfelder EUropäischer Jugend-, Beschäftigungs- oder Bildungspolitik hinausgeht. In der Debatte fällt zudem auf, dass die Idee fraktionsübergreifend auf eine hohe prinzipielle $\mathrm{Zu}$ stimmung stößt. ${ }^{3}$ Grundlegende normative Einwände gegen die Idee, mit einer Erhöhung von Mobilität ein bestimmtes Gut erreichen zu können, tauchen in keiner Rede auf.

Innerhalb der Debattenbeiträge nimmt das Narrativ der Krise eine zentrale Rolle ein. Der gegenwärtige Status Europas wird durch Merkmale wie „Populismus, Euroskeptizismus und Rückkehr des Nationalismus“" (Morgano/ $\mathrm{S} \& \mathrm{D}^{4}$ ), mediale Zerrbilder der Realität, die gravierende Jugendarbeitslosigkeit oder die mangelnde Erreichbarkeit der Bürger*innen mittels Fakten (Weber/EVP) als krisenhaft beschrieben. Auf der Grundlage dieser Gegenwartsdiagnose werden Gegenerzählungen entwickelt, welche den Weg aus der Krise weisen und eine Zukunft imaginieren, in der die beschriebenen Krisenmomente überwunden sein werden. \#FreeInterrail wird als eine solche Gegenerzählung behandelt.

„, Trotzdem müssen wir uns bewusst werden, dass wir viele Menschen beim Thema Europa nicht nur mit Fakten erreichen, nicht nur mit Gesetzen erreichen, sondern wir müssen Emotionen dahintersetzen, was Europa bedeutet. Deswegen hatte ich in der Rede vor zwei Wochen [...] einfach in

3 Einzig die EFDD-Fraktion lehnt den Vorschlag ab. Dies geschieht allerdings nicht aus inhaltlichen - ,weil es sich tatsächlich um eine lobenswerte Initiative handelt, die den Geist der Europäischen Gemeinschaft wiederbeleben würde" (Aiuto/EFDD [im Original italienisch. Eigene Übersetzung]) - sondern aus organisatorischen und strukturellen Gründen. Dies bestärkt den Eindruck, dass einer Erhöhung der Mobilität junger Menschen in der aktuellen politischen Verfasstheit des Diskurses nur schwer zu widersprechen ist.

4 Nach Zitaten wird im Folgenden jeweils der Name sowie bei der ersten Nennung die Fraktion des*r Sprecher*in genannt. 
den Raum gestellt, wie es denn wäre, wenn wir das, was junge Leute diesen Sommer mit Interrail erlebt haben, dass wir dieses Geschenk jedem jungen Menschen in Europa machen könnten, nämlich die Schönheit Europas kennenzulernen, den Nachbarn im anderen Land nicht als Nachbar zu erleben, sondern als Freund, als Freundin zu erleben, schlicht und einfach zu spüren, was es bedeutet, Europäer zu sein. "(Weber/EVP)

Die von der Initiative ausgehenden Möglichkeiten werden als Gegenmittel (Morgano) oder Geschenk (Weber) bezeichnet. In besonderem Maße wird die emotionale Komponente des Reisens hervorgehoben, wobei ein Gegenhorizont zwischen zwei verschiedenen Europabildern entworfen wird: Während das bisherige und gegenwärtige Europa als faktenbasiert und bürokratisiert dargestellt wird, dient der Impuls \#FreeInterrail als Zukunftsmodell dazu aufzuzeigen, dass ,wir Emotionen dahinter setzen [müssen], was Europa bedeutet“ (ders.). Neben einem „Europa der Verordnungen, der Richtlinien, der Gipfel“ (Lambsdorff/ALDE) müsse es auch ein „Europa der Seele, des Herzens und auch der Basis“ (ders.) geben. Reisen scheinen hierfür ein geeignetes Medium der Vermittlung der „Schönheit Europas“ (Weber) zu sein, um „einfach zu spüren, was es bedeutet, Europäer zu sein“"(ders.). Wim van de Camp (EVP) bringt die Verbindung von Europa, Emotion und Reisen auf einen einfachen Nenner: „Europa ist Emotion. Und Sie können Emotionen schmecken, wenn Sie herumreisen. ${ }^{\text {5 }}$ Emotionen bilden so ein entscheidendes Narrativ der Debatte, mit welchem die Zustimmung zur Grundidee von \#FreeInterrail plausibilisiert wird. Neben der Betonung der emotionalen Wirkung des Reisens auf junge Europäer*innen wird die Bedeutung von Europareisen in zwei Debattenbeiträgen (Weber; Harms/Verts) auch auf die eigene Biografie übertragen. Die biografische Erfahrung der Interrailreise in Jugendjahren wird als mitteilungswert und damit als offenbar politisch relevant eingesetzt. Aus narrativanalytischer Perspektive zeigt sich hieran erneut die plausibilisierende Kraft der in den Erzählungen vollzogenen Modellierungen von Zeit: Die narrative Konstruktion von Legitimität für eine zukünftig zu vollziehende politische Handlung speist sich sowohl aus der Skizzierung einer von Krisen gekennzeichneten Gegenwart wie auch aus der vergangenheitsbezogenen Darstellung biographischer Erfahrungen. War-, Ist- und Soll-Zustände werden dabei narrativ miteinander verwoben und sequenzialisiert, was zur narrativen Konstruktion politischer Plausibilität des intendierten Ziels beiträgt.

Was in der Debatte neben den skizzierten Narrativen allerdings kaum eine Rolle spielt, sind individuelle Entwicklungen, welche durch Mobilität initiiert werden könnten. Pädagogische Leitkategorien wie Bildung, Reflexion, Wissen, Kompetenz etc. spielen eine untergeordnete Rolle oder werden zumindest nicht expliziert. Von Reisen zu initiierende Bildungsprozesse als potentielle

Im Original niederländisch. Eigene Übersetzung. 
Irritationen subjektiver Welt- und Selbstverständnisse werden nicht thematisiert. Vielmehr scheint das intendierte Lernziel eines positiveren Europaverständnisses und -verhältnisses handlungsleitend zu sein: „Man kann mit relativ wenig Aufwand viel europäisches Zusammenwachsen und Verständigung erreichen" (Harms).

\section{Fazit: (Lern-)Mobilität zwischen Entgrenzung und Einordnung}

Narrative der Mobilität können als historisch wandelbare Konstruktionen des Zusammenhangs von Bildung und Reisen verstanden werden (vgl. Zick 2018). EUropäische Mobilitätspolitik nimmt dabei gängige Verheißungen des bildenden Charakters des Reisens auf. Bildungstheoretisch knüpft sie häufig an einem Verständnis von Bildung als transformativem Prozess an, innerhalb dessen sich Mobilität als Transformationsanlass betrachten lässt. Mobilität wird in diesem Kontext als Scharnier zwischen bildungs- und identitätspolitischem Engagement in Form eines ,positiveren“ Europabildes eingesetzt. \#FreeInterrail knüpft an dieser Verbindung an, erzeugt gerade dadurch Anschlussfähigkeit an den EUropäischen Diskurs und wird politisch entsprechend wohlwollend rezipiert und partiell umgesetzt. Moving Europe forward als Slogan der Initiative schlägt dabei in eine ähnliche Kerbe wie bestehende Narrative EUropäischer Mobilitätspolitik wie z.B. Wer sich bewegt, bewegt Europa.

Die Förderung von Mobilität imaginiert dabei zunächst Prozesse der Entgrenzung auf geographischer, sozialer, mentaler, psychischer, politischer und individueller Ebene. Wo Mobilität als Entgrenzung beschrieben wird, gehen mit den insinuierten grenzüberschreitenden Bewegungen zumeist Chancen zur Weiterentwicklung einher. Und tatsächlich eröffnen sich durch das mobilitätspolitische Wirken der EU auf vielen Ebenen des Bildungs- und Arbeitswesens Möglichkeiten für Auslandsaufenthalte und damit Zugänge zu ansonsten tendenziell verschlossenen Lern- und Arbeitswelten. Gleichzeitig offenbart sich anhand der Narrativanalyse ein Spannungsfeld von Entgrenzung und Einordnung, innerhalb dessen sich Erzählungen über Sinn und Nutzen von Mobilität im politischen Diskurs verstehen lassen. Indem Mobilitätsaktivitäten durch EUropäische Mobilitätspolitik vermeintlich für alle ermöglicht werden, werden sie gleichsam zur Norm erhoben - „Beweglichkeit wird zum Imperativ“ (Liesner 2006: 174). Individuelle und europäische Bewegungen werden dabei miteinander verknüpft. Bewegung in Europa wird als Bewegung für Europa narrativ zur Chance für eine Erhöhung der Zustimmung $z u$ Europa. Individueller Bewegung wird entsprechend nicht nur ein entgrenzendes, sondern auch 
ein einordnendes Potential zuteil, indem anhand geförderter Bewegungen durch Europa die Europäizität der Bewegten in die richtigen Bahnen gelenkt werden kann.

Die mit \#FreeInterrail politisch verbundenen Ziele lassen sich als Lernaufträge hin zu einer stärkeren Identifikation mit dem europäischen Projekt interpretieren. Sie erinnern darin an Konzepte des politischen Reisens des 19. Jahrhunderts oder an die Grand Tour, bei der mit Mobilität einerseits die Zugehörigkeit zu einer Gruppe sozialisatorisch hergestellt wurde und gleichzeitig die normative Übereinstimmung der Reisenden mit den Werten der Gruppe erhöht werden sollte (vgl. Hlavin-Schulze 1998: 34f.; 62f.). Die mit Mobilität verbundene Entgrenzung ist entsprechend stark mit Momenten der Einordnung in bestehende Ordnungen verknüpft, ohne dass dadurch ihr transformatives Potential narrativ verloren ginge. Im Gegenteil: Im Rahmen der Narrativanalyse zeigte sich, dass im Kontext von \#FreeInterrail in starkem Maße politische Hoffnungen auf Veränderung artikuliert werden und damit auf spezifische Effekte von Mobilität abgezielt wird. Bei den anvisierten Transformationen stehen jedoch nicht entgrenzende Bildungserfahrungen im Sinne veränderter Subjekt-Welt-Verhältnisse im Vordergrund, sondern primär Veränderungen der normativen Einstellungen der Reisenden zu Europa. Entsprechend passt sich \#FreeInterrail ein in eine EUropäische Mobilitätspolitik, welche Mobilität eben primär mit Lern- und nicht mit Reflexionszwecken versieht.

Weiterbildung und Erwachsenenbildung wiederum sind in einer Vielzahl an Aktivitäten in Praxis und Forschung in die Unterstützung, Beratung, Evaluation und institutionelle Rahmung von Mobilität in und durch EUropa involviert. Sie tragen entsprechend dazu bei, dass sich ein Mobilitätsimperativ etablieren konnte, welcher Fortschritt, Europäisierung und Mobilität narrativ eng miteinander verbindet. Eine stärkere Rezeption und Anwendung narrativanalytischer Ansätze könnte hier gewinnbringend sein, um bildungspolitisch relevante Diskurse methodisch fundiert hinsichtlich der sie leitenden Erzählungen kritisch zu beleuchten und dabei auch die den Disziplinen in den jeweiligen Narrativen zugedachten Rollen zu hinterfragen. Narrativanalytische Mobilitätsforschung in der Erwachsenenbildung könnte so angelegt zu einer reflexiven Distanzierung vom EUropäischen Mobilitätsimperativ führen, ohne dabei in eine bloße Opposition zur Ermöglichung von Auslandsaufenthalten zu geraten. 


\section{Literatur}

Alheit, Peter / Dausien, Bettina (2000): Die biographische Konstruktion der Wirklichkeit. Überlegungen zur Biographizität des Sozialen. In: Hoerning, E. (Hrsg.): Biographische Sozialisation. Stuttgart: Lucius\&Lucius, S. 257-283.

Amtsblatt der Europäischen Union (2011): Empfehlung des Rates vom 28. Juni 2011 - Jugend in Bewegung - die Mobilität junger Menschen zu Lernzwecken fördern (2011/C 199/01).

Europäisches Parlament: Protokoll der Debatte „Zum 18. Geburtstag ein InterrailPass für Europa" vom 04.10.2016. Online: http://www.europarl.europa.eu/sides/getDoc.do?type $=$ CRE\&reference $=20161004 \&$ secondRef $=$ ITEM-015\& language $=\mathrm{DE}[09.04 .2019]$.

Fegter, Susann et al. (2015): Erziehungswissenschaftliche Diskursforschung. Theorien, Methodologien, Gegenstandskonstruktionen. In: Dies. (Hrsg.): Erziehungswissenschaftliche Diskursforschung. Wiesbaden: Springer VS, S. 9-57.

FreeInterrail. Homepage: http://freeinterrail.eu/ [09.04.2019].

Gadinger, Frank / Jarzebski, Sebastian / Yildiz, Taylan (2014): Politische Narrative. Konturen einer politikwissenschaftlichen Erzähltheorie. In: Dies. (Hrsg.): Politische Narrative. Wiesbaden: Springer VS, S. 3-38.

Herr \& Speer: Lasst uns reisen. Kostenloses Interrail für Junge als EU-Projekt. In: DIE ZEIT (2015), Nr. 34. Online: https://www.zeit.de/2015/34/interrail-europa-kennenlernen-kostenlos-jugend [09.04.2019].

Horn, Eva (2014): Zukunft als Katastrophe. Frankfurt a.M.: S. Fischer.

Hlavin-Schulze, Karin (1998): „Man reist ja nicht, um anzukommen“. Reisen als kulturelle Praxis. Frankfurt a.M. / New York: Campus Verlag.

Koller, Hans-Christoph (2012): Bildung anders denken - Einführung in die Theorie transformatorischer Bildungsprozesse. Stuttgart: Kohlhammer.

Liesner, Andrea (2006): Mobilität. In: Dzierzbicka, A. / Schirlbauer, A. (Hrsg.): Pädagogisches Glossar der Gegenwart. Wien: Löcker, S. 172-182.

Münch, Sybille (2016): Interpretative Policy-Analyse. Eine Einführung. Wiesbaden: Springer Fachmedien.

Rothe, Daniela (2015): „Lebensbegleitendes Lernen für alle“. Chancengleichheit Chancengerechtigkeit - Bildungsgerechtigkeit. Zur ambivalenten Produktivität einer begrifflichen Transformation. In: Fegter, S. et al. (Hrsg.): Erziehungswissenschaftliche Diskursforschung. Wiesbaden: Springer VS, S. 325-346.

Schäfer, A. (2011): Irritierende Fremdheit: Bildungsforschung als Diskursanalyse. Paderborn: Ferdinand Schöningh.

Viehöver, Willy (2014): Erzählungen im Feld der Politik, Politik durch Erzählungen. Überlegungen zur Rolle der Narrationen in den politischen Wissenschaften. In: Gadinger, F. et al. (Hrsg.): Politische Narrative. Konzepte - Analysen Forschungspraxis. Wiesbaden: Springer VS, S. 67-92. 
Yildiz, Taylan / Gadinger, Frank / Jarzebski, Sebastian (2015): Das narrative Element des Politischen: Überlegungen zu einer Poetologie des Wissens in der Politikwissenschaft. In: Zeitschrift für Politikwissenschaft 25. Jg., Heft 3, S. 421-431.

Zick, Sebastian (2018): Bildung, Beratung, Bewegung. Die Steuerung der Bildungsreise zwischen Apodemik und europäischer Mobilitätspolitik. In: Dörner, O. et al. (Hrsg.): Beratung im Kontext Lebenslangen Lernens - Konzepte, Organisation, Politik, Spannungsfelder. Leverkusen: Barbara Budrich, S. 75-87. 

Eva Heinrich

\section{Veränderung von Praktiken beim Wiedereinstieg in eine entgrenzte Arbeitswelt}

\section{Hinführung und Rahmung}

Übergänge in der Gesellschaft, zwischen Kulturen, in institutionellen Strukturen, organisationalen Zusammenhängen, aber auch individuellen Lebenslagen sind nicht nur in unserem Leben und Erleben zur Selbstverständlichkeit geworden, sondern beschäftigen in ihren Dynamiken, ihrer Diversifikation, Komplexität, Fragilität und Kontingenz auch Forschende verschiedenster Disziplinen $^{1}$ (vgl. von Felden/Schmidt-Lauff 2015: 11). Während insbesondere stark institutionalisierte und regulierte Übergänge wie der Übergang aus dem Kindergarten in die Schule (u.a. Oehlmann et al. 2011, Griebel et al. 2013), aus der Schule in die Berufstätigkeit (u.a. Benner/Galyschew 2013, von Felden/Schiener 2010) oder auch aus der Berufstätigkeit in die Nacherwerbsphase (u.a. Gebrande/Tippelt 2015, Costard 2010) wissenschaftlich beschrieben sind, so bedarf es einer Sensibilität gegenüber gesellschaftlich weniger exponierten und wissenschaftlich noch lückenhaft beschriebenen Übergängen. Als ein Beispiel hierfür kann der Übergang aus der Elternzeit zurück in die Erwerbstätigkeit betrachtet werden, welcher im Kontext der wissenschaftlichen Forschung zu Berufsrückkehrerinnen und -rückkehrern zwar oftmals quantitativ (u.a. Wippermann 2011) und in Bezug auf seine Einflussfaktoren (Frodermann et al. 2018), jedoch kaum in seiner relationalen Herstellung und seinem praktischen Vollzug beschrieben wurde.

Dieser Lücke widmet sich ein im Rahmen des DFG-Graduiertenkolleg Doing Transitions angesiedeltes Dissertationsprojekt, welches gezielt die Herstellung und den Vollzug des beruflichen Wiedereinstiegs nach der Elternzeit innerhalb der hochdynamischen Branche der Unternehmensberatungen in den Blick nimmt. Durch Experteninterviews (Bogner/Littig/Menz 2014) mit organisational Verantwortlichen und problemzentrierte Interviews (Witzel 1982) mit Führungskräften und Personen im Übergang, jeweils in und nach der Elternzeit, soll der Übergang in seiner Herstellung und seinem prozesshaften Vollzug durch Analysen in Anlehnung an die Methodologie der Grounded Theory (Glaser/Strauß 1967) erschlossen werden. In diesem Beitrag wird der

1 Hier ist u.a. das DFG-geförderte Graduiertenkolleg Doing Transitions (Projektnummer 2105) zu nennen, welches sich seit 2017 mit Formen der Gestaltung von Übergängen im Lebenslauf beschäftigt. 
Übergang zunächst in seinem Kontext und seinem relationalen Gefüge systematisiert. Durch die anschließende Einnahme einer praxeologischen Perspektive auf Übergänge werden Implikationen herausgearbeitet, die sich auf dem gewählten theoretischen Hintergrund aufbauend für die Analyse von Herstellung und Vollzug des fokussierten Übergangs ergeben.

\section{Heuristik zur Beschreibung des Übergangs in seiner Relationalität}

Untersucht man den Übergang aus der Elternzeit zurück in die Erwerbstätigkeit, so wird schnell offensichtlich, dass dieser in ein multidimensionales Geflecht verschiedenster Beziehungen eingebettet ist. Möchte man eben diese Relationalität von Einflüssen und Abhängigkeiten einfangen, so können Ebenen vorerst als semantische Einheiten dienen, um sich der Komplexität des Feldes und der Kontextualität des Übergangs zu nähern.

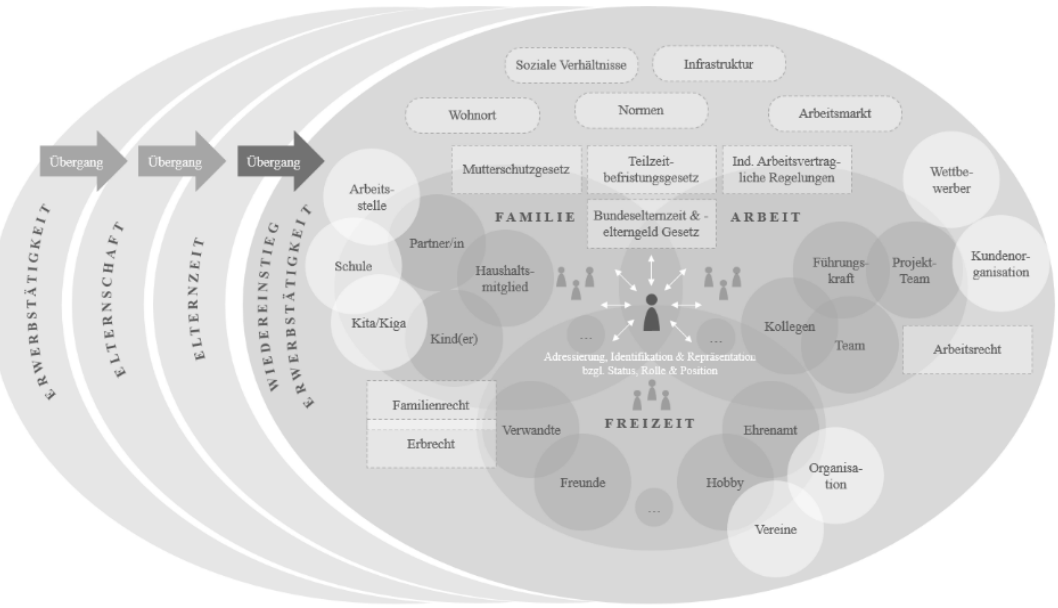

Abb. 1 Ökosystemische Darstellung des Übergangs Elternzeit-Erwerbstätigkeit (Quelle: Eigene Darstellung in Anlehnung an Bronfenbrenner, 1981)

Als Heuristik zur Beschreibung des Übergangs in seiner Relationalität kann der ökosystemische Ansatz nach Bronfenbrenner (1981) genutzt werden. In Anlehnung an Bronfenbrenner können die Ebenen des Mikro-, Meso-, Exo-, Makro und Chronosystems differenziert und als aufeinander folgend, immer größer und umfassender werdend begriffen werden (Oerter/Montada 1995: 
88). Die kleinste semantische Einheit, die Mikroebene, stellt hierbei das Individuum dar. Als solches kann sowohl die Person, die den Übergang aus der Elternzeit zurück in die Erwerbstätigkeit vollzieht, als auch die einzelnen Personen ihres sozialen Umfelds betrachtet werden. Diese adressieren die Person im Übergang im Hinblick auf ihren Status, ihre Rolle und ihre Funktion auf unterschiedliche Weise, zum Beispiel als Vater oder Mutter, Partner oder Partnerin, als Kollege oder Kollegin, als Vorgesetzten oder Vorgesetzte, Freund oder Freundin oder auch als Vereinsmitglied. Diesen unterschiedlichen Adressierungen liegen jeweils bestimmte Beziehungen und damit einhergehende Erwartungen zu Grunde.

Während manche Personen in engerer Beziehung zueinander stehen, scheinen andere Personenkonstellationen weniger direkt miteinander verknüpft. So ist es beispielsweise naheliegender, dass das Kind der Person im Übergang eine engere Verbindung zu seinem anderen Elternteil unterhält als zu den Kolleginnen und Kollegen des Elternteils im Übergang. Stehen einzelne Personen bzw. Mikrosysteme in einer solch engeren Verbindung und beeinflussen sich gegenseitig, so kann dies als Mesoebene betrachtet werden. Im beschriebenen Beispiel lassen sich diese über die unterschiedlichen Lebensbereiche wie den der Familie, der Arbeit oder der Freizeit differenzieren. Die Person im Übergang beteiligt sich folglich an verschiedenen Lebensbereichen und ist damit Teil mehrerer Mesosysteme.

Ergeben sich Ereignisse, die das Mesosystem beeinflussen, so können sich diese u.a. auf untergeordnete Mikrosysteme auswirken. Gleichzeitig ist es jedoch auch denkbar, dass Ereignisse in Mesosystemen passieren, welchen das Individuum nicht unmittelbar angehört, dieses jedoch trotzdem beeinflussen. Ein anschauliches Beispiel für das bisher skizzierte Bild könnte ein Streik von Betreuungsfachkräften der Betreuungseinrichtung des Kindes sein. Auch wenn die Person im Übergang kein unmittelbarer Teil dieses Mesosystems ist, würde sich ein solcher Vorfall dennoch auf sie auswirken. Bronfenbrenner (1981: 42) bezeichnet „Lebensbereiche, an denen die [...] Person nicht selbst beteiligt ist, in denen aber Ereignisse stattfinden, die beeinflussen, was in ihrem Lebensbereich geschieht, oder die davon beeinflusst werden" als Exosysteme.

Weiterhin ist die Person im Übergang in eine Gesellschaft und Kultur eingebunden, die über bestimmte Weltanschauungen, Ideologien, Normen, Werte, Konventionen, Traditionen und Gesetze verfügt und dadurch Rahmungen und Orientierungen schafft. Diese gesellschaftlichen, politischen und kulturellen Verankerungen können als Makroebene betrachtet werden.

Die Visualisierung von Mikro-, Meso-, Exo- und Makroebene kann als Momentaufnahme und somit als Querschnitt verstanden werden. Durch die Einführung der Begrifflichkeit des Chronosystems, welche die zeitliche Veränderung eines Konglomerats an Systemen beschreibt (vgl. Bronfenbrenner 1986), wird eine chronologische Dimension eröffnet, die die Beschreibung von Ver- 
änderungen im Längsschnitt ermöglicht. Dem fokussierten beruflichen Wiedereinstieg gehen notwendigerweise die Situation der Elternzeit und weiter zurückliegend das Elternwerden sowie die Situation einer vorangegangenen Erwerbstätigkeit voraus.

Wenngleich die gewählte heuristische Darstellung die Person im beruflichen Wiedereinstieg in ihrem relationalen Gefüge abbildet und zum Ausgangspunkt der Erschließung macht, so rückt rekurrierend auf den Fokus der Herstellung und des Vollzugs des Übergangs der transformative Prozess über das Chronosystem ins Zentrum der Betrachtung.

\section{Der Übergang als raum-zeitlich strukturierter Praxiskomplex}

Besonders anschlussfähig an die vorgenommene Systematisierung scheint van Genneps (1909) Verständnis eines Übergangs als Prozess zwischen zwei genau definierten Situationen. Basierend auf dieser Idee könnte im gewählten Beispiel als erste Situation die der Elternzeit und als zweite Situation die der wiederaufgenommenen Erwerbstätigkeit betrachtet werden. Der Prozess dazwischen, die Transformation des relationalen Gefüges über das Chronosystem, könnte dann als Übergang verstanden werden. Van Gennep (1909) führt die Systematisierung weiter, indem er in seinem Phasenmodell eine idealtypische Abfolge von Übergängen skizziert. Als erste Phase des Übergangsprozesses betrachtet er die Ablösungsphase, in welcher Rituale der Trennung vollzogen werden. Um dies am Beispiel des Rückübergangs aus der Elternzeit greifbar zu machen, wäre die Ablösungsphase noch in der Situation der Elternzeit anzusiedeln und könnte durch die Planung des bevorstehenden Übergangs initiiert werden. Auf der familiären Seite könnte dies die Organisation der Kinderbetreuung betreffen, auf organisationaler Seite die Kontaktaufnahme zum Arbeitgeber, um den bevorstehenden Wiedereinstieg zu besprechen. Die Abkehr von der noch aktuellen Situation, der Elternzeit, wird angestoßen.

Als zweite Phase des Übergangsprozesses charakterisiert van Gennep die Phase der Schwelle, die von Ritualen der Umwandlung begleitet wird. Unter Rückbezug auf den Wiedereinstieg könnte die Schwellenphase den Vorgang der Transformation des Konglomerats an Systemen, also das Geschehen zwischen zwei genau definierten Situationen darstellen. Ein wesentlicher Bestandteil dieses Geschehens könnte auf der beruflichen Seite der erste Arbeitstag sein, an welchem der Mitarbeiter bzw. die Mitarbeiterin in irgendeiner Form Präsenz zeigt. Die vorangegangene Situation der Elternzeit wurde verlassen und ein vollständiges Ankommen in der Situation der Erwerbstätigkeit unter 
neuen lebensweltlichen Bedingungen ist noch nicht gegeben. Die Person im Übergang befindet sich in einer Phase des „Dazwischen“ oder unter Rückgriff auf die Systematisierung nach Bronfenbrenner inmitten der Transformation über das Chronosystem. Diese verläuft nicht zwingend parallel und zeitgleich auf allen Ebenen, sondern kann als sukzessiver Wandlungsprozess angesehen werden.

Als dritte Phase des Übergangsprozesses betrachtet van Gennep die Integrationsphase, welche durch Rituale der Angliederung begleitet wird. Die Person im Übergang eignet sich unter Berücksichtigung des neuen Verhältnisses ihrer Lebensbereiche neue Routinen an. Familiäre und organisationale Belange gehen einen geregelten Gang - ein neuer Alltag ist geschaffen. Das in der Elternzeit bestehende Konglomerat an Systemen hat sich verändert und stellt sich nun in einer neuen Konstellation dar.

Auch wenn das Phasenmodell nach van Gennep zunächst eine gewisse Linearität impliziert, so gilt es eben diese vor dem Hintergrund empirischer Analysen zu reflektieren und nach Wanka (2018: 12) Brüche („Scheitern“) und Diskontinuitäten (,Steckenbleiben“) in Betracht zu ziehen.

Versteht man einen Übergang nun als prozesshaftes, kontingentes Phänomen, so liegt dem eine sozialkonstruktivistische Theorietradition zugrunde. Vor diesem Hintergrund werden Übergänge weniger als Ausgangspunkt einer Fragestellung nach Funktion und Wirkungsweise verstanden, als vielmehr zum Ziel einer Fragestellung nach Herstellung und Gestaltung erklärt. Wenngleich das Erkenntnispotenzial anderer Ansätze nicht bestritten wird, so scheint die praxeologische Rekonstruktion von Übergängen durch ihren expliziten Fokus auf Praktiken fruchtbar, um diese in ihrem konkreten Vollzug und ihrer Phänomenologie zu ergründen.

In einem praxistheoretischen Verständnis wird eine "flache Ontologie“ (Schatzki 2016: 30) und damit die analytische Auflösung von semantisch gefassten Ebenen des Sozialen proklamiert (vgl. Nicolini 2017). Auf Basis dieser Vorstellung sind alle sozialen Kategorien und Phänomene in Praktiken auflösbar, sodass nichts außerhalb dieser existiert. Die Praktiken selbst wiederum bestehen dabei aus Teilnehmenden, über die Handlungsfähigkeit und Handlungsmacht als „distributed agency“ verteilt sind (Enfiel/Kockelman 2017). Blickt man aus dieser Perspektive auf den Wiedereinstieg nach der Elternzeit in die Erwerbstätigkeit, so kann der Übergang als aus Praktiken bestehend, durch Praktiken hergestellt, vollzogen und reguliert verstanden werden. Dabei können die verschiedenen semantisch gefassten Einheiten (Mikro-, Meso-, Exo- und Makrosystem) als Teilnehmende betrachtet werden, über die sich die Handlungsmacht und Handlungsfähigkeit verteilt.

Weiterhin wird aus einer praxeologischen Perspektive nicht gefragt, warum oder wozu etwas getan wird, sondern wie es getan wird (vgl. Reckwitz 2003: 290, Hui et al. 2017). Bezüglich des Wiedereinstiegs sind somit weder divergierende Interpretationen von Individuen noch deren subjektives Erleben von 
Interesse, sondern vielmehr die inhärent materiellen Praktiken, die sich durch implizites Wissen und Können, Bedeutungen und diskursive Elemente manifestieren (Shove et al. 2012). Dabei wird zwischen der Performanz und Entität von Praktiken unterschieden, welche gleichzeitig wie zwei Seiten einer Medaille miteinander verbunden sind (vgl. Wanka 2018: 5). Die Performanz beschreibt eine Praktik in ihrem Vollzug und damit mit hohem Veränderungspotenzial. Untersucht man den fokussierten Übergang in seiner Performanz, so interessiert man sich für den individuellen Vollzug: Es wird danach gefragt, wie die Person im Übergang diesen durchlaufen hat, durch die Anpassung, Aufgabe oder Entwicklung welcher Praktiken der Übergang hergestellt und gestaltet wurde. Die individuelle Ausführung und damit der Übergang in seiner Einzigartigkeit stehen im Fokus. Die Entität wiederum stellt eine Praktik in ihrer Struktur dar, die routiniert reproduziert wird. Untersucht man den Übergang in seiner Entität, so fokussiert man sich auf dahinterliegende latente Strukturen des Übergangs im Allgemeinen: Es wird nach dem impliziten Wissen und Können, den Bedeutungen und Diskursen, die mit diesem Übergang in Verbindung stehen, gefragt (Shove et al. 2012). Die Entität des Übergangs existiert, auch wenn gerade niemand den Übergang tut, sodass der Übergang in seiner Phänomenologie im Fokus steht.

Übergänge sind jedoch nicht als unabhängige, isolierte Prozesse zu betrachten, sondern sie knüpfen das soziale Gewebe im Nexus zwischen anderen Praktiken (vgl. Wanka 2018: 4). Sie sind als in ein Netz anderer Praktiken eingebettet anzusehen, stehen in Beziehung zueinander und schließen mehr oder weniger aneinander an. Um auf das Beispiel des Wiedereinstiegs ins Berufsleben zurückzukommen, so verläuft dieser im Anschluss an eine Elternzeit oftmals in enger Verknüpfung mit Praktiken der Eingewöhnung des Kindes in eine Betreuungsstätte. Dabei finden Praktiken nicht in „,neutralen“ Räumen statt, sondern sind durch den sozialen Kontext der Teilnehmenden bedingt (vgl. Wanka 2018: 7). So muss beispielsweise beim Betreten der Kindertagesstätte nicht täglich erneut ausgehandelt werden, wer zu den Betreuenden und wer zu den Betreuten gehört.

Bestehen nun stärkere Zusammenhänge zwischen einzelnen Praktiken, so formen diese Praxisbündel und Praxiskomplexe (Shove et al. 2012: 81). Praxisbündel beschreiben dabei die Zerlegung von Praktiken in kleinere Bestandteile. Wenn die Person im Übergang an ihrem ersten Arbeitstag nach der Elternzeit beispielsweise berichtet, sie habe sich zunächst einen Überblick über eingetroffene Nachrichten verschafft, so kann dies zunächst als Praktik betrachtet werden. Berichtet sie weiterhin, wie sie bei der Ausführung dieser Praktik vorgegangen ist, z.B. indem erst alle Nachrichten durchgelesen und die unwichtigen gelöscht wurden, anschließend priorisiert und dann abgearbeitet wurde, so stellen diese einzelnen Aktivitäten jeweils Praxisbündel dar. Praxiskomplexe wiederum beschreiben das Zusammenfassen von Praktiken zu grö- 
Beren Einheiten (Reckwitz 2003). So kann beispielsweise die Vielzahl an Aktivitäten, die rund um den beruflichen Wiedereinstieg nach der Elternzeit stattfinden, zum Praxiskomplex des Übergangs zusammengefasst werden.

Verknüpft man nun praxeologische Überlegungen mit den vorangegangenen Systematisierungen in Anlehnung an Bronfenbrenner und van Gennep, so lässt sich ein Übergang als raum-zeitlich strukturierter Praxiskomplex innerhalb eines Netzes von vor- und nachgelagerten, aber auch parallel verlaufenden Praktiken verstehen (vgl. Wanka 2018: 12). Der Übergang wird dadurch nicht als isolierte Einheit betrachtet, sondern in seiner Kontextualität und Relationalität begriffen. Die zunächst als Ebenen gefassten semantischen Einheiten können als Teilnehmende des Übergangs betrachtet werden, über welche sich die Handlungsmacht und -fähigkeit verteilt. Der Fokus auf konkrete Praktiken des Übergangs aus der Elternzeit zurück in die Erwerbstätigkeit ermöglicht die Erfassung, aber vor allem ein Verständnis der wechselseitigen Verschränkung organisationaler und individueller Modi der Gestaltung und Herstellung des Übergangs.

\section{Erste Ergebnisse zu Praktiken des beruflichen Wiedereinstiegs nach der Elternzeit}

Aus dieser praxeologischen Perspektive heraus getätigte erste Analysen des empirischen Materials lassen sich unter Rückgriff auf van Genneps (1909) idealtypisches Phasenmodell von Übergängen beschreiben. So können noch in der Elternzeit Praktiken wie beispielsweise die der Übergabe der Kindesbetreuung, der Kontaktaufnahme zwischen Mitarbeiter oder Mitarbeiterin und Führungskraft, aber auch Praktiken der Projekteinsatzplanung und der ersten Wiederaufnahme erwerbsbezogener Tätigkeiten wie das Wiederaufklappen des Laptops und Lesen eingetroffener Mails der Phase der Ablösung zugeordnet und der Einleitung des Übergangs zugeschrieben werden. Betrachtet man den ersten Arbeitstag nach der Elternzeit als Schwelle, an welcher der vorangegangene Alltag verlassen und ein neuer noch nicht geschaffen wurde, so scheint sich diese Phase beim beruflichen Wiedereinstieg unter anderem durch Praktiken der Schaffung von Schonräumen auszuzeichnen. Diese Schonräume werden zum Teil gezielt durch die Lage der Elternzeit hervorgerufen, indem das Elternzeitende auf einen klassischen Urlaubszeitraum, in welchem weniger Kundennachfrage besteht, gelegt wird. Weitere Optionen zur Schaffung von Schonräumen stellen das Wahrnehmen von an die Elternzeit anschließenden Urlaubstagen oder die vorläufige Möglichkeit zur Arbeit aus dem Homeoffice dar. Auch dedizierte Zeiträume zur Wiedereinarbeitung können die Phase der 
Schwelle markieren. In der anschließenden Integrationsphase lassen sich unter anderem das Aushandeln von Akzeptanz, Zutrauen und Verständnis, aber auch der formelle (,heads-up geben“) und informelle (,gemeinsames Kaffeetrinken") Austausch als Praktiken der Angliederung klassifizieren. Des Weiteren zeigen sich über die verschiedenen idealtypischen Phasen und Teilnehmenden hinweg wiederkehrend Praktiken des Ergreifens und/oder Verweigerns von Lernanlässen als zentrales Kriterium für das Gelingen oder Scheitern des Übergangs.

In weiteren Analysen soll der praktische Vollzug des fokussierten Übergangs im Detail untersucht und durch die Verschränkung der Perspektiven von betroffenen Mitarbeitern und Mitarbeiterinnen, Vorgesetzten und Personalverantwortlichen plastisch gemacht werden. Die daraus resultierende Darstellung des Übergangs in seinem relationalen Praxisnetz kann weiterführend als Basis für die Annäherung an zahlreiche spannende Fragestellungen dienen. Das Dissertationsprojekt fokussiert weiterführend die Frage nach Handlungsfähigkeit und Handlungsmacht im Spannungsfeld dualer Aushandlungen der Teilnehmenden und soll dadurch Implikationen zur Optimierung für die pädagogische Praxis in wirtschaftlichen Organisationen bieten.

\section{Literatur}

Benner, Ilka / Galyschew, Alexandra (2013): Hindernisse und Anforderungen an der ersten Schwelle - Hinweise aus der Studie „Zufriedenheit mit der Berufsorientierung aus Sicht Jugendlicher im Übergangssystem“, in: Friese, Marianne / Benner, Ilka / Galyschew, Alexandra (Hrsg.): bwp@ Spezial 6-Hochschultage Berufliche Bildung 2013, S. 1-18. http://www.bwpat.de/ht2013/ft02/ benner_galyschew_ft02-ht2013.pdf [22.01.2019].

Bogner, Alexander / Littig, Beate / Menz, Wolfgang (Hrsg.) (2002): Das Experteninterview. Theorie, Methode, Anwendung. Wiesbaden: VS Verlag für Sozialwissenschaften.

Bronfenbrenner, Urie (1981): Die Ökologie der menschlichen Entwicklung. Stuttgart: Klett-Cotta.

Bronfenbrenner, Urie (1986): Recent Advances in Research on the Ecology of Human Development, in: Silbereisen, Rainer K. / Eyferth, Klaus / Rudinger, Georg (Hrsg.): Development as Action in Context. Berlin, Heidelberg: Springer, S. 137-164.

Costard, Astrid (2010): Der Übergang in den Ruhestand als Bezugspunkt für Bildungsangebote. https://www.die-bonn.de/doks/costard0601.pdf [22.01.2019].

Enfield, Nick J. / Kockelman, Paul (2017): Distributed Agency. Oxford: Oxford University Press. 
Felden, Heide von / Schiener, Jürgen (Hrsg.) (2010): Transitionen - Übergänge vom Studium in den Beruf. Zur Verbindung von qualitativer und quantitativer Forschung. Lernweltforschung Band 6, Wiesbaden: VS Verlag.

Felden, Heide von / Schmidt-Lauff, Sabine (2015): Transitionen in der Erwachsenenbildung: Übergänge im gesellschaftlichen Wandel, im Fokus von Forschung und aus Sicht pädagogischer Professionalität, in: Schmidt-Lauff, Sabine / Felden, Heide von / Pätzold, Henning (Hrsg.): Transitionen in der Erwachsenenbildung: Gesellschaftliche, institutionelle und individuelle Übergänge. Schriftenreihe der Sektion Erwachsenenbildung der Deutschen Gesellschaft für Erziehungswissenschaft. Opladen, Berlin, Toronto: Verlag Barbara Budrich, S. $11-16$.

Frodermann, Corinna / Bächmann, Ann-Christin / Hagen, Marina / Grunow, Daniela / Müller, Dana (2018): Betriebliche Angebote zur Vereinbarkeit von Familie und Beruf: Mütter kehren schneller zu familienfreundlichen Arbeitgebern zurück. In: IAB-Kurzbereicht 18/2018 Aktuelle Analysen aus dem Institut für Arbeitsmarkt- und Berufsforschung. http://doku.iab.de/kurzber/2018/kb1818. pdf [22.01.2019].

Gebrande, Johanna / Tippelt, Rudolf (2015): Basiskompetenzen am Übergang in die Nacherwerbsphase. In: Schmidt-Lauff, Sabine/Felden, Heide von / Pätzold, Henning (Hrsg.) (2015): Transitionen in der Erwachsenenbildung. Gesellschaftliche, institutionelle und individuelle Übergänge. Opladen, Berlin, Toronto: Verlag Barbara Budrich. S. 189-201.

Gennep, Arnold van (1909): Les rites de passage. Paris: Ohne Verlag.

Gennep, Arnold van (aus dem Französischen von Klaus Schomburg) (1986): Übergangsriten. Frankfurt: Campus Verlag.

Glaser, Barney G. / Strauß, Anselm (1967): The Discovery of Grounded Theory: Strategies for Qualitative Research. Chicago: Aldine.

Griebel, Wilfried / Wildgruber, Andreas / Held, Julia / Schuster, Andrea / Nagel, Bernhard (2013): Partizipation im Übergangsmanagement von Kitas und Schulen: Eltern als Ressource, in: Bildungsforschung 10 (2013) 1, S. 26-44.

Hui, Allison / Schatzki, Theodore R. / Shove, Elizabeth (Hrsg.) (2016): The Nexus of Practices. Connections, constellations, practitioners. London: Routledge.

Nicolini, Davide (2016): Is small the only beautiful? Making sense of „large phenomena" form a practice-based perspective. In: Hui, Allison / Schatzki, Theodore R. / Shove, Elizabeth (Hrsg.): The Nexus of Practices. Connections, constellations, practitioners. London: Routledge, S. 98-113.

Oehlmann, Sylvia/Manning-Chlechowitz, Yvonne/Sitter, Miriam (Hrsg.) (2011): Frühpädagogische Übergangsforschung. Von der Kindertageseinrichtung in die Grundschule. Weinheim/München: Juventa.

Oerter, Rolf / Montada, Leo (Hrsg.) (1995): Entwicklungspsychologie: ein Lehrbuch. 4. Auflage. Weinheim, Basel: Beltz.

Reckwitz, Andreas (2003): Grundelemente einer Theorie sozialer Praktiken. Eine sozialtheoretische Perspektive. In: Zeitschrift für Soziologie, Jg. 32, Heft 4, S. 282-301. 
Schatzki, Theodore R. (2016): Praxistheorie als flache Ontologie, in: Schäfer, Hilmar (Hrsg.): Praxistheorie. Ein soziologisches Forschungsprogramm. Bielefeld: transcript. S. 29-44.

Shove, Elizabeth / Pantzar, Mika / Watson, Matt (2012): The Dynamics of Social Practice: Everyday Life and How it Changes. London: Sage.

Wanka, Anna (2018): Grundzüge einer praxeologischen Übergangsforschung. Nicht veröffentlicht.

Witzel, Andreas (1982): Verfahren der qualitativen Sozialforschung. Überblick und Alternativen. Frankfurt am Main: Campus.

Wippermann, Carsten (2011): Zeit für Wiedereinstieg - Potenziale und Perspektiven. https://www.bmfsfj.de/blob/95560/a08012dla9bfad7639490c9be04e1f44/ zeit-fuer-wiedereinstieg-potenziale-und-perspektiven-data.pdf [06.04.2019]. 


\section{Die Rekonstruktion betrieblicher Weiterbildungsentscheidungen in Klein- und Kleinstunternehmen}

\section{Einleitung}

Erwachsenenbildung und Lernen in Zeiten von Globalisierung, Transformation und Entgrenzung lässt sich konsequenterweise auch im Kontext der betrieblichen Weiterbildung thematisieren: Wenn sich Arbeitsmarktstrukturen, Beschäftigungssysteme und Qualifikationsbedarfe verändern, sich das Verhältnis von Arbeit, Beruf, Freizeit u.v.m. transformiert, so ist das Grund genug, über das Handeln und Entscheiden der Protagonisten, im vorliegenden Fall die Klein- und Kleinstunternehmen, informieren zu wollen. Hierfür werden betriebliche Weiterbildungsentscheidungen in den Blick genommen. Betriebliche Weiterbildungsentscheidungen werden letztlich immer dann untersucht, wenn betriebliche Weiterbildung als Untersuchungsgegenstand ausgewiesen ist. Dies geschieht in den meisten Fällen jedoch indirekt, denn Entscheidungshandlungen und ebenso Handlungen, die entweder Entscheidungen ähnlich sind (Langenheder 1975: 39f.) oder retrospektiv als Entscheidungen kommuniziert werden, sind betrieblicher Alltag. So werden Ergebnisse prominenter Surveys und Statistiken wie das AES und CVTS auf europäischer ebenso wie beispielsweise das IAB Betriebspanel auf der nationalen Ebene als Resultate u.a. der Entscheidungshandlungen verschiedener Akteure inner- und außerhalb der Betriebe interpretiert. Kurzum werden den Ergebnissen Unternehmensentscheidungen zugeordnet, die beispielsweise in einen hypothetischen Zusammenhang mit Umwelteinflüssen gebracht werden. So könnte die ökonomische Situation eines Landes als Prädiktor für die Weiterbildungsabstinenz oder die Steigerung des betrieblichen Weiterbildungsengagements beschrieben oder die Finanzierung der Weiterbildung durch die Betriebe als abhängig vom nationalen Fachkräftemangel dargestellt werden. Implizit geht es zudem um betriebliche Weiterbildungsentscheidungen, wenn auf den Anstieg weiterbildungsanbietender Unternehmen (Krekel 2018: 341), bevorzugte Personalstrategien (Janssen/Leber 2015) oder diverse Einflussfaktoren auf das Weiterbildungsangebot (Dummert 2018: 348ff.) usw. verwiesen wird.

Wie genau jedoch die organisationalen Entscheidungspfade aussehen, vermögen diese Untersuchungen kaum zu sagen. Vielmehr wird aus den Ergebnissen von Entscheidungen auf dieselben und die ihnen vorausgegangenen 
Prozesse geschlossen. Im vorliegenden Beitrag geht es darum, den Blick auf die mikropolitischen Aushandlungs- und Entscheidungsprozesse zu lenken und den genannten Perspektiven eine sinnvolle Ergänzung zu bieten. Der Beitrag folgt der Annahme, dass betriebliche Weiterbildungsentscheidungen in Klein- und Kleinstunternehmen (KKU) das Ergebnis von einerseits branchenund unternehmensspezifischen Einflüssen und andererseits Werthaltungen der Akteure sind, die jeweils in Entscheidungssituationen durch Relevanzsetzungen Bedeutung gewinnen oder verlieren. Weiterbildungsentscheidungen in Klein- und Kleinstunternehmen sind darum nicht das Ergebnis rationaler Kalkulation oder eines wie auch immer gearteten Bildungscontrollings, aber dennoch weder beliebig noch unprofessionell. Die für den Beitrag zentrale Fragestellung lautet: Wodurch werden die betrieblichen Weiterbildungsentscheidungen in Klein- und Kleinstunternehmen beeinflusst und wie ließen sich Entscheidungspfade beschreiben und unterscheiden? Der Beitrag bietet einen theoretischen Zugang zur Beantwortung dieser Fragestellung an und schließt mit dem Ausblick auf eine empirische Analyse.

\section{Diskurse über betriebliche Weiterbildungsentscheidungen}

Betriebliche (Weiter-)Bildungsentscheidungen $\mathrm{zu}$ thematisieren ist keineswegs neu, es existieren einige empirische Untersuchungen allein im deutschsprachigen Raum mit unterschiedlichen Fokussierungen. Hierunter lassen sich die Arbeiten von Weber et al. (1994) fassen, die auf der Basis von Fallstudien insgesamt 31 Entscheidungsprozesse in 28 Unternehmen (davon nur fünf in KMU und ein Kleinstunternehmen) betrachten. Der Fokus liegt hier auf den Verlaufsformen von Entscheidungsprozessen, weniger auf den Entscheidungen selbst. ${ }^{1}$ Ferner erörtern die Autoren, wie unterschiedliche Verlaufsformen die Ergebnisse der Entscheidungen beeinflussen. Die zentralen Variablen der erörterten kollektiven Entscheidungsprozesse sind Interessenbetroffenheit sowie Konfliktausmaß und Konfliktoffenheit (ebd.: 117). Es gelingt ihnen darüber hinaus vier Prozesstypen zu identifizieren, die als inkrementeller (1), Routine- (2), verdeckt-politischer (3) bzw. offen-politischer (4) Prozess bezeichnet werden (ebd., S. 132).

1 Entscheidungen, verstanden als Auswahl, sind im Vergleich zu den ihnen vorausgegangenen (Entscheidungs-)Prozessen unterkomplex. Wenn davon gesprochen wird, Entscheidung zu fokussieren, dann meint das immer auch den ihr vorausgegangenen Prozess. 
Entscheidungsprozesse können aber auch als Ausdruck einer betrieblichen Handlungslogik (Harney 1998) verstanden werden, d.h. der Verbindung zwischen der ,formalen, nach außen hin dokumentierten Rationalität von Organisationen einerseits und ihrer materiellen Umsetzung in die alltägliche Organisationswirklichkeit andererseits" (ebd.: 115). Aus dieser Perspektive reagieren Betriebe auf die äußere Bedrohung ihrer Ressourcen mithilfe von Herstellung und Tausch. Betriebliche Handlungslogik wird dann nicht als rational choice verstanden, denn hier stünde das individuelle Nutzenkalkül im Vordergrund, sondern als Präferenz, die jenem Nutzenkalkül vorangestellt wird (ebd.: 116). Das spezifisch betriebliche an dieser Handlungslogik liegt dann keineswegs nur in den interindividuellen Unterschieden verschiedener Betriebe begründet, sondern muss als ,gemeinsamer Nenner' verstanden werden, auf welchen Betriebe als Entscheidungsagenturen (Harney 1998: 124) Bezug nehmen. Hierunter fällt besonders das Risiko der permanenten Ersetzbarkeit betrieblicher Weiterbildung sowie des Unternehmens selbst. Betriebliche Handlungslogik kann sich demnach gegen die betriebliche Weiterbildung oder das Unternehmen wenden und trotzdem Programmerfüllung anstatt Programmkrise sein (Harney 1998: 129).

Betriebliche Weiterbildungsentscheidungen finden ihren Ausdruck ferner in expliziten Weiterbildungsstrategien (Weil et al. 2007). Auf der Grundlage einer quantitativen Befragung von deutsch-schweizer Weiterbildungsverantwortlichen in KMU sowie einer sich anschließenden qualitativen Interviewstudie schlagen Weil et al. eine Typologie der Weiterbildung in KMU vor. Hierzu zählen organisationsorientierte (1), defizit-ausgleichende mitarbeiterorientierte (2), problemlösend-bedarfsorientierte (3) sowie aufgaben- und kundenorientierte (4) Betriebe (ebd.: 5). Zum Sample der qualitativen Vertiefungsstudie gehörten allerdings ausschließlich Unternehmen, die in den vergangenen drei Jahren vor der Erhebung formale Weiterbildung anboten (ebd.: 10).

Betriebliche Weiterbildungsentscheidungen werden nicht zuletzt auch im Kontext von Bildungscontrolling und Aushandlungsprozessen erörtert (Heuer 2010; Käpplinger 2009a, 2009b, 2010a). Untersuchungen, deren Datengrundlage einerseits die RBS-Umfrage (Referenz-Betriebs-System), Expertengespräche sowie Fallstudien sind, kommen zu dem Schluss, dass Bildungscontrolling (häufig in Form des Kostencontrollings) (Käpplinger 2010b: 10) überwiegend von großen Unternehmen und dort multipolar praktiziert wird (ebd.: 11). Auf der Grundlage von vergleichenden Fallstudien in mehreren Großunternehmen identifiziert Heuer (2010: 20ff.) außerdem zwei Ebenen von Weiterbildungsentscheidungen: Einerseits die grundlegenden „Entscheidungen zur Weiterbildung" (ebd.: 20), d.h. die Frage, ob Weiterbildung angeboten wird oder nicht, und Weiterbildungsentscheidungen, die sich auf Organisation, Ablauf und Akteure beziehen (ebd.). Als wesentliche Einflussfaktoren darauf, ob Weiterbildung für den Betrieb infrage kommt, stellten sich heraus: der Fokus auf Nutzenmaximierung, die Übernahme von Legitimation, die Verteilung von 
Gratifikation sowie der Umgang mit der Dynamik betrieblicher Anforderungen (ebd.: 21).

Aufbauend auf der Erkenntnis, dass Weiterbildungsentscheidungen folglich auf unterschiedlichen Ebenen getroffen werden und von einer starken Heterogenität von Entscheidungsprozessen auszugehen ist, erörtert Käpplinger mithilfe eines konfigurationstheoretischen Ansatzes die verschiedenen Konfigurationen von Weiterbildungsentscheidungen auf der Makro-Ebene (Entscheidung über Weiterbildung) sowie der Meso-Ebene (Konfigurationen der Weiterbildungskompetenz) (Käpplinger 2016: 141ff.). Mithilfe einer Re-Analyse zahlreicher Interviews wurden ferner Weiterbildungsentscheidungen auf der Mikro-Ebene rekonstruiert. Hier konnten Konfigurationen gemäß der Funktion $^{2}$ von Weiterbildung (Anpassung, Motivation, Akquisition etc.) (Käpplinger 2016: 188) ebenso wie Spezifika von betrieblichen und individuellen Perspektiven auf diese Funktionen (ebd.: 217ff.) beschrieben werden.

Auf die Funktionen, allerdings im Sinne von akteursabhängigen Zuschreibungen in der Programmplanung, heben auch die Arbeiten von Hippel und Röbel (Hippel/Röbel 2016) ab. Deren Datengrundlage bilden drei Unternehmensfallstudien mit je 9-12 Experten- und problemzentrierten Interviews sowie eine Programmanalyse. Im Fokus steht hierbei der besondere Einfluss der Akteure (Macht, Interessen und letztlich deren Funktionszuschreibungen) auf die Programmplanung im Kontext betrieblicher Weiterbildung. Die Programmplanungsperspektive macht diesen Ansatz außerdem für die Diskurse in der Erwachsenenbildung besonders anschlussfähig.

Nicht zuletzt werden die betrieblichen Bedarfsbestimmungen im Zuge von Weiterbildungsentscheidungen in Großbetrieben (Röbel 2017) und KMU (Lohaus/Habermann 2011) thematisiert. Entscheidungen zu den Bedarfen sind folglich in Großbetrieben abhängig von der Programmart (Katalog, maßgeschneidert, bereichsintern, -extern usw.) (Röbel 2017: 32ff.), Bedarfe finden sich aber auch in KMU. Diese jedoch werden allzu häufig ausgeschlossen, wenn es zu einer Engführung des Weiterbildungsbegriffs kommt (Lohaus/Habermann 2011: 51).

Darüber hinaus formulieren Dobischat und Düsseldorf (2013: 253) im Anschluss an ihre Erörterungen zur betrieblichen Weiterbildung von KMU auf der Grundlage des AES, des Datenreports zum Berufsbildungsbericht sowie des Bildungsberichts das Desiderat, betriebliche Weiterbildung in KMU genauer und ,gegen den Mainstream der großbetrieblichen Weiterbildungsforschung [zu analysieren sowie, $\mathrm{CM}][\ldots]$ die Ursachen der Weiterbildungszurückhaltung in ihrer Verbindung zu betrieblichen Entscheidungsprozessen gegen Weiterbildung [...]“" (ebd.) zu erforschen.

2 Ein Nachteil liegt m.E. in der Anwendung eines im Wesentlichen deduktiven Verfahrens, bei dem es eher zu einer Bestätigung der angenommenen Funktionszuweisungen kam zu einer Etablierung eines neuen Ordnungsmusters betrieblicher Weiterbildungsentscheidungen. 
Der Forschungsstand zeigt, dass bei zahlreichen unterschiedlichen Fokussierungen (Verlaufsformen, betriebliche Handlungslogik, Weiterbildungsstrategien, Bildungscontrolling, Aushandlungsprozesse, Entscheidungsebenen, Konfigurationen, Funktionen und Funktionszuschreibungen, Programmplanung, Bedarfe etc.) der Entscheidungsbegriff und die Entscheidungsprozesse selbst (insbes. Präferenzordnungen, Prozesse der Auswahl, Rationalitätskonzepte etc.) ausgeblendet bleiben und insgesamt drei dominante Perspektiven auf Weiterbildungsentscheidungen existieren: eine deskriptive, die Typen und Verlaufsformen formuliert; eine ökonomisch-inspirierte, die betriebswirtschaftliche Konzepte thematisiert und u.a. dadurch normative Positionen beleuchtet sowie eine interaktionelle Perspektive, die die sozialen Aushandlungsprozesse zwischen Individuen im Kontext Unternehmen fokussiert.

Der Forschungsstand zeigt weiterhin, dass kaum ein spezifischer Fokus auf Klein- und Kleinstunternehmen gelegt wird, wenngleich sie gemessen an ihrem Anteil von 96,4 \% an allen Unternehmen (Statistisches Bundesamt 2019) besondere Aufmerksamkeit verdienen. In der deutschen Unternehmenslandschaft kommt kleinen und mittleren Unternehmen (KMU) insgesamt eine doppelte Bedeutung zu: So offenbaren die Unternehmensstrukturstatistiken des Statistischen Bundesamtes einerseits deren hohen Anteil an allen Unternehmen $(99,3 \%)$ (ebd.) und etwa $60 \%$ der über 26 Millionen Erwerbstätigen sind in ihnen beschäftigt (Söllner 2016: 109). Andererseits resultiert ihre wirtschaftliche Bedeutung insbesondere aus ihrem Beschäftigungsbeitrag: KMU generieren überproportional häufig Arbeitsplätze und wirken auch in Krisenzeit stabilisierend auf das Beschäftigungssystem (ebd.: 110).

Für eine Perspektive auf die organisationalen Entscheidungspfade der betrieblichen Weiterbildung besitzen Klein- und Kleinstunternehmen ein weiteres Spezifikum: Entscheidungsträger der betrieblichen Weiterbildung sind die Unternehmensleitungen in Personalunion. Das bedeutet, Entscheidungen können sehr viel direkter und ohne Rücksicht auf Legitimationsinteressen gegenüber Vorgesetzten getroffen werden - können zugleich aber nicht mit Verweis auf Formalisierungen des Unternehmens gegenüber den Mitarbeiterinnen und Mitarbeitern legitimiert werden. Es ist demzufolge anzunehmen, dass diese spezifische Konstellation die Entscheidungsprozesse in KKU von denen in mittleren und Großunternehmen unterscheidet.

\section{Theoretische Perspektiven}

Betriebliche Weiterbildungsentscheidungen als gemeinsamer Nenner der eben dargestellten Untersuchungen können unter Berücksichtigung der drei genann- 
ten dominanten Perspektiven mithilfe von drei theoretischen Perspektiven untersucht werden: Zur Berücksichtigung der Perspektive auf soziale Aushandlungsprozesse nutze ich den kultur-politischen Ansatz von P. Faulstich, zur Berücksichtigung der betriebswirtschaftlichen Logik sowie um normative Positionen erörtern und strukturieren zu können und Strategien von Weiterbildungsentscheidungen zu diskriminieren bietet sich der konfigurationstheoretische Ansatz betrieblicher Weiterbildung von B. Käpplinger an. Zuletzt nutze ich die verhaltenswissenschaftlich orientierte Entscheidungstheorie von James G. March, um die Verlaufsformen betrieblicher Weiterbildungsentscheidungen aus einer entscheidungstheoretischen Perspektive zu beschreiben. Ohne auf diese Ansätze umfassend einzugehen, sollen dennoch im Folgenden kurz die Kernelemente nachgezeichnet werden, um die es in der Betrachtung geht: Faulstich (1998) postulierte ein kultur-politisches Theoriekonzept, das einerseits in der Lage ist, dem unternehmenskulturellen Kontext inklusive seiner ökonomischen Rationalitäten wie auch den individuellen Interessen und Werten der Akteure Rechnung zu tragen (ebd.: 4). Hierfür ist es nötig die politische Dimension im Sinne der Machtkämpfe und Interessenskonflikte der Akteure und die kulturelle Dimension im Sinne ihrer je besonderen sozialen Strukturen zu antizipieren (ebd.: 5). Faulstich wendet sich damit gegen den Ansatz, Unternehmen nur idealtypisch mithilfe ökonomischer Rationalität zu fassen, und versteht stattdessen Interaktions- und Entscheidungsprozesse von Unternehmen vor dem Hintergrund des wechselseitigen Einflusses von informellem und formalem Organisationsaufbau mit den individuellen Interessen der Akteure (Faulstich 1998: 6). An diese Perspektive können für die Untersuchung betrieblicher Weiterbildungsentscheidungen sehr gut weitere (nicht nur) in der Erwachsenenbildung prominente Theorien und Ansätze angeknüpft werden, wie Programmentwicklung und Programmplanung in der Erwachsenenbildung (Fleige et al. 2018) und einige weitere.

Die konfigurationstheoretische Perspektive hilft indes, , ,...] die Heterogenität betrieblicher Weiterbildung und ihre differenten Ausprägungen aufzuzeigen" (Käpplinger 2016: 113) und eine theoretische Differenzierung betrieblicher Weiterbildung zu ermöglichen, die bisher weitestgehend ausblieb. Was vorliegt, sind Unterscheidungen auf der Grundlage politischer Steuerungsgrößen, wie es beispielsweise die KMU sind. Jedoch ist offensichtlich, ,[...] dass ein mittelständisches Unternehmen von zum Beispiel ca. 240 Beschäftigten teilweise mehr strukturelle Ähnlichkeiten mit einem Großunternehmen aufweist als mit einem Mikrounternehmen mit vier Beschäftigten“" (ebd.: 215). Konfiguration bedeutet in diesem Zusammenhang die Verbindung von Organisationsmerkmalen, die Mintzberg (1991: 107f.) als ein Netzwerk verschiedener Variablen versteht, die sich für eine Organisation gegenüber ihrer Umwelt als passend erweisen. Sie sorgen für eine organisationale Konsistenz von Entscheidungen und zielen dabei noch stärker auf Harmonie als auf Perfektion. Käpplinger verfolgt die Idee, beispielsweise die Weiterbildungsbeteiligung 
von Betrieben mittels dieses Ansatzes so strukturiert beschreiben zu können wie es auf der individuellen Ebene durch Konzepte wie Schicht, Milieu und Klasse längst schon möglich ist (Käpplinger 2016: 114ff.). Betriebliche Weiterbildungsentscheidungen sollten demzufolge als Konfigurationen beschreibbar sein.

Zur Beschreibung betrieblicher Weiterbildungsentscheidungen aus einer entscheidungstheoretischen Perspektive, die zugleich aber nicht normativ nach der rationalen Entscheidung in Unternehmen fahndet, sondern sich an die empirischen Realitäten hält und in der Lage ist, Entscheidungshandlungen zu beschreiben, werden verhaltenswissenschaftlich orientierte Konzepte von Entscheidungen in Organisationen herangezogen. Allen voran dienen hier die Arbeiten von James G. March als Zugang. Marchs Ansätze stehen für eine rigorose Abkehr von rationalistischen Entscheidungsparadigmen, die damit einhergehende Steigerung des Einflusses von Intuition, Glaube und Tradition, stellen die Statik von Präferenzordnungen infrage (March 1990: 283) und gehen statt von linearen Entscheidungsprozessen von organisierter Anarchie und der Simultanität von Problemen, Lösungen und Entscheidern aus (ebd.: 14). Ferner berücksichtigt March sich verändernde Zwecke von Unternehmen ebenso wie die Notwendigkeit, von konsistentem Entscheiden abzuweichen, obwohl dies für Unternehmen oft eine wesentliche Legitimationsgrundlage darstellt (ebd.: 283). Anders als anzunehmen wäre, liefert March für die Rekonstruktion betrieblicher Weiterbildungsentscheidungen weder eine Struktur, die sie erfassen, noch ein Instrument, das sie gestalten könnte. Stattdessen wird der Blick auf den Umgang mit Präferenzen sowie Zielsystemen und deren strategischen Einsatz im Unternehmen gelenkt. Es werden ferner verschiedene Rationalitäten der Entscheidungsfindung ebenso wie der Umgang mit Widersprüchen und Inkonsistenzen berücksichtigt.

\section{Eine empirische Aussicht}

Vor dem Hintergrund, dass Pfade betrieblicher Weiterbildungsentscheidungen offensichtlich deskriptiv formuliert werden müssen, normativ-betriebswirtschaftliche Logik nachzuvollziehen haben, den Blick auf die sozialen Aushandlungsprozesse berücksichtigen sollten und es das Ziel ist, über Weiterbildungsentscheidungen in KKU durch Rekonstruktion ebendieser zu informieren, ist ein qualitativer Zugang angemessen. Hierzu nutze ich Experteninterviews mit den Geschäftsführern von KKU mit dem Ziel, das „Betriebswissen“ (Meuser/Nagel 2002: 76) bezogen auf Entscheidungsabläufe und -begründungen zur betrieblichen Weiterbildung zu verstehen. Das Sample besteht aus KKU verschiedener Branchen gemäß der Klassifikation der Wirtschaftszweige 
(Statistisches Bundesamt 2009). Zudem besteht der Anspruch, möglichst unvoreingenommen ${ }^{3}$ in die Analyse des Materials zu gehen und keine Kategorien im Sinne einer deduktiven Analyse anzulegen. Folglich werden die Transkripte in der Philosophie der Grounded Theory (Strauss/Corbin 1996) zunächst offen, anschließend axial und selektiv codiert. Bisher konnten neun Interviews realisiert werden. Wenngleich die Kodierung parallel stattfindet, ist es für die Darstellung erster Ergebnisse aber noch zu früh.

\section{Zusammenfassung}

Sollen betriebliche Weiterbildungsentscheidungen analysiert werden, braucht es den Blick auf die Akteure ebenso wie auf die sozialen Strukturen der Unternehmen. Beides konnte im vorliegenden Beitrag nur sehr rudimentär erfolgen und muss dringend weiter ausgebaut werden. Es lohnt sich, die eruierten Entscheidungsmuster in Konfigurationen mit Blick auf die dargestellten entscheidungstheoretischen Aspekte (Zwecke, Präferenzen etc.) zusammenzufassen, um die innere Logik der Entscheidungshandlungen nachvollziehen zu können. Letztlich könnten auf dieser Grundlage Angebote und Formate betrieblicher Weiterbildung angepasst, Fragen nach Gründen der Nicht-Teilnahme an betrieblicher Weiterbildung aus organisationaler wie sogar aus individueller Perspektive erörtert und die Besonderheiten ebenso wie die Heterogenität von $\mathrm{KKU}$ in Bezug auf Weiterbildung besser verstanden werden.

\section{Literatur}

Arnold, Rolf (1983): Pädagogische Professionalisierung betrieblicher Bildungsarbeit. (Studien zur Erziehungswissenschaft, 17). Frankfurt a.M., Bern u.a.: P. Lang.

Dobischat, Rolf / Düsseldorff, Karl (2013): Betriebliche Weiterbildung in Kleinund Mittelbetrieben (KMU). Forschungsstand, Problemlagen und Handlungserfordernisse. Eine Bilanz. In: WSI Mitteilungen 4, S. 247-254.

3 Soweit dies in Anbetracht der theoretischen Zugänge maximal möglich ist. 
Dummert, Sandra (2018): Betriebliche Weiterbildung. In: Bundesinstitut für Berufsbildung (Hrsg.): Datenreport zum Berufsbildungsbericht 2018. Informationen und Analysen zur Entwicklung der beruflichen Bildung. Bonn: Budrich, S. 348-350.

Faulstich, Peter (1998): Strategien der betrieblichen Weiterbildung. Kompetenz und Organisation. Vahlens Handbücher der Wirtschafts- und Sozialwissenschaften. München: Vahlen.

Fleige, Marion et al. (2018): Programm- und Angebotsentwicklung in der Erwachsenen- und Weiterbildung. UTB Erwachsenenbildung, Weiterbildung, Band 4966. Bielefeld: wbv.

Harney, Klaus (1998): Handlungslogik betrieblicher Weiterbildung. Weiter lernen. Stuttgart: Hirzel.

Heuer, Ulrike (2010): Weiterbildungsentscheidungen: Aushandlungsprozesse und Bildungscontrolling - Handreichung. In: Käpplinger, B. (Hrsg.): Weiterbildungsentscheidungen und Bildungscontrolling. Impulse aus der Bildungsforschung für die Bildungspraxis. Wissenschaftliche Diskussionspapiere aus dem BIBB, Band 117. Bonn: BIBB, S. 18-49.

Hippel, Aiga von / Röbel, Tina (2016): Funktionen als akteursabhängige Zuschreibungen in der Programmplanung betrieblicher Weiterbildung. In: Zeitschrift für Weiterbildungsforschung - Report 1, S. 61-81.

Janssen, Simon/Leber, Ute (2015): Engagement der Betriebe steigt weiter. http://doku.iab.de/kurzber/2015/kb1315.pdf [Zugriff: 31.01.2019].

Käpplinger, Bernd (2009a): Bildungscontrolling: Vor allem in Großbetrieben ein Thema. BIBB-Umfragen von 1997 und 2008 im Vergleich. In: BIBB REPORT 3,13, S. $1-8$.

Käpplinger, Bernd (2009b): Forschungsprojekt. Betriebliche Weiterbildungsentscheidungen: Aushandlungsprozesse und Bildungscontrolling. Abschlussbericht. http://www2.bibb.de/bibbtools/tools/dapro/data/documents/pdf/eb_2220 3.pdf [Zugriff: 31.01.2019].

Käpplinger, Bernd (2010a): Weiterbildungsentscheidungen auf Grundlage von Bildungscontrolling und jenseits von Bildungscontrolling - Empirische Ergebnisse eines BIBB-Forschungsprojektes. In: Käpplinger, B. (Hrsg.): Weiterbildungsentscheidungen und Bildungscontrolling. Impulse aus der Bildungsforschung für die Bildungspraxis. Wissenschaftliche Diskussionspapiere aus dem BIBB, Band 117. Bonn: BIBB, S. 6-17.

Käpplinger, Bernd (Hrsg.) (2010b): Weiterbildungsentscheidungen und Bildungscontrolling. Impulse aus der Bildungsforschung für die Bildungspraxis. Wissenschaftliche Diskussionspapiere aus dem BIBB, Band 117. Bonn: BIBB.

Käpplinger, Bernd (2016): Betriebliche Weiterbildung aus der Perspektive von Konfigurationstheorien. 1. Auflage. Theorie und Praxis der Erwachsenenbildung. Bielefeld: Bertelsmann.

Krekel, Elisabeth M. (2018): Indikatoren zur beruflichen Weiterbildung. In: Bundesinstitut für Berufsbildung (Hrsg.): Datenreport zum Berufsbildungsbericht 2018. Informationen und Analysen zur Entwicklung der beruflichen Bildung. Bonn: Budrich, S. 341. 
Langenheder, Werner (1975): Theorie menschlicher Entscheidungshandlungen. Sozialisation und Kommunikation, Bd. 3. Stuttgart: Ferdinand Enke Verlag.

Lohaus, Daniela / Habermann, Wolfgang (2011): Weiterbildung im Mittelstand. Personalentwicklung und Bildungscontrolling in kleinen und mittleren Unternehmen. München: Oldenbourg.

March, James G. (Hrsg.) (1990): Entscheidung und Organisation. Kritische und konstruktive Beiträge, Entwicklungen und Perspektiven. Wiesbaden: Gabler.

Meuser, Michael / Nagel, Ulrike (2002): ExpertInneninterviews - vielfach erprobt, wenig bedacht. Ein Beitrag zur qualitativen Methodendiskussion. In: Bogner, A. / Littig, B. / Menz, W. (Hrsg.): Das Experteninterview. Theorie, Methode, Anwendung. Opladen: Leske und Budrich, S. 71-93.

Mintzberg, Henry (1991): Mintzberg über Management. Führung und Organisation Mythos und Realität. Wiesbaden: Gabler Verlag.

Röbel, Tina (2017): Bildung im Betrieb? Empirische Betrachtung der Bedarfsbestimmung im Großunternehmen: Prozesse, Akteure und Begründungen. In: Zeitschrift für Weiterbildungsforschung - Report 1, S. 25-39.

Söllner, René (2016): Der deutsche Mittelstand im Zeichen der Globalisierung. In: Statistisches Bundesamt (Hrsg.): WISTA 2/2016. Wirtschaft und Statistik. Neue Ausgabe. Wiesbaden: Statistisches Bundesamt, S. 107-119.

Statistisches Bundesamt (2008): Klassifikation der Wirtschaftszweige. https:// www.destatis.de/DE/Methoden/Klassifikationen/Gueter-Wirtschaftsklassifikationen/Downloads/klassifikation-wZ-2008-3100100089004.pdf?_blob=publicationFile\&v $=5$ [Zugriff: 08.04.2019].

Statistisches Bundesamt (2019): Unternehmensstrukturstatistik. Anteile kleiner und mittlerer Unternehmen an ausgewählten Merkmalen 2015. https://www. destatis.de/DE/ZahlenFakten/GesamtwirtschaftUmwelt/UnternehmenHandwerk/KleineMittlereUnternehmenMittelstand/Tabellen/Insgesamt.html $\quad[\mathrm{Zu}-$ griff: 31.01.2019].

Strauss, Anselm L. / Corbin, Juliet M. (1996): Grounded theory. Grundlagen qualitativer Sozialforschung. Unveränd. Nachdr. Weinheim: Beltz.

Weber, Wolfgang (1994): Betriebliche Bildungsentscheidungen. Entscheidungsverläufe und Entscheidungsergebnisse. Empirische Personal- und Organisationsforschung, Bd. 1. München, Mering: Hampp.

Weil, Markus et al. (2007): Best-Practice-Weiterbildung in KMU. Eine Befragung von Deutschschweizer KMU-Weiterbildungsverantwortlichen zu Strategien, Lernformen und Kooperationen in der Weiterbildung. http://www.weiterbildung-in-kmu.ch/images/pdf/best_practice_wb_kmu.pdf [Zugriff: 31.01.2019]. 
Martin Reuter, Stefan Koscheck, Andreas Martin

\section{Qualitätsmanagement und organisationale Felder in der Weiterbildung}

\section{Qualitätsmanagement im Feld der Weiterbildung}

Die Beschäftigung mit Qualität in der Erwachsenenbildung kann als semantische Erscheinung struktureller Veränderungen im Bildungssystem (Kuper 2002: 536) verstanden werden. Das Thema Qualität ist in der Weiter-/Erwachsenenbildung seit längerem zentral und eng mit dem Thema Professionalisierung verbunden (vgl. Gieseke 1997). Dabei wird die Beschäftigung mit dem Weiterbildungspersonal (vgl. Martin et al. 2016; Dobischat et al. 2018) zunehmend um die Organisationsebene ergänzt. Dass 2017 laut dem wbmonitor 80\% der Einrichtungen ein Qualitätsmanagementsystem (QMS) nutzten (vgl. Ambos et al. 2018: 12), verdeutlicht, dass der Organisation eine zentrale Rolle bei der Qualitätssicherung und -entwicklung von Weiter-/Erwachsenenbildung zugemessen wird. Das Besondere am Qualitätsdiskurs in der Weiterbildung, dessen Ursprung im ökonomischen Sektor lag, ist, dass er bereichsübergreifend geführt (vgl. Schrader 2011: 72) und ein spezifischer Fokus auf die Organisationsebene gelegt wird (Ehses 2016: 151). Dies kann als Suche nach einem „für die Gesamtheit der Bildungsorganisationen geltenden Organisations- und Handlungssystem“ (Meisel 2002: 9f.) gedeutet werden, in dem Kriterienkataloge der Profession, (pädagogische) Evaluationsmethoden und weitere Instrumente zur Qualitätssicherung genutzt werden. Aus erwachsenenpädagogischer Perspektive rückt dabei jedoch die plurale Verfasstheit der Weiterbildung in den Blick. Bisherige Untersuchungen fokussierten spezifische QMS (Hartz 2011), Qualitätsindikatoren (Töpper 2012), QMS aus mikropolitischer Perspektive (Käpplinger 2017), QMS aus machttheoretischer Sicht (Forneck/Wrana 2005: 174) oder die Inhalte der QM-Modelle verbunden mit der Frage nach Steuerung (Aust/Schmidt-Hertha 2012). Der vorliegende Beitrag hingegen nimmt im Anschluss an Schrader (2011) und DiMaggio/Powell (2009) das organisationale Feld als Einflussfaktor auf den Umgang mit der Thematik $\mathrm{QM}^{1}$ in den Blick. Es kann davon ausgegangen werden, dass der Umgang mit der Thematik QM in den jeweiligen Kontexten unterschiedlich ist, da sich Einrichtungen an ihrem organisationalen Feld orientieren. Soll die Erforschung des Themas QMS der Pluralität der Weiterbildung gerecht werden und zu differenzierten Erkenntnissen führen, ist es wichtig, Einrichtungen

1 Ansätze hierzu mit dem Fokus auf LQW finden sich bei Hartz 2011. 
in einem ersten Schritt komplexitätsreduzierend zu systematisieren, damit typische Merkmalskombinationen sichtbar werden. Da die QM-Debatte und Transformationsprozesse in engem Zusammenhang stehen, gilt es diese Aspekte auch gemeinsam zu analysieren.

Daraus leitet sich folgende Forschungsfrage ab: Wie lässt sich der Bereich der organisierten Weiterbildung mehrdimensional und empirisch so klassifizieren, dass die für den Weiterbildungsbereich typische Komplexität deutlich reduziert wird? Im Zentrum stehen dabei Einrichtungsmerkmale, die besonders im Kontext von QMS relevant sind.

Dabei wird der Neo-Institutionalismus (NI) für die theoretische Fundierung und Strukturierung des ,parzellierten Feldes“ (Forneck/Wrana 2005) genutzt. Zur Bestimmung der organisationalen Felder der Weiterbildung wird auf Daten der wbmonitor Umfrage zurückgegriffen. Methodisch wird mithilfe einer Clusteranalyse der Bereich der Weiterbildung empirisch systematisiert. Nach Vorstellung der Cluster gilt es, Unterschiede im Kontext Qualitätsmanagement bzw. der zugrundeliegenden Qualitätsverständnisse zu diskutieren. Im Fazit werden zentrale Rückschlüsse festgehalten und ein Ausblick mit Anschlussmöglichkeiten für weitere Forschungen gegeben.

\section{Theoretische Aspekte im Anschluss an den Neo-Institutionalismus}

Mit dem Neo-Institutionalismus ist ein theoretischer Anschluss gegeben, der sich explizit mit der Schnittstelle Organisation und Gesellschaft befasst. Mit den klassischen Konzepten Legitimität, organisationales Feld und Isomorphie bietet er Erklärungs- und Analysemöglichkeiten für Feldbildungsprozesse in der Weiterbildung. „Im Kern geht es in der soziologischen Variante des NeoInstitutionalismus darum, Organisationen und ihre Strukturen in einem Bedingungsfeld unterschiedlicher Institutionen zu erklären" (Herbrechter/Schemmann 2010: 128).

Institutionen wurden in der neo-institutionalistischen Organisationsforschung von Beginn an eng mit dem beschriebenen Begriff bzw. Konzept der Legitimität verbunden. Zentral ist dabei die entstehende überindividuelle Verhaltensnorm, die in einem bestimmten Kontext als legitim erachtet wird. Entsprechend nutzen Organisationen diese, um ihr Handeln zu legitimieren. So können Institutionen als Schnittstelle zu dem Konzept des organisationalen Feldes gedeutet werden, das als zentrale Analyseeinheit im NI gesehen wird 
(Senge 2005). Kennzeichen des Feldes ${ }^{2}$ sind, dass es nicht nur andere Organisationen mit ähnlichen Leistungen, sondern die Gesamtheit relevanter Akteure (z.B. Regulierungsbehörden) umfasst. Organisationen beobachten ihr organisationales Feld und schließen an dortige Verhaltenserwartungen (Wettbewerb, Staat usw.) an, um ihre Legitimität zu sichern. Dies ist für Organisationen fundamental, da sie sich so ihre vitalen Ressourcen erschließen (Meyer/Rowan 2009). Durch den Anschluss an Verhaltenserwartungen des organisationalen Feldes gleichen sich Organisationen in ihren Strukturen wechselseitig an, was mit dem Konzept der Isomorphie beschrieben wird. ${ }^{3}$

\section{Forschungsdesign und Datengrundlage}

Als Datengrundlage wird auf die wbmonitor Umfrage 2017 zurückgegriffen. Dabei handelt es sich um eine vom Bundesinstitut für Berufsbildung (BIBB) und dem Deutschen Institut für Erwachsenenbildung - Leibniz-Zentrum für Lebenslanges Lernen (DIE) durchgeführte Online-Umfrage unter Anbietern allgemeiner und beruflicher Weiterbildung. Unter Weiterbildung wird dort in Anlehnung an den Deutschen Bildungsrat (1970) ein organisiertes Bildungsangebot verstanden, das sich an ausgebildete oder erfahrene Erwachsene richtet. Dazu gehören auch Fortbildungen, Umschulungen oder Maßnahmen der beruflichen Rehabilitation - ebenso wie Angebote der allgemeinen, politischen oder kulturellen Erwachsenenbildung. Nicht zur Weiterbildung gezählt werden jedoch Ausbildung, berufsvorbereitende Maßnahmen oder Vermittlung in Arbeit. Gemeint ist ein Angebot für externe Personen bzw. Betriebe/Organisationen, nicht deren innerbetriebliche Weiterbildung für die eigenen Mitarbeitenden (vgl. Koscheck/Ohly 2017: 7). Mit ihrer Ausrichtung auf die gesamte Anbieterlandschaft in diesem Bereich (nicht zur Zielgruppe zählen Betriebe, deren Weiterbildungen nicht öffentlich zugänglich sind bzw. für externe Kunden angeboten werden) stellt sie die größte bundesweit regelmäßig durchgeführte Anbieterbefragung dar. ${ }^{4}$ Der Themenschwerpunkt 2017

2 Um die empirische Anschlussmöglichkeit zu gewährleisten, wird an das Konzept des organisationalen Feldes von DiMaggio und Powell (2009) angeschlossen. Zur Übersicht von Weiterführungen des Konzepts vgl. Becker-Ritterspach/Becker-Ritterspach 2006b: 118-136.

3 DiMaggio und Powell (2009) unterschieden dabei Zwang, Norm und Mimese. Zur kritischen Diskussion der Reichweite der Isomorphie vgl. Becker-Ritterspach/Becker-Ritterspach 2006a.

4 Zur konzeptionellen Vertiefung vgl. Koscheck 2010. 
zu Qualitätsmanagementsystemen wurde in Kooperation mit der Justus-Liebig-Universität Gießen entwickelt. An der Umfrage beteiligten sich 1.755 Einrichtungen ( $9,0 \%$ Rücklaufquote).

Die Systematisierung der heterogenen Anbieterlandschaft erfolgt meist deskriptiv anhand bestimmter Differenzierungsmerkmale, beispielsweise zwischen öffentlichem und privatem Sektor bzw. Staat und Markt. Ein theoretisch hergeleitetes, idealtypisches Modell stellen die - ebenfalls an den Neo-Institutionalismus anknüpfenden - Reproduktionskontexte der Weiterbildung von Schrader (2011) dar. Nach DiMaggio und Powell lässt sich jedoch „die Struktur eines organisationalen Feldes [...] nicht a priori bestimmen, sondern muss auf der Grundlage empirischer Untersuchungen definiert werden“ (DiMaggio/Powell 2009: 60). Um dem Rechnung zu tragen, wurde eine Clusteranalyse durchgeführt. Ziel dieses Verfahrens ist es, Untersuchungsobjekte zu Gruppen (Clustern) zusammenzufassen, die im Hinblick auf die betrachteten Merkmale möglichst homogen sind, d.h. möglichst ähnliche Strukturen aufweisen gleichzeitig sollen die Gruppen untereinander möglichst verschieden sein (vgl. Bacher 1996).

Mehrdimensionale Gruppierungen von Einrichtungen der Weiter- bzw. Erwachsenenbildung liegen national beispielsweise von Käpplinger (2007), Herbrechter und Schemmann (2010) oder auch Schrader (2011) vor und konnten wichtige Forschungsbeiträge leisten. Kennzeichen dieser Arbeiten ist die Nutzung von Daten regionaler Einrichtungen. Die vorliegende Systematisierung beansprucht Gültigkeit für den gesamten Weiterbildungsbereich Deutschlands, wobei, wie beschrieben, die betriebliche Weiterbildung nicht erschöpfend berücksichtigt werden kann. Um eine möglichst reliable und reproduzierbare Klassifikation zu erhalten, wurden jeweils nur Merkmale verwendet, welche im zeitlichen Verlauf sehr stabil sind. Unter diesem Gesichtspunkt wurden folgende Merkmale zur Bestimmung der organisationalen Felder genutzt:

- Art der Einrichtung

- Ausrichtung des Angebots auf allgemeine und/oder berufliche Weiterbildung

- Thematische Breite des Angebots (Anzahl der Themenfelder)

- Personalstruktur (Anteil Angestellte (inkl. Beamte) am Gesamtpersonal (Angestellte/Honorarkräfte/Ehrenamtliche))

- Finanzierungsstruktur (Finanzierungsanteile von Teilnehmenden; Betrieben; Arbeitsagenturen bzw. Jobcentern; Gebietskörperschaften (Kommune, Land, Bund, EU); nicht öffentlichen Trägern; Sonstigen) ${ }^{5}$

5 Bezüglich der Finanzierungsstruktur sind - zumindest über einen längeren Zeitraum Veränderungen als wahrscheinlicher anzusehen als bei den anderen aufgeführten Merkmalen. Da Kunden einer Organisation nach DiMaggio und Powell als Bestandteil des organisationalen Feldes sind (vgl. DiMaggio/Powell 2009: 60) und hinsichtlich der Res- 
- Verhältnis Weiterbildungsbereich zu ggf. anderen Geschäfts-/Tätigkeitsfeldern (Anteil Weiterbildung am Gesamtumsatz)

Die aufgeführten Merkmale haben sich in der Bildungsberichterstattung des wbmonitor als zentrale Informationen zur Charakterisierung der organisationalen Heterogenität des Bildungsbereichs Weiterbildung bewährt (vgl. Bundesinstitut für Berufsbildung 2018; 2017; 2016; 2015). Die Berechnung der Clusteranalyse erfolgte nach dem robusten und bewährten hierarchisch-agglomerativen Ward-Verfahren (vgl. Bacher 1996: 158). ${ }^{6}$

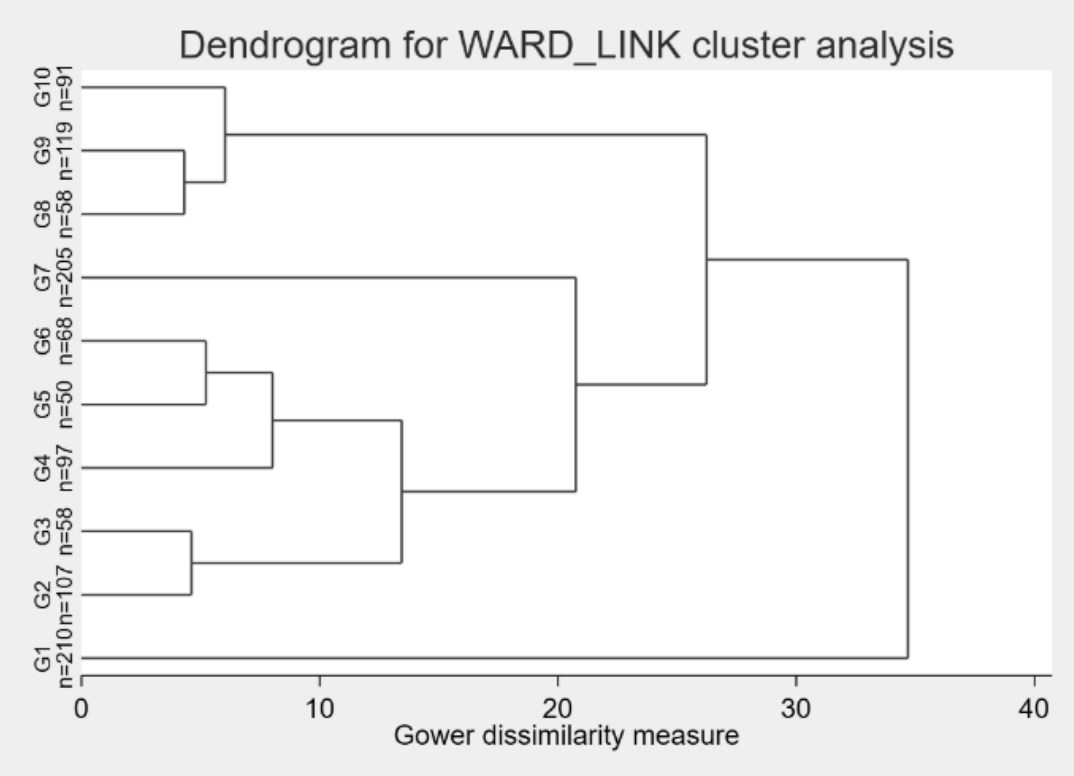

Abbildung 1: Dendrogram-Cluster. Quelle: eigene Berechnung auf Basis der wbmonitor Umfrage 2017; eigene Darstellung

Das Dendrogram visualisiert den Vereinigungsvorgang der Cluster, ausgehend von einer 10-Cluster-Lösung (Abb. 1). Die als optimal anzusehende Clusterzahl wurde statistisch (Abb. 2, Duda-Hart-Index) in Kombination mit der inhaltlichen Plausibilität der auf dieser Basis infrage kommenden Clusterlösungen identifiziert. Kriterium für die Entscheidung der Clusterwahl ist beim

sourcenbeschaffung einer Einrichtung bei der Entscheidung, ein QMS zu implementieren, relevant sein können, war die Berücksichtigung der Finanzierungsstruktur in der Clusteranalyse unverzichtbar.

6 Zuvor wurden mittels des Single-Linkage-Verfahrens Ausreißer identifiziert und aus der Analyse ausgeschlossen (vgl. Backhaus et al 2016: 501). 
Duda-Hart-Index ein möglichst hohes Verhältnis je 2/je 1 bei gleichzeitig möglichst niedrigem pseudo T-squared (vgl. Milligan/Cooper 1985). Damit wären nach statistischen Kriterien sowohl eine drei, vier als auch acht Cluster Lösung möglich. Bezüglich der Überprüfung der Clusterlösung kommen Weidenbeck/Züll zu folgendem Schluss: ,letztendlich bleibt dem Anwender nur sein theoretisches Wissen über mögliche Clusterstrukturen, mit dem er die Ergebnisse validieren kann“ (Weidenbeck/Züll 2010: 547). Damit kommt der sachlogischen Bestimmung der Clusterzahl eine bedeutende Rolle zu (vgl. Backhaus et al. 2016: 494).

\begin{tabular}{|c|c|c|}
\hline \multirow{2}{*}{$\begin{array}{c}|c| \\
\text { Number of }\end{array}$} & \multicolumn{2}{|c|}{ Duda /Hart } \\
clusters & Je (2)/Je (1) & $\begin{array}{c}\text { pseudo } \\
\text { T-squared }\end{array}$ \\
\hline 1 & 0.9394 & 68.39 \\
2 & 0.9653 & 30.62 \\
3 & 0.9800 & 11.91 \\
4 & 0.9718 & 10.98 \\
5 & 0.9222 & 17.98 \\
6 & 0.6758 & 127.60 \\
7 & 0.8886 & 14.54 \\
8 & 0.9689 & 5.23 \\
9 & 0.9499 & 9.23 \\
10 & 0.9552 & 9.53 \\
\hline
\end{tabular}

Abbildung 2: Duda/Hart-Index-Cluster . Quelle: eigene Berechnung auf Basis der wbmonitor Umfrage 2017; eigene Darstellung

Um vor diesem Hintergrund eine Systematisierung zu entwickeln, die neben der Deskription auch anschlussfähig für weitere empirische Analysen sein kann, wurde sich gegen die Acht-Cluster-Lösung aufgrund zu niedriger Fallzahlen pro Cluster entschieden. Besonders vor dem Hintergrund, auch belastbare Ergebnisse bei der Analyse von Merkmalen mit hoher Standardabweichung zu ermöglichen, erschien dies sinnvoll. Um dabei trotzdem möglichst differenziert und inhaltlich angemessen zu bleiben, wurde sich für die Vier- 
Cluster-Lösung entschieden, welche - gegenüber der Drei-Cluster-Lösung die Cluster der Gemeinschaften und gemeinnützig/öffentlichen Einrichtungen unterscheidet. $^{7}$

Das Erfordernis der Zeitstabilität wurde anhand vorliegender Panelfälle bis 2007 rekonstruiert und anhand der Variable „Art der Einrichtung“ geprüft. In der Summe lässt sich das Ergebnis als ausreichend zuverlässig beschreiben. ${ }^{8}$

\section{Das komplexe Feld - Organisationstypen in der Weiterbildung}

Im Ergebnis konstituierten sich die vier organisationalen Felder der Weiterbildung primär nach den dort anzutreffenden Einrichtungstypen, was auch für die Zeitstabilität spricht. Cluster 1 enthält die Volkshochschulen, nur wenige Einrichtungen sind anderer Art. In Cluster 2 befinden sich verschiedene Anbietertypen mit vorrangig gemeinnütziger Ausrichtung sowie staatliche Einrichtungen der beruflichen Weiterbildung und Bildungszentren von Kammern. Cluster 3 besteht vor allem aus Einrichtungen in der Trägerschaft einer Kirche, Partei, Gewerkschaft, eines Verbandes oder Vereins, deren Weiterbildungsangebote vor allem werte- und interessengebunden sind. In Anlehnung an die Reproduktionskontexte (Schrader 2011) wird dieses Cluster als ,Gemeinschaften" bezeichnet. Im Cluster 4 schließlich haben sich die privat kommerziellen Weiterbildungsanbieter gruppiert. Eine detaillierte Beschreibung der Cluster zentrale Strukturmerkmale der vier organisationalen Felder enthält Tabelle 1 kann an dieser Stelle nicht erfolgen.

7 Für Fälle mit fehlenden Werten - die Clusteranalyse setzt vollständige Daten voraus wurde die Clusterzugehörigkeit anschließend mithilfe der linearen und multinominalen Regression imputiert (692 Fälle).

8 Von den nicht imputierten einbezogenen Fällen $(n=1063)$ konnten je nach Jahrgang zwischen 213 (2009) und 549 (2016) Fälle analysiert werden. Dabei lag die Stabilität in diesem Zeitraum - bezogen auf die im Cluster befindlichen Anbietertypen - in Cluster 1 zwischen $98 \%$ bis $100 \%$, in Cluster 2 zwischen $78 \%$ bis $95 \%$, in Cluster 3 zwischen $79 \%$ und $97 \%$ und bei Cluster 4 zwischen $86 \%$ und $100 \%$. 


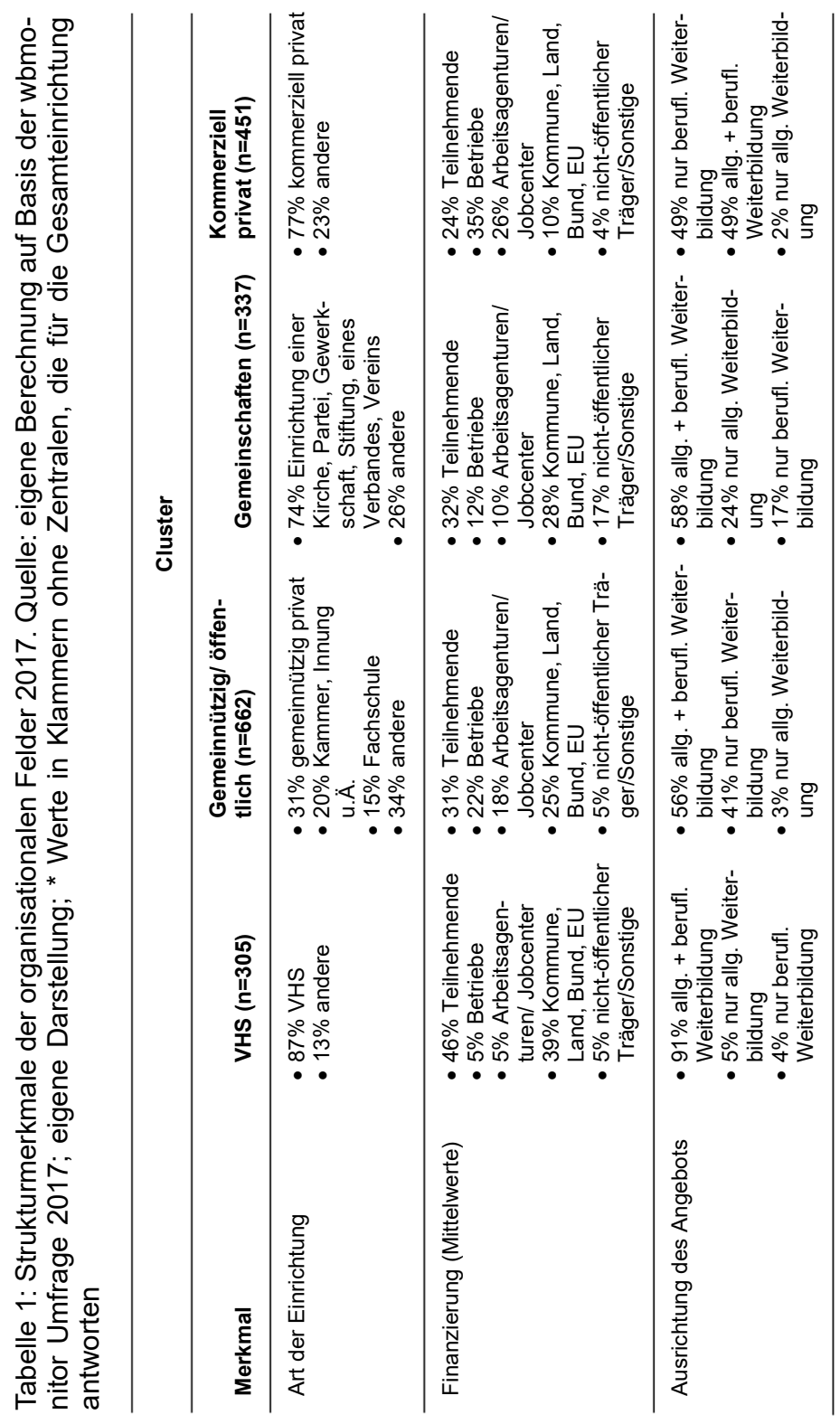




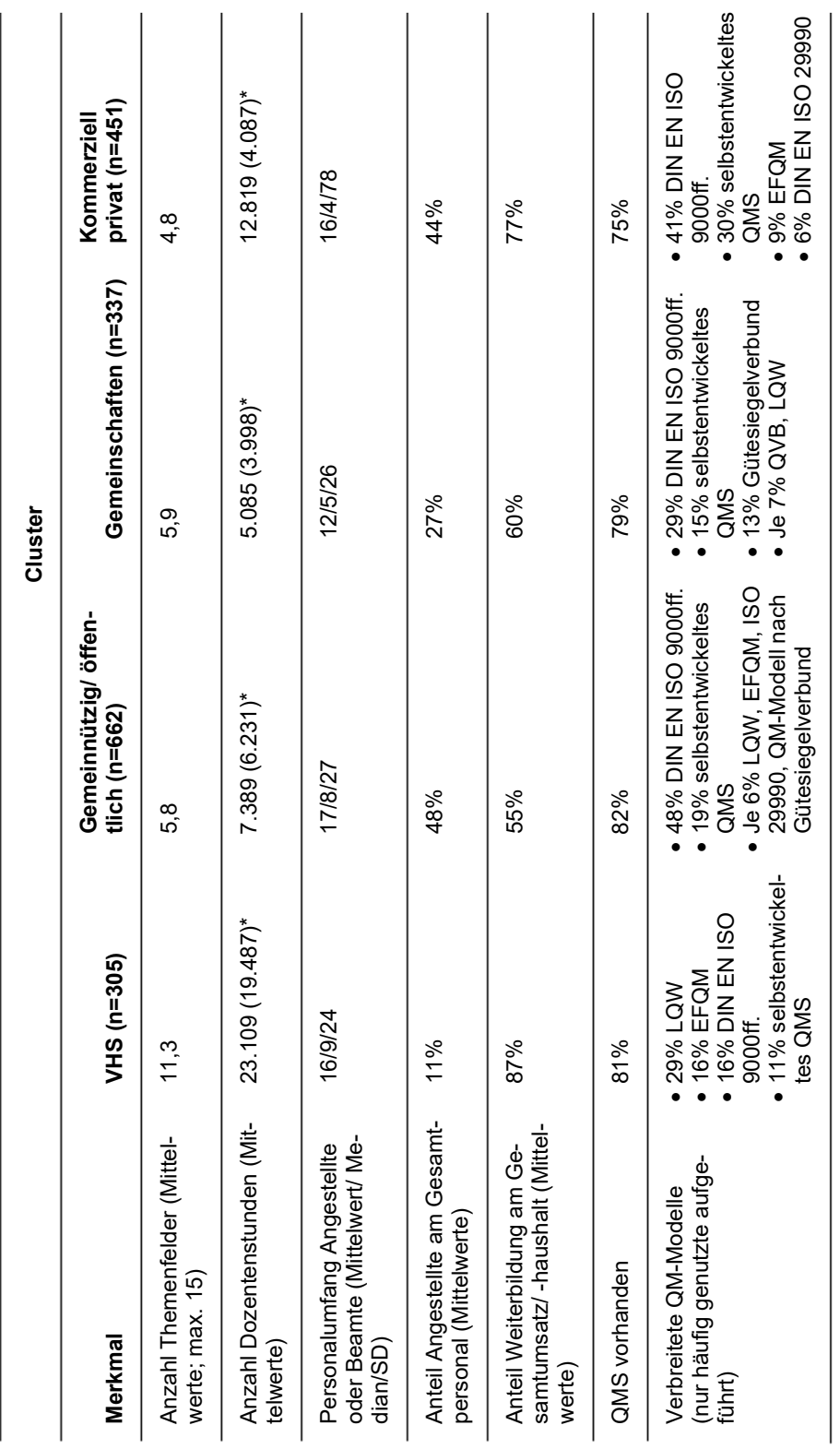


Im Folgenden wird das Potential der Clusteranalyse anhand der Aspekte Komplexität und der Nutzung von QMS aufgezeigt.

Bilden Modelle wie beispielsweise die Reproduktionskontexte von Schrader (2011) Weiterbildungskontexte in idealtypischer Weise ab, so zeigt die empirische Analyse auch Komplexität innerhalb der Cluster auf. Es wird deutlich, dass in allen Clustern Mischformen bei der Finanzierung bestehen, auch wenn einzelne Cluster spezifische Schwerpunkte zeigen. Da den jeweiligen Financiers spezifische Interessen unterstellt werden können, sind Einrichtungen in allen Clustern gefordert, mit unterschiedlichen Interessen umzugehen und vielfältige Koordinationsleistungen zu vollbringen. Dies multipliziert sich, wenn z.B. die Ausdifferenzierung im öffentlich finanzierten Bereich mit in den Blick genommen wird. So kann hier nicht von einem Akteur mit einheitlichen Interessen ausgegangen werden, sondern den Ministerien der Länder im Kontext der allgemeinen Weiterbildung, der Bundesagentur für Arbeit oder dem Bundesamt für Migration und Flüchtlinge können unterschiedliche Interessen und Bildungsziele unterstellt werden. Damit bleibt festzuhalten, dass sich auch innerhalb der Cluster komplexere Strukturen zeigen als idealtypisch angenommen. Als Herausforderung für die Theorienentwicklung kann im Anschluss an diese Befunde die präzise Abbildung und Erklärung der Komplexität, wie im Sinne der Mischfinanzierung und Interessen beschrieben wurde, festgehalten werden.

Hinsichtlich der Nutzung von QMS fällt auf, dass in allen Feldern ein hoher Abdeckungsgrad zu beobachten ist. Unterschiede bestehen jedoch hinsichtlich der genutzten QM-Modelle: ${ }^{9}$ Während im Cluster VHS die Lernerorientierte Qualitätstestierung in der Weiterbildung (LQW) das am häufigsten genutzte QMS ist, hat sich in den anderen drei Clustern die jeweils größte Anbietergruppe für Qualitätsmanagement nach der DIN EN ISO 9000-Normenreihe entschieden. Darüber hinaus fällt auf, dass im Cluster „Kommerziell privat“ mit 26\% der größte Finanzierungsanteil von Arbeitsagenturen und Jobcentern vorliegt und auch mit 30\% der höchste Anteilswert von selbstentwickelten QMS. Einen möglichen Erklärungsansatz bietet dabei die AZAV-Zulassung, die Voraussetzung ist, um im Bereich der Arbeitsmarktförderung tätig werden zu können. In ihr wird ein QMS vorgeschrieben, ohne dass dies zwangsläufig auch zertifiziert sein muss. Entsprechend naheliegend scheint es, den individuellen Ansprüchen entsprechend ein selbstentwickeltes QMS zu nutzen. Ein weiterer Hinweis, der für ein selbstentwickeltes QMS spricht, ist die Größe der Einrichtungen gemessen am Personalumfang. Der Median von 4 Angestellten (bei einem MW von 16 und einer SD von 78) zeigt auf, dass in diesem Cluster viele kleine Einrichtungen vertreten sind, für die ein QMS daher grundsätzlich von eingeschränkterem Nutzen sein dürfte als in größeren und komplexeren Organisationen. Entsprechend scheint die mit 75\% niedrigste Dichte an QMS

9 Für eine Übersicht und einen Vergleich zentraler QMS vgl. Käpplinger/Reuter 2017: 12f. oder Ambos et al. 2018: 17-19. 
plausibel. Vor dem Hintergrund des ökonomischen Ursprungs der QM-Debatte könnte dieser Befund jedoch auch irritieren, da gerade in diesem kommerziell ausgerichteten Cluster eine besonders hohe Verbreitung erwartbar gewesen wäre. Vor dem Hintergrund der geringen durchschnittlichen Themenfelder jedoch, welche Einrichtungen in diesem Cluster anbieten, kann von relativ vielen Nischenanbietern ausgegangen werden, die für sich keinen Bedarf für ein QMS sehen (vgl. auch Ambos et al. 2018: 20), bzw. für die auch kein Erfordernis von Seite der Kunden (Teilnehmende/Betriebe) besteht.

Die mit 29\% besonders große Verbreitung von LQW im Cluster VHS scheint vor dem Hintergrund der bildungspolitischen Forcierung plausibel. Hartz benennt als Ziel des Bund-Länder-Verbundprojekts „Qualitätstestierung in der Weiterbildung“, „LQW bundesweit in Einrichtungen der Weiterbildung einzuführen“" (Hartz 2011: 42). Mit dem Auftrag der Offenheit des Angebots und der Gemeinwohlorientierung (vgl. DVV 2011; Schöll 2005: 42ff.) ist bei Volkshochschulen ein normativer Auftrag gegeben, der gut mit dem in LQW formulierten Ziel ,gelingendes Lernen“ (Zech 2017: 55) zu fördern kompatibel zu sein scheint. Mit 39\% finanziert sich dieser Cluster auch am stärksten durch Mittel von Kommune, Bund, Land und/oder EU, was die Bedeutsamkeit der Bezugnahme auf staatliche Maximen unterstreicht. Trotz der augenscheinlichen Passung von LQW zu den VHS bilanziert Ehses 2016 diesbezüglich eine Entwicklung ,[v]on der Euphorie des Anfangs zu pragmatischen Strategien“, womit auf das Prozesshafte und den stetigen Wandel auch innerhalb des (QM) Diskurses aufmerksam gemacht werden soll.

Damit bleibt festzuhalten, dass sich die eingangs erwähnten heterogenen Ziele von Einrichtungen in spezifischen Umgangsweisen in einem empirisch systematisierten Feld der Weiterbildung mit der Thematik QM aufzeigen lässt. Dabei scheinen komplexe Verhältnisse dahingehend vorzuherrschen, dass der Bereich nicht einfach kompliziert ist, sondern, dass Qualität im Sinne der Organisation sowohl von Programmen als auch der Lehr-Lernprozesse vielschichtig, verflochten und verschlungen sind. Kausales Denken ist demnach durch ein Denken zu ergänzen, das davon ausgeht, dass die Elemente eines Forschungsgegenstandes in komplexe Wechselwirkungen zueinander treten und nicht ohne weiteres kausal erklärt werden können (vgl. Nassehi 2017).

\section{Fazit}

Mit dem wbmonitor konnte eine Datengrundlage genutzt werden, die es ermöglicht organisationale Felder mehrdimensional und relativ zeitstabil zu systematisieren. Dabei konnten Unterschiede bei der verwendeten Art des QMS 
gezeigt, clusterspezifische Einflüsse skizziert und als Herausforderungen für die Theorieentwicklung benannt werden.

Im Anschluss an diese Befunde ergeben sich damit folgende Forschungsperspektiven:

Die hier benannten ersten Thesen zur Erklärung der Clusterdifferenzen im Kontext QM gilt es weiter kritisch zu analysieren, sowohl methodisch im Sinne einer erweiterten Gültigkeitsprüfung der Cluster (Bacher et al. 2010: 493ff.), als auch inhaltlich. Hierzu liegt eine Möglichkeit im Anschluss an den NI in der Rekonstruktion von Isomorphismen (Zwang, Norm, Mimese: DiMaggio/Powell 2009), die zur Implementierung aber auch zur Aufrechterhaltung des QMS geführt haben könnten. Eine weitere Anschlussmöglichkeit liegt darin, die Diffusionsprozesse der QMS in die Weiterbildung zu rekonstruieren. Um hier möglichst belastbare Ergebnisse zu produzieren, bietet sich methodisch die Nutzung der Ereignisdatenanalyse an. Weiterhin scheint die Berücksichtigung des Konzepts der „losen Kopplung“ (Weick 2009) vielversprechend. So wäre hier der Frage nach differenten Umgangsweisen zwischen einer formalen oder aktiven Integration nachzugehen. Ansatzpunkt hierzu kann in Wirksamkeitszuschreibungen gesehen werden (Hartz 2015). Dabei scheint die Frage zentral, ob sich die Wirkungen eher auf die Feldzugehörigkeit oder auf das jeweils vorhandene QMS zurückführen lassen.

\section{Literatur}

Ambos, Ingrid / Koscheck, Stefan / Martin, Andreas / Reuter, Martin (2018): Qualitätsmanagementsystem in der Weiterbildung. Ergebnisse der wbmonitor Umfrage 2017. Bonn: BIBB. https://www.die-bonn.de/doks/2018-qualitaetsmanagement-01.pdf [Zugriff: 17.01.2019].

Aust, Kirsten / Schmidt-Hertha, Bernhard (2012): Qualitätsmanagement als Steuerungsinstrument im Weiterbildungsbereich. In: REPORT Zeitschrift für Weiterbildungsforschung, 2/2012, S. 43-55. https://www.die-bonn.de/doks/report/ 2012-Weiterbildungspolitik-01.pdf [Zugriff: 29.01.2019].

Bacher, Johann (1996): Clusteranalyse. München/Wien: Oldenbourg.

Bacher, Johann / Pöge, Andreas / Wenzig, Knut (2010): Clusteranalyse. Anwendungsorientierte Einführung in Klassifikationsverfahren. 3., erg., vollst. überarb. u. neu gest. Aufl. München: Oldenbourg Wissenschaftsverlag.

Backhaus, Klaus / Erichson, Bernd / Plinke, Wulff / Weiber, Rolf (2016): Multivariate Analysemethoden. Eine anwendungsorientierte Einführung. 14. überarb. u. akt. Aufl. Berlin, Heidelberg: Springer Gabler.

Becker-Ritterspach, Florian / Becker-Ritterspach Jutta (2006a): Isomorphie und Entkopplung im Neo-Institutionalismus. In: Senge, Konstanze / Hellmann, Kai- 
Uwe (Hrsg.): Einführung in den Neo-Institutionalismus. Berlin: VS, S. 102-117.

Becker-Ritterspach, Jutta / Becker-Ritterspach, Florian (2006b): Organisationales Feld und Gesellschaftlicher Sektor im Neo-Institutionalismus. In: Senge, Konstanze / Hellmann, Kai-Uwe (Hrsg.): Einführung in den Neo-Institutionalismus. Berlin: VS, S. 118-136.

Bundesinstitut für Berufsbildung (Hrsg.) (2015/2016/2017/2018): Datenreport zum Berufsbildungsbericht 2015/2016/2017/2018. Informationen und Analysen zur Entwicklung der beruflichen Bildung. Bonn.

DiMaggio, Paul J. / Powell, Walter W. (2009): Das „,stahlharte Gehäuse“ neu betrachtet: Institutionelle Isomorphie und kollektive Rationalität in organisationalen Feldern. In: Koch, Sascha / Schemmann, Michael (Hrsg.): Neo-Institutionalismus in der Erziehungswissenschaft. Wiesbaden: VS, S. 57-84.

Dobischat, Rolf / Elias, Arne / Rosendahl, Anna (Hrsg.) (2018): Das Personal in der Weiterbildung. Im Spannungsfeld von Professionsanspruch und Beschäftigungsrealität. Wiesbaden: Springer VS.

DVV - Deutscher Volkshochschul-Verband e.V. (Hrsg.) (2011): Die Volkshochschule - Bildung in öffentlicher Verantwortung. Bonn.

Ehses, Christiane (2016): Von der Euphorie des Anfangs zu pragmatischen Strategien. Bilanz der langjährigen Qualitätsentwicklung der hessischen Volkshochschulen im Kontext von LQW. In: Hessische Blätter für Volksbildung 02/2016, S. $150-160$.

Forneck, Hermann-Joseph / Wrana, Daniel (2005): Ein parzelliertes Feld: eine Einführung in die Erwachsenenbildung. Bielefeld: Bertelsmann.

Gieseke, Wiltrud (1997): Die Qualitätsdiskussion aus erwachsenenpädagogischer Sicht. Was bedeutet Qualität als Erwachsenenpädagoge? In: Arnold, Rolf (Hrsg.): Qualitätssicherung in der Erwachsenenbildung. Opladen: Leske + Budrich, S. 29-47.

Hartz, Stefanie (2011): Qualität in Organisationen der Weiterbildung. Eine Studie zur Akzeptanz und Wirkung von LQW. Wiesbaden: VS.

Hartz, Stefanie (2015): Wirksamkeitszuschreibungen von Weiterbildungseinrichtungen zur Lernerorientierten Qualitätstestierung. In: Zeitschrift für Erziehungswissenschaft, 18(2), S. 303-325.

Herbrechter, Dörthe / Schemmann, Michael (2010): Organisationstypen in der Weiterbildung. Eine empirische Analyse aus der Perspektive des Neo-Institutionalismus. In: Dollhausen, Karin / Feld, Timm C. / Seitter, Wolfgang (Hrsg.): Erwachsenenpädagogische Organisationsforschung. Wiesbaden: VS, S. $125-141$.

Käpplinger, Bernd (2007): Abschlüsse und Zertifikate in der Weiterbildung. Bielefeld: Bertelsmann.

Käpplinger, Bernd (2017): Qualitätsentwicklung in der Weiterbildung: Fluch oder Segen? Eine mikropolitische Analyse. In: Bolder, Axel / Bremer, Helmut / Epping, Rudolf (Hrsg.): Bildung für Arbeit unter neuer Steuerung. Wiesbaden: VS, S. $167-184$. 
Käpplinger, Bernd / Reuter, Martin (2017): Qualitätsmanagement in der Weiterbildung. In: Wiso Diskurs 15/2017. http://library.fes.de/pdf-files/wiso/ 13905.pdf [Zugriff: 23.01.2019].

Koscheck, Stefan (2010): wbmonitor 2007-2009. BIBB-FDZ Daten- und Methodenbericht Nr. 4/2010. Bonn. https://metadaten.bibb.de/download/642 [Zugriff: 17.01.2019].

Koscheck, Stefan / Ohly, Hana (2017): wbmonitor 2016. BIBB-FDZ Daten- und Methodenbericht 4/2017. Bonn. https://www.bibb.de/veroeffentlichungen/de/ publication/download/8509 [Zugriff: 01.02.2019].

Kuper, Harm (2002): Stichwort: Qualität im Bildungssystem. In: Zeitschrift für Erziehungswissenschaft Volume 5, Issue 4, S. 533-551.

Martin, Andreas / Lencer, Stefanie / Schrader, Josef / Koscheck, Stefan / Ohly, Hana / Dobischat, Rolf / Elias, Arne / Rosendahl, Anna (2016): Das Personal in der Weiterbildung. Arbeits- und Beschäftigungsbedingungen, Qualifikationen, Einstellungen zu Arbeit und Beruf. Bielefeld: wbv. https://www.diebonn.de/doks/2017-weiterbildner-01.pdf [Zugriff: 07.02.2019].

Meisel, Klaus (2002): Qualitätsentwicklung im Aufbruch. In: Heinold-Krug, E. / Meisel, Klaus (Hrsg.): Qualität entwickeln - Weiterbildung gestalten. Handlungsfelder der Qualitätsentwicklung. Bielefeld: Bertelsmann, S. 9-19.

Meyer, John / Rowan, Brian (2009): Institutionalisierte Organisationen. Formale Struktur als Mythos und Zeremonie. In: Koch, Sascha / Schemmann, Michael (Hrsg.): Neo-Institutionalismus in der Erziehungswissenschaft. Grundlegende Texte und empirische Studien. Wiesbaden: VS, S. 28-56.

Milligan, Glenn W. / Cooper, Martha C. (1985): An examination of procedures for determining the number of clusters in a data set. In: Psychometrika, Vol. 50, No. 2, 159-179.

Nassehi, Armin (2017): Die sieben Paradoxien modener Gesellschaften. Paradox 17. https://www.youtube.com/watch?v=R16FYluOoBE [Zugriff: 31.01.2019].

Schöll, Ingrid (2005): Marketing in der öffentlichen Weiterbildung. Bielefeld: wbv.

Schrader, Josef (2011): Struktur und Wandel der Weiterbildung. Bielefeld: wbv.

Senge, Konstanze (2005): Der Neo-Institutionalismus als Kritik der ökonomistischen Perspektive. Dissertation. TU Darmstadt, Fachbereich Gesellschafts- und Geschichtswissenschaften. http://elib.tu-darmstadt.de/diss/000620/ [Zugriff: 23.01.2019].

Töpper, Alfred (2012): Ausgangslage und Qualitätsbegriff. In: Töpper, Alfred (Hrsg.): Qualität von Weiterbildungsmaßnahmen. Einflussfaktoren und Qualitätsmanagement im Spiegel empirischer Befunde. Bielefeld: wbv, S. 11-16.

Weick, Karl E. (2009): Bildungsorganisationen als lose gekoppelte Systeme. In: Koch, Sascha / Schemmann, Michael (Hrsg.): Neo-Institutionalismus in der Erziehungswissenschaft. Wiesbaden: VS, S. 85-109.

Weidenbeck, Michael / Züll, Cornelia (2010): Clusteranalyse. In: Wolf, Christof / Best, Henning (Hrsg.): Handbuch der sozialwissenschaftlichen Datenanalyse. Wiesbaden: Springer VS. S. 525-552. 
Zech, Rainer (2017): Lernerorientierte Qualitätsentwicklung in der Weiterbildung. Leitfaden für die Praxis Modellversion 3. 6. korrigierte Auflage Januar 2017. Hannover: artset. http://www.qualitaets-portal.de/wp-content/uploads/LQW-3Leitfaden-201701.pdf [Zugriff: 23.01.2019]. 

Lisa Lorenz, Eike Asche

\section{Zwischen Fluidität und Kontinuität - Planung von Bildungsangeboten für die Digitalisierung}

\section{Einleitung}

Der Prozess der Digitalisierung vollzieht sich in unserem Verständnis weniger disruptiv, sondern als evolutionärer, kontinuierlicher Wandel, der jedoch tiefgreifende Veränderungspotenziale für einzelne Tätigkeitsprofile und Berufsgruppen sowie ganze Branchen in sich birgt (Brandt, Polom \& Danneberg 2016, Dengler \& Matthes 2015, 2018). Es entstehen veränderte Bildungsbedarfe und -bedürfnisse, die in der Programmplanung interpretativ zu erschließen und in institutionalisierte Bildungsangebote u.a. zur beruflichen Weiterbildung zu transferieren sind, um den Lernenden sowie Adressatinnen und Adressaten eine aktive Auseinandersetzung mit neuen beruflichen Anforderungen und eine individuelle berufliche Entwicklung zu ermöglichen (Gieseke 2003, 2008, Siebert 2009). Denn neben informellen Aneignungsformen braucht Lernen „,verlässliche Angebote zu zentralen Wissensbereichen“ (Gieseke 2003: 209).

Der digitale Wandel wird auf politischer Ebene unter anderem durch das Aufsetzen von Förderprogrammen forciert. Darüber sollen kleine und mittlere Unternehmen (KMU) befähigt werden, die Potenziale der Digitalisierung für sich zu nutzen (BMWi 2015). In diesem Kontext verorten sich die Mittelstand 4.0-Kompetenzzentren, die vom Bundesministerium für Wirtschaft und Energie seit Ende 2015 projektförmig ${ }^{1}$ gefördert werden.

Über die öffentliche Finanzierung werden eigene institutionelle Gebilde geschaffen, deren Dienstleistungen für KMU auch die berufliche Weiterbildung umfassen. Erwachsenenbildung wird damit beigeordnet (Börjesson \& Zimmermann 2005, Gieseke \& Opelt 2005), um bei den Unternehmen eine wirtschaftlich orientierte Organisationsentwicklung u.a. über die Veränderung von Prozessen und Strukturen oder die Erschließung neuer Geschäftsfelder zu initiieren. Hierdurch findet eine „Indienstnahme“ der Erwachsenenbildung statt, die nicht per se zu verurteilen, aber kritisch zu reflektieren und mit pädagogisch-didaktischen Ansätzen und Überlegungen in Einklang zu bringen ist (Siebert 2009). Im hier beschriebenen Anwendungsfall deutet die geschaffene

1 Insgesamt bestehen 25 Kompetenzzentren (Stand: 04/19) mit differierenden thematischen Schwerpunkten in einem bundesweiten, flächendeckenden Netzwerk. 
Position der ,,pädagogisch-didaktischen Begleitung“2 darauf hin, dass bei der Konzipierung und Durchführung beruflicher Weiterbildungsangebote explizit auch erwachsenenpädagogische Fragestellungen berücksichtigt bzw. bearbeitet werden.

Im vorliegenden Beitrag gibt die Gründung des Mittelstand 4.0-Kompetenzzentrums Hannover ${ }^{3}$ den Anlass, ein für die Erwachsenenbildung zentrales Thema in der aktuellen digitalen Transformation zu untersuchen: das Verhältnis von Fluidität und Kontinuität von Planungsprozessen in einem wandlungsintensiven Inhaltsbereich wie der Digitalisierung. Hierfür wird exemplarisch die im Programm der Falleinrichtung erkennbare Nutzung von Planungsstrategien im Zeitverlauf von zwei Jahren analysiert.

\section{Planungsstrategien in der Digitalisierung}

Zur Gestaltung von Bildungsangeboten und -programmen agieren Planende unter dem Leitmotiv der Teilnehmerorientierung in einem Feld, das durch verschiedene Akteure und Interessen geprägt ist. Sie handeln kommunikativ die verschiedenen Positionen aus und beziehen sich flexibel auf pädagogische und interdisziplinäre Wissensressourcen (Gieseke 2008, 2015). Die Bildungsangebote werden entsprechend antizipierter und erschlossener Bedarfe geplant sowie inhaltsbereichs- und einrichtungsspezifisch zu einem Programm zusammengestellt (Gieseke 2003, 2015, Schlutz 2006). Somit kommt der Bedarfsanalyse im Prozess der Programmplanung eine besondere Bedeutung zu.

(Weiter-)Bildungsbedarfe sind zu verstehen als Lernerfordernisse, die sich aus der Diskrepanz zwischen benötigten bzw. wünschenswerten Kompetenzen (Soll) und bereits vorhandenen Kompetenzen (Ist) ergeben (Schlutz 2006) und in verschiedene Bedarfsdimensionen differenziert werden können. Im regionalen Kontext sind aus Sicht eines Bildungsanbieters Bedarfe auf individueller (z.B. Einzelkunde) oder institutioneller Ebene (z.B. im Unternehmen) relevant, die aktuell vorhanden sind oder zukünftig (d.h. prospektiv) zum Tragen kommen (Merk 1998). Daneben existiert der politische/gesellschaftliche Be-

2 Die Stelle ,pädagogisch-didaktische Begleitung“ ist mit Personen besetzt, die über ein erwachsenenpädagogisches Studium verfügen. Diese können auf wissenschaftliches Wissen zu den von Programmplanung betroffenen Themengebieten zurückgreifen und Planungsentscheidungen in ihren komplexen Bezügen wahrnehmen bzw. begründen (Gieseke 2003, 2015, 2018, Gieseke \& Hippel 2018).

3 Das Zentrum ist angesiedelt an der Leibniz Universität Hannover. Die pädagogische Begleitung obliegt dem Institut für Berufspädagogik und Erwachsenenbildung (IfBE) unter der Leitung von Prof. Dr. Steffi Robak. 
darf, der als Ergänzung der individuellen und institutionellen Bedarfe zu verstehen ist und sich u.a. in einer politischen Willensbildung ausdrückt (Ortner 1981, Bardeleben et al. 1990). Die Analyse von Bildungsbedarfen ist aufgrund der immanenten Vielschichtigkeit ein mehrschrittiger, oft kontinuierlicher Prozess, bei dem Weckung und Ermittlung des Bedarfs mitunter fließend ineinander übergehen (Müller \& Stürzl 1992, Gieseke 2008). Schlutz spricht daher auch von „Bedarfserschließung“ (Schlutz 2006: 38).

Um eruierte oder antizipierte Bedarfe in Bildungsangebote zu überführen, greifen Planende auf verschiedene Planungsstrategien zurück. Diese können als übergreifende Entscheidungsmuster über Bildungsangebote verstanden werden, mit denen Planende das Bildungsprogramm (weiter-)entwickeln (Lorenz 2019). Sie lassen sich institutionen- und fachbereichsübergreifend in Neuentwicklung, Fortschreibung und Streichung unterteilen (Pohlmann 2018). Bei der Neuentwicklung werden neue Bildungsangebote gemeinsam mit Dozierenden, Kooperationspartnern und Teilnehmenden geplant, die fortgeschrieben und im nächsten Halbjahr weiter angeboten werden, sofern weiterhin Nachfrage besteht oder es sich um ein für den Bildungsauftrag der Einrichtung relevantes Thema handelt. Die Fortschreibung kann entweder mit demselben Angebot erfolgen oder eine Anpassung/Modifikation beispielsweise der Veranstaltungsdauer beinhalten. Schließlich verschwinden Angebote, die über längere Zeit nicht (mehr) nachgefragt werden, über die Streichung aus dem Programm (ebd.).

Die Planungsstrategien sind eng mit den Strategien zur BedarfserschlieBung verbunden, die auch als „Bedarfsstrategien“ (Schlutz 2006: 45, Herv.i.O.) bezeichnet werden und die Wechselbeziehung von Angebot und Nachfrage unterstreichen:

- Bei der angebotsorientierten Vorgehensweise wird das Angebot entsprechend antizipierter Bedarfe und ausgehend von Bedarfshypothesen für eine breite und offene Zielgruppe erstellt. Über beidseitige Suchbewegungen und flankierende Maßnahmen stößt das Angebot auf Nachfrage - oder auch nicht.

- Die nachfrageorientierte Vorgehensweise kommt zur Anwendung, wenn die Zielgruppe relativ klar einzugrenzen ist. Der Angebotserstellung ist eine dezidierte Bedarfserkundung vorgeschaltet (Gieseke 2008, Schlutz 2006).

In der Praxis sind die Bedarfsstrategien nicht klar zu trennen. Es sind zwar Schwerpunkte im jeweiligen Vorgehen zu erkennen, die sich jedoch gegenseitig durchdringen (Schlutz 2006).

Will man die Programmplanung des Kompetenzzentrums Hannover näher betrachten, so bedarf es der stärkeren Herausarbeitung von dessen Kontext. Dieser wird 1) durch die Schnittstelle der wissenschaftlichen und beruflichen 
Weiterbildung, 2) durch die Digitalisierung als inhaltlicher Schwerpunkt des Programms und 3) durch die Institutionalform ,Einrichtung der beigeordneten Bildung' charakterisiert.

Die betrachtete Institution basiert auf interorganisationaler Kooperation (Tippelt \& Kadera 2016), bei der Partner aus Universitäten, Hochschulen und Forschungsinstituten interdisziplinär zusammenarbeiten, um u.a. Bildungsangebote zu offerieren. Diese Angebote lassen sich an der Schnittstelle zwischen beruflicher und wissenschaftlicher ${ }^{4}$ Weiterbildung verorten, wobei Beruf und Beruflichkeit im Sinne einer beruflichen Handlungskompetenz (Dehnbostel 2008) Bezugspunkte der beruflichen Weiterbildung darstellen (Arnold \& Pätzold 2011). Die Teilnehmenden qualifizieren sich für die Digitalisierung überwiegend aus beruflichen Motiven sowohl während der Arbeits- als auch in der Freizeit. Im Kompetenzzentrum liegen betriebliche Weiterbildung (während der Arbeitszeit mit Kostenübernahme durch den Arbeitgeber) und individuelle berufsbezogene Weiterbildung (nicht-betriebliche Weiterbildung aus beruflichen Gründen) (Bilger \& Kuper 2012) vor.

Der Megatrend Digitalisierung beeinflusst die Programm- und Angebotsplanung inhaltlich und/oder methodisch. Über den verstärkten Einsatz von Technologien am Arbeitsplatz verändern sich zum einen Bildungsbedarfe und damit Lerninhalte bzw. Themen von Bildungsangeboten. Beim hier betrachteten Fallbeispiel stellt Digitalisierung das Leitmotiv dar und wirkt primär inhaltlich auf die Erstellung des Programms, mit dem digitalisierungsspezifische Weiterbildungsangebote für Fach- und Führungskräfte primär aus Unternehmen des produzierenden Gewerbes offeriert werden.

Digitalisierung wird für den produzierenden Bereich unter Industrie 4.0 gefasst: „Objekte, Maschinen, Geräte werden intelligent, nutzen das Internet der Dinge und kommunizieren mit Menschen auf natürliche Weise" (Reinhart 2017: VII). Die treibenden Technologien werden in immer kürzeren Innovationszyklen weiterentwickelt, ihre Leistungsfähigkeit nimmt zu und die Automatisierung beschleunigt sich (ebd.). Die damit einhergehenden Anforderungen an das Verständnis einzelner Technologien, ihrer Verknüpfung untereinander und nicht zuletzt deren Auswirkungen auf die Arbeitsprozesse führen in ihrer Gesamtheit zu einer steigenden Komplexität auf Seiten der Beteiligten und wandelnden Qualifikationsanforderungen (Brandt et al. 2016). Die kurzen Innovationszyklen der Digitalisierung können zu fluiden Bildungsbedarfen führen, die sich dynamisch ändern und in der Programmplanung aufzunehmen sind.

4 Wir fassen wissenschaftliche Weiterbildung als Teilbereich der beruflichen Weiterbildung (Dehnbostel 2008), die sich daraus ableitet, dass die Bildungsangebote wesentlich von wissenschaftlichen Mitarbeitenden geplant und durchgeführt werden, die an universitären bzw. forschungsnahen Einrichtungen tätig sind (Wolter 2011). 
Die angedeutete Fluidität wird unterstrichen durch die Institutionalform der beigeordneten Bildung, bei der Institutionen Weiterbildungsangebote offerieren, ohne sich selbst als Bildungseinrichtung zu verstehen. Bildung wird in diesen beigeordnet zur Unterstützung des Hauptgeschäfts angeboten (Gieseke \& Opelt 2005). Das Kompetenzzentrum Hannover kann als Einrichtung der beigeordneten Bildung betrachtet werden, da Bildungsangebote nur einen Aspekt des Unterstützungsspektrums für Unternehmen darstellen, die neben anderen Dienstleistungen wie Firmengesprächen und Demonstrationen offeriert werden.

Analysen zur Planung in der beigeordneten Bildung aus dem kulturellen Bereich deuten darauf hin, dass eine Differenz zwischen der Kontinuität im Programm von Erwachsenenbildungseinrichtungen und den wandelnden als „fluide“ gekennzeichneten Angeboten der Einrichtungen beigeordneter Bildung besteht (Börjesson \& Zimmermann 2005, Gieseke \& Opelt 2005). Diese Fluidität entsteht einerseits durch die Kontingenz von Bildung in den Einrichtungen, da sie nicht das primäre Organisationsziel darstellt, und andererseits aus den oftmals nicht ausreichend gesicherten finanziellen Ressourcen zur Durchführung der Angebote (Börjesson \& Zimmermann 2005, Gieseke \& Heuer 2011). Befunde zur betrieblichen Weiterbildung in Großunternehmen, die ebenfalls der beigeordneten Bildung zuzuordnen sind, zeigen jedoch auch ein Programmplanungshandeln unter Aushandlung verschiedener subjektiv zugeschriebener Funktionen von Weiterbildung (Hippel 2016, Hippel \& Röbel 2016). Diese Aushandlungsprozesse münden in unterschiedliche Programmarten wie z.B. dem Programmkatalog (ebd.) und verweisen damit auf eine kontinuierliche Bildungsarbeit. Vor diesem Hintergrund wird das Verhältnis zwischen Kontinuität und Fluidität in der Digitalisierung erklärungsbedürftig und zur erkenntnisleitenden Perspektive, unter der das Programm in der vorliegenden Ausarbeitung analysiert wird.

\section{Methodisches Vorgehen}

Um zu klären, inwieweit die Programm- und Angebotsplanung des Kompetenzzentrums eher fluide oder von einer Kontinuität geprägt ist, werden die Planungsstrategien der Neuentwicklung, Fortschreibung und Streichung im Zeitverlauf nachvollzogen. Hierfür wird auf alle Programme seit Bestehen des Zentrums zurückgegriffen, die den Zeitraum von zwei Jahren (08/16-07/18) abbilden. Dieses Vorgehen wird gewählt, da das „Programm [...] der Spiegel einer Bildungsorganisation oder -institution [ist]. Es gibt Aufschluss über einen Wandel und/oder eine Kontinuität, die durch Forschung zugänglich, benennbar und erklärbar werden" (Gieseke 2015: 170). Dies gilt gleichermaßen 
für Einrichtungen der beigeordneten Bildung, wenn die Akteure Bildungsangebote in Programmstrukturen konzipieren.

Folgende Forschungsfragen sind Gegenstand der Untersuchung:

1. Welche Planungsstrategien werden im Zeitverlauf sichtbar?

2. Wie werden Angebot und Nachfrage aufeinander bezogen?

Zur Beantwortung der Forschungsfragen wurde eine organisationsbezogene „qualitativ-quantifizierende Programmanalys[e]“ (Käpplinger \& Robak 2018: 68) durchgeführt. Die Programmanalyse stellt eine genuin erwachsenenpädagogische Erhebungs- und Auswertungsmethode dar (Käpplinger 2008), die methodologisch betrachtet eine spezielle Form der Dokumentenanalyse und damit ein non-reaktives Verfahren ist (Hoffmann 2012), mit dem die Ergebnisse des planerischen Handelns als Ausschnitte der Weiterbildungspraxis untersuchet werden (Käpplinger \& Robak 2018). Die Angebote wurden im Rahmen der Programmanalyse entlang der Kategorien Themen, Inhaltsbereiche und Veranstaltungsdauer klassifiziert und quantitativ analysiert (ebd.). Weiterhin wurden die Planungsstrategien über den Nachvollzug und Abgleich von Angeboten zwischen den einzelnen Programmen herausgearbeitet.

Da im Rahmen der Ausarbeitung auch auf die Teilnahmestatistiken der Angebote zugegriffen werden konnte, war es ebenfalls möglich, Angebot und Nachfrage aufeinander zu beziehen. Hierdurch wird die vielfach beschriebene kritische Einschränkung der Programmanalyse, sie ließe keine Aussagen über die tatsächliche Durchführung der analysierten Angebote zu (Käpplinger 2008, Nolda 2011), aufgehoben.

\section{Zwischen Fluidität und Kontinuität - Ergebnisse der Programmanalyse}

Das analysierte Programm besteht zum 30.06.2018 aus insgesamt 25 Präsenzveranstaltungen, die sich rund um das Thema Digitalisierung und Industrie 4.0 gruppieren. Es sind vor allem kurze Veranstaltungsformate, von denen ca. $3 / 4$ eintägig (19/25) und knapp 1/4 Zweitägig (6/25) angelegt sind. Im Programm existiert eine Basisschulung (,Generalschulung“), in der Grundlagen der Digitalisierung im Produktionsbereich sowie Potenziale für Produktionsprozesse thematisiert werden. Neben der Basisschulung finden sich weitere 24 Themen, die drei Inhaltsbereichen zugeordnet sind. 


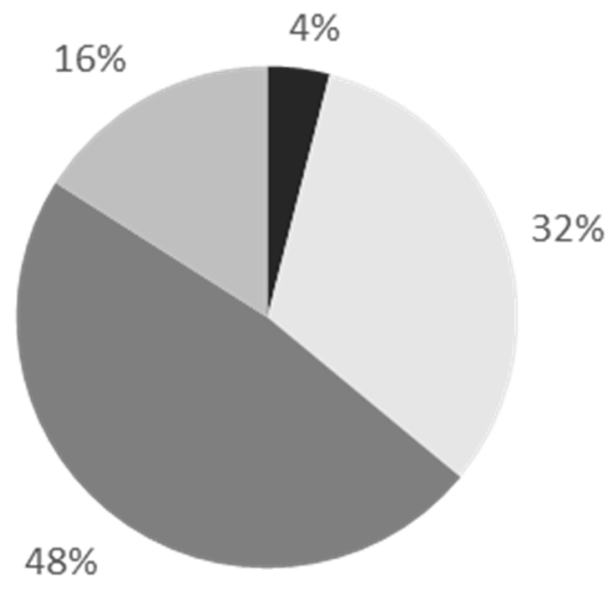

- Generalschulungen

- Produktivität
Unternehmensdaten

Arbeitswelt

Abbildung 1: Verteilung der Angebote auf die Inhaltsbereiche des Programms (Quelle: eigene Darstellung)

Der größte Inhaltsbereich gemessen an der Anzahl der Angebote ist der „Produktivitätsbereich“ (vgl. Abb. 1), in dem 12 Bildungsangebote (48\%) das Potenzial der Produktivitätssteigerung durch den Einsatz von Technologien in Produktion und Lager thematisieren. Durchgängige Produktentwicklung, ITSicherheit und Lean Production in der Industrie 4.0 sind drei Beispiele für dieses Segment. Bei den „Unternehmensdaten“ werden die Bedeutung und der Nutzen von Daten für die Digitalisierung herausgestellt. Ihre Aufnahme, Verarbeitung und Auswertung ist elementar, um Transparenz im Produktionsprozess bzw. im Unternehmen zu erhalten und zu steigern. Acht Schulungen (32\%), darunter Datenakquise und Data Mining zur Auswertung von Big Data, entfallen auf diesen Inhaltsbereich. Die verbleibenden vier Angebote (16\%) sind im Inhaltsbereich „Arbeitswelt“ zusammengefasst, in dem Auswirkungen der Digitalisierung auf Arbeit und Organisation aus einer soziologischen Perspektive betrachtet, Arbeitnehmerrechte kritisch hinterfragt und Möglichkeiten der verbesserten Ergonomie am Arbeitsplatz über den Einsatz innovativer Technologien behandelt werden.

Es zeigt sich, dass Digitalisierung bzw. Industrie 4.0 im Programm des Kompetenzzentrums überwiegend hinsichtlich seiner Potenziale für Unternehmen des produzierenden Gewerbes konzeptionell gestaltet wird. Gleichzeitig weist aber vor allem das Segment „Arbeitswelt“ auf die vielfältigen Bezüge 
der Digitalisierung und die damit einhergehenden Anforderungen aus einer interdisziplinären Perspektive hin, wodurch den Lernenden eine kritisch reflexive Perspektive auf die Digitalisierung und deren Folgen ermöglicht werden soll.

Wie bereits einleitend beschrieben, wurde das Kompetenzzentrum Ende 2015 gegründet und bietet seit Juli 2016 ein von Grund auf neu entwickeltes Programm an, das sich zunächst etablieren musste. Betrachtet man die einzelnen Angebote des Programms im Zeitverlauf, so werden nachfrageorientierte Anpassungen und angebotsorientierte Kontinuität ersichtlich.

Abbildung 2 zeigt ein Beispiel für eine im Schwerpunkt angebotsorientierte Vorgehensweise, bei der die zunächst ausbleibende Nachfrage für das Angebot Digitalisierung in der Prozesskette nicht zur Streichung führte, wie es von einer (rein) marktgetriebenen Praxis anzunehmen wäre. Stattdessen wurde es kontinuierlich zweimal im Halbjahr angeboten, bis Ende 2017 das erste Mal eine Durchführung (nach insgesamt fünf ausgefallenen Veranstaltungen in Folge) zu verzeichnen war. Dieser Verlauf kann als typisch für die Institution angesehen werden und findet sich in einer Reihe weiterer Veranstaltungen wieder - bis hin zu einer Veranstaltung, die im betrachteten Zeitraum kein einziges Mal realisiert werden konnte, aber dennoch Teil des Programms geblieben ist. Ein Grund für diese Kontinuität ist darin zu sehen, dass mit den offerierten Bildungsangeboten vor allem prospektive Bedarfe angesprochen sind, die mitunter in den adressierten Unternehmen selbst (noch) nicht wahrgenommen werden.

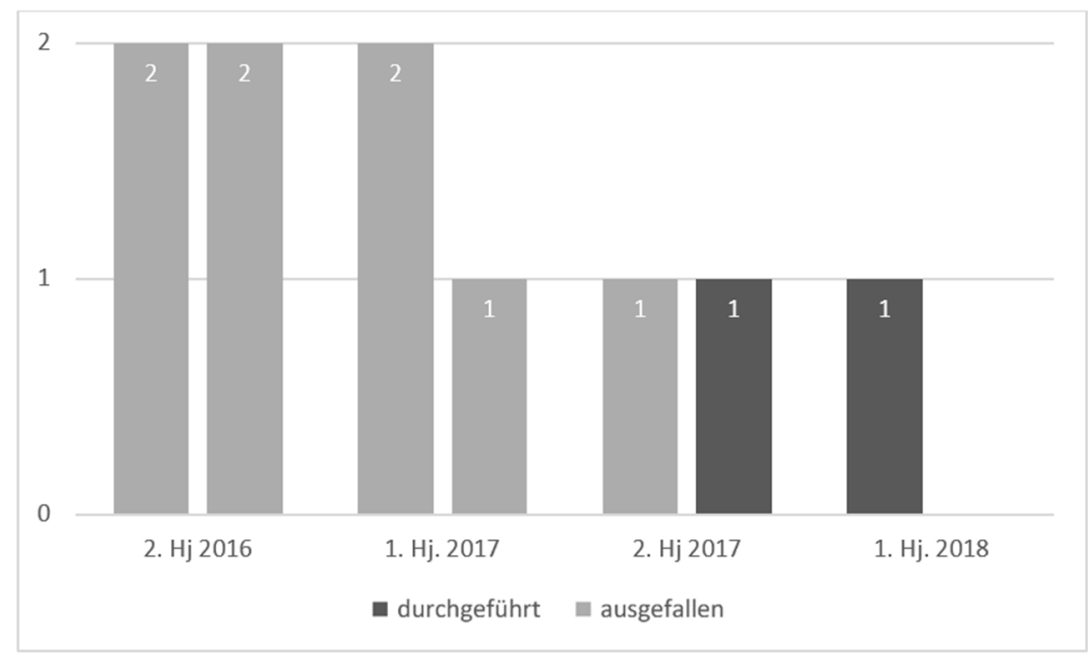

Abbildung 2: Angebot und Nachfrage der Veranstaltung „Digitalisierung in der Prozesskette" (Quelle: eigene Darstellung) 
Obwohl es im betrachteten Zeitraum keine Streichungen im Programm gab, wurden dennoch Anpassungen hinsichtlich der Veranstaltungsdauer (im 2. Halbjahr 2017) vorgenommen, wie die vormals zweitägige Veranstaltung $D i$ gitalisierung in der Prozesskette verdeutlicht, die nach drei ausgefallenen Terminen auf einen Tag gekürzt wurde. Dieser Effekt geht mit der allgemeinen Tendenz zu kürzeren Formaten in der betrieblichen Weiterbildung einher (Schönfeld \& Behringer 2017) und könnte aufgrund der entstehenden (indirekten) Kosten der Teilnahme für Unternehmen eine nachfrageorientierte Anpassung darstellen.

Ein anderes Beispiel beschreibt eine stärker nachfrageorientierte Vorgehensweise. Das Angebot IT-Sicherheit in der Produktion stieß von Beginn an auf eine hohe Nachfrage und wurde bereits im ersten Jahr regelmäßig durchgeführt. Die stetige Nachfrage führte zu einem zusätzlichen Veranstaltungstermin pro Halbjahr, wobei die Veranstaltungsdauer unverändert blieb. Zusätzlich wurde resultierend aus der vorhandenen Nachfrage ein neues Angebot zur IT-Sicherheit entwickelt, das sowohl die Produktion als auch die Verwaltung adressiert und damit eine Erweiterung der Zielgruppe darstellt.

Die bei der Veranstaltung „Digitalisierung der Prozesskette“ angedeutete Kontinuität des Programms spiegelt sich auch bei den Planungsstrategien wider. Über die betrachteten zwei Jahre wurde kein einziges Angebot trotz ggf. (anfänglich) ausbleibender Nachfrage gestrichen. Stattdessen wurden alle anfangs neu entwickelten Angebote (22 von 25 Angebote) fortgeschrieben. Diese Fortschreibung beinhaltet sowohl 13 Angebote, die in Thema und Veranstaltungsdauer unverändert blieben, als auch neun Angebote, bei denen entweder die Veranstaltungsdauer auf einen Tag reduziert (7/25) oder das Angebot in einzelne Module segmentiert wurde (2/25). Zudem sind drei Angebote zu den Themen Produktionsplanung und -steuerung, virtuelle Realitäten in der Produktion und das bereits genannte Thema IT-Sicherheit für das ganze Unternehmen neu entwickelt worden.

\section{Fazit}

Die Ergebnisse der Programmanalyse zeigen, dass Programmplanung für die durch Veränderungen geprägte, dynamische Digitalisierung nicht per se mit punktueller und fluider Angebotsentwicklung einhergeht. Stattdessen ermöglichen eine gesicherte Finanzierung und die Beteiligung von Erwachsenenbildnern abseits einer einseitigen Marktorientierung die Etablierung von Programmstrukturen in Einrichtungen der beigeordneten Bildung und damit die Auseinandersetzung der Lernenden mit beruflich relevantem wissenschaftli- 
chem Wissen zur Digitalisierung. Durch die Planenden werden über Fortschreibungen von Angeboten und partielle Neuentwicklungen kontinuierliche, institutionalisierte Lernräume geschaffen.

Obwohl das Kompetenzzentrum im Vergleich mit etablierten Bildungseinrichtungen wie Volkshochschulen nur ein relativ kleines Programm (25 Angebote) aufweist, ist es dennoch für die Reflexion von institutionalisierter Erwachsenenbildung relevant. Denn mit den Kompetenzzentren entstehen deutschlandweit neue Akteure im Feld der Erwachsenen- und Weiterbildung, die spezialisierte Bildungsangebote für die Digitalisierung konzipieren und damit wissenschaftliches Wissen für den „Wissens- und Technologietransfer“ (Höffer-Mehlmer 2011: 991) zielgruppengerecht aufbereiten und zugänglich machen (Gieseke 2015).

Um die Anforderung ,Aktualität vs. Kontinuität" (Siebert 2009: 77, Herv. i.O.) in den Programmen auszubalancieren wird eine im Schwerpunkt angebotsorientierte Vorgehensweise umgesetzt, die den politisch gesetzten bzw. definierten Bedarf ,Industrie 4.0“ über prospektiv aus der Forschung abgeleitete Bedarfe ausgestaltet. Damit weisen die Bedarfe einen anderen Zeithorizont auf als die im Schwerpunkt aktuellen Bedarfe der Unternehmen. Hieraus resultiert eine Bedarfsdiskrepanz zwischen politisch definiertem Bedarf auf der einen und dem von KMU für sich selbst festgestellten Bedarf auf der anderen Seite. Die (anfänglich) ausbleibende Nachfrage führt zu modifizierten Fortschreibungen einzelner Angebote, bei denen die Veranstaltungsdauer und -formate angepasst werden. Die Wechselbeziehung zwischen Angebot und Nachfrage wird genutzt, um Bedarfe zu wecken, d.h. Nachfrage durch wiederholte Ankündigungen und Maßnahmen der Teilnehmergewinnung zu erzeugen. Die Fragen, wie solche Bedarfe geweckt und in Nachfrage überführt werden können, berühren die Teilnehmenden- und Adressatenforschung, zeigen darüber hinaus aber auch Desiderate im Bereich der Bedarfsforschung auf.

\section{Literatur}

Arnold, R. \& Pätzold, H. (2011): Weiterbildung und Beruf. In: Tippelt, R. \& Hippel, A. v. (Hrsg.): Handbuch Erwachsenenbildung/Weiterbildung. Wiesbaden: VS Verlag, S. 653-664.

Bardeleben, R., Böll, G., Drieling, C., Gnahs, D., Seusing, B. \& Walden, G. (1990): Strukturen beruflicher Weiterbildung. Analyse des beruflichen Weiterbildungsangebots und -bedarfs in ausgewählten Regionen. Berichte zur beruflichen Bildung. Heft 114. Berlin, Bonn: BIBB. 
Bilger, F. \& Kuper, H. (2012): Weiterbildungssegmente: Teilnahme und Aktivitäten. In: Bilger, F., Gnahs, G., Hartmann, J. \& Kuper, H. (Hrsg.): Weiterbildungsverhalten in Deutschland. Resultate des Adult Education Survey 2012. Bielefeld: Bertelsmann Verlag, S. 36-49.

Börjesson, I. \& Zimmermann, U. (2005): „Und dann gibt es noch Bildungsangebote ...". Regionalanalysen zu beigeordneten kulturellen Bildungsangeboten im Berliner Stadtbezirk Friedrichshain-Kreuzberg und in der brandenburgischen Uckermark. In: Gieseke, W., Opelt, K., Stock, H. \& Börjesson, I.: Kulturelle Erwachsenenbildung in Deutschland. Empirische Analyse Berlin/Brandenburg. Münster: Waxmann, S. 131-202.

Brandt, A., Polom, L. \& Danneberg, M. (2016): Gute digitale Arbeit. Auswirkungen der Digitalisierung im Dienstleistungsbereich. library.fes.de/pdf-files/wiso/12786.pdf [Zugriff: 03.01.19].

Bundesministerium für Wirtschaft und Energie (BMWi) (2015): Bekanntmachung „Mittelstand 4.0 - Digitale Produktions- und Arbeitsprozesse“" vom 19. Juni 2015. https://www.bundesanzeiger.de/ebanzwww/wexsservlet?page.navid= official_starttoofficial_print\&genericsearch_param.edition $=\mathrm{BA} n \mathrm{n}+\mathrm{AT}+23.06 .2015 \&$ global_data.language $=$ [Zugriff: 21.08 .18$]$.

Dehnbostel, P. (2008): Berufliche Weiterbildung. Grundlagen aus arbeitnehmerorientierter Sicht. Berlin: edition sigma.

Dengler, K. \& Matthes, B. (2015): In kaum einem Beruf ist der Mensch vollständig ersetzbar. Folgen der Digitalisierung für die Arbeitswelt. doku.iab.de/kurzber/2015/kb2415.pdf [letzter Zugriff: 03.04.19].

Dengler, K. \& Matthes, B. (2018): Wenige Berufsbilder halten mit der Digitalisierung Schritt. Substituierbarkeitspotenziale von Berufen. doku.iab.de/kurzber/2018/kb0418.pdf [letzter Zugriff: 03.04.19].

Gieseke, W. (2003): Programmplanungshandeln als Angleichungshandeln. In: Gieseke, W. (Hrsg.): Institutionelle Innensichten der Weiterbildung. Bielefeld: Bertelsmann, S. 189-211.

Gieseke. W. (2008): Bedarfsorientierte Angebotsplanung in der Erwachsenenbildung. Bielefeld: Bertelsmann.

Gieseke, W. (2015): Programme und Angebote. In: Dinkelaker, J. \& Hippel, A. v. (Hrsg.): Erwachsenenbildung in Grundbegriffen. Stuttgart: Kohlhammer, S. $165-173$

Gieseke W. (2018): Forschungen zum Programmplanungshandeln. In: Fleige, M. Gieseke, W., Hippel, A. v., Käpplinger, B. \& Robak, S. (Hrsg.): Programm- und Angebotsplanung in der Erwachsenen- und Weiterbildung. utb, S. 52-63.

Gieseke, W. \& Heuer, U. (2011): Weiterbildungsentscheidungen und beigeordnete Bildung. In: R. Arnold \& A. Pachner (Hrsg.): Lernen im Lebenslauf. Baltmannsweiler, S. 107-127.

Gieseke, W. \& Hippel, A. v. (2018): Programmplanung als Konzept und Forschungsgegenstand. In: Fleige, M. Gieseke, W., Hippel, A. v., Käpplinger, B. \& Robak, S. (Hrsg.): Programm- und Angebotsplanung in der Erwachsenenund Weiterbildung. utb, S. 38-51. 
Gieseke \& Opelt (2005): Zusammenfassung: Bildungstheoretische Ansätze für kulturelle Bildung - Aspekt Programm. In: Gieseke, W., Opelt, K., Stock, H. \& Börjesson, I.: Kulturelle Erwachsenenbildung in Deutschland. Empirische Analyse Berlin/Brandenburg. Münster: Waxmann, S. 317-334.

Hippel, A. v. (2016). Programmplanungskulturen in der betrieblichen Weiterbildung. Eine Programmanalyse zu Funktionen betrieblicher Weiterbildungsprogramme. In: Dörner, O., Iller, C., Pätzold, H. \& Robak, S. (Hrsg.): Differente Lernkulturen - regional, national und transnational. Opladen, Berlin \& Toronto: Barbara Budrich, S. 139-159.

Hippel, A. v. \& Röbel, T. (2016): Funktionen als akteursabhängige Zuschreibungen in der Programmplanung betrieblicher Weiterbildung, Zeitschrift für Weiterbildungsforschung 39, S. 61-81.

Höffer-Mehlmer, M. (2011): Programmplanung und -organisation. In: Tippelt, R. \& Hippel, A. v. (Hrsg.): Handbuch Erwachsenenbildung/Weiterbildung. Wiesbaden: VS Verlag, S. 989-1002.

Hoffmann, N. (2012): Dokumentenanalyse. In: Schäffer, B. \& Dörner, O. (Hrsg.): Handbuch Qualitative Erwachsenen- und Weiterbildungsforschung. Opladen, Berlin \& Toronto: Babara Budrich, S. 395-405.

Käpplinger, B. (2008): Programmanalysen und ihre Bedeutung für pädagogische Forschung. In: Forum Qualitative Sozialforschung / Forum, Qualitative Social Research, 9 (1). http://nbn-resolving.de/urn:nbn:de:0114-fqs0801379 [Zugriff: 04.01.18].

Käpplinger, B. \& Robak, S. (2018): Forschen mit Programmen: Orientierungen für studentische Arbeiten. In: Fleige, M. Gieseke, W., Hippel, A. v., Käpplinger, B. \& Robak, S. (Hrsg.): Programm- und Angebotsentwicklung in der Erwachsenen- und Weiterbildung. utb, S. 64-75.

Lorenz, L. (2019): Planungsstrategien in der Programm- und Angebotsplanung bei Bildungsdienstleistern der beruflich-betrieblichen Weiterbildung. In: Hessische Blätter für Volksbildung. Im Erscheinen.

Merk, R. (1998): Weiterbildungsmanagement. (2., überarbeitete Auflage). Neuwied.

Müller, J. \& Stürzl, W. (1992): Dialogische Bildungsbedarfsanalyse - eine zentrale Aufgabe des Weiterbildners. In: Geißler, H.: Neue Qualitäten betrieblichen Lernens. Frankfurt am Main, S. 103-141.

Nolda, S. (2011): Programmanalyse - Methoden und Forschungen. In: Tippelt, R. \& Hippel, A. v. (Hrsg.): Handbuch Erwachsenenbildung/Weiterbildung. Wiesbaden: VS Verlag, S. 293-307.

Ortner, G. E. (1981): Bedarf und Planung in der Weiterbildung. Zur Differenzierung des Bedarfsbegriffs für die Weiterbildung. In: Bayer, M., Ortner, G. \& Thunemeyer, B. (Hrsg.): Bedarfsorientierte Entwicklungsplanung in der Weiterbildung. Opladen, S. 24-46.

Pohlmann, C. (2018): Bildungsurlaub - Vom gesellschaftspolitischen Anliegen zum Instrument beruflicher Qualifizierung? Eine Analyse der Bildungsurlaubsdiskurse in der Weiterbildung. Berlin: Peter Lang. 
Reinhart, G. (2017): Handbuch Industrie 4.0. Geschäftsmodelle, Prozesse, Technik. München: Carl Hanser Verlag.

Schlutz, E. (2006): Bildungsdienstleistungen und Angebotsentwicklung. Münster: Waxmann.

Schönfeld, G. \& Behringer, F. (2017): Betriebliche Weiterbildung. In: Bilger, F., Behringer, F., Kuper, H. \& Schrader, J. (Hrsg.): Weiterbildungsverhalten in Deutschland 2016 - Ergebnisse des Adult Education Survey (AES), S. 56-73. http://www.die-bonn.de/doks/2017-weiterbildungsforschung-01.pdf [Zugriff: 04.01.18].

Siebert, H. (2009): Didaktisches Handeln in der Erwachsenenbildung. Didaktik aus konstruktivistischer Sicht (6. überarbeitete Auflage). Augsburg: ZIEL.

Tippelt, R. \& Kadeira, S. (2016): Netzwerke und lernende Regionen als Orte organisationspädagogischer Forschung und Praxis. In: Göhlich, M., Schröer, A. \& Weber, S. (Hrsg.): Handbuch Organisationspädagogik. Wiesbaden: Springer VS. https://doi.org/10.1007/978-3-658-07746-4_76-1 [Zugriff: 04.01.18].

Wolter, A. (2011): Die Entwicklung wissenschaftlicher Weiterbildung in Deutschland: Von der postgradualen Weiterbildung zum lebenslangen Lernen. In: Beiträge zur Hochschulforschung 33. München, S. 8-35. 

Gregor Dutz, Lisanne Heilmann

\section{Lesekompetenz und politische Partizipation: Empirische Befunde aus PIAAC-L}

\section{Einleitung}

Die Frage von gesellschaftlicher und politischer Teilhabe ist in Zeiten tiefgreifender Veränderungen von großer Bedeutung: Vor dem Hintergrund von Globalisierung, Digitalisierung, Klimawandel und Flucht sind die durch politische Bildung vermittelten Fähigkeiten zur Identifikation und Artikulation von Interessen wichtige Grundkompetenzen aller Bürger*innen. Auch das Erkennen von und die Kritik an ökonomischen und Machtinteressen, etwa in Bezug auf Weiterbildung selbst, ist eine wichtige Kompetenz, um gesellschaftliche Ursachen individualisierter Probleme aufzudecken.

Angebote politischer Grundbildung richten sich oft an sogenannte politikferne oder bildungsferne Gruppen, deren vermeintlichen geringen politischen Kenntnisse und ihre benachteiligte soziale Lage volle gesellschaftliche und politische Teilhabe verhindern (vgl. Zeuner 2017: 45). Ziel politischer Grundbildung ist, benachteiligte Menschen zu befähigen, ,ihre Interessen zu klären und ihre Stimme zu Gehör zu bringen“" (Grotlüschen 2016: 200).

Im Kontext des deutschsprachigen Grundbildungsdiskurses liegt der Fokus dabei häufig auf der Gruppe der gering literalisierten Erwachsenen ${ }^{1}$ : Viele politikbezogene Praktiken erfordern schriftsprachliche Kenntnisse, etwa das Lesen der Tageszeitung oder das Verfassen von Einladungen zu politischen Veranstaltungen, so dass die Annahme eines Zusammenhangs von Lese- und Schreibkompetenz und dem Grad der politischen Partizipation nahe liegt (vgl. Korfkamp 2016: 462). In quantitativen Studien wurde dieser Zusammenhang bisher nur selten betrachtet. Es ist das Ziel dieser Auswertung, zur Verkleinerung dieser Forschungslücke beizutragen.

Im Folgenden gehen wir dazu zunächst auf die individuellen und gesellschaftlichen Bestimmungsfaktoren politischer Partizipation ein, dann auf den Forschungsstand $\mathrm{zu}$ politischer Partizipation von Menschen mit geringen schriftsprachlichen Fähigkeiten. Anschließend beschreiben wir kurz die verwendeten Daten und Methoden, gefolgt von der Darstellung und Diskussion der Ergebnisse.

1 Die Bezeichnung ,funktionale*r Analphabet*in“ gilt als stigmatisierend und erklärungsbedürftig. Wir versuchen daher, auf diese Bezeichnung zu verzichten, und sprechen von gering literalisierten Erwachsenen (vgl. Grotlüschen et al. 2019). 


\section{Soziale Selektivität politischer Partizipation}

Die soziale Selektivität politischer Partizipation wurde in zahlreichen Studien nachgewiesen. Böhnke (2011) zeigt, dass ehrenamtliches und politisches Engagement sowie das politische Interesse mit sinkendem Einkommen abnehmen. Die längsschnittliche Analyse zeigt außerdem, dass der Abstieg in Armut zu einer Abnahme der politischen Partizipation führt (ebd.: 21-23).

Neben den sozioökonomischen Erklärungsfaktoren wie Bildungsniveau oder Einkommen haben auch Werte und Einstellungen gegenüber Politik einen Einfluss auf die politische Partizipation und die Wahlbeteiligung. Milieus mit einem niedrigen sozialen Status weisen eine geringere Wahlbeteiligung auf als Milieus mit höherem Status (Schäfer/Vehrkamp/Gagné 2013: 16).

Einstellungen gegenüber Politik können Ausdruck finden in der politischen Wirksamkeitserwartung. Das Konzept der politischen Wirksamkeitserwartung wird in der Partizipationsforschung als eine der Partizipation vorgelagerte Einstellung verstanden und gibt die subjektive Einschätzung der eigenen Einflussmöglichkeiten auf politische Entscheidungen und die Politik im Allgemeinen wieder (Vetter 1997: 34-39). Als wichtigste Erklärungsgröße für die politische Wirksamkeitserwartung hat sich der Bildungsabschluss erwiesen; aber auch Einkommen, Werteorientierungen und politische Kontextfaktoren sind relevant (Bödeker 2012; Vetter 1997: 26-32).

\section{Die gesellschaftlichen Bedingungen geringer politischer Partizipation}

Um die soziale Selektivität politischer Partizipation zu erklären, werden darüber hinaus häufig auf individueller Ebene Unfähigkeit oder Unwillen zur politischen Partizipation unterstellt. Damit geht einher, dass die Fähigkeit zur politischen Partizipation grundsätzlich vorausgesetzt wird und ein Abweichen vom Idealbild des*der aktive* $n$ Bürger*in als fehlende politische Kompetenz oder politisches Desinteresse gedeutet wird (Bremer/Kleemann-Göhring 2010: 14-16). Das Defizit wird dann in erster Linie beim Individuum gesehen. Nach Bremer (2010) kann dies unter Bezugnahme auf Bourdieus Theorien der symbolischen Herrschaft und des politischen Feldes jedoch auch als vorweggenommene Fremdausschließung interpretiert werden: Diese Form der Selbstausschließung ,sagt weniger über vorhandenes politisches Wissen, sondern mehr über den Zustand und die Grenzen des politischen Feldes“ (Bremer 2010: 
187). Auch „Laien“ besitzen politisches Wissen, welches ,aber eher ein praktisches Gespür, ein latentes, vorreflexives, alltagsweltlich erworbenes Wissen [ist], das mehr Ethos als Logos, mehr Haltung als Bewusstsein ist" (Bremer/Kleemann-Göhring 2010: 18).

So zeigt sich in qualitativen Studien, dass Menschen aus bildungsfernen Milieus Interesse an politischen Themen haben und über politisches Wissen verfügen. Jedoch fehlt bei diesen Gruppen das Gefühl der Legitimität der eigenen politischen Ansprüche oder sie haben das Gefühl, „die da oben“würden keine Rücksicht auf sie nehmen (Korfkamp 2016: 461). Pape (2011) kommt zu dem Schluss, dass gering literalisierte Erwachsene ,nicht die erforderlichen schriftsprachlichen Kompetenzen [haben], um sich über politische Themen zu informieren“ und sie ,durchaus über ein politisches Wissen und politische Einstellungen verfügen, aber dass ihnen die Teilnahme am politischen Diskurs im engeren Sinne praktisch verwehrt ist" (Pape 2011: 22; vgl. Bremer/Pape 2017). $\mathrm{Zu}$ ähnlichen Ergebnissen kommen Studien über bildungsbenachteiligte Jugendliche (Calmbach/Borgstedt 2012).

Es ist also festzuhalten, dass für die Erklärung geringer politischer Partizipation nicht nur die individuellen Kompetenzen, sondern immer auch die gesellschaftlichen Umstände im Blick behalten werden sollten, die zu einem Teilhabeausschluss bestimmter Gruppen führen können.

\section{Politische Partizipation und schriftsprachliche Kompetenz}

In quantitativen Kompetenzstudien spielt die politische Bildung bisher eine untergeordnete Rolle. Zwar enthält der PISA-Jahrgang 2018 den neuen Kompetenzbereich Global Competencies (kritisch dazu Grotlüschen 2018), das Fragenprogramm der internationalen Erwachsenen-Kompetenzstudie Programme for the International Assessment of Adult Competencies (PIAAC) enthält jedoch nur Fragen zur politischen Selbstwirksamkeit (Political Efficacy), zum freiwilligen Engagement (Voluntary Work) und dem gesellschaftlichen Vertrauen (Social Trust). Diese werden von Bremer/Ludwig (2015) in ihrer Bedeutung für die politische Bildung untersucht. Sie kritisieren die ,eher unterkomplexe Operationalisierung" und halten die Beurteilungsmöglichkeit der „Handlungsrelevanz von Kompetenz in Bezug auf Politik und Partizipation [...] aufgrund von PIAAC“ für spekulativ (ebd.: 39). Grotlüschen (2016) wertet die politische Selbstwirksamkeit, das freiwillige Engagement und das soziale Vertrauen in Bezug auf die PIAAC-Kompetenzstufen aus und kommt zu dem Ergebnis, dass eine niedrigere Kompetenzstufe mit weniger freiwilligem 
Engagement, geringerer politischer Selbstwirksamkeitserwartung und weniger sozialem Vertrauen einhergeht.

\section{Fragestellung und Hypothesen}

Unsere Fragestellung lautet: Welchen Einfluss haben die schriftsprachlichen Kenntnisse (Literalität) und politische Einstellungen auf bestimmte politische Handlungsweisen? Ausgehend von Theorie und Forschungsstand gehen wir dabei von den folgenden Hypothesen aus:

1. Je höher das schriftsprachliche Kompetenzlevel, desto häufiger wird eine politische Praktik ausgeübt.

2. Es gibt einen positiven Zusammenhang zwischen politischer Wirksamkeitserwartung und Häufigkeit von politischen Verhaltensweisen.

3. Menschen mit höherem Schulabschluss üben häufiger politische Praktiken aus.

\section{Daten und Methode}

Für die Analyse nutzen wir Daten aus der PIAAC-L-Studie (GESIS/LIFBI 2017). Bei PIAAC-L handelt es sich um die deutsche längsschnittliche Fortführung von PIAAC 2012, die drei zusätzliche Wellen in den Jahren 2014, 2015 und 2016 umfasst. Der PIAAC-Kompetenztest wurde erneut in der Welle 2015 durchgeführt.

Die Operationalisierung politischer Handlungsweisen erfolgt dabei über die in PIAAC-L erfassten politischen Praktiken. Im Fragebogen des Jahres 2015 wurden die folgenden vier politischen Praktiken erfasst, die hier ausgewertet werden sollen:

- Frage 1 (Politische Beteiligung): Wie oft machen Sie das: Beteiligung in Parteien, in der Kommunalpolitik, Bürgerinitiativen?

- Frage 2 (Ehrenamtliche Tätigkeit): Wie oft machen Sie das: Ehrenamtliche Tätigkeiten in Vereinen, Verbänden oder sozialen Diensten?

- Frage 3 (Zeitung lesen): Wie oft machen Sie das: Zeitung lesen (in Papierform oder im Internet)?

- Frage 4 (Diskutieren): Wie oft kommt es im Allgemeinen vor, dass Sie mit Ihrem Partner über politische und soziale Fragen diskutieren? 
Die Fragen nach politischer Beteiligung und ehrenamtlichem Engagement (Fragen 1 und 2) sind etablierte Fragen aus der Partizipationsforschung. Diese Formen von politischen Handlungen erfordern schriftsprachliche Kenntnisse, etwa für das Lesen oder Verfassen von Anträgen, Einladungen oder Protokollen, so dass von vergleichsweise hohen Zugangsbarrieren für gering literalisierte Erwachsene ausgegangen werden kann. Das Lesen von Zeitungen (Frage 3) erfordert Lesefähigkeiten, die je nach Art der Zeitung unterschiedlich hoch sein können. Mit dem*der Partner*in über politische Fragen zu diskutieren erfordert keine schriftsprachlichen Kenntnisse und findet in geschützten Bereichen statt, so dass es hier auch möglich ist, mit vorreflexivem und alltagsweltlichem Wissen (vgl. Korfkamp 2016: 461) über Politik zu sprechen.

Die Operationalisierung der Literalität erfolgt über die in PIAAC-L 2015 gemessene Lesekompetenz, verstanden als ,das Verstehen, Nutzen und Interpretieren von geschriebenen Texten" (Rammstedt 2013: 12). Die auf einer Skala von 0-500 gemessene Lesekompetenz kann in sechs Kompetenzstufen von unter Level 1 bis Level 5 eingeteilt werden (Zabal et al. 2013: 37f.).

Wir führen mehrere logistische Regressionen durch, um unsere Hypothesen zu überprüfen. Die Regressionen werden unter Berücksichtigung der zehn plausiblen Werte durchgeführt und gepoolt (Rubin 1987: 76). Die abhängige Variable ist dabei jeweils die dichotomisierte Häufigkeit der Ausübung einer der vier politischen Handlungsweisen $(0=$ nie oder seltener als einmal pro Monat, $1=$ mindestens einmal pro Monat). In einem ersten Modell dient ausschließlich die Literalitäts-Kompetenzstufe als Prädiktorvariable. In einem zweiten Modell ergänzen wir als unabhängige Variablen die politische Wirksamkeitserwartung, Geschlecht, Alter, Bildungsabschluss, Beschäftigungsstatus, Migration und Sprache.

\section{Grenzen der Untersuchung}

Auf Grund der verwendeten Daten und Methode sind einige forschungsmethodische Einschränkungen zu erwähnen.

Auf dem obersten und dem untersten Literalitätslevel befindet sich eine kleine Anzahl an Personen. In der Gesamtbevölkerung bestehende Zusammenhänge könnten in dieser Untersuchung wegen eines zu kleinen Samples nicht signifikant sein. Dies betrifft insbesondere die Analyse von Subgruppen oder Fragen mit geringer Antwortvarianz. Der Schwerpunkt von PIAAC(-L) ist nicht die politische Partizipation gering literalisierter Menschen. Insofern ist die Auswahl an Fragen zu politischen Praktiken limitiert. Die vier ausgewerteten Fragen decken aber ein breites Spektrum möglicher politischer Praktiken 
ab, die gleichzeitig unterschiedliche Anforderungen an die Lese- und Politikkompetenz stellen.

Die Einschätzung des eigenen Handelns als politisch ist voraussetzungsreich (vgl. Calmbach/Borgstedt 2012). Gerade in quantitativen Studien besteht daher die Gefahr, dass politisches Interesse oder politische Kenntnisse nur unzureichend erfasst werden.

\section{Darstellung der Ergebnisse}

Auf eine detaillierte deskriptive Darstellung der oben genannten Fragen bzw. eine bivariate Untersuchung unter Einschluss des Literalitätslevels muss hier aus Platzgründen verzichtet werden. ${ }^{2}$ Dennoch sollen zwei Erkenntnisse aus dieser Analyse hier genannt werden: Zum einen sind die Beteiligung in Parteien/Kommunalpolitik/Bürgerinitiativen (Frage 1) und das ehrenamtliche Engagement (Frage 2) in der Gesamtbevölkerung nur gering ausgeprägt. Dies relativiert Ansprüche an sogenannte politikferne Gruppen, sich am Idealbild des*der Aktivbürger*in zu orientieren. Es lässt sich außerdem ein Zusammenhang zwischen Lesekompetenz und Häufigkeit der Ausübung einer politischen Praktik vermuten: Je höher die gemessene Kompetenz, desto häufiger wird eine Praktik ausgeübt.

Für die multivariate Analyse führen wir mehrere logistische Regressionen durch, bei denen die abhängige Variable jeweils die dichotomisierte Häufigkeit der Ausübung einer der vier politischen Handlungsweisen ist. Die Koeffizienten für alle vier abhängigen Variablen werden dabei jeweils gemeinsam als odds ratios in einer Tabelle dargestellt. Werte größer eins bedeuten eine höhere Wahrscheinlichkeit, eine politische Praktik mindestens einmal pro Monat auszuüben, Werte kleiner eins eine geringere Wahrscheinlichkeit - jeweils im Vergleich zur Referenzgruppe.

2 Diese Zahlen waren Teil der Präsentation auf der Sektionstagung und können gern bei den Autor*innen angefordert werden. 


\section{Modell ohne Kontrollvariablen}

Tabelle 2: Modell ohne Kontrollvariablen (Koeffizienten als odds ratios, Referenzgruppe in Klammern, $\left.{ }^{*} p<0,05,{ }^{* *} p<0,01,{ }^{* * *} p<0,001\right)$

\begin{tabular}{lllll}
\hline & $\begin{array}{l}\text { Politische } \\
\text { Beteiligung }\end{array}$ & $\begin{array}{l}\text { Ehrenamtliche } \\
\text { Tätigkeit }\end{array}$ & $\begin{array}{l}\text { Zeitung } \\
\text { lesen }\end{array}$ & Diskutieren \\
\hline \multicolumn{5}{c}{ PIAAC Level (Level 2) } \\
\hline $\begin{array}{l}\text { Unter } \\
\text { Level 1 }\end{array}$ & 0,00 & 0,36 & 0,53 & $0,25^{\star * *}$ \\
\hline Level 1 & 0,96 & 0,71 & 0,93 & 0,71 \\
\hline Level 3 & 1,12 & 1,24 & $1,40^{*}$ & $1,83^{* * *}$ \\
\hline Level 4 & 1,44 & $1,52^{*}$ & $2,35^{* * *}$ & $2,79^{* * *}$ \\
\hline Level 5 & 0,12 & 2,17 & 4,36 & 24,42 \\
\hline
\end{tabular}

In einem ersten Modell führen wir eine logistische Regression mit den Lesekompetenzstufen als Prädiktorvariable durch. Die Regressionskoeffizienten (Tabelle 1) der logistischen Regression zeigen, dass Personen auf einer höheren Kompetenzstufe in der Regel eine höhere Wahrscheinlichkeit besitzen, eine politische Handlungsweise mindestens einmal pro Monat auszuüben, als die Referenzgruppe. Umgekehrt ist dies bei den Personen unter Level 2: Deren Wahrscheinlichkeit eine politische Handlungsweise auszuüben ist geringer.

Es zeigt sich jedoch auch, dass vor allem bei den Fragen zur Beteiligung in Parteien, in der Kommunalpolitik und Bürgerinitiativen sowie zum Engagement in Vereinen, Verbänden oder sozialen Diensten diese Effekte zum Großteil nicht signifikant sind.

Das Diskutieren mit dem*der Partner*in über politische und soziale Fragen weist für Personen unter Level 1 eine signifikant niedrigere und für Personen auf Level 3 und Level 4 eine signifikant höhere Wahrscheinlichkeit im Vergleich zur Referenzgruppe (Level 2) auf, diese Handlung mindestens einmal pro Monat auszuüben.

Bei der logistischen Regression ohne Kontrollvariablen zeigen sich also Effekte in der erwarteten Richtung. Ein signifikanter Effekt der Lesekompetenz auf die Häufigkeit, eine politische Handlungsweise auszuführen, zeigt sich jedoch nicht bei allen Praktiken und Levels. 



\begin{tabular}{lcccc}
\hline & $\begin{array}{l}\text { Politische } \\
\text { Beteiligung }\end{array}$ & $\begin{array}{l}\text { Ehrenamtliche } \\
\text { Tätigkeit }\end{array}$ & $\begin{array}{l}\text { Zeitung } \\
\text { lesen }\end{array}$ & Diskutieren \\
\hline \multicolumn{5}{c}{ Erstsprache Deutsch (Ja) } \\
\hline Nein & $3,60^{*}$ & 0,77 & 0,92 & $0,58^{* *}$ \\
\hline \multicolumn{5}{c}{ Menschen wie ich haben keinen Einfluss auf die Regierung } \\
(Stimme voll und ganz zu)
\end{tabular}

Bei allen vier Fragen zeigt sich, dass die Stärke des Einflusses des Literalitätslevels in diesem Modell zurückgeht. Außerdem verringert sich die Signifikanz, so dass nicht mehr von einem signifikanten Zusammenhang von Literalität und Häufigkeit der Ausübung politischer Praktiken gesprochen werden kann.

Bei den Kontrollvariablen zeigen sich verschiedene Effekte. Das Geschlecht hat einen signifikanten Einfluss auf die Häufigkeit der Beteiligung in einer Partei oder der Kommunalpolitik und auf die Häufigkeit des Zeitunglesens. In beiden Fällen ist die Wahrscheinlichkeit der Ausübung der Praktik bei Frauen geringer als bei Männern.

Der Bildungsabschluss ist ein signifikanter Einflussfaktor bei drei von vier Fragen. Ein hoher Bildungsabschluss erhöht die Wahrscheinlichkeit, eine der vier Praktiken auszuüben, im Vergleich zur Referenzgruppe mit mittlerem Bildungsabschluss, ist bei Frage 2 (Ehrenamtliche Tätigkeit) jedoch nicht signifikant. Ein niedriger Bildungsabschluss verringert diese Wahrscheinlichkeit, ist aber im Fall von Frage 1 (Politische Beteiligung) und 2 (Ehrenamtliche Tätigkeit) nicht signifikant.

Der Beschäftigungsstatus hat in unserem Modell nur in Frage 2 (Ehrenamtliche Tätigkeit) und nur für die Gruppe der nicht mehr Erwerbstätigen einen signifikanten Einfluss. Für die Wahrscheinlichkeit der Ausübung politischer Handlungsweisen sind auch die Kontrollvariablen zu Sprache und Migration größtenteils nicht signifikant. Auffallend ist, dass die Wahrscheinlichkeit, sich in einer Partei zu engagieren, für Personen mit einer anderen Erstsprache als Deutsch höher ist als für deutsche Erstsprachler*innen. Wir führen diesen Effekt auf die Zusammensetzung des Samples zurück, in welchem sowohl die Gruppe derjenigen, die diese Praktik ausüben, als auch derjenigen mit anderer Erstsprache als Deutsch recht klein sind. Sollte sich solch ein Effekt jedoch 
mit anderen Daten replizieren lassen, ist er sicherlich einer näheren Betrachtung wert.

Die politische Selbstwirksamkeit wird in PIAAC mit einem Item erfragt. Dieses Item fragt nach der Zustimmung zu der Aussage „Menschen wie ich haben keinerlei Einfluss darauf, was die Regierung macht" (GESIS o.J.: 139). Mit Ausnahme der Frage zum Zeitungslesen zeigen alle Praktiken zumindest in den zwei ablehnenden Antwortkategorien einen signifikanten Einfluss auf die Wahrscheinlichkeit, eine der Praktiken auszuüben.

Besonders deutlich zeigt sich dies beim freiwilligen Engagement und dem Diskutieren mit dem*der Partner*in. Je stärker Personen ihren Einfluss auf die Regierung einschätzen, desto wahrscheinlicher ist es, dass sie sich in Vereinen engagieren oder mit der*dem Partner*in über politische und soziale Fragen diskutieren.

Beim Zeitunglesen hat im Modell die politische Selbstwirksamkeit keinen signifikanten Einfluss auf die Wahrscheinlichkeit der Häufigkeit.

\section{Diskussion der Ergebnisse}

Der in der bivariaten Auswertung vermutete Zusammenhang von Lesekompetenz und politischen Praktiken hat sich in der logistischen Regression ohne Kontrollvariablen nur für einige Fragen und Kompetenzstufen nachweisen lassen. Im zweiten Modell mit Kontrollvariablen konnte diese Vermutung nicht weiter aufrechterhalten werden. Hypothese 1 - ein direkter Einfluss der Lesekompetenz - konnte also nicht bestätigt werden.

Hypothese 2 und damit die Vermutung, dass die politische Selbstwirksamkeitserwartung einen Effekt aufweist, lässt sich für die Fragen 1, 2 und 4 bestätigen. Lediglich das Zeitunglesen ist hiervon nicht abhängig.

Hypothese 3, der Einfluss des Bildungsniveaus auf politische Partizipation, lässt sich ebenfalls bestätigen, lediglich Frage 2, das ehrenamtliche Engagement, zeigt hier keine signifikanten Ergebnisse.

Es zeigt sich also, dass für die Ausübung der vier ausgewählten politischen Praktiken nicht die Lesekompetenz, sondern der Bildungsabschluss und die politische Wirksamkeitserwartung ausschlaggebend sind.

Der Einfluss des Bildungsabschlusses kann zum einen als höhere Politikkompetenz der besser gebildeten Personen gewertet werden, welche im Rahmen einer längeren (Berufs-)Ausbildung eine umfassendere politische Bildung erfahren haben, die sich in einer höheren politischen Partizipation zeigt.

Sowohl der Bildungsabschluss als auch die Selbstwirksamkeitserwartung können jedoch auch als indirekte Indikatoren für den Einfluss von Einstellun- 
gen und Werten auf die politische Partizipation interpretiert werden. Dies ermöglicht die Rückbindung der Ergebnisse an die Bourdieusche Milieutheorie und die Theorie vom politischen Feld. Das Engagement in einer Partei oder einem Verein geschieht in Feldern, an denen ein Teil der Bevölkerung nicht teilhat, dessen Zugangsmöglichkeit jedoch unabhängig von der Lesekompetenz ist.

Bezogen auf die eingangs beschriebenen globalen Entwicklungen wirft milieuspezifisches politisches Handeln das Problem auf, dass eine Gruppe von Menschen ihre Interessen nur unzureichend artikulieren und durchsetzen kann. Gleichzeitig ist diese Gruppe von den negativen Folgen der beschriebenen Entwicklungen überproportional stark betroffen und von gesellschaftlicher Exklusion stärker bedroht. Zwar sind die Angehörigen dieser Gruppe nicht grundsätzlich von Teilhabe ausgeschlossen, weisen aber in verschiedenen Bereichen (wie etwa dem Arbeitsmarkt) ein höheres Risiko auf exkludiert zu werden.

Die Bedeutung von Lesekompetenz für die alltägliche politische Partizipation scheint uns auf Grund der obigen Ergebnisse weniger entscheidend als häufig dargestellt. Dagegen besteht die Gefahr, durch die Betonung benötigter schriftsprachlicher Kompetenzen gering literalisierte Gruppen von politischer Teilhabe eher auszuschließen, statt sie zu inkludieren (vgl. Bremer/KleemannGöhring 2010: 18). Interpretiert man die spezifische Ausübung bestimmter politischer Praktiken als milieuspezifisch, so deuten die Ergebnisse darauf hin, dass die geringere Ausübung der politischen Praktiken weniger in der gemessenen Lesekompetenz begründet liegt. Viel entscheidender erscheint uns die Frage, ob das eigene politische Handeln als legitim und die eigene (Politik-)Kompetenz als ausreichend eingeschätzt werden. Zukünftige (quantitative) Studien sollten neben einer breiteren Auswahl politischer Praktiken daher auch in den Blick nehmen, ob die Befragten ihr politisches Handeln als legitim einschätzen oder überhaupt als politisch begreifen.

\section{Literatur}

Bödeker, Sebastian (2012): Soziale Ungleichheit und politische Partizipation in Deutschland. Grenzen politischer Gleichheit in der Bürgergesellschaft. OBS Arbeitspapier.

Böhnke, Petra (2011): Ungleiche Verteilung politischer und zivilgesellschaftlicher Partizipation. In: Aus Politik und Zeitgeschichte, 1-2, S. 18-25.

Bremer, Helmut (2010): Symbolische Macht und politisches Feld. Der Beitrag der Theorie Pierre Bourdieus für die politische Bildung. In: Lösch, B. / Thimmel, A. (Hrsg.): Kritische politische Bildung. Ein Handbuch. Reihe Politik und Bildung, Bd. 54. Schwalbach/Ts.: Wochenschau Verlag, S. 181-192. 
Bremer, Helmut / Kleemann-Göhring, Mark (2010): „Defizit“ oder „Benachteiligung“. Zur Dialektik von Selbst- und Fremdausschließung in der politischen Erwachsenenbildung und zur Wirkung symbolischer Herrschaft. In: Zeuner, C. (Hrsg.): Demokratie und Partizipation. Beiträge der Erwachsenenbildung. Hamburg, S. 12-28.

Bremer, Helmut / Ludwig, Felix (2015): Herausforderungen der Ergebnisse der Studie „Programme for the International Assessment of Adult Competencies“ (PIAAC) für die politische Bildung. Expertise.

Bremer, Helmut / Pape, Natalie (2017): Literalität und Partizipation als milieuspezifische soziale Praxis. In: Menke, B./ Riekmann, W. (Hrsg.): Politische Grundbildung. Inhalte - Zielgruppen - Herausforderungen. Non-formale politische Bildung, Band 12. Schwalbach/Ts.: Wochenschau Verlag, S. 56-73.

Calmbach, Marc / Borgstedt, Silke (2012): „Unsichtbares“ Politikprogramm? Themenwelten und politisches Interesse von „bildungsfernen“ Jugendlichen. In: Kohl, W. / Seibring, A. (Hrsg.): „Unsichtbares“ Politikprogramm? Themenwelten und politisches Interesse von „bildungsfernen“ Jugendlichen. Schriftenreihe / Bundeszentrale für Politische Bildung, Band 1138. Bonn: Bundeszentrale für Politische Bildung, S. 43-80.

GESIS (o. J.): PIAAC Deutschland 2012 - Hintergrundfragebogen.

GESIS/LIFBI (2017): PIAAC-Longitudinal (PIAAC-L), Germany. ZA5989 Datenfile Version 3.0.0. Köln: GESIS Data Archive.

Grotlüschen, Anke (2016): Politische Grundbildung - Theoretische und empirische Annäherungen. In: Zeitschrift für Weiterbildungsforschung - Report, 2, S. 183-203.

Grotlüschen, Anke (2018): Global Competence - Does the new OECD competence domain ignore the Global South? In: Studies in the Education of Adults.

Grotlüschen, Anke et al. (2019): LEO 2018 - Leben mit geringer Literalität. Pressebroschüre. Hamburg.

Korfkamp, Jens (2016): Politische Grundbildung. In: Löffler, M. / Korfkamp, J. (Hrsg.): Handbuch zur Alphabetisierung und Grundbildung. UTB, Band 8683. Stuttgart: Waxmann, S. 458-466.

Pape, Natalie (2011): Politische Partizipation aus der Sicht funktionaler Analphabet/inn/en. In: Report - Zeitschrift für Weiterbildungsforschung 34, 3, S. 1523.

Rammstedt, Beatrice (2013): PIAAC 2012: Die wichtigsten Ergebnisse im Überblick. In: Rammstedt, B. (Hrsg.): Grundlegende Kompetenzen Erwachsener im internationalen Vergleich. Ergebnisse von PIAAC 2012. Münster: Waxmann, S. 11-29.

Rubin, Donald B. (1987): Multiple imputation for nonresponse in surveys. Wiley series in probability and mathematical statistics. Applied probability and statistics. New York: Wiley.

Schäfer, Armin / Vehrkamp, Robert / Gagné, Jérémie Felix (2013): Prekäre Wahlen. Milieus und soziale Selektivität der Wahlbeteiligung bei der Bundestagswahl 2013. Gütersloh: Bertelsmann Stiftung. 
Vetter, Angelika (1997): Political efficacy - Reliabilität und Validität. Alte und neue Messmodelle im Vergleich. DUV. Sozialwissenschaft. Wiesbaden: Deutscher Universitäts-Verlag.

Zabal, Anouk et al. (2013): Grundlegende Kompetenzen der erwachsenen Bevölkerung in Deutschland im internationalen Vergleich. In: Rammstedt, B. (Hrsg.): Grundlegende Kompetenzen Erwachsener im internationalen Vergleich. Ergebnisse von PIAAC 2012. Münster: Waxmann, S. 31-59.

Zeuner, Christine (2017): Was ist / was gehört zur politischen Grundbildung? In: Menke, B. / Riekmann, W. (Hrsg.): Politische Grundbildung. Inhalte - Zielgruppen- Herausforderungen. Non-formale politische Bildung, Band 12. Schwalbach/Ts.: Wochenschau Verlag, S. 34-55. 



\section{Weiterbildungsteilnahme und subjektives Alter - Kann das subjektive Alter helfen, die Funktion und Motivation einer Teilnahme zu erklären?}

\section{Einleitung}

Das Alter als Variable in der Weiterbildungsforschung zu erfassen ist weitverbreitet, wie die EdAge-Studie (Tippelt et al. 2009) oder das Adult Education Survey (AES) (Bilger et al. 2017) zeigen. In Zeiten von Globalisierung, Transformation und Entgrenzung scheinen sich Altersbilder und -rollen allerdings $\mathrm{zu}$ verändern und womöglich sogar aufzulösen. Darüber hinaus ist aufgrund der zu erwartenden demographischen Entwicklung als Form gesellschaftlicher Transformation mit einem sich verstärkenden Fachkräftemangel zu rechnen. Die Bereitschaft aller Altersklassen sich weiterzubilden ist eine zentrale Möglichkeit, diesem Mangel entgegenzuwirken. Daneben scheint das Alter allerdings keine Größe mehr zu sein, an der entscheidende Lebensphasen gemessen werden. Dies wird durch Entgrenzungsprozesse wie die Destandardisierung von Lebensläufen und Selbstkonzepten verstärkt. Mit steigendem Alter gehen eine zunehmende Differenzierung und Heterogenität persönlicher Erfahrungen und Selbstbilder einher, was zu einer nachlassenden Erklärkraft des chronologischen Alters führt (Carstensen 2006; Staudinger 2012). Eine Alternative zur Erfassung des chronologischen Alters, d.h. der Erhebung des Lebensalters, kann die Messung des subjektiven Alters bieten, welche das Alter abbildet, mit dem sich Individuen selbst identifizieren (Goldsmith/Heiens 1992; Rubin, D. C. \& Berntsen, D. 2006). Die erste Frage, die im Folgenden zu behandeln ist, lautet demnach: Ist das gefühlte, subjektive Alter einer Person relevanter für Lebensentscheidungen als das chronolgische Alter? Konkreter ist zu fragen, inwiefern sich das subjektive Alter als Erklärungsansatz auch für die Einschätzung und Teilnahme an einer Weiterbildung eignet. Abschließend ist die Frage zu stellen, ob das subjektive Alter ebenso oder vielleicht sogar statt des chronologischen Alters routinemäßig in der Weiterbildungsforschung erfasst werden sollte.

Zur Behandlung dieser Fragen wird im Folgenden (3) das theoretische Modell vorgestellt, welches Grundlage der weiteren Betrachtung ist. Hieraus werden anschließend die zu prüfenden Hypothesen abgeleitet. In Abschnitt 4 werden die Daten genauer beschrieben, die Operationalisierung verwendeter Konstrukte wird in Abschnitt 5 erläutert und danach (6) entsprechende Ergebnisse 
vorgestellt. Zuletzt erfolgt eine Zusammenfassung und kritsche Betrachtung des Vorgehens sowie ein Ausblick (7). Zunächst wird nun ein Überblick über die Beteiligungsforschung vor dem Hintergrund des Alters gegeben.

\section{Teilnahme und Alter}

Daten des AES zeigen, dass die älteste befragte Gruppe (60-64 Jahre) in geringstem Maße an Weiterbildung teilnimmt. Gegenüber der 2016 am stärksten beteiligten Gruppe der 45- bis 49-Jährigen (Teilnahmequote $46 \%$ ), hat die älteste Gruppe nur mit einer Quote von $38 \%$ teilgenommen (Bilger et al. 2017, S. 42). Besonders in der betrieblichen Weiterbildung sind Ältere (60-64 Jahre) und Jüngere (18/19-24 Jahre) in geringerem Maße vertreten als andere Altersgruppen. Die individuell-berufsbezogene Weiterbildung wird hingegen von jüngeren Altersgruppen etwas stärker wahrgenommen als von älteren, die nicht-berufsbezogene Weiterbildung ist in jüngeren Altersgruppen mit einer Quote von $22 \%$ bei den 18/19- bis 24-Jährigen stärker besucht als im mittleren Alter ab 35 Jahren (9 bis $10 \%$ ) und nimmt dann ab 55 Jahren wieder etwas zu (12-14 \%) (ebd., S. 42). An diesen Quoten hat sich in den letzten zehn Jahren wenig verändert, wenngleich die Teilnahme der älteren Befragten (ab 55 Jahren) insgesamt zugenommen hat. Die Zunahme der Beteiligung Älterer zeigt sich auch in der Volkshochschulstatistik und der EdAge-Studie (Huntemann/Reichart 2017; Tippelt et al. 2009). Laut Tippelt et al. (2009) nehmen vor allem ältere Befragte (65-80 Jahre) dabei eher aus privaten als aus beruflichen Gründen an Weiterbildung teil.

Die Beteiligung scheint mit dem chronologischen Alter zusammenzuhängen, auch wenn hier inferenzstatistische Aussagen großteils fehlen. Zum Einfluss des subjektiven Alters auf die Beteiligung konnte bereits gezeigt werden, dass ein positives Altersbild mit einer stärkeren Wahrnehmung von Bildungsangeboten einhergeht (Sachverständigenkomission 2010). Darüber hinaus fehlt es aber aktuell an Studien, die die Beziehung zwischen empfundenem Alter und Weiterbildung behandeln. Um diesen theoretisch angenommenen Zusammenhang zu prüfen bietet sich ein quantitatives, hypothesenprüfendes Vorgehen an. 


\section{Theoretisches Modell}

Der Arbeit liegt ein theoretisches Modell zugrunde, welches die Zusammenhänge verschiedener Altersvariablen als unabhängige Variablen (UV) mit relevanten Variablen der Einschätzung und Wahrnehmung einer Weiterbildung mit den Konstrukten Funktion und Motivation als abhängige Variablen (AV) beschreibt (Abb. 1). Daneben werden Kontrollvariablen berücksichtigt.

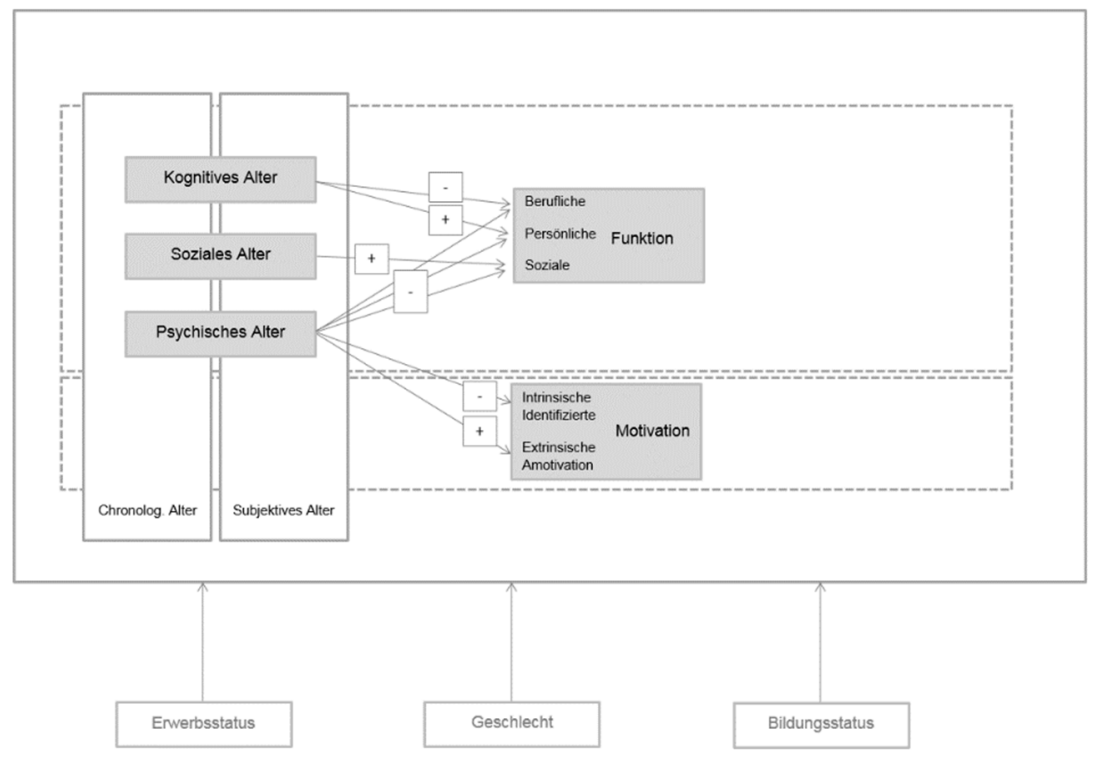

Abbildung 1: Theoretisches Modell zum Zusammenhang von Alter und Funktion sowie Motivation einer Weiterbildungsteilnahme (eigene Darstellung)

In Anlehnung an Filipp, Ferring und Klauer (1989) sowie Kade (2009) wird davon ausgegangen, dass das subjektive Alter die Konstrukte kognitives, soziales und psychisches subjektives Alter umfasst.

Das kognitive subjektive Alter fokussiert, wie sich Personen hinsischtlich geistiger Fähigkeiten wie Konzentration und Aufmerksamkeit persönlich einschätzen. Es ist anzunehmen, dass die persönliche Einschätzung eines Nachlassens bzw. einer zufriedenstellenden geistigen Kapazität dazu beiträgt, ob Lernanstrengung angestrebt oder eher vermieden wird.

Das soziale subjektive Alter beschäftigt sich mit der altersbedingten Veränderung des sozialen Umfelds. Rückgang sozialer Kontakte und Rollenverluste durch den Berufsausstieg können hiermit einhergehen (Kade 2009; Künemann/Kohli 2010). Da eine solche Veränderung des sozialen Umfelds aber 
nicht zwingend mit dem chronologischen Alter auftritt, ist die persönliche Einschätzung des eigenen sozialen Umfelds besonders relevant.

Zuletzt wird das psychische subjektive Alter betrachtet. Dieser Faktor des Alters bezieht sich auf die psychische Spannkraft und Belastbarkeit einer Person (Weinert 1994; Kade 2009). Das psychische Alter umfasst die persönliche Einschätzung der vorhandenen Kompetenzen, Einstellungen und Motive einer Person (Staudinger 2012).

Die Funktion einer Weiterbildungsteilnahme und die Motivation zu ihr werden als abhängige Variablen betrachtet, die entscheidenden Einfluss auf die Wahrnehmung und Einschätzung einer Teilnahme besitzen.

Die Motivation zu einer Teilnahme wird nach der Selbstbestimmungstheorie (SDT) (Deci/Ryan 2008, 2012) in selbstbestimmte (intrinsische und identifizierte) und extrinsische Motivation bzw. Amotivation aufgeteilt. Insbesondere aufgrund der Freiwilligkeit der Teilnahme der Befragten erscheint die Frage nach selbst- bzw. fremdbestimmten Teilnahmegründen besonders relevant.

Unter der Funktion einer Weiterbildung wird die subjektive Bedeutung einer Teilnahme (von Hippel/Röbel 2016) verstanden. Sie beschreibt die Aufgabe, die einer Weiterbildungseilnahme im Lebenslauf zugesprochen wird. Insbesondere berufliche, aber auch persönliche Gründe werden damit erfasst. Zusätzlich ist davon auszugehen, dass eine Teilnahme mit einem Interesse an sozialem Austausch mit anderen Teilnehmenden oder der Bedeutung einer Lerngruppe einhergehen kann (soziale Funktion).

Zusätzlich werden Erwerbsstatus, Geschlecht sowie Bildungsstatus kontrolliert.

\section{Hypothesen}

Im Folgenden werden Hypothesen aus dem theoretischen Modell abgeleitet, die relevant für die Beantwortung der leitenden Fragen dieses Beitrags sind.

$\mathrm{H}_{1}$ : Das subjektive Alter teilt sich in drei Bereiche (kognitiv, sozial, psychisch) auf.

Da die Überprüfung der $\mathrm{H}_{1}$ Konsequenzen für die UVs hat, die für die weiteren Annahmen zur Verfügung stehen, wird auf die Ergebnisse hierzu bereits an dieser Stelle verwiesen. Die Faktorenstuktur wurde mittels Hauptkomponentenanalysen und explorativer Faktorenanalysen mit orthogonaler Varimax-Rotation untersucht (Bühner 2011; Werner 2014). Nach Kaiser-Guttmann-Kriterium wird die Anzahl zu extrahierender Faktoren festgelegt (Döring/Bortz 2016). Die Analyse ergibt, dass das subjektive Alter nicht wie angenommen 
drei, sondern nur zwei Faktoren (kognitives Alter und sozial-psychisches Alter) umfasst.

$\mathrm{H}_{2 \mathrm{a}}$ : Mit steigendem subjektivem sozial-psychischem Alter nimmt die intrinsische Motivation zur Teilnahme an einer Weiterbildung ab.

$\mathrm{H}_{2 \mathrm{~b}}$ : Mit steigendem subjektivem sozial-psychischem Alter nimmt die extrinsische Motivation zur Teilnahme an einer Weiterbildung zu.

Es ist zu erwarten, dass die Planung und Teilnahme an einer Weiterbildung mit Anstrengungen einhergeht, die sozial-psychisch Ältere nur aufgrund extrinsischen Drucks in Angriff nehmen. Daher wird von einer Abnahme der intrinsischen und einer Zunahme der extrinsischen Motivation ausgegangen.

$\mathrm{H}_{3 \mathrm{a}}$ : Mit steigendem subjektivem kognitivem Alter nimmt die berufliche Funktion einer Weiterbildungsteilnahme ab.

$\mathrm{H}_{3 \mathrm{~b}}$ : Mit steigendem subjektivem sozial-psychischem Alter nimmt die berufliche Funktion einer Weiterbildungsteilnahme ab.

In Anlehnung an das Modell der Optimierung durch Selektion und Kompensation (SOK) (Baltes/Baltes 1989b, 1989a) ist zu erwarten, dass mit steigendem Alter die Bedeutung der beruflichen Funktion abnimmt und dies durch persönliche Teilnahmegründe kompensiert wird. Darüber hinaus wird angenommen, dass Personen, die sich kognitiv älter fühlen als sie chronologisch sind, weniger berufliche Chancen mit der Teilnahme an Weiterbildung verbinden. Auch hier ist erneut davon auszugehen, dass sich mit der nachlassenden psychischen Spannkraft des sozial-psychischen Alters die Bedeutung, die einer Weiterbildung zugesprochen wird, verringert.

$\mathrm{H}_{4}$ : Das subjektive sozial-psychische Alter hängt mit der sozialen Funktion einer Weiterbildungsteilnahme zusammen.

Anhand der ursprünglich erwarteten dreistufigen Faktorenstruktur des subjektiven Alters war anzunehmen, dass mit steigendem sozialem Alter und dem damit einhergehenden schrumpfenden sozialen Umfeld die soziale Funktion einer Weiterbildung zunimmt. Daneben wird für das psychische Alter ein generelles Nachlassen der Funktionen einer Weiterbildung, somit auch der sozialen Funktion, erwartet. Da nun nur ein Faktor zur Verfügung steht, ist die Hypothese ungerichtet zu testen. 


\section{Datengrundlage}

Zur Prüfung der Hypothesen wurden zwischen Mai und Oktober 2015 zwei Stichproben erfasst. Die erste Stichprobe (im Folgenden Stichprobe Theologie) umfasst Teilnehmende des Kurses „Theologie im Fernkurs - Grundkurs Theologie“ $(\mathrm{n}=265)$. Der Kurs wird von der Domschule Würzburg koordiniert, die Teilnehmenden stammen aus dem gesamten Bundesgebiet und sind zum Zeitpunkt der Befragung chronologisch durchschnittlich 46,44 Jahre alt (SD = 10,08). Das kognitive subjektive Alter liegt bei 40,82 Jahren ( $\mathrm{SD}=14,52)$, das sozial-psychische subjektive Alter bei 40,00 Jahren $(\mathrm{SD}=9,76)$. Diese jüngere Einschätzung des subjektiven gegenüber dem chronologischen Alter ist mit Ergebnissen der Studien von Goldsmith und Heiens (1992) sowie Rubin und Berntsen (2006) konform. Der Anteil weiblicher Befragter liegt bei 51,6\%. Personen mit akademischem Bildungsabschluss sind mit 57,21 \% in der Mehrzahl. Mit 76,68 \% sind die meisten Personen der Stichprobe erwerbstätig. Da es sich bei Stichprobe Theologie um einen Fernkurs und ein konfessionell orientiertes Bildungsangebot handelt, ist die Stichprobe sowohl methodisch als auch inhaltlich selektiv. Der Kurs verfügt über Präsenzphasen, die in der Befragung ebenfalls berücksichtigt wurden, methodisch steht allerdings die Bearbeitung von Lehrbriefen im Vordergrund.

Um dieser Selektivität zu begegnen, wurde eine zweite Stichprobe aus Teilnehmenden verschiedener Kurse an Industrie- und Handelskammern (IHK) und Volkshochschulen (VHS) erfasst (im Folgenden Stichprobe IHK/VHS) (n =441). Das durchschnittliche chronologische Alter liegt hier bei 44,79 Jahren $(\mathrm{SD}=14,52)$. Erneut weichen das kognitive subjektive Alter $(39,79 ; \mathrm{SD}=$ $13,01)$ und das sozial-psychische subjektive Alter $(38,72 ; \mathrm{SD}=11,73)$ hiervon nach unten ab. Mit 68,83\% ist die Mehrzahl der Befragten weiblich. Dies ist vermutlich auf die VHS-Teilnehmenden zurückzuführen, die insgesamt vorrangig weiblich sind (Huntemann/Reichart 2017). Auch die Anzahl von $67,38 \%$ Teilnehmenden mit einem nicht-akademischen als höchstem Abschluss ist auffällig, wenn man dies etwa mit den Daten des AES vergleicht, das Personen mit höheren Abschlüssen als größte Teilnehmendengruppe der Weiterbildung aufzeigt (Bilger et al. 2017). Es kann vermutet werde, dass diese hohe Repräsentation von Personen mit mittleren Bildungsabschlüssen vor allem auf die IHK-Teilnehmenden zurückzuführen ist, da die IHK insbesondere Personen mit mittleren Abschlüssen für Aufstiegsfortbildungen adressiert. 


\section{Operationalisierung}

Das subjektive Alter wird mit der Selbsteinschätzung der Befragten anhand kontinuierlicher, metrischer Altersangabe zum kognitiven und sozial-psychischen Alter erfasst. Das kognitive Alter wird anhand der Bereiche Konzentration, Gedächtnis und Aufmerksamkeitsspanne ermittelt. Das sozial-psychische Alter umfasst die Aspekte Freizeitaktivitäten, Freundschaften, Teilnahme an gesellschaftlichen Ereignissen, Lebensfreude, Seelische Belastbarkeit und Ausgeglichenheit.

Um die Funktion einer Weiterbildungsteilnahme zu erfassen, schätzen die Befragten verschiedene Aussagen auf einer vierstufigen Skala $(1=$ stimmt überhaupt nicht bis $4=$ stimmt völlig) ein. Hauptkomponenten- und explorative Faktorenanalysen ergeben drei Faktoren: Berufliche, soziale und persönliche Funktion. Das Cronbachs $\alpha$ der drei Skalen liegt in beiden Studien zwischen 0,77 und 0,96, die Reliabilität ist somit gegeben.

Die Motivation der Teilnehmenden wurde mittels Situational Motivation Scale (SIMS) (Guay/Vallerand/Blanchard 2000) gemessen. Die Skala erfasst die Konstrukte intrinsische, identifizierte, extrinsische und Amotivation. Auch hier liegen Werte des Cronbachs $\alpha$ zwischen 0,74 und 0,87. Einzig die Reliabilität der Variable identifizierte Motivation ist in Stichprobe Fernkurs $(\alpha=$ $0,68)$ und Stichprobe VHS/IHK $(\alpha=0,52)$ eingeschränkt, was bei der Auswertung und Interpretation der Ergebnisse zu berücksichtigen ist.

\section{Ergebnisse}

Die Auswertung der Daten erfolgte mit Strukturgleichungsmodellen (SEM). Fehlende Werte wurden mittels Full information maximum likelihood (FIML) geschätzt (Lüdtke et al. 2007; Geiser 2010).

Die Güte der einzelnen Pfadmodelle wurde anhand verschiedener Modellindizes überprüft und ist in nahezu allen Fällen als gegeben anzusehen (Wollschläger 2014; Backhaus et al. 2016). Einzig das Modell „Berufliche Funktion" weist eine verringerte Modellgüte auf, die bei der Interpretation zu berücksichtigen ist.

Die in Abbildung 2 bis 5 dargestellten Modelle stellen die Pfade dar, die untersucht wurden. Die Pfeilrichtung gibt dabei die Richtung des untersuchten Zusammenhangs an. 


\section{Motivation}

Der Zusammenhang des chronologischen Alters und der intrinsischen Motivation wird in Abbildung 2 deutlich. Sowohl in Stichprobe Fernkurs als auch in $I H K / V H S$ zeigt sich ein signifikant positiver Zusammenhang, der veranschaulicht, dass mit höherem chronologischem Alter die intrinsische Motivation zunimmt. Darüber hinaus ergibt sich nur in Studie Fernkurs ein signifikant negativer Zusammenhang zwischen subjektivem Alter und intrinsischer Motivation.

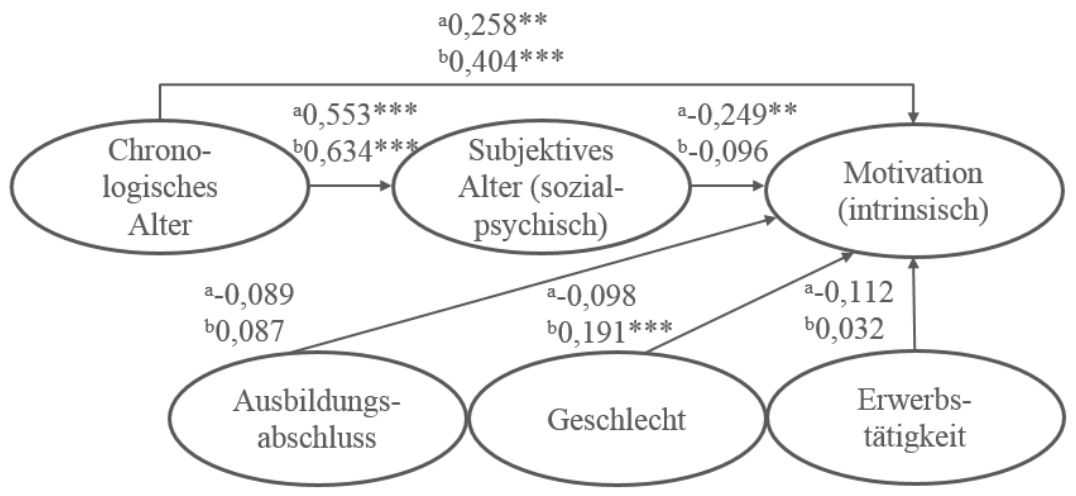

Abbildung 2: Zusammenhang Alter und intrinsische Motivation (eigene Darstellung); Anmerkungen: aStudie Fernkurs, bStudie IHK/VHS; ${ }^{*} p \leq 0,05 ;{ }^{* *} p \leq 0,01$; ${ }^{* * *} p \leq 0,001$

Während die intrinsische Motivation in Zusammenhang mit den Altersvariablen steht, zeigt sich dies für die extrinsische Motivation nicht. Weder das chronologische noch das subjektive Alter zeigt einen überzufälligen Zusammenhang. Es lässt sich vermuten, dass das Alter sich auf selbstbestimmte, aber weniger auf fremdbestimmte Motivation auswirkt. 


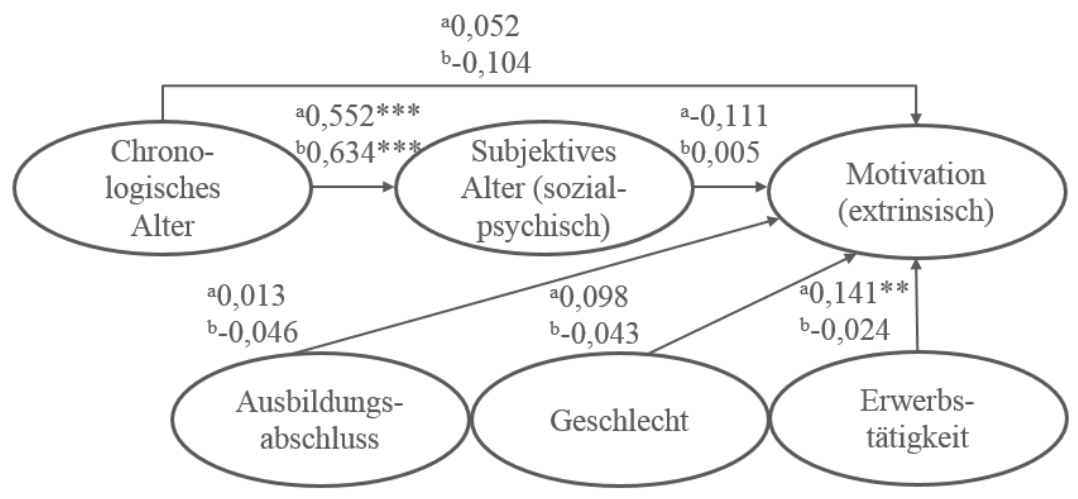

Abbildung 3: Zusammenhang Alter und extrinsische Motivation (eigene Darstellung): Anmerkungen: aStudie Fernkurs, bStudie IHK/VHS; ${ }^{*} p \leq 0,05 ;{ }^{* *} p \leq 0,01$; ${ }^{* * *} p \leq 0,001$

\section{Funktion}

Die berufliche Funktion steht in keiner der beiden Stichproben in Zusammenhang mit den subjektiven Altersvariablen. Darüber hinaus zeigt sich, dass nur in Stichprobe IHK/VHS ein signifikant negativer Zusammenhang zwischen chronologischem Alter und beruflicher Funktion besteht. Dies ist überraschend, da auf Grundlage bisheriger Studien zu erwarten war, dass das Lebensalter die Teilnahme an Weiterbildung aus beruflichen Gründen deutlich negativ beeinflusst (Tippelt/Schmidt/Kuwan 2009; Bilger et al. 2017).

In beiden Stichproben steht die soziale Funktion sowohl mit dem chronologischen als auch mit dem subjektiven Alter in signifikantem Zusammenhang. Mit höherem chronologischem Alter nimmt die soziale Funktion zu, mit höherem sozial-psychischem Alter hingegen ab. 
${ }^{a}-0,167$

b- $0,437 * * *$

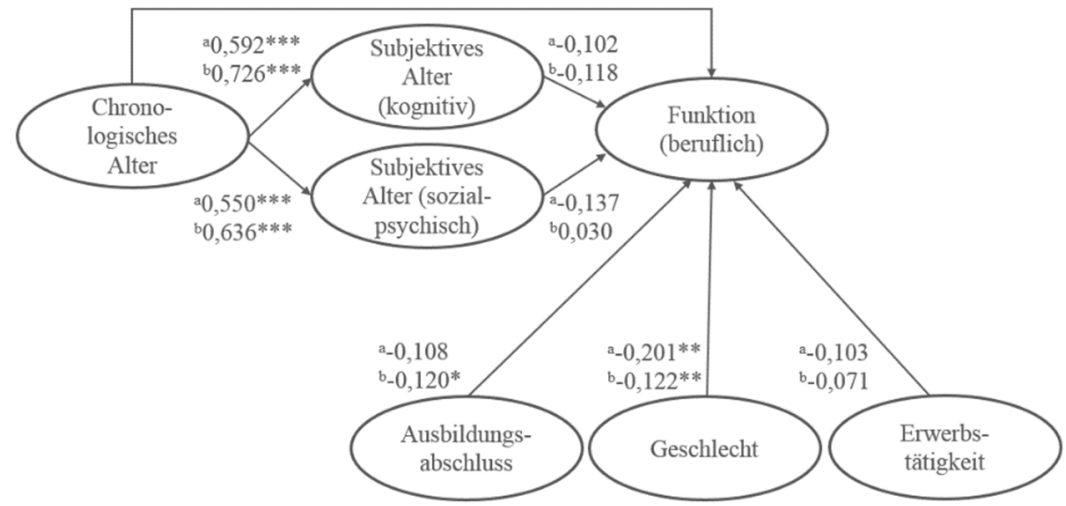

Abbildung 4: Zusammenhang Alter und berufliche Funktion (eigene Darstellung); Anmerkungen: a Studie Fernkurs, bStudie IHK/VHS; ${ }^{*} p \leq 0,05 ;{ }^{* *} p \leq 0,01$; ${ }^{* * *} p \leq 0,001$

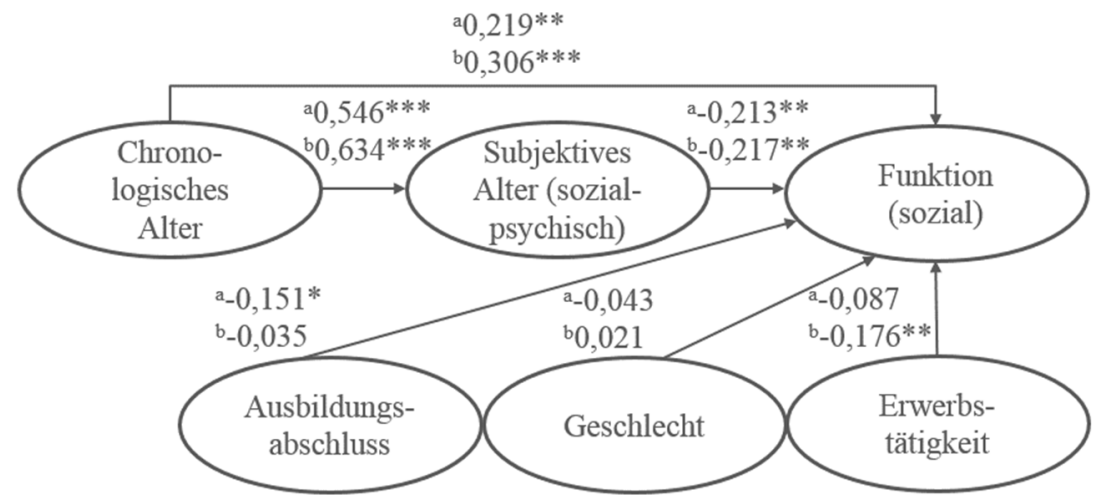

Abbildung 5: Zusammenhang Alter und soziale Funktion (eigene Darstellung) ; Anmerkungen: aStudie Fernkurs, ${ }^{b}$ Studie IHK/VHS; ${ }^{*} p \leq 0,05$; ${ }^{* *} p \leq 0,01$; ${ }^{* * *} p \leq 0,001$ 


\section{Diskussion}

Mit höherem chronologischem Alter geht eine Zunahme an intrinsischer Motivation einher. Mit steigendem subjektivem sozial-psychischem Alter nimmt die intrinsische Motivation hingegen ab. Dies ergibt sich allerdings nur in Stichprobe Fernkurs. Über $\mathrm{H}_{2 \mathrm{a}}$ kann daher nicht eindeutig entschieden werden, der Zusammenhang scheint kontextabhängig zu sein. $\mathrm{H}_{2 \mathrm{~b}}$ ist abzulehnen, da die extrinsische Motivation weder mit dem subjektiven noch dem chronologischen Alter in Zusammenhang steht.

Die berufliche Funktion steht mit keiner der betrachteten subjektiven Altersvariablen in Zusammenhang, $\mathrm{H}_{3 \mathrm{a}}$ und $\mathrm{H}_{3 \mathrm{~b}}$ sind daher abzulehnen. Auffällig ist hierbei vor allem, dass nur in Stichprobe IHK/VHS ein negativer Zusammenhang des chronologischen Alters mit der beruflichen Funktion besteht. Aufgrund bisheriger Forschung (Tippelt/Schmidt/Kuwan 2009; Bilger et al. 2017) wurde ein deutlicherer und kontextunabhängiger Zusammenhang vermutet.

Hypothese $\mathrm{H}_{4}$ kann angenommen werden, da das subjektive sozial-psychische Alter in beiden Stichproben mit einer negativen sozialen Funktion einhergeht, während das chronologische Alter hiermit in positivem Zusammenhang steht.

Die Ergebnisse verdeutlichen, dass die subjektiven Altersvariablen das chronologische Alter nicht ersetzen können, aber eine zusätzliche Erklärkraft mit sich bringen. Besonders bei der Betrachtung sozialer Aspekte der Weiterbildung, aber auch hinsichtlich methodischer Überlegungen kann die Betrachtung des empfundenen Alters erkenntnisvertiefend sein. Mit Rückgriff auf die eingangs formulierten Forschungsfragen ist also zu sagen, dass das subjektive Alter nicht relevanter für Lebensentscheidungen ist als das chronologische Alter. Es kann aber als zusätzlicher Erklärungsansatz in der Weiterbildungsforschung herangezogen werden, insbesondere bei der Betrachtung sozialer Aspekte einer Teilnahme.

Da es sich bei Stichprobe Fernkurs aufgrund des didaktischen Settings und der konfessionellen Ausrichtung um eine methodisch und inhaltlich selektive Stichprobe handelt, ist dies bei der Interpretation der Ergebnisse zu berücksichtigen. Auch die anhand der Modellindizes eingeschränkte Güte des SEM zur beruflichen Funktion (vgl. Abb. 4) ist unter Vorbehalt zu interpretieren. Das Modell ermöglicht eine Vielzahl weitere Pfade, die die Güte beeinträchtigen, die aber vor dem theoretischen Hintergrund nicht sinnvoll sind. Der eher kleine Umfang der Stichproben ist zu berücksichtigen. Da die Befunde vorheriger Arbeiten aber repliziert werden konnten (z.B. zum Einfluss des chronolgischen Alters auf die intrisische Motivation), ist nicht von einer starken Verzerrung der Ergebnisse auszugehen. 
Die Ergebnisse legen nahe, dass die Entgrenzung im Bereich des Alters noch nicht so weit fortgeschritten ist, wie sich auf Basis des gesellschaftlichen Dikurses und theoretischer Überlegungen vermuten ließe. In Bezug auf die große Bedeutung von Weiterbildung vor dem Hintergrund gesellschaftlicher Transformation hin zum Fachkräftemangel ist zu berücksichtigen, dass nicht allein das (chronologische) Alter die Teilnahme aus beruflichen Gründen beeinflusst.

Da sich die theoretisch erwartete dreifaktorielle Struktur des subjektiven Alters empirisch nicht zeigt, bietet sich eine Weiterentwicklung des Konstrukts an. Hierzu wird die Forschung zu Altersbildern herangezogen. Altersbilder umfassen Vorstellungen und Erwartungen an den Altersprozess, Altersselbstbilder fokussieren dabei vor allem den eigenen Altersprozess (SchmidtHertha/Mühlbauer 2012; Staudinger 2012). Der Vorteil der Erfassung von Altersselbstbildern liegt in der auf die Zukunft gerichteten Perspektive, die mit diesem Konstrukt einhergeht. Es ist davon auszugehen, dass zukunftsorientierte Erwartungen die Handlung und Planung, die z.B. mit der Aufnahme einer Weiterbildung einhergehen, stärker beeinflussen als die Einschätzungen der gegenwärtigen Situation (Bandura 1986). Es konnte bereits gezeigt werden, dass negative Altersselbstbilder zu weniger positiver Einflussnahme auf den eigenen Altersprozess führen (Schmidt-Hertha/Mühlbauer 2012; Staudinger 2012). Hieran lässt sich die Vermutung anschließen, dass eine solche Einflussnahme sich auch bei Weiterbildungsteilnahmen zeigt und diese positiv mit positiven Altersselbstbildern zusammenhängen. Weitere Forschung hierzu erscheint sinnvoll, da die vorliegende Arbeit bereits die eigene Erklärkraft und Bedeutung des subjektiven Alters in Ansätzen zeigen konnte und mit einer Überarbeitung des Konstrukts vertiefende Erkenntnisse zu erwarten sind.

\section{Literatur}

Backhaus, K. et al. (2016): Multivariate Analysemethoden. Eine anwen-dungsorientierte Einführung. 14. Aufl. Berlin \& Heidelberg: Springer Gabler.

Baltes, P. B. / Baltes, M. M. (1989a): Erfolgreiches Altern: Mehr Jahre und mehr Leben. In: Baltes, M. M. / Kohli, M. / Sames, K. (Hrsg.): Erfolgrei-ches Altern. Bedingungen und Variationen. Bern, Stuttgart, Toronto: Verlag Hans Huber, S. $5-10$.

Baltes, P. B. / Baltes, M. M. (1989b): Optimierung durch Selektion und Kompensation - Ein psychologisches Modell erfolgreichen Alterns. In: Zeitschrift für Pädagogik 35, S. 85-105.

Bandura, A. (1986): Social foundations of thought and action: A social cognitive theory. Englewood Cliffs: Prentice-Hall. 
Bilger, F. et al. (Hrsg.) (2017): Weiterbildungsverhalten in Deutschland 2016. Ergebnisse des Adult Education Survey (AES). Survey - Daten und Berichte zur Weiterbildung. Bielefeld: wbv.

Bühner, M. (2011): Einführung in die Test- und Fragebogenkonstruktion. 3. Aufl. München: Pearson Studium.

Carstensen, L. L. (2006): The influence of a sense of time on human de-velopment. In: Science, 312, S. 1913-1915.

Deci, E. L. / Ryan, R. M. (2008): Self-Determination Theory: A macrotheory of human motivation, development, and health. In: Canadi-an Psychology 49, 3, S. $182-185$.

Deci, E. L. / Ryan, R. M. (2012): Self-Determination Theory. In: Van Lange, P. A. M. / Kruglanski, A. W. / Higgins, E. T. (Hrsg.): Handbook of theories of social psychology. Volume 1. London, Thousand Oaks, New Delhi, Singapore: Sage Publications, S. 416-437.

Döring, N. / Bortz, J. (2016): Datenanalyse. In: Döring, N. / Bortz, J. (Hrsg.): Forschungsmethoden und Evaluation in den Sozial- und Humanwis-senschaften. 5. Auflage. Berlin, Heidelberg: Springer, S. 597-784.

Filipp, S.-H. / Ferring, D. / Klauer, T. (1989): Subjektives Alterserleben - ein Merkmal erfolgreichen Alterns? In: Baltes, M. M. / Kohli, M. / Sames, K. (Hrsg.): Erfolgreiches Altern. Bedingungen und Variationen. Bern, Stuttgart, Toronto: Verlag Hans Huber, S. 296-300.

Geiser, C. (2010): Datenanalyse mit Mplus. Eine anwendungsorientierte Einführung. 2. Auflage. Wiebaden: VS Verlag für Sozialwissenschaften.

Goldsmith, R. E. / Heiens, R. A. (1992): Subjective age: A test of five hy-potheses. In: The Gerontologist 32, 3, S. 312-317.

Guay, F. / Vallerand, R. J. / Blanchard, C. (2000): On the assessment of situational intrinsic and extrinsic motivation: The Situational Motivation Scale (SIMS). In: Motivation and Emotion 24, 3, S. 175-213.

Hippel, A. von / Röbel, T. (2016): Funktionen als akteursabhängige Zu-schreibung in der Programmplanung betrieblicher Weiterbildung. In: Report Zeitschrift für Weiterbildungsforschung 39, 1, S. 61-81.

Huntemann, H. / Reichart, E. (Hrsg.) (2017): Volkshochschul-Statistik. 55. Folge, Arbeitsjahr 2016. DIE Survey Daten und Berichte der Weiterbil-dung. Bielefeld: wbv.

Kade, S. (2009): Altern und Bildung: Eine Einführung. 2., aktualisierte und überarbeitete Auflage. Bielefeld: Bertelsmann.

Künemann, H. / Kohli, M. (2010): Soziale Netzwerke. In: Aner, K. / Karl, U. (Hrsg.): Handbuch Soziale Arbeit und Alter. Wiesbaden: VS Verlag für Sozialwissenschaften, S. 309-313.

Lüdtke, O. et al. (2007): Umgang mit fehlenden Werten in der psychologi-schen Forschung. Probleme und Lösungen. In: Psychologische Rundschau 58, 2, S. 103-117.

Rubin, D. C. \& Berntsen, D. (2006): People over forty feel $20 \%$ younger than their age: Subjective age across the lifespan. In: Psychonomic Bulletin \& Review 13, 5, S. 776-780. 
Sachverständigenkomission. (2010). Sechster Bericht zur Lage der älte-ren Generation in der Bundesrepublik Deutschland. Altersbilder in der Ge-sellschaft, Bundesministerium für Familie, Senioren, Frauen und Jugend.

Schmidt-Hertha, B. / Mühlbauer, C. (2012): Lebensbedingungen, Lebens-stile und Altersbilder älterer Erwachsener. In: Berner, F. / Rossow, J. / Schwitzer, K.-P. (Hrsg.): Individuelle und kulturelle Altersbilder. Expertisen zum Sechsten Altenbericht der Bundesregierung, Band 1. Wiesbaden: VS Verlag für Sozialwissenschaften, S. 109-149.

Staudinger, U. M. (2012): Fremd- und Selbstbild im Alter. Innen- und Au-ßensicht und einige der Konsequenzen. In: Graf Kielmansegg, P. / Häfner, H. (Hrsg.): Alter und Altern. Wirklichkeit und Deutung. Berlin, Heidelberg: Schneider Verlag, S. 187-200.

Tippelt, R. et al. (2009): Bildungsverständnis und -motivation Älterer. In: Tippelt, R. et al. (Hrsg.): Bildung Älterer. Chancen im demografischen Wan-del. Bielefeld: Bertelsmann, S. 174-187.

Tippelt, R. / Schmidt, B. / Kuwan, H. (2009): Bildungsteilnahme. In: Tip-pelt, R. et al. (Hrsg.): Bildung Älterer. Chancen im demografischen Wandel. Bielefeld: Bertelsmann, S. 32-45.

Weinert, F. E. (1994): Altern in psychologischer Perspektive. In: Baltes, P. B. / Mittelstraß, J. / Staudinger, U. M. (Hrsg.): Alter und Altern: Ein interdis-ziplinärer Studientext zur Gerontologie. Berlin, New York: Walter de Gruyter, S. 180-203.

Werner, C. (2014): Explorative Faktorenanalyse: Einführung und analyse mit R. http://www.psychologie.uzh.ch/fachrichtungen/methoden/team/christinawerner/faktorenanalyse/explorative_faktorenanalyse_mit_r_cswerner.pdf $\quad[\mathrm{Zu}-$ griff: 16.02.2017].

Wollschläger, D. (2014): Grundlagen der Datenanalyse mit R. Eine an-wendungsorientierte Einführung. 3. Aufl. Berlin, Heidelberg: Springer Spekt-rum. 
Frank Behr, Christiane Hof

\section{Habituelle Grundlagen des Studierverhaltens. Annäherungen an die Bedingungen universitären Lernens}

Der vorliegende Band fragt nach „Erwachsenenbildung und Lernen in Zeiten von Globalisierung, Transformation und Entgrenzung." Die damit beschriebenen Veränderungen können Anlass sein, neue pädagogische Konzepte zur Unterstützung des Lernens von Erwachsener an verschiedenen Lernorten und in unterschiedlichen institutionellen Kontexten zu entwickeln. Sie können aber auch Ausgangspunkt für empirische Studien sein, die sich mit den spezifischen Bedingungen und Formen des Lernens von Erwachsener befassen. Anknüpfend an die Diagnose einer Entgrenzung bzw. Vervielfältigung der Lernorte widmet sich die Erwachsenenbildungsforschung zunehmend auch der Untersuchung der Formen und Bedingungen des Lernens Erwachsener jenseits der klassischen Institutionen der Weiterbildung. Damit kommen auch andere Lernorte - etwa das Lernen am Arbeitsplatz, im Verein, im Rahmen bürgerschaftlichen Engagements und nicht zuletzt auch an der Universität - in den Blick. Durch die empirische Untersuchung des hochschulischen Lehrens und Lernens kann die Erwachsenenbildungswissenschaft somit auch einen Beitrag zur Analyse des Lebenslangen Lernens leisten (Rhein 2015).

Der vorliegende Beitrag beleuchtet das Erwachsenenlernen anhand einer Studie zum Studierverhalten an der Universität. Der Fokus wird dabei auf die habituellen Bedingungen des Studierens gelegt. Die Ergebnisse weisen aber darauf hin, das Wechselverhältnis zwischen individuellen Lernvoraussetzungen und institutionellen Möglichkeitsräumen stärker in den Blick zu nehmen.

\section{Fragestellung und Konzeption der Untersuchung}

Die Hochschul- und Studierendenforschung verweist immer wieder auf ausgeprägte Unterschiede im Studierverhalten (z. B. Schulmeister 2014; Vogel \& Woisch 2013). Auf der Suche nach Erklärungen werden studienbezogene Einstellungen (Brahm \& Jenert 2015) und Kompetenzen (Helm 2015), fachkulturelle und didaktisch-methodische Rahmenbedingungen (Hebecker u.a. 2016) 
sowie sozio-ökonomische Bedingungen (Lörz 2012; Maaz, Watermann \& Daniel 2013) bzw. milieuspezifische und habituelle Bedingungen (Lange-Vester \& Sander 2016; Lörz 2017) herausgearbeitet.

Die Praxis des Studierens selbst wird allerdings nur selten in den Blick genommen. Diese wird vor allem durch die Erfassung von Studienzeiten (Zeitbudgets bzw. Studienworkload), die für Lehrveranstaltungen, selbstorganisiertes Lernen, Prüfungsvorbereitung etc. aufgewendet werden, beleuchtet (Lüders, Eisenacher \& Plessmann 2006; Schulmeister \& Metzger 2011). Näher an den Studieraktivitäten sind Arbeiten, die das Engagement der Studierenden in den Blick nehmen: So kommt etwa der Literaturreport von Kuh et al. $2006 \mathrm{zu}$ folgendem Schluss: „In fact, the best predictor of college grades is the combination of an individual student's academic preparation, high school grades, aspirations, and motivation. Once students start college, however, another key factor in their success ... is, ,student engagement', or the extent to which they take part in educationally effective practices" (Kuh et al. 2006: 31). Auch die Untersuchung von Georg und Bargel (2012: 396), zeigt, dass ein Studium vor allem dann zu höheren Erträgen führt, wenn es aktiv und engagiert, autonom und zielgerichtet betrieben wird".

Vor diesem Hintergrund scheint es sinnvoll, das Lernen an der Universität nicht anhand der Learning-Outcomes - und damit anhand des Studienerfolgs zu erfassen, sondern das Studierverhalten selbst in den Blick zu nehmen. Theoretisch ist dabei anzuknüpfen an Konzepte, die das Studieren als soziale Praxis beschreiben (vgl. Lave \& Wenger 1991; Hof 2018: 194ff.) und dabei die Aktivitäten und Praktiken der Lernenden in den Blick nehmen (zur praxistheoretischen Fundierung dieser Perspektive vgl. Schatzki 2001, 2002).

Solche Studierpraktiken können sich konkret beziehen auf formale Studienorte (Partizipation an und Engagement in Lehrveranstaltungen, z.T. unter Präsenzpflicht), auf non-formale Unterstützungsangebote (außerfachliche Lernangebote, Vorträge, Auslandssemester etc.) oder auf informelle Lern- und Studienaktivitäten jenseits formaler Lernangebote im Rahmen des Selbststudiums (selbstorganisierte Lerngruppen, studentische Arbeitsgemeinschaften etc.). Studierverhalten wird in diesem Sinne verstanden als aktive Nutzung formaler, non-formaler und informeller Lerngelegenheiten im Studium und an der Universität. 


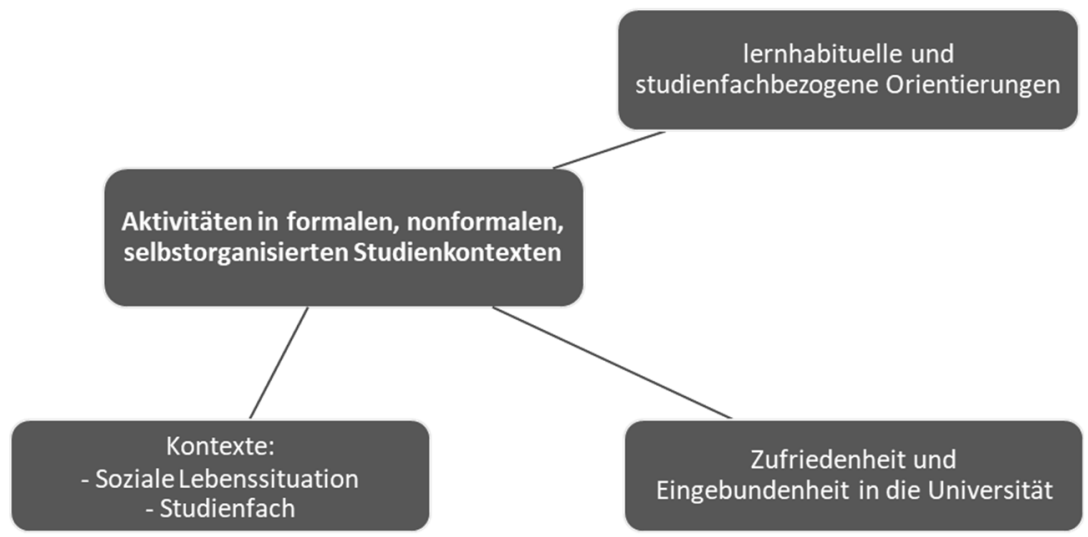

Abbildung 1. Theoretisches Modell zum Studierverhalten (eigene Darstellung).

Das Studierverhalten steht - so unsere Annahme - in einem Zusammenhang mit fachkulturellen und lebensweltlichen Rahmenbedingungen ebenso wie mit individuellen lernhabituellen und studienfachbezogenen Orientierungen. Die Bedingungen und studentischen Orientierungen beeinflussen die Studienzufriedenheit bzw. das Gefühl der Eingebundenheit in die Universität. Im vorliegenden Betrag wird der Fokus auf die Beschreibung des Studierverhaltens gelegt und dabei der Einfluss lernhabitueller Orientierungen der Studierenden auf das Studierverhalten untersucht. Im Mittelpunkt stehen damit Fragen nach konkreten Praktiken des Studierens sowie nach dem Einfluss lernhabitueller Orientierungen auf das Studierverhalten. ${ }^{1}$

Damit knüpfen wir auf der einen Seite an diejenigen Arbeiten der Studierendenforschung an, die nach den personalen Bedingungen des Studierens fragen. Auf der anderen Seite aber eröffnen der Rekurs auf Bourdieu und praxistheoretische Konzepte auch die Möglichkeit bzw. die Notwendigkeit, die Frage $\mathrm{zu}$ stellen, welche lernhabituellen Orientierungen eine bessere Passung zu den im Feld der Universität erwarteten Verhaltensweisen bzw. Studierpraktiken aufweisen.

Zur empirischen Klärung der Forschungsfragen wurde 2018 eine standardisierte Online-Befragung von Studierenden verschiedener Studiengänge an der Goethe-Universität in Frankfurt und der Universität Koblenz-Landau durchgeführt. Die vorliegende Untersuchung basiert auf einer Stichprobe von insgesamt 1.234 Studierenden (Goethe-Universität Frankfurt am Main: 1.100; Universität Koblenz-Landau: 134).

1 Die gesamte Studie zum Studierverhalten untersucht darüber hinaus noch weitere Fragen - etwa den Einfluss fachkultureller Rahmungen und sozialer Lebensbedingungen auf das Studierverhalten. Darüber hinaus suchen wir nach Bedingungen erfolgreichen Studierens sowie den Veränderungen von Studierpraktiken über die Zeit. 


\section{Lernhabituelle Orientierungen als Grundlage für Studierverhalten}

Sozialstrukturellen Bedingungen und den dadurch vermittelten habituellen Orientierungen wird eine besondere Bedeutung für die Lebensgestaltung - und damit eben auch für bildungsbezogene Aktivitäten - zugewiesen (Bremer 2007; Bourdieu \& Passeron 1971). Im Anschluss an Bourdieu wird davon ausgegangen, dass die Mehrzahl aller Lernaktivitäten - und damit auch das individuelle Studierverhalten - habitualisiert sind. Das Habituskonzept fokussiert dabei die inkorporierten gesellschaftlichen Strukturen und sozial erworbenen Wahrnehmungs-, Bewertungs- und Handlungsschemata, die das Studierverhalten beeinflussen. Ihm wird als durch Sozialisationsprozesse erworbene „strukturierende Struktur“ (Bourdieu 1987: 98) eine besondere Bedeutung für die Erklärung der Unterschiede im Lern- und damit eben auch Studierverhalten zugeschrieben (vgl. Behr 2017: 104ff.; Göhlich \& Zirfas 2007: 51). Dies erklärt sich insbesondere dadurch, dass Lernende anhand ihrer habituellen Orientierungen unbewusst vielfältige Lerngelegenheiten erkennen, bewerten und nutzen. In diesem Zusammenhang betont Bourdieu wiederholt die gewichtige Bedeutung der frühen „Erfahrungen“ in der Kindheit (Bourdieu 1987: 113), wobei er auch immer wieder darauf hinweist, dass eine Modifikation des Habitus im Rahmen der bestehenden Grundstrukturen und Grenzen möglich ist (Bourdieu 2001: 207).

Die Frage nach dem Einfluss lernhabitueller Orientierungen auf das Studierverhalten erfordert eine Operationalisierung dieser Variable. Für die vorliegende Untersuchung wurden die Skalen des Lernhabitus eingesetzt, die Frank Behr in Anlehnung an die theoretische Dimensionierung von Bourdieus entwickelt hat (Behr 2017). Die Anzahl der Items wurde für die hier vorliegende Untersuchung auf 14 reduziert und die Bezeichnung der Skalen namentlich entsprechend angepasst. Diese fünf lernhabituellen Skalen basieren auf den Dimensionen: familiäre Lernunterstützung (Beispielitem: „Meine Eltern waren mir in Lernangelegenheiten oft ein Vorbild“", Cronbachs $\alpha=.84$ ), Lernfreude (Beispielitem: „Seit früher Kindheit lerne ich, weil es mir Freude macht", Cronbachs $\alpha=.66$ ), Lernfokus (Beispielitem: „Lernen bedeutet für mich hauptsächlich, mit Situationen und Problemen im eigenen Leben umgehen zu können“, Cronbachs $\alpha=.78$ ), Lernpräferenzen (Beispielitem: „Schon als Kind lernte ich generell lieber gemeinsam mit anderen in einer Gruppe“, Cronbachs $\alpha=.76$ ) und Strukturiertheit (Beispielitem: „Wenn ich lerne, dann ist der Vorgang immer sehr geordnet und strukturiert", Cronbachs $\alpha=.77$ ). Fast alle Items weisen hohe Trennschärfen auf. Die Items wurden in Form von Statements formuliert, denen die Probanden auf einer 4-stufigen Ratingskala mehr oder weniger zustimmen konnten. 


\section{Darstellung der Ergebnisse}

\section{Lernhabituelle Orientierungen}

Um sinnvoll zu unterscheidende Gruppen zu bestimmen und die Komplexität des explorativen Datenmaterials zu reduzieren wurde mit den lernhabituellen Skalen als Klassifizierungsmerkmalen eine Two-Step Clusteranalyse in SPSS durchgeführt. Dadurch konnten insgesamt sieben Cluster identifiziert werden, die als unterschiedliche lernhabituelle Orientierungen interpretiert werden (s. Abbildung 2).

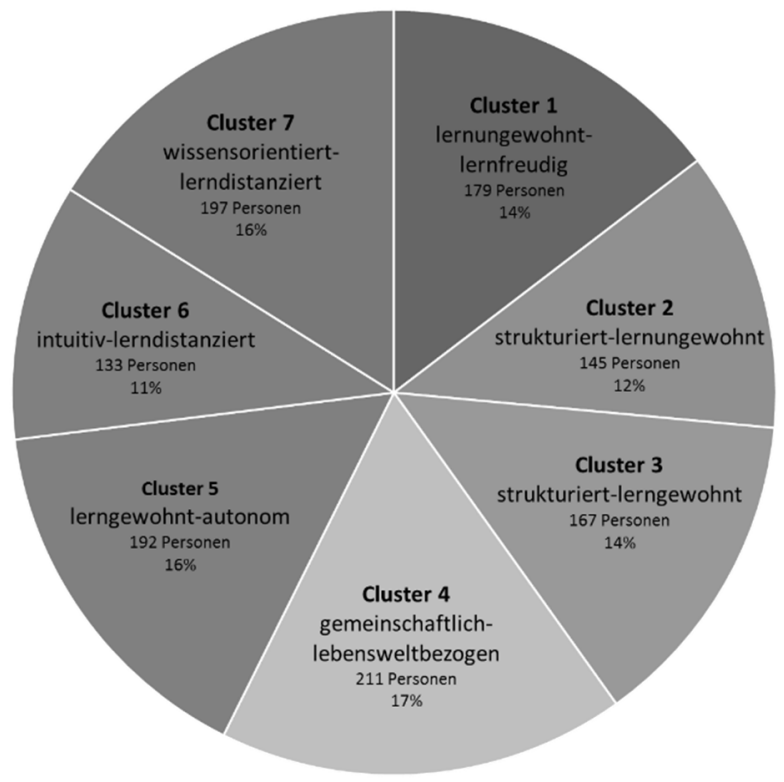

Abbildung 2. Größen der lernhabituellen Cluster; $n=1224$

Für die inhaltliche Beschreibung der Cluster wird auf die Verteilung der Mittelwerte der lernhabituellen Skalen innerhalb der einzelnen Cluster zurückgegriffen. Dabei fällt auf, dass diese Dimensionen für die Konstitution der einzelnen Cluster unterschiedlich ins Gewicht fallen und zwischen den einzelnen Clustern unterschiedliche Werte aufweisen.

Als besonders herausstechende Differenzierungsmerkmale erweisen sich die Skalen bezüglich der familiären Lernunterstützung, der Strukturiertheit beim Lernen sowie der Lernfreude. So verweisen hohe Mittelwerte bei der 
Skala Lernunterstützung in den Clustern 1 und 2 auf eine geringe Förderung des Lernens durch die Eltern (lernungewohnt). Niedrige Werte in den Clustern 3 und 5 drücken hingegen generell positive Lernerfahrungen in der Familie aus; die Eltern waren ihren Kindern bei Lernangelegenheiten oft ein Vorbild (lerngewohnt). Hinsichtlich der Lernfreude wird deutlich, dass die Personen aus Cluster 1 gewöhnlich gerne lernen (lernfreudig). Dagegen lernen Personen der Cluster 6 und 7 eher mit wenig Freude und meistens nur, wenn es unbedingt notwendig ist (lerndistanziert). Mit Blick auf die Strukturiertheit beim Lernen zeigt sich, dass Personen der Cluster 2 und 3 ihre Lernaktivitäten vorbereiten und beim Lernen sehr geordnet vorgehen (strukturiert). Im Gegensatz dazu zeichnen sich Personen des Clusters 6 eher durch unvorbereitete und unstrukturierte Lernhandlungen aus; der Vorgang des Lernens wird dementsprechend mehr dem Zufall überlassen und erfolgt eher spontan (intuitiv). Insgesamt unterscheiden sich die Profile der gebildeten Cluster hinsichtlich ihrer Einschätzungen bzw. Antwortmuster bei den lernhabituellen Dimensionen deutlich voneinander, weisen aber in Teilen auch Parallelen auf.

Im Hinblick auf eine weitere Interpretation wurden für die namentliche Bezeichnung nur die beiden wichtigsten lernhabituellen Dimensionen eines Clusters berücksichtigt, da die Unterschiede in der Werteverteilung bei diesen Skalen am größten ausfallen. Die Vergabe der Cluster-Namen stützte sich auf begriffliche Gegensatzpaare, die den diametralen Charakter der lernhabituellen Orientierungen bezeichnen.

\section{Aktivitäten in Lehrveranstaltungen}

Die Einschätzungen der Studierenden hinsichtlich der Relevanz verschiedener Handlungen und Aktivitäten in den Lehrveranstaltungen zeigen, dass vor allem aktives Mitdenken von über $90 \%$ als wichtig bzw. sehr wichtig angesehen wird. Auch das Anwenden und Einüben von Praktiken ist für vier Fünftel dort essentiell. Über $70 \%$ der Befragten ist es wichtig, in den Lehrveranstaltungen mitzuschreiben oder hauptsächlich zuzuhören. Sehr wichtig und wichtig sind für die Mehrheit auch das Stellen von Fragen (68 \%), das Besprechen von Texten $(61 \%)$, das Zuschauen bei Experimenten und Vorführungen (61 \%) sowie das Diskutieren der Lehrinhalte (55\%). In den Veranstaltungen selbst forschen und Texte lesen werden als wichtige Tätigkeiten gesehen (42\% und $32 \%$ ). Gruppenarbeit ist für ein Drittel von hoher Relevanz, d. h. für zwei Drittel ist dies weniger oder gar nicht wichtig. Ein Viertel der Befragten möchte in den Veranstaltungen ausschließlich anwesend sein und etwa ein Fünftel möchte dort wenig tun. Dem Halten von Referaten messen über vier Fünftel der Studierenden keine große Bedeutung zu. 
Die Vielzahl der unterschiedlichen Tätigkeiten in den Lehrveranstaltungen konnte mittels einer Faktorenanalyse auf vier Faktoren reduziert werden (Gesamtvarianzaufklärung: 55 \%). Auf den ersten Faktor laden performative Aktivitäten: referieren, selbst etwas vorführen, demonstrieren, zeigen, in Kleingruppen arbeiten, diskutieren und selbst forschend tätig sein. Mit dem zweiten Faktor werden passive Verhaltensweisen zusammengefasst: wenig tun müssen, nicht aktiv mitzudenken, ausschließlich anwesend sein, keine Fragen stellen. Der dritte Faktor bündelt textbezogene Aktivitäten: Texte besprechen, Texte lesen, mitschreiben. Rezeptive Aktivitäten sind kennzeichnend für den vierten Faktor: vorwiegend zuschauen und zuhören. Mittels Varianzanalysen wurde geprüft, ob sich Studierende mit verschiedenen lernhabituellen Orientierungen in ihren Aktivitäten während der Lehrveranstaltungen unterscheiden bzw. ob die Orientierungen einen Einfluss auf ihre Aktivitäten haben. Die simultane Überprüfung der vier Aktivitäten mittels einer multivariaten (einfaktoriellen) Varianzanalyse (MANOVA) bescheinigt einen signifikanten Gesamteffekt der lernhabituellen Cluster. Anschließende univariate Varianzanalysen ergeben signifikante Mittelwertunterschiede bei den Faktoren performatives Verhalten, textbezogene und rezeptive Aktivitäten (s. Tabelle 1). Posthoc-Mittelwertvergleiche mit dem Student-Newman-Keuls-Test zeigen, dass performative Handlungen (wie z. B. referieren und diskutieren) den Personen der Cluster 1 (lernungewohnt-lernfreudige Orientierungen), 3 (strukturiertlerngewohnte Orientierungen) und 4 (gemeinschaftlich-lebensweltbezogene Orientierungen) wichtiger sind als Personen, die zum Cluster 7 (wissensorientiert-lerndistanzierte Orientierungen) gehören. Studierende aus den Clustern 2 (strukturiert-lernungewohnt) und 3 (strukturiert-lerngewohnt) messen textbezogenen Aktivitäten mehr Bedeutung zu als Studierende mit wissensorientiertlerndistanzierten Orientierungen (Cluster 7). Bei rezeptiven Aktivitäten unterscheiden sich entsprechend Personen aus Cluster 7 und 6 (intuitiv-lerndistanziert) signifikant von der Personengruppe von Cluster 3 (strukturiert-lerngewohnt). 


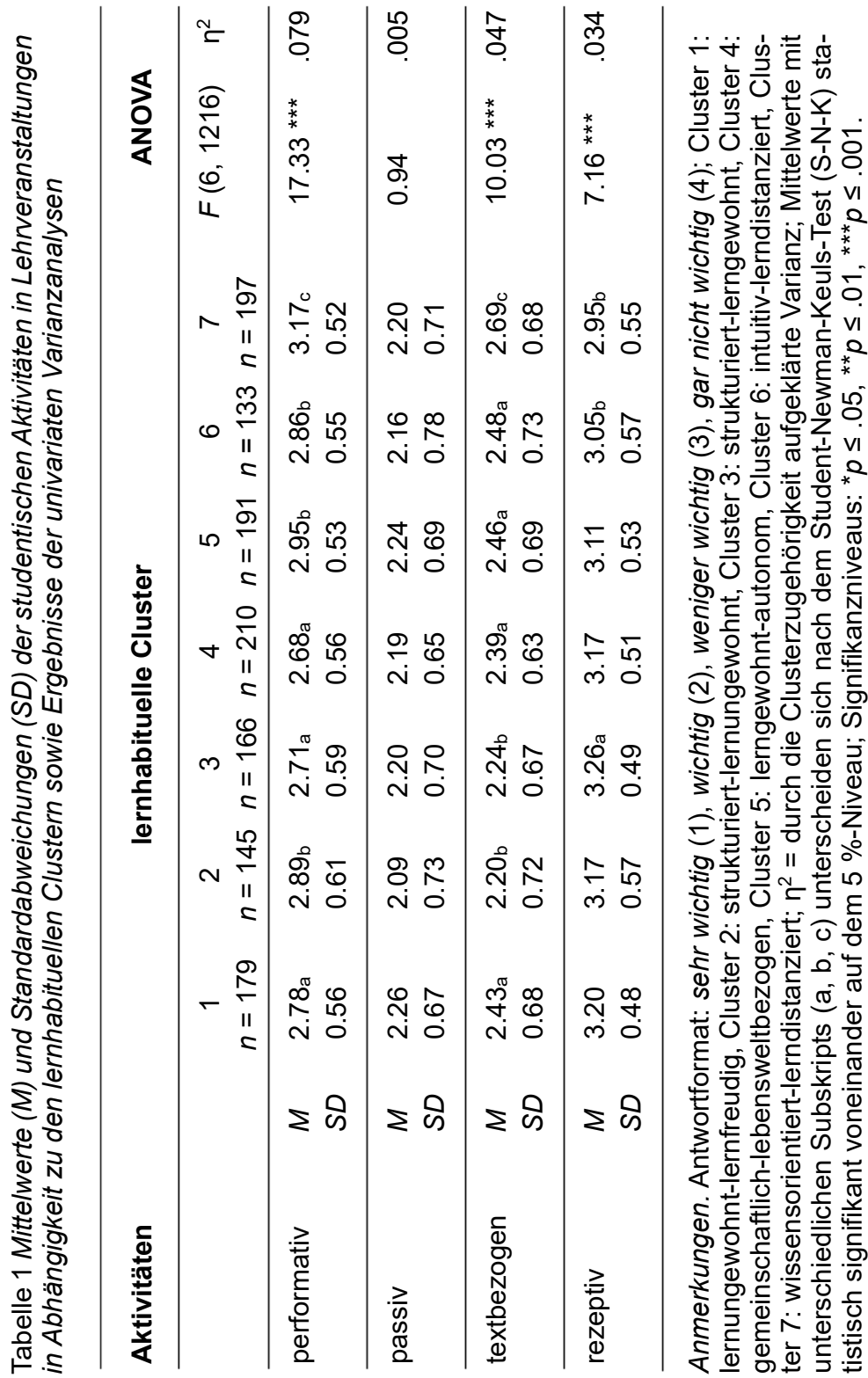




\section{Engagement in selbstorganisierten Lerngruppen}

Etwa die Hälfte der befragten Studierenden trifft sich häufig bis sehr häufig in selbstorganisierten Lerngruppen, um Prüfungen vorzubereiten (51 \%), um Referate oder Hausarbeiten gemeinsam zu erstellen (44 \%) und um Informationen zu beschaffen (46\%). Ungefähr $40 \%$ der Befragten engagieren sich in studentischen Gruppen, um sich bei Studienarbeiten gegenseitig zu unterstützen und um Studieninhalte zu diskutieren. Ein Drittel nutzt diese Treffen, um Arbeit aufzuteilen. Ungefähr jeder vierte Studierende gibt an, sich häufig bis sehr häufig mit Kommilitoninnen und Kommilitonen zu treffen, um gemeinsam Fachpraktiken zu üben oder gemeinsame Forschungsprojekte zu bearbeiten. Die Vor- und Nachbereitung von Seminaren wird nur für von jedem bzw. jeder sechsten Befragten als häufiger Grund angegeben.

Aus den 12 Variablen zum Engagement in Lerngruppen ließen sich mittels einer Faktorenanalyse exakt zwei Faktoren extrahieren, die insgesamt $62 \%$ der Varianz der Ausgangsvariablen erklären. Die höchsten Faktorladungen auf dem Faktor allgemeine Zusammenarbeit haben die Items „Studieninhalte diskutieren“, „Informationen beschaffen“ und „Prüfungen vorbereiten“. Auf den Faktor konkrete Projekte laden die Items, gemeinsam Referate und Hausarbeiten erstellen“, „gemeinsam Forschungsprojekte“ bearbeiten, „Aufteilen von Arbeit". Die Ergebnisse der multivariaten Varianzanalysen (MANOVA) ergeben einen statistisch signifikanten Gesamteffekt der lernhabituellen Cluster auf das Engagement in studentischen Lerngruppen. Univariate (einfaktorielle) Varianzanalysen (ANOVA) verdeutlichen, dass sich die Cluster hinsichtlich der allgemeinen Zusammenarbeit und des Engagements bei konkreten Projekten statistisch signifikant unterscheiden (s. Tabelle 2). Vergleiche der Mittelwerte über Post-hoc-Tests (S-N-K) verdeutlichen insbesondere, dass sich Studierende des Clusters 4 mit gemeinschaftlich-lebensweltbezogen Orientierungen eher häufig in Lerngruppen engagieren. Dagegen sind Personen aus dem Cluster 7 mit wissensorientiert-lerndistanzierten Orientierungen selten in Lerngruppen aktiv. Ihnen geht es - so könnte man interpretieren - um die Aneignung, aber weniger um die Verarbeitung von Wissen. 


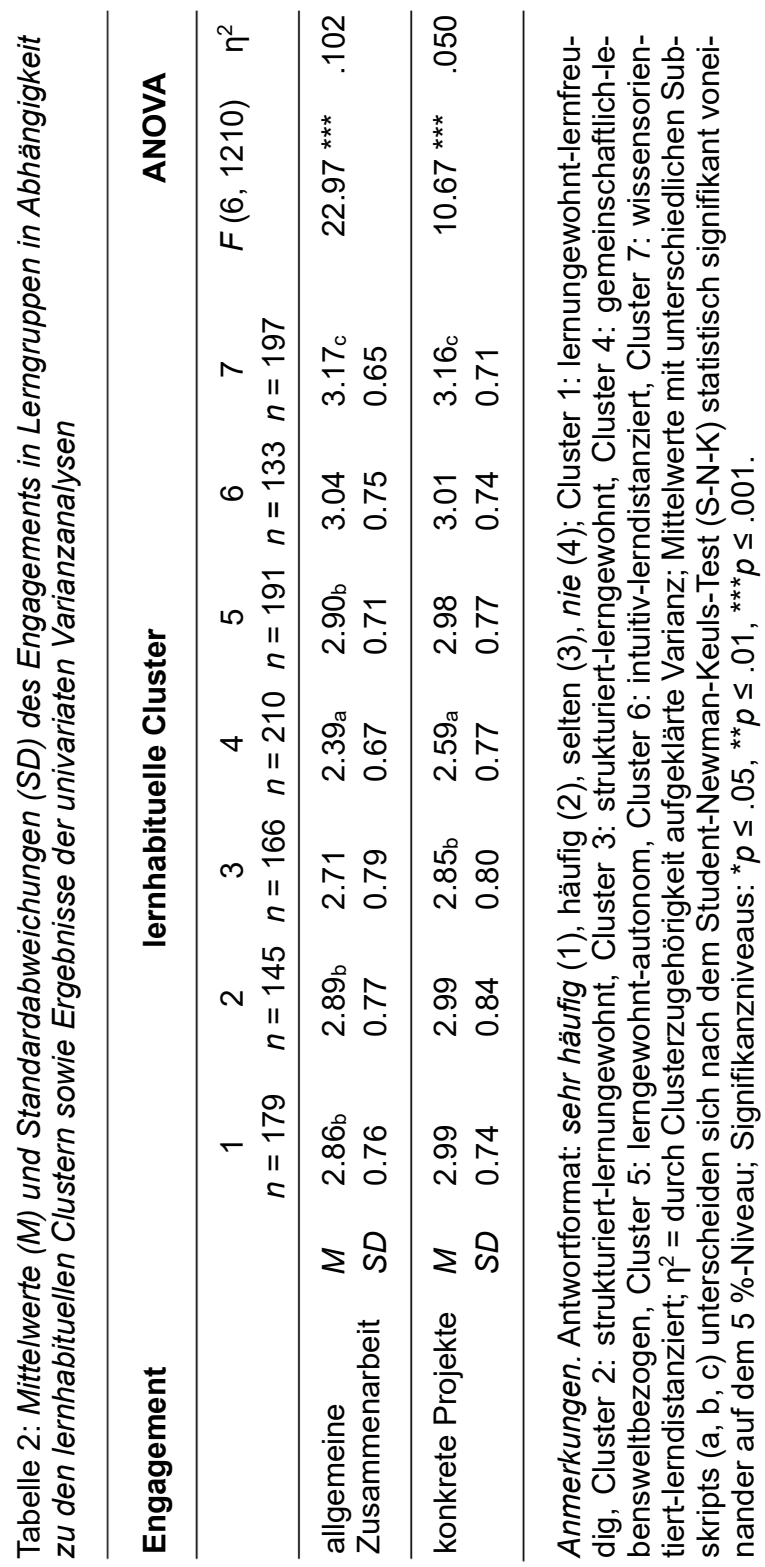




\section{Diskussion}

Mit Blick auf die formulierten Forschungsfragen brachte die Untersuchung des Studierverhaltens auf Basis einer standardisierten Befragung von Studierenden zusammenfassend drei wichtige Befunde:

Die deskriptiven Auswertungen der Querschnittsdaten verweisen zunächst einmal auf ausgeprägte Unterschiede im Studierverhalten und in den Lernaktivitäten. Die Studentinnen und Studenten unterscheiden sich deutlich hinsichtlich der vielfältigen Aktivitäten in Lehrveranstaltungen sowie hinsichtlich des Engagements in selbstorganisierten Lerngruppen.

Zweitens konnten die befragten Studierenden mittels einer clusteranalytischen Datenanalyse hinsichtlich ihrer lernhabituellen Orientierungen in unterschiedliche Cluster gruppiert werden. Die einzelnen Cluster unterscheiden sich in Bezug auf die lernhabituellen Dimensionen: familiäre Lernunterstützung, Lernfreude, Lernfokus, Lernpräferenzen und Strukturiertheit beim Lernen. Die Differenzen zwischen den Clustern sind allerdings nicht besonders deutlich, was auch ein Hinweis darauf sein könnte, dass andere Faktoren, etwa die Fachkulturen der verschiedenen Studiengänge oder die sozialen Lebenssituationen der Studierenden relevante Faktoren zur Beschreibung unterschiedlicher Studierendengruppen sind.

Ungeachtet dieser problematisierenden Hinweise ist festzuhalten, dass einzelne Cluster besonders auffallen: Cluster 3 (strukturiert-lerngewohnt) zeichnet sich durch sehr strukturierte Lernprozesse, frühe familiäre Lernunterstützung sowie durch eine hohe Lernfreude aus. Außerdem lernen Personen dieses Clusters am liebsten allein, d. h. ohne die Unterstützung von anderen. ${ }^{2}$ Cluster 4 (gemeinschaftlich-lebensweltbezogen) ist dagegen vor allem dadurch charakterisiert, dass diese Studentinnen und Studenten das gemeinsame Lernen in einer Gruppe präferieren. Zudem legen sie beim Lernen großen Wert auf ihre eigenen Erfahrungen. Der Fokus beim Lernen liegt bei diesen Personen auf der Entwicklung ihrer Persönlichkeit und auf der Anwendung von Wissen in konkreten Praxissituationen. Während diese beiden Cluster dem Lernen gegenüber im Allgemeinen sehr positiv eingestellt sind, ist Cluster 7 (wissensorientiertlerndistanziert) gekennzeichnet durch eine geringe Lernfreude und der Präferenz des Allein-Lernens ohne Unterstützung von anderen. Studierende aus diesem Cluster verfügen auch nicht über positive Erfahrungen einer familiären Lernunterstützung. Sie interessieren sich darüber hinaus nicht dafür, Wissen auf konkrete Situationen bzw. Probleme beziehen zu können.

2 Cluster 2 (strukturiert-lernungewohnt) zeigt ganz ähnliche Ausprägungen, ohne allerdings über die positive Erfahrung einer Lernunterstützung seitens der Familie zu verfügen. 
Die Ergebnisse der verschiedenen Varianzanalysen konnten drittens zeigen, dass Studierende der verschiedenen Cluster mit ihren unterschiedlichen lernhabituellen Orientierungen sich hinsichtlich der Aktivitäten in Lehrveranstaltungen und des Engagements in selbstorganisierten Lerngruppen signifikant unterscheiden. Die varianzanalytischen Befunde verweisen damit auf einen signifikanten Einfluss lernhabitueller Orientierungen auf das Studierverhalten.

Relationiert man diese lernhabituellen Cluster nun zusätzlich mit den im Rahmen dieses Beitrags untersuchten Studieraktivitäten, dann fällt besonders ins Auge, dass Cluster 3 (strukturiert-lerngewohnte Orientierungen) sich durch performatives Lernverhalten und textbezogene Aktivitäten auszeichnet, wogegen Cluster 7 mit wissensorientiert-lerndistanzierten Orientierungen dies nicht tut. Cluster 7 wiederum zeigt im Gegensatz zu Cluster 3 ein rezeptives und passives Lernverhalten in Lehrveranstaltungen und hat kein Interesse an sichtbaren Aneignungsaktivitäten (etwa die Arbeit am Text oder die Präsentation von erarbeitetem Wissen). In gleicher Weise grenzen sich Studierende des Clusters 4 mit ihren gemeinschaftlich-lebensweltbezogenen Orientierungen von anderen Gruppen ab, indem sie sich besonders häufig in studentischen Lerngruppen engagieren. Die lernbezogenen Präferenzen und Erfahrungen scheinen also mit bestimmten Studierpraktiken einherzugehen. Zugespitzt formuliert: wenig Lernfreude, unstrukturierte Vorgehensweisen, kaum gemeinschaftliches Lernen und negative familiäre Lernerfahrungen bedingen ein wenig aktives Studierverhalten.

Neben Lernfreude und Lernstrategien scheint aber auch die unterschiedliche Perspektive auf Wissen einen Einfluss auf das Studierverhalten zu haben. Denn Studierende, die primär an einer Aneignung von Wissen (und nicht der Verarbeitung von Wissen, der Erarbeitung von Problemlösungen) orientiert sind - und im Schelerschen Sinne (Scheler 1925) - kein Bildungswissen intendieren, präsentieren eher ein passives und rezeptives Studierverhalten. Umgekehrt zeigen Studierende, die sich nicht nur Wissen und Kenntnisse aneignen wollen, sondern diese als Grundlage für die Bearbeitung von Fragen und Probleme ansehen und Bezüge zur eigenen Lebenssituation herstellen wollen, signifikant höhere Zustimmung zu selbstorganisierten Lerngruppen (gemeinschaftliches Lernen) und Austausch/Diskussion/Kleingruppenarbeit im Seminar.

Man könnte diese Befunde so interpretieren, dass es Personen gibt, die den Anforderungen eines universitären Studiums sehr viel besser entsprechen (Cluster 2 und 3) als andere (Cluster 6 und 7), weil sie über die notwendigen Lernstrategien, die erforderliche Lernfreude und das ,richtige' Bildungsverständnis verfügen. Die Universität könnte diese Erkenntnisse als Aufforderung deuten, Eignungstests einzuführen oder Beratung anzubieten, um den Studienbewerberinnen und -bewerbern gegebenenfalls vom Studium abzuraten oder 
ihnen die erforderlichen Studierkompetenzen in besonderen Vorkursen zu vermitteln. Zudem bieten hochschuldidaktische Programme zur Professionalisierung und Verbesserung der Lehre vielfältige Möglichkeiten, die individuellen Kompetenzen der Lehrpersonen für eine studierendenzentrierte Lehre zu stärken (Rheinländer 2015).

Während diese Sichtweise das Studierverhalten in erster Linie als Ergebnis individueller Handlungsintentionen und -kompetenzen ansieht, verweisen etwa praxistheoretische Konzepte auf die Notwendigkeit, Aktivitäten und institutionelle Bedingungen in ihrem Wechselverhältnis zu betrachten. Entsprechend wird davon ausgegangen, dass das Studierverhalten nicht primär als Ausdruck individueller Handlungskompetenzen anzusehen ist, sondern als in spezifischen Handlungskontexten mögliche und sozial ,erlaubte' Aktivitäten. Die Frage „In Lehrveranstaltungen ist es mir wichtig ..." verweist demzufolge nicht nur auf die individuellen Wünsche und Erwartungen der Befragten, sondern auch auf die bisherigen Erfahrungen mit universitären Lehrveranstaltungen. Denn wenn ich vor allem in Vorlesungen sitze, dann dürfte die Arbeit in Kleingruppen oder das Halten von Referaten keine Relevanz haben. Die Antworten der Studierenden sind demzufolge nicht allein als individuelle Einschätzung zu werten, sondern auch als Beschreibung der (fachkulturell) vorhandenen Lehrformate. Mit anderen Worten: das Studierverhalten ist eingelagert in universitären Praktiken, verstanden ,als sozial geregelte, kulturell typisierte und organisierte Bündel menschlicher Aktivitäten“ (Alkemeyer 2013: 44 mit Bezug auf Schatzki 2002). Studierpraktiken hängen demzufolge nicht allein von den personalen Voraussetzungen der Studierenden ab, sondern sind eingelagert in gesellschaftliche Wissens- und Sinnordnungen, die sich in räumlichen Ordnungen, Idealbildern der Studierenden und spezifischen Kommunikations- und Interaktionsformen manifestieren. Sie determinieren nicht das Verhalten, begrenzen aber die Verhaltensspielräume bzw. legen spezifische Verhaltensweisen nahe. Für die Beteiligten bedeutet dies die Notwendigkeit, „Mitspielfähigkeit“ (Alkemeyer \& Buschmann 2016) zu erlangen. Um die Frage zu beantworten, welche individuellen und sozialen bzw. institutionellen Voraussetzungen auch jenseits lernhabitueller Orientierungen hilfreich sind, um erfolgreich an der Universität ,mitspielen' und damit auch Lernen zu können, bedarf es noch weiterer Auswertungen (nicht nur) unserer Daten.

\section{Literatur}

Alkemeyer, Thomas (2013): Subjektivierung in sozialen Praktiken: Umrisse einer praxeologischen Analytik. In: Gelhard, A. / Budde, G. / Freist, D. (Hrsg.): 
Selbst-Bildungen: soziale und kulturelle Praktiken der Subjektivierung. Bielefeld: Transcript-Verlag, S. 33-68.

Alkemeyer, Thomas / Buschmann, Nikolaus (2016): Praktiken der Subjektivierung - Subjektivierung als Praxis. In: Schäfer, H. (Hrsg.): Praxistheorie. Ein soziologisches Forschungsprogramm. Bielefeld: Transcript-Verlag, S. 115-136.

Behr, Frank (2017): Lernhabitus und Weiterbildung. Determinanten des Weiterbildungsverhaltens von Lehrerinnen und Lehrern. Studien zur Professionsforschung und Lehrerbildung. Bad Heilbrunn: Verlag Julius Klinkhardt.

Bourdieu, Pierre (1987): Sozialer Sinn. Kritik der theoretischen Vernunft. Frankfurt am Main: Suhrkamp.

Bourdieu, Pierre (2001): Meditationen. Zur Kritik der scholatischen Vernunft. Frankfurt am Main: Suhrkamp.

Bourdieu, Pierre / Passeron, Jean-Claude (1971): Die Illusion der Chancengleichheit. Untersuchungen zur Soziologie des Bildungswesens am Beispiel Frankreichs. Stuttgart: Ernst Klett.

Brahm, Taiga / Jenert, Tobias (2015): On the assessment of attitudes towards studying. Development and validation of a questionnaire. Learning and Individual Differences, 30, S. 233-242.

Bremer, Helmut (2007): Soziale Milieus, Habitus und Lernen. Zur sozialen Selektivität des Bildungswesens am Beispiel der Weiterbildung. Weinheim: Juventa.

Georg, Werner / Bargel, Timo (2012): Individuelle und institutionelle Bedingungen für den Studienertrag. In: ZSE Zeitschrift für Soziologie der Erziehung und Sozialisation, 4, S. 396-413.

Göhlich, Michael / Zirfas, Jörg (2007): Lernen. Ein pädagogischer Grundbegriff. Stuttgart: Kohlhammer.

Hebecker, Eike / Szczyrba, Birgit / Wildt, Beatrix (Hrsg.) (2016): Beratung im Feld der Hochschule. Formate - Konzepte - Strategien - Standards. Wiesbaden: Springer.

Helm, Christoph (2015): Determinants of competence development in accounting secondary education. In: Empirical Research in Vocational Education and Training 7: 1. DOI 10.1186/s40461-015-0022-8.

Hof, Christiane (2018): Der Lebenslauf als Rahmen für Lern- und Bildungsprozesse. In: Hof, C. / Rosenberg, H. (Hrsg.): Lernen im Lebenslauf. Theoretische Perspektiven und empirische Zugänge. Wiesbaden: Springer VS, S. 181-204.

Kuh, George D. / Kinzie, Jillian / Buckley, Jennifer A. / Bridges, Brian K. / Hayek, John C. (2006): What Matters to Student Success: A Review of the Literature. Commissioned Report for the National Symposium on Postsecondary Student Success: Spearheading a Dialog on Student Success. (https://nces.ed.gov/npec/ pdf/kuh_team_report.pdf).

Lange-Vester, Andrea / Sander, Tobias (Hrsg.) (2016): Soziale Ungleichheiten, Milieus und Habitus im Hochschulstudium. Beltz Verlag: Weinheim Basel.

Lave, Jean / Wenger, Etienne (1991): Situated learning. Legitimate peripheral participation. Cambridge: Cambridge University Press. 
Lörz, Markus (2017): Soziale Ungleichheiten beim Übergang ins Studium und im Studienverlauf. In: Baader, M. / Freytag, T. (Hrsg.): Bildung und Ungleichheit in Deutschland. Wiesbaden: Springer Fachmedien, S. 311-338.

Lüders, Manfred / Eisenacher, Sabine / Plessmann, Steffen (2006): Der Umgang mit Studienzeit. Eine empirische Untersuchung bei Studierenden der Lehrämter und im Diplom-Studiengang Erziehungswissenschaft. In: Allemann-Ghionda, C. / Terhart, E. (Hrsg.): Kompetenzen und Kompetenzentwicklung von Lehrerinnen und Lehrern. Zeitschrift für Pädagogik, 51. Beiheft. Weinheim: Beltz, S. $116-129$.

Maaz, Kai / Watermann, Rainer / Daniel, Annabell (2013): Effekte sozialer Herkunft auf den Übergang zur Hochschule. In: Asdonk, J. / Kuhnen, S. / Bornkessel, P. (Hrsg.): Von der Schule zur Hochschule: Analysen, Konzeptionen und Gestaltungsperspektiven des Übergangs. Münster: Waxmann, S. 50-76.

Rhein, Rüdiger (2015): Hochschulisches Lernen - eine analytische Perspektive. In: Zeitschrift für Weiterbildung, 38, 3, S. 347-363.

Rheinländer, Kathrin (2015): Von der Bedeutung und der Möglichkeit einer ungleichheitssensiblen Hochschullehre. In: Rheinländer, K. (Hrsg.): Ungleichheitssensible Hochschullehre. Positionen, Voraussetzungen, Perspektiven. Wiesbaden: Springer VS, S. 46-69.

Schatzki, Theodore R. (2001): Introduction: practice theory. In: Schaftzki, Th. R. / Knorr Cetina, K. / Savigny, E. von (Hrsg.): The Practice Turn in contemporary Theory. London/New York: Routhledge, S. 1-14.

Schatzki, Theodore R. (2002): The Site of the Social. A Philosophical Account of the Constitution of Social Life and Change. Pennsylvania: State University Press.

Scheler, Max (1925): Die Formen des Wissens und die Bildung. In: Ders.: Philosophische Weltanschauung. 3. Auflage 1968. Bern/München: Francke, S. $16-48$.

Schulmeister, Rolf (2014): Auf der Suche nach Determinanten des Studienerfolgs. In: Brockmann, J. / Pilniok, A. (Hrsg.): Studieneingangsphase in der Rechtswissenschaft. Baden-Baden: Nomos, S. 72-205.

Schulmeister, Rolf / Metzger, Christiane (2011): Die Workload im Bachelor: Zeitbudget und Studienverhalten. Eine empirische Studie. Münster: Waxmann.

Vogel, Bernd / Woisch, Andreas (2013): Orte des Selbststudiums. Eine empirische Studie zur zeitlichen und räumlichen Organisation des Lernens von Studierenden. In: HIS Forum Hochschule, 7, S. 12-13. 

Franziska Schulze-Stocker, Christian Schäfer-Hock

\section{Transformation von Hochschulen - \\ Transformation von Bildungswegen junger \\ Erwachsener: Frühwarnsysteme an Hochschulen in Deutschland}

\section{Einleitung}

Frühwarnsysteme stellen ein neues Angebot für Studierende dar. An Hochschulen werden sie genutzt, um anhand der Studienverlaufs- und Prüfungsdaten abbruchgefährdete Studierende zu identifizieren. Durch ein Signal, von außen“ (Hinweise auf Unterstützungs- und Beratungsangebote per E-Mail) soll die Reflexion und Aktivität der Studierenden angeregt werden (allgemein zu Frühwarnsystemen: Hahn/Krystek 1984; zur Situation an Hochschulen: Schulze-Stocker/Schäfer-Hock/Pelz 2017, 2019).

Diese Art von Frühwarnsystemen wird im vorliegenden Beitrag als Folge der Digitalisierung des Bildungssektors konzeptualisiert; zudem werden Ergebnisse einer qualitativen Befragung von Studierenden der Technischen Universität Dresden (TU Dresden) vorgestellt, die dazu dienen, den Aufbau eines solchen Frühwarnsystems evidenzbasiert zu steuern. Im Mittelpunkt steht der Umgang der Studierenden mit identifizierten Schwierigkeiten im Studium. Zentrale Fragen sind: Welche Typen von Problemlagen und Problembewältigungsstrategien lassen sich identifizieren? Und: Welche Identifizierungsmerkmale müssten darauf aufbauend in ein Frühwarnsystem aufgenommen werden?

\section{Rahmende Theorien und Entwicklungen}

\section{Transformation des Bildungssystems}

Deutschlands Bildungs- und Hochschulsystem lässt sich als ,soziotechnisches Gebilde“ (Dolata 2008: 44) konzeptualisieren, das im Zuge des „Megatrends 
[...] Digitalisierung“ (Käpplinger 2019: 19) eine technische Revolution erfährt. Der Begriff ,Revolution“ ist deshalb treffend, weil die Digitalisierung eine enorm hohe Eingriffstiefe hat und einen hohen Anpassungsdruck erzeugt. ${ }^{1}$ Es findet ein Wandel auf mindestens sechs Ebenen statt (Dolata 2008): Neben (1) dem technischen Profil des Bildungs- und Hochschulsystems bewirkt die Digitalisierung Änderungen (2) der Distributionsbedingungen (z.B. neue Lehrformate und neue Publikationsformen), (3) der Akteurskonfigurationen (z.B. Fortbildung des Personals), (4) der Interaktions- und Kommunikationsbedingungen (z.B. Social-Media-Kanäle zur ,Kundenbindung 'im Hochschulmarketing), (5) der Steuerungssysteme (z.B. Datenschutzregeln) und schließlich (6) der Systemgrenzen selbst (z.B. die Debatte über eine Datafizierung/Ökonomisierung; siehe unten).

Mit diesen tiefgreifenden Folgen der Digitalisierung lassen sich Phänomene auf der Makro-, Meso- und Mikroebene beobachten, die eine Implementierung von Frühwarnsystemen an Hochschulen ermöglichen bzw. beeinflussen (siehe Tabelle 1). ${ }^{2}$

Tabelle 1: Folgen des Wandels durch die Digitalisierung des Bildungs- und Hochschulsystems

\begin{tabular}{lll}
\hline Makro & Meso & Mikro \\
\hline $\begin{array}{l}\text { Mehr Regeln zum } \\
\text { Datenschutz }\end{array}$ & $\begin{array}{l}\text { (Live-)Studiengangsmoni- } \\
\text { toring und -management }\end{array}$ & $\begin{array}{l}\text { Online-Self- } \\
\text { Assessments zur } \\
\text { Unterstützung der } \\
\text { Studienwahl }\end{array}$ \\
\hline $\begin{array}{l}\text { Mehr Statistik- und } \\
\text { Berichtspflichten }\end{array}$ & $\begin{array}{l}\text { Gestiegener technischer, } \\
\text { personeller und finanziel- } \\
\text { ler Aufwand zur Etablie- } \\
\text { rung von Campus- } \\
\text { Management-Systemen }\end{array}$ & $\begin{array}{l}\text { (Live-)Stud- } \\
\text { ierendenmonitor- } \\
\text { ing }\end{array}$ \\
\hline $\begin{array}{l}\text { Einfachere } \\
\text { Quantifizierbarkeit der } \\
\text { Leistungen von Ver- } \\
\text { waltung, Organisation, } \\
\text { Lehre und Forschung }\end{array}$ & $\begin{array}{l}\text { Nehe Lehrformatter (z.B. } \\
\text { Open Online Courses) }\end{array}$ & $\begin{array}{l}\text { Großskalige } \\
\text { Einzelansprache } \\
\text { Studierender nach } \\
\text { einem Screening }\end{array}$ \\
& & $\begin{array}{l}\text { zur Intervention in } \\
\text { den Studienverlauf } \\
\text { (um z.B. über }\end{array}$ \\
& & \begin{tabular}{l} 
Frühwarnsysteme \\
\hline
\end{tabular} \\
\hline
\end{tabular}

1 Letztlich ist die Digitalisierung ein von Menschen gesteuerter und gewollter Veränderungsprozess (vgl. schon Eurich 1998). In seinen Folgen erscheint er Mitgliedern der Hochschulen dennoch als exogen angetriebener Wandel.

2 Das sind keineswegs alle Veränderungen des Hochschul- und Bildungssystems. Eine umfassende Analyse der Veränderungen durch die Digitalisierung kann hier jedoch nicht geleistet werden. 


\begin{tabular}{lll}
\hline & Bessere präventive Di- & $\begin{array}{l}\text { Studien- } \\
\text { abbrüche zu } \\
\text { vermeiden) }\end{array}$ \\
\hline $\begin{array}{l}\text { Möglichkeit zur } \\
\begin{array}{l}\text { Einführung bundes- } \\
\text { Meiter }\end{array}\end{array}$ & $\begin{array}{l}\text { Vergleichbarkeit } \\
\text { der Leistung von } \\
\text { agnostik auch für sel- } \\
\text { tene Problemlagen auf- } \\
\text { grund der Nischenwir- } \\
\text { kung digitaler Informati- } \\
\text { onsspeicher (Anderson } \\
\text { 2007) }\end{array}$ & $\begin{array}{l}\text { Studierenden mit } \\
\text { ihren Peers wird } \\
\text { größer }\end{array}$ \\
& $\begin{array}{l}\text { Neue Kommunikations- } \\
\text { wege (Ticketsysteme, } \\
\text { Push-Nachrichten an Stu- } \\
\text { dierende aus der Verwal- } \\
\text { tung) }\end{array}$ & $\begin{array}{l}\text { Mehr Selbststeue- } \\
\text { rung des Studiums } \\
\text { durch Studierende } \\
\text { möglich (Wahl von } \\
\text { Kursen, Prüfungen }\end{array}$ \\
& \begin{tabular}{l} 
online von daheim) \\
\hline
\end{tabular} & $\begin{array}{l}\text { Neue Informations- } \\
\text { wege für Studie- } \\
\text { rende durch die }\end{array}$ \\
& $\begin{array}{l}\text { massenhafte Ver- } \\
\text { fügbarkeit von In- } \\
\text { formationen im }\end{array}$ \\
& Web \\
\hline
\end{tabular}

Anm.: fett $=$ Die wichtigsten Veränderungen, die den Aufbau von Frühwarnsystemen aufgrund der technischen Transformation ermöglichen

\section{Studienerfolg und Studienabbruch}

Das Verlassen des Hochschulsystems ohne (ersten) Abschluss und ohne Wiederaufnahme eines Studiums wird als ,Studienabbruch' bezeichnet (Heublein/Wolter 2011; Lewin 1999). Er ist das Ergebnis eines multifaktoriellen (Passungs-)Prozesses (Blüthmann/Lepa/Thiel 2008; Isleib 2015). Zur Erklärung dieses Phänomens werden Theorien aus verwandten Disziplinen herangezogen (vgl. etwa Sarcletti/Müller 2011). Über alle Theorien hinweg lassen sich ähnliche Faktoren identifizieren, die Einfluss auf die Entscheidung für den Studienabbruch bzw. Studienerfolg haben können. So schlagen Blüthmann et al. (2008) in einem theoretischen Modell des Studienerfolgs die folgenden Bedingungsfaktoren vor: Eingangsvoraussetzungen, Studienbedingungen, Studier- und Lernverhalten sowie Kontextbedingungen (Abbildung 1). 


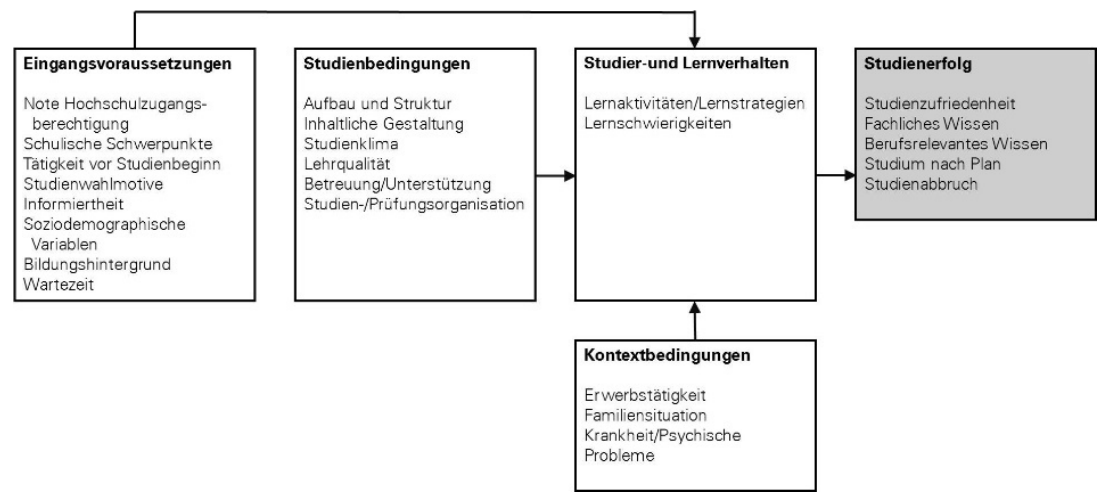

Abbildung 1: Allgemeines theoretisches Modell des Studienerfolgs (Blüthmann et al. 2008: 415)

\section{Präventions- \& Interventionsmaßnahmen}

Ein möglicher Umgang der Studierenden mit Problemen im persönlichen und hochschulischen Bereich ist die Inanspruchnahme von Unterstützungsangeboten. $\mathrm{Zu}$ den Möglichkeiten der Hochschulen, intervenierend und präventiv den Studienerfolg zu fördern, gehören folgende konkrete Maßnahmen: Orientierungsveranstaltungen, Einführungs- und Vorbereitungskurse, Mentoring, (angeleitete) Lerngruppen, Seminare zum Studienmanagement, persönliche Studienberatung, Teilzeitstudium, Flexibilisierung des Studiums, Online-Self-Assessments, Diagnostik-Tools und Frühwarnsysteme (Ahles/Köster/Vetter/Wulff 2016; Cesca/Schulze-Stocker/Pelz 2019; Kolb/Kraus/Pixner/Schüpbach 2006). In vielen dieser Angebote steht eine Intervention mittels Digitalisierung im Fokus. Bei Frühwarnsystemen an deutschen Hochschulen zeigt sich dies in der automatisierten Identifizierung etwaiger Studienabbrüche anhand vorab bestimmter (Studienverlaufs-)Merkmale und dem Kontaktieren der Studierenden über E-Mails (Förderrichtlinie des BMBF 2016; TU Dresden 2017). Diese Form der Intervention bedarf einer genauen Analyse der an der jeweiligen Hochschule vorherrschenden Probleme. 


\section{Rolle der Erwachsenenbildung}

Technische Systeme zur Erfassung der Leistung Studierender sind mitunter dem Vorwurf ausgesetzt, mit ihrer quantitativen Logik den Steuerungsinteressen der Wirtschaft zu folgen. Frühwarnsysteme könnten für Ökonomisierungstendenzen in den Feldern der Studienberatung und der Erwachsenenbildung stehen (Wilhelm 2017) ${ }^{3}$. Um dennoch sicherzustellen, dass Frühwarnsysteme sich an Evidenzen orientieren und so das Wohl der Studierenden berücksichtigen, sollte die Erprobung und Einführung von Frühwarnsystemen durch Forscherinnen und Forscher begleitet werden. Ihre Rolle liegt - quasi als inhärenter Gegenpol - in der kritischen und experimentellen Begleitung der angestoßenen Transformationsprozesse der Lehr- und Lernkultur an Hochschulen (Rhein 2016; Schüßler 2016) sowie in der konkreten Entwicklung und Evaluation der Präventions- und Interventionsmaßnahmen. Das ist die Perspektive, die langfristig den Studierenden, den Hochschulen, der Hochschulpolitik und der Gesellschaft insgesamt nützt.

\section{Fragestellungen und Ziele der vorliegenden Studie}

Im Zuge des Aufbaus eines Frühwarnsystems an der TU Dresden wurde als erstes eine quantitative Befragung der TU-Studierenden vorgenommen, die Auskunft über die Schwierigkeiten und Problemlösungsstrategien gibt (Pelz/ Schulze-Stocker 2018). In einem zweiten Schritt wurde eine Gruppe Studierender aus der ersten Befragung für eine teilstandardisierte und leitfadengestützte qualitative Befragung ausgewählt. Der vorliegende Artikel soll mithilfe dieser klären:

1. Welche Probleme haben Studierende an der TU Dresden? Lässt sich das der quantitativen Auftaktbefragung zugrunde liegende Studienerfolgs-Modell (Blüthmann et al. 2008) durch eine qualitative Erhebung bestätigen und ggf. ergänzen?

2. Wie genau gehen Studierende der TU Dresden mit Schwierigkeiten im Studium um? Welche Typen von Problembewältigungsstrategien lassen sich unterscheiden?

3 Häufig wird der Prozess der Ökonomisierung auf die Einführung des New Public Managements an Hochschulen zurückgeführt (Oechsler 2008). Ob die Verwendung des Begriffs ,Ökonomisierung' hierbei allerdings angebracht ist, darf bezweifelt werden (Schäfer-Hock 2015). 


\section{Methode}

\section{Kontext: Das PASST?!-Programm der TU Dresden}

Das Frühwarnsystem an der TU Dresden trägt den Namen PASST?! Partnerschaft $\cdot$ Studienerfolg $\cdot$ TU Dresden. Schwerpunkte der Programmarbeit sind die Identifizierung der Risikogruppen anhand von Merkmalen des Studienund Prüfungsverlaufs ${ }^{4}$, aber auch die Beratung und Unterstützung der Studierenden, die an Studienabbruch, Studienunterbrechung, Studiengangs- oder Hochschulwechsel denken (Risikogruppen). Darüber hinaus gehören die Begleitforschung zu den Bedingungen für erfolgreiche Studienverläufe, die Programmevaluation sowie die (wissenschaftliche) Öffentlichkeitsarbeit und Vernetzung zum PASST?!-Programm. Zusammen mit den Erkenntnissen aus der Beratungspraxis und der Begleitforschung sollen die Identifizierungsmerkmale weiterentwickelt werden sowie Befunde in das Qualitätsmanagement der TU Dresden einfließen.

\section{Anlage, Stichprobe \& Auswertung}

Die vorliegenden Daten stammen aus der Interviewstudie des PASST?!-Programms (Erhebungszeitraum Mai bis Juni 2017). Qualitative Interviews zeichnen sich vor allem durch ihre inhaltliche Offenheit aus. Dabei haben die Interviewten den Raum, die Antworten in ihrer eigenen Logik zu artikulieren. $\mathrm{Zu}$ dem können ,Narrationen' angeregt werden, d.h., dass die Setzung eigener Relevanzen in der jeweils eigenen Sprache erfolgen kann (Helfferich 2014). Die durchgeführten Interviews zielten auf die Erforschung von Problemlagen sowie unterschiedlicher Strategien des Umgangs mit unsicheren Entscheidungssituationen im Hinblick auf den Verbleib oder das Verlassen der Hochschule ab. Es wurden 17 Interviews mit denjenigen Teilnehmenden der Auftaktbefragung geführt, die eine Studienabbruchneigung geäußert (insgesamt 72 Perso-

4 Studierende (1) haben im ersten Semester nicht mindestens zwei Prüfungsleistungen bestanden, (2) haben in den vorangegangenen zwei Semestern in der Summe weniger als 30 Leistungspunkte erbracht, (3) haben eine erste Wiederholungsprüfung nicht bestanden, (4) sind im vorangegangenen Semester mindestens drei Mal von Prüfungsleistungen zurückgetreten bzw. haben sich drei Mal abgemeldet, (5) haben die Regelstudienzeit um zwei oder mehr Semester überschritten. Insgesamt wurden seit dem 1. April 20167.980 E-Mails anlässlich der Identifizierung verschickt (Stand 24.05.2019 bei 7.859 Teilnehmenden). 
nen) und ihre Bereitschaft zur Teilnahme an der qualitativen Erhebung signalisiert hatten. Als Incentive erhielten die Teilnehmenden einen Büchergutschein in Höhe von 25 Euro. Die Befragten stammen aus acht der elf Studiengänge, die in der Auftaktbefragung vertreten waren. Der Frauenanteil liegt bei 52,9\%. Die interviewten Personen sind im Mittel 24 Jahre alt. Zwölf der 17 befragten Personen studieren in Studiengängen aus den Bereichen Mathematik, Informatik, Naturwissenschaft und Technik (MINT).

Für die Auswertung wurden die Interviews transkribiert und Kurzbeschreibungen für die Grobanalyse erstellt. Das Kategoriensystem wurde aus deduktiv sowie induktiv hergeleiteten Kategorien gebildet: Für die Differenzierung der Problemlagen konnte auf das Modell von Blüthmann et al. (2008; siehe Abbildung 1) zurückgegriffen werden, welches anhand des Materials weiter konkretisiert wurde. ${ }^{5}$ Die Kategorien zum Umgang mit den Problemen (systematische Studienplanung, Alternativen zum jetzigen Studium, Einbezug des sozialen Umfelds und Beratung) stammen aus den Interviews. Anschließend wurde das Datenmaterial inhaltsanalytisch ausgewertet (Mayring 2010).

\section{Ergebnisse}

\section{Differenzen in den Problemlagen}

Die Übertragung der in den Interviews genannten Probleme auf das Studienerfolgs-Modell von Blüthmann et al. (2008; Abschnitt 2.2) macht deutlich, dass alle in den Interviews geäußerten Schwierigkeiten den Kategorien zugeordnet werden können. Die Kategorie Studienbedingungen (Aufbau und Struktur, inhaltliche Gestaltung ...) ist am stärksten vertreten. Innerhalb dieser Kategorie fällt die hohe Anzahl der Codierungen der Subkategorien Studienorganisation, Ausstattung und Betreuung/Unterstützung auf.

Bei der Analyse des Materials stellten sich zwei Differenzierungsaspekte als interessant heraus: Studium eines MINT-Faches und Position im Studienverlauf. Das soll im Folgenden mithilfe illustrierender Beispiele näher ausgeführt werden.

- MINT-Differenzierung: Nicht-MINT-Studierende nennen mehr Problemlagen im Zusammenhang mit der Prüfungsorganisation (Kategorie Studienbedingungen): „Ich finde, wir haben ziemlich viele, also gerade so im

5 Neue Aspekte waren: Berufswunsch und Studienortmotivation (Eingangsvoraussetzungen) sowie Ausstattung und Zufriedenheit mit den Studienbedingungen (Studienbedingungen). 
Hauptstudium, ziemlich viele Seminararbeiten zu schreiben und da das hauptsächlich natürlich auf die Ferien konzentriert ist, ist es schon so, dass man das Semester hat, dann kommen die Ferien, aber so richtig Ferien sind es eigentlich auch nicht, weil man bis zum Ende des Semesters an den Arbeiten schreibt und am nächsten Tag geht es dann direkt wieder los. " (I1).

- Differenzierung nach Studienposition: Studierende beschreiben vermehrt Probleme zum Studienanfang: ,, Aber wir waren halt am Anfang natürlich auch sehr, sehr viele Studenten, wo dann auch sehr, sehr viele rausgeworfen wurden oder selbst gegangen sind. “(I2). , Jetzt in den höheren Semestern wird es deutlich besser, also wirklich viel besser. “(I3). Diese Differenzierung zeigt sich bzgl. des Studienerfolgs-Modells von Blüthmann et al. (2008) besonders in den Subkategorien Prüfungs- und Studienorganisation, Lernstrategien, Studieninhalte und -klima. Dieser Befund verwundert nicht, da der Eintritt in die Hochschule eine Transformation der Lebenslage darstellt, die eine hohe Adaptionsfähigkeit erfordert.

Ein weiterer interessanter Befund ist die Rückkoppelung der Problemzuschreibung seitens der Studierenden, d.h., selbst bei strukturellen Problemen und Transformationsprozessen wird die individuelle Schuld- und Problemzuschreibung gesucht: Individualverantwortung statt Strukturopfer.

\section{Differenzen im Problemumgang}

Anhand des Datenmaterials wurden vier unterschiedliche Lösungsstrategien, d.h. Strategien des Umgangs mit Problemen, identifiziert:

- Unter systematischer Studienplanung wird z.B. die Teilnahme an einer Lerngruppe oder das bewusste Einlegen einer Pause verstanden.

- Die Alternativen zum jetzigen Studium beschreiben andere Wege, dazu zählen Studiengangwechsel oder Studienabbruch.

- Der Einbezug des sozialen Umfelds in Form eines (Informations-)Austausches, bspw. mit Peers oder Fachfremden, stellt eine weitere Strategie dar.

- Ein wichtiger Aspekt ist die Inanspruchnahme von Beratung (Lehrende, TU-Beratungsstellen, andere Stellen etc.).

In der Kategorie Beratung wird mit Blick auf die Zentrale Studienberatung das Gefühl als nichtgeprüfte Vorannahme geäußert, dort keine Hilfe für konkrete Problemlagen zu erhalten. Möglicherweise ist das Wort „zentrale“ für Studierende irreführend. Hingegen spielt das soziale Umfeld eine große Rolle (sowohl inner- als auch außerhalb der Universität), was sich in der Kategorie 
Austausch zeigt. Universitätsintern wird dabei vor allem der Erfahrungsaustausch mit Gleichgesinnten als relevant und sehr sinnvoll wahrgenommen:

„Im ersten Semester haben wir unsere Mathe-Prüfung auch schon nur mit einer Repetitorin bestanden und da das ziemlich gut geklappt hat, haben wir jetzt, ein Freund und ich, halt einfach gesagt: ,Komm, die macht das zwar nicht mehr, aber lass uns irgendwas anderes suchen, ansonsten packen wir das jetzt schon wieder nicht. "“(I4.)

„Und ja, was dann halt hilft, ist, dass es den anderen genauso geht und dass man dann sich mit Freunden trifft und gemeinsam irgendwie lernt, Übungen macht. "(I5).

Auch im Einbezug des sozialen Umfelds zeigt sich eine Differenz zwischen Studierenden aus MINT- und Nicht-MINT-Studiengängen: Das gemeinsame Lernen steht eher bei MINT-Studierenden im Fokus, was auch an unterschiedlichen Prüfungsmodi liegen könnte. Des Weiteren wird die systematische Studienplanung hervorgehoben: , [...] im letzten Semester war ich im Ausland und habe mir da so ein bisschen eine Pause eingelegt und bin jetzt wieder motivierter. “(I5). Auffällig ist, dass diese Studienplanung eher bei Studierenden aus MINT-Studiengängen als Form des Umgangs mit Problemen im Studium vorkommt.

Die angesprochene Rückkopplung struktureller zu individuellen Problemlagen hat auch Einfluss auf die Lösungsstrategien, denn es werden Individuallösungen für wenig beeinflussbare, strukturelle Probleme gesucht und gefunden.

\section{Schnittstelle zwischen Problemlagen und -umgang}

Aus den Daten wurde ersichtlich, dass Motivation eine hohe Relevanz hat. Diese kommt in den Interviews in zweierlei Hinsicht vor:

- Motivation für das Studienfach und für den -ort (sog. Interesse)

- Motivation im Zusammenspiel zwischen Problemen und Lösungsstrategien (sog. Antrieb)

In der zweiten Lesart hat die Kategorie Motivation besondere Bedeutung, da diese quer zu anderen Kategorien des Studienerfolgs-Modells nach Blüthmann et al. (2008) liegt. Die vielen Überschneidungen mit anderen Kategorien zeugen davon, dass Motivation einen Schnittpunkt zwischen Problemen im Studium und dem Umgang mit ihnen darstellt.

„,Dann irgendwie so rund um Weihnachten [...] war ich mit meinen Eltern im Urlaub. [...] dann hat mein Vater den alles entscheidenden Satz quasi 
gebracht: ,Und was passiert, wenn du einfach ein Jahr länger studierst? . So, ja. Und dann so langsam ging es mit der Motivation wieder bergauf." (I4).

\section{Diskussion der Ergebnisse \& Fazit}

Die Einführung von Frühwarnsystemen an Deutschlands Hochschulen ist ein Beispiel dafür, wie ein Makro-Prozess in Form der digitalen Transformation auch auf der Mikro-Ebene des bzw. der einzelnen Studierenden ankommt. Dort ergibt sich die Möglichkeit, einzelne Studierende in großer Zahl anhand spezifischer Merkmale zu identifizieren und mittels einer Intervention transformierend in ihren Studien- und damit letztlich Bildungsverlauf einzugreifen. Um Frühwarnsysteme erfolgreich aufzubauen, bedarf es hochschulspezifischer Forschung wie der hier vorgestellten an der TU Dresden: Mithilfe der vorliegenden Daten konnte das Modell von Blüthmann et al. (2008) auch mit einer qualitativen Erhebung bestätigt ${ }^{6}$ und erweitert werden. Es gibt anhand des Datenmaterials (siehe illustrierende Beispiele Abschnitt 4.1 und 4.2) Hinweise darauf, dass sich die identifizierten Schwierigkeiten nach Studienposition sowie MINT-Zugehörigkeit differenzieren lassen und Motivation eine moderierende Schlüsselkategorie zwischen Schwierigkeit(en) und Umgang ist.

Somit lassen sich drei Hypothesen formulieren, die der zielgruppenspezifischeren Weiterentwicklung der Beratungsformate und Identifizierungsmerkmale dienen könnten:

- Gemäß der Position im Studienverlauf zeigen sich unterschiedliche Gewichtungen der Gelingensbedingungen für Studienerfolg.

- Studierende aus MINT-Studiengängen unterscheiden sich hinsichtlich der Problemlagen und des Problemumgangs von den Studierenden aus NichtMINT-Studiengängen.

- Die Kategorie Motivation hat einen moderierenden Einfluss auf das Zusammenspiel zwischen Problemwahrnehmung und Problemumgang.

Diese Befunde stellen Beratungsinstitutionen und Frühwarnsysteme an Hochschulen vor komplexe Herausforderungen; das gilt zudem besonders für das Ergebnis „Individualverantwortung statt Strukturopfer“. Hier könnten die Angebote des Frühwarnsystems entlastend wirken.

6 Quantitativ ist das bereits in der Untersuchung der Auftakterhebung (Pelz/SchulzeStocker 2018) geschehen. 
Die in der qualitativen Studie ersichtlichen Merkmale lassen sich nur in einer zusätzlichen Befragung zu Beginn des Studiums und im Weiteren parallel dazu erfassen und an das Frühwarnsystem koppeln, sodass man neben den Identifizierungsmerkmalen aus Studienverlaufs- und Prüfungsdaten auch immer noch persönliche Angaben der Studierenden hat, um sie gezielt zu identifizieren und anzusprechen. Neben einer regelmäßigen Befragung der Studierenden müssten die Ergebnisse des Qualitätsmanagements und des Frühwarnsystems zusammengeführt werden (vgl. als Beispiel den Beitrag der Absolventenstudien für das QM der TU Dresden: Frohwieser/Herklotz/Szymenderski 2018).

Ebenso wie Blüthmann et al. (2008) ihre Kategorien als generelles Konzept des Studienerfolgs verfasst haben und diese damit ortsunabhängig anwendbar sind, sind die Ergebnisse der vorliegenden Studie keinesfalls allein gültig für den Studienstandort Dresden, auch wenn sie zur Verbesserung des dortigen Frühwarnsystems erhoben wurden. Zumindest weisen die Ergebnisse der vorangegangenen quantitativen Erhebung (Schulze-Stocker et al. 2019) nicht darauf hin, dass sich die Studierenden der TU Dresden in ihren Problemlagen von Studierenden anderer Hochschulen unterscheiden.

Im Rahmen weiterer Untersuchungen ist der Einfluss existierender Frühwarnsysteme auf Bildungswege der Studierenden von Interesse. Zuvor könnte eine Sekundärauswertung der qualitativen Interviews Aussagen über die Komplexität und Verschränkung etwaiger Probleme im Studium ermöglichen. Zudem scheint das Bild von Beratung Relevanz zu haben, da in den Daten ein Einfluss der Wahrnehmung auf die Nutzung von Beratung an Hochschulen deutlich wurde. Zusätzlich könnte von Interesse sein, ob und wie ein Frühwarnsystem die Wahrnehmung bzw. Nutzung von Beratungsangeboten durch Ansprache im richtigen Moment verbessern könnte. Diesen Forschungsdesideraten gilt es weiter nachzugehen.

\section{Literatur}

Ahles, Lisa / Köstler, Ursula / Vetter, Nicole / Wulff, Anne (2016): Studienabbrüche an deutschen Hochschulen. Stand der Thematisierung und strategische Ansatzpunkte. Baden-Baden: Nomos.

Anderson, Chris (2007): The Long Tail. Der Lange Schwanz. Nischenprodukte statt Massenmarkt. Das Geschäft der Zukunft. München: Hanser.

Blüthmann, Irmela / Lepa, Steffen / Thiel, Felicitas (2008): Studienabbruch und wechsel in den neuen Bachelorstudiengängen. Untersuchung und Analyse von Abbruchgründen. In: Zeitschrift für Erziehungswissenschaft, 11(3), S. 406-429. 
BMBF (2016): Richtlinie zur Förderung von Forschung über „Studienerfolg und Studienabbruch“. Bundesanzeiger vom 24.02.2016. https://www.bmbf.de/foerderungen/bekanntmachung-1151.html [Zugriff: 29.03.2018].

Cesca, Stephanie K. / Schulze-Stocker, Franziska / Pelz, Robert (2019): Welches Ziel verfolgt die Beratung von Studienabbrecher/innen in Deutschland? In: Zeitschrift für Beratung und Studium, 14(1), S. 7-14.

Dolata, Ulrich (2008): Technologische Innovationen und sektoraler Wandel. Eingriffstiefe, Adaptionsfähigkeit, Transformationsmuster: Ein analytischer Ansatz. In: Zeitschrift für Soziologie, 37(1), S. 42-59.

Eurich, Claus (1998): Mythos MultiMedia. Über die Macht der neuen Technik. München: Kösel.

Frohwieser, Dana / Herklotz, Markus / Szymenderski, Peggy (2018): Die Dresdner Absolventenstudie und ihr Beitrag für die Studiengangsevaluation an der TU Dresden. In: Falk, S. / Reimer, M. / Schmidt, U. (Hrsg.): Absolventenstudien und Qualitätsmanagement. Best Practices an deutschen und österreichischen Hochschulen. Münster: Waxmann, S. 139-153.

Hahn, Dietger / Krystek, Ulrich (1984): Frühwarnsysteme als Instrument der Krisenerkennung. In: W. H. Staehle / H. Albach (Hrsg.): Betriebswirtschaftslehre und ökonomische Krise: Kontroverse Beiträge zur betriebswirtschaftlichen Krisenbewältigung. Wiesbaden: Gabler, S. 3-24.

Helfferich, Cornelia (2014): Leitfaden- und Experteninterviews. In: N. Baur/J. Blasius (Hrsg.): Handbuch Methoden der empirischen Sozialforschung. Wiesbaden: Springer VS, S. 559-574.

Heublein, Ulrich / Wolter, Andrä (2011): Studienabbruch in Deutschland. Definition, Häufigkeit, Ursachen, Maßnahmen. In: Zeitschrift für Pädagogik, 57(2), S. 214-236.

Isleib, S. (2015): Neue Theorieströmungen zum Studienabbruch. Herkunft, Genese und Potenziale für die Studienabbruch- und Hochschulforschung. http://www. dzhw.eu/pdf/pub_vt/21/2015-04-10_gfhf_studienabbruch_isleib.pdf [Zugriff: 21.08.2019].

Käpplinger, Bernd (2019): Beratung im Rampenlicht - Von Sisyphos zu Prometheus? In: O. Dörner / C. Iller / I. Schüßler / C. Maier-Gutheil / C. Schiersmann (Hrsg.): Beratung im Kontext des Lebenslangen Lernens. Konzept, Organisation, Politik, Spannungsfelder. Opladen: Budrich, S. 17-32.

Kolb, Monika / Kraus, Michael / Pixner, Johann / Schüpbach, Heinz (2006): Analyse von Studienverlaufsdaten zur Identifikation von studienabbruchgefährdeten Studierenden. In: Das Hochschulwesen, 54(6), S. 196-201.

Lewin, Karl (1999): Studienabbruch in Deutschland. In: M. Schröder-Gronostay / H.-D. Daniel (Hrsg.): Studienerfolg und Studienabbruch. Beiträge aus Forschung und Praxis. Neuwied/Kriftel/Berlin: Luchterhand, S. 17-50.

Mayring, Philipp (2010): Qualitative Inhaltsanalyse - Grundlagen und Techniken (11., akt. u. überarb. Ausg.). Weinheim/Basel: Beltz Verlag.

Oechsler, Walter A. (2008): Anwendung von betriebswirtschaftlichen Verfahren in der öffentlichen Verwaltung - Gefahren, Risiken und Nebenwirkungen am Beispiel der neuen Steuerungsinstrumente Baden-Württemberg. In: R. Fisch / 
A. Müller / D. Beck (Hrsg.): Veränderungen in Organisationen. Stand und Perspektive. Wiesbaden: VS Verlag für Sozialwissenschaften, S. 53-63.

Pelz, Robert / Schulze-Stocker, Franziska (2018): Determinanten der Studienabbruchneigung von Studierenden. Ergebnisse einer Befragung im Rahmen des PASST?!-Programms der TU Dresden. Arbeitspapier Nr. 2018-01. http://t1p. de/p19w [Zugriff: 30.01.2019].

Rhein, Rüdiger (2016): Die Universität als Lernort. In: O. Dörner / C. Iller / H. Pätzold / S. Robak (Hrsg.): Differente Lernkulturen - regional, national, transnational. Opladen: Budrich, S. 205-216.

Sarcletti, Andreas / Müller, Sophie (2011): Zum Stand der Studienabbruchforschung. Theoretische Perspektiven, zentrale Ergebnisse und methodische Anforderungen an künftige Studien. In: Zeitschrift für Bildungsforschung, 1(3), S. 235-348.

Schäfer-Hock, Christian (2015): Der Ökonomisierungssprung des Journalismus im digitalen Zeitalter. Identifizierung eines Umbruchs mittels klarer Indikatoren. In: S. Pagel (Hrsg.): Schnittstellen (in) der Medienökonomie. Baden-Baden: Nomos, S. 164-187.

Schüßler, Ingeborg (2016): Lernkulturen in Transformationsgesellschaften. Paradoxien, Herausforderungen und Gestaltungsoptionen. In: O. Dörner / C. Iller / H. Pätzold / S. Robak (Hrsg.): Differente Lernkulturen - regional, national, transnational. Opladen: Budrich, S. 15-26.

Schulze-Stocker, Franziska / Schäfer-Hock, Christian / Pelz, Robert (2017): Weniger Studienabbruch durch Frühwarnsysteme - Das Beispiel des PASST?!Programms an der TU Dresden. In: Zeitschrift für Beratung und Studium, 12(1), S. 26-32.

Schulze-Stocker, Franziska / Schäfer-Hock, Christian / Pelz, Robert (2019): Intervention in den Studienverlauf und Beratung. In: O. Dörner / C. Iller / I. Schüßler / C. Maier-Gutheil / C. Schiersmann (Hrsg.): Beratung im Kontext des Lebenslangen Lernens. Konzept, Organisation, Politik, Spannungsfelder. Opladen: Budrich, S. 221-233.

TU Dresden (2017): Mit Frühwarnsystemen Studienabbrüchen an Sachsens Hochschulen vorbeugen. https://tu-dresden.de/tu-dresden/newsportal/news/mitfruehwarnsystemen-studienabbruechen-an-sachsens-hochschulen-vorbeugen [Zugriff: 28.01.2018].

Wilhelm, Daniel (2017): Kritische Reflexion einiger Ökonomisierungstendenzen in der Studienberatung. In: Zeitschrift für Beratung und Studium, 12(4), S. 106-109. 



\section{Lernziele bestimmen? Kompetenzen erfassen? Prozesse imaginieren? Zur „Messung“ individueller und kollektiver Transformation im Kontext Organisationspädagogischer Professionalisierung}

\section{Einleitung}

Gerade im Horizont des Nachhaltigkeitsdiskurses werden Globalisierung, Transnationalisierung und Mehrebenen-Governance zu relevanten Referenzpunkten für Erwachsenenbildung und Organisationspädagogik. An den politischen Programmatiken der globalen Nachhaltigkeitsziele (Sustainable Development Goals, SDGs) sollen sich politisches, administratives, gesellschaftliches und ökonomisches Handeln orientieren. Um die globalen Nachhaltigkeitsziele umzusetzen, wird die Professionalisierung von Prozessbegleitung und Netzwerkberatung empfohlen (WBGU). Hier knüpfen erwachsenenpädagogische und organisationspädagogische Potenziale der Professionalisierung an (Heidelmann/Weber 2018). ${ }^{1}$ Mittels dreier Innovationslabore wurde über ein Jahr hinweg exemplarisch im Feld der nachhaltigen Ernährung das Ziel verfolgt, den Aufbau des regionalen Ernährungskreislaufes zu unterstützen. Gleichzeitig wurden Studierende verschiedener Studiengänge in einem Veranstaltungszyklus dahingehend professionalisiert, regional vernetzte Transformationsarrangements zu begleiten. Der Beitrag reflektiert und diskutiert das Analysepotenzial taxonomischer, kompetenzdiagnostischer und bildbasierter Prozessanalysen im Lichte transformationstheoretischer Perspektiven (Schäffter 2014).

Nach einer kurzen Vorstellung des kollektiven Lernarrangements (1) reflektiert der Beitrag das Potenzial der jeweiligen Zugänge bezüglich ihrer epistemischen Einbettung und forschungsmethodischen Ausgestaltung (2). Vor diesem Hintergrund wird im letzten Kapitel das im Projekt pilotierte Analyseund Auswertungskonzept organisationspädagogischer Professionalisierung als ästhetische Transformation knapp vorgestellt (3).

1 Das Ausbildungsprogramm ,Nachhaltige Zukünfte gestalten lernen“ wurde von April 2018 bis März 2019 durch den Rat für Nachhaltige Entwicklung im Programm ,Nachhaltigkeitskultur' gefördert. 


\section{Professionalisierung als Innovations- und Transformationslernen zwischen Hochschule und Region}

In einem einjährig angelegten Zertifikatsprogramm werden Studierende verschiedener Masterstudiengänge wissenschaftlich fundiert und gleichzeitig erfahrungsorientiert darauf vorbereitet, regionale Vernetzungsprozesse für Kreislaufökonomien methodisch zu unterstützen und zu begleiten. Neben Vorlesung und Seminar nutzt der hochschuldidaktisch innovative Ansatz auch offene, regional verschränkte Lernarrangements. Im Programm werden drei Innovationslabore des regionalen Ernährungskreislaufs realisiert (Weber/Heidelmann 2019), welche die Studierenden mitorganisieren, durchführen und reflektieren. Dieser Ansatz organisationspädagogischer Professionalisierung, der gleichermaßen auch für die Erwachsenenbildung furchtbar gemacht werden kann, generiert einen gemeinsamen „hybriden“ Erfahrungsraum und geht über Praxiskontakte einzelner Studierender (Singer-Brodowski 2016) hinaus. Diese Generierung eines, liminalen' und grenzbearbeitenden Raumes zwischen Hochschule und Region ist mehr als ,agile' Hochschuldidaktik (Arn 2016). Jenseits eines simulationsbasierten Ansatzes des Lernens (Bauer/Weinhardt 2014) wird Erfahrungs-Lernen in Echtzeit angelegt. Dieses Innovationsund Transformationslernen grenzt sich auch von einem dienstleistungsorientierten „Service-Learning“ ab (Weber/Heidelmann/Adler 2019).

Im regional, institutionell und intergenerational verschränkenden Lernarrangement werden die Innovationslabore zu Schlüsselstellen des Innovationslernens von Studierenden und Professionellen (Adler/Weber 2019). ${ }^{2}$ Sie bringen systematisch differente Akteure des regionalen Ernährungskreislaufs zusammen und unterstützen das vernetzte Arbeiten an spezifischen Problem- und Zielstellungen. Sie zielen auf eine integrierte Ideen- und Strategieentwicklung, die Stärkung der regional nachhaltigen Kreislaufwirtschaft und ein regionales ebenso wie universitäres Lernarrangement für die beraterische Professionalisierung Studierender. Im Projekt interessiert uns die Frage, inwiefern und auf welche Weise ein solch komplexes Lernarrangement die Professionalisierung Studierender ebenso wie regionale kollektive Transformationsprozesse unterstützen kann.

Der vorliegende Beitrag fragt nach geeigneten Analysezugängen für das einjährig über drei Innovationslabore hinweg angelegte Lernarrangement. Welchen analytischen Beitrag können Taxonomien, Kompetenzdiagnostische Ansätze und Imaginationsbezogene Prozessanalysen leisten? Im Rückgriff auf

2 Diese finden mit jeweils ca. 60-70 Teilnehmenden in regionalen Lernräumen wie einem Kreistagssitzungssaal oder einer Regionalkonferenz statt. 
die transformationstheoretischen Überlegungen Schäffters (2014) soll das Feld der Analysehorizonte auf seine Brauchbarkeit erschlossen werden.

\section{Taxonomisch messen? Kompetenzen erfassen? Prozesse imaginieren? Modellierungen der Transformation in Analyse- und Messmodellen}

Um verschiedene Zugänge auf ihr analytisches Potenzial zu untersuchen, werden im Folgenden die Zugänge kurz vorgestellt, ihre epistemische Qualität im Licht transformationstheoretischer Perspektiven reflektiert und ihr möglicher Beitrag für die Untersuchung der Professionalisierung Studierender und die Reflexivierung Professioneller diskutiert.

\section{Lernzieltaxonomien: Konversionsprozesse im Modus linearer Transformation}

Die Professionalisierung Studierender könnte beispielsweise mit Lernzieltaxonomien untersucht werden. Diese systematisierten Bestimmungen von Lehrzielen haben bereits im Kontext der Lehrerbildung besondere Resonanz entfaltet. Von einem systematisch bestimmbaren, qualifikatorisch steigerbaren Wissen ausgehend, wird hier ein linearer und kumulativer Lernzuwachs erwartet, der sich als Lernfortschritt auf Niveaustufen abbilden lässt (Winther 2010).

Für die Systematisierung bestimmbarer Lernziele wurde das Bloomsche Modell der Taxonomiestufen (Bloom/Krathwohl 1956) besonders bekannt. Lernziele werden sequenziell aufeinander aufbauend für drei Lernbereiche des kognitiven, affektiven und psychomotorischen Lernens erschlossen. Mit Blick auf eine Professionalisierung von Beratung müsste eine solche Taxonomie allerdings erst erstellt werden, da es hier bislang keine geeigneten institutionalisierten Curricula gibt. Im Fachdiskurs wird problematisiert, dass vor allem die kognitive Dimension der Taxonomien entwickelt wurde, affektive und psychomotorische Dimensionen jedoch konzeptionell vernachlässigt und lediglich rudimentär ausgearbeitet wurden (Häußler 2014: 257). Trotz vielfacher Adaptionen und Erweiterungen des Bloomschen Modells (Anderson/Krathwohl 2001) müssten für den Kontext der Organisations- und Netzwerkberatung spezifische personale, aktivitätsbezogene, fachlich-methodische oder sozial-kommunikative Dimensionen systematisiert und gestuft modelliert werden. 
Ein solcher taxonomischer Zugang könnte situative Statuserhebungen zu spezifischen Prüfzeitpunkten einholen. In kausal-teleologischer Manier würde hier der Zusammenhang zwischen erfolgreicher Niveauerreichung und Vermittlungskontext hergestellt. Taxonomische Ansätze gehen von der Beobachter- wie Beobachtenden-unabhängigen, objektiv mess- und extern überprüfbaren Lernzielerreichung aus. Erhebungs- und auswertungsparadigmatisch würde die Messbarkeit der vorab definierten Lernziele angenommen. Kognitivistische Verengungen des schulischen Kontextes finden ihre Entsprechung in quantifizierend-,,messenden“ Forschungslogiken (Erpenbeck et al. 2017: XXII).

Aus transformationstheoretischer Sicht betrachtet ist das hier eingelagerte qualifikatorische Paradigma an bekannte und bestimmbare Ausgangslagen sowie Zielstellungen des Lernens gebunden. Im Projekt geht es jedoch nicht um Konversionsprozesse im Modus einer ,linearen Transformation “ (Schäffter 2014: 115). Ist- und Sollzustand lassen sich in unserem Kontext nicht eindeutig feststellen. Auch würden quantifizierende Zugänge die Komplexität der Prozesse zu wenig einholen können. Auch wenn Vermittlung z.B. im Rahmen der Vorlesung eine Rolle spielt, knüpft ein qualifikatorischer Fokus zu wenig an der Dynamik und Emergenz der zu untersuchenden Prozesse an.

\section{Generalisiert handlungsorientierte Kompetenzmodellierung: Aufklärung im Modus zielbestimmterTransformation}

Alternativ könnten generalisiert handlungsbezogene kompetenztheoretische Konstrukte Analysepotenziale für unser Projekt eröffnen (Erpenbeck et al. 2017). Kompetenzperspektiven würden die Lernenden und ihre Ziele - und nicht Lehrziele - zum Ausgangspunkt nehmen. Relevant würden hier nicht Dispositionen der Lernenden, sondern vor allem Entwicklungsresultate, also beobachtbare „Performanz“ (ebd.: XIII) in der situativen „Realisierung der Dispositionen“ (Erpenbeck/Heise 1997: 311). „Messen“ lässt sich die Bewältigung konkreter Anforderungen hinsichtlich fachlich-methodischer (kognitiver); motivationaler; personaler (ethischer), willensmäßiger (aktivitätsbezogener) und sozialkommunikativer Kompetenzen (Erpenbeck et al. 2017: XIII). Je nach Definiertheit und Expliziertheit der Handlungsziele bzw. der Offenheit und Intransparenz von Handlungssituationen lassen sich „Kompetenztypen“ unterscheiden (ebd.: XXV).

Das ,generalisiert handlungsbezogene“ Cluster des Kompetenzdiskurses bildet sich laut Erpenbeck et al. (2017) z.B. im europäischen und deutschen Qualifikationsrahmen für lebenslanges Lernen ab. Kompetenzen werden hier verstanden als ,die nachgewiesene Fähigkeit, Kenntnisse, Fertigkeiten sowie 
persönliche, soziale und methodische Fähigkeiten in Arbeits- oder Lernsituationen und für die berufliche und/oder persönliche Entwicklung zu nutzen“" (ebd.: XXII). Kompetenzmodellierung, Kompetenzdiagnostik und Kompetenzmessung zeigen hier durchaus noch eine Nähe zu taxonomischen Ansätzen. Beobachtungsformen wie z.B. Kompetenztests, eignungsdiagnostische und andere Testverfahren verbleiben auch hier vielfach im Feld ,quantitativer Messungen“" (ebd.: XXIX). Forschungsmethodisch wären hingegen nicht nur quantifizierend-objektivierende Zugänge, sondern auch qualitative und beobachtende Zugänge denkbar.

Der normative Charakter von Qualifizierungs- und Kompetenzrahmen und die gestuften Setzungen von Kompetenznormen richten sich an die Lernenden und formulieren attraktive Ziele der möglichen (eigenen) Performanz. Insofern strukturieren Qualifikations- und Kompetenzrahmen Lernen „soghaft“ (Schäffter 2014: 118), es werden Leitnormen formuliert, die implizit den Charakter von Vorbildern annehmen. Sinnstiftung erfolgt hier normalisierend und modellhaft. Professionalisierung im Modus ,zielbestimmter Transformation “ modelliert Lernen als Orientierungslernen (ebd.: 117). Idealvorstellungen von (Handlungs-)Kompetenz knüpfen durchaus auch an „Employability“, Selbstoptimierung und ,ökonomisierte(n) [...] Varianten von Bildung“ an (Erpenbeck et al. 2017). Damit weist auch der generalisiert kompetenzorientierte Zugang eine mangelnde Passung zum Projektanliegen auf, die kontextuellen, prozessualen und relationalen Aspekte dieser Organisationspädagogischen Professionalisierung können so nicht eingeholt werden.

\section{Indikatorenbasierte Professionalisierung als Suchbewegung und zielgenerierende Transformation}

Kompetenzzugänge, die vor allem an lebensweltlichen Problemsituationen der Lernenden ansetzen, stellen eine weitere Alternative dar. Hier geht es um Fähigkeiten zur Bewältigung und Lösung variabler Situationen (Weinert 2001: 27). In den Blick kommen Selbstorganisation, subjekt- und personenbezogene Perspektiven auf individuelle Handlungsdispositionen (Erpenbeck et al. 2017). Indikatorenbasierte Ansätze der Professionalisierung (Tippelt 2018) könnten ebenfalls genutzt werden, um die Professionalisierung Studierender und Professioneller zu untersuchen. Spezifische Merkmalsausprägungen und Dimensionierungen z.B. disziplinär eigener Wissensbestände, eines seitens der Fachcommunity definierten disziplinären Ethos, fachlich definierter methodischer Zugänge sowie gemeinsam geteilter Wertbezüge könnten hier Referenzpunkte der Untersuchung studentisch-beraterischer Professionalisierung sein (Heidelmann/Weber 2018). Komplexere kompetenztheoretische Modellierungen wür- 
den z.B. an einen Humboldtschen Bildungsbegriff anschließen. Indikatorenbasierten Ansätzen wurde im professionstheoretischen Kontext jedoch der Vorwurf gemacht, lediglich additiv Kriterien abzubilden und theoretisch abstinent zu sein (Dewe/Ferchhoff/Radtke 1992; Ferchhoff/Schwarz 2014). Forschungsmethodisch könnten hier intersubjektiv validierende, selbst einschätzende und „selbstbeobachtende“ Formen gewählt werden. „Qualitative Charakterisierungen“, „komparative Beschreibungen“, aber auch ,simulative Abbildungen“ oder „observative Erfassungen“ könnten hier methodische Zugänge sein (Erpenbeck et al. 2017: XXIX). Auch die Kompetenzentwicklung in Zeitreihen könnte Entwicklungsaspekte methodisch einholen.

Professionalitätsnormen würden von der prinzipiellen Bestimmbarkeit eines ,gegenseitigen Ufers“ (Schäffter 2014: 121) ausgehen und Orientierungssicherheit für zielerschließende, diffuse Suchbewegungen bieten. Eine solche Analyse würde die Eigenbewegung der Lernenden in den Blick nehmen und sich auf den pfadabhängig und prozessual entstehenden Möglichkeitsraum richten. Die Lernorganisation würde sich auf selbstgesteuerte Suchbewegungen (Tietgens 1986) beziehen und Lernarrangements der „Unterstützung im Umgang mit Neuartigem und Fremdartigem" schaffen (Schäffter 2014: 120). In diesem Sinne würden indikatorenbasierte Analysen von Professionalisierung als zielgenerierender Transformationsmodus gefasst werden. Anhand definierter Dimensionen von Professionalität würde hier ebenfalls von der Bestimmbarkeit der Entwicklungsdesiderate ausgegangen. Eine relational und kontextuell angelegte Perspektive bleibt allerdings auch hier noch unterbelichtet.

\section{Gestaltungskompetenz als Transformationsmodus „korrelativen Matchings“}

Alternativ würde das Kompetenzmodell der Ermöglichungsdidaktik (Arnold/Schüssler 2010) an ein „tiefgründend selbstorganisationsbezogenes“ Cluster des Kompetenzdiskurses (Erpenbeck et al. 2017) anschließen, das „Selbstorganisation“, „Komplexität“ und „Kreativität“ als zentrale Referenzpunkte setzt (Ritter/Gründer 1976). Kompetenz würde hier verstanden als Fähigkeit, ,,selbstorganisiert und kreativ zu handeln und mit unscharfen oder fehlenden Zielvorstellungen und Unbestimmtheit umzugehen" (Erpenbeck et al. 2017: XII). „Kreative Selbstorganisationsfähigkeiten“ (ebd.: XXIV) und Prozessgestaltung würden ins Zentrum gerückt.

Der Ansatz der „Gestaltungskompetenz“ im Horizont einer Bildung für nachhaltige Entwicklung nach de Haan (2008) könnte zentraler Referenzpunkt 
einer organisationspädagogischen Professionalisierung sein. Der Ansatz definiert 12 Teilkompetenzen, ${ }^{3}$ die den Hauptkompetenzfeldern der ,Sach- und Medienkompetenz' (Teilkompetenzen 1 bis 4); der ,Sozialkompetenz' (Teilkompetenzen 5 bis 8) und ,Selbstkompetenz' (Teilkompetenzen 9 bis 12) zugerechnet werden (de Haan 2008). Ziel des Ansatzes ist die Befähigung Lernender, ,die Zukunft von Sozietäten, in denen man lebt, in aktiver Teilhabe im Sinne nachhaltiger Entwicklung modifizieren und modellieren zu können“ (de Haan/Harenberg 1999: 60). Der Ansatz verweist damit auf die Ermöglichung von Zukunft in offenen Gestaltungsprozessen (de Haan 2008: 31). Demnach lässt Grenzbearbeitung heuristisches Wissen entstehen, das Handeln und Handlungsfähigkeit in ständiger Veränderung ermöglicht. Kontingente Ressourcen werden fruchtbar gemacht für faktenbasiertes, hypothesenbildendes, kreativ entwickelndes Neu-Konstruieren von Entwicklungszielen (de Haan 2008: 27). Auch hier erscheinen prozessorientierte und qualitative Zugänge als besonders geeignete „Messmethoden“ (Erpenbeck et al. 2017: VVXI).

Im Sinne einer korrelativen Übergangsstruktur nach Schäffter (2014: 122) vollzieht sich Denken zwischen ungewissen Ausgangslagen und zielgenerierenden Suchbewegungen. Gestaltungskompetenz bezieht sich in diesem Sinne auf 1) die reflexive Bestimmung von Ausgangslagen, 2) die korrelative Aussteuerung im liminalen Prozess und 3) auf kompetenzbasierte Zielgenerierung. Da Übergänge im Ansatz der Gestaltungskompetenz durch reflexive Prozesspraxis entstehen sollen, schließt dieser Ansatz transformationstheoretisch an den Modus ,,korrelativer Transformation “ an.

\section{Organisationsbildung für nachhaltige Entwicklung im Modus reflexiver Transformation}

Zwar bezieht der Ansatz der Gestaltungskompetenz die methodisch unterstützte reflexive Klärung von Ausgangslagen, von Scheiterns- oder Problemerfahrungen und Zukunftsantizipationen ein. Aus organisationspädagogischer Perspektive bedarf es jedoch einer Organisationsbildung für nachhaltige Entwicklung (OBNE), die den Fokus auf das Lernen in offenen Netzwerken

3 1) Weltoffen und neue Perspektiven integrierend Wissen aufbauen; 2) Vorausschauend Entwicklungen analysieren und beurteilen können; 3) Interdisziplinär Erkenntnisse gewinnen und handeln; 4) Risiken, Gefahren und Unsicherheiten erkennen und abwägen können; 5) Gemeinsam mit anderen planen und handeln können; 6) Zielkonflikte bei der Reflexion über Handlungsstrategien berücksichtigen können; 7) An kollektiven Entscheidungsprozessen teilhaben können; 8) Sich und andere motivieren können, aktiv zu werden; 9) Die eigenen Leitbilder und die anderer reflektieren können; 10) Vorstellungen von Gerechtigkeit als Entscheidungs- und Handlungsgrundlage nutzen können; 11) Selbständig planen und handeln können; 12) Empathie für andere zeigen können. 
(Chesborough 2003; Weber 2018a,b) und der Transformationsgesellschaft (Schäffter 2001) richtet (Weber/Heidelmann 2019). Lernen fokussiert hier auch auf die Ermöglichung transformativer Forschungs- und Bildungsarrangements (WBGU 2011), die mit nichtlinearer Dynamik, emergenten Strukturen, inneren Bedingtheiten, spezifischen Koordinationsmustern und Historizität arbeiten (Erpenbeck et al. 2017: XXIV). Solche Lernarrangements realisieren und aktualisieren den Modus „reflexiver Transformation“. Kontinuierliche Veränderungen erfordern iterative Selbstvergewisserung und fortlaufende Orientierung. Das Innovationslabor ist in diesem Sinne als Lern-, Reflexions- und Übergangsraum für Professionelle und Studierende zu verstehen (Schäffter 2014: 134). Es wird zum Transformationsritual der Übergangsgestaltung (Weber 2005) sowohl hinsichtlich der Professionalisierung der Studierenden wie auch der Professionellen. Forschungsmethodisch kommt daher ein imaginativer Zugang der Prozessgestaltung zur Geltung, der Reflexivierung ebenso wie Entwurfshandeln unterstützt.

\section{Reflexive Imagination im Prozess: Die längsschnittliche Erschließung individueller und kollektiver organisationspädagogischer Professionalisierung}

Innovationslabore als Schlüsselstellen und liminale Übergangsräume werden relevant für die Professionalisierung Studierender und der Akteure des regionalen Feldes. Innovationslabore bearbeiten ungewisse Ausgangslagen und ungewisse Zielstellungen prozessual als transformative Übergangsrituale (Weber 2005). Bearbeitungsmodus und Forschungsprozess sind als ästhetischer Prozess angelegt und basieren auf dem Prinzip ästhetischer Reflexivierung.

Sowohl regionale Akteure wie auch Studierende werden über den einjährigen Prozess hinweg längsschnittlich (Brake 2018) und im Prozessverlauf vor und nach den drei durchgeführten Innovationslaboren qualitativ mittels bildbasierter Interviews und Gruppendiskussionen befragt. Im Falle der regionalen Akteure erfolgen die Erhebungen als bildbasiertes Interview, im Falle der Studierenden als bildbasierte Peer-Interviews über zehn Erhebungszeitpunkte hinweg. Die von den Studierenden moderierten Kleingruppen der Innovationslabore werden zum Referenzpunkt ihres eigenen Selbstvergewisserungsprozesses. Reflexive Orientierung erfolgt hier als Imaginations- und Entwurfsprozess.

Der Einsatz und die Analyse von Bild- und Sprachmetaphern in bildbasierten Interviews und Gruppendiskussionen ermöglicht zugleich einerseits die 
,Verflüssigung' von Welt- und Selbstbezügen und andererseits, diese Veränderungen im Prozess nachvollziehen zu können (Koller 2012: 162). Seit den 1950er Jahren (Merton/Kendall 1956) werden Bilder aufgrund ihrer ,elicitiven“, d.h. Sprechen hervorbringenden Funktion genutzt. Der Ansatz ästhetischer Transformation fasst jedoch Veränderungen der Selbst- und Weltbeziehungen insgesamt als sinnlich-ästhetischen Prozess und schlägt entsprechend auch forschungsmethodisch ästhetische Zugänge vor (Weber 2018a).

Bildbasierte Ansätze gehen über die Analyse praktischer Vollzugslogiken eines Sprechens über eigene Bildungsprozesse (Kokemohr 2003: 29) hinaus und beziehen die Imaginationen des Selbst ein. Die in den Bildwahlen eingelagerte ,diskursive Vagheit“ (Küllertz 2007: 7) der Bildmetaphern wird metaphorisch expliziert. Imaginationen werden reflexiv fruchtbar in den Narrationen der eigenen Erfahrungen mit anderen und der Grenzüberschreitung sowie des beraterischen Selbst im Werden.

Das „Konzeptsystem, nach dem wir sowohl denken als auch handeln“, ist „im Kern [als] grundsätzlich metaphorisch“ zu verstehen (Lakoff/Johnson 2004: 11). Als metaphorische Artikulationen unterstützen Bilder und Narrationen die Hervorbringung ebenso wie die Rekonstruktion transformatorischer Bildungsprozesse. Metaphern artikulieren sich in Narrationen. So legt Schmitt (2011) einen metaphernbasierten Zugang vor und Engel (2018) zeigt das Potenzial bildbasierter Zugänge im Kontext von Evaluation auf. Als metaphorische Artikulationen unterstützen Bilder die Hervorbringung ebenso wie die Rekonstruktion transformatorischer Bildungsprozesse. Bildbasiertes ebenso wie metaphorisches Sprechen lässt sich sehr gut auch längsschnittlich erschließen und rekonstruieren. So haben Kochis und Gillespie (2006) die Veränderung studentischer Metaphorik untersucht.

Karl (2007) versteht Metaphern als eingelagert in diskursive Formationen. Sie sieht daher die Anschlussfähigkeit metaphernanalytischer Zugänge für diskursmethodologische Analysen. So lassen sich Denk-, Gefühls- und Handlungsweisen Einzelner mit der Analyse historisch-kultureller, macht-wissenförmiger Symbolisierungsweisen verknüpfen. Narrationen und die sie figurierenden metaphorischen Konzepte lassen sich dann als individuelle und kollektive (Konstruktions-)Leistungen in ihrer Verschränkung mit historisch-kultureller, gesellschaftlicher und organisationaler Diskurspraxis analysieren (Weber/Wieners 2018).

Wie umrissen wurde, können mittels imaginativer Reflexivierung tiefere Sinnebenen Lernender in ihren Professionalisierungsprozessen und diskursiven Einbettungen in den Blick kommen. Jenseits qualifikatorischer oder kompetenztheoretischer Rekonstruktionen eröffnen Arrangements der Selbstvergewisserung und der Imagination einen zugleich forschenden wie transformatorischen Blick auf individuelles und kollektives Entwurfshandeln im Werden. Organisationspädagogische Professionalisierung kann so für Studierende wie auch für die Erwachsenenbildung, für Professionelle und Organisationen als 
(kollektiver) transformatorischer Bildungs- und reflexiver Transformationsprozess ganzheitlich erschlossen und untersucht werden.

\section{Literatur}

Adler, Annett / Weber, Susanne Maria (2019): Future and Innovation Labs as Heterotopic Spaces: In: Weber, S. M. / Schröder, C. / Truschkat, I. / Herz, A. / Peters, L. (Hrsg.): Organisation und Netzwerke. Wiesbaden: Springer VS, S. $375-383$.

Anderson, Lorin / Krathwohl, David (2001): A Taxonomy for Learning, Teaching, and Assessing: A Revision of Bloom's Taxonomy of Educational Objectives. London: Longman Publishing Group.

Arn, Christof (2016): Agile Hochschuldidaktik. Weinheim: Beltz Juventa.

Arnold, Rolf / Schüssler, Ingeborg (2010): Ermöglichungsdidaktik. Baltmannsweiler: Schneider.

Bauer, Petra / Weinhardt, Marc (2014): Perspektiven sozialpädagogischer Beratung. Weinheim: Beltz Juventa.

Bloom, Benjamin / Krathwohl, David (1956). Taxonomy of educational objectives: The classification of educational goals. Handbook I: Cognitive domain. New York: David McKay Company.

Brake, Anna (2018): Prozessorientierung und Längsschnittdesign als Forschungsstrategie der Organisationspädagogik. In: Göhlich, M. / Schröer, A. / Weber, S. M. (Hrsg.): Handbuch Organisationspädagogik. Wiesbaden: Springer VS. S. 307-319.

Chesbrough, Henry (2003): Open Innovation. Boston: Harvard Business School Press.

de Haan, Gerhard (2008): Gestaltungskompetenz als Kompetenzkonzept für Bildung für nachhaltige Entwicklung. In: Bormann, I. / de Haan, G. (Hrsg.): Kompetenzen der Bildung für nachhaltige Entwicklung. Wiesbaden: Springer VS, S. 23-44.

Dewe, Bernd / Ferchhoff, Wilfried / Radtke, Frank-Olaf(1992): Das „Professionswissen" von Pädagogen. Wiesbaden: Springer.

Engel, Juliane (2018): Evaluation als Methode organisationspädagogischer Praxis. In: Göhlich, M. / Schröer, A. / Weber, S. M. (Hrsg.): Handbuch Organisationspädagogik. Wiesbaden: Springer VS, S. 697-708.

Erpenbeck, John / Heise, Volker (Hrsg.) (1997): Der Sprung über die Kompetenzbarriere: Kommunikation, selbstorganisiertes Lernen und Kompetenzentwicklung von und in Unternehmen. Bielefeld: Bertelsmann.

Erpenbeck, John / Rosenstiel, Lutz von / Grote, Sven / Sauter, Werner (Hrsg.) (2017): Handbuch Kompetenzmessung. 3. Aufl. Stuttgart: Schaefer-Poeschel. 
Ferchhoff, Wilfried / Schwarz, Martin P. (2014): Zur Genese der klassischen Professionen. Bad Heilbrunn: Julius Klinkhardt.

Häußler, Peter (2014): Wie lässt sich der Lernerfolg messen? In: Kircher, E. / Girwidz, R. / Häußler, P. (Hrsg.): Physikdidaktik. Theorie und Praxis. Wiesbaden: Springer VS, S. 247-293.

Heidelmann, Marc-André / Weber, Susanne Maria (2018): Organisationspädagogisches Wissen im Praxisfeld der Organisationsentwicklung und -beratung. In: Göhlich, M. / Schröer, A. / Weber, S. M. (Hrsg.): Handbuch Organisationspädagogik. Wiesbaden: Springer VS. S. 865-877.

Karl, Ute (2007): Metaphern als Spuren von Diskursen in biographischen Texten. In: Forum Sozialforschung, 8(1).

Kochis, Bruce/Gillespie, Diane (2006). Conceptual metaphors as interpretive tools in qualitative research: A re-examination of college students' diversity discussions. In: The Qualitative Report, 11(3), S. 566-585.

Kokemohr, Rainer (2007): Bildung als Welt- und Selbstentwurf im Fremden. In: Koller, H. C. / Marotzki, W. / Sanders, O. (Hrsg.): Bildungsprozesse und Fremdheitserfahrung - Beiträge zu einer Theorie transformatorischer Bildungsprozesse. Bielefeld: transcript, S. 13-69.

Koller, Hans-Christoph (2012): Bildung anders denken. Einführung in die Theorie transformatorischer Bildungsprozesse. Stuttgart: Kohlhammer.

Küllertz, Daniela (2007): Überlegungen zu einer bildungstheoretisch inspirierten Diskursanalyse multimedialer Artikulation. In: Bildungsforschung 4(2). $26 \mathrm{~S}$.

Lakoff, George / Johnsson, Mark (2004): Leben in Metaphern: Konstruktion und Gebrauch von Sprachbildern. Heidelberg: Carl-Auer-Systeme-Verlag.

Merton, Robert / Kendall, Patricia (1956). The focused interview: A manual of problems and procedures. Glencoe: Free Press.

Ritter, Joachim / Gründer, Karlfried (1976): Historisches Wörterbuch der Philosophie. Band 4, I-K. Stuttgart.

Schäffter, Ortfried (2001): Weiterbildung in der Transformationsgesellschaft. Hohengehren: Schneider.

Schäffter, Ortfried (2014): Bildungsformate im gesellschaftlichen Strukturwandel. In: Felden, H. von / Schäffter, O. / Schicke, H. (Hrsg.): Denken in Übergängen. Wiesbaden: Springer VS, S. 111-136.

Schmitt, Rudolf (2011). Metaphernanalyse in der Erziehungswissenschaft. In: Maschke, S. / Stecher, L. (Hrsg.): Enzyklopädie Erziehungswissenschaft Online. Qualitative Forschungsmethoden. Weinheim: Juventa, S. 1-34.

Singer-Brodowski, Mandy (2016): Studierende als GestalterInnen einer Hochschulbildung für nachhaltige Entwicklung. Berlin: BWV Berliner Wissenschaftsverlag.

Tippelt, Rudolf (2018): Professionsforschung und Bildung. In: Tippelt, R. / Schmidt-Hertha, B. (Hrsg.): Handbuch Bildungsforschung, 4. Aufl., S. 649-667.

Tietgens, Hans (1986): Erwachsenenbildung als Suchbewegung. Annäherungen an eine Wissenschaft von der Erwachsenenbildung. Bad Heilbrunn/Obb.: Julius Klinkhardt. 
WBGU - Wissenschaftlicher Beirat der Bundesregierung Globale Umweltveränderungen (2011). Hauptgutachten. Welt im Wandel. Gesellschaftsvertrag für eine Große Transformation. Berlin: WBGU.

Weber, Susanne Maria (2005). Rituale der Transformation. Großgruppenverfahren als pädagogisches Wissen am Markt. Habilitationsschrift. Wiesbaden. Springer VS.

Weber, Susanne Maria (2018a): Ästhetisierung und Gestaltungsorientierung als Forschungsstrategien der Organisationspädagogik. In: Göhlich, M. / Schröer, A. / Weber, S. M. (Hrsg.): Handbuch Organisationspädagogik. Wiesbaden: Springer VS, S. 343-355.

Weber, Susanne Maria (2018b): Innovationsmanagement als Gegenstand der Organisationspädagogik. In: Göhlich, M. / Schröer, A. / Weber, S. M. (Hrsg.): Handbuch Organisationspädagogik. Wiesbaden: Springer VS, S. 517-529.

Weber, Susanne Maria / Wieners, Sarah (2018): Diskurstheoretische Grundlagen der Organisationspädagogik. In: Göhlich, M. / Schröer, A. / Weber, S. M. (Hrsg.): Handbuch Organisationspädagogik. Wiesbaden: Springer VS, S. 635-646.

Weber, Susanne Maria / Heidelmann, Marc-André / Adler, Annett (2019): Mit der Engagementwerkstatt Studierende und Freiwilligenorganisationen vereinen. In: Möller, C. / Rundnagel, H. (Hrsg): Freiwilliges Engagement von Studierenden. Wiesbaden: Springer, S. 131-152.

Weber, Susanne Maria / Heidelmann, Marc-André (2019): Towards Regional Circular Economies. In: Leal Filho, W. / Bardi, U. (Hrsg.): Sustainability in University Campuses. Cham: Springer.

Weinert, Franz (2001): Vergleichende Leistungsmessung in Schulen - Eine umstrittene Selbstverständlichkeit. In: Weinert, F. (Hrsg.): Leistungsmessungen in Schulen. Weinheim: Beltz. S. 17-31.

Winther, Esther (2010): Kompetenzmessung in der beruflichen Bildung. Bielefeld: Bertelsmann. 
Nikolaus Meyer, Dieter Nittel

\section{Transformation von Erziehung und Bildung: Begleitung als Merkmal des pädagogisch- organisierten Systems des lebenslangen Lernens}

\section{Kongruenz zwischen Selbst- und Fremdbeschreibungen: die Allgegenwart von Begleitung in Theorie und Praxis}

Bei der zusammenfassenden Analyse unterschiedlicher empirischer Qualifikations- und Forschungsprojekte mit ganz unterschiedlichen Fragestellungen und Erkenntnisinteressen (vgl. Nittel, Schütz, Tippelt 2014; Burkart, Meyer, Stemmer 2016; Meyer 2017; Nittel, Tippelt 2018) zeigt sich sehr deutlich immer wieder ein und dasselbe Phänomen: In den beruflichen Selbstbeschreibungen ${ }^{1}$ von pädagogisch Tätigen aus der Elementar- wie der Primarbildung, der Sekundarstufe I und II, der Sozialpädagogik, der Erwachsenenbildung und den Hochschulen nehmen die Begriffe Begleiten und Begleitung eine zentrale Stellung ein. ${ }^{2}$ Hier zwei Beispiele aus den Bereichen Schule und Erwachsenenbildung:

- „B: Das ist auch etwas sehr Besonderes, dass man Schüler begleitet, durch wichtige Lebensjahre. In meiner Schule war von Klasse fünf bis zur Klasse zehn äh, Unterricht und, ja das war eine Pubertätszeit, wichtige Zeit, Kinder durch die Pubertät $\mathrm{zu}$ begleiten, bis sie junge Erwachsene sind.“ (Sekundarbereich)

- „OSm: Wertschätzend sein, das ist, glaub ich, ein absolutes Muss für jeden Trainer, weil Menschen in einem Lernprozess zu begleiten, ist ja doch durchaus zuweilen doch was sehr intimes, wenn Menschen gerade in einem neuen Bereich ihre ersten Schritte machen, nicht wissen, wie, was passiert, wenn ich hinfalle.“ (Erwachsenenbildung)

1 Zum Begriff der beruflichen Selbstbeschreibung: Nittel 2002.

2 Die Autoren legen auf den qualitativen Charakter des vorliegenden Beitrags wert. Gleichwohl illustriert eine quantitative Auszählung der vorliegenden Daten (Fünf Expert*inneninterviews und drei Gruppendiskussionen) nur eines Qualifizierungsprojekts (vgl. Meyer 2017) bereits den skizzierten Befund der naturalisierten Verwendung: Während Begleiten 48- und Begleitung immerhin noch 18-mal genannt werden, liegen die zentralen erziehungswissenschaftlichen Begriffe Lernen (39) und Bildung (17) dahinter. 
Aber nicht nur die sozialen Welten pädagogischer Berufskulturen, sondern auch die Organisationssphäre nutzt die Kategorien Begleiten - verstanden als situative Praktik - und die Begleitung als Synonym für eine weitgehend habitualisierte Kernaktivität (vgl. Nittel, Meyer, Kipper 2019). In beiden Fällen dient der Begriff dazu, die eigene pädagogische Praxis zu beschreiben und dabei der notwendigen Vielfalt an Situationsfacetten gerecht zu werden. Darauf weisen die institutionellen Selbstbeschreibungen ${ }^{3}$ deutlich hin. Selbst in der Logik der Organisation nehmen also Begleiten und Begleitung eine manifeste Bedeutung an, wie die folgenden Beispiele aus der Erwachsenenbildung und der Altenhilfe zeigen:

- Das Leitbild KVHS Kassel hält fest: VHS soll „lebensbegleitendes Lernen ermöglichen und organisieren. Die Volkshochschule begleitet gesellschaftliche Entwicklungen und wirkt sozialen Ungleichheiten im Bildungsbereich entgegen." (KVHS Kassel 2016)

- „Dabei ist Hilfe immer individuell. Das kann ein Gespräch sein, die Pflege zuhause in der eigenen Wohnung, ein Begleiten von schwerkranken Menschen, eine Beratung oder einfach nur eine mitmenschliche Hilfe, damit das Leben weitergeht." (Caritas Nordhessen)

Bei den bisher präsentierten Beispielen aus den beruflichen wie den institutionellen Selbstbeschreibungen wurden die Kategorien Begleitung und Begleiten explizit genutzt. Daneben ließen sich beide Begriffe und ihre immanente Bedeutung aus den uns vorliegenden Daten auch rekonstruktiv herausarbeiten. Gleichwohl verbleibt der vorliegende Beitrag auf der explizit-manifesten Ebene, um eine Klärung des scheinbar naturwüchsig zur Anwendung kommenden Begriffspaars zu leisten. Denn: Zwar finden die Praktiker*innen und die entsprechenden Organisationen die Beschreibung der eigenen Arbeit mithilfe von Begleiten und Begleitung passend, was aber in scharfem Kontrast zur erziehungswissenschaftlichen Literatur steht. Das hat eine systematische Recherche zahlreicher Lexika, Handbücher sowie erziehungswissenschaftlicher Klassiker, sowohl historischer als auch aktueller Publikationen belegen können (Nittel, Meyer 2018, S. 1065f.).

Trotz des Fehlens einer genuinen theoretischen Dimension lässt sich sagen, dass die vorliegenden erziehungswissenschaftlichen Zeitdiagnosen - wie etwa die These von der Universalisierung des Pädagogischen (Kade, Seiter 2007) pädagogische Begleitung mindestens implizit voraussetzen oder sogar explizit zur Beschreibung moderner Konzepte wie Inklusion nutzen (vgl. Sommerfeld, Hollenstein, Calzaferri 2012). Im Ansatz der Humanontogenese von Dieter

3 Institutionelle Selbstbeschreibungen verstehen wir als kommunikative Gattung, mit der „die Organisation sich selbst identifiziert“ (Luhmann 2000, S. 417). Konzepte, Leitbilder und Konzeptionen lassen sich als eine Art einheitsstiftendes Gedächtnis der jeweiligen Einrichtung begreifen. In ihr können der organisationale Auftrag, zentrale Wertvorstellungen, die Historie oder zentrale Kooperationspartner festgehalten werden. 
Lenzen (1997), gerät die Begleitung bei der Formung des Lebenslaufs der Adressat*innen, also die lebenslange Entwicklung eines Menschen, ebenfalls in den Blick. Gerade auf der Ebene der Ontogenese ist die mit Universalisierung von Kade und Seitter (2007) beschriebene extensive Pädagogisierung der biographischen Lebensführung zu beobachten.

Auch aus der Sicht von Verfechtern der Wissensgesellschaft ist die Kategorie der Begleitung gleichsam zwingend, obwohl sie kaum unmittelbar in den entsprechenden Texten auftaucht. In diesem Wissenschaftsmilieu wird der Ausbau des Bildungsbereichs schon sehr früh prognostiziert (vgl. Machlup 1962), wobei dieser sich nicht nur auf Schule und Universitäten, sondern auf das gesamte Spektrum pädagogischer Interventionsmöglichkeiten über die Lebensspanne erstreckt. Mit der Diversifizierung von Begleitung wird Inklusion zum Normalfall, womit erneut ein Anschluss an die moderne sozialwissenschaftliche Forschung angedeutet wäre. Wir haben dabei die Theorie sozialer Systeme im Blick, wie sie von Rudolf Stichweh (2016) entwickelt wurde. Eine Dimension der sich hier anbahnenden Transformation ist die Abkehr von der „Vorbereitung“ und die gleichzeitige Hinwendung zur biographischen Begleitung. Baute die Vorbereitung noch auf der Vorstellung einer sachlichen, personellen und zeitlichen Konzentration des Lernens in bestimmten lebenszyklischen Phasen auf, so reagiert Begleitung auf die wachsende Kontingenz und Komplexität der modernen Gesellschaft, die Zunahme von Ungewissheit und eine damit verbundene neue Logik pädagogischer Interventionen über die Lebensspanne ${ }^{4}$, nämlich die arbeitsteilige Gestaltung der Humanontogenese.

\section{Begleitung - Symptom für die Formierung eines pädagogisch organisierten Systems des lebenslangen Lernens in statu nascendi?}

Wir betrachten die eben skizzierte Kongruenz von beruflichen und institutionellen Selbstbeschreibungen einerseits und der wissenschaftlichen Beobachtung andererseits als Indiz eines groß angelegten säkularen Transformationsprozesses: als Zeichen und Vorbote für eine sich anbahnende Veränderung des traditionellen Erziehungs- und Bildungssystems in Richtung eines pädagogisch organisierten Systems des lebenslangen Lernens. Das Begleiten als Prak-

4 Die Diversifizierung von pädagogischer Beratung über die gesamte Lebensspanne illustriert den Wechsel von Vorbereitung auf das Leben in Richtung seiner Begleitung (vgl. Gieseke, Nittel 2016). 
tik und die Begleitung als Kernaktivität dürfen folglich nicht nur als Handlungsphänomene, sondern müssen gleichzeitig als zentrale Operationen in der arbeitsteiligen Gestaltung der Humanontogenese betrachtet werden. Sie setzen die Mindestbedingungen dafür, dass die sequentielle Staffelung der Kooperation zwischen Erziehungs- und Bildungsorganisationen über die gesamte Lebensspanne auf Dauer gestellt werden kann.

Einer der mächtigsten Katalysatoren für den hier fokussierten Wandlungsprozess ist die Verschränkung von Phylo- und Ontogenese (vgl. Nittel 2017): So bilden die Pädagogisierung der biographischen Lebensführung - wie etwa die Gestaltung von Übergängen durch Erziehungs- und Bildungsinstitutionen und die zunehmende Selbstpädagogisierung als neoliberale Selbstoptimierungsstrategie - und die historisch noch nie dagewesene, objektivierbare Expansion pädagogischer Einrichtungen, Ämter und Dienstleistungen (vgl. Martin et al. 2016; Destatis 2018) auf der Ebene der Menschheitsgeschichte zwei Seiten derselben Medaille. Das informelle Lernen außerhalb dieses weitläufigen Handlungsrahmens fungiert als Umwelt und wird als das betrachtet, was es ist, als naturwüchsige Sozialisation der Lebenspraxis. Beide Prozessdimensionen befeuern und verstärken sich wechselseitig: der wachsende Bedarf an pädagogischer Betreuung, Beratung und Unterstützung beim Individuum lässt die Zahl der Einrichtungen anwachsen, während die Existenz bestimmter institutioneller Angebotsstrukturen auch die individuelle Nachfrage verstärkt. Das sich entfaltende System weist die Sphären Innen und Außen auf. Die Einheit des formalen- und des non-formalen Lernen bildet den Systemkern, während das informelle Lernen zur Umwelt avanciert. Momentan wird die gesellschaftliche Entwicklung maßgeblich durch die Substitution der naturwüchsigen Sozialisation - des informellen durch das organisierte Lernen - bestimmt. Als Arbeitsdefinition für das hier sich formierende System wählen wir die folgende Formulierung: „Pädagogische Praktikerinnen verrichten an ,pädagogisch Anderen' (Kinder, Schülerinnen, Studierende, Auszubildende, Klienten, Teilnehmerinnen usw.) adressierte Erziehungs- und Bildungsarbeit in Einrichtungen, die sich selbst als pädagogisch definieren“ (Nittel, Schütz, Tippelt 2014, S. 22).

In dieser tautologisch anmutenden Beschreibung zeigt sich das Alleinstellungsmerkmal von Erziehungs- und Bildungsinstitutionen: Sie begreifen sich als pädagogische soziale Einheiten, weil sie in ihren Absichtserklärungen und dem beruflichen Auftrag eine Intentionalität der rollenspezifischen Veränderung im Horizont des Lebenslaufs zur Geltung bringen. Während es auf der Ebene der Intentionalität und Programmatik um die rollenspezifische Prägung geht, vollzieht sich in der Funktionalität sehr wohl eine ,ganzheitliche“ Beeinflussung der Persönlichkeit. Die Folgen sind evident: In Erziehungs- und Bildungsfragen begleiten speziell ausgebildete Fachkräfte mit einer bestimmten beruflichen Lizenz Gesellschaftsmitglieder von Kindheit über Jugend bis zum späten Erwachsenenleben im Prozess des Älterwerdens (vgl. ebd.) und tragen 
damit zur Identitätsbildung bei. Die zentrale Leistung des Systems ist letztlich die Schaffung von Bedingungen, um Möglichkeiten der Integration in andere Funktionssysteme zu schaffen, den gesellschaftlichen Status Quo einerseits zu erhalten, andererseits aber auch Impulse zum gesellschaftlichen Fortschritt und zur Innovationsfähigkeit der Gesellschaft beizutragen. Die damit korrespondierende Funktion des Systems vollzieht sich somit in der Herstellung und Bewahrung der Lernfähigkeit und Lernbereitschaft über die gesamte Lebensspanne.

\section{Konsequenzen für die Erwachsenenbildung/Weiterbildung}

Die eben angedeuteten Transformationsprozesse haben weitreichende Konsequenzen nicht nur für die Erwachsenenbildung/Weiterbildung, sondern für alle anderen Bereiche des früheren „Erziehungs- und Bildungswesens“. Die Verhältnisse zwischen Elementarpädagogik, Primarbereich, Sekundarstufe I und II, Sozialpädagogik und Hochschulbildung stehen ebenso vor der Notwendigkeit einer Neujustierung wie auch die Bedingungen innerhalb dieser Teilbereiche. Zunächst einmal avancieren die genannten Sektoren allesamt in den Status von Subsystemen. Die Erwachsenenbildung/Weiterbildung verliert bereits auf der Ebene der Attribuierung und Benennung ihre marginalisierte Rolle als vierte Säule des Bildungssystems. Absolut zwingend ist eine solche Egalisierung schon deswegen, weil die nun etablierte funktionale Differenzierung zwischen den Subsystemen die bisher übliche Zählweise (Primarbereich, Tertiärbereich etc.) und damit die unterschwellig durch den bisher dominanten Modus der Vorbereitung transportierte Rangordnung obsolet macht. Unter Maßgabe der Funktion des Systems - zur Herstellung, Veränderung und Bewahrung der Lernfähigkeit und Lernbereitschaft über die gesamte Lebensspanne beizutragen und für demokratische Gemeinwesen funktionale Identitätsformationen zu erzeugen - sind die oben erwähnten Subsysteme prinzipiell gleich wichtig und relevant. Letztlich können, wie am Beispiel der Alphabetisierung deutlich wird, scheinbar eindeutig zugeordnete Lernleistungen - hier das Lesen und Schreiben, das eigentlich der schulischen Lernphase zugeschrieben wird auch an anderer Stelle des Systems - hier der Volkshochschule - erworben werden. Diese zunehmende Flexibilisierung, ja möglicherweise sogar Verflüssigung von Institutionsgrenzen trägt letztlich gesellschaftlichen Notwendigkeiten und Entwicklungen Rechnung, die unter den verschiedensten zeitdiagnostischen Begrifflichkeiten (Wissensgesellschaft, Risikogesellschaft etc.) gefasst werden. Unterm Strich ist ihnen allen die Erkenntnis inhärent, dass nur eine 
Phase der Vorbereitung auf das Leben und seine notwendigen werdenden Wissensbestände nicht mehr reicht. Dieser Tatbestand ist bereits im Alltagswissen der Bevölkerung präsent: Eine Untersuchung des Instituts für Demoskopie Allensbach fragte, ob ,Schüler in Deutschland ausreichend auf das Arbeitsleben vorbereitet" werden. Im Ergebnis verneinten dies 52\% der Gesamtbevölkerung und immerhin noch 49\% der Eltern schulpflichtiger Kinder (FAS 2019).

Daneben tritt im Zuge von Individualisierungsprozessen ein weiteres Charakteristikum moderner Komplexgesellschaften zutage: Zum Menschenbild demokratischer Gesellschaften zählt heute die weitgehende Gleichwertigkeit der Lebensalter. Diese vorausgesetzt, können auch nicht die pädagogischen Organisationen, die diese Lebensalter bedienen, ungleich behandelt werden. Dabei will bedacht sein, dass die Erwachsenenbildung/Weiterbildung für einen besonders großen Teil der Lebenszeit zuständig ist, stellt man die durchschnittliche Lebenserwartung von Frauen und Männer in den Gesellschaften des Westens in Rechnung. Schon dieser Umstand evoziert, wenn man an die materielle Unterversorgung dieses Bereichs denkt, eine dauerhafte Skandalisierung.

Im Zuge der gesellschaftlichen Modernisierung und unter den Bedingungen des demographischen Wandels werden sich, so unsere Annahme, die Zugänge zum System des lebenslangen Lernens in der Zukunft immer mehr in die höheren Altersklassen verschieben (vgl. Schramek et al. 2018), während sich die Zugänge und die damit verbundenen Statuspassagen in den unteren Altersklassen konsolidiert haben. Derzeit zeichnet sich die klare Zugangsverdichtung in den Altersgruppen zwischen drei bis sechs Jahren ab. Aber nicht nur die möglichst reibungslose Sozialisation von Kindern und Jugendlichen, auch die soziale Integration von Menschen in der dritten Lebensphase wird die Gesellschaft langfristig vor große Herausforderungen stellen. Die Beteiligung an Bildungs- und Freizeitprogrammen wohlfahrtsstaatlicher und privater Organisationen oder solchen der Gesundheitsbildung werden in einigen Jahrzehnten aus der Sicht vieler Experten*innen zu strategisch wichtigen Integrationsinstrumenten für die dann noch stärker angewachsenen Alterskohorten der 50- bis 70-Jährigen und der 70- bis 90-Jährigen avancieren (vgl. Aner und Karl 2010). Soziale Vereinzelung im Zuge einer gesteigerten Individualisierung, das Sinnvakuum aufgrund einer wachsenden Säkularisierung, in übersteigerten Konsum gehüllter Hedonismus, rastlose Reisetätigkeiten und andere soziale Phänomene schaffen einen objektiven Bedarf an neuen Formen der Vergemeinschaftung im Medium der Bildung. Kulturelle Teilhabe ist am ehesten über Partizipation an Bildung realisierbar. Zugänge zu Angeboten im pädagogisch organisierten System des lebenslangen Lernens könnten dann gleichzeitig auch die Pforten zu nicht-pädagogischen Programme öffnen, wie etwa zu Vorbereitungskursen für bürgerschaftliches Engagement im Umweltschutz oder zu einem freiwilligen sozialen Jahr für Senior*innen. Im Zeichen 
einer gewandelten Generationenordnung wird es dann auch um die Möglichkeit der Übernahme von bislang unzugänglichen sozialen Rollen (als Seniorberater*in in Firmen) oder die Begleitung von Aktivitäten im Sinne von Mentor*innen gehen müssen, die dazu beitragen, dass der Innovationsbedarf moderner Gesellschaften nicht mehr allein von der jungen Generation bedient werden muss.

Begleitet von dieser Entwicklung sich verändernder Verhältnisse innerund außerhalb des Subsystems Erwachsenenbildung einerseits sowie gesellschaftlichen Transformationsprozessen andererseits wird sich auch das Verhältnis zur Sozialpädagogik wandeln müssen. Zunächst gelte es, Synergieeffekte zwischen beiden Bereichen zu nutzen, und zwar im Sinne von „Was kann die Erwachsenenbildung von der Sozialpädagogik lernen und inwiefern kann die Sozialpädagogik von der Erwachsenenbildung profitieren?“. Fachkräfte aus beiden Subsystemen arbeiten schließlich teilweise in den gleichen Praxisfeldern wie etwa in der Alphabetisierungsarbeit, bei der Betreuung von Migranten und/oder bei der Vermittlung von Schlüsselqualifikationen sowie Alltagskompetenzen.

Flankiert wird dieser größere Bedarf eines interdisziplinären Austauschs zwischen Sozialpädagogik und Erwachsenenbildung durch übergreifende Prozesse. So wird die Kooperation im System noch weiter zunehmen, und zwar nicht nur im horizontalen und vertikalen Sinne. Vielmehr werden auch Crossover-Kooperationen an Boden gewinnen: zwischen Kindergärten und Hochschulen, zwischen Einrichtungen der Seniorenarbeit und der Elementarpädagogik. Die Erwachsenenbildung wird - so unsere Prognose - noch viel mehr als bisher auch andere pädagogische Berufsgruppen als Adressat*innen der Fort- und Weiterbildung entdecken und gewinnen müssen. Sträflich vernachlässigt wurde bisher die Fortbildung von Lehrer*innen als originäre Aufgabe der Erwachsenenbildung.

Erziehung und Bildung werden langfristig ihre Funktion als Einheitsformel verlieren. Wenn es ein Funktionssystem gibt, können zur Beschreibung nicht zwei Einheitsformeln (Erziehung und Bildung) dienen. Der gesellschaftliche Wandlungsprozess, der auch die Formierung eines Systems des lebenslangen Lernens langfristig evozieren wird, führt zur notwendigen Anerkennung, dass lebenslanges Lernen als widersprüchliche Einheit von Erziehung und Bildung verstanden werden muss. Dabei wird allerdings das verbreitet Paradox berührt, keine Erziehungsprozesse bei Erwachsenen zu leisten. Sowohl Erwachsenenbildung als auch Sozialpädagogik werden sich der Tatsache, die durchaus bereits auch in renommierten Analysen enthalten ist (vgl. Luhmann 2015), stellen müssen, dass objektiv auch Erziehungsfunktionen im System wahrgenommen werden. So zeigen sich erzieherische Funktionen in den Handlungsweisen von sozialpädagogischen Mitarbeiter*innen des Sozialdienstes, wenn diese die 
Lernprozesse ihre Klient*innen mithilfe der Kernaktivität des Sanktionierens ${ }^{5}$ unterstützen. Hier schildert beispielsweise eine Praktikerin die positive Untermalung durch entsprechende Laute, wenn eine neue Bewohnerin sich die ersten Male im Plenum der Wohngruppe zu Wort meldet. Ebenso zeigen sich Erziehungsprozesse in DaF-Kursen oder auch in der VHS-Theatergruppe. Die Praktiker*innen der Erwachsenenbildung betonen dagegen noch sehr deutlich, dass zentrales Güte- und Kernkriterium erwachsenenpädagogischer Arbeit die Freiwilligkeit der Teilnehmenden sei (vgl. Meyer 2017). Diese Perspektive schildern Sozialarbeiter*innen dagegen oft eher als Ziel ihres Handelns, denn als seine Grundlage (vgl. Pantuček-Eisenbacher 2001). Im Zuge des Wandlungsprozesses hin zum Begleiten von Lernen, wird die Erwachsenenbildung ebenfalls nicht an einer Änderung dieses Paradigmas vorbeikommen. Sie wird vielmehr auch Zielgruppen ansprechen (müssen), die weniger aus Ablehnung denn aus Nicht-Wissen die Bildungsmöglichkeiten des Systems des lebenslangen Lernens nicht kennen (vgl. VHS Offenbach 2018). Insofern wächst die Bedeutung der Erwachsenenbildung, weil sie einerseits über alle Funktionen des Systems des lebenslangen Lernens in Beratungsprozessen Auskunft geben und andererseits alle Funktionen auch weitgehend selbst anbieten kann. Insofern wäre die Entwicklung eines disziplinären Stolzes durchaus angebracht. Daneben gilt es wahrzunehmen, dass sowohl in der Sozialpädagogik als auch in der Erwachsenenbildung Freiwilligkeit nicht ontologisiert werden kann, sondern interaktiv hergestellt werden muss.

Gleichwohl gilt, was am Beispiel Alter angedeutet wurde: Fortschritte in der weiteren Systembildung werden nur dann zu erzielen sein, wenn es - unter Maßgabe von technokratisch nicht verkürzter Konzept und Ansätze - zu einer weiteren und gemeinsamen pädagogischen Erschließung des späten Erwachsenenalters und der hohen Altersphase gerade auch in scheinbar klassischen Pflegesettings kommt. Sollte dies gelingen, ist das pädagogisch organisierte System des lebenslangen Lernens nicht nur im metaphorischen, sondern in einem realen Sinne ,geschlossen“, da ein solches Szenario tatsächlich eine mehr oder weniger lückenlose Versorgung der Lebensspanne mit pädagogischen Angeboten implizieren würde. Das späte Erwachsenenalter und die hohen Altersphasen dürfen aus erziehungswissenschaftlicher Perspektive nicht nur auf offene oder subtile Defizitetikettierungen verkürzt werden, sondern müssen, gerade weil sich hier viele erzieherische Komponenten finden lassen (RehaMaßnahmen, paternalistische Versorgungsstrukturen in manchen Heimen), viel stärker unter dezidiert bildungstheoretischen Vorzeichen als potentielle Phasen pädagogischen Handelns ins Blickfeld gerückt werden. Dabei ist ein

5 Damit ist sowohl das Missbilligen als auch das Billigen von Leistungen und des Sozialverhaltens der pädagogischen Anderen durch die pädagogischen Leistungsrollenträger (Lehrer*in, Dozent*in usw.) gemeint. Die Formulierung von Lob und Tadel, die Betonung von Stärken und Schwächen, die positive oder negative Bewertung einer Reaktion gehören in das Spektrum dieser Kernaktivität (vgl. Nittel, Meyer, Kipper 2019). 
Festhalten an einem emphatischen Autonomiebegriff unerlässlich. Diese anspruchsvolle Aufgabenstruktur ist nur von beiden Subsystemen, der Sozialpädagogik und der Erwachsenenbildung, gemeinsam zu bewältigen und darf nicht anderen Berufsgruppen, wie der Pflegewissenschaft und der Psychologie, überlassen werden. Ein Schulterschluss von Sozialpädagogik und Erwachsenenbildung könnte in der theoretisch anspruchsvollen und gleichzeitig praxisorientierten Formulierung eines Handlungsfeldes „Alter“ bestehen. Die dabei zur Anwendung kommende gleichzeitige Wahrnehmung von Hilfs- als auch Bildungs- und Erziehungsaspekten könnte dann - diese Perspektive verspricht am meisten Erfolg - unter dem Dach der von beiden Berufsgruppen realisierten pädagogischen Kernaktivitäten (Beraten, Unterrichten, Begleiten, Sanktionieren und Organisieren) und Technologien (Programme, Veranstaltungsformen, Methoden, Medien) realisiert werden (vgl. Nittel, Meyer, Kipper 2019).

\section{Literatur}

Aner, K. \& Karl, U. (Hrsg.) (2010): Handbuch Soziale Arbeit und Alter. Wiesbaden: Springer VS.

Burkart, G., Meyer, N., Stemmer, R. (2016): Organisationales Lernen in Einrichtungen der stationären Altenhilfe als Ermöglichung von Autonomie und Partizipation. Der pädagogische Blick (4), S. 209-217.

Frankfurter Allgemeine Sonntagszeitung (FAS). (2018): Lernen fürs Leben (16.12.2018).

Gieseke, W., Nittel, D. (Hrsg.) (2016): Handbuch Pädagogische Beratung über die Lebensspanne. Weinheim: Beltz.

Kade, J., Seitter, W. (Hrsg.) (2007): Pädagogisches Wissen. Opladen: Barbara Budrich.

Lenzen, D. (1997): Lebenslauf oder Humanontogenese? In: D. Lenzen, N. Luhmann (Hrsg.), Bildung und Weiterbildung im Erziehungssystem. Lebenslauf und Humanontogenese als Medium und Form. Frankfurt am Main: Suhrkamp, S. 228-247.

Luhmann, N. (2015): Die Wissenschaft der Gesellschaft. Frankfurt am Main: Suhrkamp.

Machlup, F. (1962): The production and distribution of knowledge in the United States. Princeton, NJ: Princeton Univ. Press.

Martin, A., Lencer, S., Schrader, J., Koscheck, S., Ohly, H., Dobischat, R., Elias, A., Rosendahl, A. (2016): Das Personal in der Weiterbildung. Bielefeld: wbv.

Meyer, N. (2017): Komparative pädagogische Berufsgruppenforschung: Erwachsenenpädagogen und Journalisten im Vergleich. Bielefeld: wbv.

Nittel, D. (2002): Berufliche Selbstbeschreibungen im Spiegel von Praxisberichten. Hessische Blätter für Volksbildung (2), S. 137-152. 
Nittel, D. (2017): Ein System in statu nascendi. Auf dem Weg zum pädagogisch organisierten System des lebenslangen Lernens? In: R. Arnold, W. Neuser (Hrsg.), Beobachtung des Wissens - Das Wissen des Beobachters. Annäherung an eine systemische Hermeneutik. Baltmannsweiler: Schneider Hohengehren, S. 119-166.

Nittel, D., Schütz, J., Tippelt, R. (2014). Pädagogische Arbeit im System des lebenslangen Lernens. Ergebnisse komparativer Berufsgruppenforschung. Weinheim: Beltz.

Nittel, D., Meyer, N. (2018): Pädagogische Begleitung: Handlungsform und Systemmerkmal. Zeitschrift für Erziehungswissenschaft 21 (5), S. 1063-1082.

Nittel, D., Tippelt, R. (2018): Die Resonanz des lebenslangen Lernens im Erziehungs- und Bildungswesen. Eine vergleichende Untersuchung beruflicher und institutioneller Selbstbeschreibungen. Düsseldorf: Hans-Böckler-Stiftung.

Nittel, D., Meyer, N., Kipper, J. (2019): Ordnungsdimensionen des pädagogischen Handelns: Technologien und Kernaktivitäten. (Unveröffentlichtes Manuskript)

Pantuček-Eisenbacher, P. (2001): Under Pressure oder: Von der gefährlichen Illusion, Menschen zur Lebenstüchtigkeit zwingen zu können. Erziehung heute (4).

Schramek, R., Kricheldorff, C., Schmidt-Hertha, B., Steinfort-Diedenhofen, J. (Hrsg.) (2018): Alter(n) - Lernen - Bildung. Ein Handbuch. Stuttgart: Verlag W. Kohlhammer.

Sommerfeld, P., Hollenstein, L., Calzaferri, R. (2012): Integration und Lebensführung. Wiesbaden: Springer VS.

Stadt Offenbach am Main (Hrsg.) (2018): Projekt „, Mein Ort für Bildung “: vhs will Migranten über den Deutschkurs hinaus erreichen. https://www.offenbach.de/bildung/vhs/vhs-ort-fuer-bildung02.11.2018.php, zugegriffen: 19. Dezember 2018.

Statistisches Bundesamt (Destatis) (Hrsg.) (2018): Beschäftigte in Kindertageseinrichtungen. https://www.destatis.de/DE/ZahlenFakten/GesellschaftStaat/Soziales/Sozialleistungen/Kindertagesbetreuung/Tabellen/Tabellen_Beschaeftigte.html, zugegriffen: 28. November 2018.

Stichweh, R. (2016): Inklusion und Exklusion. Bielefeld: transcript. 
Steffi Robak, Marion Fleige, Christian Kühn, Stephanie Freide, Jessica Preuß

\section{Kulturelle Erwachsenenbildung im Zeichen digitaler Transformation: Theoretische Perspektiven und forschungsleitende Zugänge}

\section{Ausgangslage und Grundannahmen}

Ausgangspunkt des Beitrags ist die Frage danach, wie kulturelle Transformationsprozesse, insbesondere die Digitalisierung, die Kulturelle Bildung mit ihren Angeboten und Programmen und damit auch das zugrundeliegende Planungshandeln verändern. Anknüpfend an bereits vorliegende bildungswissenschaftliche Befunde zur Kulturellen Bildung (Fleige/Gieseke/Robak 2015) und unter Einbezug kulturwissenschaftlicher und -soziologischer Erkenntnisse wird eine theoretische Erweiterung des Verständnisses Kultureller Erwachsenenbildung in einer , Kultur der Digitalität “ (Stalder 2016) verfolgt. ${ }^{1}$ Drei wesentliche Grundannahmen zum Zusammenhang kultureller Transformationsprozesse und Programmplanung (von Kultureller Bildung) sowie deren Folgen für einen analytischen Blick auf die Kulturelle Erwachsenenbildung rahmen den nachfolgenden Beitrag: a) Die kulturellen Transformationsprozesse führen zu einer Ausweitung der Prozesse der Kulturformung. Dies lässt sich bereits mit den Ansätzen zur Transkulturalität und Hybridisierung (Bhabha 2000; Reckwitz 2006; Welsch 2011) beschreiben und kann mit der ,, Gesellschaft der Singularitäten “ (Reckwitz 2017) und der „,Kultur der Digitalität“ (Stalder 2016) für den digitalen Transformationsprozess weiterentwickelt werden; b) Gerade die Kulturelle Erwachsenenbildung reagiert aktiv auf diese kulturellen Transformationen, indem sie veränderte Bedürfnisse und Interessen der Lernenden antizipiert sowie bildungspolitisch gesetzte Bedarfe im Prozess der Programmplanung aufnimmt und neuartige Zugangsweisen zur Deutung von Partizipation an und (De-)Konstruktion von Kultur offeriert. Die Programmplanung wird zur Transformationsinstanz (Kühn/Robak/Fleige 2018), da sie über die fortlaufende Planung die gesellschaftlichen Entwicklungen in Bildungsmöglichkeiten überführt, die mit verschiedenen Zugängen zu Lernen und Bildung unterlegt sind; c) Infolgedessen differenzieren sich die thematischen

1 Die Behandlung dieser und weiterer Fragen sind eingebettet in ein vom BMBF gefördertes Grundlagenforschungsprojekt: „Funktionen und Bildungsziele der Digitalisierung in der Kulturellen Bildung: Systematisierung und Analyse aktueller VHS-Angebote“" (https://www.die-bonn.de/id/35729/about/html/). 
sowie didaktischen Programm- und Angebotsstrukturen der jeweiligen Einrichtungen der Erwachsenen-/Weiterbildung weiter aus.

\section{Theoretische Zugänge zur Kulturellen Erwachsenenbildung}

Allein die Vielfalt des Kulturbegriffs verdeutlicht, dass es kein allgemeingültiges Konzept Kultureller Bildung geben kann. Die verschiedenen Ansätze, „Kultur“ zu definieren, sind Ausdruck divergierender Interessen unterschiedlicher gesellschaftlicher Akteure, die um die Auslegung des Kulturbegriffs ringen (Überblick: Fuchs 2012; Reckwitz 2000, 2017). In Bezug auf die Erforschung Kultureller Bildung kann es also nicht darum gehen, sich einer spezifischen Auslegung des Kulturbegriffs zu verschreiben. Vielmehr muss erkannt werden, dass das Verhältnis von Kultur und Bildung auslegungsbedürftig ist. Diese Auslegungen lassen sich zeit- und institutionsspezifisch über die Angebote und Programme Kultureller Bildung zeigen (Überblick zu Befunden: Fleige et al. 2018b; Fleige/Gieseke/Robak 2015). Für die bildungswissenschaftliche Erforschung zur Kulturellen Erwachsenenbildung bieten vor allem die Kulturwissenschaft und -soziologie theoretische Anschlussmöglichkeiten, da diese für sich wandelnde Interessen an Kultur und Bildung offen sind (Gieseke 2003a: 208f.) und eine Auseinandersetzung mit kulturellen Transformationsprozessen vorantreiben.

\section{Transkulturalität und Hybridisierung}

Spätmoderne Gesellschaften sind von einer zunehmenden Heterogenisierung geprägt, die sich etwa in der Ausdifferenzierung verschiedener Migrationsformen bis hin zur Migrationsgesellschaft, aber vor allem auch millieuspezifischer (,singularisierter") Lebensstile (Reckwitz 2017: 273ff.) zeigt. Darin eingebunden sind neue und veränderte Erfahrungen von Fremdheit, die durch die global-vernetzte Kommunikation, die weltweite Zirkulation von Kultur- und Wirtschaftsgütern und Migrationsbewegungen getragen und somit alltäglich für die Subjekte erfahrbar werden. Welsch $(2011 ; 2012)$ verweist hier mit dem Konzept der Transkulturalität darauf, dass sich Kulturen wechselseitig durchdringen und die Idee in sich abgeschlossener und nach außer abgegrenzter „kultureller Kugeln“ nicht mehr aufrechterhalten werden kann. In einem dritten Raum (Bhabha 2000), der sich mit der Wahrnehmung kultureller Differenz 
ausbildet, kommt es seit Langem zu Vermischungen (Hybriden). Der Idee einer Reinheit von Kulturen kann damit eine klare Absage erteilt werden. Reckwitz (2006) beschreibt dies übergreifend als Hybridisierung, die als Prozess der kulturellen Neuformung die Strukturen der Modernisierung durchzieht. Das führt nicht nur zu einer permanenten Veränderung von Kultur, die in der Spätmoderne als Subjektkultur konzeptualisiert wird, sondern auch zu einer oft in sich widerstreitenden Vielfalt kultureller Praktiken, Deutungs- und Sichtweisen. In diesem kulturellen Raum des Dazwischen können sich gegenkulturelle Bewegungen ausformen, die Anlass für Protest geben (ebd.). In den Zwischenräumen kommt es zu kulturellen Neuformungen, die eine wesentliche Grundlage für die Singularisierung schaffen. Die sich bildenden Subjekte wirken hierbei zentral über ihre Praktiken, Interaktionen, wirklichkeitskonstruierenden Erfahrungen, Deutungen, aber auch Lern- und Bildungsaktivitäten, die sie als Ressourcen einbringen, an der Neuformung von Kultur mit (Robak 2017; 2018). Bedeutung, Zugehörigkeit und auch Identität werden damit verhandelt und können neu ausgeformt werden. Die Planung von entsprechenden Programmen und Angeboten Kultureller Bildung wirkt aus Perspektive der Transkultur als Transformationsinstanz, indem die Planenden die beschriebenen Phänomene als gesellschaftliche Entwicklungen wahrnehmen und deuten, darauf bezogene Bedarfe ableiten sowie individuelle Bedürfnisse und Interessen antizipieren und im Hinblick auf passfähige Bildungsangebote aufeinander beziehen.

\section{Digitalisierung als kultureller Transformationsprozess}

Im Rückgriff auf die Hybridisierung als Modernisierungsmerkmal lässt sich Digitalisierung als kultureller Transformationsprozess konzipieren, der im Kern kein von Technik evozierter oder vorangetriebener Prozess ist (technokratische Sichtweise) (Allert/Asmussen 2017: 29), sondern sich in schon länger stattfindende gesellschaftliche Veränderungen einordnet. Wenngleich ein komplexes Zusammenspiel von Technik- und Medienwandel sowie Veränderungen der Art und Weise der Lebensführung und der Ökonomie in der Spätmoderne zu verzeichnen sind, so muss doch festgehalten werden, dass gerade diejenigen Formen der Techniknutzung und -hervorbringung auf kulturelle Praktiken zurückgehen, die lange vor dem Internet oder dem Smartphone existierten (Stalder 2016). Nach Reckwitz (2017) muss hierbei unterschieden werden zwischen einer technischen Entwicklung, die auf die Produktion standardisierter Massengüter ausgerichtet ist, und einer digitalen Technologie, die zur Umwelt der Kulturalisierung wird. 
„Technik ist immer weniger Werkzeug, sondern wird immer mehr zu einer technologischen Umwelt, in der sich die Subjekte bewegen. Bei dieser Umwelt handelt es sich im Kern um eine kulturelle Umwelt, welche die Subjekte immerfort affiziert.“ (Reckwitz 2017: 237, Hervorhebung i.O.)

Dies stellt für Reckwitz einen Strukturbruch in der Moderne dar, welcher die Logik des Allgemeinen ablöst, die auf die Standardisierung, Massenproduktion und Angleichung ausgerichtet ist. Stattdessen stützen für ihn die digitalen Technologien eine Logik des Besonderen, die veränderte soziale Einheiten (Subjekte, Objekte, Zeitlichkeit, Örtlichkeit, Kollektive) hervorbringt. Kennzeichnendes Merkmal ist, dass diesen Einheiten nicht nur gesellschaftlicher Nutzen, sondern auch ein Wert zugeschrieben oder aberkannt wird. Mit dieser In-Wert-Setzung (Valorisierung) ist verbunden, dass die singulären Subjekte von diesen kulturalisierten sozialen Einheiten angezogen, also affiziert werden (ebd.: 27ff.). Die Subjekte binden an diese Einheiten Emotionen und bauen Beziehungen zu ihnen auf, was ein konstituierendes Merkmal von Lern- und Bildungsprozessen darstellt (Gieseke 2007). Digitalisierung erzeugt damit also keine digitale Welt neben der analogen oder eine digitalisierte Gesellschaft, sondern sie bringt eine technologische Umwelt hervor, in der sich bestimmte Formen der Bedeutungsproduktion und -verwaltung, also kulturelle Prozesse, ausformen.

Eng damit verbunden ist, dass sich die soziale Basis der Kultur erweitert ein kultureller Wandlungsprozess, der etwa in den 1950er Jahren beginnt. Der Weg dahin wurde entscheidend geebnet durch das Wachstum der Wissensökonomie, die Erosion der Heteronormativität und die Auflösung der Asymmetrie zwischen einem kulturellen Zentrum und der Peripherie (Stalder 2016: $21 \mathrm{ff}$.). Stalder (2016: 10) spricht hier von der „,Kultur der Digitalität “, in der sich die kulturellen Möglichkeiten eben jener Bedeutungsproduktion und -aushandlung enorm vervielfältigt haben. Soziales Handeln ist zunehmend in komplexe Technologien eingebettet, aktive partizipative und kreative Gestaltung von kulturellen Prozessen findet vermehrt über sowie durch digitale Medien statt und weitet sich dadurch aus (ebd.: 58). Damit schafft die Digitalisierung, anknüpfend an Bhabhas Theorem eines „Dritten Raumes“ (2000), neue Zwischenräume, die in einer technologischen Umwelt hervorgebracht werden und auf diese angewiesen sind. Hier finden Aushandlungen und Neuformungen kultureller Praktiken, Deutungs- und Sichtweisen statt, die als Gegenstandsbereich Kultureller Bildung einzubeziehen sind. In dieser Breite beschäftigt sich Kulturelle Bildung also nicht nur mit Fragen der künstlerisch-ästhetischen Produktion und Wahrnehmung, sondern eben auch mit dem Verhältnis von „fremd“ und „eigen“ sowie mit kulturformenden Prozessen innerhalb technologischer Umwelten.

Dabei ist Kulturelle Erwachsenenbildung immer auch in ihrer gesellschaftlichen Verwobenheit und in Bezug auf ihr ,gesellschafts-, kultur- und geschichtskritisches Potenzial“" (Fleige/Gieseke/Robak 2015: 17) zu befragen 
und in ihren offerierten Zugangsweisen wie strukturellen Platzierungen näher zu beschreiben. Forschungen zur Kulturellen Bildung zeigen in diesem Zusammenhang, wie sich der Bildungsbereich zunehmend mit anderen Bereichen (z.B. Gesundheitsbildung, Berufliche Bildung) verschränkt. Mit Blick auf die Digitalisierung diffundiert Kulturelle Bildung hier nun stärker in technische (z.B. EDV, Programmieren, Industrie 4.0), vor allem aber auch politische und ganz grundlegend in ethisch-normative Themenbereiche.

\section{Programm(-planungs-)forschungen zur Kulturellen Erwachsenenbildung}

Über die empirischen Programmforschungen im Feld der Kulturellen Erwachsenenbildung (z.B. Gieseke et al. 2005; Robak et al. 2015a; Robak/Petter 2014; Forschungsbericht WB-Kultur 2018 ${ }^{2}$ ) kann nachvollzogen werden, welche Auslegungen des Verhältnisses von Kultur, Bildung und Kunst sowohl institutionen- als auch themenbereichs- und zielgruppenbezogen in den Angeboten sedimentiert sind. Beim Blick auf die zugrundeliegenden Angebots- und Programmplanungsprozesse zeigt sich, dass der Kulturellen Bildung dabei unterschiedliche Funktionen und Bildungsziele (Robak/Fleige 2017; Hippel/Röbel 2016) zugewiesen werden. Dies verweist auf die Autonomie des Planungshandelns als Kern pädagogischen Handelns in den Einrichtungen der Erwachsenen-/Weiterbildung. Dabei werden die verschiedenen Perspektiven beteiligter Akteurinnen und Akteure auf die entwickelten und zu entwickelnden Bildungsangebote im Modus der Angleichung kommunikativ abgestimmt (Gieseke 2003b, 2008) bzw. die vielfältigen Erwartungshaltungen vor dem Hintergrund trägerspezifischer und institutioneller Rahmungen balanciert (Hippel 2013) und so aushandelnd eigenständige Entscheidungen getroffen (Fleige et al. 2018: 59). Die Planenden sind demnach trotz der komplexen einrichtungsbezogenen Einflüsse relativ autonom, um die einzelnen Planungsschritte kreativ auszugestalten (Robak et al. 2015b). Neben bewährten Angebotsformen und einer nachfrageorientierten Programmplanung entstehen dabei innovative Angebote, die die aufgespürten gesellschaftlichen Trends und feldspezifischen Entwicklungen prospektiv aufgreifen (können). Programmplanung ist somit

2 Spezifisch für die Weiterbildung von in Kunst und Kultur Tätigen konnte mit dem Projekt ,WB-Kultur“ (https://www.die-bonn.de/id/32311/about/html/) eine institutionenspezifische Auslegung offengelegt werden, die zum einen die Entwicklung professioneller Handlungsstrukturen in den Tätigkeitsfeldern stützt und gleichzeitig biografisch sich entwickelnde Interessenlagen der Teilnehmenden adressiert; dieses vor dem aktuellen Hintergrund der Ausdifferenzierung der gesellschaftlichen Erwartungen an Kunst, Kultur und Kulturelle Bildung. 
notwendige Bedingung und Transformationsinstanz, da sie maßgeblich mitentscheidet, welche Themen und Inhalte in den Bildungsangeboten behandelt werden, wie diese im Lehr-/Lernarrangement didaktisch umgesetzt und welche Möglichkeiten der Erweiterung von Wissen und Können damit geschaffen werden.

\section{Portale als pädagogisch gestaltete Zugänge zur Kulturellen Bildung}

Das Spektrum Kultureller Bildung kann mit dem Theorem der „Portale Kultureller Bildung " beschrieben werden. Sie sind das empirische Ergebnis einer trägerübergreifenden Programmanalyse zur Kulturellen Erwachsenenbildung in Berlin und Brandenburg in den Jahren 1996 und 2001 (Gieseke et al. 2005) und dienen vor allem der Programmforschung als Kategorien zur Analyse von Zugängen zur Kulturellen Bildung. In ihrer konzeptionellen Anlage verbinden die Portale Kultureller Bildung offerierte Themenstrukturen und Lern- und Wissensformen mit angebotenen Vermittlungs- sowie Aneignungs- und Rezeptionswegen in den unterschiedlichen Sparten und Feldern von Kunst und Kultur (grundlegend: Gieseke et al. 2005; Gieseke 2003a; weiterentwickelt z.B.: Robak/Petter 2014). Dadurch werden akzentuierte Inhalte und Themen der Kulturellen Bildung in ihrer jeweiligen Verschränkung mit spezifischen didaktisch-methodischen Ausformungen kategoriengeleitet erfass- und erschließbar sowie Innovationen, Veränderungen und Entwicklungen empirisch sicht- und erkennbar.

Grundlegend zeigen sich aus den bisherigen Studien für die Kulturelle Bildung drei Portale: Das systematisch-rezeptive Portal ist auf die Deutung(smöglichkeiten) von Kunst und Kultur ausgerichtet. Über die Ausbildung und Aktivierung von Deutungs-, Interpretations- und Urteilsfähigkeit (i.d.R. über die Vermittlung kunst-/kulturtheoretischen Wissens, das systematisch aufbereitet ist) und eines entsprechenden kritischen Bewusstseins „erweitern sich die eigenen Spielräume zur Betrachtung von Welt und für das eigene Handeln“ (Fleige/Gieseke/Robak 2015: 85). Das selbsttätig-kreative Portal ist auf das Erwerben und Erlernen von Methoden, Techniken und Arbeitsweisen sowie auf das Suchen und Ausprobieren verschiedener Darstellungs- und Ausdrucksformen ausgerichtet, die rückgekoppelt werden an eigene und alternative Wahrnehmungsweisen. Damit wird die Möglichkeit eröffnet, sowohl zu sich selbst (neu) in Beziehung zu treten als auch sich der Welt mitzuteilen und damit zu einer eigenständigen Wirklichkeits- und Selbsterkenntnis zu gelangen (ebd.: 130). Dem verstehend-kommunikativen Portal geht es um das Begreifen und Verständigen, Erkennen und Diskutieren differenter kultureller Praxis und kulturellen Wissens im Hinblick auf die Gestaltung und Formung von Kultur 
(Gieseke/Opelt 2005: 43; Robak 2017: 334). Indem man sich kommunikativ über das Eigene und das Fremde (interkulturelle Bildung) und deren kulturelle Verflechtungen (transkulturelle Bildung) verständigt, wird in entsprechenden Angeboten Welt über gemeinsame Erfahrungen gedeutet und (de-)konstruiert (Robak/Fleige 2017; Robak 2017: 333f.). Gerade in diesem Portal zeigt sich, dass die Ausformungen von Transkulturalität und Hybridisierung zu veränderten Zugängen zu Kultureller Bildung führen, die sich - auch mit Bezug auf den kulturwissenschaftlichen Diskurs (z.B. Keuchel/Wagner 2012) - auf eine breite Auslegung von Kultur beziehen und sich nicht nur auf die künstlerischästhetischen Themen beschränken.

\section{Das analytisch-reflexive Portal als neuer Zugang zur Kulturellen Bildung}

Vor dem Hintergrund der theoretischen Ausführungen zu Digitalisierung als kultureller Transformation ist in aktuellen VHS-Programmen ein weiteres Portal erkennbar, in dem sich die gestiegenen Partizipationsinteressen der Individuen ausdrücken, die über digitale Kommunikations- und Gestaltungsmöglichkeiten ihre Lebenswelten mitgestalten und an der Bedeutungsproduktion teilhaben wollen: das analytisch-reflexive Portal. Das Portal deckt auf, wie neue und veränderte Formen der Kulturgestaltung in Bildungsinstitutionen unter Einbindung verschiedenster Wissensressourcen im Prozess der Programmplanung in Angebote transformiert werden. In diesem Prozess kommt - ähnlich dem systematisch-rezeptiven Portal - der Vermittlung strukturiert aufbereiteten Wissens eine wichtige Rolle zu. Im Zentrum stehen allerdings nicht die Wahrnehmung und Bearbeitung der Differenz verschiedener Kulturen sowie deren Deutung aus inter- und transkultureller Perspektive (verstehend-kommunikativ) oder die Interpretationsbedürftigkeit und -möglichkeiten der Artefakte künstlerisch-ästhetischen Handelns (systematisch-rezeptiv). Vielmehr geht es um das Verstehen von und die Teilhabe an Kulturformungsprozessen, die auf eine digitale, technologiebasierte Umwelt angewiesen sind bzw. in ihr hervorgebracht werden. Das Selbst bekommt damit Möglichkeiten alternativer Umgangs-, Zugangs- und Gestaltungsformen aufgezeigt, erarbeitet sich diese und wird dazu angeregt, alltägliche Routinen des Technikumgangs und -einsatzes zu hinterfragen.

In diesem Sinne ermöglichen die entsprechenden Angebote Kultureller Bildung über den Aufbau von Wissen und Können einerseits die Partizipation an jenen Zwischenräumen, die von einer technologischen Umwelt abhängig sind. Hier sind die Forderungen nach Erweiterung von Medien- bzw. Digitalkompetenzen (Carretero/Vuorikari/Punie 2017) zu verorten - mitsamt den verschiedenen Aspekten von Mündigkeit wie Urteilsfähigkeit, Selbstbestimmung und 
Eigenverantwortung. Es ist aber auch kritisch zu fragen, ob diese Angebote selbst neue Zwischenräume sein können, in denen, gerade mit Blick auf die Entwicklung mediengestützter Bildungsformate, die Kulturen des Lernens und Lehrens (Fleige/Robak 2018) neu ausgeformt werden und gar von einer digitalen Bildungsrevolution (Dräger/Müller-Eiselt 2015) gesprochen werden kann. In jedem Fall schafft die Digitalisierung veränderte Möglichkeiten der Umsetzung und Steuerung von Lernprozessen, in denen sich verschiedene Szenarien mediengestützen Lernens (Kerres 2018) verwirklichen. Die technologische Umwelt ist dann die kulturelle Bedingung dafür, dass sich bestimmte lernkulturelle Regime, die zunehmend auf den Konzepten von Eigenverantwortung, Selbststeuerung und Output-Orientierung beruhen, umsetzen lassen.

\section{Schlussbemerkung: Digitalisierung der Kulturellen Bildung als Anforderung an professionelles Handeln}

Mit der digitalen Transformation bildet sich eine technologische Umwelt heraus, in der sich die gesellschaftsformenden Praktiken der Verhandlung und Realisierung von Bedeutung wandeln. Daraus resultieren aufseiten der Individuen veränderte Bildungsbedürfnisse/-bedarfe und Interessen. Programm- und Angebotsplanung im Handlungsmodus der Angleichung avanciert hier zu einer zentralen Transformationsinstanz. Sie gestaltet über die Erweiterung von Wissen und Können in Bildungsangeboten Partizipationswege an kulturellen Formungsprozessen, die in den sich in technologischen Umwelten entwickelnden Zwischenräumen stattfinden. Gerade Kulturelle Bildung ist hierbei der zentrale Ort für eine kritisch-reflexive Auseinandersetzung mit diversen Formen des gesellschaftlichen Zusammenlebens, den sich immer weiter ausdifferenzierenden kulturellen Gemeinschaften und veränderten Praktiken in einer technologischen Umwelt. Ein Verständnis Kultureller Bildung, das die Verwobenheit mit gesellschaftlichen Entwicklungen erkennt und reflektiert, befindet sich stets selbst im Wandel und bleibt auslegungsbedürftig. Die Ausformung des analytisch-reflexiven Portals als pädagogisch gestalteter Zugang zur Kulturellen Bildung liefert eine erste wichtige Erkenntnis, wohin sich die Angebots- und Programmstrukturen für die Kulturelle Bildung mit der Digitalisierung entwickeln. Darüber hinaus wird es darauf bezogener Programmforschung darum gehen, weitere Strukturen in den (VHS-)Programmen offenzulegen, die mit Blick auf die technologischen Umwelten sich verändernde Inhalte und Formen offerierten Lehrens und Lernens erfassen. Die Herausforde- 
rung besteht hier darin, die unterschiedlichen Auslegungen Kultureller Bildung, die zum einen eng auf künstlerisch-ästhetische Bildung (Fuchs 2012) und zum anderen eher weit auf den Menschen als Gestalter seiner Welt (Liebau 2012) gefasst sind, zu berücksichtigen. Ebenso gilt es, die verschiedenen Szenarien mediengestützten Lernens (Kerres 2018) - gerade im Hinblick auf die Entwicklung von Lehr-Lernkulturen (Fleige/Robak 2018) - mit einzubeziehen. Die tiefreichenden und neuartigen Anforderungen an die Angebots- und Programmplanenden (Albrecht/Revermann 2016: 18f.; Schmidt-Hertha et al. 2017: 35) und das Bildungsmanagement (Robak 2018) sind unter Rückgriff auf empirisch gesichertes Wissen zu bearbeiten, damit die Bildungseinrichtungen die Prozesse der digitalen und auch transkulturellen Transformation in professionelle erwachsenenpädagogische und profilbildende lernkulturelle Entwicklungen münden lassen können.

\section{Literatur}

Albrecht, Steffen / Revermann, Christoph (2016): Zusammenfassung. In: Albrecht, Steffen; Revermann, Christoph: Digitale Medien in der Bildung. TABArbeitsbericht Nr. 171. Berlin: Büro für Technikfolgen-Abschätzung beim Deutschen Bundestag.

Allert, Heidrun / Asmussen, Michael (2017): Bildung als produktive Verwicklung. In: Allert, Heidrun / Asmussen, Michael / Richter, Christoph (Hrsg.): Digitalität und Selbst: Interdisziplinäre Perspektiven auf Subjektivierungs- und Bildungsprozesse. Bielefeld: transcript Verlag, S. 27-68.

Bhabha, Homi K. (2000): Die Verortung der Kultur. Tübingen: Stauffenburg.

Carretero, Stephanie / Vuorikari, Riina / Punie, Yves (2017): DigComp 2.1. The Digital Competence Framework for Citizens. With eight proficiency levels and examples of use. Publications Office of the European Union.

Dräger, Jörg / Müller-Eiselt, Ralph (2015): Die digitale Bildungsrevolution. Der radikale Wandel des Lernens und wie wir ihn gestalten können. München: DVA.

Fleige, Marion / Gieseke, Wiltrud / Hippel, Aiga von / Käpplinger, Bernd / Robak, Steffi (Hrsg.) (2018a): Programm- und Angebotsentwicklung in der Erwachsenen- und Weiterbildung. Bielefeld: wbv Publikationen.

Fleige, Marion / Gieseke, Wiltrud / Robak, Steffi (2015): Kulturelle Erwachsenenbildung. Strukturen - Partizipationsformen - Domänen. Bielefeld: W. Bertelsmann.

Fleige, Marion / Hippel, Aiga von / Stimm, Maria / Gieseke, Wiltrud / Robak, Steffi (2018b): Befunde der Programmforschung. In: Fleige, Marion / Gieseke, Wiltrud / Hippel, Aiga von / Käpplinger, Bernd / Robak, Steffi (Hrsg.) (2018): 
Programm- und Angebotsentwicklung in der Erwachsenen- und Weiterbildung. Bielefeld: wbv Publikationen, S. 76-89.

Fleige, Marion / Robak, Steffi (2018): Lehr-Lernkultur in der Erwachsenenbildung. In: Tippelt, Rudolf / Hippel, Aiga von (Hrsg): Handbuch Erwachsenenbildung/Weiterbildung. Wiesbaden: VS-Verlag, S. 623-642.

Forschungsbericht WB-Kultur (2018): Robak, Steffi / Gieseke, Wiltrud / Fleige, Marion / Heidemann, Lena / Kühn, Christian / Preuß, Jessica / Krueger, Anneke / Freide, Stephanie: Abschlussbericht. Berufliche Weiterbildung für professionelles Handeln in Kunst, Kultur und kultureller Bildung - empirische Analyse zu Passungsprofilen von Angebotsstrukturen, Verwertungsinteressen und Nutzen am Beispiel der Bundesakademie für Kulturelle Bildung Wolfenbüttel. (unveröffentlicht; Publikation in Planung)

Fuchs, Max (2012): Kulturbegriffe, Kultur der Moderne, kultureller Wandel. In: Bockhorst, Hildegard / Reinwand-Weiss, Vanessa-Isabelle / Zacharias, Wolfgang (Hrsg.): Handbuch Kulturelle Bildung. Schriftenreihe Kulturelle Bildung, Vol. 30. München: Kopaed, S. 63-67.

Gieseke, Wiltrud (2003a): Kulturelle Bildung zwischen Bildungswirklichkeit und Veränderungen. In: Brödel, Rainer / Siebert, Horst (Hrsg.): Ansichten zur Lerngesellschaft. Festschrift für Josef Olbrich. Unter Mitarbeit von Josef Olbrich. Baltmannsweiler: Schneider-Verlag Hohengehren, S. 200-210.

Gieseke, Wiltrud (2003b): Programmplanungshandeln als Angleichungshandeln. Die realisierte Vernetzung in der Abstimmung von Angebot und Nachfrage. In: Gieseke, Wiltrud (Hrsg.): Institutionelle Innensichten der Weiterbildung Bielefeld: W. Bertelsmann.

Gieseke, Wiltrud (2007): Lebenslanges Lernen und Emotionen. Wirkungen von Emotionen auf Bildungsprozesse aus beziehungstheoretischer Perspektive. Bielefeld: W. Bertelsmann.

Gieseke, Wiltrud (2008): Bedarfsorientierte Angebotsplanung in der Erwachsenenbildung. Bielefeld: W. Bertelsmann.

Gieseke, Wiltrud / Opelt, Karin (2005): Programmanalyse zur kulturellen Bildung in Berlin/Brandenburg. In: Gieseke, Wiltrud / Opelt, Karin / Stock, Helga / Börjesson, Inge: Kulturelle Erwachsenenbildung in DeutschlandExemplarische Analyse Berlin/Brandenburg. Münster: Waxmann, S. 43-130.

Gieseke, Wiltrud / Opelt, Karin / Stock, Helga / Börjesson, Inge (2005): Kulturelle Erwachsenenbildung in Deutschland - Exemplarische Analyse Berlin/Brandenburg. Münster: Waxmann.

Hippel, Aiga von (2013): Programmplanung als professionelles Handeln - „Angleichungshandeln“ und „Aneignungsmodi“ im aktuellen Diskurs der Programm- und Professionsforschung. In: Käpplinger, Bernd / Robak, Steffi / Schmidt-Lauff, Sabine (Hrsg.): Engagement für die Erwachsenenbildung Ethische Bezugnahmen und demokratische Verantwortung Wiesbaden: VS Verlag, S. 131-143.

Hippel, Aiga von / Röbel, Tina (2016): Funktionen als akteursabhängige Zuschreibungen in der Programmplanung betrieblicher Weiterbildung. In: Zeitschrift für Weiterbildungsforschung, 2016 (1): Politische Bildung, S. 61-81. 
Kerres, Michael (2018): Mediendidaktik: Konzeption und Entwicklung digitaler Lernangebote. Berlin: De Gruyter.

Keuchel, Susanne / Wagner, Ernst (2012): Poly-, Inter- und Transkulturalität. In: Bockhorst, Hildegard / Reinwand, Vanessa-Isabelle / Zacharias, Wolfgang (Hrsg.): Handbuch Kulturelle Bildung. München: kopaed, S. 52-57.

Kühn, Christian / Robak, Steffi / Fleige, Marion (2018): Programmplanung in einer Kultur der Digitalität. In: EB Erwachsenenbildung: Lernen und leben in der digitalen Welt. 2018 (4), S. 184-185.

Liebau, Eckart (2012): Anthropologische Grundlagen. In: Bockhorst, Hildegard / Reinwand-Weiss, Vanessa-Isabelle / Zacharias, Wolfgang (Hrsg.): Handbuch Kulturelle Bildung. Schriftenreihe Kulturelle Bildung, Vol. 30. München: Kopaed, S. 29-35.

Reckwitz, Andreas (2000): Die Transformation der Kulturtheorien. Zur Entwicklung eines Theorieprogramms. Weilerswist: Velbrück Wissenschaft.

Reckwitz, Andreas (2006): Das hybride Subjekt. Weilerswist: Velbrück Wissenschaft.

Reckwitz, Andreas (2017): Die Gesellschaft der Singularitäten. Zum Strukturwandel der Moderne. Berlin: Suhrkamp.

Robak, Steffi (2017): Entwicklungen und Akzente in der Inter- und Transkulturellen Bildung. Der verstehend-kommunikative Zugang kultureller Bildungsangebote. Hessische Blätter für Volksbildung, 2017 (4), S. 333-351.

Robak, Steffi (2018): Weiterbildungsmanagement. In: Fleige, Marion / Gieseke, Wiltrud / Hippel, Aiga von / Käpplinger, Bernd / Robak, Steffi (Hrsg.): Programm- und Angebotsentwicklung in der Erwachsenen- und Weiterbildung. Bielefeld: wbv Publikationen.

Robak, Steffi / Fleige, Marion (2017): Kulturelle Erwachsenenbildung: (Bildungs-)Interessen, Strukturen, Partizipationsformen - und ihre Übersetzung in Wissensstrukturen für Programmentwicklung. In: Literatur Kulturelle Bildung Online. Verfügbar unter: https://www.kubi-online.de/autorinnen/fleige-marion.

Robak, Steffi / Fleige, Marion / Sterzik, Linda / Seifert, Jennifer / Teichmann, Anne-Kristin / Krueger, Anneke (2015a): Die Konstitution Kultureller Bildungsräume. Fünf überregionale Institutionen der non-formalen Kulturellen (Erwachsenen-)Bildung: Bildungsangebotsentwicklungen, innovative Impulse, Planungsspielräume. Recherche und Analyse im Auftrag des Rates für Kulturelle Bildung, durchgeführt an der Leibniz Universität Hannover und der Humboldt-Universität zu Berlin im Zeitraum April-Juni 2015. Essen: Rat für Kulturelle Bildung.

Robak, Steffi / Petter, Isabelle (2014): Programmanalyse zur interkulturellen Bildung in Niedersachsen, Bielefeld: W. Bertelsmann.

Robak, Steffi / Rippien, Horst / Heidemann, Lena / Pohlmann, Claudia (Hrsg.) (2015b): Bildungsurlaub - Planung, Programm, Partizipation. Eine Studie zur Perspektivverschränkung. Frankfurt a.M.: Peter Lang.

Schmidt-Hertha, Bernd / Rohs, Matthias / Rott, Karin Julia / Bolten, Ricarda (2017): Fit für die digitale (Lern-)Welt? Medienpädagogische Kompetenzan- 
forderungen für Erwachsenenbildner/innen. In: Schrader, Josef (Hrsg.): Digital/Analog. DIE Zeitschrift für Erwachsenenbildung. 24. Jahrgang, 2017 (3), S. 35-37.

Stalder, Felix (2016): Kultur der Digitalität. Berlin: Suhrkamp.

Welsch, Wolfgang (2011): Immer nur der Mensch? Entwürfe zu einer anderen Anthropologie. Berlin: De Gruyter.

Welsch, Wolfgang (2012): Homo mundanus. Jenseits der anthropologischen Denkform der Moderne. Weilerswist: Velbrück Wissenschaft. 


\section{Heimat, Globalisierung und Erwachsenenbildung: Befunde einer Regionalstudie}

\section{Einleitung}

Heimat könnte eine Art Gegenbegriff zu Begriffen wie Globalisierung und Entgrenzung sein, die bei der Sektionstagung in Mainz neben Transformation zentrale Themen im Tagungstitel waren. Entsprechend wird in politischen Diskursen versucht den Heimatbegriff als Gegenbegriff bzw. -reaktion zu bestimmen: „Wir würden nicht über Zusammenhalt, wir würden nicht über Heimat, wir würden nicht über das Bedürfnis nach Gemeinschaft und die Bewahrung kultureller Identitäten sprechen, wenn es nicht eine tiefe und spürbare Verunsicherung über die mannigfaltigen Folgen einer nun seit dem Ende des Kalten Krieges sich vollziehenden und Globalisierung genannten Entgrenzung aller Lebensverhältnisse gäbe. Eine Entgrenzung, deren gesellschaftliche Folgen alle westlichen Gesellschaften erfasst und zu politischen Verwerfungen geführt haben" (Seehofer 2018: 6). Man könnte die aktuelle Konjunktur des Heimatdiskurses mit seinen neuen Heimatministerien in Bund und Ländern sowie Heimatkongressen schnell als ein reflexhaftes, regressives Unwohlsein in der Moderne und einer komplexen Welt, die immer weniger in simplen Kausalitäten zu begreifen ist, abtun. Die Sehnsucht nach einfachen Lösungen ist nachvollziehbar, aber wahrscheinlich werden sich viele Probleme in ihrer Komplexität und Vernetztheit durch sie nicht lösen lassen.

Kann man aber den Heimatbegriff und damit verbundene Verständnisse und Assoziationen auch anders verstehen? Kann man den Heimatbegriff gar klar definieren, wie es sich manche vielleicht wünschen mögen? Wissenschaftliche Einschätzungen sind hier eher skeptisch: „Die Diskussion um den Heimatbegriff ist mittlerweile so weitverzweigt, dass er sich jeder eindeutigen Definition entzieht. Zudem spiegelt die z. B. ,von Max Frisch attestierte Unübersetzbarkeit des deutschen Wortes Heimat' (Cremer/Klein 1990: 35) dessen zahlreiche Bedeutungsfacetten und Verwendungszusammenhänge wider.“ (Peißker-Meyer 2002: 17) Andere und uns adäquat erscheinende Zugänge zum Heimatbegriff verweisen ebenfalls auf dessen Vielschichtigkeit und seine hochgradig differenten Nutzungen hin, die sich wissenschaftlich eindeutigen Begriffsbestimmungen entziehen: „Heimat wurde und wird bis zur Unkenntlichkeit verklärt und verkitscht, von den unterschiedlichsten Ideologien stili- 
siert, benutzt und missbraucht. Mythologisch aufgeladene, sentimentale Erinnerung gehört ebenso zum Begriffsfeld Heimat wie beinahe banale geografische Ortsbestimmung. Heimat ist Projektionsfläche der unterschiedlichsten Sehnsüchte [...] Während die einen an der Enge der Heimat leiden, kranken die anderen am Verlust derselben, werden heimwehkrank“" (Reusch 2007: 5). Börjesson (2005: 352) zeigt in einem der wenigen Beiträge aus der Erwachsenenbildungsforschung zum Heimatbegriff des Weiteren auf, dass seit den 1970er Jahren der Begriff von sozial-ökologischen Bewegungen für eine Kapitalismuskritik, Alternativökonomien, Gegenkulturen und generell für eine Rückgewinnung regionaler Gestaltungsmöglichkeiten eingesetzt wird. Insgesamt kann der Heimatbegriff politisch weder grundsätzlich ,progressiv“ noch „konservativ“", sondern ambivalent und vielgestaltig begriffen werden. Intellektuell besteht durchaus die Gefahr, den Begriff Heimat zu schnell in eine bestimmte „Schublade“ der Rückwärtsgewandtheit und Nostalgie zu stecken.

Dieses eher offene Begriffsverständnis aus der Literatur passt gut zu unseren empirischen Ergebnissen, wo wir eher zufällig in der Empirie auf die häufige und differente Verwendung des Begriffs Heimat durch die Befragten aufmerksam wurden und induktiv nachspürten, wie der Begriff unserer eher offenen Bedarfserhebungen und im Kontext von Erwachsenenbildung thematisiert wird. Im Folgenden werden somit die Ergebnisse eines kleineren regionalen Projektes im Bereich der Anwendungsforschung in diesen Kontext eingebettet. Das Projekt wurde u.a. aus Mitteln des Europäischen Landwirtschaftsfonds für die Entwicklung des ländlichen Raums gefördert und im Rahmen einer Kooperation der Volkshochschule Landkreis Gießen mit der Professur für Weiterbildung der Justus-Liebig-Universität Gießen durchgeführt. Der Heimatbegriff war dabei kein konzeptioneller Ausgangspunkt oder Bestandteil der Forschungsfrage für das Projekt, sondern seine Relevanz war ein unerwartetes Teilergebnis der Bedarfserhebungen vor Ort.

\section{Allgemeiner Forschungsstand: Heimat und Erwachsenenbildung im ländlichen Raum als Stiefkind der Weiterbildungsforschung}

Die katholische Arbeitertochter vom Land war eine von Ralf Dahrendorf der breiten Öffentlichkeit bekannt gemachte Kunstfigur, welche die Bildungsdiskussionen in den 1960er Jahren maßgeblich beeinflusste und heute wohl als eine frühe Form einer intersektionistischen Betrachtung von Bildungsbenachteiligungen eingeordnet werden würde. Diese Denkfigur suggeriert eine Homogenität ländlicher Räume, die so nicht gegeben ist. Ländliche Räume sind 
vielmehr differenziert zu betrachten, „um der Vielfalt räumlicher Funktionen und Entwicklungen sowie wachsender Disparitäten Rechnung zu tragen“" (Franzen et al. 2008: 2). Auch Heimat ist wie bereits erwähnt nichts Monolithisches, sondern kann und wird oft subjektiv sehr unterschiedlich erlebt, da Heimat oft mit sehr persönlichen Emotionen, Bedeutungen und Erfahrungen besetzt ist. Umso erstaunlicher ist es, wenn Heimat in politischen Diskursen manchmal als etwas Gefestigtes mit einer speziellen Identität oder gar einer kollektiven Gemeinschaft definiert werden soll. Ländlichen Räumen im Allgemeinen und der Erwachsenenbildung im ländlichen Raum im Speziellen werden schon lange einerseits eine Krise und andererseits eine Renaissance zugesprochen (vgl. Faber 1981). Dezidierte sowie empirische Publikationen sind jedoch selten (vgl. Gieseke et al. 2005; Klemm 2004). Zumeist stehen städtische Räume im Blickpunkt einer alltags-/partizipationsorientierten Erwachsenenbildung. Aktuell kann jedoch ein neues Interesse an Erwachsenenbildung in ländlichen Räumen festgestellt werden. So richtete der letzte hessische Weiterbildungsbericht sein Augenmerk auf diese Räume und forderte:

„Auf der Grundlage dieser Befunde empfehlen wir eine weitere Stärkung der öffentlichen Verantwortung und Förderung für bzw. von Weiterbildung. Insbesondere mit Blick auf die herausgearbeiteten räumlichen Disparitäten hinsichtlich der Anbieterdichte sollte diese Stärkung auch der Aufrechterhaltung des Angebotes in den ländlichen Räumen zugutekommen." (Hessisches Kultusministerium 2016: 11)

„Um in ganz Hessen zukünftig gleiche Zugangschancen zur Weiterbildung sicherstellen zu können, ist besonderes Augenmerk auf die Beteiligung und die Beteiligungschancen in ländlichen Gebieten zu richten, insbesondere vor dem Hintergrund des zu erwartenden demografischen Wandels." (ebd.: 39)

„Die angesichts des demografischen Wandels zu erwartende sich vergrößernde Kluft zwischen Zentrum und Peripherie legt nicht nur eine Stabilisierung, sondern vielmehr eine Erhöhung der öffentlichen Förderung nahe. Diese sollte auch dazu verwendet werden, das von den öffentlichen Einrichtungen erbrachte Angebot in den ländlichen Räumen zu stärken." (ebd.: 122)

In diesem hessenweiten Diskurs ist das Entwicklungsprojekt im Landkreis Gießen von der Anlage sowie von der Zielstellung her einzuordnen. Auch in anderen Regionen in Deutschland ist ein dezidiertes und wachsendes Interesse an Erwachsenenbildung im ländlichen Raum zu beobachten, wobei es stellenweise um die Sicherung bereits im Rückbau befindlicher Strukturen geht (vgl. Beetz et al. 2018). Ökonomen medial präsenter Einrichtungen wie dem IfoInstitut formulieren dagegen drastische Alternativen: „Manche Dörfer sollten wir besser schließen [...] Gibt es ein Recht auf Heimat? Die Politik sollte den 
Menschen gegenüber ehrlich sein. Bei insgesamt stark schrumpfender Bevölkerung kann sie nicht jedes Dorf auf Dauer aufrechterhalten. Natürlich können dort weiter Menschen wohnen, aber dann eben ohne Internet, ohne Busanbindung und mit dem Risiko, dass die Feuerwehr im Notfall eine halbe Stunde braucht, bis sie dort ist" (Ragnitz 2018: 17). Es ist jedoch darauf hinzuweisen, dass Artikel 72 des Grundgesetzes eine „Gleichwertigkeit der Lebensverhältnisse“" als Aufgabe und Ziel vorsieht und insofern die ökonomische Perspektive als mit der Verfassung der Bundesrepublik Deutschland schwer vereinbar einzustufen ist. Der oben zitierte Vorschlag ist zudem für Sachsen formuliert, wobei sich die Frage stellt, ob andere ländliche Regionen in den nächsten Dekaden nicht vor ähnlichen Entwicklungen und Diskussionen stehen werden, die in Sachsen früher als in anderen ländlichen Regionen Deutschlands auftreten. Zwar wächst Deutschland als Ganzes aktuell entgegen demografischen Prognosen aufgrund von Zuwanderung weiterhin, aber ländliche Räume sind doppelt problematisch in ihrer Entwicklung, da sie oft nicht von Migrationsbewegungen aus dem In- oder Ausland profitieren. In unseren Interviews vor Ort wurde beispielsweise darauf hingewiesen, dass die Geflüchteten mittlerweile alle nach Gießen wollten bzw. gegangen seien.

Dies alles ist keine Entwicklung der letzten Jahre. Schon seit der ersten Industrialisierung im 18./19. Jahrhundert lässt sich eine Longue durée der Urbanisierung beobachten; so lebten um 1800 noch ca. 75\% der Deutschen auf dem Land. Auch die Globalisierung ist kein Phänomen der letzten Dekaden. Bereits Marx/Engels beschrieben 1848 für ihre Zeit Phänomene, die an heutige Gegenwartsdiagnosen zumindest erinnern: „Die uralten nationalen Industrien sind vernichtet worden und werden noch täglich vernichtet. Sie werden verdrängt durch neue Industrien, deren Einführung eine Lebensfrage für alle zivilisierten Nationen wird, durch Industrien, die nicht mehr einheimische Rohstoffe, sondern den entlegensten Zonen angehörige Rohstoffe verarbeiten und deren Fabrikate nicht nur im Lande selbst, sondern in allen Weltteilen zugleich verbraucht werden. [...] Und wie in der materiellen, so auch in der geistigen Produktion. Die geistigen Erzeugnisse der einzelnen Nationen werden Gemeingut. Die nationale Einseitigkeit und Beschränktheit wird mehr und mehr unmöglich, und aus den vielen nationalen und lokalen Literaturen bildet sich eine Weltliteratur.“ (Marx/Engels 1848) Vielleicht ist eher das Neue, dass die Geschwindigkeit der Globalisierung rasant zugenommen hat und dass natürlich die technischen, wirtschaftlichen und politischen Situationen heute andere sind?

Bezogen auf die Weiterbildung liefert der sogenannte Weiterbildungsatlas bundesweite Vergleichsdaten auf Basis von Raumordnungsregionen (Martin et al. 2015). Dabei sind Teilhabe- und Gerechtigkeitsprobleme von zentralem Interesse. In der interkulturellen Bildung befasst man sich mit der alten und „neuen Heimat“ von Migrantinnen und Migranten (Robak/Petter 2014: 36). Globalisierung bedeutet theoretisch (Schemmann 2006; Klemm 2009) u.a. 
eine Gleichzeitigkeit von Homogenisierung und Heterogenisierung. Ist also der Heimatsdiskurs Anzeichen für eine rückwärtsgewandte Lokalisierung als begrenzende Gegenbewegung zur Globalisierung? Oder ist Heimat geselliger und individueller (Lern-)Raum für Identitätsentwicklung und der Entwicklung von Solidarität fern von einer Abwehr des Neuen?

\section{Methodisches Vorgehen}

Die Empirischen Daten wurden im Projekt triangulativ erhoben und ausgewertet. $\mathrm{Zu}$ Beginn wurden sieben leitfadengestützte Experteninterviews mit neun regionalen Akteurinnen und Akteuren mit vertiefter Orts- und Personenkenntnis geführt. Hierfür wurde vom Bürgermeister über Vereinsvorsitzende bis zu Lehrkräften in der Erwachsenenbildung ein breites Spektrum vor Ort befragt. In einer nachfolgenden in der Lokalpresse angekündigten quantitativen Straßenbefragung mit vielen offenen, nicht-standardisierten Fragen wurden zudem vor einem gut frequentierten Supermarkt in der Region 90 Personen aus der Bevölkerung zu ihren Bildungsbedarfen befragt. In einem nächsten Schritt wurden die Experteninterviews mit der quantitativen Befragung vor Ort verbunden und verglichen. Dies ermöglichte eine Kontrastierung der Ergebnisse, was sowohl eine wechselseitige Bestätigung als auch differente Bedarfsprognosen zur Folge haben kann. Die auf Basis der erhobenen Bedarfe entstandenen vier Pilotkurse mit insgesamt 57 Kursteilnehmenden wurden sodann über einen Kurzfragebogen, teilnehmende Beobachtungen sowie Gruppendiskussionen evaluiert. Ziel war es dabei die Akzeptanz dieser neuen Angebote bei der Bevölkerung bzw. bei den Kursteilnehmenden zu ergründen. Die vielfältigen Ergebnisse dieser Erhebungen sind im Rahmen von formativen Evaluationen in verschiedenen Phasen des Projekts entwicklungsorientiert eingeflossen und durch u.a. aufgrund von Anonymisierungsnotwendigkeiten unveröffentlichte Projektberichte dokumentiert. Diese Anonymisierungsnotwendigkeiten sind hier besonders wichtig für ethisch verantwortungsbewusste Forschung, da aufgrund der relativ geringen Bevölkerungszahl in dem beforschten ländlichen Raum eine Re-Identifikation von Befragten, die wir verhindern wollen, leicht möglich wäre. Der Fokus der nachfolgenden Betrachtung liegt nun allein auf den Teilergebnissen zum Begriff „Heimat“, der im Rahmen der qualitativen wie quantitativen Bedarfserhebungen eine unerwartet große Relevanz bekam, obwohl nicht explizit danach gefragt wurde. Dies führte letztendlich dazu, dass die Forschenden verstärkt der Frage nachgingen, was seitens der Befragten mit dem Heimatbegriff verbunden wird und worin die Konjunktur des Heimatbegriffes begründet liegt. 
Aus Platzgründen und wegen des hohen De-Anonymisierungsrisikos wird im Folgenden nicht detailliert und mit ausgiebigen Belegstrukturen differenziert, was mit welcher Methode wann im Projektverlauf erhoben wurde. Die im Folgenden gewählte Darstellungsform mag gemäß Begutachtung pointillistisch wirken, aber für eine elaborierte Belegstruktur bietet dieser Sammelband keinen ausreichenden Platz. Zudem wollen wir eher das sich uns darstellende Gesamtbild zur Diskussion stellen als nur einige wenige Zitate zu diskutieren.

\section{Ergebnisse}

\section{Heimat als alte Handarbeits- und Handwerkstechniken}

Eine erste und deutlich sichtbare Schicht an Bezügen der Befragten zum Heimatthema hat viel mit traditionellen Handwerken und Techniken zu tun. So werden in den Befragungen kulturelle Aktivitäten vorgeschlagen, beispielswiese das Brotbacken in noch vorhandenen Backhäusern zu lehren und zu praktizieren. Butter, Käse, Marmelade, Pflaumenmus könnten selbst gemacht werden. Man könnte Weben lernen am Webstuhl aus dem Heimatmuseum, der noch funktionstüchtig sei. Auch Schmiedearbeiten werden als Thema genannt. Generell erinnert vieles an die allgemeine Bewegung, die in den letzten Jahren mit „DIY - Do-it-yourself“ (Kuni 2014) bezeichnet wird und nicht allein in ländlichen Räumen zu beobachten ist. Bei den in unserer Studie befragten Personen scheint die Nennung dieser Bildungsbedarfe nicht durch ökonomischen Mangel, der zum Selbermachen aus materieller Not führt, motiviert. Im Gegenteil könnte es sogar mehr Zeit und Geld kosten, diese Produkte selbst herzustellen, statt sie günstiger zu kaufen. Interessante, dies unterstützende ethnografische Beobachtungen ließen sich teilnehmend beim geselligen Ausklingen einer Bildungsveranstaltung anstellen, bei dem einzelne Teilnehmende von ihren Renovierungs-/Umbauarbeiten an alten Fachwerkhäusern berichteten, wo bewusst alte Handwerkstechniken wie z. B. das Streichen mit Schlämmkreide eingesetzt und Modernisierungen wie Tapezierungen der 1960er/70er-Jahre bewusst rückgebaut wurden, um den historischen Zustand wiederherzustellen. Den Berichten war zu entnehmen, dass sehr viel Zeit und Geld in diese und andere Arbeiten investiert wird. Über dieses Beispiel hinaus sollten Ziele von Bildungsveranstaltungen sein, dass ,man am Ende was in der Hand hat" und das „Brauchtum aufleben lassen“ kann. Zum einen soll an vergangenes Tun erinnert werden und zum anderen wird eine Sinngebung im sichtbaren und sinnvollen Produzieren gesucht. Ob dies bewusst und explizit als ein „Unbe- 
hagen bezüglich globalisierter Märkte“, „Konsum-Kritik“ (Kuni 2014: 20) intendiert ist oder ökologische Gründe hat, mag man eher bezweifeln, da solche Überlegungen in den Interviews nicht explizit benannt wurden. Als Hintergrund sind diese aber ggf. mitzudenken, da niemand direkten Zugang zu all seinen Motivlagen hat. Relevanter dürfte dagegen folgender Punkt sein: „Wer schon im Beruf den ganzen Tag vor dem Bildschirm sitzt, will dort nicht noch seine Freizeit verbringen, sondern etwas tun, bei dem man selbst Hand anlegen kann. Das braucht der Mensch, um sich und die Welt zu begreifen und zu gestalten. Darum kommen wir von den Gegenständen, den Dingen und dem Material nicht los.“ (ebd.: 21)

\section{Heimat und Sprache: Erzählungen, Brüche und Legenden}

Es wurden von Befragten auch Wünsche artikuliert, in Sprachkursen den heimischen Dialekt erklärt zu bekommen. Heimatliche Gedichte und Literatur von einem regionalen Autor wurden als Themen genannt, die man bei Einzelveranstaltungen wie Lesungen in einem geselligen Rahmen präsentieren könnte. Teilweise erfolgte dies wohl aus einer defensiven Haltung heraus, weil gerade die Älteren ein Schwinden des Dialekts beobachten und dies als Verlust ansehen.

Generell werden zudem ein gewisser Bedarf und ein Interesse an Heimatgeschichte(n) bekundet. Bei der Begehung von historischen Kellern im Ort und dem damit verbundenen Spaziergang von Keller zu Keller war es aufgrund unseren teilnehmenden Beobachtungen vielfach Gesprächsthema, was an Geschichten - durchaus auch im Sinne von Klatsch und Tratsch - über Vergangenes bekannt ist. Dabei stellten sich Geschichten bzw. Gerüchte über ein noch existentes geheimes Tunnelnetzwerk bzw. einem Tunnel bis zur nächsten Burg als Mythos heraus. Die durch vielfaches Nacherzählen und wohl gelegentlich durch Ausschmücken berichteten Ortsgeschichten entsprechen nicht immer der historischen Wahrheit, sondern stellen subjektiv gefärbte Erzählungen dar, die wie andere Berichte der Oral History mit methodischer Vorsicht zu sondieren sind. Große historische Ereignisse spielen in diesen Erzählungen kaum eine Rolle oder stellen eher die allgemeine Hintergrundfolie dar, wenn es um die Ereignisse im Ort und die Menschen geht. So ist es eher die Ausnahme, dass der 2. Weltkrieg und die Judenverfolgung im Ort als mögliches Bildungsthema von Befragten benannt werden. Dies ist aber trotzdem bemerkenswert, da hier der eigene Ort und die Geschichte kritisch reflektiert werden und Heimat in einer aufklärerischen Art und Weise fern von Verkitschung und Idealisierung Thema ist. Mehr und häufiger präsent ist jedoch die teilweise historische Entstehung des Ortes als mittelalterliches Wehrdorf und dann in den 1970er Jahren die diversen freiwilligen oder auch unfreiwilligen Eingemeindungen. Bis 
heute prägen gewisse Konflikte zwischen den Ortsteilen das Leben der Stadt, was in einigen Ortsteilen zu unterschiedlichen politischen Mehrheiten bei Wahlen führt. Dies und anderes erinnert an Befunde anderer Regionalstudien der Weiterbildungsforschung, wo ebenfalls deutlich wurde, dass Heimat kein allein harmonischer Ort ist, sondern dass vielmehr realistisch mit Konflikten umgegangen werden muss: „Ich kann Ihnen ein Beispiel geben: Dargersdorf, das ist ein Ortsteil von Vietmannsdorf. Und es gibt eigentlich schon seit Jahrhunderten diesen Streit. Dargersdorf und Vietmannsdorf. Also die beiden Dörfer kommen irgendwo nicht zusammen. Es gibt irgendwie kein gemeinsames Dorfleben, Dargersdorf ist ein Dorf, die machen ihre eigenen Feste. Vietmannsdorf ist ein Dorf, die machen ihre eigenen Feste. [...] Also insofern kann man sagen, wir haben hier eine Heimat Vietmannsdorf und da schon wieder eine Heimat Dargersdorf" (Börjesson/Zimmermann 2005: 187). In einigen Experteninterviews oder bei der Begrüßungsrede zur Kellerbegehung im Rahmen der Pilotbildungsangebote wies ein Vereinsvorsitzender auf die bestehenden Spannungen zwischen den Ortsteilen hin. Von gemeinsamen Bildungs- und Kulturveranstaltungen erhoffen sich Vereine und der Bürgermeister, diese Spannungen zu überwinden, wenngleich sie nicht zu explizit thematisiert werden sollen. So wurde sich in Interviews und Befragungen mehrfach explizit und deutlich gegen Veranstaltungen der politischen Bildung ausgesprochen, die man eher, ,den Parteien überlassen“"wolle, obwohl der Ort z. B. ein Rechtsradikalenproblem hat. Bildung und Kultur sollen das Leben vor Ort verbessern, ohne dass die Probleme oder Konflikte direkt Thema sein sollen. Dies stellt eine große Herausforderung für die lokale Bildungsarbeit vor Ort dar und erfordert eine große programmplanerische Sensibilität beim Interagieren mit Akteurinnen und Akteuren vor Ort.

\section{Heimat als physischer Ort im Wandel der Zeit}

Heimat ist in der Literatur und in der alltäglichen Begriffsverwendung nicht nur ein Gefühl, sondern auch ein konkreter Ort oder Raum. Als Ideen zu Bildungsbedarfen wurden u.a. Kräuterwanderungen rund um das Dorf genannt. Die historische Kellerführung im Ort als ein Pilotangebot bezieht sich ebenfalls auf reale, physische Charakteristika der Heimat mit ihren Besonderheiten und Alltäglichkeiten. Allerdings ist Heimat sicherlich mehr als die Topografie des physischen Ortes. Heimat ist in vielfacher Hinsicht ein emotionaler Ort, der mit verschiedenen Zeiten zwischen Gestern, Heute und Morgen erinnernd wie in die Zukunft blickend verbunden wird. Es war in vielen Gesprächen Thema, wie der Ort gestern war, heute ist und morgen sein sollte. Dabei spielten Gefühle eine große Rolle, da Heimat mit sehr persönlichen Erfahrungen 
und Haltungen verbunden ist. Über Bildung und Kultur wird diskutiert. Welchen Beitrag leisten sie, um den Ort noch lebenswerter zu machen? Interessant ist dabei jedoch auch, dass Heimat als Ort manchmal mit lediglich einem Ortsteil, nur einer Straße oder einem bestimmten Platz verbunden wird. Heimat muss nicht den ganzen Ort oder die Region bedeuten, sondern kann viel kleinteiliger bzw. subjektiver verstanden werden. Eine Außensicht, die sich an eher administrativ definierten Räumen orientiert, stößt hier auf Alltagsperspektiven und subjektive Deutungen, die Heimat anders begreifen und kleinteiliger zuschneiden. So war in der Studie zu beobachten, dass viele Befragte nicht den ganzen Ort, sondern z. B. nur bestimmte Ortsteile für sich als Heimat verstehen.

\section{Heimat als intergenerationales Thema?}

Bemerkenswert ist, dass Heimat sich uns als ein Thema darstellte, das vor allem von den Älteren aktiv thematisiert wurde. Zumindest war gerade bei der Straßenbefragung festzustellen, dass primär ältere Befragte den Heimatbegriff dafür verwenden, intergenerationale Bildungsangebote anzusprechen und anzuregen. Das Zusammenspiel der Generationen vor Ort war dabei explizit oder implizit oft Thema. Es gab Anregungen für intergenerationale Angebote zum Thema, wie das Leben früher war und heute ist. Bei den Jüngeren erfolgte dies nicht in gleicher Intensität. Möglicherweise drückt dies somit nur den einseitigen Wunsch der älteren Generation aus, der nicht auf Gegenseitigkeit beruht. Jüngere bezeichneten dagegen den Ort z. T. lediglich als „Schlafstadt“. Die Älteren scheinen dies durchaus auch wahrzunehmen und manchmal scheint über „Heimat“ der Wunsch der Älteren thematisiert werden, dass sich die Jüngeren mehr mit dem Ort identifizieren und mehr zum Dorfleben beitragen sollten. Diese Aussage lässt sich nicht näher quantifizieren, aber die Reziprozität des Wunsches nach intergenerationalem Lernen dürfte eine Herausforderung für die Bildungsarbeit darstellen. Gegebenenfalls wird über „Heimat“ auch implizit ein Generationenkonflikt thematisiert.

\section{Fazit}

So manche soziologische Diagnose scheint überzogen zu sein: „Wenn die These richtig ist, dass sich aufgrund der Dynamiken der globalisierten Welt keine dauerhafte Heimat in der sozialen Welt zu finden ist, dann haben wir 
womöglich nur die Chance, zu postmodernen „Wellenreitern“ zu werden, die ohne Heimat und Zielbestimmung glücklich werden, oder uns in einen ,überzeitlichen", aber im Beschleunigungszeitalter doch auch anachronistisch anmutenden Anker in der Transzendenz, das heißt im Übersinnlich-Jenseitigen zu suchen" (Rosa 2007: 18). Wenn wir unsere - für uns selbst überraschenden - empirischen Ergebnisse sondieren, dürfte es jedoch viel zu früh sein, den Heimatbegriff in der Bildungsarbeit aufzugeben und ,postmoderne Wellenreiter" pauschal zu postulieren. Dies dürfte sowohl für ländliche wie für städtische Räume eine Rolle spielen. Im Ergebnis unserer Regionalstudie zeigt sich vielmehr, dass der Heimatbegriff sehr wohl eine große Bedeutung für die Bevölkerung hat. Dies wollten wir hier zumindest zur Diskussion stellen und weitere Forschungen anregen, da es sich letztlich um ein relativ kleines und kurzes Projekt aus dem Bereich der Anwendungsforschung handelte. Allerdings ist diese Resonanz des Begriffs Heimat wie hier skizziert vielschichtig und sollte keinesfalls in einem politischen Lagerdenken vorschnell ablehnend oder zustimmend eingeordnet werden. Vielversprechender scheint es, sich vorurteilsfrei mit den Assoziationen und Bedürfnissen auseinanderzusetzen und sie in ihrer Bildungsrelevanz zu sondieren.

Für die Weiterbildungsarbeit bietet der Heimatbegriff eine Reihe von Ansatzpunkten, da unterschiedliche Bedarfe und Bedürfnisse über diesen Begriff transportiert werden. Die definitorische Unklarheit mag wissenschaftlich unbefriedigend sein, sich aber für die Praxis als eher hilfreich erweisen. Vieles bewegt sich dabei zunächst im Bereich der kulturellen Bildung (Handwerken, Gestalten, Sprache, Geschichte etc.), wobei Intergenerationalität und Emotionen wichtige Querschnittsthemen sind. Bemerkenswert ist einerseits die deutlich bekundete Distanz gegenüber Themen der politischen Bildung - wobei die programmplanerische Arbeit in den nachgefragten Themenbereichen auch politische Bezüge ermöglichen kann: In einem Brotbackkurs kann die Frage nach der Herkunft der Zutaten dazu führen, dass Fragestellungen der Bildung für nachhaltige Entwicklung eingebunden und diskutiert werden; in Seminaren zu lokal-historischen Themen wären Bezüge zu politischen, sozialen und wirtschaftlichen Kontexten herstellbar. Andererseits ist der ländliche Raum keinesfalls nur harmonisch, denn regionale Konflikte und ein nicht immer konstruktiver Umgang damit fallen auf. Hiermit muss die Weiterbildungsarbeit vor Ort diplomatisch und sensibel umgehen können. Das enge Geflecht an Vereinen vor Ort ist zum einen sehr hilfreich, um Kooperationspartner für Bildungsveranstaltungen zu finden. Zum anderen existieren oft Konflikte zwischen oder innerhalb dieser Vereine. Die Bildungsarbeit einer Kreisvolkshochschule kann hier leicht in ortsinterne Konflikte geraten. Es ist wichtig bis unerlässlich, vor Ort Ansprechpartnerinnen und Ansprechpartner zu haben, aber dadurch nicht die eigene Neutralität zu gefährden. 
In diesem Zusammenhang kann die Frage der lokalen Organisation von Erwachsenenbildung neu gestellt werden. Ist das tradierte, weitverbreitete Modell ehrenamtlicher Außenstellenleitungen noch zeitgemäß? Wären die klassischen Aufgabenbereiche (Bedarfsweitergabe, Ansprechpartner/in für Schlüssel- und Raumfragen, Öffentlichkeitsarbeit vor Ort) im Sinne eines breiteren lokalen Netzwerks auf mehrere Personen aufteilbar, um niedrigschwelligere „Partizipationsmöglichkeiten (zu) schaffen, die man annehmen kann, aber nicht annehmen muss" (Bundesministerium für Familie, Senioren, Frauen und Jugend 2015: 33) und um den jeweiligen Interessen und Kompetenzen freiwillig Engagierter Rechnung zu tragen? Wäre hauptamtliches Engagement auf programmplanerischer Ebene notwendig, um Bedarfserschließungsprozesse systematisch zu moderieren und ehrenamtliches Engagement stärker zu begleiten? Im Rahmen einer Pilotierung möchte die VHS eine hauptberufliche, programmplanerische Zuständigkeit für den Ort etablieren und freiwilliges Engagement gewinnen. Dieses Steuerungsmodell soll inhaltlich ausgestaltet und formativ evaluiert werden. Ziel der Initiative ist letztlich die Steigerung der Weiterbildungsbeteiligung vor Ort.

Bezogen auf die Ausgangsthematisierung, die Heimat als Gegenbegriff zur Globalisierung begreift, stellt sich vor Ort die Situation als wesentlich banaler dar. Es geht weniger um große gesellschaftliche Entwicklungen, Migrationsbewegungen oder Kapitalismus - dies sind eher Hintergrundfolien für ein Geschehen vor Ort, was zum einen oft lange Vorgeschichten hat und zum anderen sehr persönliche Konstellationen berührt. Grenzlinien und Abgrenzungen innerhalb der Ortsgemeinschaft sind teilweise viel größer als diejenigen gegenüber dem Außen oder Neuankömmlingen. Man sollte mit Heimat - konzipiert und instrumentalisiert - als Gegenbegriff zur Globalisierung nicht ablenken von dem, was vor Ort an Herausforderungen und Entwicklungsmöglichkeiten besteht. Für die Weiterbildungsforschung könnte es aktuell interessant sein, sich gerade in ländlichen Räumen mit dem Begriff Heimat, seiner Relevanz und seinen Bedeutungsschichten näher zu befassen. Wir hoffen dazu eine kleine Anregung mit diesem Text zu bieten, der dieses Thema allein schon aus Platzgründen sicherlich nicht erschöpfend behandelt.

\section{Literatur}

Beetz, Stephan / Bender, Pauline / Haubold, Friederike (2018): Erwachsenenbildung im ländlichen Raum. Chemnitz: Sächsischer Volkshochschulverband e.V. Börjesson, Inga (2005): Ökologische Anfragen. In: Gieseke, W. / Opelt, K. / Stock, H. / Börjesson, I. (Hrsg.): Kulturelle Erwachsenenbildung in Deutschland. Münster: Waxmann, S. 350-357. 
Börjesson, Inga / Zimmermann, Ulrike (2005): „Und dann gibt es noch Bildungsangebote ..." Regionalanalysen zu beigeordneten kulturellen Bildungsangeboten im Berliner Stadtbezirk Friedrichshain-Kreuzberg und in der brandenburgischen Uckermark. In: Gieseke, W. / Opelt, K. / Stock, H. / Börjesson, I. (Hrsg.): Kulturelle Erwachsenenbildung in Deutschland. Münster: Waxmann, S. 131-201.

Bundesministerium für Familie, Senioren, Frauen und Jugend (2015): Kooperation von Haupt- und Ehrenamtlichen als Gestaltungsaufgabe - Ein Leitfaden für die Praxis. Berlin: BMFSFJ.

Faber, Werner (1981): Das Dorf ist tot - es lebe das Dorf. Erwachsenenbildung im ländlichen Raum. Düsseldorf: Patmos-Verlag.

Franzen, Nathalie / Hahne, Ulf / Hartz, Andrea / Kühne, Olaf / Schafranski, Franz / Spellerberg, Annette / Zeck, Holger (2008): Herausforderung Vielfalt Ländliche Räume im Struktur- und Politikwandel. Hannover: Akademie für Raumforschung und Landesplanung.

Gieseke, Wiltrud / Opelt, Karin / Stock, Helga / Börjesson, Inga (2005): Kulturelle Erwachsenenbildung in Deutschland. Münster: Waxmann.

Hessisches Kultusministerium - Landeskuratorium für Weiterbildung und Lebensbegleitendes Lernen (Hrsg.) (2016): Weiterbildungsbericht Hessen 2015. Wiesbaden: Hessisches Kultusministerium.

Käpplinger, Bernd (2016): Lernarchitekturen und Antinomien. In: Weiterbildung 27,5 , S. 26-28.

Klemm, Ulrich (2006): Bildung als gesellschaftsverändernde Praxis. In: Zeitschrift für internationale Bildungsforschung und Entwicklungspädagogik 29, 1/2, S. 39-44.

Klemm, Ulrich (2009): Erwachsenenbildung zwischen Globalisierung und Regionalisierung. Zeitschrift für internationale Bildungsforschung und Entwicklungspädagogik 32, 4, S. 4-9.

Kuni, Verena (2014): Stichwort DIY - Do It Yourself. In: DIE-Zeitschrift für Erwachsenenbildung 21, 4, S. 20-21.

Martin, Andreas / Schömann, Klaus / Schrader, Josef / Kuper, Harm (Hrsg.) (2015): Deutscher Weiterbildungsatlas. Bielefeld: wbv.

Marx, Karl / Engels, Friedrich (1848): Das Manifest der Kommunistischen Partei. London. URL: http://www.mlwerke.de/me/me04/me04_459.htm [Abrufdatum: 26.06.2019]

Peißker-Meyer, Cosima (2002): Heimat auf Zeit. Europäische Frauen in der arabischen Welt. Bielefeld: transcript-Verlag.

Reusch, Siegfried (2007): Editorial. In: der blaue reiter 12, 23, S. 4-5.

Robak, Steffi / Petter, Isabell (2014): Programmanalyse zur interkulturellen Bildung in Niedersachsen. Bielefeld: wbv.

Rosa, Hartmut (2007): Heimat im Zeitalter der Globalisierung. In: der blaue reiter 12,23, S. 13-18.

Schemmann, Michael (2006): Bildung und Globalisierung. DIE-Zeitschrift für Erwachsenenbildung 13,2 , S. 24-25 
Seehofer, Horst (2018): Heimat. In: Frankfurter Allgemeine Zeitung vom 30.04.2018, S. 6. 



\section{Transformations- und Lernprozesse alleinerziehender Mütter. Eine qualitative Studie}

Transitionen in der Erwachsenenbildung - gleich ob auf individueller oder auf institutioneller Ebene - sind Gegenstand des Diskurses in der Erwachsenenbildung (vgl. Schmidt-Lauff, von Felden, Pätzold 2015). Unterschiedliche Lebensphasen und biographische Übergänge stellen den Menschen, der sie durchläuft, vor unterschiedliche Herausforderungen und Hürden. Ein Blick auf die grundlagentheoretischen Überlegungen innerhalb der Erziehungswissenschaft macht deutlich, dass vor allem die Bewältigung von Brüchen und Diskontinuitäten Anlässe für Bildungsprozesse sind (vgl. von Felden 2009; Benner 2005; Koller 2005).

Der folgende Beitrag fokussiert spezielle Übergangssituationen. Analysiert wurden dabei die biografischen Lern- und Bildungsprozesse alleinerziehender Mütter. Diese Gruppe bildet besonders deutlich einen gesellschaftlichen Wandel $\mathrm{ab}$ - die Veränderung traditioneller Familienformen (Rinken 2010). Der Umgang der Alleinerziehenden mit den sich entwickelnden Ambivalenzen und Widersprüchen im Zuge dieser Transformationsphase soll näher beleuchtet werden, indem danach gefragt wird, welche Lernerfahrungen dabei als bedeutsam wahrgenommen werden.

Dazu wird zunächst in den thematischen Bezug der Studie eingeführt, der Forschungsstand zu der fokussierten Zielgruppe der Alleinerziehenden vorgestellt und das entsprechende Forschungsdesiderat aufgezeigt. Ausgehend davon werden die Fragestellung der Untersuchung und das darauf bezogene methodische Vorgehen dargelegt. Den Schwerpunkt des Beitrages bildet die Vorstellung der empirischen Befunde, welche abschließend theoretisch kontextualisiert werden.

\section{Thematischer Kontext}

In der Studie stehen Alleinerziehende als Zielgruppe für die Erwachsenenbildung im Fokus. Diese Gruppe ist im Kontext von Fragen zu Transformationen und Übergängen insofern interessant, weil sie besonders deutlich mit Transformationen im Kontext von Familienformen konfrontiert ist. Mit der Plurali- 
sierung der Lebensformen - und dem damit implizierten Wandel der Familienformen, ist ein stetiger Anstieg von Alleinerziehenden zu verzeichnen (vgl. Höhn, Dorbritz 1995; Klein 1999; Hammer 2002; Wagner 2008; Nave-Herz 2009). Die Zahlen des Statistischen Bundesamts (2010) belegen außerdem, dass rund 90 Prozent aller Alleinerziehenden Frauen sind. Alleinerziehen stellt also eine typisch weibliche Lebensform dar, die zudem einem vierfach erhöhten Armutsrisiko ausgesetzt ist (Lenze 2014). Zusammenfassend betrachtet, handelt es sich bei Alleinerziehenden um eine Gruppe, deren besondere (Krisen-)Situation spezifische Transformations- und Lernprozess erwarten lässt. Vor diesem Hintergrund wird im Folgenden beleuchtet, wie diese gesellschaftlich hochrelevante Gruppe in der Forschung thematisiert wird.

\section{Forschungsstand und Forschungsdesiderat}

Das Forschungsinteresse an der Zielgruppe lässt sich schwerpunktmäßig an Untersuchungen zu Heterogenität, sozioökonomischen Aspekten und möglichen Risiken verorten. Ein Blick auf den Forschungsstand zur Lebensform oder Lebensphase der Alleinerziehenden zeigt, dass die Situation von Ein-Eltern-Familien sehr vielfältig ist (Schneider, Krüger, Lasch, Limmer, MatthiasBleck 2001; Brand, Hammer 2002; Brand 2006).

Finanzielle Risiken und darauf reagierende Reformansätze, um die sozioökonomische Situation von Ein-Eltern-Familien in Deutschland zu verbessern, hat Lenze (2014) in einer Studie aufgezeigt. Neben den finanziellen Unsicherheiten finden in der Forschung ebenso generelle Herausforderung der Alleinerziehenden Beachtung. Dabei werden sowohl gesundheitliche Risiken (vgl. Köngeter 2011; Hancioglu 2014) als auch Unterstützungsmöglichkeiten alleinerziehender Mütter in den Blick genommen, mit denen die Bedeutung institutioneller Hilfeleistungen und soziale Netzwerkstrukturen herausgearbeitet wird (vgl. Nestmann, Stiehler 1998; Brand, Hammer 2002; Bundesministerium für Familie, Senioren, Frauen und Jugend 2011).

Die Betrachtung von Alleinerziehenden aus der Perspektive der Biografieforschung bezieht sich zum großen Teil auf die Erforschung der subjektiv wahrgenommenen Ressourcen. Dabei zeigt sich, dass die von außen oft als defizitäre, „objektiv prekär“ beschriebene Lage Alleinerziehender nicht zwangsläufig so von den Befragten wahrgenommen wird (Mädje, Neusüss 1996; Brand, Hammer 2002; Meier, Preusse, Sunnus 2003; Ott Strohmeier 2003). Vielmehr deutet sich an, dass durch die Bewältigung prekärer finanzieller Situationen Alltags- und Haushaltsführungskompetenzen als Ressource genutzt werden 
Die Bedeutung biografischer Lernprozesse, wie sie zum Beispiel in Studien zu transformativen Lernprozessen von Unternehmerinnen mit Migrationsgeschichte (Laros 2015) oder Lernprozessen von Lehrenden in Pflegeberufen (Ostermann-Vogt 2011) herausgearbeitet wurden, ist für die Gruppe der Alleinerziehenden noch nicht empirisch betrachtet worden. Zwar lassen sich aus den oben genannten Studien zu den eigenen Ressourcen implizite Hinweise auf Lernerfahrungen finden, explizit wurden diese jedoch noch nicht zum Gegenstand empirischer Forschung. Vor diesem Hintergrund kann festgehalten werden, dass bisher keine Studie über die explizit beschriebenen Lern- und Bildungserfahrungen alleinerziehender Mütter in der Reflexion der eigenen biografischen Übergangsphase existiert. Diesem Forschungsdesiderat entsprechend wird im Beitrag die Fragestellung verfolgt, welche Lern- und Bildungserfahrungen alleinerziehende Mütter in der expliziten Reflexion dieser eigenen biografischen Übergangsphase beschreiben und welche Herausforderungen mit dieser Phase verbunden sind.

\section{Forschungsdesign}

Ausgehend von diesen Fragestellungen wurde ein qualitativ-inhaltsanalytischer Forschungszugang gewählt. Im Mittelpunkt des Erkenntnisinteresses der Studie stand die subjektive Sichtweise der Befragten auf die - ihnen explizit verfügbaren - Wissensbestände zu den je eigenen biographischen Lernerfahrungen im Kontext des Lebens als Alleinerziehende. Damit bleibt das Erkenntnisinteresse auf der Ebene des expliziten Wissens der Subjekte, wozu, wie der Forschungsstand zeigt, noch keine empirischen Ergebnisse vorliegen. Im Folgenden werden die Datenerhebung, die Zusammenstellung des Samples und die Auswertungsmethode skizziert.

\section{Datenerhebung}

Als Datenerhebungsverfahren kam das leitfadengestützte Interview mit offenen Erzählimpulsen zum Einsatz (Kaiser 1992; Schütze 1983; Glinka 1998). In Anlehnung an die Interviewtechnik des narrativen Interviews wurden im ersten Teil des Interviews ein erzählgenerierender offener Impuls zur Phase des Alleinerziehens gestellt. Im Anschluss daran folgte ein fokussierter Nachfrageteil zu den Bereichen Lernerfahrungen, besondere Herausforderungen sowie Bewältigungsstrategien während dieser Lebensphase. Die Mütter wurden 
durch diese Art der Frageformulierungen als „Expertinnen“ des eigenen Lebensverlaufs (Dausien 1994: 143; Becker-Schmidt 1994: 175) angesprochen. Die gewählte Form des Interviews macht es möglich, die Sichtweise der befragten Subjekte auf die eigenen Lern- und Bildungsprozesse zu erhalten (Schütze 1980). Durch die Entscheidung für eine inhaltsanalytische Auswertung kommen insbesondere diejenigen Wissensbestände in den Blick, die den Subjekten selbst explizit verfügbar sind. In der Erhebungssituation zeigte sich, dass auch eine Explikation von impliziten Wissensbestandteilen über das eigene Lernen bzw. die eigenen Lernprozesse durch das Sprechen über die eigene Situation in Gang gesetzt und bewusstgemacht wurde. ${ }^{1}$

\section{Sample}

Als Methode wurde das sogenannte selektive Sampling gewählt. Durch diese wird zum einen in der Stichprobe der Kern des Feldes gut repräsentiert, zum anderen sind aber auch die abweichenden Fälle vertreten (Merkens 2008: 291). Insgesamt soll damit eine „maximale Variation angestrebt werden“ (ebd.).

Die Hauptkriterien für die Auswahl der Fälle orientierten sich an der Definition des statistischen Bundesamts. Dieses definiert Alleinerziehende als ,alle Mütter und Väter, die ohne Ehe- oder Lebenspartner mit mindestens einem ledigen Kind unter 18 Jahren in einem Haushalt zusammenleben. Unerheblich ist dabei, wer im juristischen Sinn für das Kind sorgeberechtigt ist. Im Vordergrund steht [...] vielmehr der aktuelle und alltägliche Lebens- und Haushaltszusammenhang. Aus diesem Grund wird auch nicht zwischen leiblichen, Stief, Pflege- und Adoptivkindern unterschieden“ (Statistisches Bundesamt 2010: 7). Das Sample umfasst zehn Interviews mit alleinerziehenden Frauen, die hinsichtlich der Hauptsamplekriterien Alter, Lebenssituation, Bildungsabschluss, berufliche Situation und Sorgerechtsregelung variieren. Die Varianz wurde dadurch erreicht, dass innerhalb dieser Kriterien auf eine möglichst große Bandbreite geachtet wurde. Dies lässt sich exemplarisch am Beispiel der Lebenssituation verdeutlichen: so wurden nicht nur geschiedene (3) oder getrenntlebende (3), sondern auch ledige (4) Mütter befragt. Auch beim Bil-

1 Beispielsweise unterbricht Frau B den Erzählverlauf und stellt fest, dass sie sich durch das Sprechen mit einem Außenstehenden über ihre eigene Situation noch bewusster über die eigenen Erfahrungen wird (,,̈̈h, ich krieg Gänsehaut, ich hab’ da jetzt so noch nie wirklich drüber gesprochen. (Pause). Da sprichst du halt immer nur mit Freundinnen, die halt irgendwie involviert sind“", Frau B: 70). Es gab jedoch auch Situationen, in denen erst im informellen Teil des Interviews, nach Ausschalten des Diktiergerätes, berichtet wurde, wie hilfreich die Erzählung über das Erlebte für die Reflexion der Lebenssituation sowie für die Selbstreflexion gewesen sei. 
dungsabschluss zeigte sich eine breite Varianz: fünf Mütter hatten einen Berufs- oder Realschulabschluss, eine die Allgemeine Hochschulreife und eine weitere hatte ein Studium absolviert. Tatsächlich zeigte sich in der Studie, dass die Varianz in den Lebenssituationen auch eine Heterogenität in den Deutungen nach sich zieht. Die Lebenssituationen scheinen eine große Bedeutung dafür zu haben, wie die Subjekte selbst explizit ihre biografischen Lernerfahrungen einordnen und beschreiben.

\section{Auswertungsmethode: qualitative Inhaltsanalyse}

Die Auswertung der Daten erfolgte mittels der qualitativen Inhaltsanalyse in Anlehnung an das Verfahren nach Kuckartz (2016). Die Auswertungsstrategie lehnte sich an ein deduktiv-induktives Verfahren der Kategorienbildung an. Dabei konnten deduktiv abgeleitete Oberkategorien induktiv durch Subkategorien ausdifferenziert werden und auch zusätzliche Oberkategorien induktiv gebildet werden. Aus der Analyse des Forschungsstandes sowie der theoretischen Beschäftigung mit dem Diskurs um alleinerziehende Mütter wurde deutlich, dass beispielsweise die Vereinbarkeit von Familie und Beruf für Alleinerziehende eine zentrale Herausforderung für die Frauen darstellt. Entsprechend wurde diese Perspektive in die Leitfragen des Interviews integriert und als Kategorie im Prozess der Codierung genutzt. Diese Aspekte wurden in der Erhebung aufgegriffen und in einem ersten Kodierdurchgang als deduktive Beobachtungskategorien auf das Material angelegt. In weiteren Materialdurchgängen konnte in Ergänzung der deduktiven Kategorien aus dem Material selbst eine induktive Kategorie gebildet werden. Das hierarchisch deduktivinduktive Kategoriensystem mit Ober- und verschiedenen Formen von Unterkategorien wurde abschließend erneut am Material überprüft und intersubjektiv über eine Doppelcodierung abgesichert (vgl. Kuckartz 2016; Mayring 2008).

Auf Basis des Kategoriensystems ließen sich vier typische Themenbereiche verdichten, mit denen die subjektive Bedeutung der Bildungs- und Lernerfahrungen abgesteckt werden kann. Diese werden im Folgenden als Ergebnisse der Untersuchung näher betrachtet. 


\section{Ergebnisse der Untersuchung}

In der Verdichtung der typischen - d.h. für die Lernprozesse Alleinerziehender relevanten - Lernerfahrungen zeigt sich, dass Herausforderungen als potenzielle Lernanlässe wahrgenommen werden und dass Unterstützungsperspektiven als soziale Lernprozesse genutzt werden. Außerdem wird deutlich, dass die ambivalente Deutung der eigenen Situation die Wahrnehmung des Lernens Deutungshorizonte beeinflussen kann und dass das Konstrukt des Lernens von den Befragten explizit sehr differenziert beschrieben wird.

\section{Herausforderungen als Lernanlässe}

Im Themenbereich Herausforderungen schreiben die Befragten unterschiedlichen Aspekten Relevanz zu. Insbesondere finanzielle Herausforderung führen für die Alleinerziehenden zu einem wahrgenommenen Spannungsfeld zwischen Unterstützung und Abhängigkeit. Der Umgang mit dieser Herausforderung wird explizit als Lernanlass thematisiert, wie Frau E erzählt: „,Ich musste da auch für mich lernen, alleine damit umzugehen [...], alles zu managen, dass das läuft, dass immer genug zu essen da ist, dass das Geld irgendwo nicht einfach so rausfliegt" (Frau E: 355-357).

Auch Herausforderungen hinsichtlich einer subjektiv bedeutsamen Doppelbelastung von Berufstätigkeit und Familienarbeit und Phasen der Erwerbslosigkeit werden für die Befragten zum Lernanlass. So berichtet Frau H, dass aufgrund beruflicher Diskriminierungserfahrungen Lern- und Entwicklungsprozesse initiiert werden:

„Immer wenn ich mich auf eine feste Stelle beworben hab, haben sie mir schon am Telefon erklärt, dass Stelle und alleinerziehende Mama nicht kompatibel sind [...] da bin ich froh über meine Selbstständigkeit, auch wenn's unsicher ist. Ich brauch einfach dieses... ja, mal herausgefordert zu sein, kognitiv. Ich lern auch immer was Neues dazu, les ich mir auch viel selbst an. Geht nicht anders in meinem Bereich“ (Frau H: 146-181).

Frau $\mathrm{H}$ nimmt die Herausforderung zum Lernanlass und durchläuft dadurch eine Entwicklungsphase, in der sie die beruflichen Herausforderungen als Chance begreift.

Auch der Umgang mit dem Vater der Kinder wird von den Befragten als Herausforderung beschrieben. Das Verhalten des Vaters kann für die Frauen auf vielfältige Art belastend wirken: von fehlender finanzieller und praktischer Unterstützung bei der Erziehung bis hin zu ausgeprägten Konflikten. Gerade 
dadurch erleben sich die Befragten als ,allein“ im Prozess der Erziehung. Diese Herausforderungen werden von einigen Müttern als Lernanstoß genutzt, sich reflexiv mit den eigenen Handlungsstrategien auseinanderzusetzen. Frau $\mathrm{H}$ nimmt den Umgang mit der Alleinverantwortlichkeit dabei bewusst an:

„Und ansonsten hat mich echt genervt, dass mein Ex jede Entscheidung von mir torpediert hat. Das erzeugt so ein Ohnmachtsgefühl [...]. Da hab ich jetzt beschlossen, ich entscheide einfach alles alleine“" (Frau H: 239-244).

Frau $\mathrm{H}$ empfindet einen starken Widerstand durch ihren ehemaligen Partner, durch den sie sich in ihrem Handlungs- und Entscheidungsspielraum stark eingeschränkt fühlt. Dies wird wiederum als Anstoß eines Lernprozesses beschrieben, nämlich zu lernen eigene Entscheidungen zu treffen.

In der Zusammenschau der beschriebenen Herausforderungen zeigt sich, dass die subjektiv empfundenen Problemlagen als Anstoß genommen werden, um sich mit der eigenen Selbstorganisation reflexiv auseinanderzusetzen, was von Frau A exemplarisch wie folgt beschrieben wird.

,$[\ldots]$ dass man da einfach lernt, wie man damit am besten umgeht [...] Auch halt, das Organisieren [...], dass man einfach schaut, wie man das alles zum Positiven, irgendwie ... wenden kann oder rausfinden, wie man es halt am besten alles einrichtet und macht. Es ... ist einfach mit dabei, Es ... ist einfach mit dabei, weil man einfach als Person, glaub ich, anders geworden ist" (Frau A: 454-461).

Frau A beschreibt als Lernergebnis nicht nur die gesteigerte Organisationsfähigkeit, sondern darüber hinaus einen biografischen Entwicklungsprozess, der dazu geführt hat, dass sie ,als Person anders geworden ist“.

\section{Unterstützung als sozialer Lernprozess}

Neben dem Lernfeld der Herausforderungen wurden auch die Möglichkeiten der Unterstützung für soziale Lernprozesse genutzt. $\mathrm{Zu}$ den Unterstützungsmöglichkeiten zählen die Frauen nicht nur Familienangehörige, den Freundesund Kollegenkreis, Nachbarinnen, sondern auch das Agieren in Internetforen. Dabei werden die Unterstützungsmöglichkeiten als Bewältigungsressource und Lernprozess während dieser Lebensphase genutzt. Die kooperativen Lernprozesse innerhalb der Unterstützungsmöglichkeiten zeigen sich vor allem hinsichtlich der Suchbewegungen hinsichtlich der Bewältigung der Herausforderungen dieser spezifischen Lebensphase. Erreicht wird das - wie Frau H feststellt - durch Austausch mit anderen, der einen Perspektivwechsel ermöglicht. 
„Irgendwie sind alle Trennungen anders. Oder jede Alleinerziehende hat andere Probleme. [...] Aber ganz, ganz wichtig: viel reden! Also, nicht nur einfach rumjammern, sondern zielorientiert reden. Manchmal kriegt man dadurch nämlich auch ne andere Perspektive“ (Frau H: 247-252).

Doch nicht nur konsistente soziale Beziehungen nutzen die Frauen zu reflexivem Lernen und Identitätsbildung, auch Ambivalenz- bzw. Differenzerfahrungen können als Lernanlass genutzt werden, was sich exemplarisch im Austausch mit dem Vater der gemeinsamen Kinder offenbart. Er kann in seiner Rolle gleichermaßen als Unterstützer - z.B. durch die Beteiligung an der Kindererziehung - und als Belastung - vor allem nach einer konfliktreichen Trennung - wahrgenommen werden. Frau E beschreibt das Verhältnis zum Vater der gemeinsamen Kinder ambivalent. Auf die Frage, ob der Kontakt zum Vater für sie hilfreich sei, antwortet sie: ,Wenn es die Kinder betrifft, ist es ok. Wenn es mich persönlich betrifft, dann nicht" (Frau E: 434-437). Diese Ambivalenz regt aus Sicht der Befragten Lernprozesse hinsichtlich des eigenen Selbstverständnisses an.

Neben diesen privaten sozialen Konstellationen wird auch die Unterstützung durch Institutionen als soziales Lernen erlebt: institutionelle Beratungsangebote bei Sozialpädagoginnen und Therapeuten ermöglichen eine explizite Reflexion über die eigene Situation. Exemplarisch lässt sich das bei Frau A zeigen. Sie hat eine Beratungsstelle der Diakonie aufgesucht und berichtet von einem gravierenden Schritt in ihrer persönlichen Entwicklung, den sie durch den intensiven Austausch mit der dort arbeitenden Sozialpädagogin erreicht hat. ,Und sie hat mir dann gezeigt, ja, wie ich dann eigentlich meine Grenzen steck [...] Ich muss sagen, sie hat mir dann soooo die Augen geöffnet" (Frau A: 255-257).

Nicht nur persönliche Gespräche, auch virtueller Austausch - z.B. in Internetforen speziell für alleinerziehende Mütter - kann das Herausbilden eines gemeinsamen Selbst- und Weltbilds ermöglichen. Frau H erlebt den interaktiven Austausch mit anderen Alleinerziehenden in ähnlichen lebensweltlichen Verhältnissen als eine anregende Lernumgebung, welche die Reflexion über die eigene Identität bzw. Persönlichkeitsbildung unterstützt:

„Und es tut gut zu hören, wenn man sieht: Man ist nicht allein in dieser Lage. Da gibt's noch mehr Frauen, die kämpfen genau denselben Kampf! Das kann auch helfen“ (Frau H: 239-243).

Insgesamt lässt sich festhalten, dass der soziale Austausch mit anderen den Frauen einen Raum der (Selbst-)Reflexion eröffnet, in dem sie ihre eigenen Perspektiven kritisch hinterfragen und durch die Kommunikation mit anderen Personen neue Sichtweisen entwickeln können. Das unterstützende soziale Umfeld kann ein anregendes Lernambiente bieten, indem dort Reflexionsräume geschaffen werden. 


\section{Wahrnehmung und Deutung der eigenen Situation}

Bei der Wahrnehmung und Deutung der eigenen Situation zeigt sich hinsichtlich des eigenen Selbstbildes eine deutliche Ambivalenz der Mütter, da sie sowohl positive als auch negative Selbstzuschreibungen nutzen. Obwohl sie sich auf der einen Seite als zufrieden beschreiben und sich dagegen wehren, in einer Opferrolle wahrgenommen zu werden, empfinden sie auch negative Gefühle dem eigenen Selbst gegenüber, die sich beispielweise in Schuldgefühlen äußern. Der Eindruck, gesellschaftlichen Erwartungen nicht entsprechen zu können, führt bei den Frauen zu Verunsicherung hinsichtlich der eigenen Rolle bzw. Identität. Diese ambivalente Beschreibung zeigt sich ebenfalls in der Beziehung zu ihren Kindern. Die als eng und vertrauensvoll beschriebenen Beziehung wird zugleich durch Abgrenzungsprobleme belastet. Beispielsweise sieht Frau F die Gefahr, das Kind zum „Partnerersatz “ (Frau F: 412) werden zu lassen. Durch diese ambivalenten Voraussetzungen können bei den Frauen Selbstbildungsprozesse angeregt werden. Dies zeigte sich im Material an der positiven Bilanzierung der Gesamtsituation, was einen expansiven Lernmodus bedingte. Die Frauen, die ihre Situation positiv bilanzieren, strebten danach, ihre Handlungsumwelt mithilfe von Lernhandlungen zu erweitern und ihre Entwicklung voranzubringen. Durch diese Wandlungsprozesse konnten sie sich gleichermaßen in ihrer Identität als Alleinerziehende stabilisieren, was in einer positiven Bilanzierung der eigenen Entwicklung mündete. Hier kristallisierte sich durchgehend ein positives Resümee der eigenen Entwicklung als strategischer Umgang mit Ambivalenz der Frauen heraus. Sie beschreiben neben der Empfindung von Unabhängigkeit und Entscheidungsfreiheit auch persönliche Vorteile wie, im Vergleich zu früher mehr Kraft und mehr Zeit zu haben. In der Phase des Alleinerziehens erleben die Mütter bei sich Veränderungen, die retrospektiv als persönliche Weiterentwicklung gedeutet werden. Beispielhaft beschreibt Frau D ihre persönliche Entwicklung als ein ,,komplettes Umdenken“ (Frau D: 344). Das „,komplette Umdenken“ markiert einen langdauernden Lernprozess bei Frau D, der in einer Neuorientierung ihres biografischen Entwurfs gipfelt. Diese Selbstbildungsprozesse haben eine transitorische Ausprägung, was Frau B verdeutlicht, wenn sie davon erzählt, wie sehr sich ihre subjektive Sichtweise geändert hat, nachdem sich ihre biografische Planung durch die Trennungssituation änderte:

„Diese Trennung jetzt zum Beispiel hat irgendwie mein komplettes Weltbild, Mannbild, Familienbild erschüttert. Das war halt so, ich hatte da so Grundfeste, ich war da altmodisch, Familie und ... Ich habe halt lernen müssen, dass es nicht so ist, auch wenn ich's gewollt hab. Und ich hab' eigentlich am Anfang lang dran festgehalten, weil, das will man ja eigentlich nicht wahrhaben dann und ... aber das musst ich halt lernen, dass es 
halt nicht so ist und scheinbar nicht den einen für einen gibt und ... ja. Also die Trennung war definitiv 'ne Lernsituation“ (Frau B: 971-977).

Diesem ,expansiven Deutungsmodus“, der zu Lern- und Entwicklungsprozessen führt, steht ein defensiver Modus gegenüber. Dieser äußert sich bei den Müttern, die ausschließlich in negativen Selbstzuschreibungen verharren. Hier wird Lernen eher blockiert, eine biografische Umorientierung ist für die Frauen damit nicht möglich.

Entscheidend dafür, die Deutung der eigenen Situation als Lernanlass zu nutzen, ist bei den Müttern eine reflexive Bearbeitung dieser spezifischen Lebensphase. Durch die Reflexion darüber, die - wie bereits weiter oben beschrieben beispielsweise mit institutioneller Unterstützung - stattfand, konnte der eigenen Deutungshorizont erweitert und damit ein Perspektivwechsel angestoßen werden.

\section{Lernprozesse und Lernerfahrungen}

Der vierte Themenbereich beschreibt, wie die Befragten ihre Wissensbestände $\mathrm{zu}$ unterschiedlichen Lernprozessen und -erfahrungen explizieren. Über das gesamte Sample wird deutlich, dass die Frauen hier insbesondere das Lernen als identitätsbildenden Entwicklungsprozess markieren. So resümiert Frau B für sich sehr deutlich „Lernen ist für mich [...] 'ne Entwicklung “ (Frau B: 939-940). Lernen wird in dieser Perspektive als beiläufiger Entwicklungsprozess gesehen, der en passant im Verlauf der Übergangsphase stattfindet und zur Persönlichkeitsveränderung beiträgt. Diesen Prozess der Identitätsveränderung beschreibt beispielsweise Frau A ganz explizit, wenn sie von ihrem Persönlichkeitsbildungsprozess durch das Alleinerziehen berichtet: „Es ... ist einfach mit dabei, weil man einfach als Person, glaub ich, anders geworden ist" (Frau A: 458-459).

Dafür nutzen die Alleinerziehenden formale und nonformale Lernsettings sowie informelle soziale Lerngelegenheiten wie zum Beispiel das Lernen von den eigenen Kindern. Frau E stellt im Interview fest, dass ,man auch viel von den Kindern (lernt). Finde ich jetzt. Also ich hab von meinen Kinder auch viel gelernt" (Frau E: Z 864-865).

Hinsichtlich der Beschreibung der Lernprozesse spielt für die Befragten der Modus der Reflexion eine besondere Rolle. Die Fähigkeit, über Lernanlässe und Lernprozesse reflektieren zu können, scheint bei den Befragten entscheidend für das Lernen aus Erfahrungen und das Lernen in Übergängen zu sein. Das Lernen findet bei den Frauen erst durch die Reflexion darüber bzw. durch 
seine Bewusstmachung statt. Durch die eröffneten reflexiven Lernarrangements können die Frauen ihre Erfahrungen des Alleinerziehens biografische bearbeiten.

Die Ergebnisse lassen sich folgendermaßen bündeln: Im Datenmaterial wird sichtbar, dass die alleinerziehenden Frauen die zentralen Herausforderungen ihrer Lebenssituation als Lernanlass für sich deuten und nutzen. Innerhalb der Unterstützungsmöglichkeiten finden die Mütter ebenfalls Lernanlässe, beispielsweise soziales Lernen. Die Herausforderungen und Deutungen der eigenen Situationen stellen die Rahmenbedingungen für die biografischen Lernprozesse bereit, die laut den Befragten unterschiedliche Formen annehmen.

\section{Diskussion: ambivalente Transformationen}

In der theoretischen Kontextualisierung der empirischen Befunde kann zunächst festgehalten werden, dass die biografischen Lernerfahrungen der alleinerziehenden Mütter Anschlussmöglichkeiten zum transformativen Lernen nach Mezirow $(2000,2009,2012)$ sowie zur Theorie der Deutungsmuster und des Deutungslernens nach Arnold und Schüßler (1996) aufzeigen.

In den Konzepten der Deutungsmuster und des transformativen Lernens wird davon ausgegangen, dass die Individuen sinnstiftende Muster in sich tragen, die zur Orientierung im Alltag dienen. Die Muster (oder auch Bedeutungsperspektiven) sind dabei nicht starr, sondern variabel und werden im Lauf des Lebens erworben. Veränderbar sind sie durch das Auftreten eines Ereignisses, das Irritation hervorruft und das Individuum handlungsfähig macht. Erst durch dieses „desorientierende Dilemma“ (Mezirow 2000: 22) können Deutungsstrukturen aufgebrochen und verändert werden.

Bei den Müttern ist dieses krisenhafte Ereignis die Trennung vom Vater der gemeinsamen Kinder. Sie stellen fest, dass im Übergang zum Alleinerziehen die Bedeutungsperspektiven, die sinnhaft schienen, nicht mehr funktionieren und neu gebildet werden müssen. Diese Um- oder Neudeutung stellt wiederum den Ausgangspunkt für den Lernprozess dar. Jedoch sind die erlebten Irritationen bzw. „Diskrepanzerfahrungen nur dann lernförderlich, wenn sie als solche anerkannt und reflexiv verarbeitet werden" (Schüßler 2008: 14). Die bekannte Bedeutung von Differenzerfahrungen zeigt sich mit dieser Studie auch und gerade für die Gruppe alleinerziehender Mütter, was bisher noch nicht in den Blick genommen wurde. Bisherige Erkenntnisse lassen sich damit bestätigen, wobei dies erstmals differenzierter gezeigt werden kann. Darüber hinaus kann die defizitäre Sichtweise auf diese Gruppe, die im Diskurs überwiegt, entkräftet werden. 
Die Frauen beschreiben den Lernprozess anhand unterschiedlicher Erlebnisse, in der sie rückblickend eine grundlegende Veränderung bei sich und in ihren Verhaltensweisen feststellen. Eine besondere Rolle spielt dabei die Reflexivität des Lernenden. Lernen - und damit ein tiefgreifender Bildungsprozess - kann nur in der eigenen reflexiven Auseinandersetzung stattfinden (Lerch 2010: 159). Da kritische (Selbst-)Reflexion durch den Austausch mit Dritten entsteht, spielen im Sample die Unterstützungsmöglichkeiten durch soziale Netzwerke oder durch institutionelle Beratung eine große Rolle.

Besonders interessant erscheint bei den Ergebnissen vor allem, dass die Beschreibungen der Übergangssituationen Ambivalenzen beinhalten. Deutlich wird das in den Ergebnissen beispielsweise bei der Selbstbeschreibung der Mütter, wenn sie zwischen positivem Selbstbild und negativer Selbstzuschreibung pendeln. Aber auch bei der Darstellung der Beziehung zu den eigenen Kindern schwanken die Frauen zwischen positiven und negativen Zuschreibungen. Scheinbar bestehen Wechselbeziehungen zwischen positiv und negativ wahrgenommenen Ereignissen, die sich bei den Müttern während der als krisenhaft erlebten Passage zum Alleinerziehen herausbilden. Aus diesem Blickwinkel betrachtet, bedeutet Bildung nicht wie im klassischen Sinn die Suche nach der eigenen Identität. „Bildung bedeutet in dieser Hinsicht gerade nicht Selbstfindung, Selbsterhaltung oder Selbstverwirklichung auf dem Grunde einer Überfülle an Möglichkeiten, die nur noch zu verwirklichen sind, sondern eine konflikthafte Lebensführung, einen spezifischen Prozess der Subjektivation, der eingespannt bleibt zwischen reiner Autonomie und bloßer Heteronomie.“ (Meyer-Drawe 2007: 85). Ambivalenz ist damit eine „Impulskraft von transformatorischen Bildungsprozessen (Haller 2011: 369).

Ambivalenz wird in den Ansätzen zum transformativen Lernen oder zu Deutungsmustern nur implizit erwähnt. Vor diesem Hintergrund könnte es gewinnbringend sein, das heuristische Konstrukt der Ambivalenz (Lüscher 2016) als ergänzende Theorieperspektive in den Blick zu nehmen. Kurt Lüscher beschreibt Ambivalenzerfahrungen im Kontext von Prozessen der Identitätsentwicklung des Individuums: Von Ambivalenzen spricht er, ,wenn Menschen auf der Suche nach der Bedeutung von Personen, sozialen Beziehungen und Tatsachen, die für Facetten ihrer Identität und dementsprechend für ihre Handlungsbefähigung wichtig sind, zwischen polaren Widersprüchen des Fühlens, Denkens, Wollens oder sozialer Strukturen oszillieren, die zeitweilig oder dauernd unlösbar scheinen“ (Lüscher 2011: 378).

Aus der Perspektive des heuristischen Konstrukts können die in den Daten ersichtlichen Pendelbewegungen zwischen unterschiedlichen Polen - wie zum Beispiel positiven und negativen Selbstbeschreibungen - theoretisch kontextualisiert werden, indem diese als Ausdruck einer ,reflexive[n] Ambivalenznormalität“" (Welser 2015: 75) interpretiert werden können. 
Zudem wird es mit dieser Theorieperspektive möglich, den Auslöser transformativer Lernprozesse („Dilemma“), nicht ausschließlich negativ zu konnotieren, sondern ihn vielmehr als Ausgangspunkt eines Entwicklungsprozesses zu betrachten, in dem sowohl negative als auch positive Perspektiven enthalten sind. In diesem Zusammenhang kann auch dem Umgang mit Ambivalenz eine zentrale Bedeutung in transformativen Lernprozessen zugeschrieben werden. Dabei erscheint wiederum die zuvor erwähnte Reflexionsfähigkeit als bedeutsame Komponente, um Ambivalenzen im eigenen Erleben als solche zu identifizieren und entsprechende Umgangsformen zu entwickeln, wie zum Beispiel eine gewisse Ambivalenztoleranz (Jekeli 2002) als Möglichkeit, oszillierende biografische Spannungsfelder ,auszuhalten“.

\section{Literatur}

Arnold, Rolf, Schüßler, Ingeborg (1996): Deutungslernen - ein konstruktiver Ansatz lebendigen Lernens. In: Arnold, Rolf (Hrsg.): Lebendiges Lernen. Baltmannsweiler: Schneider Hohengehren, S. 184-206.

Becker-Schmidt, Regina (1994): Diskontinuität und Nachträglichkeit - Theoretische und methodische Überlegungen zur Erforschung weiblicher Lebensläufe. In: Diezinger, Angela, Kitzer, Hedwig, Anker, Ingrid, Bingel, Irma, Haas, Erika, Odierna, Simone (Hrsg.): Erfahrung mit Methode -Wege sozialwissenschaftlicher Frauenforschung. Freiburg: Kore. S. 155-183.

Benner, Dietrich (2005): Erziehung - Bildung - Negativität. In: Zeitschrift für Pädagogik. 49. Beiheft. Weinheim: Beltz, S. 7-21.

Brand, Dagmar (2006): Alleinerziehende mit volljährigen Kindern. Über den Wandel von Lebenslagen und Lebensformen. Wiesbaden: VS Verlag für Sozialwissenschaften.

Brand, Dagmar, Hammer, Veronika (2002): Balanceakt Alleinerziehend. Lebenslagen, Lebensformen, Erwerbsarbeit. Wiesbaden: Westdeutscher Verlag.

Bundesministerium für Familie, Senioren, Frauen und Jugend - BMFSFJ (2011): Lebenswelten und -wirklichkeiten von Alleinerziehenden. http://www.bmfsfj. de/RedaktionBMFSFJ/Broschuerenstelle/Pdf-Anlagen/Lebenswelten-_20undwirklichkeiten-von-Alleinerziehenden, property $=$ pdf,bereich $=$ bmfsfj,,$-\overline{2 p r a c h e}=$ de,rwb=true.pdf [Zugriff: 08.11.2018].

Dausien, Bettina (1994): Biographieforschung als „Königinnenweg“? Überlegungen zur Relevanz biographischer Ansätze in der Frauenforschung. In: Diezinger, Angela, Kitzer, Hedwig, Anker, Ingrid, Bingel, Irma, Haas, Erika, Odierna, Simone (Hrsg.). Erfahrung mit Methode - Wege sozialwissenschaftlicher Frauenforschung. Freiburg: Kore, S. 129-153. 
Felden, Heide von (2009): Auf sich selbst verwiesen. Reflexive Modernisierung und neue Lernanforderung. In: DIE Zeitschrift für Erwachsenenbildung, Band II/2009, Bonn, S. 34-37.

Glinka, Hans-Jürgen (1998): Das narrative Interview. Eine Einführung für Sozialpädagogen. Weinheim/München: Juventa.

Haller, Miriam (2011): Dekonstruktion der „Ambivalenz“. Poststrukturalistische Neueinschreibungen des Konzepts der Ambivalenz aus bildungstheoretischer Perspektive, in: Forum der Psychoanalyse 27, H. 4, S. 359-371.

Hammer, Veronika (2002): Frauen nach der Familienphase. Ein Orientierungskurs in der Praxis. Oldenburg: Verlag Dialogische Erziehung.

Hancioglu, Mine (2014): Alleinerziehende und Gesundheit. Die Lebensphase ,alleinerziehend" und ihr Einfluss auf die Gesundheit. Inaugural-Dissertation: Ruhr-Universität Bochum.

Höhn, Charlotte, Dorbritz, Jürgen (1995): Zwischen Individualisierung und Institutionalisierung - Familiendemographische Trends im vereinten Deutschland. In: Nauck, Bernhard, Onnen-Isemann, Corinna (Hrsg.): Familie im Brennpunkt von Wissenschaft und Forschung. Neuwied: Luchterhand. S. 149-174.

Jekeli, Ina (2002): Ambivalenz und Ambivalenztoleranz. Soziologie an der Schnittstelle von Psyche und Sozialität. Osnabrück: Der Andere Verlag.

Kaiser, Ruth (1992): Narrativ-fokussiertes Interview in der Bildungsforschung. Merkmale, Anwendung, Auswertung. In: Grundlagen der Weiterbildung - Zeitschrift, 3 (6), S. 361-364.

Klein, Thomas (1999): Pluralisierung versus Umstrukturierung am Beispiel partnerschaftlicher Lebensformen. In: Kölner Zeitschrift für Soziologie und Sozialpsychologie, 51 (3), S. 469-490.

Koller, Hans-Christoph (2005): Negativität und Bildung. Eine bildungstheoretisch inspirierte Lektüre von Kafkas Brief an den Vater. In: Zeitschrift für Pädagogik, 49. Beiheft, S. 136-149.

Köngeter, Anja (2011): Macht Alleinerziehen krank? Der psychische Gesundheitszustand alleinerziehender Mütter unter besonderer Berücksichtigung heterogener Lebenslagen in Deutschland. München: Grin.

Kuckartz, Udo (2016): Qualitative Inhaltsanalyse: Methoden, Praxis, Computerunterstützung. 3. durchges. Auflage. Weinheim: Beltz Juventa.

Laros, Anna (2015): Transformative Lernprozesse von Unternehmerinnen mit Migrationsgeschichte. Wiesbaden: Springer.

Lenze, Anne (2014): Alleinerziehende unter Druck. Rechtliche Rahmenbedingungen, finanzielle Lage und Reformbedarf. Gütersloh: Bertelsmann Stiftung.

Lerch, Sebastian (2010): Lebenskunst lernen? Lebenslanges Lernen aus subjektwissenschaftlicher Sicht. Bielefeld: Bertelsmann.

Lüscher, Kurt (2011): Ambivalenz weiterschreiben. Eine wissenssoziologischpragmatische Perspektive. In: Forum Psychoanalyse 27(4), S. 373-393.

Lüscher, Kurt (2016): Sozialisation und Ambivalenzen. Bausteine eines Vademekums. In: ZSE 36/2016 (2), S. 116-138.

Mayring, Phillip (2008): Qualitative Inhaltsanalyse. Grundlagen und Techniken. Weinheim/Basel: Beltz. 
Meier, Uta, Preusse, Heide, Sunnus, Eva Maria (2003): Steckbriefe von Armut. Haushalte in prekären Lebenslagen. Wiesbaden: Westdeutscher Verlag.

Merkens, Hans (2008) Auswahlverfahren, Sampling, Fallkonstruktion. In: Flick, Uwe, Kardorff, Ernst von, Steinke, Ines (Hrsg.): Qualitative Forschung. Ein Handbuch. Reinbek: Rowohlt, S. 286-298.

Meyer-Drawe, Käte (1982): Lernen als Umlernen. In: Lippitz, Wilfried, MeyerDrawe, Käte (Hrsg.): Lernen und seine Horizonte. Phänomenologische Konzeptionen menschlichen Lernens - didaktische Konsequenzen. Sriptor: Königstein, S. $19-45$.

Meyer-Drawe, Käte (2007): „Du sollst Dir kein Bildnis noch Gleichnis machen ...“ Bildung und Versagung. In: Koller, Hans-Christoph, Marotzki, Winfried, Sanders, Olaf (Hrsg.): Bildungsprozesse und Fremdheitserfahrung. Beiträge zu einer Theorie transformatorischer Bildungsprozesse. Bielefeld: transcript, S. 8393.

Mezirow, Jack (2000): Learning to Think Like an Adult: Core Concepts of Transformational Theory. In: Mezirow, Jack \& Associates (Hrsg.): Learning as Transformation: Critical Perspectives on a Theory in Progress. San Francisco: Jossey-Bass, S. 3-33.

Mezirow, Jack (2009): Transformative Learning Theory. In: Mezirow, Jack, Taylor, Edward W. (Hrsg.): Transformative learning in practice. San Francisco: Jossey-Bass, S. 18-32.

Mezirow, Jack (2012): Learning to Think Like an Adult. Core Concepts of Transformation Theory. In: Taylor, Edward W., Cranton, Patricia and Associates (Hrsg.): The Handbook of Transformative Learning. San Francisco: Jossey Bass, S. 73-95.

Nave-Herz, Rosemarie (2009): Familie heute. Wandel der Familienstrukturen und Folgen für die Erziehung. 4. Auflage. Darmstadt: WBG.

Nestmann, Frank, Stiehler, Sabine (1998): Wie allein sind Alleinerziehende? Soziale Beziehungen alleinerziehender Frauen und Männer in Ost und West. Opladen: Leske + Budrich.

Ostermann-Vogt, Bettina (2011): Biographisches Lernen und Professionalitätsentwicklung. Lernprozesse von Lehrenden in Pflegeberufen. Wiesbaden: VS Verlag für Sozialwissenschaften.

Ott, Notburga, Strohmeier, Klaus Peter (2003): Alleinerziehende im Sozialhilfebezug - Risiken und Chancen im Leben zwischen Familie und Erwerbstätigkeit. Forschungsbericht, Ruhr-Universität Bochum.

Rinken, Barbara (2010): Spielräume in der Konstruktion von Geschlecht und Familie? Alleinerziehende Mütter und Väter mit ost- und westdeutscher Herkunft. Wiesbaden: VS Verlag für Sozialwissenschaften.

Schmidt-Lauff, Sabine, Felden, Heide von, Pätzold, Henning (2015): Transitionen in der Erwachsenenbildung. Gesellschaftliche, institutionelle und individuelle Übergänge. Opladen/Berlin/ Toronto: Verlag Barbara Budrich.

Schneider, Norbert F., Krüger, Dorothea, Lasch, Vera, Limmer, Ruth, MatthiasBleck, Heike (2001): Alleinerziehen. Vielfalt und Dynamik einer Lebensform. Schriftenreihe des BMFSFJ. Band 199. Stuttgart/Berlin/Köln: Kohlhammer. 
Schüßler, Ingeborg (2008): Reflexives Lernen in der Erwachsenenbildung - zwischen Irritation und Kohärenz. In: Bildungsforschung, 5 (2), S. 12-18.

Schütze, Fritz (1980) Narrative Repräsentation kollektiver Schicksalsbetroffenheit. In: Lämmert, Eberhard (Hrsg.); Erzählforschung: Ein Symposion. Stuttgart: Metzler, S. 568-590.

Schütze, Fritz (1983): Biographieforschung und narratives Interview. In: Neue Praxis, 13 (9), S. 283-293.

Statistisches Bundesamt (2010): Alleinerziehende in Deutschland. Ergebnisse des Mikrozensus 2009. Wiesbaden. https:/www.destatis.de/DE/PresseService/ Presse/Pressekonferenzen/2010/Alleinerziehende/pressebroschuere_Alleinerziehende2009.pdf?_blob=publicationFile [Zugriff: 20.01.2019].

Statistisches Bundesamt (2010): Alleinerziehende in Deutschland. Ergebnisse des Mikrozensus 2009. Wiesbaden. https://www.destatis.de/DE/PresseService/ Presse/Pressekonferenzen/2010/Alleinerziehende/pressebroschuere_Alleinerziehende2009.pdf?_blob=publicationFile [Zugriff: 05.10.2018].

Wagner, Michael (2008): Entwicklung und Vielfalt der Lebensformen. In: Schneider, Norbert F. (Hrsg.): Lehrbuch Moderne Familiensoziologie. Opladen/Farmington Hills: Barbara Budrich. S. 99-120.

Welser, Stephanie (2015). Interaktion zwischen Pädagogin und Mädchen unter widersprüchlichen Bedingungen. In: Betrifft Mädchen., 02, S. 71-76. 


\section{Transformation des Subjekts im Kontext des Übergangs in die Hochschule}

Der vorliegende Beitrag beschäftigt sich aus der Sicht des Subjekts mit Transformationen und nimmt die dabei (potentiell) stattfindenden Bildungsprozesse in den Blick. Diese werden im spezifischen Kontext des Übergangs in die Hochschule empirisch analysiert. Durch diesen Fokus wird es möglich, unterschiedliche Facetten von transformatorischer Bildung sowie Hinweise auf selbstbezogene Veränderungen in den Eigentheorien der Subjekte herauszuarbeiten. Darüber hinaus lässt sich die Funktion dieses spezifischen Übergangs als Generator solcher Bildungsprozesse rekonstruieren.

Vor diesem Hintergrund werden folgende Fragestellungen bearbeitet, die über ihren Bezug zur subjektiven Gestaltung des Übergangs miteinander verbunden sind:

- Welche Dimensionen charakterisieren den Übergang in die Hochschule aus Sicht der Betroffenen?

- Welche selbstbezogenen Veränderungen lassen sich in den Eigentheorien der Befragten identifizieren?

Um einen Beitrag zur Diskussion dieser Fragestellungen zu leisten, wird zunächst das Forschungsfeld der Hochschullandschaft dargestellt. Nach der Rahmung der zentralen theoretischen Konzepte wird das Forschungsdesign erläutert, auf dessen Ergebnissen der vorliegende Beitrag beruht. Dem schließen sich die zentralen empirischen Ergebnisse sowie deren Rückkopplung an den Forschungsstand an.

\section{Veränderungen in der Hochschullandschaft}

Wie jede pädagogische Organisationsform hat auch die Hochschule im historischen Verlauf vielfältige Veränderungen erfahren. Dies betrifft bspw. nicht nur die Ausdifferenzierung in verschiedene Institutionalisierungsformen, sondern auch die Zielsetzungen, mit denen der Besuch von Hochschulen verbunden war und ist (Fisch 2015). Auch der Blick auf die jüngere Geschichte der Hochschule offenbart substantielle Veränderungen, die anhand unterschiedlicher Phänomene sichtbar werden. 
Als eines dieser Phänomene kann die angebotsseitige Ausdifferenzierung von Hochschulen identifiziert werden. Exemplarisch wird dies anhand der Anzahl der Studiengänge in Deutschland deutlich. So stieg ihre Anzahl in den letzten zehn Jahren um etwas mehr als 59\% auf insgesamt 19.011 derartiger hochschulischer Angebote (Hochschulrektorenkonferenz 2017: 9). Die angesprochenen Veränderungen zeigen sich darüber hinaus im Anstieg der totalen Studierendenzahlen. Betrachtet man die Entwicklung seit dem Jahr 2000, so lässt sich auch hier ein deutlicher Anstieg um knapp 62\% auf 2.844.978 Personen verzeichnen. Eine besondere Rolle unter den verschiedenen Institutionalisierungsformen der Hochschulen spielen hierbei die Fachhochschulen. Deren Anteil an der Gesamtzahl der Studierenden in Deutschland stieg zwischen dem Wintersemester 1999/2000 und dem Wintersemester 2017/2018 von rund 24\% auf knapp 35\% an (Statistisches Bundesamt 2018a: 13). Jenseits des genuinen Anstiegs der Studierendenzahlen zeigt sich eine deutliche Erhöhung der Studienanfänger*innenquote. Je nach Berechnungsweise ist der Anteil der Studienanfänger*innen an der Bevölkerung des entsprechenden Geburtsjahres zwischen den Jahren 2000 und 2017 von etwa 33\% auf 56\% angestiegen (Statistisches Bundesamt 2018b).

Neben dem angebotsseitigen Ausbau der Hochschullandschaft und einer steigenden Nachfrage durch eine Erhöhung der Studierendenzahlen bzw. der entsprechenden Quote lassen sich die Veränderungen in der Hochschullandschaft auch anhand der Zusammensetzung der Studierendenschaft erkennen. Dies wird anhand zweier Kennzahlen deutlich. Erstens sind hier die berufsbiografischen Vorerfahrungen zu nennen: Inzwischen verfügen $22 \%$ der Studienanfänger*innen über eine abgeschlossene Berufsausbildung (Autorengruppe Bildungsberichterstattung 2018: 157). Dies führt unter anderem auch zu einer Erhöhung des Lebensalters von beruflich Qualifizierten zu Studienbeginn (Middendorf et al. 2017: 29). Zweitens wird dieses Phänomen auch anhand der zuvor erworbenen Zugangsberechtigungen sichtbar. Nach wie vor bildet die allgemeine Hochschulreife den klassischen Zugang in die Hochschule, doch zeigt sich beispielsweise anhand der zunehmenden Nutzung des Dritten Bildungswegs eine Tendenz der Öffnung gegenüber anderen Wegen in diesen Bereich des Bildungssystems. Im Vergleich zum Jahr 2000 haben 2017 bereits siebenmal mehr Personen diesen Zugangsweg in Hochschulen gewählt, wobei anzumerken ist, dass ihr Anteil an den Studienanfänger*innen mit 3\% weiterhin eher überschaubar ausfällt (Autorengruppe Bildungsberichterstattung 2018: 157).

Insgesamt lässt sich festhalten, dass die Hochschullandschaft, exemplarisch dargestellt anhand ihrer Entwicklungen seit dem Jahr 2000, hinsichtlich ihrer Angebote von expansiven Tendenzen geprägt ist. Ihre Angebote werden von immer mehr Personen nachgefragt und im Hinblick auf die Studierendenschaft lässt sich zudem eine Pluralisierung diagnostizieren. Vor diesem Hin- 
tergrund eröffnen sich mehr Möglichkeiten im Erwachsenenalter, um Hochschulen als formale Lernkontexte zu nutzen. Der vorliegende Beitrag thematisiert in diesem Zusammenhang die subjektiven Veränderungen der Studierenden im Rahmen der Aufnahme eines Hochschulstudiums, wobei der Übergang in die Hochschule als potentiell kritisches Lebensereignis verstanden wird.

\section{2 Übergangs- und Transformationsprozesse}

$\mathrm{Zu}$ den unterschiedlichen Facetten von Übergängen können theoretische Bezüge hergestellt werden, die je eigene Schwerpunkte auf derartige Prozesse legen. Im vorliegenden Beitrag wird das Konzept der Life-Trajectory (Schäffter 2015) zur Rahmung von Übergängen gewählt, das sich aufgrund verschiedener Aspekte für die Analyse von biografischen Prozessen besonders eignet: Aus dieser Perspektive heraus werden Übergänge im Sinn einer Life-Trajectory zunächst als soziale Prozesse verstanden, die jedoch nicht primär durch institutionalisierte Rahmungen strukturiert, sondern vielmehr ohne eine solche Steuerungsinstanz zu denken sind. Der Prozesscharakter wird in diesem Konzept zudem damit unterstrichen, dass Übergänge als eine pfadabhängige Abfolge von einzelnen Lebensereignissen verstanden werden, was die Prognosemöglichkeit für den Eintritt spezifischer Ereignisse erschwert (Schäffter 2015: 27f.). Gleichzeitig werden dadurch Phänomene vielfältiger Verstrickungen und Verknüpfungen zwischen Lebensereignissen, beispielsweise zwischen privaten und beruflichen Entwicklungen oder persönlichen und familienbiografischen Erfahrungen, theoretisch aufgegriffen und empirisch zugänglich. Ein weiteres Kennzeichen der Life-Trajectory, das für die Analyse von biografischen Veränderungen und vor allem den damit verbundenen Bildungsprozessen relevant ist, bildet die Zieloffenheit dieses Zugangs. Die damit verbundene prospektive Ausrichtung verweist auf einen Möglichkeitsraum für zukünftige Entwicklungen, der dem Subjekt einen multioptionalen Spielraum zur Gestaltung der eigenen Übergangsprozesse und damit des eigenen Lebensverlaufs ermöglicht. Um die entsprechenden Übergänge zu realisieren, wird im vorgestellten Konzept auch eine zentrale Bedingung an das Subjekt formuliert. Um beispielsweise Kontexterschließung zu ermöglichen, wie sie bei der Aufnahme eines Hochschulstudiums im Sinn einer transformativen Aneignung von Lernkontexten nötig wird, bedarf es vonseiten des Subjekts einer permanenten Selbstvergewisserung über die Zieloffenheit des eigenen Handelns (Schäffter, 2015: 29).

Der im Zusammenhang von Life-Trajectory bereits am Beispiel der transformativen Aneignung von Lernkontexten angesprochene Aspekt der Trans- 
formation findet sich in einem weiteren anschlussfähigen Konzept. Dessen Relevanz ergibt sich vor dem Hintergrund der Diagnose einer Transformationsgesellschaft (Schäffter 2014: 37) und den damit korrespondierenden Auswirkungen auf die Anforderungsstrukturen und Möglichkeitsräume der subjektiven Lebensführung. Daran anschließend lässt sich das Konzept der transformatorischen Bildungsprozesse (Koller 2018) vor allem hinsichtlich zweier Facetten für die Analyse von Übergängen in die Hochschule nutzbar machen. Erstens wird Bildung aus dieser Perspektive als „Prozess der Transformation grundlegender Figuren des Selbst- und Weltverhältnisses“ (Koller 2018: 17) verstanden, wodurch die Auswirkungen hochschulischer Lernkontexte jenseits von Wissensvermittlungs- und -aneignungsprozessen fassbar werden. Zweitens lassen sich aus dieser Sicht Übergänge als Krisen greifen, die als Anlässe zur Transformation durch Infragestellung etablierter Welt- und Selbstbezüge verstanden werden können.

Die zwei vorgestellten Bezüge ermöglichen die Analyse von selbstbezogenen Veränderungen, die Subjekte im Kontext des Übergangs in die Hochschule erleben. Um diese Erfahrungen empirisch zu erfassen, wurde ein spezifischer Zugang zum Forschungsfeld gewählt, der die Eigentheorien der Subjekte in den Mittelpunkt rückt.

\section{Erforschung der Wege in die Hochschule}

Um die oben formulierten Fragestellungen vor dem Hintergrund empirischer Ergebnisse diskutieren zu können, wurde ein monomethodisches und explorativ-rekonstruktives Design gewählt. Im Fokus stehen dabei die verschiedenen Phänomene des Übergangsprozesses, der von den befragten Subjekten im Kontext ihrer Aufnahme eines Hochschulstudiums durchlaufen wird. Als Studienteilnehmende wurden dabei studienberechtigte Personen, eingeschriebene Studierende, exmatrikulierte Personen sowie Absolvent*innen von hochschulischen Studiengängen in Betracht gezogen. Der Forschungsprozess beruht auf den method(olog)ischen Prämissen der Grounded Theory, was sich vor allem in Bezug auf das Sampling sowie die Verwobenheit von Datenerhebung, -analyse und Theoriebildung auswirkt (Strübing 2014: 9ff.). Die Datenerhebung geschah durch problemzentrierte Interviews (Witzel/Reiter 2012: 15), sodass einerseits der thematische Fokus des Gesprächs mittels eines Leitfadens gerahmt und andererseits die Relevanzsetzungen der Befragten zur Geltung kommen konnten. Um die interessierenden Phänomene zu rekonstruieren, wurde auf das theoretische Kodieren der Grounded Theory zurückgegriffen (Strübing 
2014: 15f.). Dadurch wird auch auf der Ebene der Datenauswertung gewährleistet, dass die Perspektivität der Subjekte auf den eigenen Übergang in die Hochschule mit in den Prozess der Theoriebildung einfließt.

Die nachfolgend dargestellten Ergebnisse basieren auf einem kontrastiv ausgewählten Bestandteil des Samples. Es wurden drei Interviews mit Personen ausgewählt, die sich hinsichtlich ihrer Generationslagerung, des Geschlechts, der gewählten Studiengänge, der Studiengestaltung hinsichtlich der dabei realisierten Hochschulwechsel sowie der jeweiligen Studienphase unterscheiden. Diese Fälle werden im Rahmen der Ergebnisdarstellung als P1, P2 und $\mathrm{P} 3$ bezeichnet.

\section{Empirische Ergebnisse}

Aufgrund der geringen Fallzahl und des damit korrespondierenden Stands der Theoriebildung sind die nachfolgenden Ergebnisse als noch vorläufig zu betrachten. Trotz dieses frühen Auswertungsstadiums lassen sich jedoch bereits einige zentrale Facetten beschreiben, die sich mit Blick auf die oben ausgeführten Fragestellungen als instruktiv erweisen. Die mit ihnen korrespondierenden Resultate werden im Folgenden nach den beiden Fragestellungen getrennt dargestellt.

\section{Konturierung des Übergangs in die Hochschule}

Im Hinblick auf die thematisierten Dimensionen des Übergangs in die Hochschule aus Sicht der Betroffenen kann zunächst der multioptionale Spielraum zur Gestaltung der eigenen Übergangsprozesse konturiert werden. Bei der damit verbundenen Frage, in welchen Kontexten der Übergang realisiert wird, verweisen die Studienteilnehmenden auf den Umstand, dass neben der Aufnahme eines Hochschulstudiums weitere Übergangsprozesse vollzogen werden. In diesem Kontext verorten sie den Wohnortwechsel, die Aufnahme einer Erwerbstätigkeit, den Zusammenzug mit der*dem Partner*in, die Familiengründung sowie die damit nicht zwangsläufig verbundene Kindererziehung. Bei genauerer Betrachtung dieser Konstellationen fällt zudem die Präsenz simultaner Übergänge im Erwachsenenalter (Hof/Maier-Gutheil 2014: 147) ins Auge. Exemplarisch lässt sich dies anhand der Angaben von P2 beschreiben: Diese Person verbindet die Aufnahme eines Hochschulstudiums mit einem Wechsel des Wohnorts in ein anderes Bundesland und zieht simultan dazu mit 
ihrer*ihrem Partner*in zusammen. Dieses Phänomen zeigt nicht nur die Vielschichtigkeit des vollzogenen Übergangsprozesses auf, sondern verweist auf zwei weitere Aspekte: Erstens verdeutlicht die erläuterte Simultanität das Potential empirischer Analysen für die Erweiterung theoretischer Perspektiven auf Übergangsprozesse. Der bereits in Beschreibungen von schrittweisen Übergängen oder auch Ereignisketten aufscheinende Fokus auf Sequenzialität lässt sich vor dem Hintergrund der empirischen Ergebnisse zumindest kritisch hinterfragen. Zweitens leitet sich aus der Diagnose simultaner Übergänge eine potentiell steigende Komplexität der Anforderungsstrukturen für das Subjekt ab. Je nach Lebenssituation und Ressourcen erhöht sich dabei die Wahrscheinlichkeit für die Einstufung der Konstellation als Krise (Koller 2018: 71ff.).

\section{Entscheidung für die Aufnahme eines Hochschulstudiums}

Fragt man vor dem Hintergrund des Prozesscharakters von Übergängen in die Hochschule nach dessen Beginn, so wird im empirischen Material die Studienentscheidung in den Vordergrund der Sachverhaltsdarstellungen gerückt. Es zeigt sich in diesem Zusammenhang einerseits, dass der konkrete Zeitpunkt zur Aufnahme eines Hochschulstudiums nach dem Erwerb der Hochschulzugangsberechtigung variabel ausgestaltet wird. So entscheiden sich manche Personen für einen mehrmonatigen Auslandsaufenthalt, während andere einen unmittelbaren Anschluss nach dem Verlassen der Institution Schule anstreben. Allen Fällen gemeinsam ist andererseits die Kopplung der grundsätzlichen Entscheidung für die Aufnahme eines Hochschulstudiums an den Erwerb der Hochschulzugangsberechtigung in Form der allgemeinen Hochschulreife. Diese Verknüpfung von grundsätzlicher Berechtigung und faktischer Entscheidung zeigt sich exemplarisch anhand des folgenden Datenauszugs.

„Das war vorgegeben, mehr oder weniger von den Eltern. [...] Also das, das war nie so die Sache mit (.) die haben mir jetzt keine Uni ausgesucht, wie sie in der vierten Klasse mir, in der fünften Klasse mir das Gymnasium ausgesucht haben. Aber es war einfach, ja, du studierst. Es ist einfach so, eine GOTTgebene grundsätzliche Sache, du studierst. Das ist ja so, deswegen machst du ja überhaupt ein Abi.“ (P3-178-180)

In diesem Datenbeispiel dokumentiert sich über den Verweis auf den Einfluss der Eltern die Orientierung der Person an fremdinduzierten Erwartungen. Dieser Eindruck verstärkt sich noch dadurch, dass dieser Verbindung durch den Einbezug einer metaphysischen Instanz eine nahezu absolute Legitimität beigemessen wird. Etwas schwächer thematisiert dieses Phänomen auch P1: 
„Da war eigentlich, glaube ich, das war nicht die Frage, was man macht. [...] ich glaube, das ist einfach bei den Abimäusen so [...] Ja, waren wir so, was sollen wir sonst machen, also alle aus dem Klassenverband haben halt irgendwas studiert" (P1-149-151)

Dieser Auszug verdeutlicht aus der Perspektive der Betroffenen heraus die Selbstverständlichkeit bzw. Unhinterfragtheit der beschriebenen Kopplung. Die Anschlussfähigkeit dieses Phänomens zeigt sich im Vergleich mit empirischen Ergebnissen zum Übergang zwischen Schule und Arbeit. Dabei wird die Prägekraft gesellschaftlicher Erwartungen an Jugendliche herausgearbeitet, die sich in einem Übergangsregime manifestieren (Walther 2014: 91). Auch im Fall des Übergangs in die Hochschule wird dieses Phänomen deutlich, wobei die Personen ebenfalls Normalitäten konstatieren und eigene Entscheidungen tendenziell in den Hintergrund treten.

\section{Unterstützungskontexte beim Übergang in die Hochschule}

Auch vor dem Hintergrund von Fragen nach unterstützenden Kontexten, die die Subjekte zur Übergangsgestaltung in Anspruch nehmen, zeigen sich anschlussfähige Ergebnisse. In Ergänzung zu Ansätzen, den Übergang in die Hochschule mittels organisationaler Angebote zu rahmen (Driesen/Ittel 2019), verweisen die empirischen Daten auf eine Priorisierung lebensweltnaher Beratungskontexte. Deutlich wird dies anhand des nachfolgenden Auszugs:

„Ich sehe mich irgendwie noch, wie ich dieses Z4 Campusding wälze und nebenbei, ich glaube, mein Schwager und meine Schwester auch noch mit dabei saßen [...] Und gefragt haben, warum ich eigentlich so diesen Studiengang in R13 zum Beispiel, der irgendwie ich weiß gar nicht mehr warum der weggefallen ist, warum ich mich für den interessiere oder (.) ja allgemein meine Geschwister, die immer noch Input geliefert haben und überlegt haben, hey, wie wäre es denn noch damit" (P2-184-186)

Wie auch in weiteren Datenbeispielen scheint hier jenseits der Frage, ob organisationale Angebote in Anspruch genommen werden, vor allem das soziale Umfeld bei der Auswahl von Studienort und Studiengang relevant zu sein. Insbesondere dokumentiert sich in den analysierten Daten die deutliche Präferenz, sich im Entscheidungsprozess auf die Empfehlungen des sozialen Umfelds und hier vor allem der Familie sowie enger Freunde zu stützen. Demgegenüber werden Unterstützungskontexte von Hochschulen oder anderen Beratungseinrichtungen dethematisiert. Im Rahmen dieser spezifischen Konstellation beziehen sich die Studienteilnehmenden vor allem dann auf die Empfehlungen ihres sozialen Umfelds, wenn diese auf eigenen Studiererfahrungen beispielsweise 
von Geschwistern beruhen, die bereits ein Hochschulstudium abgeschlossen haben.

\section{Selbstbezogene Veränderungen im Kontext des Übergangs in die Hochschule}

Mit Blick auf die zweite Fragestellung und die theoretische Rahmung der transformatorischen Bildung lässt sich zunächst festhalten, dass im Zusammenhang mit der Aufnahme eines Hochschulstudiums keine Hinweise auf biografische Wandlungsprozesse (Schütze 1984: 92ff.) rekonstruiert werden konnten. Vielmehr zeigt sich, dass der Übergang in die Hochschule in den drei Fällen auf je unterschiedliche Weise gerahmt wird und spezifische Irritationen auftauchen, die als Anlässe für transformatorische Bildung gewertet werden können.

Die Erläuterungen einer Person verdeutlichen die Möglichkeit, mittels der Aufnahme eines Hochschulstudiums den multioptionalen Spielraum im mittleren Erwachsenenalter zu bewahren. So eröffnen sich durch diesen Schritt nicht nur neue berufliche Perspektiven, sondern es findet auch eine Erweiterung der sozialen Kontakte statt, die wiederum zu Irritationen des eigenen Selbst- und Weltverhältnisses führen kann. In diesem Zusammenhang dokumentiert sich zudem der Aspekt der Pfadabhängigkeit von Übergängen. Im konkreten Beispiel geschieht dies, indem durch die Familiengründung die Aufnahme eines Hochschulstudiums angeregt wird.

Aus der Perspektive einer anderen Person werden sowohl die Entscheidung für den gewählten Studiengang als auch der faktische Studienverlauf als krisenhafte Negativerfahrung konstruiert. Zwar wird von der befragten Person durchaus ein Zuwachs an spezifischem Fachwissen und bestimmten Kompetenzen diagnostiziert, doch führt der Verlauf des eigenen Studiums dazu, dass eine negativ konnotierte biografische Suchbewegung initiiert wird.

Auch die dritte Person konstatiert Fremdheitserfahrungen, die jedoch nicht auf die gesamte Studienphase bezogen werden. Stattdessen werden diese mit dem Hochschulwechsel nach Abschluss des Erststudiums verbunden (Hilgemann 2017: 59), die zusammen mit dem Aufbau eines als neu deklarierten sozialen Umfelds zu Irritationen führen. Sie dokumentieren sich in verschiedenen Phänomenen wie etwa der Rekontextualisierung retrospektiver Erfahrungen und prospektiver Zielsetzungen. Exemplarisch zeigt sich dies bei der Bewertung der eigenen Studienerfahrungen und der Ableitung von Handlungsempfehlungen für den eignen Nachwuchs zur zukünftigen Entscheidungsfindung. In diesem Zusammenhang finden sich starke reflexive Anteile, die überwiegend im Medium transgenerativer Projektionen realisiert werden. 


\section{Transformatorische Bildung im Kontext des Übergangs in die Hochschule}

In der Rückschau zeigen sich anhand der drei ausgewählten Fälle Phänomene, die einen Beitrag zur erziehungswissenschaftlichen Erforschung von Übergängen leisten können. Hinsichtlich der ersten Fragestellung nach den Dimensionen des Übergangs in die Hochschule lässt sich neben den gewählten Unterstützungskontexten vor allem die Existenz eines spezifischen Übergangsregimes zwischen weiterführender Schule und Hochschule rekonstruieren. Dieses manifestiert sich in den Sachverhaltsdarstellungen der befragten Personen primär in der Verknüpfung vom Erwerb der allgemeinen Hochschulreife und der Entscheidung für die Aufnahme eines Hochschulstudiums.

Mit Fokus auf die Bedingungen transformatorischer Bildung lassen sich zudem die simultanen Übergänge im Erwachsenenalter rekonstruieren. Diese scheinen im Datenmaterial am Beispiel des Übergangs in die Hochschule als Wohnortwechsel, Aufnahme einer Erwerbstätigkeit, Zusammenzug mit der*dem Partner*in, Familiengründung und der Kindererziehung auf. Hinsichtlich der zweiten Fragestellung nach selbstbezogenen Veränderungen in den Eigentheorien der Befragten konnten in den Interviews primär Anlässe für transformatorische Bildungsprozesse rekonstruiert werden. Dabei erwies sich vor allem die steigende Komplexität dieser simultanen Übergänge nicht nur aus theoretischer Perspektive, sondern auch mit Blick auf die empirischen Daten als Bedingung für potentiell krisenhafte Erfahrungen und damit als möglicher Anlass für transformatorische Bildungsprozesse. Während die Aufnahme eines Hochschulstudiums aus einer fallübergreifenden Perspektive dementsprechend genuin als ein solcher Anlass begriffen werden kann, zeigt sich mit Fokus auf die untersuchten Fälle ein differenziertes Bild. Dabei lässt sich ein Kontinuum an retrospektiven Einschätzungen feststellen, das von der Bestätigung des eigenen multioptionalen Spielraums durch den Übergang in die Hochschule einerseits bis hin zur Negativkonnotation des Studiums andererseits reicht.

\section{Literatur}

Autorengruppe Bildungsberichterstattung (2018): Bildung in Deutschland 2018. Ein indikatorengestützter Bericht mit einer Analyse zu Wirkungen und Erträgen von Bildung. Bielefeld: wbv. 
Driesel, Cornelia / Ittel, Angela (Hrsg.) (2019): Der Übergang in die Hochschule. Strategien, Organisationsstrukturen und Best Practices an deutschen Hochschulen. Münster: Waxmann.

Fisch, Stefan (2015): Geschichte der europäischen Universität. Von Bologna nach Bologna. München: Beck.

Hilgemann, Meike (2017). Der Übergang vom Bachelor zum Master. Bildungsentscheidungen im Schnittfeld von Gender und Fachkultur. Opladen: Budrich.

Hochschulrektorenkonferenz (2017): Statistische Daten zu Studienangeboten an Hochschulen in Deutschland. Studienangebote, Studierende, Absolventinnen und Absolventen. Wintersemester 2017/2018. Bonn: Hochschulrektorenkonferenz.

Hof, Christiane / Maier-Gutheil, Cornelia (2014): Übergänge im Erwachsenenalter. Befunde und offene Fragen für die Erwachsenenbildung. In: Hof, C. / Meuth, M. / Walther, A. (Hrsg.): Pädagogik der Übergänge. Übergänge in Lebenslauf und Biografie als Anlässe und Bezugspunkte von Erziehung, Bildung und Hilfe. Weinheim: Beltz Juventa, S. 146-167.

Koller, Hans-Christoph (2018): Bildung anders denken. Einführung in die Theorie transformatorischer Bildungsprozesse (2. Auflage). Stuttgart: Kohlhammer.

Middendorff, Elke/ Apolinarski, Beate / Becker, Karsten / Bornkessel, Philipp / Brandt, Tasso / Heißenberg, Sonja / Poskowsky, Jonas (2017): Die wirtschaftliche und soziale Lage der Studierenden in Deutschland 2016. 21.Sozialerhebung des Deutschen Studentenwerks durchgeführt vom Deutschen Zentrum für Hochschul- und Wissenschaftsforschung. Bonn /Berlin: Bundesministerium für Bildung und Forschung.

Schäffter, Ortfried (2014): Navigieren durch vernetzte Bildungslandschaften. Zum impliziten Erwerb von Übergangskompetenz in Lernbiographien. In: Felden, H. v. / Schäffter, O. / Schicke, H. (Hrsg.): Denken in Übergängen. Weiterbildung in transitorischen Lebenslagen. Wiesbaden: Springer VS, S. 37-59.

Schäffter, Ortfried (2015): Übergangszeiten - ,Transitionen' und ,Life Trajectories'. Navigieren durch Bildungslandschaften im Lebensverlauf. In: SchmidtLauff, S. / Felden, H. v. / Pätzold, H. (Hrsg.): Transitionen in der Erwachsenenbildung. Gesellschaftliche, institutionelle und individuelle Übergänge. Opladen: Budrich, S. 19-34.

Schütze, Fritz (1984): Kognitive Figuren des autobiographischen Stegreiferzählens. In: Kohli, M. / Robert, G. (Hrsg.): Biographie und Soziale Wirklichkeit: neue Beiträge und Forschungsperspektiven. Stuttgart: Metzler, S. 78-117.

Statistisches Bundesamt (2018a): Bildung und Kultur. Studierende an Hochschulen. Wintersemester 2017/2018. Wiesbaden: Statistisches Bundesamt.

Statistisches Bundesamt (2018b): Entwicklung der Studienanfängerquote* in Deutschland von 2000 bis 2017. https:/de.statista.com/statistik/daten/studie/ 72005/umfrage/entwicklung-der-studienanfaengerquote/ [Zugriff 22.09.18].

Strübing, Jörg (2014): Grounded Theory. Zur sozialtheoretischen und epistemologischen Fundierung eines pragmatistischen Forschungsstils (3. Auflage). Wiesbaden: VS. 
Walther, Andreas (2014): Übergangsregimes. Übergänge zwischen Schule und Arbeit im internationalen Vergleich. In: Hof, C. / Meuth, M. / Walther, A. (Hrsg.): Pädagogik der Übergänge. Übergänge in Lebenslauf und Biografie als Anlässe und Bezugspunkte von Erziehung, Bildung und Hilfe. Weinheim: Beltz Juventa, S. $78-103$.

Witzel, Andreas / Reiter, Herwig (2012): The Problem-Centred Interview. Los Angeles: SAGE. 

Joachim Ludwig

\section{Geht das unter die Haut? Zur Rolle der Gefühle in der Erwachsenenbildung}

\section{Problemstellung und Fragestellung}

Transformationsprozesse, die Subjekte im Kontext der Erwachsenenbildung vollziehen, sind in der Regel schwierige und komplexe Welt- und Selbstverständigungsprozesse, da sie oft nur schwer veränderbare Deutungsmuster und Sinnhorizonte tangieren. Solche teils habituellen Transformationen umfassen nicht allein die Verstandesebene, sondern immer auch Gefühle bzw. Empfindungen. Empfindungen sind Ausdruck ästhetischer Wahrnehmungen und Erfahrungen. Ästhetik wird in diesem Beitrag in Anlehnung an Welsch (2010) im Sinne von Aisthetik verstanden ,als Thematisierung von Wahrnehmungen aller Art, sinnenhaften ebenso wie geistigen, alltäglichen wie sublimen, lebensweltlichen wie künstlerischen" (Welsch 2010, 9f.). Ästhetisches Wahrnehmen kann so gesehen überall auftauchen (Reckwitz 2015, 25). Welche Rolle spielen sie in der Praxis der Erwachsenenbildung?

Für die politische Bildung sind Emotionen und Gefühle aktuell ein wichtiges Thema sowohl auf Tagungen (Bundeskongress Politische Bildung 2019: Was uns bewegt. Emotionen in Politik und Gesellschaft) als auch in der neueren Literatur (Schwerpunktheft Emotionen des Journals für politische Bildung 2/2018; Huber/Krause 2018). Für die Kulturelle Bildung sind Körperempfindungen und Gefühle im Kontext ästhetischer Wahrnehmungen und Erfahrungen ebenfalls ein Charakteristikum. Im Verbund mit Gefühlen und Empfindungen werden dort sachlich-soziale Themen wie z.B. biographische Übergänge oder Hate-Speech im Internet reflektiert. Die Verbindung von Gefühlen und sachlich-sozialen Diskursen wird in vielen Bildungsfeldern intendiert. Dies ist aber schon allein begrifflich schwierig, da Emotionen und Kognitionen in der Psychologie unterschieden und nur selten wieder zusammengeführt werden. Emotionen gelten meist als nicht-rational. Deshalb werden in diesem Beitrag statt des psychologischen Begriffs „Emotion“ die ästhetischen Begriffe „Gefühl“ oder „Empfindung“" verwendet. Empfindungen/Gefühle beschreiben zusammen mit den sachlich-sozialen Diskursen die beiden Seiten des Verstandes und sind Teil des ästhetischen Diskurses, auf den in diesem Beitrag Bezug genommen wird. Empfindungen sind Ausdruck ästhetischer Wahrnehmungen. Spielen ästhetische Wahrnehmungen und die damit verbundenen Empfindun- 
gen in der Erwachsenenbildungspraxis eine Rolle, damit komplexe Transformationsprozesse angemessen unterstützt werden können? Geht dort Bildung unter die Haut?

Nach der Relevanz von Gefühlen für die Praxis der Erwachsenenbildung befragt, verweisen Kursleitende regelmäßig auf den zentralen Stellenwert der Atmosphäre: Eine gute Atmosphäre sorge für eine enge Bindung der Teilnehmenden an den Kurs und für das Gelingen des Kurses insgesamt. Angesprochen wird hier die Atmosphäre als ein gestaltbares soziales Medium, das eine förderliche Lernkultur im Kurs hervorbringen soll. In erwachsenenpädagogischen Praxisanleitungen findet man Vorschläge für die Steuerung der ,sozialemotionalen Gruppenprozesse" (Nuissl/Siebert 2013, 124) durch die Kursleitenden, die den Kurs zu einer sozialen Einheit formen (a.a.O., 122) und eine gute Kursatmosphäre schaffen sollen. Gefühle werden hier nicht direkt thematisiert, sondern indirekt über die Gestaltung der Kursatmosphäre als eine Art Rahmenbedingung für die Gefühlswelt der Teilnehmenden. Empfindungen und Gefühle der Teilnehmenden werden in Praxisanleitungen aber auch direkt angesprochen, denn ,alle Lernprozesse sind emotional gefärbt“" (Nuissl/Siebert 2013, 104). Empfindungen spielen direkt eine Rolle, wenn es z.B. um das Erzeugen von Motivation und Interesse geht (Nuissl/Siebert 2013, 34; Szepansky 2010, 34ff.). Vorgeschlagen wird beispielsweise das Mittel der Provokation, um Teilnehmende betroffen zu machen (Allespach u.a. 2009, 127). Gefühle werden auch direkt angesprochen, wenn darauf hingewiesen wird, dass Lernen Spaß machen soll (Becker u.a. 1980, 296). Gemeinsam ist diesen Hinweisen und Strategien aus der Praxis, dass Gefühle die Sacharbeit unterstützen sollen. Der Grundtenor in der Literatur zur pädagogischen Praxisanleitung ist: Gefühle werden für die Sacharbeit funktionalisiert und haben keinen eigenen systematischen Ort im didaktischen Denken. Eine Kurssitzung ,sollte möglichst spannend beginnen, die Neugierde der Lernenden wecken, sie motivieren, [um; jl] sich im Verlaufe des Lernens möglichst rational mit dem Lerngegenstand auseinanderzusetzen“" (Brauer 2018, 25).

Gefühle haben in der Praxisliteratur eine sachlich-unterstützende Funktion. Wenig reflektiert werden im didaktischen Diskurs Gefühle als systematischer Teil des Welt- und Selbstverhältnisses der Teilnehmenden, das transformiert werden soll. Gefühle haben keinen systematischen Ort beim Lernen (Brauer 2018, 25). Das im Folgenden vorgestellte Projekt sinnbild (Sinn und ästhetische Erfahrung in der Erwachsenenbildung) fragt danach, ob sich bestimmte Sichtweisen und ästhetische Praktiken oder Methoden der Kursleitenden identifizieren lassen, mit denen sie die Gefühle und Empfindungen der Teilnehmenden für die didaktische Unterstützung von Transformationsprozessen in Lehr-Lernverhältnissen aufgreifen. In welcher Weise nehmen Kursleitende in ihrer professionellen Planungsarbeit und Durchführungspraxis auf Gefühle 
und Empfindungen der Teilnehmenden Bezug? Vorgestellt werden im Folgenden der Forschungsstand, das Forschungsdesign des Projekts sinnbild und erste Zwischenergebnisse.

\section{Forschungsstand}

Wie stellt sich der Forschungsstand zu Gefühlen und Empfindungen, die überwiegend als Emotionen diskutiert werden, in der Erwachsenenbildung dar? Dazu liegen im Vergleich zu anderen Aspekten des Lehr-Lernverhältnisses nur wenige Arbeiten vor. Im Folgenden wird auf die wichtigsten Studien in chronologischer Reihenfolge eingegangen. Eine nicht mehr wegzudenkende Perspektivenerweiterung auf das Denken zum Lernen und Lehren stellt das Buch „Gruppendynamik und Erwachsenenbildung“ dar, das 1967 von Tobias Brocher veröffentlicht wurde. Brocher bringt erstmals in der Erwachsenenbildung die affektive Seite der Lernenden und des Lehr-Lernverhältnisses zur Sprache. Reflektiert wird der Zusammenhang von Sachbezügen und affektiven Bezügen: Frühere Lernerfahrungen der Teilnehmenden und Kursleitenden sowie affektive gruppendynamische Prozesse führen zu latenten Spannungen und wirken begrenzend bzw. fördernd auf das Lehr-Lernverhältnis.

Im Kontext eines erfahrungsbezogenen Lernens und Lehrens reflektiert Günther Holzapfel (1982, S. 68f.) im Anschluss an Klaus Holzkamp sowie Ute Holzkamp-Osterkamp den Stellenwert von subjektiven Befindlichkeiten als Ausdruck gesellschaftlicher Strukturen. Seine Arbeiten münden 2002 in die Monographie „Leib, Einbildungskraft, Bildung“, in der er vor dem Hintergrund der humanistischen Psychologie sowohl den Dualismus von Verstand und Emotion kritisiert als auch den rationalistischen Monismus, der selbst einen Mythos darstellt und somit begrenzend wirkt. Um diese Grenzen zu überwinden, betont er die leibliche Situiertheit des Erfahrens, Erkennens und Lernens. Bildung hätte als eine Art Dialyseprozess die Aufgabe, den Menschen zu helfen, sich ihrer Phantasiewelt bewusster zu werden, indem sie leibliche Spür, Wahrnehmungs- und Ausdrucksprozesse anstößt (Holzapfel 2004, 159ff.).

Aus einer konstruktivistischen Perspektive zielt Rolf Arnold (2005) auf ein ähnliches Konzept in seinem Buch „Die emotionale Konstruktion der Wirklichkeit“. Arnold verweist auf ,emotionale Matrixen“ $(2005,7)$, die es bewusst $\mathrm{zu}$ machen gilt. Seine Grundthese besagt, dass diese Matrixen es uns immer wieder erlauben ,die bekannten - schlechten oder guten - Gefühle zu aktivieren" $(2005,2)$. Erwachsenenbildung hat die Einbindung des Rationalen in das Emotionale beim Wahrnehmen, Denken und Handeln in Rechnung zu stellen und darf sich nicht kognitiv verengen $(2005,3)$. Ziel soll die Vermittlung reflexiven Wissens sein, d.h. ,ein Wissen über das eigene Verhalten in Prozessen 
der Wissensaneignung und der Kooperation“ $(2005,69)$. „Muster des Sich-inder-Welt-Fühlens" $(2005,70)$ sollen bewusst erlebt und reflektiert werden. Es gilt Emotionen zur Sprache zu bringen und eine ,emotionale Selbstreflexivität"“ $(2005,255)$ zu entwickeln.

Eine beziehungstheoretische Perspektive auf Emotionen und Bildung nimmt Wiltrud Gieseke (2007) in ihrem Buch „Lebenslanges Lernen und Emotionen“ ein. Aus ihrer Sicht eröffnen Emotionen eine erweiterte Perspektive auf Beziehungen in Bildungsprozessen: „Erst mit dem Bedürfnis die anderen zu verstehen, die anderen zu lieben - übrigens der einzige Weg, sich zu lieben und zu verstehen - beginnt Bildung“" $(2007,54)$. Emotionen schaffen - so eine ihrer Hauptthesen - die für das lebenslange Lernen notwendige Haltung von Offenheit und Neugierde $(2007,89)$. Der ,sich involvierende Bezug zur Welt“ $(2007,54)$ wird als Welt- und Selbstverhältnis in den Emotionen zum Ausdruck gebracht. Ohne Emotionen wären Lern- und Bildungsprozesse nicht denkbar. „Bindungen und Beziehungen ... sind emotionstheoretisch die Bedingungen und Voraussetzungen von Lernen“" $(2007,107)$. Emotionen sind dabei sowohl Seismographen der Weltverarbeitung als auch Orientierung gebende Bewertungsmaßstäbe, in denen sich alte Erfahrungen und neue Erlebnisse verbinden. Emotionen gelten als lernbar. Die emotionalen Schemata bedürfen einer erzieherischen Grundlegung (2007, 90ff.), entwickeln sich implizit und verändern sich sehr langsam $(2007,132)$.

Die hier vorgestellten Arbeiten sind stark emotionspsychologisch geprägt und stellen die wechselseitige Verbindung von Emotion und Kognition heraus bzw. kritisieren Einseitigkeiten. Sie versuchen die Wirksamkeit menschlicher Emotionen in Bildungsprozessen zu beleuchten und tiefer zu verstehen. Sie liefern wichtige Einsichten in die Fundierung von Erkenntnis- und Bildungsprozessen durch Gefühle. Sie machen deutlich, dass Empfindungen in Bildungs- und Lernprozessen immer im Spiel sind, auch und gerade dann, wenn wir sie nicht thematisieren. Die vorgestellten Arbeiten bieten darüber hinaus viele Anregungen für ein gefühlssensibles und selbstreflexives pädagogisches Handeln. Sie machen aber auch auf Desiderate aufmerksam. Es fehlen immer noch empirische Arbeiten zur Rolle und zum Umgang mit Gefühlen in LehrLernverhältnissen. Über die emotionspsychologische Perspektive hinaus fehlt schließlich auch der Anschluss an den ästhetischen Diskurs. Dort werden Gefühle und Empfindungen nicht im Kontext tiefenpsychologischer Grundmuster reflektiert, sondern als ästhetische Wahrnehmung und Erfahrung von Gegenständen, Beziehungen und Situationen. 


\section{Gegenstand und theoretischer Zugang}

Das Projekt sinnbild greift vor allem zwei Desiderate auf: Es will zum einen einen empirischen Beitrag zur Rolle der Gefühle in erwachsenenpädagogischen Lehr-Lernverhältnissen leisten und zum zweiten die ästhetische Perspektive als eine gesellschaftliche und soziale Perspektive im Unterschied zur psychologischen Perspektive stark machen. Im Mittelpunkt stehen Kursleitende mit ihrem didaktischen Denken über die Rolle der Gefühle und Empfindungen im Lehr-Lernverhältnis. Untersucht wird, in welcher Weise Kursleitende die Gefühle und Empfindungen der Teilnehmenden in ihre Kursplanung und Praxis einbeziehen und welche Erwartungen bzw. welche Wirkungsannahmen sie damit verbinden. Wir untersuchen nicht die Teilnehmenden und ihre emotionalen Befindlichkeiten oder Schemata, auch nicht ihre leiblich-affektiven Handlungen/Reaktionen. Wir untersuchen nicht, ob und wie ästhetische Wahrnehmungen bei den Teilnehmenden stattfinden. Dies wäre sehr interessant, kann aber im Rahmen dieser Untersuchung nicht geleistet werden, weil es eine aufwändige Datenerhebung in den Kursen erfordern würde. Dazu müssten ästhetische Feldforschungen durchgeführt werden. Wir beschränken uns auf die Kursleitenden und wollen mit Hilfe ihrer Sichtweisen einen vertieften Einblick in ihr Denken über die Rolle der Gefühle und Empfindungen der Teilnehmenden in der Seminarpraxis gewinnen. Unser Gegenstand ist somit auch nicht der in Kursen beobachtbare Umgang der Kursleitenden mit Gefühlen im Kurs. Wir erheben ausschließlich die Sichtweisen der Kursleitenden auf ihre Interaktion mit den Teilnehmenden und auf die damit verbundene Rolle der Gefühle und Empfindungen. Auf diese Weise sollen typische Interaktionsweisen und Praktiken identifiziert werden, die Gefühle und Empfindungen der Teilnehmenden bearbeiten.

Wenn hier von den Sichtweisen der Kursleitenden auf Gefühle und Empfindungen die Rede ist, dann ist damit eine bestimmte Vorstellung von Bildungsprozessen und der Rolle der Ästhetik in diesen Bildungsprozessen verbunden. Bildungs- und Lernprozesse werden in dieser Untersuchung als Weltund Selbstverständigungsprozesse der Teilnehmenden verstanden, die sich auf konkrete sachlich-soziale Erkenntnisgegenstände beziehen und eine erweiterte gesellschaftliche Teilhabe zum Ziel haben. Solche Lern- und Bildungsprozesse nehmen in Handlungsproblematiken, Krisen, Irritationen, d.h. immer auch in den damit verbundenen Gefühlen ihren Ausgang. Auch das Ziel dieser Bildungs- und Lernprozesse, die Erweiterung der eigenen gesellschaftlichen Handlungsfähigkeit und Teilhabe, ist mit den Vorstellungen und Empfindungen eines guten Lebens verbunden. Kursleitende etablieren ganz unterschiedlich geformte Lehr-Lernverhältnisse, um solche Bildungsprozesse aufzugreifen, anzustoßen, zu unterstützen usw. Die Beziehungen zwischen den Lernenden und den Kursleitenden sowie der Kursgruppe spielen dabei eine zentrale 
Rolle. Darauf weist Wiltrud Gieseke (2007) hin. Auch dort, wo es auf den ersten Blick nur um Wissensaneignung geht, setzen sich die Lernenden immer zum Bildungsgegenstand, d.h. zum gesellschaftlich produzierten sachlich-sozialen Wissen und zur sozialen Situation im Kurs in ein Verhältnis. Sie prüfen und bewerten für sich sowohl die Beziehungen als auch die Relevanz des bearbeiteten Wissens für ihre eigenen Lebensinteressen und ihren weiteren Bildungsprozess. Die Positionierungen zu diesem neuen Wissen erfolgen auf der Basis biographisch erworbener ästhetischer Erfahrungen. Mit ihren Verständigungsprozessen über die Lern- und Bildungsgegenstände setzen sich die Menschen immer auch mit ihren Gefühlen ins Verhältnis zur Welt: Sie nehmen eine Haltung zu diesem Lern- und Bildungsgegenstand (Ernährung, Hate-Speech, Fremdsprachen usw.) ein und entwickeln ggf. eine neue Position und Perspektive auf ihn - oder auch nicht und lassen es beim Alten. In jedem Falle sind Bildungs- und Transformationsprozesse mit ästhetischen Wahrnehmungen und Empfindungen verbunden.

Bildung als Fortentwicklung des eigenen Welt- und Selbstverhältnisses ist an Vernunft und Urteilsfähigkeit gebunden. Wer urteilen will, kann dies nur von einer bestimmten Position aus tun. Urteilsfähigkeit ist an Positionierungen gebunden, die angeben, was gültig ist, was wie bewertet und empfunden wird. Insofern sind Bildungsprozesse und die Entwicklung von Handlungsfähigkeit an ästhetische Wahrnehmungen und Erfahrungen geknüpft, auf deren Basis es erst zu Positionierungen kommen kann (Ittner/Ludwig 2019). Ohne ästhetisch gewonnene Gefühle und Empfindungen sind keine Bildungsprozesse möglich. Andererseits gilt es aber, Meinungen/Positionierungen in Bildungsprozessen zu überwinden, um Distanz zu schaffen, Perspektiven zu öffnen oder neue zu finden und wieder handlungsfähig zu werden.

Bildungs- und Lernprozesse sind mit zwei unterschiedlichen Erfahrungsmodi verbunden: erstens mit einer Diskrepanzerfahrung und zweitens mit einer ästhetischen Erfahrung. Bei der Diskrepanzerfahrung wird eine sachlichsoziale Diskrepanz in Form einer eingeschränkten Handlungs- und Denkfähigkeit erlebt. Sie ist eine praktische Erfahrung, die sich auf eingeschränkte sachlich-soziale Bedeutungen bezieht. Die Diskrepanzerfahrung bildet den Ausgangspunkt für die Erweiterung des vorhandenen Orientierungs- und Anwendungswissens. Bei der ästhetischen Erfahrung setzen sich die Menschen zum Bildungsgegenstand in ein ästhetisches Verhältnis. Die mit der ästhetischen Erfahrung einhergehende Empfindsamkeit gegenüber dem Lern- und Bildungsgegenstand entscheidet darüber, ob der Lern- und Bildungsprozess, d.h. die Welt- und Selbstverständigung überhaupt begonnen wird, ob der Diskrepanzerfahrung Relevanz zukommt. Die Möglichkeit zur ästhetischen Erfahrung ist so gesehen eine zentrale Voraussetzung für Bildungs- und Transformationsprozesse.

Für den theoretischen Zugang zur Rolle der Gefühle und Empfindungen in Lehr-Lernverhältnissen greifen wir auf den Ästhetikdiskurs zurück, da er 
- die Körperlichkeit und Räumlichkeit betont,

- sich mit dem Bildungsdiskurs verbinden lässt und

- Gefühle in einen gesellschaftlichen Zusammenhang stellt.

Das Modell ästhetischer Wahrnehmung und Erfahrung von Harry Lehmann (2016) erscheint für den Zweck dieser Untersuchung besonders geeignet, weil es die Entwicklung ästhetischer Erfahrenheit als Lernprozess entwirft und Ästhetik ohne normative Festlegung des Schönen und Guten versteht (man muss deshalb nicht alle Voraussetzungen teilen, die Lehmann mit seiner Ästhetiktheorie entwirft). Menschen entwickeln nach Lehmann ihr Empfindungsvermögen lernend im Verlauf ihrer Biographie. Das Empfindungsvermögen ist biographisch und gesellschaftlich gerahmt und an bestimmte Handlungsfelder gebunden (Kleidung, Essen, Getränke, Literatur, Gesellschaftspolitik, pädagogisches Handeln usw.). Die Praxis der Ausprägung von Empfindungswerten ist eine Praxis der Wahrnehmungsvergleiche, die zu immer differenzierteren Empfindungen führen.

Die ästhetische Wahrnehmung ist im Unterschied zur sinnlichen Wahrnehmung keine direkte Beobachtung. Sie entsteht erst aus dem Vergleich zweier oder mehrerer Wahrnehmungen (a.a.O., 21). Während die sinnliche Wahrnehmung erster Ordnung eine direkte Beobachtung ist und mittels der Sinne Objekte unterscheidet (also beispielsweise einen Walzer vom Tango, die Farbe Rot von der Farbe Blau), unterscheidet die ästhetische Wahrnehmung zweiter Ordnung die Empfindungen von Wahrnehmungen. Eine wahrgenommene Kursatmosphäre wird mit einer anderen Wahrnehmung verglichen und im Rahmen eines Erfahrungsfeldes ,Kursatmosphären' bewertet. Mit der ästhetischen Wahrnehmung werden wahrgenommene Empfindungen verglichen und differenziert. Es entstehen ein größeres Unterscheidungsvermögen in der Selbstwahrnehmung und eine erweiterte Achtsamkeit für die eigenen Empfindungen. „Man könnte auch sagen, dass der ästhetisch Wahrnehmende eine Selbstbeobachtung durchführt, denn letztendlich dient ihm die Differenzwahrnehmung in der äußeren Welt dazu, eine Differenz in sich selbst aufzuspüren: Er erlebt, dass er zwei ähnliche Wahrnehmungseindrücke unterschiedlich empfindet" (a.a.O., 26). Lehmann versteht die Anhäufung von Wahrnehmungsvergleichen als einen Lernprozess, in dessen Verlauf ein relationales Modell ästhetischer Erfahrung entsteht. Es lässt sich mit einem Koordinatensystem vergleichen, in dem die Enden der verschiedenen Vektoren positiv und negativ bewertet sind. Es stellt eine ästhetische Erfahrenheit dar, vor deren Hintergrund ästhetisch wahrgenommen wird. Dabei sind die Empfindungen als Ergebnis der ästhetischen Wahrnehmungen und Erfahrungen immer unmittelbar mit den praktischen gesellschaftlichen Feldern verknüpft, d.h. ein Weinkenner muss kein Kenner gesellschaftspolitischer Modelle sein. Lehmann weist damit darauf hin, dass ästhetische Erfahrungen die Kehrseite der praktischen Erfahrungen sind und umgekehrt. 
Das Projekt sinnbild untersucht, ob und wie aus Sicht der Kursleitenden didaktische Settings geplant und beschrieben werden, die ästhetische Wahrnehmungs- und Erfahrungsräume für die Teilnehmenden eröffnen sollen. Anders formuliert: Wie praktizieren und verstehen Kursleitende die Inszenierungen ästhetischer Erfahrungsmöglichkeiten in ihren Kursen, um komplexe Welt- und Selbstverständigungsprozesse nicht nur in sachlich-sozialer Hinsicht, sondern auch hinsichtlich der eigenen Empfindungen zu unterstützen?

\section{Untersuchungsdesign}

Das Projekt sinnbild untersucht Kursleitende aus dem gesamten Spektrum der Erwachsenenbildung, d.h. aus dem Bereich der politischen Bildung, aus Sprachkursen, Gesundheitskursen, Grundbildungskursen, Kursen der betrieblichen und beruflichen Weiterbildung usw. Das sind Kurse, in denen Körperlichkeit und Gefühle mit Blick auf den Gegenstand unterschiedliche Bedeutung besitzen (beispielsweise kommt dem Körper in Gesundheits- oder Ernährungskursen eine andere Bedeutung zu als in Kursen zur Kindererziehung).

Es sind 2 Untersuchungsphasen geplant. Aktuell befinden wir uns in der ersten qualitativen Phase. Zunächst wurde didaktische Ratgeberliteratur aus dem Bereich der Erwachsenenbildung mit Blick auf die dort stattfindende Thematisierung von Emotionen, Gefühlen und Empfindungen ausgewertet. Die Analyse der Dokumente erfolgte in Anlehnung an die qualitative Inhaltsanalyse nach Mayring (2015). Das Material wurde auf die zentralen Inhalte reduziert und strukturiert, so dass Oberkategorien herausgefiltert wurden. Basierend auf dieser Auswertung ist ein Interviewleitfaden für problemzentrierte Interviews entstanden.

Die Fallauswahl soll ein möglichst breites Spektrum an Kursleitenden abdecken. Diese sollen sich hinsichtlich Ausbildung, Erfahrungshintergrund, Alter, Geschlecht, beruflichem Status, allgemeiner und betrieblich/beruflicher Bildung, Kursthemen, Bildungseinrichtungen und der Rolle der ästhetischen Wahrnehmung im Kurs unterscheiden. Diese erste Fallauswahl wird durch ein theoretical sampling fortgesetzt, das durch die ersten Zwischenergebnisse gesteuert wird und möglichst kontrastive Fälle sucht.

Die Kursleitenden werden bundesweit akquiriert. Gegenwärtig liegen 40 Telefoninterviews vor. Ca. 80-100 Interviews sollen insgesamt erhoben und inhaltsanalytisch (Mayring 2015, Kukartz 2012) ausgewertet werden. Ziel dieser Phase ist es, typische didaktische Umgangsweisen mit den Empfindungen der Teilnehmenden und entsprechende Wirkungserwartungen der Kursleiten- 
den zu rekonstruieren. Ästhetische Praktiken, d.h. Praktiken, in denen die ästhetische Wahrnehmung und Erfahrung der Teilnehmenden zum Gegenstand im Kurs gemacht wird, interessieren uns besonders.

Für die zweite Untersuchungsphase ist eine quantitative bundesweite Online-Befragung geplant. Hierzu erstellen wir auf der Grundlage unserer Erkenntnisse aus der ersten Phase einen Online-Fragebogen und wollen repräsentative Ergebnisse zu didaktischen Umgangsweisen mit Gefühlen und Empfindungen in Kursen der Erwachsenenbildung vorlegen.

\section{Vorläufige Ergebnisse}

Im Folgenden werden erste Ergebnisse aus den Interviews vorgestellt. Wir haben dazu das didaktische Handeln der Kursleitenden in zwei Kernaufgaben unterschieden:

1. Arbeitsbündnis mit den Teilnehmenden herstellen, aufrechterhalten und beenden

2. Bearbeitung des Bildungsgegenstandes (z.B. Sprachen, Gesundheit, Politik, Ernährung, Umwelt, Ökonomie)

Die Kursleitenden und die didaktische Ratgeberliteratur nennen für die Umsetzung dieser Aufgaben eine Fülle von Methoden und Praktiken, die aber hier nicht vorgestellt werden. Im Sinne der Fragestellung dieser Untersuchung geht es vielmehr um die exemplarische Darstellung der seitens der Kursleitenden erwarteten Gefühle und Empfindungen der Teilnehmenden sowie ihre Rolle im Kontext der beiden didaktischen Kernaufgaben.

\section{Zu 1: Arbeitsbündnis mit den Teilnehmenden herstellen}

Um was geht es bei dieser didaktischen Aufgabe? Benannt werden in der Literatur und von den Kursleitenden

a) eine positive Atmosphäre schaffen,

b) gute Beziehungen herstellen,

c) auf den Bildungsgegenstand hinführen.

\section{Zu a): eine positive Atmosphäre schaffen}

Im Anschluss an Andreas Rauh lassen sich Atmosphären als Relation zwischen Umgebungsqualitäten und subjektivem Empfinden verstehen (2012, 90f.). Ein 
Kirchenraum hat eine andere Atmosphäre als ein Seminarraum. Menschen sind in Atmosphären, der Gestimmtheit eines Raumes, ergriffen und betroffen. Atmosphären bilden so den Hintergrund, vor dem etwas analytisch wahrgenommen wird. Die Entwicklung einer guten Atmosphäre ist oft das Ziel der Kennenlernphase im Kurs. Die Atmosphäre soll nach dem Verständnis der interviewten Kursleitenden den Hintergrund bilden, damit z.B. Kreativität und Phantasie im Kurs möglich werden, um Perspektivwechsel zu unterstützen oder auch, damit eine wertschätzende Fehlerkultur entsteht. „Wahrnehmung ist qua Spüren eine Erfahrung davon, dass ich selbst da bin und wie ich mich, wo ich bin, befinde" (Rauh 2012, 116). Zu diesem Zweck wird der Kursraum oft durch Bewegung angeeignet und in verschiedenster Weise ausgestaltet. Es sollen positive körperliche Empfindungen möglich und eine positive Gestimmtheit für das gemeinsame Arbeitsbündnis erzeugt werden. Diese Gestimmtheit als relativ distanzlose atmosphärische Wahrnehmung des Kurses wird im weiteren Kursverlauf durch die Kursleitenden selten reflektiert.

\section{Zu b): gute Beziehungen herstellen}

Die didaktische Aufgabe hat hier das Verhältnis von Einzelnen und Gruppe, insbesondere zwischen Kursleitenden und Teilnehmenden zum Gegenstand. Es soll ein Vertrauensverhältnis und offenes Klima, ein Wohlgefühl in der Seminargruppe geschaffen werden. Beispielsweise sollen durch gemeinsam vereinbarte Regeln ein respektvoller Umgang abgesichert, Verbindlichkeit hergestellt und so Störungen vermieden werden. Dieses Verhältnis untereinander ist Voraussetzung für und Ergebnis von Teilnehmenden, die sich selbst als Individuum anerkannt und wertgeschätzt fühlen. D.h., die Teilnehmenden sollen ihre Wünsche, Erwartungen und Empfindungen im Kurs aufgenommen sehen und einen Einblick in die Gemeinsamkeiten und Differenzen im Kurs gewinnen. Es soll ein Team entstehen, in dem sich jede/r soweit anerkannt fühlt, dass sie/er angstfrei Differenzen bearbeiten kann.

Gute Beziehungen verbinden die Kursleitenden mit Wohlbefinden, dem Gefühl der Teilnehmenden, anerkannt zu sein, und mit einem Sicherheitsgefühl. All dies soll Vertrauen aufbauen, Ängste sowie Anspannung reduzieren und schließlich Mut machen, sich den anderen gegenüber zu öffnen, sich näher zu kommen und auch über Facetten der eigenen Person zu sprechen, die üblicherweise nicht öffentlich sind. Gute Beziehungen umfassen auch Zuneigung und ein Zusammengehörigkeitsgefühl, das die Grundlage für Freude an der gemeinsamen Arbeit schafft. Wer sich anerkannt und sicher fühlt, riskiert es auch, seine eigene Position und Haltung zu einem bestimmten Aspekt des Bildungsgegenstandes zu zeigen, d.h. Position gegenüber anderen zu beziehen. 


\section{Zu c): auf den Bildungsgegenstand hinführen}

Hier geht es den Kursleitenden darum, auf den Bildungsgegenstand hinzuführen, die Aufmerksamkeit auf bestimmte Aspekte zu lenken und ggf. die Teilnehmenden für den Gegenstand zu interessieren. Die Orientierung und Hinführung folgt entweder den Zielen der Kursleitenden oder es werden die verschiedenen Interessen und Problemstellungen der Teilnehmenden zur Sprache gebracht. Interesse kann durch ein Betroffenheitsgefühl erzeugt werden. Die unterschiedlichen Gefühle und Empfindungen, welche die Teilnehmenden mit ihren Interessen und Problemstellungen bezüglich des Bildungsgegenstands verbinden, werden oft durch ihre Erzählungen zum Ausdruck gebracht (Fallerzählungen). Auch mit Irritationen wird versucht ein Gefühl der Betroffenheit und damit Interesse für den Gegenstand zu wecken.

\section{Zu 2): Bearbeitung des Bildungsgegenstandes}

Diese didaktische Aufgabe umfasst in der Regel mehrere Teilaufgaben:

a) neues Wissen erschließen oder einführen

b) neues Wissen mit vorhandenem vergleichen und kritisch reflektieren

c) Positionierungsmöglichkeiten zum neuen Wissen schaffen

d) Praxistransfer herstellen

Im Folgenden wird ein Beispiel zu c) gegeben. Positionierung beschreibt das Ins-Verhältnis-setzen zum Bildungsgegenstand, eine Haltung, die immer eine Bewertung umfasst. Ohne Positionierung und das durch sie erzeugte Verhältnis zum Bildungsgegenstand kommt kein Bildungsprozess zustande. Wer sich nicht ins Verhältnis setzt, bringt die Bedeutungslosigkeit des Gegenstandes für sie*ihn zum Ausdruck, misst ihm einen geringen Wert zu. Trotz der Relevanz dieser Bewertung wird die Bearbeitung des Bildungsgegenstandes häufig der sogenannten Sacharbeit zugerechnet, in der Gefühle keinen Platz haben sollen. Kursleitende thematisieren im Kontext inhaltlicher Arbeit selten Gefühle und Empfindungen der Teilnehmenden. Teilnehmende positionieren sich aber immer zu den Bildungsgegenständen - und dies nicht nur im Feld der politischen Bildung. Man kann sich nicht nicht positionieren.

Es gibt Fälle, in denen sich Teilnehmende gegen die Kursleitenden positionieren. So geschieht es z.B. in einer pädagogischen Weiterbildung zu digitalen Medien. Technikskeptische Teilnehmende bringen ihre Position argumentativ zum Ausdruck und sehen zugleich den Kursleiter, der die Technik befürwortet, als argumentativen Gegenpart. Der Kursleiter fühlt sich in dieser Situation angegriffen und interpretiert die Positionierung der Teilnehmenden als Widerstand und nicht als Ausdruck einer Empfindungen gegenüber dem Bil- 
dungsgegenstand. Es folgt in der Regel ein Störungsmanagement, das die Störenden zurückdrängt und zugleich die anderen Teilnehmenden und ihre sehr verschiedenen Positionen und Perspektiven auf den Bildungsgegenstand nicht zur Sprache kommen lässt. Ein inhaltliches Problem wird so durch die Ausklammerung der Empfindungsseite zu einem persönlichen Problem der Widerstandleistenden umgedeutet.

\section{Schlussfolgerungen und Perspektiven}

Bildungsprozesse sind mit ästhetischer Wahrnehmung und Erfahrung, d.h. mit Gefühlen und Empfindungen untrennbar verbunden. Betrachtet man die vorliegenden 40 Interviews, dann ist auffällig, dass Gefühle und Empfindungen vor allem im Kontext des Arbeitsbündnisses thematisiert werden. Bei der Bearbeitung des Bildungsgegenstandes werden dagegen Gefühle und Empfindungen selten explizit thematisiert. Um auszuschließen, dass diese Beobachtung dem Datenmaterial oder der Interpretationsweise geschuldet ist, werden bei der weiteren Datenerhebung und -auswertung kontrastierende Kurstypen erhoben. Typisch ist auch, dass dort, wo die Kursleitenden explizit Wert auf ästhetische Wahrnehmungen legen (z.B. bei der Herstellung des Arbeitsbündnisses), die damit verbundenen Gefühle und Empfindungen selten zur Sprache gebracht und reflektiert werden. Sie wirken als eine implizite Wohlfühlfolie.

Das Projekt sinnbild will den systematischen Ort der Gefühle und Empfindungen für die Unterstützung von Bildungs- und Transformationsprozessen ausleuchten. Es geht darum, kognitiv-rationale Einseitigkeiten im didaktischen Diskurs zu überwinden und für die zentrale Rolle der Gefühle und Empfindungen in Transformationsprozessen zu sensibilisieren. Darauf sollte vor allem das Beispiel mit den Positionierungsmöglichkeiten gegenüber neuem Wissen hinweisen. Der Zugang über die Sichtweisen der Kursleitenden kann nur eingeschränkte Erkenntnisse zum Ergebnis haben. Sie sind kein Ersatz für ästhetische Feldforschungen, welche die ästhetischen Wahrnehmungen der Teilnehmenden zum Gegenstand haben könnten. Ein tieferes Verständnis der Rolle von Gefühlen und Empfindungen im didaktischen Handeln der Kursleitenden ist aber eine wichtige Voraussetzung für quantitative Erhebungen, die dann repräsentative Aussagen über den Umfang didaktisch-ästhetischer Praktiken erlauben. Schließlich sind die im Projekt sinnbild gesammelten Sichtweisen der Kursleitenden auf Gefühle und Empfindungen hilfreiches Material und Grundlage für pädagogische Aus- und Weiterbildungen. 


\section{Literatur}

Allespach, Martin; Meyer, Hilbert; Wentzel, Lothar (2009): Politische Erwachsenenbildung. Ein subjektwissenschaftlicher Zugang am Beispiel der Gewerkschaften. Marburg: Schüren.

Arnold, Rolf (2005): Die emotionale Konstruktion von Wirklichkeit. Beiträge zu einer emotionspädagogischen Erwachsenenbildung. Baltmannsweiler: Schneider.

Becker, Georg E.; Clemens-Lodde, Beate; Köhl, Karl (1981): Unterrichtssituationen. Ein Trainingsbuch für Lehrer und Ausbilder. 2. Aufl. München/Wien: Urban \& Schwarzenberg.

Brauer, Juliane (2018): Fühlen und Lernen. Ein Blick auf Emotionen im Klassenzimmer und darüber hinaus. In: Emotionen. Journal für politische Bildung 8 (2), S. 24-28.

Gieseke, Wiltrud (2007): Lebenslanges Lernen und Emotionen. Wirkungen von Emotionen auf Bildungsprozesse aus beziehungstheoretischer Perspektive. Bielefeld: Bertelsmann.

Holzapfel, Günther (1982): Erfahrungsorientiertes Lernen mit Erwachsenen. München/Wien: Urban \& Schwarzenberg.

Holzapfel, Günther (2002): Leib, Einbildungskraft, Bildung. Nordwestpassagen zwischen Leib, Emotion und Kognition. Bad Heilbrunn: Klinkhardt.

Holzapfel, Günther (2004): Nordwestpassagen zwischen Leib, Emotion und Kognition in der Pädagogik. Zur Einheit und Differenz von Wahrnehmen, Fühlen, Denken und Einbildungskräften in Lern- und Bildungsprozessen. In: Report (1), S. 157-163.

Huber, Matthias; Krause, Sabine (Hg.) (2018): Bildung und Emotion. Wiesbaden: Springer VS.

Ittner, Helmut; Ludwig, Joachim (2018): Bedeutungs-Begründungs-Zusammenhänge des künstlerisch-pädagogischen Handelns. In: Joachim Ludwig und Helmut Ittner (Hg.): Forschung zum pädagogisch-künstlerischen Wissen und Handeln. Pädagogische Weiterbildung für Kunst- und Kulturschaffende, Band 2 Forschung. Wiesbaden: Springer VS.

Kuckartz, Udo (2012). Qualitative Inhaltsanalyse. Methoden, Praxis, Computerunterstützung. Weinheim/Basel: Beltz Juventa

Lehmann, Harry (2016): Gehaltsästhetik. Paderborn: Wilhelm Fink Verlag.

Mayring, Philipp (2015). Qualitative Inhaltsanalyse. Grundlagen und Techniken. Weinheim/Basel: Beltz Juventa.

Nuissl, Ekkehard; Siebert, Horst (2013): Lehren an der VHS. Ein Leitfaden für Kursleitende. Bielefeld: Bertelsmann.

Rauh, Andreas (2012): Die besondere Atmosphäre. Bielefeld: transcript.

Reckwitz, Andreas (2015): Ästhetik und Gesellschaft - ein analytischer Bezugsrahmen. In: Andreas Reckwitz (Hg.): Ästhetik und Gesellschaft. Grundlagentexte aus Soziologie und Kulturwissenschaften. Berlin: Suhrkamp, S. 13-54. 
Szepansky, Wolf P. (2010): Souverän Seminare leiten. 2. Aufl. Bielefeld: Bertelsmann.

Welsch, Wolfgang (2010): Ästhetisches Denken. 7. Aufl. Stuttgart: Reclam. 
Tim Stanik, Lisa Marie Fritsch

\section{Theoretische Begründungen und empirische Erfassbarkeit von Teilnehmerorientierung in der institutionalisierten Erwachsenenbildung}

\section{Einleitung}

Wenn die Rolle der Erwachsenenbildung im Kontext aktueller gesellschaftlicher Veränderungsprozesse zur Sprache kommt, wird nicht nur im Call zu dieser Jahrestagung, sondern auch im bildungspolitischen Diskurs reflexartig auf das Konzept des Lebenslangen Lernens rekurriert. Dabei wird insbesondere die Notwendigkeit von informellen, selbstgesteuerten Lernprozessen betont, wenngleich über 50 Prozent der deutschen Bevölkerung im Jahr 2016 an institutionellen Weiterbildungsmaßnahmen teilgenommen haben (vgl. Bilger \& Strauß 2017: 32). Diese Beteiligungsquote soll mit dem jüngst verabschiedeten Qualifizierungschancengesetz gestärkt bzw. erhöht werden. Jenseits der durch das Gesetz geregelten Finanzierungen von und der Beratungen über Weiterbildungsmaßnahmen stellt sich auch die Frage, wie sowohl die Weiterbildungseinrichtungen makro- und mesodidaktisch als auch die Lehrenden mikrodidaktisch in ihren Kursen, Seminaren, Trainings etc. den aktuellen gesellschaftlichen Herausforderungen begegnen können. Dabei scheint es unstrittig zu sein, dass beispielsweise Destandardisierungen von (Erwerbs-)Biografien, die Individualisierung sowie die vielschichtigen und häufig ungewissen Kompetenzanforderungen eine verstärkte Berücksichtigung der jeweiligen Lebenslagen, Lerninteressen, Lernvoraussetzungen etc. der Teilnehmenden erfordern. Hierauf hatte und hat die Erwachsenenbildung mit ihrem Leitprinzip der Teilnehmerorientierung (TNO) eine - wenngleich vornehmlich präskriptive - didaktische Antwort.

Der vorliegende Beitrag beschäftigt sich einerseits mit der empirischen Erfassbarkeit der Umsetzung des Prinzips der TNO und andererseits mit dessen Relevanz im Hinblick auf gesellschaftliche Transformationsprozesse. Zunächst werden traditionelle Begründungslinien und aktuelle Anschlussmöglichkeiten von TNO skizziert sowie Befunde des Forschungsstandes aufgearbeitet. Daraufhin wird die mittlerweile 35 Jahre alte Forderung aufgegriffen, dass TNO nicht nur auf der Ebene des Wollens und des Wünschens zu diskutieren, sondern als etwas Beobachtetes und Beobachtbares zu reflektieren sei (vgl. Tietgens 1983: 67). Hierzu wurde in einem mehrstufigen Prozess ein Beobachtungsinstrument zur hoch-inferenten Einschätzung der Umsetzung von 
TNO in Kursen der Erwachsenenbildung entwickelt. Anhand eines Analysebeispiels, in dem die Ratings eines Kurses den Selbstbeschreibungen der Kursleiterin gegenübergestellt werden, werden Grenzen und Herausforderungen einer ausschließlichen hoch-inferenten Erfassung von TNO diskutiert. Abgeschlossen wird der Beitrag mit einem Ausblick, in dem empirische Anschlussmöglichkeiten aufgezeigt werden.

\section{Gegenstandstheoretische Annäherungen an TNO}

Erste explizite Ansätze von TNO im deutschsprachigen Raum finden sich bereits in der neuen Richtung der Weimarer Republik, in der die konkreten Lebenserfahrungen und Interessen der Teilnehmenden zentrale Ansatzpunkte der Lernprozesse werden sollten (vgl. Olbrich 2001: 202). Nach dieser ersten eher praktischen Auseinandersetzung setzte Ende der 1970er Jahre ein breiter theoretisierender Diskurs um TNO ein, wobei das Thema in den letzten Dekaden wieder in den Hintergrund gerückt ist. So weist z. B. die Literaturdatenbank Fis-Bildung für die Jahre 2012 bis 2017 lediglich 24 Treffer unter dem Schlagwort der TNO auf, während für die Jahre 1980 bis 1982 allein 75 Artikel unter dem Begriff verschlagwortet wurden.

In Anschluss an Tietgens (1980: 216) bedeutet TNO grundlegend, eine Passung zwischen objektiven Lernanforderungen und individuellen Voraussetzungen der Teilnehmer*innen herzustellen, wobei TNO nicht als einmalige Lernzieldiskussion am Kursanfang zu realisieren (vgl. Siebert 1981: 126), sondern als ein die Veranstaltungen durchlaufendes didaktisch-methodisches Prinzip zu verstehen sei (vgl. Holm 2012: 6). So umfasse TNO eine an den Teilnehmenden ausgerichtete Kursplanung, -gestaltung und -evaluation (vgl. Breloer 1980: 32).

\section{Traditionelle Begründungen von TNO}

Oben sind bereits die beiden traditionellen Begründungslinien von TNO angesprochen. So dient TNO zur Selbstvergewisserung der Erwachsenenbildung, um sich gegenüber anderen Bildungsbereichen wie Schule oder Hochschule abzugrenzen (vgl. Schrader 2010). Das Leitprinzip fungiert dabei als programmatische Orientierung (vgl. Fuhr 2001), es wird als Grundlage für eine professionelle Ethik der Erwachsenenbildung entworfen (vgl. Arnold 1991: 22) oder 
als Fundament für pädagogisches Handeln von Erwachsenenbildner*innen gesehen (vgl. Peters 1991: 99). Außerdem kann TNO nach Armutat (1996) zur (bildungstheoretischen) Selbstvergewisserung der Erwachsenenbildung insofern einen Beitrag leisten, als durch sie Lebenswelten und Sichtweisen der Teilnehmenden zum Ausgangspunkt von institutionalisierten Lernprozessen gemacht werden.

Hieran schließt die zweite Begründungslinie an, die TNO als didaktischmethodisches Prinzip zur Planung und Gestaltung von erwachsenengerechten Lehr-/Lernangeboten betrachtet. TNO umfasst in dieser Perspektive eine an den Teilnehmenden ausgerichtete Auswahl von Lerninhalten, einen teilnehmerorientierten Methodeneinsatz sowie eine Beteiligung der Lernenden an allen didaktischen Entscheidungen. Im Hinblick auf eine teilnehmerorientierte Aufbereitung von Lerninhalten gehe es z.B. darum, Inhalte mithilfe didaktischer Reduktionen und Rekonstruktionen so aufzubereiten, dass Passungen zu den Lernvoraussetzungen der Teilnehmenden hergestellt (vgl. Tietgens 1980: 219) und mit ihren Erfahrungen in Bezug gebracht werden (vgl. Holm 2012: 11). Außerdem sollten die eingesetzten Lehr-/Lernmethoden sich an Lebenswelten und an vorhandenen Interaktionsmustern der Teilnehmenden orientieren (vgl. Breloer 1980: 58) sowie hinreichend abwechslungsreich sein, um ihre Aufmerksamkeit zu gewinnen bzw. aufrecht zu erhalten (vgl. Tietgens \& Weinberg 1975: 238).

Aufgrund der konzeptionellen Unschärfe von TNO wird jedoch auch kritisch hinterfragt, inwiefern das Prinzip überhaupt ein handlungsleitender Ansatz für die Erwachsenenbildung sein kann (vgl. Faulstich \& Zeuner 1999: $100)$ oder ob es nicht aufgrund der zuweilen sehr heterogenen Teilnehmenden zu Überforderungen bei den Kursleitenden führe (vgl. Kade 1989a: 326). Nittel (1997: 182) hebt dagegen in Bezug auf die Uneindeutigkeit von TNO gerade das Potenzial des Prinzips hervor. So sei TNO keine alltagsweltliche Begrifflichkeit, sondern könne als ein Äquivalent zum Begriff des Klient*innenbezugs anderer Professionen betrachtet werden, der gerade aufgrund seiner Unschärfe zu einer Selbstverständigung der professionellen Berufskultur der Erwachsenenbildung auffordere.

\section{Aktuelle Relevanz von TNO}

In Bezug auf das Thema dieser Sektionstagung kann TNO auch als potenzielle didaktische Antwort auf Fragen, die sich im Kontext der aktuellen gesellschaftlichen Veränderungsprozesse stellen, betrachtet werden. So erfordern beispielsweise diskontinuierliche (Erwerbs-)Biografien, die sich immer weiter in- 
dividualisierenden Lebensentwürfe, die vielschichtigen und häufig ungewissen Kompetenzanforderungen aufgrund von Digitalisierung und Globalisierung einerseits die Gestaltung von reflexiven und andererseits von zieloffenen Lehr-/Lernprozessen in der institutionalisierten Erwachsenenbildung.

In diesem Zusammenhang werden transformative Bildungs- und Lernprozesse zentral, da tradierte Bedeutungsstrukturen irritiert werden und sich zunehmend als dysfunktional erweisen können. Oder, um es in direkter Anlehnung an Mezirow (1994) auszudrücken, es bedarf einer Transformation der „habits of minds“ (Mezirow 1994: 223). Kurse der Erwachsenenbildung können dafür geeignete Lernorte sein, an denen Teilnehmende ihre unhinterfragten Annahmen gemeinsam reflektieren und ggf. auch transformieren, um sich neue Handlungsoptionen zu erschließen. So bedeutet TNO bereits bei Tietgens (1980: 208ff.), vorhandene Deutungsmuster der Teilnehmenden zwar zur Kenntnis zu nehmen, diese aber nicht einfach zu verlängern, sondern sie selbst zu (Lern-)Gegenständen der Kurse werden zu lassen.

Eine weitere Anschlussmöglichkeit von TNO liegt in den kaum mehr vorhersehbaren Kompetenzanforderungen, die insbesondere zieloffene Lernprozesse notwendig werden lassen. Zieloffene Lernprozesse sind dadurch gekennzeichnet, dass den Teilnehmenden mehrere Lernzieloptionen eröffnet und diese pädagogisch begleitet werden, aber durch die Teilnehmenden auf individuellen Lernwegen selbst zu erschließen sind (vgl. Schäffter 2001: 23f.). Auch hier sind die Anschlussmöglichkeiten zum Prinzip der TNO offensichtlich. Gerade wenn Lernziele nicht mehr von außen festzulegen und Zugangswege zu diesen mit den Teilnehmenden in Suchbewegungen erst zu erschließen sind, stellt das Prinzip der TNO eine potentielle didaktische Antwort dar.

Unter diesen Perspektiven scheint das Prinzip relevanter denn je, wenngleich nur wenige Erkenntnisse darüber vorliegen, inwiefern Kurse der Erwachsenenbildung teilnehmerorientiert geplant und gestaltet werden.

\section{Empirische Befunde zur TNO}

Befunde der unmittelbaren Lehr-/Lernforschung zeigten, dass z.B. Kurse an (Heim-) Volkshochschulen in den 1970er Jahren ,überwiegend stofforientiert“ waren und die Kursleitenden fast so viele Redebeiträge wie alle Teilnehmenden zusammen aufwiesen (vgl. Siebert 1975: 47). Befragungen von Lehrenden als das dominierende Format einer mittelbaren Lehr-/Lernforschung in der Erwachsenenbildung kommen zu den Ergebnissen, dass TNO für Kursleitende wenn überhaupt eine randständige Kategorie ist und insbesondere im Kontext der didaktisch-methodischen Planungen thematisiert wird (vgl. Hof 2001: 89). Dass TNO insbesondere auf methodische Entscheidungen reduziert wird, 
Teilnehmerorientierung und institutionalisierte Erwachsenenbildung 345

konnte durch eine weitere Interviewstudie belegt werden (vgl. Luchte 2001: 156). Zieht man die Studien von Kade (1989b) und Bastian (1997) heran, wird deutlich, dass das Handeln von Kursleitenden offensichtlich weniger an ihren Teilnehmenden orientiert als vielmehr durch die (berufs-)biografische Bedeutung der Lehrtätigkeit und/oder das fachbezogene Selbstverständnis geprägt war.

Befragungen von Teilnehmenden konnten zeigen, dass Beteiligungsmöglichkeiten der Lernenden über methodisch-didaktisch Entscheidungen angenommen und positiv beurteilt werden (vgl. Kejcz u.a. 1981: 121f.; Packebusch 1987: 145). Eine Befragung von Teilnehmenden von Integrationskursen ergab, dass diese die Kurse als teilnehmerorientiert wahrnehmen, da sie sich maßgeblich von ihren schulischen Erfahrungen in ihren Herkunftsländern unterscheiden (z.B. egalitärer Umgang zwischen Lehrenden und Lernenden, die Aufforderungen und Möglichkeiten der aktiven Beteiligung) (vgl. Zimmer 2013: 120ff.).

Einschränkend muss hierzu angemerkt werden, dass sowohl habituelle Milieuzugehörigkeiten (vgl. Barz \& Tippelt 2004) als auch kognitive Lerntypen (vgl. Schrader 2008) darauf Einfluss haben können, was von Lernenden als teilnehmerorientiert angesehen wird.

\section{Entwicklung eines hoch-inferenten Beobachtungsinstruments zur Erfassung von TNO}

Wenngleich ein breiter theoretischer Diskurs und einige empirische Befunde zur TNO vorliegen, existiert bislang noch kein empirisches Verfahren zur systematischen Erfassung des Prinzips in den Kursen. In Anlehnung an die schulische Unterrichtsforschung wurde daher ein hoch-inferentes Ratingverfahren entwickelt, um TNO in Kursen der Erwachsenenbildung durch externe Beobachter*innen erfassen bzw. einschätzen zu können. Das Instrument entstand in einem mehrstufigen Entwicklungs- und Erprobungsprozess. Zuerst wurden auf der Grundlage einer Literaturrecherche aus theoretischen Beiträgen, didaktischen Handreichungen und empirischen Untersuchungen zur TNO potentiell beobachtbare Kriterien des Prinzips ermittelt. Diese wurden in einem zweiten Schritt in fünf Dimensionen mit je drei Beobachtungsitems überführt und in Anlehnung an die schulische Unterrichtsforschung mit Hilfe einer vierstufigen Likert-Skala abgestuft. Die Dimensionen beziehen sich u.a. auf die Lehr/Lernmethoden und auf die Auswahl der Lerninhalte. Mit Hilfe weiterer Items wird eingeschätzt, inwieweit den Teilnehmenden alle didaktischen Entschei- 
dungen transparent gemacht werden und ob ihnen kontinuierlich die Möglichkeit gegeben wird, sich daran zu beteiligen. Eine letzte Beobachtungsdimension bezieht sich auf Einschätzungen, inwiefern Lernergebnisse und Lernprozesse reflektiert bzw. inwiefern Lernschwierigkeiten zum Gegenstand der Kurse gemacht werden (vgl. Tab. 1).

Das vorläufige Beobachtungsinstrument und das zugehörige Kodiermanual sind in einem dritten Schritt von Expert*innen aus Wissenschaft und Praxis kommunikativ validiert und daraufhin überarbeitet worden. In Anlehnung an Untersuchungen zur Bedeutungsüberschneidung von Beschreibungskategorien bei Ratingverfahren (vgl. Beck 1980) und zum Rater-Bias (vgl. Praetorius 2014) ist das Instrument in einem vierten Schritt nochmals empirisch weiterentwickelt worden. Anhand einer 25-minütigen Kurssequenz wurde der Ratingbogen von Studierenden der Erwachsenenbildung erprobt und bewertet. Dabei wurden sowohl die Verständlichkeit als auch die Beobachtbarkeit der einzelnen Items mit Hilfe einer 6-stufigen Likert-Skala (von 6=sehr leicht/gut bis $1=$ sehr schwer/schlecht verständlich bzw. beobachtbar) eingeschätzt. Außerdem wurden die Probanden dazu befragt, inwiefern sie für die Ratings der einzelnen Items auf das Kodiermanual zurückgegriffen haben.

Insgesamt wurde die Verständlichkeit der Items höher eingeschätzt als deren Beobachtbarkeit (vgl. Tab. 1), wobei die (Nicht-)Nutzung des Manuals keinen Einfluss auf diese Bewertungen hatte. Auf Basis dieser Testratings wurden die Items mit einer niedrig eingeschätzten Beobachtbarkeit (unter 3,5 im Mittel) bzw. Items mit einer niedrig eingeschätzten Verständlichkeit (unter 5,0 im Mittel) samt der entsprechenden Abschnitte im Manual überarbeitet (siehe die hervorgehobenen Items 1.3, 4.2, 5.2, 5.3).

Obwohl mit dem so überarbeiteten Instrument in ersten Ratings $(n=8$ Kurse) durch die beiden Autor*innen eine zufriedenstellende Inter-Rater-Reliabilität erzielt werden konnte, werden hier bereits generelle Herausforderungen und Grenzen einer ausschließlichen hoch-inferenten Erfassung von Sichtstrukturen der TNO deutlich, insbesondere wenn die Ratings mit den Perspektiven der Lehrenden der untersuchten Kurse trianguliert werden.

In der Folge werden diese Herausforderungen anhand eines Analysebeispiels illustriert und es werden methodische Überlegungen zu deren Überwindung zur Diskussion gestellt. 
Teilnehmerorientierung und institutionalisierte Erwachsenenbildung 347 Tabelle 1: Verständlichkeit und Beobachtbarkeit der Items im Mittelwert, $n=31$ (Quelle: eigene Berechnungen, Darstellung)

\begin{tabular}{|c|c|c|c|}
\hline Nr. & Item & $\begin{array}{l}\text { Verständ- } \\
\text { lichkeit (M) }\end{array}$ & $\begin{array}{l}\text { Beobacht- } \\
\text { barkeit (M) }\end{array}$ \\
\hline \multicolumn{4}{|c|}{ 1. Lehr-/Lernmethoden ... } \\
\hline 1.1 & fördern die Aufmerksamkeit der TN & 5,13 & 4,35 \\
\hline 1.2 & $\begin{array}{l}\text { unterstützen die aktive Auseinander- } \\
\text { setzung mit den Lerninhalten der TN }\end{array}$ & 5,23 & 4,42 \\
\hline 1.3 & $\begin{array}{l}\text { Lehr-/Lernmethoden fördern } \\
\text { selbstgesteuerte Auseinandersetzung } \\
\text { mit den Lerninhalten }\end{array}$ & 4,32 & 3,84 \\
\hline \multicolumn{4}{|c|}{ 2. Lerninhalte ... } \\
\hline 2.1 & $\begin{array}{l}\text { werden mit Erfahrungen/Vorwissen } \\
\text { der TN verknüpft }\end{array}$ & 5,52 & 3,87 \\
\hline 2.2 & $\begin{array}{l}\text { beziehen sich auf potenzielle } \\
\text { Verwendungssituationen der TN }\end{array}$ & 5,42 & 4,74 \\
\hline 2.3 & $\begin{array}{l}\text { können mit Hilfe von Materialien } \\
\text { außerhalb des Kurses vertieft werden }\end{array}$ & 5,45 & 3,68 \\
\hline \multicolumn{4}{|c|}{ 3. Transparenz ... } \\
\hline 3.1 & über Lehr-/Lernziele wird hergestellt & 5,42 & 4,23 \\
\hline 3.2 & über Lerninhalte wird hergestellt & 5,61 & 5,10 \\
\hline 3.3 & $\begin{array}{l}\text { über Lehr-/Lernmethoden wird } \\
\text { hergestellt }\end{array}$ & 5,32 & 4,35 \\
\hline \multicolumn{4}{|c|}{ 4. Partizipation ... } \\
\hline 4.1 & über Lerninhalte wird ermöglicht & 5,10 & 3,77 \\
\hline 4.2 & über Lehr-/Lernziele wird ermöglicht & 5,13 & 3,39 \\
\hline 4.3 & $\begin{array}{l}\text { über Lehr-/Lernmethoden wird } \\
\text { ermöglicht }\end{array}$ & 5,68 & 4,00 \\
\hline \multicolumn{4}{|c|}{ 5. Lernreflexion und Lernberatung } \\
\hline 5.1 & Lernergebnisse werden thematisiert & 5,26 & 4,55 \\
\hline 5.2 & Lernprozesse werden reflektiert & 4,74 & 3,48 \\
\hline 5.3 & $\begin{array}{l}\text { Lernschwierigkeiten werden zum } \\
\text { Gegenstand des Kurses gemacht }\end{array}$ & 4,32 & 2,81 \\
\hline
\end{tabular}




\section{Herausforderungen der hoch-inferenten Erfassung von TNO - ein Analysebeispiel}

Die folgenden Darstellungen beziehen sich auf einen Englisch-Konversationskurs, der sich laut Kursankündigung an ,junge Senior*innen“ richtet, die ihre vorhandenen Fremdsprachenkenntnisse vertiefen möchten und auf deren Wünsche eingegangen werden soll. Die Kursleiterin (KL) ist ein Native Speaker, die nach eigenen Angaben besonderen Wert auf ein gutes Verhältnis zu ihren Teilnehmerinnen legt (KL, Z. 148-160).

Bei der aufgezeichneten bzw. gerateten Kurssitzung waren sechs Teilnehmerinnen anwesend. Die Sitzung wurde durch die KL interaktiv gestaltet, indem z.B. in mehreren Kursgesprächen Vokabeln gemeinsam erarbeitet wurden und unterschiedliche spielerische Übungen zum Einsatz kamen, die die TN zum Sprechen anregten. So ist es auch nicht überraschend, dass der Methodeneinsatz im Hinblick auf TNO hoch geratet wird (die entsprechenden Items erzielen die höchsten Ausprägungen), wohingegen die Items, mit deren Hilfe die Transparenzherstellung und Partizipationsmöglichkeiten erfasst werden, niedrig geratet werden. Dies ist insofern auffällig, als der Kursankündigungstext ein anderes Ergebnis erwarten ließ. Konfrontiert man nun die Ratingergebnisse mit den Aussagen der KL zu der Kurssitzung, die im Anschluss mit Hilfe eines Stimulated-recall-Interviews erhoben wurden, besteht eine Kongruenz zwischen den Selbsteinschätzungen der KL und den Fremdeinschätzungen der Rater. So wurde z.B. das Item „Lernmethoden regen zu einer aktiven Auseinandersetzung mit den Lerninhalten an" mit der höchsten Ausprägung im Rating eingeschätzt. Die KL gibt im Interview an, dass es ihr besonders wichtig sei, mit den Teilnehmenden einen alltagsweltlichen Wortschatz aufzubauen, damit diese sowohl in der Kurssituation als auch bei potenziellen Auslandsaufenthalten keine Hemmungen haben, sich in der Fremdsprache zu verständigen (vgl. KL, Z. 86-92).

Dass die niedrig bewertete Herstellung von Transparenz über das didaktisch-methodische Vorgehen auch von der KL als nicht notwendig erachtet wird, zeigt das folgende Interviewzitat: „Ich denke, die die merken, dass mein Ziel ist, dass sie sich einfach trauen die Vokabeln zu sagen. Ich glaube, dass das ganz klar ist" (KL, Z. 139). An diesem Beispiel werden Grenzen einer ausschließlichen externen Einschätzung der Umsetzung von TNO auf Basis der Sichtstrukturen schnell offensichtlich, da nicht überprüft werden kann, ob den Teilnehmerinnen die Lernziele tatsächlich hinreichend transparent sind und ob diese auch ihren Erwartungen entsprechen. Um dies zu klären, bedarf es ergänzender Befragungen der Teilnehmer*innen. Eine solche Perspektivenverschränkung lässt sich ferner damit begründen, dass zwischen den Ratings anderer Kurse und den Selbsteinschätzungen dieser Kursleiterin Diskre- 
panzen festzustellen sind. So berichtet z.B. die Kursleiterin eines Kommunikationskurses, ihre Methoden besonders teilnehmerorientiert einzusetzen, während die Ratings der entsprechenden Items (1.1-1.3) dies nicht abbilden.

Bei dem Analysebeispiel des Englischkurses konnte keine Beteiligung der Teilnehmerinnen an der Festlegung der Lernziele beobachtet werden, weshalb das entsprechende Item mit der geringsten Ausprägung geratet wurde. Im Interview wird jedoch deutlich, dass die KL klare Vorstellungen darüber hat, wofür und in welchen konkreten Kontexten die Teilnehmerinnen ihre Englischkenntnisse in Zukunft anwenden werden: So hätten die Teilnehmerinnen das Interesse, die finanziellen und zeitlichen Ressourcen für Urlaube und viele Restaurantbesuche im Ausland. Daher vermittele sie ihnen ein entsprechendes Fremdsprachenwissen für diese Kontexte (vgl. KL, Z. 86-92). Es wird deutlich, dass TNO immer auch Antizipation ist (vgl. Tietgens 1980) und darum nicht ausschließlich über Sichtstrukturen erfasst und bewertet werden kann. Es scheint daher notwendig, neben Sichtstrukturen und Befragungen der Beteiligten auch das vorbereitende mikrodidaktische Planungshandeln in die Analysen einzubeziehen (vgl. Stanik 2016).

\section{Empirische Anschlussmöglichkeiten}

In weiteren Studien ist außerdem zu prüfen, in welchen Zusammenhängen die Umsetzung von TNO in Kursen der Erwachsenenbildung mit niedrig-inferent zu erfassenden Sichtstrukturen (z.B. Verteilungen von Redebeiträgen, Einsatz von Unterrichtsmedien oder der Wechsel von Arbeits- und Sozialformen) der Kurse stehen. Darüber hinaus ist empirisch zu ermitteln, inwiefern teilnehmerorientiertes Kurshandeln in Zusammenhang mit weiteren empirisch bereits fundierten Qualitätsmerkmalen ,guten Unterrichts“ steht (z.B. effektive Nutzung von Unterrichtszeit oder kognitive Aktivierung der Teilnehmenden) (vgl. Meyer 2004).

Auf Basis einer empirischen Erfassung von TNO wird es möglich, das Prinzip und dessen unterstellte Bedeutung für die Gestaltung (non-)formaler Lernprozesse mit Erwachsenen zu überprüfen. So müssten Wirkungen der (Nicht)Beachtung des Prinzips z.B. auf die Kurszufriedenheit, die Verminderung von Drop-outs oder auf die Einflüsse der Lernergebnisse der Lernenden erfasst werden. Schließlich wäre, wie das obige Fallbeispiel illustriert, systematisch zu untersuchen, inwiefern TNO als Antizipation bei der mikrodidaktischen Kursplanung berücksichtigt und schließlich in der situativen Lehr-/Lerninteraktion realisiert wird. 
Auf Basis solcher triangulativer Analysen wird es möglich, TNO für die unmittelbare Lehr-/Lernforschung weiter nutzbar zu machen (vgl. Schrader 2010: 284) und empirisch zu validieren oder ggf. zu modifizieren.

\section{Literatur}

Armutat, Sascha (1996). Zur unerkannten fundamentalanthropologischen Dimension einer teilnehmerorientierten Erwachsenenbildung. In: GDWZ - Grundlagen der Weiterbildung, H. 3, S. 161-164.

Arnold, Rolf (1991). Ethische und instrumentelle Professionalität in der Erwachsenenbildung. In: Gieseke W. / Meueler, E. / Nuissl, E. (Hg.). Ethische Prinzipien der Erwachsenenbildung. Verantwortlich für was und vor wem?. Mainz: Deutsche Gesellschaft für Erziehungswissenschaft, S. 22-29.

Barz, Heiner/ Tippelt, Rudolf (2004). Weiterbildung und soziale Milieus in Deutschland: Band 1. Praxishandbuch Milieumarketing. Bielefeld: Bertelsmann.

Bastian, Hannelore (1997). Kursleiterprofile und Angebotsqualität. Bad Heilbrunn: Klinkhardt.

Beck, Klaus (1980). Die Bedeutungsüberschneidung von Beschreibungskategorien als Problem der Unterrichtsforschung. Eine methodenkritische Untersuchung am Beispiel des Ratingverfahrens. Forschungsbericht Nr. 6. Otto-Selz-Institut für Psychologie und Erziehungswissenschaft. Universität Mannheim.

Bilger, Frauke / Strauß, Alexandra (2017). Beteiligung an non-formaler Weiterbildung. In: Bilger, F. / Behringer, F. / Kuper, H. / Schrader, J. (H.). Weiterbildungsverhalten in Deutschland 2016. Ergebnisse des Adult Education Survey (AES). Bielefeld: WBV, S. 25-55.

Breloer, Gerhard (1980). Aspekte einer teilnehmerorientierten Didaktik der Erwachsenenbildung. In: Breloer, G. / Dauber, H. / Tietgens, H. (Hg.). Teilnehmerorientierung und Selbststeuerung in der Erwachsenenbildung. Braunschweig: Westermann, S. 8-112.

Faulstich, Peter / Zeuner, Christine (1999). Erwachsenenbildung. Eine handlungsorientierte Einführung. Weinheim: Juventa.

Fuhr, Thomas (1991). Kompetenzen und Ausbildung des Erwachsenenbildners. Eine Studie zur Professionalisierung der Erwachsenenbildung. Bad Heilbrunn: Klinkhardt.

Hof, Christiane (2001). Konzepte des Wissens. Eine empirische Studie zu den wissenstheoretischen Grundlagen des Unterrichtens. Bielefeld: Bertelsmann.

Holm, Ute (2012). Teilnehmerorientierung als didaktisches Prinzip der Erwachsenenbildung - aktuelle Bedeutungsfacetten. Bonn. https://www.die-bonn.de/ doks/2012-teilnehmerorientierung-01.pdf [Zugriff: 10.01.2019]. 
Kade, Jochen (1989a). Kursleiter und die Bildung Erwachsener. Fallstudien zur biographischen Bedeutung der Erwachsenenbildung. Bad Heilbrunn: Klinkhardt.

Kade, Jochen (1989b). Erwachsenenbildung und Identität. Eine empirische Studie zur Aneignung von Bildungsangeboten. Weinheim: Deutscher Studien-Verlag.

Kejcz, Yvonne u.a. (1981). Bildungsurlaub: Angebotsplanung und Teilnehmermotivation. BUVEP -Endbericht Band II (unveränderte Neuauflage). esprint: Heidelberg.

Luchte, Katja (2001). Teilnehmerorientierung in der Praxis der Erwachsenenbildung. Weinheim: Deutscher Studien-Verlag.

Meyer, Hilbert (2004). Was ist guter Unterricht? Berlin: Cornelsen-Scriptor.

Mezirow, Jack (1994). Understanding Transformative Theory. In: Adult Education Quarterly, 44, 4, S. 222-232.

Nittel, Dieter (1997). Teilnehmerorientierung - Kundenorientierung - Desorientierung ...? Votum zugunsten eines ,einheimischen Begriffs“. In: Arnold, R. (Hg.). Qualitätssicherung in der Erwachsenenbildung. Opladen: Leske u. Budrich, S. 163-184.

Olbrich, Josef (2001). Geschichte der Erwachsenenbildung in Deutschland. Opladen: Leske u. Budrich.

Packebusch, Lutz (1987). Teilnehmerorientierung in der betrieblichen Weiterbildung. Entwicklung, Erprobung und Evaluation eines Seminarprogrammes zum kommunikativen Handeln in einem Dienstleistungsunternehmen. Frankfurt a.M.: Peter Lang.

Peters, Roswitha (1991). Erwachsenenbildungs-Ethik und ErwachsenenbildungsProfessionalität - eine zwingende Zusammengehörigkeit?. In: Gieseke, W. / Meueler, E. / Nuissl, E. (Hg.). Ethische Prinzipien der Erwachsenenbildung. Verantwortlich für was und vor wem? Mainz: Deutsche Gesellschaft für Erziehungswissenschaft, S. 99-105.

Praetorius, Anna-Katharina (2014). Messung von Unterrichtsqualität durch Ratings. Münster: Waxmann.

Schäffter, Ottfried (2001). Weiterbildung in der Transformationsgesellschaft: Zur Grundlegung einer Theorie der Institutionalisierung. Hohengehren: Schneider Verlag.

Schrader, Josef (2010). Teilnehmerorientierung. In: Arnold, R. / Nolda, S. / Nuissl, E. (Hg.). Wörterbuch Erwachsenenbildung. 2., überarbeitete Auflage. Bad Heilbrunn: UTB, S. 284-285.

Schrader, Josef (2008). Lerntypen bei Erwachsenen. Empirische Analysen zum Lernen und Lehren in der beruflichen Weiterbildung. 2. Ergänzte Auflage. Bad Heilbrunn: Klinkhardt.

Siebert, Horst (1981). Teilnehmerorientierung als eine didaktische Legitimationsgrundlage. In: Olbrich, J. (Hg.). Legitimationsprobleme in der Erwachsenenbildung. Stuttgart: Kohlhammer, S. 113-133.

Siebert, Horst (1975). Probleme, Ergebnisse und Konsequenzen einer empirischen Untersuchung. In: Siebert, H. / Gerl, H. (Hrsg.): Lehr- und Lernverhalten bei Erwachsenen. Braunschweig: Westermann, S. 13-123. 
Stanik, Tim (2016). Mikrodidaktisches Planungshandeln von Lehrenden in der Erwachsenenbildung - theoretische und empirische Annäherungen an ein Desiderat. In: Zeitschrift für Weiterbildungsforschung, 39,3, S. 317-330. http://www. die-bonn.de/zfw/32016/stanik.pdf [Zugriff: 10.01.2019].

Tietgens, Hans (1983). Teilnehmerorientierung in Vergangenheit und Gegenwart. Bonn: Deutscher Volkshochschul-Verband.

Tietgens, Hans (1980). Teilnehmerorientierung als Antizipation. In: Breloer, G. / Dauber, H. / Tietgens, H. (Hg.). Teilnehmerorientierung und Selbststeuerung in der Erwachsenenbildung. Braunschweig: Westermann, S. 177-235.

Tietgens, Hans / Weinberg, Johannes (1975). Erwachsene im Feld des Lehrens und Lernens. Braunschweig: Westermann.

Zimmer, Veronika (2013). Anspruch und Wirklichkeit von Integrationskursen im Lichte erwachsenenpädagogischer Forschung. Bonn. www.die-bonn.de/doks/ 2013-inklusion-01.pdf [Zugriff: 10.01.2019]. 
Klaus Buddeberg, Christopher Stammer

\section{Schließt der digitale Wandel ältere und gering literalisierte Erwachsene aus?}

\section{Einleitung}

Der Prozess der Digitalisierung beinhaltet vor allem bezogen auf die Arbeitswelt (Carstensen 2016, S. 41) ein erhebliches Potenzial räumlicher und zeitlicher Entgrenzung. Die Kehrseite dieses Prozesses ist das Risiko, dass bestimmte Bevölkerungsgruppen von solchen Entwicklungen abgekoppelt werden (könnten). Die von Bund und Ländern ausgerufene „Nationale Dekade für Alphabetisierung und Grundbildung" verfolgt das Ziel, „die Lese- und Schreibkompetenzen sowie das Grundbildungsniveau Erwachsener in Deutschland“ (BMBF/KMK 2016, S. 4) substanziell zu verbessern. Unter dem Begriff Grundbildung werden dabei auch grundlegende Fähigkeiten im IT-Bereich verstanden, wenn die Bundesregierung beispielsweise „die Vermittlung von digitalen Fähigkeiten als Schlüsselkompetenz für alle Altersgruppen“ (Bundesregierung 2018) fordert.

Kompetenzstudien wie PIAAC (Programme for the International Assessment of Adult Competences) weisen jedoch auf geringe Grundkompetenzen bei größeren Bevölkerungsanteilen hin (OECD 2013). Bezogen auf die „Problemlösekompetenzen in technologiereichen Umgebungen“"zeigt PIAAC für Deutschland, dass mehr als 14 Prozent der Erwachsenen mit ihren Kompetenzwerten nicht einmal die niedrigste Stufe (Level 1) im Bereich technologiebasierten Problemlösens erreichen (Rammstedt/Ackermann 2013).

PIAAC stellt einen deutlichen Zusammenhang zwischen dem Vorhandensein von Grundkompetenzen einerseits und der Nutzung dieser Kompetenzen in Alltagspraktiken andererseits heraus. Je höher die Lesekompetenz ist, desto häufiger nutzen Menschen diese Kompetenz in diversen Situationen des Alltags (Reder 2017; OECD 2013). Demzufolge sollten auch schriftbezogene digitale Alltagspraktiken bei gering literalisierten Erwachsenen hinsichtlich der Arten und der Häufigkeit der Nutzung gegenüber höher Literalisierten eingeschränkt sein. Gleichzeitig lässt sich ein besonders starker Einfluss des Alters auf die Nutzung digitaler Medien erwarten, der möglicherweise sogar wirkmächtiger ist als die digitalen und literalen Grundkompetenzen (SchmidtHertha 2014). 
Mit diesem Beitrag gehen wir der Frage nach, in welchem Umfang Personen mit geringer Literalität und ältere Menschen Informations- und Kommunikationstechnik (IKT) nutzen. Im Detail untersuchen wir, ob allgemeine Aussagen zu diesen beiden Subgruppen zulässig sind oder ob sich, je nach Art der digitalen Praktiken, verschiedene Nutzungsschemata erkennen lassen.

\section{Kontext Grundbildung}

In der spezifischen Fragestellung dieses Beitrags verorten wir die Voraussetzungen für digitales Lernen im Bereich der Grundbildung. Um zu bestimmen, was Grundbildung bedeutet, arbeitet Euringer (2016) einen modularen Grundbildungsbegriff heraus, der neben den Aspekten Zielgruppen, Angebotsformate und Mindestniveaus als zentrales Element eine inhaltliche Beschreibung enthält. Grotlüschen nimmt ebenfalls eine inhaltliche Orientierung vor und benennt in Abgrenzung von dem seit den 1970er Jahren dominanten Begriff der Alphabetisierung mehrere Inhaltsbereiche von Grundbildung, unter anderem die digitale, politische, finanzielle und gesundheitsbezogene Grundbildung (Grotlüschen 2016). Diese Themenbereiche sind auch im Grundsatzpapier von BMBF und KMK zur Nationalen Dekade für Alphabetisierung und Grundbildung präsent (BMBF/KMK 2016), die sich wiederum mit den 2006 von der Europäischen Kommission definierten Schlüsselkompetenzen für lebensbegleitendes Lernen überschneiden (Europäische Kommission 2017).

\section{Kontext Digitalisierung}

Digitale Technologien halten Einzug in diverse Lebensbereiche (Wolf/Koppel 2017) und führen dort zu räumlicher und zeitlicher Entgrenzung. So lässt sich eine Ablösung des klassischen Bankgeschäfts in einer Filiale durch die Abwicklung von Zahlungsvorgängen über das Internet oder durch SmartphoneApps dokumentieren und quantifizieren (Bundesverband deutscher Banken 2017). Der Rückbau personaler Interaktion findet eine Analogie im Bereich des E-Government. Bei Verwaltungsprozessen sind immer mehr Schritte durch Bürgerinnen und Bürger selbst zu erledigen, weil in die Prozesse immer weniger Menschen eingebunden sind (Smythe 2018). Die schrift- und technologiebasierte Abwicklung wird dadurch in zunehmendem Maße alternativlos 
(Wolf /Koppel 2017). Für Personen mit entweder geringer Schriftsprachkompetenz oder mit Schwierigkeiten im Umgang mit digitalen Technologien bzw. beidem kann dies eine systematische Benachteiligung darstellen.

\section{Einflussfaktoren Alter und Literalität}

Die Lese- und Schreibkompetenz wurde als möglicher Bedingungsfaktor für digitale Alltagspraktiken in den bisherigen Studien zur Nutzung digitaler Technologie selten explizit betrachtet. Ausnahmen bilden die LEO - Level-One Studie aus dem Jahr 2011 (Grotlüschen /Riekmann 2012) und die PIAAC-Studie der OECD (Rammstedt /Ackermann 2013). Analysen zum Zusammenhang von Kompetenzstufen und der Häufigkeit der Kompetenznutzung legen grundsätzlich die Annahme nahe, dass mit geringer Lesekompetenz (PIAAC) bzw. geringer Lese- und Schreibkompetenz (LEO) auch eine seltenere Nutzung schriftbezogener digitaler Praktiken einhergeht. Als zusätzliche Variable ziehen wir das Alter heran. Zusammenhänge zwischen Alter und digitaler Aktivität sind in diversen Studien belegt (Koch/Frees 2017), besonders bei der Nutzung mobiler Endgeräte (vgl. Bitkom Research 2018). Laut Wolf und Koppel kann es ,als berechtigte Annahme gelten, dass insbesondere erwachsene Personen mit geringer Lese- und Schreibkompetenz auch weniger Computererfahrung und somit eine geringe Medienkompetenz besitzen“" (2017, S. 4; vgl. Rosenbladt /Bilger 2011).

\section{Alter}

Aus der demografischen Entwicklung ergibt sich eine wachsende Relevanz des Alters als Einflussgröße auf die Nutzung digitaler Technologie. Eine bildungswissenschaftliche Einordnung und eine Klassifizierung des Altersbegriffs leistet Iller (2018). Für die hier vorgelegten Analysen liegen mit den beiden maßgeblichen Datenquellen LEO-Studie (Geburtsjahrgänge 1945-1992) und PIAAC (Geburtsjahrgänge 1947-1996) Daten von Erwachsenen bis zu einem Alter von 64 Jahren (LEO) bzw. 65 Jahren (PIAAC) vor.

Um die altersbezogenen Verteilungen zu illustrieren, greift dieser Beitrag auf das Konzept der Generationen zurück. Generation wird hier im Sinne einer historisch-gesellschaftlichen Generation verstanden, die nah beieinanderliegende Geburtsjahrgänge bei einer gleichzeitigen Verortung in einem sozialen 
Raum vereint (Rump/Eilers 2013). Maßgeblich bei der Wahl des Generationenkonzepts ist die Debatte um eine Aufteilung in Personengruppen, die vor der Digitalisierung und nach der Digitalisierung geboren wurden. Letztere werden als „Digital Natives“ oder „Generation Y“ bezeichnet. Pietraß setzt sich kritisch mit dem Generationenansatz bezogen auf den Begriff der „Digital Natives“ (Prensky 2001) auseinander, weist aber dennoch auf die grundsätzliche Eignung des Ansatzes für bildungswissenschaftliche Fragen hin (Pietraß 2013). Die Einteilung in Generationen erfolgte auf Basis der Klassifizierung von Hurrelmann und Albrecht (2014) sowie Scholz (2014). Bei der Einteilung in die Generationen der 68er (bis Geburtsjahr 1949), der Babyboomer (19501964), der Generation X (1965-1979) und der Generation Y (1980-2000) lässt sich der Begriff der Digital Natives auf die jüngste dieser Gruppen, die Generation $\mathrm{Y}$ beziehen.

\section{Literalität}

Lese- und Schreibkompetenz wird im Kontext von Assessment-Studien auch als Literalität (Literacy) bezeichnet. In diesem Zusammenhang dominiert ein gegenwärtig vor allem durch die OECD geprägtes Verständnis von Literalität als einer messbaren individuellen Kompetenz. Demnach lässt sich Literalität präzise anhand definierter Benchmarks bestimmen, die wiederum auf einer Übereinkunft bestimmter als notwendig erachteter Minimalanforderungen an die Lese- und Schreibkompetenz basieren. Eine Gegenposition zu dieser Lesart von Literalität wird von Autor/inn/en in der Tradition der so genannten New Literacy Studies vertreten. Aus dieser Perspektive ist Literalität als soziale Praxis zu verstehen, die sich interpersonell, regional und sozial unterscheidet und sich somit der Möglichkeit der Messbarkeit prinzipiell entzieht (Hamilton et al. 2015).

Das Konzept von Literalität, das diesem Beitrag zugrunde liegt, bedient sich bei beiden Positionen. Die Verwendung von Assessmentdaten impliziert die Bereitschaft, die Messbarkeit von Literalität grundsätzlich anzuerkennen. Gleichzeitig greift die Analyse auf Variablen zur praktischen Kompetenznutzung (Skill Use) zurück und anerkennt so die Interpretation von Literalität als soziale Alltagspraxis. Der Skill-Use-Ansatz hat in den letzten Jahren an Bedeutung gewonnen. In der PIAAC-Studie wurden Alltagspraktiken bezogen auf Literalität, Numeralität und digitale Problemlösekompetenz in ihrer Häufigkeit erfasst (Reder 2017). 


\section{Datengrundlage}

Die Beantwortung der Forschungsfragen erfolgt durch die Sekundäranalyse der Datensätze der PIAAC-Studie und der LEO - Level-One Studie bezogen auf die IKT-Nutzung in Abhängigkeit von literalen Kompetenzstufen und vom Alter.

Verwendete Variablen aus dem PIAAC-Datensatz (Scientific Use File, $\mathrm{N}=5.400$ ) sind die Literalitätsscores, die Altersvariablen sowie Variablen über die Verwendung von IKT in der Freizeit. Variablen zur Nutzung von Computern und verwandten Praktiken liegen für einen Teildatensatz der LEO-Studie vor $(\mathrm{N}=1.401)$. Er umfasst Personen, deren höchster Schulabschluss ein Hauptschul-, Volksschul- oder vergleichbarer Abschluss ist. Diese Teilstichprobe des LEO-Datensatzes ist demnach nicht bevölkerungsrepräsentativ, sondern fokussiert auf den unter Bildungsbereich. Auch bei den LEO-Daten greift die Auswertung auf die Literalitätsscores und die Altersvariablen zurück.

In LEO wurde die Kompetenznutzung dichotom erfasst, es wurde also erfragt, ob eine Person eine bestimmte Aktivität ,schon einmal durchgeführt“ hat oder nicht. In PIAAC wurde die Kompetenznutzung differenzierter in einer Häufigkeitsabstufung erfasst (Reder 2017). Die PIAAC-Variablen wurden zur unmittelbaren Vergleichbarkeit ebenfalls dichotomisiert.

Die sechsstufige Kompetenzstufung in PIAAC ist zu einer dreistufigen Einordnung komprimiert (Level 1 und darunter; Level 2 und 3; Level 4 und darüber). Die Alpha-Level aus LEO sind ebenfalls auf drei Stufen komprimiert (Alpha-Level 1 bis 3; Alpha-Level 4; Alpha-Level 5 und darüber) (zur Systematik der Alpha-Levels vgl. Grotlüschen et al. 2012).

Unter Verwendung der jeweiligen Altersvariablen der Studien wurden die Generationen gebildet. In einem weiteren Schritt wurden die Generationengruppen (Babyboomer usw.) nach den jeweiligen Kompetenzabstufungen (PIAAC-Level bzw. LEO-Alpha-Level) unterteilt.

\section{Ergebnisse}

\section{Computernutzung}

Die PIAAC-Studie zeigt bei der Frage nach Computernutzung im Allgemeinen eine weite Verbreitung über die Generationen hinweg. Im Bevölkerungsdurchschnitt nutzen rund 92 Prozent einen Computer in der Freizeit. Die jüngeren 
Generationen nutzen den Computer mehr als die Älteren (Generation Y: 98\%; Generation X: 93\%; Babyboomer: 87\%; 68er-Generation: 81\%). Die Computernutzung steht zudem in einem Zusammenhang mit der literalen Kompetenz (PIAAC Level 4/5: 99\%; PIAAC-Level 2/3: 93\%; PIAAC-Level 1 und darunter: $83 \%$ ).

Die LEO-Teilstichprobe repräsentiert den unteren Bildungsbereich. Die Computernutzung steht auch hier in Zusammenhang mit Alter und Literalität. Die Unterschiede zwischen den Generationen und zwischen den Kompetenzstufen sind deutlicher ausgeprägt als bei PIAAC. Insgesamt nutzen rund 70 Prozent der Stichprobe einen Computer bei der Arbeit oder in der Freizeit. Innerhalb der Generation Y nutzen 85 Prozent einen Computer (Generation X: 78\%; Babyboomer: 62\%; 68er-Generation: 47\%). Personen mit Lese- und Schreibkompetenzen auf Alpha-Level 5 und darüber nutzen zu 79 Prozent einen Computer (Alpha-Level 4: 72\%; Alpha-Level 1-3: 55\%). Hinsichtlich beider Faktoren (Alter und Literalität) sind die Unterschiede bei der Computernutzung statistisch signifikant, $\mathrm{r}(1376)=-.273, \mathrm{p}<.01$ (Alter) und $\mathrm{r}(1376)=.191$, $\mathrm{p}<.01$ (Literalität).

Bei gleichzeitiger Betrachtung von Alter und Literalität zeigen beide Studien in Bezug auf die allgemeine Nutzung eines Computers einen ähnlichen Zusammenhang: Die Nutzung nimmt mit dem Alter ab und innerhalb der Generationen ${ }^{1}$ nutzen höher Literalisierte eher einen Computer als gering Literalisierte. Der Unterschied in der Computernutzung entlang der Lese- und Schreibkompetenz steigt mit jedem Schritt zur nächstälteren Generation. Innerhalb der Generation Y beträgt der Unterschied zwischen höher Literalisierten und gering Literalisierten 6 Prozentpunkte. In der Generation X beträgt die Differenz 28 Prozentpunkte, in der Gruppe der Babyboomer 26 Prozentprozentpunkte. Je älter jemand ist, so wäre eine erste Schlussfolgerung, desto stärker wirkt sich der Literalitätslevel auf die Computernutzung aus. Je jünger jemand ist, umso mehr schwindet der Einfluss der Literalität.

Für verschiedene Arten der IKT-Nutzung können verschiedene Schemata erstellt werden, die Unterschiede in der Nutzung von IKT-Anwendungen in Abhängigkeit vom Alter sowie in Abhängigkeit von literalen Kompetenzen darlegen.

1 Aufgrund sehr geringer Fallzahl werden im Folgenden keine separaten Ergebnisse für die 68er-Generation ausgewiesen. 
Schema 1: Abstufung zwischen den Generationen und zwischen den literalen Kompetenzstufen

Beschreibung: Jüngere nutzen die entsprechenden Anwendungen in einem größeren Ausmaß als Ältere. Höher literalisierte Erwachsene nutzen die IKT-Anwendungen in größerem Ausmaß als gering Literalisierte. Beispiel: Nutzung von Textverarbeitung (PIAAC) ${ }^{2}$.

Bevölkerungsweit nutzen rund 83 Prozent derjenigen, die in ihrer Freizeit einen Computer nutzen, dabei ein Textverarbeitungsprogramm. Mit Blick auf die Generationen zeigt sich, dass mit steigendem Alter die Nutzung von Textverarbeitungsprogrammen, also eine explizit schriftliche Praktik, abnimmt. Die Generation Y nutzt zu fast 90 Prozent Textverarbeitungsprogramme (Generation X: 83\%; Babyboomer: 79\%). Innerhalb der Generationen kommt es zu einer Abstufung bei der Nutzung in Abhängigkeit von den literalen Kompetenzstufen. So nutzen zwischen 94 Prozent und 98 Prozent der Personen auf den PIAAC Kompetenzstufen 4 und darüber Textverarbeitungsprogramme (Generation Y: 98\%, Generation X: 96\%, Babyboomer: 94\%). Für Personen mit einer mittleren Lesekompetenz (PIAAC Kompetenzstufen 2 und 3) sinken die Werte um 7 bis 11 Prozentpunkte im Vergleich zu den höher Literalisierten (Generation Y: 91\%; Generation X: 85\%; Babyboomer: 83\%). Einen weiteren Rückgang in der Anzahl der Nutzerinnen und Nutzer zeigt sich für gering Literalisierte. Die Generationen der Babyboomer und der Generation X weisen Werte von 54 Prozent bzw. 53 Prozent auf. Bei den gering Literalisierten der Generation Y liegt die Anzahl derjenigen, die bereits ein Textverarbeitungsprogramm genutzt haben, bei rund 67 Prozent.

Schema 2: Abstufung zwischen den Generationen, aber keine eindeutige Tendenz zwischen den literalen Kompetenzstufen

Beschreibung: Ältere nutzen die IKT-Anwendungen weniger als Jüngere. Ein eindeutiger Trend für die Nutzung in Abhängigkeit vom Literalitätslevel für alle Generationen ist nicht auszumachen. Beispiel: Echtzeitdiskussionen im Internet, z.B. Onlinekonferenzen und Chatgruppen (PIAAC) $)^{3}$.

Eine ungefähre Analogie zur Nutzung sozialer Netzwerke ist die Frage danach, ob Befragte ,an Echtzeitdiskussionen im Internet [...], z.B. an Onlinekonfe-

2 Diesem Schema folgen weitere digitale Praktiken: Nutzen eines Tabellenkalkulationsprogramms; Nutzen eines Textverarbeitungsprogramms (alle aus PIAAC), Dateien kopieren/verschieben; Textteile in einem Dokument kopieren / verschieben; Geräte an Computer anschließen und installieren; mit einem Tabellenkalkulationsprogramm rechnen (alle aus LEO).

3 Diesem Schema folgen weitere digitale Praktiken: Chatrooms, Foren oder Newsgroups besuchen; Computerspiele (beide aus LEO). 
renzen oder Chatgruppen“ (Wortlaut des Fragentextes) teilnehmen. Im Bevölkerungsdurchschnitt nutzt jede dritte Person (35\%) diese Art interaktiver Kommunikation. In der Betrachtung der Generationen lassen sich deutliche Abstufungen erkennen. Für die Generation Y liegt der Anteil der Nutzenden mit 64 Prozent fast doppelt so hoch wie im Bevölkerungsdurchschnitt (Generation X: 30\%; Babyboomer: 13\%). Dagegen ist kein eindeutiger Trend für den Zusammenhang zwischen digitaler Praktik und Literalität festzustellen. Innerhalb der Generation X haben 32 Prozent der höher literalisierten Personen schon einmal auf diese Art kommuniziert (gering Literalisierte: 29\%). Die Generation Y differenziert etwas deutlicher entlang der Literalitätslevel. Hoch literalisierte Personen nutzen zu 71 Prozent, Personen mit Kompetenzen auf den Stufen 2 und 3 zu 64 Prozent und gering literalisierte Personen zu 55 Prozent entsprechende Technik.

Schema 3: Abstufung zwischen den literalen Kompetenzstufen, aber keine oder geringfügige Abstufung zwischen den Generationen

Beschreibung: Zwischen den Generationen ist keine (oder nur geringe) unterschiedliche Nutzung von IKT-Anwendungen festzustellen. Von höher Literalisierten nutzen mehr Personen die entsprechenden IKT-Anwendungen als von gering Literalisierten. Beispiele: E-Mail (PIAAC) ${ }^{4}$.

Bei der Nutzung von E-Mail gibt es insgesamt nur einen geringen altersbedingten Unterschied. Unabhängig von ihrem Literalitätslevel nutzen 97 Prozent der Generation Y E-Mail als Kommunikationsmittel, innerhalb der Generation X beträgt der Anteil 94 Prozent und innerhalb der Gruppe der Babyboomer 91 Prozent. Bei der Nutzung von E-Mail schlägt sich hingegen die Lesekompetenz deutlicher nieder als das Alter. Die höher Literalisierten der Generation Y nutzen ausnahmslos E-Mail als Kommunikationsmittel, die gering Literalisierten dieser Generation zu 93 Prozent. In der Generation X reicht die Spanne von 99 Prozent (hohe Literalität) bis 79 Prozent (geringe Literalität) und bei den Babyboomern von 97 Prozent (hohe Literalität) bis 79 Prozent (geringe Literalität).

Schema 4: Keine oder geringfügige Abstufung zwischen den Generationen sowie zwischen den literalen Kompetenzstufen

Beschreibung: Unterschiede in der Anwendung von IKT sind weder in Abhängigkeit vom Alter noch in Abhängigkeit von den literalen Kompetenzen festzustellen. Beispiel: Nutzung von Suchmaschinen (LEO) ${ }^{5}$.

4 Diesem Schema folgen weitere digitale Praktiken: E-Mails mit Anhang senden/empfangen; E-Mails oder SMS versenden (beide aus LEO); Transaktionen im Internet durchführen (aus PIAAC).

5 Diesem Schema folgt ebenfalls: Nutzen des Internets, um Sachverhalte oder Themen besser zu verstehen (aus PIAAC). 
Die Nutzung von Suchmaschinen erwies sich bereits 2010 bzw. 2012 (Zeitpunkte der Datenehebungen von PIAAC und LEO) als eine digitale Praktik, die weder hinsichtlich des Alters noch hinsichtlich der Literalität größere Unterschiede erkennen lässt. Im Durchschnitt der Teilstichprobe der LEO-Studie aus dem unteren Bildungssegment nutzten rund 98 Prozent schon einmal Suchmaschinen. Die einzelnen Generationen liegen in etwa auf der Höhe dieses Durchschnittswerts (Generation Y: 98\%; Generation X: 99\%; Babyboomer 97\%). Bezogen auf das Literalitätslevel bewegen sich die Unterschiede ebenfalls im Bereich weniger Prozentpunkte.

\section{Ausblick}

Chancen auf Entgrenzung durch die Digitalisierung für bestimmte Bevölkerungsgruppen führen auch das Risiko des Ausschlusses anderer Gruppen mit sich. Das gilt, wie gezeigt werden konnte, allgemein für ältere Personen und für Personen, die Schwierigkeiten beim Lesen und Schreiben haben. Grundsätzlich bestätigt die Analyse von PIAAC- und LEO-Daten bezogen auf die allgemeine Computernutzung den Zusammenhang zwischen Alter und Literalität einerseits und der Nutzung digitaler Technologien andererseits. Allerdings ändern sich die Zusammenhänge bei der Betrachtung von Einzelvariablen. Dies ist als ein Hinweis darauf zu lesen, dass zusammengefasste Indizes oder sehr allgemein formulierte Variablen zur Häufigkeit von Alltagspraktiken zwar plausible allgemeine Trends ermöglichen, spezifische Abweichungen vom generellen Trend bezogen auf einzelne Praktiken hingegen verdecken.

Der Blick auf die Nutzung spezifischer Kompetenzen erscheint im gewählten Kontext Digitalisierung, der erheblichen raschen Veränderungen unterliegt, besonders geboten. So wurde deutlich, dass in einigen Aspekten der grundsätzlich gültige limitierende Einfluss geringer Literalität auf die jeweilige Art der Kompetenznutzung weitgehend aufgehoben ist. Dies betrifft mit der Nutzung sozialer Netzwerke (PIAAC-Variable zu Echtzeitdiskussionen, Onlinekonferenzen, Chatgruppen), die sich gegenüber der reinen Computernutzung durch E-Mail oder Textverarbeitung als fortgeschrittene interaktive Praktiken beschreiben lässt. Die hier verwendeten Variablen haben innerhalb weniger Jahre einen Prozess der Veralterung durchlaufen. Im Rahmen der LEO-Grundbildungsstudie (im Feld 2018, Ergebnisse Mitte 2019) werden auch neuere Formen von IKT-Nutzung in Zusammenhang mit Literalität und anderen persönlichen Faktoren untersucht.

Der beschriebene verschwindende Einfluss der Literalität gegenüber dem Alter bei einigen Praktiken lässt die Vermutung zu, dass gering literalisierte Erwachsene keineswegs grundsätzlich von digitaler Praxis ausgeschlossen 
sind. Das gilt besonders, wenn sie einer der jüngeren Kohorten angehören. So bestätigt eine Studie zur „Netzgesellschaft“, dass Personen mit niedrigem Schulabschluss (Volks- und Hauptschulabschluss) den geringsten Anteil von Computern am Arbeitsplatz hatten. Beim Besitz von Mobiltelefonen hingegen war der Unterschied zwischen den formal niedrig Gebildeten und höher Gebildeten weniger groß (Bitkom Research 2011).

Digital literacy (verstanden als umfassende Kompetenzen in diesem Bereich) lässt sich auf Basis der vorgestellten Ergebnisse als eine soziale Praxis interpretieren, die in einigen - wenn auch nicht in allen - Facetten weitgehend unabhängig vom gemessenen Kompetenzniveau der Literalität (im Sinne der Lese- und Schreibkompetenz) ist. Die Untersuchung schriftbezogener oder digitaler Praktiken ist unverzichtbar, um ein umfassendes Bild der Grundkompetenzen in einer Gesellschaft zu zeichnen. Die ausschließliche Analyse von Kompetenzen unterstützt die Annahme, dass größere Subpopulationen von erheblichem Teilhabeausschluss bedroht sind. Der Blick auf Alltagspraktiken zeigt hingegen, dass trotz geringer Kompetenzen die alltägliche Anwendung dieser Praktiken in sozialen Kontexten durchaus üblich und verbreitet ist (vgl. Reder 2017). Im vorliegenden Beitrag wurde dies auf Basis einer vereinfachten dichotomisierten Betrachtungsweise illustriert, die sich aufgrund der Vergleichbarkeit der beiden Hauptdatenquellen anbot. Eine differenziertere Betrachtungsweise auf Basis von Häufigkeitsabstufungen dürfte den Erklärungswert in weiterer Forschung noch erhöhen.

Von Interesse wird sein, wie sich die demografische Entwicklung in diesem spezifischen digitalen Kontext auswirken wird. Grundsätzlich wird der Anteil der Digital Natives innerhalb der Gesellschaft immer größer. Aber wird damit die Altersschere bei der Nutzung sich weiterhin verändernder digitaler Technologien ebenfalls abnehmen? Weitere Forschung und Beobachtung wird mittelfristig zeigen, ob sich neue digitale Spaltung entlang der Altersachse ergeben wird.

\section{Literatur}

Bitkom Research (2011): Netzgesellschaft. Eine repräsentative Untersuchung zur Mediennutzung und dem Informationsverhalten der Gesellschaft in Deutschland. Online: www.bitkom.org/noindex/Publikationen/2011/Studie/StudieNetzgesellschaft/BITKOM-Publikation-Netzgesellschaft.pdf, zuletzt geprüft am 31.07.2019.

Bitkom Research (Hg.) (2018): Social Media Social Messaging. Berlin. Online: www.bitkom-research.de/epages/63742557.sf/de_DE/?ObjectPath=/Shops/63 742557/Products/SM2018, zuletzt geprüft am 31.07.2019. 
Bundesministerium für Bildung und Forschung; Kultusministerkonferenz (2016): Grundsatzpapier zur Nationalen Dekade für Alphabetisierung und Grundbildung 2016-2026. Den funktionalen Analphabetismus in Deutschland verringern und das Grundbildungsniveau erhöhen. Online: www.alphadekade.de/files/01_Grundsatzpapier\%20zur\%20Nationalen\%20Dekade\%20Alphabetisierung\%20und\%20Grundbildung_final.pdf, zuletzt geprüft am 31.07.2019.

Bundesregierung (2018): Ein neuer Aufbruch für Europa. Eine neue Dynamik für Deutschland. Ein neuer Zusammenhalt für unser Land. Koalitionsvertrag zwischen CDU, CSU und SPD. 19. Legislaturperiode. Online: www.bundesregierung.de/resource/blob/656734/847984/5b8bc23590d4cb2892b31c987ad672b 7/2018-03-14-koalitionsvertrag-data.pdf?download=1, zuletzt geprüft am 31. 07.2019.

Bundesverband deutscher Banken (2017): Online-Banking in Deutschland. Online: https://bankenverband.de/media/files/Umfrageergebnis_LeTZrCy.pdf, zuletzt geprüft am 31.07.2019.

Carstensen, Tanja (2016): Ambivalenzen digitaler Kommunikation am Arbeitsplatz. In: Aus Politik und Zeitgeschichte 66 (18-19), S. 39-46.

Euringer, Caroline (2016): Das Grundbildungsverständnis der öffentlichen Bildungsverwaltung. Definitionen, Interessen und Machtverhältnisse. Bielefeld: W. Bertelsmann Verlag.

Europäische Kommission (2017): Schlüsselkompetenzen für lebensbegleitendes Lernen. Amt für amtliche Veröffentlichungen der Europäischen Gemeinschaften. Luxemburg.

Grotlüschen, Anke (2016): Grundbildung von Erwachsenen. In: Rudolf Tippelt; Bernhard Schmidt-Hertha (Hg.): Handbuch Bildungsforschung. Heidelberg: Springer VS.

Grotlüschen, Anke; Riekmann, Wibke (Hg.) (2012): Funktionaler Analphabetismus in Deutschland. Ergebnisse der ersten leo. - Level-One Studie. Münster [u.a.]: Waxmann.

Grotlüschen, Anke; Riekmann, Wibke; Buddeberg, Klaus (2012): Hauptergebnisse der leo. - Level-One Studie. In: Anke Grotlüschen; Wibke Riekmann (Hg.): Funktionaler Analphabetismus in Deutschland. Ergebnisse der ersten leo. - Level-One Studie. Münster: Waxmann, S. 13-53.

Hamilton, Mary; Maddox, Bryan; Addey, Camilla (Hg.) (2015): Literacy as numbers. Cambridge: Cambridge University Press.

Hurrelmann, Klaus; Albrecht, Erik (2014): Die heimlichen Revolutionäre. Wie die Generation Y unsere Welt verändert. Weinheim: Beltz.

Iller, Carola (2018): Höheres Erwachsenenalter und Bildung. In: Rudolf Tippelt; Bernhard Schmidt-Hertha (Hg.): Handbuch Bildungsforschung. Wiesbaden: Springer VS, S. 845-860.

Koch, Wolfgang; Frees, Beate (2017): ARD/ZDF-Onlinestudie 2017: Neun von zehn Deutschen online. In: Media Perspektiven (9), S. 434-446. Online: www.ard-zdf-onlinestudie.de/files/2017/Artikel/917_Koch_Frees.pdf, zuletzt geprüft am 31.07.2019. 
Kultusministerkonferenz (2016): Bildung in der digitalen Welt. Beschluss der Kultusministerkonferenz vom 08.12.2016. Online: www.kmk.org/fileadmin/Dateien/veroeffentlichungen_beschluesse/2016/2016_12_08-Bildung-in-der-digitalen-Welt.pdf, zuletzt geprüft am 31.07.2019.

OECD (2013): OECD Skills Outlook 2013. First Results from the Survey of Adult Skills. Paris: OECD Publishing. Online: www.oecd.org/skills/piaac/Skills\%20 volume \%201\%20(eng)--full\%20v12--eBook\%20(04\%2011\%202013).pdf, zuletzt geprüft am 31.07.2019.

Pietraß, Manuela (2013): Medienwandel und Generationenunterschiede. Zur Haltbarkeit der These von den „Digital Natives“. In: Ulrike Wagner (Hg.): Familienleben: Entgrenzt und vernetzt? München: kopaed (Interdisziplinäre Dikurse, 7), S. 77-86.

Prensky, Marc (2001): Digital Natives, Digital Immigrants Part 1. In: On the Horizon 9 (5), S. 1-6. DOI: 10.1108/10748120110424816.

Rammstedt, Beatrice; Ackermann, Daniela (Hg.) (2013): Grundlegende Kompetenzen Erwachsener im internationalen Vergleich. Ergebnisse von PIAAC 2012. Münster: Waxmann.

Reder, Stephen (2017): Adults' Engagement in Reading, Writing and Numeracy Practices. Portland State University. Portland (Applied Linguistics Faculty Publications and Presentations, 22). Online: https://pdxscholar.library.pdx.edu/cgi/ viewcontent.cgi? article $=1022 \&$ context $=$ ling_fac, zuletzt geprüft am 31.07. 2019.

Rosenbladt, Bernhard von; Bilger, Frauke (2011): Erwachsene in Alphabetisierungskursen der Volkshochschulen. Ergebnisse einer repräsentativen Befragung (AlphaPanel). Bonn: DVV.

Rump, Jutta; Eilers, Silke (2013): Die jüngere Generation in einer alternden Arbeitswelt. Sternenfels: Verlag Wissenschaft \& Praxis.

Schmidt-Hertha, Bernhard (2014): Kompetenzen und Kompetenzförderung bei gering Qualifizierten über die gesamte Lebensspanne. In: Projektträger im Deutschen Zentrum für Luft- und Raumfahrt e.V. (Hg.): Kompetenzen von gering Qualifizierten. Bielefeld: wbv, S. 205-221.

Scholz, Christian (2014): Generation Z. Wie sie tickt, was sie verändert und warum sie uns alle ansteckt. Weinheim: Wiley-VCH Verlag.

Smythe, Suzanne (2018): Adult Learning in the Control Society: Digital Era Governance, Literacies of Control, and the Work of Adult Educators. In: Adult Education Quarterly 21 (1). DOI: 10.1177/0741713618766645.

Stammer, Christopher (2017): Das Verhalten junger Erwachsener mit unterschiedlichen literalen Kompetenzen bei der Nutzung von Computer und Internet. Masterarbeit. Universität Hamburg. Fakultät für Erziehungswissenschaft.

Wolf, Karsten D.; Koppel, Ilka (2017): Digitale Grundbildung: Ziel oder Methode einer chancengleichen Teilhabe in einer mediatisierten Gesellschaft? In: erwachsenenbildung.at (30), 1-11. 


\section{Angaben zu den Autorlnnen}

Asche, Eike, M.A., Institut für Berufspädagogik und Erwachsenenbildung (IfBE) der Leibniz Universität Hannover, Bedarfsforschung, Bedarfsanalyse in der beruflichen Weiterbildung, Qualifizierung für die digitalisierte Arbeitswelt

asche@mitunsdigital.de

Bauer, Stefanie, Dr. phil., an der Otto-Friedrich-Universität Bamberg - Professur für Erwachsenenbildung und Weiterbildung, Arbeitsschwerpunkte: Didaktisches Handeln, Biografische und transformatorische Lernprozesse, Zielgruppenforschung stefanie.bauer@uni-bamberg.de

Baust, Christina, M. A., Universität Tübingen, Arbeitsschwerpunkt: Professionalisierung von Lehrenden in Schule und Erwachsenenbildung christina.baust@uni-tuebingen.de

Behr, Frank, Dr., Universität Koblenz Landau, Elternberatung, Lehrerbildung und Lehrerexpertise, habituelle Bedingungen von Lern- und Bildungsprozessen, digitale Bildung und E-Learning behr@uni-landau.de

Bolten, Ricarda, Dipl.-Pädagogin, Digitalisierung, Medialer Habitus, Professionalisierung, Medienpädagogische Kompetenz Lehrender in der Erwachsenenbildung

bolten@uni-bremen.de

Buddeberg, Klaus, Dr., Universität Hamburg, Literalitätsforschung, Numeralitätsforschung, Large-Scale-Assessment, Einfache Sprache, Inklusionsforschung

klaus.buddeberg@uni-hamburg.de

Denker, Torsten, Dipl.-Päd., Kreisvolkshochschule Landkreis Gießen. Arbeitsschwerpunkte: Management von Weiterbildungseinrichtungen, Qualitätsmanagement, Weiterbildung im ländlichen Raum, Gesellschaftlich-politische Bildung torsten.denker@lkgi.de 
Denninger, Anika, M.A., Wissenschaftliche Mitarbeiterin an der Professur für Weiterbildung, Institut für Erziehungswissenschaft, Justus-Liebig-Universität Gießen. Arbeitsschwerpunkte: wissenschaftliche Weiterbildung, betriebliche Weiterbildung, Bedarfsforschung, Weiterbildungsteilnahme, (Lern-)Zeitbudgets

anika.denninger@erziehung.uni-giessen.de

Dinkelaker, Jörg, Martin-Luther-Universität Halle-Wittenberg, Arbeitsschwerpunkte: Formen des Lernens Erwachsener, Empirie der Erwachsenenbildung/Weiterbildung, Umgang mit Wissen, Interaktion und Lebenslauf, Rekonstruktive Forschung.

joerg.dinkelaker@paedagogik.uni-halle.de

Fleige, Marion Dr., Deutsches Institut für Erwachsenenbildung, Leibniz-Zentrum für Lebenslanges Lernen e. V. Arbeitsschwerpunkte: Forschung zu Programmen/Programmplanung, Lernkulturen und Institutionalformen in der Erwachsenen- und Weiterbildung; vertieft zur Kulturellen und Beruflichen Bildung

fleige@die-bonn.de

Freide, Stephanie M.A., Deutsches Institut für Erwachsenenbildung, LeibnizZentrum für Lebenslanges Lernen e. V. Arbeitsschwerpunkte: Forschung zu Programmen/ Programmplanung; vertieft zur Kulturellen (Erwachsenen-)Bildung

Freide@die-bonn.de

Fritsch, Lisa, Marie, M.A. Universität Hohenheim, Lernen mit digitalen Medien, Didaktik, Professionalisierung von Lehrenden sa.fritsch@uni-hohenheim.de

Heidelmann, Marc-André, Philipps-Universität Marburg, Institut für Erziehungswissenschaft, Organisationspädagogik, Organisations- und Netzwerkberatung, Professionalisierungsforschung, Lern- und Bildungsprozesse marc-andre.heidelmann@staff.uni-marburg.de

Heilmann, Lisanne, M.A., Universität Hamburg, Fachbereich Lebenslanges Lernen, Arbeitsschwerpunkte: Grundbildung Erwachsener, Literacy, Numeracy, Gesundheitskompetenzen, Migration und Sprache lisanne.heilmann@uni-hamburg.de

Heinrich, Eva, M.A., Graduiertenkolleg Doing Transitions, Organisationales Lernen, Personalentwicklung, Übergangsforschung heinrich.eva@gmx.de 
Hof, Christiane, Prof. Dr., für Erwachsenenbildung/Weiterbildung. Goethe Universität Frankfurt, aktuelle Arbeitsschwerpunkte: Theorie und Empirie Lebenslangen Lernens, Analyse von Übergängen im Lebenslauf, soziale Praktiken als Rahmen für Lernprozesse, Universität als Lernort hof@em.uni-frankfurt.de

Käpplinger, Bernd, Prof. Dr., Professur für Weiterbildung an der Justus-Liebig-Universität Gießen. Arbeitsschwerpunkte: Programmplanung, Bildungsberatung, Volkshochschule, Betriebliche Weiterbildung, Internationaler Vergleich.

Bernd.Kaepplinger@erziehung.uni-giessen.de

Klinge, Denise, Dr., Universität der Bundeswehr München, Arbeitsschwerpunkte: Bildungsforschung und digitale Technologie, dokumentarische Methode, Bildungsentscheidungen im Kontext von Übergangsforschung und Ungleichheit.

denise.klinge@unibw.de

Koscheck, Stefan, Bundesinstitut für Berufsbildung, Weiterbildungsanbieter, Weiterbildungspersonal, öffentliche Weiterbildungsförderung 'Koscheck@bibb.de'

Kühn, Christian M.A., Leibniz Universität Hannover, Institut für Berufspädagogik und Erwachsenenbildung; Arbeitsschwerpunkte: Methodologie der Empirischen Bildungsforschung, Angebots- und Programmplanung, Bildungsmanagement, Kulturelle Transformationen (insbesondere Digitalisierung)

christian.kuehn@ifbe.uni-hannover.de

Lorenz, Lisa, M.A., Institut für Berufspädagogik und Erwachsenenbildung (IfBE) der Leibniz Universität Hannover, Programm- und Angebotsplanung, Programmforschung, Berufliche Weiterbildung

lorenz@mitunsdigital.de

Ludwig, Joachim, Dr. phil. habil., Professor für Erwachsenenbildung/Weiterbildung und Medienpädagogik an der Universität Potsdam, Bildungsprozessforschung, ästhetische Erfahrung, professionelles pädagogisches Handeln, Beratung.

ludwig@uni-potsdam.de

Martin, Andreas, Dr., Deutsches Institut für Erwachsenenbildung, Wirkungen und Erträge Bildungspolitischer Interventionen, Regionale Disparitäten, Personal in der Weiterbildung martin@die-bonn.de 
Maßmann, Alexandra, Dr. phil., Bergische Universität Wuppertal, Arbeitschwerpunkte: Weiterbildung und Alternsprozesse, Motivation im Weiterbildungskontext, Berufliche Begabung.

massmann@uni-wuppertal.de

Meyer, Nikolaus, Prof. Dr., IUBH University of Applied Sciences, komparative Berufsgruppenforschung, Professionstheorie, Soziale Arbeit und Alter(n), Soziale Arbeit und Wohnungslosigkeit sowie „Erziehung nach Auschwitz“als Mandat pädagogischen Handelns.

ni.meyer@iubh-dualesstudium.de

Müller, Christian, M.A., wissenschaftlicher Mitarbeiter an der „Professur für Erwachsenenbildung, Schwerpunkte berufliche Weiterbildung und komparative Bildungsforschung“, mein Schwerpunkt: berufliche Weiterbildung christian.mueller13@tu-dresden.de

Niemeyer, Beatrix, Prof. Dr., Europa-Universität Flensburg, Institut für Erziehungswissenschaften, Arbeitsbereich Erwachsenenbildung, Arbeitsschwerpunkte: Europäische Bildungspolitik, Europäische Erwachsenenbildungsforschung; Übergänge zwischen Bildung und Erwerb über die Lebenszeit; subjektorientierte Mobilitätsforschung

niemeyer@uni-flensburg.de

Nittel, Dieter, Prof. Dr., Goethe-Universität Frankfurt am Main, Professionsund Organisationsforschung, Theorie und Empirie des lebenslangen Lernens, Biographieforschung, qualitative Bildungsforschung, pädagogische Gesundheitsforschung

Nittel@em.uni-frankfurt.de

Pachner, Anita, Dr., Universität Tübingen, Arbeitsschwerpunkte: Lehr-Lernforschung, Begleitung lebenslangen Lernens, Pädagogische Professionsforschung, Kompetenzforschung, Internationale Bildungszusammenarbeit anita.pachner@uni-tuebingen.de

Preuß, Jessica M.A., Leibniz Universität Hannover, Institut für Berufspädagogik und Erwachsenenbildung; Arbeitsschwerpunkte: Kulturelle Transformationen (insbesondere Digitalisierung), Angebots- und Programmplanung, Kulturelle Bildung jessica.preuss@ifbe.uni-hannover.de 
Reuter, Martin, M.A., Justus-Liebig-Universität Gießen, Struktur und Steuerung des Weiterbildungssystems, Qualitätsmanagement, päd. Organisationsforschung, Lehren und Lernen in der Erwachsenenbildung Martin.Reuter@erziehung.uni-giessen.de

Robak, Steffi Prof. Dr., Leibniz Universität Hannover, Lehrstuhl Bildung im Erwachsenenalter; Arbeitsschwerpunkte: Kulturelle/Transkulturelle Bildung, Bildungsmanagement und Professionalisierung in der EB/WB, Digitalisierung in der EB/WB, Lernkulturforschung in der Erwachsenenbildung und betrieblichen Weiterbildung/Unternehmen steffi.robak@ifbe.uni-hannover.de

Rohs, Matthias, Dr. phil., Professor für Erwachsenenbildung, Technische Universität Kaiserslautern, Arbeitsschwerpunkte: Digitalisierung, Professionalisierung, informelles Lernen

'Matthias.Rohs@sowi.uni-kl.de'

Schäfer-Hock, Christian, Dr. phil., Dresden, Arbeitsschwerpunkte: Medien, Journalismus, Bildung

christian.schaefer-hock@emailkontakt.net

Schmidt-Lauff, Sabine, Prof. Dr. habil., Helmut-Schmidt-Universität / Universität der Bundeswehr Hamburg, Professur für Weiterbildung und lebenslanges Lernen. Professionalisierung, Professionalität und Professionelles Sein in der Erwachsenenbildung; internationalvergleichende Forschung zum lebensbegleitenden Lernen; temporale und zeitbezogene Phänomene in der Erwachsenenbildung.

schmidt-lauff@hsu-hh.de

Schulze-Stocker, Franziska, Dr. phil., TU Dresden, Arbeitsschwerpunkte: Studienerfolgsforschung, Forschung zur Lehrerbildung

franziska.schulze-stocker@tu-dresden.de

Schwarz, Jörg, Dr.phil., Helmut-Schmidt-Universität / Universität der Bundeswehr Hamburg, Arbeitsschwerpunkte: Erwachsenenpädagogische Professionalität, Zeit in der Erwachsenenbildung, Organisationspädagogik, Karriere und fachlich-berufliche Sozialisation, Methoden der empirischen Sozialforschung joerg.schwarz@hsu-hh.de

Stammer, Christopher, M.A., Universität Hamburg, Literalitätsforschung, Digital Literacy, Workforce Literacy, Family Literacy, Large-Scale Assessment christopher.stammer@uni-hamburg.de 
Stanik, Tim, Dr. phil., Universität Tübingen, Bildungsberatung, Lehr-Lernforschung, Professionalisierung, Grundbildung, Qualitative Forschungsmethodik timstanik@gmx.de

Von Felden, Heide, Univ. Prof.in Dr., Johannes-Gutenberg-Universität-Mainz, Bildungsforschung, Biographieforschung, Übergänge-Forschung, Forschung zum Lebenslangen Lernen, Genderforschung.

heide.von.felden@uni-mainz.de

Wahl, Johannes, Dr. phil., Goethe-Universität Frankfurt am Main, Arbeitsschwerpunkte: Lernen Erwachsener im Zeitalter der Digitalität, komparative pädagogische Berufsgruppen- und Organisationsforschung, lebenslanges Lernen, Methoden der empirischen Sozialforschung

wahl@em.uni-frankfurt.de

Weber, Susanne Maria, Prof. Dr. phil. habil., Philipps-Universität Marburg, Institut für Erziehungswissenschaft, Pädagogische Organisationsforschung, Organisations- und Netzwerkberatung, Innovationslabore, Großgruppenverfahren, Partizipative Forschung

susanne.maria.weber@uni-marburg.de

Wienberg, Jana, Dr., Universität Hamburg, Arbeitsbereich Berufliche Bildung \& Lebenslanges Lernen, Resonanztheorie, Beschleunigung und zeitdiagnostische Themen, Bildung und Lernen im höheren Erwachsenenalter, Lernen in digitalen Umwelten, Berufliche und betriebliche Erwachsenenbildung/Weiterbildung jana.wienberg@uni-hamburg.de

Wittpoth, Jürgen, Dr., Prof.i.R., Bochum, Ruhr-Universität Bochum, Institut für Erziehungswissenschaft juergen.Wittpoth@ruhr-uni-bochum.de

Zick, Sebastian, Dipl.-Päd., Europa-Universität Flensburg, Erwachsenenbildung in EUropa, Bildungstheorie, Erziehungswissenschaftliche Mobilitätsforschung Sebastian.Zick@uni-flensburg.de 
O. Dörner, C. Iller, I. Schüßler, H. von Felden, S. Lerch (Hrsg.)

Erwachsenenbildung und Lernen in Zeiten von Globalisierung, Transformation und Entgrenzung

Schriftenreihe der Sektion Erwachsenenbildung der

Deutschen Gesellschaft für Erziehungswissenschaft (DGfE)

Ausgehend von Gesellschaftsanalysen zu Transformation, reflexiver Modernisierung und flüchtiger Moderne ist die Erwachsenenbildung gefordert, sich den gesellschaftlichen Veränderungs- und Wandlungsprozessen, Entgrenzungen und strukturellen Umbrüchen zu stellen. In welchem Verhältnis wird Erwachsenenbildung zu diesen Bedingungen gesehen? Inwieweit sind welche Veränderungen in Struktur und Praxis der Bildung und des Lernens von Erwachsenen zu beobachten? Der Band versammelt Beiträge, die sich Phänomenen von Globalisierung, Transformation und Entgrenzung und deren Auswirkungen auf die Erwachsenenbildung und insbesondere auf das Lernen von Erwachsenen widmen.

\section{Die HerausgeberInnen:}

Prof. Dr. Olaf Dörner, Professor für Erziehungswissenschaft, SP Wissenschaftliche Weiterbildung und Weiterbildungsforschung, Otto-von-Guericke-Universität Magdeburg Prof. Dr. Carola Iller, Professorin für Fort- und Weiterbildung,

Stiftung Universität Hildesheim

Prof. Dr. Ingeborg Schüßler, Professorin für Erwachsenenbildung und Bildungsmanagement, PH Ludwigsburg

Prof. Dr. Heide von Felden, Professorin für Erziehungswissenschaft mit dem Schwerpunkt Erwachsenenbildung, Johannes Gutenberg-Universität Mainz Prof. Dr. Sebastian Lerch, Juniorprofessor für Lebenslanges Lernen, Johannes Gutenberg-Universität Mainz 